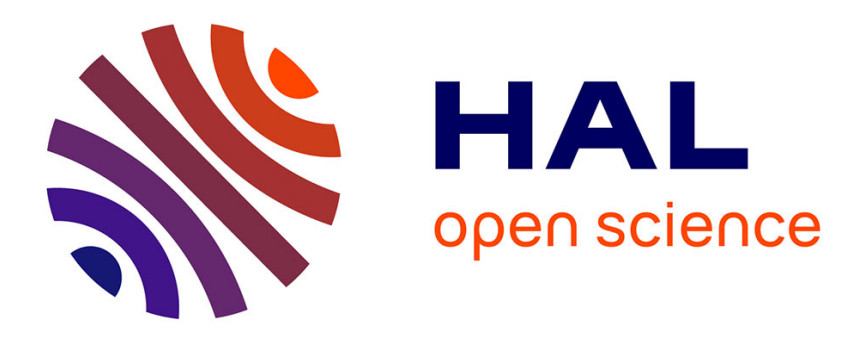

\title{
Etude expérimentale de la transition métal-isolant en dimension deux
}

\author{
Renaud Leturcq
}

\section{To cite this version:}

Renaud Leturcq. Etude expérimentale de la transition métal-isolant en dimension deux. Matière Condensée [cond-mat]. Université Paris Sud - Paris XI, 2002. Français. NNT : . tel-00002374

\section{HAL Id: tel-00002374 \\ https://theses.hal.science/tel-00002374}

Submitted on 10 Feb 2003

HAL is a multi-disciplinary open access archive for the deposit and dissemination of scientific research documents, whether they are published or not. The documents may come from teaching and research institutions in France or abroad, or from public or private research centers.
L'archive ouverte pluridisciplinaire HAL, est destinée au dépôt et à la diffusion de documents scientifiques de niveau recherche, publiés ou non, émanant des établissements d'enseignement et de recherche français ou étrangers, des laboratoires publics ou privés. 


\section{Étude expérimentale de la transition métal-isolant en dimension deux}

\section{THÈSE}

présentée et soutenue publiquement le 30 septembre 2002

pour l'obtention du

Doctorat de l'université Paris XI Orsay

(Physique des Solides)

par

Renaud LETURCQ

Composition du jury

$\begin{array}{lll}\text { Président : } & \text { Thierry Giamarchi } & \text { DPMC-Genève } \\ \text { Rapporteurs : } & \begin{array}{l}\text { Jean-Claude Portal } \\ \text { Marc Sanquer }\end{array} & \begin{array}{l}\text { INSA-Toulouse } \\ \text { CEA-Grenoble }\end{array} \\ \text { Examinateurs : } & \begin{array}{l}\text { Hélène Bouchiat } \\ \text { Dominique Mailly } \\ \text { Denis L'Hôte }\end{array} & \begin{array}{l}\text { CNRS-Orsay } \\ \text { CNRS-Marcoussy }\end{array} \\ & \text { CEA-Saclay, directeur de thèse }\end{array}$

Service de Physique de l'État Condensé — CEA Saclay — 91191 Gif-sur-Yvette
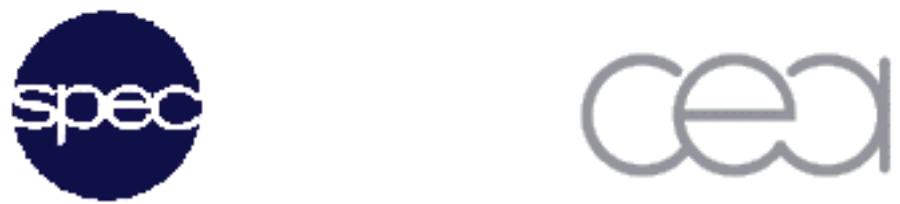
Mis en page avec la classe thloria. 


\section{Remerciements}

Cette thèse s'est déroulée au Service de Physique de l'État Condensé du CEA, à Saclay, et je voudrais tout d'abord remercier Jacques Hammann pour m'y avoir accueilli.

Mais pendant ces trois années, celui qui m'a aidé, soutenu, encouragé et motivé, enfin celui qui a été mon directeur de thèse, c'est Denis L'Hôte. Il m'a permis de découvrir un sujet passionnant de la physique de la matière condensée, il m'a appris tout ce que je sais actuellement sur les techniques de mesure et de cryogénie, et avec lui j'ai toujours eu l'impression de faire un travail intéressant.

Bien sûr, il n'est pas le seul responsable de la réussite de ma thèse, et je remercie toutes les personnes avec lesquelles j'ai pu collaborer :

Roland Tourbot qui n'était jamais loin pour résoudre toutes sortes de problèmes techniques, et sans lequel je n'aurais jamais eu autant de facilité et autant de plaisir à faire mes mesures. C'est malheureusement en quittant le laboratoire que je me rend compte de la véritable importance d'un tel soutient.

Houman Falakshahi et Claire Kikuchi, pour leur bref passage dans le groupe. Gérard Deville qui a rejoint le groupe à la fin de ma thèse, et avec lequel j'aurais eu vraiment plaisir à collaborer plus longtemps.

Les chercheurs de l'ETH Zürich et de PSI Villigen avec lesquels j'ai pu collaborer, de près ou de loin. Il y a eu en particulier Ulf Gennser, qui a servi d'intermédiaire entre la Suisse et la France, et Volkmar Senz qui est même venu me rendre visite, puis qui m'a fait découvrir la Salsa Zürichoise. Ensuite il y a eu la collaboration avec les chercheurs de l'Université de Nottingham, dont Christopher Mellor a été le représentant à Saclay.

Dominique Mailly qui m'a fait découvrir le travail en salle blanche et les bars les mieux famés de Prague.

Je remercie les membres du jury, et en particulier Jean-Claude Portal et Marc Sanquer qui ont accepté de rapporter mon manuscrit malgré sa longueur, mais aussi Hélène Bouchiat, Thierry Giamarchi et Dominique Mailly pour l'attention qu'ils ont porté à mon travail.

Puis je remercie tous les résidents du SPEC, et plus particulièrement parmi eux : Franck, tout d'abord, qui tient une place particulière car il m'a recueilli dans son bureau alors que je n'avais aucun endroit où me réfugier. C'est lui qui m'a soutenu dans les moments les plus durs, lui qui m'a démoralisé quand je débordais d'optimisme, enfin c'est lui qui me montrera la voie à suivre pour gagner plein d'argent dans la banque... à moins que ce ne soit moi qui lui montre comment gagner de l'argent dans la recherche.

Valentin pour nos déambulations à Prague et la visite des bars les moins bien famés.

Fabien qui a bien voulu attendre, pour finir sa thèse, que je termine la mienne... ce qui est une véritable preuve d'amitié.

Houman, dont j'ai déjà parlé, et qui a préféré se ranger dans le camp des théoriciens. Je n'ai malheureusement pu profiter de ses connaissances baristiques qu'en fin de rédaction de thèse.

Laure-Hélène pour nos descentes des hauteurs de Chamonix, car nous étions trop paresseux pour faire la montée à pieds.

Patrice et ses blagues, pas toujours drôles, mais dites avec tellement de cœur que j'en avais souvent les larmes aux yeux. 
Xavier qui me faisait croire qu'il était un grand sportif quand nous allions courir, mais qui avais du mal à cacher son bide de buveur de bière, et qui ne me fera jamais croire qu'il a fait le Mont-Blanc.

Je remercie les résidents de la salle café du troisième, sans lesquels une journée ne pouvait pas bien commencer. On pouvait y trouver les théoriciens, et parmi eux j'ai une pensée toute particulière pour Julien, sa guitare et son accent du nord. Les théoriciennes aussi... merci à Hugues pour avoir apporté cette touche d'originalité dans le laboratoire. Dans cette salle café, il y avait ceux que l'on était sûr d'y trouver, et ceux qui n'y ont passé que quelques mois, mais dont le souvenir reste souvent impérissable.

Je remercie tous mes amis, ma famille, et particulièrement ceux qui ont réussi à se libérer pour suivre ma soutenance (et ils étaient nombreux!). Aussi ceux qui n'ont pas pu venir, mais qui m'ont encouragé de près ou de loin tout au long de ces trois années.

Enfin je remercie mes parents et ma sœur, sans lesquels je ne serais sûrement pas arrivé jusque là : ils m'ont soutenu depuis le début, et continuent à m'encourager à continuer dans une voie que d'autres, moins courageux, ont abandonnée. 


\section{Résumé}

Dans des hétérostructures semiconductrices à basse température, il est possible de réaliser des systèmes d'électrons purement bidimensionnels de densité électronique variable. A basse densité, la théorie prédit que les corrélations entre électrons dominent les fluctuations quantiques, conduisant à un état collectif tel le cristal de Wigner. Dans les systèmes réels, la présence de désordre rend la situation plus complexe : l'observation récente d'une transition métal-isolant, non prévue par les théories d'électrons indépendants, pose la question de la compétition entre les interactions et le désordre en dimension deux. Ce travail présente des mesures de transport en fonction du champ électrique, et des mesures de fluctuations de résistance, à très basse température dans des gaz bidimensionnels de trous formés dans des puits quantiques SiGe et GaAs. Dans ces systèmes, la masse effective élevée des porteurs et la faible densité permettent d'atteindre un régime pour lequel les interactions ne sont plus négligeables. Dans les échantillons désordonnés (SiGe), pour lesquels les lois de transport peuvent être expliquées dans le cadre de particules indépendantes, les effets de champ électrique sont dus au chauffage des porteurs. Par contre, dans les échantillons moins désordonnés (GaAs), les lois de transport en température et en champ électrique à très faible densité sont en accord avec l'existence d'une phase collective. Dans les échantillons GaAs, les mesures de fluctuations de résistance, ou bruit en $1 / f$, montrent l'existence d'une transition de phase à basse densité. Ces résultats sont compatibles avec les scénarios de formation d'une phase collective en présence de désordre par l'intermédiaire d'une transition de percolation.

Mots-clés: transition métal-isolant; gaz bidimensionnel d'électrons; semiconducteurs ; mesures de transport; effet du champ électrique ; couplage électron-phonon; fluctuations de résistance; bruit en $1 / f$; transition de percolation

\section{Abstract}

One can make a two dimensional electron system with a tunable electronic density in semiconductor heterostructures at low temperature. At low density, the theory predicts that electron correlations should overcome the quantum fluctuations, leading to a correlated electronic state such as the Wigner crystal. In real systems, disorder can lead to a much intricate problem : the recent observation of a metal-insulator transition has raised the question of interactions and disorder in two dimensions. This study concerns transport and resistance fluctuations measurements, at very low temperature in two-dimensional hole gases formed in GaAs and SiGe quantum wells. The high effective mass and low density in these systems lead to strong interactions that cannot be neglected any more. In the most disordered samples ( $\mathrm{SiGe),} \mathrm{where} \mathrm{transport} \mathrm{can} \mathrm{be} \mathrm{explained} \mathrm{in}$ the independent particles' approximation, electric field effects are due to carriers heating. On the contrary, in less disordered samples (GaAs), transport laws as a function of 
the temperature and the electric field at low density can be related to transport in the case of a collective state. In GaAs samples, resistance fluctuations measurements, or 1/f noise, demonstrate the existence of a phase transition at low density. These results are compatible with scenarios predicting that the Wigner transition with disorder could be a percolation transition.

Keywords: metal-insulator transition ; two-dimensional electron gaz ; transport measurements ; electric-field effect; electron-phonon coupling; resistance fluctuations ; $1 / f$ noise 
"Une fausse science fait des athées, une vraie science prosterne l'homme vers la divinité." Voltaire, Dialogues, XXIV. 


\section{Table des matières}

\section{Partie I Systèmes d'électrons en dimension deux}

1 Désordre et interactions dans un système électronique 17

1.1 Introduction . . . . . . . . . . . . . . 18

1.2 Définitions et concepts de base . . . . . . . . . . . 18

1.3 Système électronique en présence de désordre . . . . . . . . . . . 23

1.4 Électrons en interactions . . . . . . . . . . . . . . 28

1.5 Électrons en dimension deux . . . . . . . . . . . . . 33

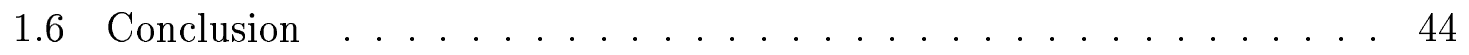

2 La transition métal-isolant en dimension deux $\quad 47$

2.1 Introduction . . . . . . . . . . . . . . . 48

2.2 Comportement métallique inattendu . . . . . . . . . . . . . 48

2.3 Observations expérimentales . . . . . . . . . . . . 52

2.4 Phénoménologie de la transition métal-isolant . . . . . . . . . . 64

2.5 Interprétations théoriques de la transition métal-isolant . . . . . . 78

2.6 Conclusion . . . . . . . . . . . . . . . . . 92 
3 Systèmes étudiés et dispositif expérimental $\quad 93$

3.1 Gaz bidimensionnels de porteurs dans les semiconducteurs . . . . . . 94

3.2 Description des échantillons utilisés . . . . . . . . . . . . 96

3.3 Techniques expérimentales . . . . . . . . . . . . . 102

Partie II Transport non-ohmique autour de la transition métalisolant

4 Lois de transport autour de la transition métal-isolant 107

4.1 Introduction . . . . . . . . . . . . . . . . 108

4.2 Transport pour des électrons localisés . . . . . . . . . . . 108

4.3 Transport d'une phase collective . . . . . . . . . . . 121

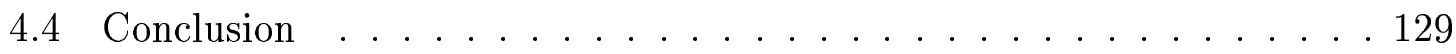

5 Transport dans les puits quantiques $p$-SiGe et $p$-GaAs 131

5.1 Introduction . . . . . . . . . . . . . . . . . 132

5.2 Présentation générale . . . . . . . . . . . . . . . . 132

5.3 Analyse du comportement métallique . . . . . . . . . . . 134

5.4 Analyse du comportement isolant . . . . . . . . . . . 138

5.5 Conclusion . . . . . . . . . . . . . . . 147

6 Transport non-ohmique dans les puits quantiques $p$-SiGe 149

6.1 Introduction . . . . . . . . . . . . . . . 150

6.2 Etude du chauffage des porteurs . . . . . . . . . . 150

6.3 Dépendances en champ électrique expliquées par le chauffage . . . . . 160




7 Transport non-ohmique dans les puits quantiques $p$-GaAs $\quad 169$

7.1 Introduction . . . . . . . . . . . . . 170

7.2 Etude des effets de chauffage dans les puits quantiques $p$-GaAs . . . . 170

7.3 Effets collectifs à faible densité de porteurs . . . . . . . . . . . 173

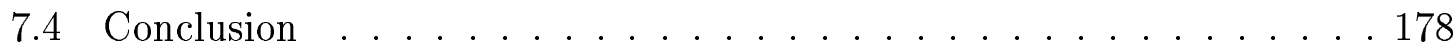

Partie III Fluctuations temporelles de résistance dans un gaz bidimensionnel de trous

8 Fluctuations de résistance dans un système d'électrons $\quad 181$

8.1 Introduction . . . . . . . . . . . . . . . . 182

8.2 Généralités sur les fluctuations de résistance . . . . . . . . . . . 182

8.3 Description classique des fluctuations de résistance . . . . . . . . . 184

8.4 Fluctuations de résistance dans un isolant . . . . . . . . . . . 188

8.5 Fluctuations de résistance et interférences quantiques . . . . . . . . . 191

8.6 Fluctuations de résistance et transition de phase . . . . . . . . . . 195

8.7 Conclusion . . . . . . . . . . . . . . . . . 201

9 Dispositif expérimental pour la mesure de bruit 203

9.1 Introduction . . . . . . . . . . . . . . . . 204

9.2 Principe de la mesure . . . . . . . . . . . . . . . . 204

9.3 Bruits parasites . . . . . . . . . . . . . 206

9.4 Suppression des bruits parasites . . . . . . . . . . . . 211

9.5 Conclusion . . . . . . . . . . . . . . . . 217

10 Fluctuations de résistance dans les puits quantiques $p$-SiGe $\quad 219$

10.1 Introduction . . . . . . . . . . . . . . . 220

10.2 Mesures sur la partie avec grille . . . . . . . . . . . . 220

10.3 Mesures sur la partie sans grille . . . . . . . . . . . . 220

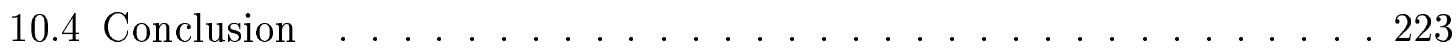


11 Fluctuations de résistance dans les puits quantiques $p$-GaAs $\quad 225$

11.1 Introduction . . . . . . . . . . . . . . . 226

11.2 Mesures du bruit en $1 / f \ldots \ldots . \ldots . \ldots . \ldots 226$

11.3 Dépendances du bruit en température et en densité . . . . . . . . 231

11.4 Observation de lois d'échelle de percolation . . . . . . . . . . 236



$\begin{array}{ll}\text { Annexes } & 247\end{array}$

A Couplage électron-phonon dans un gaz bidimensionnel de trous $\quad 247$

A.1 Introduction . . . . . . . . . . . . . . . 248

A.2 Couplage électron-phonon dans un gaz bidimensionnel de porteurs . . . 248

A.3 Détermination de la température effective des porteurs . . . . . . 253

B Diffusion de chaleur vers les contacts $\quad 259$

B.1 Loi de Wiedemann-Franz . . . . . . . . . . . . . . . 260

B.2 Modèle unidimensionnel . . . . . . . . . . . . . . . . 260

B.3 Modèle bidimensionnel . . . . . . . . . . . . . . . . . . . 261

$\begin{array}{ll}\text { Conclusion générale } & 263\end{array}$

$\begin{array}{lr}\text { Liste des publications } & 267\end{array}$

$\begin{array}{lr}\text { Bibliographie } & 269\end{array}$ 


\section{Introduction générale}

\section{Désordre et interactions dans un gaz bidimensionnel d'électrons}

Le sujet de cette thèse est l'étude expérimentale de systèmes bidimensionnels d'électrons de faible densité, pour lesquels les interactions entre électrons ne peuvent pas être négligées. Dans ces systèmes expérimentaux, on s'attend à ce que le désordre joue également un rôle important. Lorsque les interactions entre électrons sont faibles, la théorie du liquide de Fermi permet de se ramener à un système de quasi-particules indépendantes. Dans cette limite, il est possible d'étudier l'effet du désordre : le traitement quasi-classique du transport des électrons en présence de désordre conduit au calcul de la résistance d'un métal. En prenant en compte les effets d'interférences quantiques, Anderson a montré qu'un désordre suffisamment fort localise les électrons [1], et conduit à un système isolant. Dans la limite opposée, lorsque les interactions entre électrons sont très fortes, et en l'absence de désordre, Wigner a montré que les électrons s'organisent sur les nœuds d'un réseau périodique, ou cristal de Wigner [2].

Ces deux limites, d'une part le système d'électrons indépendants en présence de désordre, et d'autre part le système d'électrons fortement corrélés avec un désordre faible, ont été largement étudiés. Lorsqu'on sort de ces limites, et en particulier lorsque le désordre et les interactions entrent en compétition, on s'attend à un problème plus compliqué. Alors que les interactions tendent à organiser les électrons sur un cristal, le désordre tend à détruire cet ordre entre les électrons. Les deux questions fondamentales abordées dans cette thèse sont les suivantes :

- Que se passe-t-il lorsque le désordre et les interactions entrent en compétition? Peut-on encore appliquer avec une bonne approximation les théories de localisation par le désordre, ou ces théories sont-elles fortement modifiées par la présence des interactions?

- La seconde question concerne la transition entre le liquide de Fermi à faible interaction et le cristal de Wigner à forte interaction. Est-ce que la théorie du liquide de Fermi est valable jusqu'à la cristallisation de Wigner, ou existe-t-il un nouvel état électronique intermédiaire pour des interactions modérées, comme il a été suggéré par Benenti et al. [3]. L'introduction du désordre complique ce problème. On s'attend à une phase cristal désordonné, ou à un verre, à suffisamment forte interaction. D'autre part la présence de désordre peut modifier la nature de la transition.

Nous nous intéressons essentiellement au cas de la dimension deux. Du point de vue théorique, Abrahams et al. ont montré, à l'aide d'une théorie d'échelle, qu'un système d'électrons indépendants en présence de désordre est toujours isolant en dimension deux [4]. Du point de vue expérimental, il est possible de réaliser des gaz bidimensionnels 
d'électrons confinés à l'interface entre différents matériaux, comme par exemple des semiconducteurs. A suffisamment basse densité d'électrons, les interactions entre électrons sont fortes, et ces systèmes sont de très bons candidats pour étudier le problème des interactions et du désordre.

\section{La transition métal-isolant en dimension deux}

Jusqu'au début des années 90, les analyses des mécanismes de transport dans des systèmes bidimensionnels d'électrons, tels les transistors à effet de champ à base de silicium (MOSFET Si), étaient en accord avec la théorie d'échelle de la localisation, confirmant l'absence de transition métal-isolant en dimension deux. Dans ce contexte, les observations de Kravchenko et al. sur des MOSFETs Si ont soulevé de nombreuses questions [5]. Dans les mesures présentées, à haute densité d'électrons, la résistivité du système décroît quand la température diminue et semble saturer vers une valeur finie à température nulle. C'est la caractéristique d'un système métallique, et ce résultat expérimental semble donc contredire la théorie d'échelle de la localisation. A faible densité de porteurs, la résistivité croît quand la température diminue, ce qui est caractéristique d'un comportement isolant. Ces mesures semblent donc indiquer l'existence d'une transition métal-isolant en dimension deux. Ces résultats ont ensuite été confirmés dans d'autres systèmes bidimensionnels de porteurs, dont la caractéristique commune est de présenter de fortes interactions entre électrons.

Les moyens utilisés pour caractériser cette transition sont principalement des mesures de transport en fonction de la température, du champ électrique et du champ magnétique. Cependant ces mesures ne donnent pas toutes les informations sur un système électronique. Des mesures de compressibilité, de fluctuations de résistance et de pouvoir thermoélectrique sont venues compléter les mesures de transport, et ont montré le caractère nouveau de la transition. Cependant ces mesures restent peu nombreuses, car plus difficiles à réaliser.

\section{Objectif de la thèse}

Cette thèse a eu pour objectif d'étudier deux systèmes bidimensionnels de porteurs présentant les caractéristiques de la transition métal-isolant. Le premier est un gaz bidimensionnel de trous dans un puits quantique $\mathrm{SiGe} / \mathrm{Si}(p$-SiGe), le second un gaz bidimensionnel de trous dans un puits quantique GaAs/AlGaAs ( $p$-GaAs). L'étude comparée de ces deux systèmes, possédant des caractéristiques communes ainsi que des différences, est un bon moyen de tester l'universalité de la transition métal-isolant, ainsi que les caractéristiques liées au système. Au-delà de l'étude même de la transition métal-isolant, en raison de la masse effective élevée des trous et de la faible densité de porteurs, ces deux systèmes sont de très bons candidats pour l'étude du comportement d'un gaz bidimensionnel de porteurs en fortes interactions et en présence de désordre. Nous verrons en particulier que les puits quantiques $p$-SiGe sont plus désordonnés que les puits quantiques $p$-GaAs, ce qui permet d'étudier l'effet du désordre dans ces systèmes.

Nous avons étudié principalement deux grandeurs pendant cette thèse. Dans un premier temps nous avons fait des mesures de transport en fonction du champ électrique. 
Autour de la transition métal-isolant, il existe de fortes non-linéarités de la caractéristique $I-V$, qui ont été attribuées à un comportement critique autour d'une transition de phase, et à l'existence d'une phase collective accrochée à très faible densité de porteurs. Ensuite nous avons étudié les fluctuations de résistance à basse fréquence dans ces systèmes. Ces fluctuations sont liées à la réponse du système électronique aux fluctuations extérieures, et apportent des informations complémentaires par rapport aux mesures de transport pour étudier la physique du système. Il existe peu de mesures de bruit de résistance autour de la transition métal-isolant en dimension deux. Nous verrons que les mesures que nous avons réalisées permettent d'ouvrir une voie tout-à-fait nouvelle dans le domaine. Elles fournissent en effet une indication expérimentale forte qu'il existe bien une transition de phase continue, mais à une densité plus faible que ce qui était admis auparavant. Il pourrait s'agir d'une transition de percolation d'un liquide dans un cristal ou un verre de Wigner, scénario qui déjà a été évoqué pour décrire la cristallisation de Wigner en présence de désordre.

\section{Plan du manuscrit}

Dans le premier chapitre sont rappelées les propriétés d'un système électronique dans les limites de fort désordre, puis de fortes interactions. Étant donnée l'importance du sujet de la transition métal-isolant en dimension deux, le chapitre 2 contient une revue des résultats expérimentaux, des analyses faites et des théories proposées. Le chapitre 3 décrit les systèmes bidimensionnels de porteurs que nous avons étudié, ainsi que les techniques expérimentales.

La deuxième partie concerne les mesures de transport dans les gaz bidimensionnels de trous. Dans le premier chapitre sont rappelés les comportements attendus dans un système électronique désordonné et en présence de fortes interactions (chapitre 4). Les résultats expérimentaux sont ensuite répartis dans trois chapitres. Le chapitre 5 présente les analyses des mesures de transport réalisées à faible champ électrique (dans le régime ohmique) dans les deux systèmes étudiés, avec une présentation des deux comportements métallique et isolant. Dans le chapitre 6 sont décrits les effets du champ électrique dans les puits quantiques $p$-SiGe. Nous verrons que ces effets peuvent être interprétés par des effets de chauffage des porteurs, ce qui nous a conduit à étudier plus particulièrement le couplage électron-phonon dans ces systèmes, dont le mécanisme est développé dans l'annexe A. Nous prouvons en particulier l'existence d'un effet piézoélectrique dans ce système. Outre l'intérêt technologique que peut apporter cette connaissance, nous verrons qu'elle conduit à des informations sur la physique du système d'électrons. De plus, la connaissance du couplage électron-phonon dans ces systèmes permet de mieux interpréter les mesures de non-linéarités dans le transport. Le chapitre 7 concerne les effets de champ électrique dans les puits quantiques $p$-GaAs, pour lesquels l'effet de chauffage des porteurs ne peut pas être négligé. Cependant, à très faible densité, les effets de chauffage ne peuvent pas tout expliquer. D'autres effets du champ électrique, qui peuvent être reliés à l'existence d'une phase électronique fortement corrélée, ou d'une transition de type percolation, peuvent être évoqués.

La troisième partie concerne les mesures de fluctuations de résistance à basse fréquence. En raison de la dépendance spectrale de ces fluctuations, ce bruit est souvent appelé "bruit 
en $1 / f^{\prime \prime}$. Dans le chapitre 8 sont revues les principales interprétations des fluctuations de résistance dans un système électronique. Le point important est que ce bruit dépend fortement de la nature de la phase électronique. De plus le bruit est un bon moyen d'étude d'une transition de phase. Le chapitre 9 présente les techniques que nous avons mises au point au laboratoire pour effectuer la mesure de fluctuations de résistance de très faible amplitude à basse fréquence. Dans le chapitre 10 sont présentées les mesures effectuées sur les puits quantique $p$-SiGe. Dans ce système, le courant de fuite grillecanal n'a pas permis d'étudier le bruit dans la partie de l'échantillon muni d'une grille, et donc de varier la densité. Cependant il a été possible de faire une mesure dans une partie sans grille, a haute densité électronique. Cette mesure a permis de valider la bonne résolution du montage expérimental, et la comparaison avec d'autres mesures a permis d'interpréter ce bruit par des théories classiques de fluctuations de mobilité dues aux fluctuations du désordre. L'utilisation d'une autre technologie pour fabriquer la grille dans les puits quantique $p$-GaAs nous a permis d'étudier, dans ce système, les variations du bruit sur une large gamme de températures et de densités de porteurs à la traversée de la transition métal-isolant (chapitre 11). Le bruit augmente fortement quand on diminue la densité, et il présente de très fortes dépendances en température. De plus, la puissance de bruit suit une loi d'échelle en fonction de la résistance du système, caractéristique d'une transition de phase du deuxième ordre, et en particulier d'une transition de percolation. En complément, la conductivité du système a été étudiée dans la même gamme de densité et suit également une loi d'échelle caractéristique d'une transition de percolation. Ces résultats sont compatibles avec certaines théories décrivant le transition métal-isolant en dimension deux, et semblent dus aux fortes interactions entre électrons. 


\section{Première partie}

\section{Systèmes d'électrons en dimension deux}





\section{Chapitre 1}

\section{Désordre et interactions dans un système électronique}

\section{Sommaire}

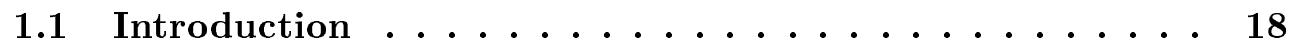

1.2 Définitions et concepts de base . . . . . . . . 18

1.2.1 Physique d'un système d'électrons . . . . . . . . . . . . 18

1.2.2 Propriétés de conduction . . . . . . . . . . . . 20

1.2.3 Théorie de Landau du liquide de Fermi . . . . . . . . . . 22

1.3 Système électronique en présence de désordre . . . . . . 23

1.3 .1 Localisation faible . . . . . . . . . . . . . . 23

1.3.2 Localisation d'Anderson . . . . . . . . . . . . . . . . 24

1.3.3 Théories d'échelle . . . . . . . . . . . . . . . . 26

1.4 Électrons en interactions . . . . . . . . . . . . 28

1.4 .1 Isolant de Mott . . . . . . . . . . . . . . . . . . . . . . . . . . . . . . .

1.4.2 Cristal de Wigner . . . . . . . . . . . . . . . 30

1.5 Électrons en dimension deux ............. 33

1.5.1 Particularités de la dimension deux . . . . . . . . . 34

1.5.2 Transport en dimension deux . . . . . . . . . . . . . . . . 35

1.5.3 Effet d'un fort champ magnétique . . . . . . . . . . 38

1.5.4 Cristallisation de Wigner . . . . . . . . . . . . . . . . . . 40

1.6 Conclusion .................... 44 


\subsection{Introduction}

Les systèmes étudiés expérimentalement pendant cette thèse sont des gaz d'électrons en dimension deux. De part les techniques de fabrication utilisées, les électrons sont en présence d'un potentiel aléatoire de désordre, et de part l'interaction Coulombienne, ces électrons interagissent fortement entre eux. Dans ce chapitre nous allons voir qu'il est possible d'avoir une bonne description de ces systèmes dans deux cas limites, le cas des faibles interactions, où le désordre domine le comportement du système électronique, et le cas du faible désordre, où ce sont les interactions qui dominent. On s'attardera plus particulièrement sur les effets du désordre et des interactions sur les propriétés de transport du système, qui peut alors être soit isolant, soit métallique.

Dans la première section $(\S 1.2)$ sont rappelées quelques définitions et concepts de base de la physiques des systèmes électroniques en matière condensée. Nous verrons en particulier comment décrire l'électrons dans un cristal, puis en présence de désordre, et nous abordons l'effet des interactions entre électrons. Enfin la théorie du liquide de Fermi, qui permet de décrire des particules en interactions par des quasi-particules indépendantes, sera présenté brièvement. Dans la deuxième section (\$1.3) est discutée le cas d'un système électronique sans interactions (modèle de particules indépendantes) en présence de désordre. Dans les modèles présentés, les interactions ne sont pas toujours absentes, mais elles sont en général traitées en champ moyen, permettant ainsi de toujours se ramener à des particules indépendantes. Dans la troisième section nous verrons le cas du système d'électrons en interactions fortes $(\S 1.4)$. Lorsque les interactions sont suffisamment fortes, les particules sont fortement corrélées et il n'est plus possible de se ramener à un problèmes de quasi-particules indépendantes. On est donc confronté au problème à $N$ corps, dont la résolution est une question majeure de la physique de la matière condensée. Enfin nous verrons que le rôle de la dimensionnalité du système est importante. En particulier on détaillera dans la dernière section (§1.5) le cas particulier de la dimension deux, son originalité face aux autres dimensionnalités.

Dans ce chapitre ont volontairement été séparés le problème du désordre et celui des interactions. Dans un système réel, ces deux ingrédients ne peuvent pas être dissociés. Le problème de la compétition entre désordre et interactions est encore vivement débattu, et il constitue le fondement des interrogations portées sur l'existence d'une transition métal-isolant en dimension deux, comme nous le verrons dans le chapitre 2.

\subsection{Définitions et concepts de base}

\subsubsection{Physique d'un système d'électrons}

\section{Electrons dans un cristal}

Dans toute la thèse, on considère des électrons dans un cristal. Le potentiel cristallin est considéré dans l'approximation de la masse effective, pour laquelle les solutions de l'équation de Schrödinger sont des fonctions de Bloch :

$$
\Psi_{n, \vec{k}}(\vec{r})=\frac{1}{\sqrt{V}} u_{n, \vec{k}}(\vec{r}) e^{i \vec{k} \cdot \vec{r}}
$$


où $u_{n, \vec{k}}(\vec{r})$ est une fonction qui a la périodicité du cristal, $\vec{k}$ est appelé vecteur d'onde de l'électron, $n$ est un indice caractérisant la bande et le spin, et $V$ est le volume du cristal. $\mathrm{Au}$ voisinage de $\vec{k}=\overrightarrow{0}$, l'énergie propre associée prend une forme semblable à l'énergie d'une particule libre :

$$
E_{n}(\vec{k})=\frac{\hbar^{2} k^{2}}{2 m^{*}}
$$

où $m^{*}$ est la masse effective des électrons dans le réseau cristallin.

Dans la suite on aura besoin de considérer le cas d'un potentiel extérieur au potentiel cristallin, comme le potentiel de confinement dans les hétérostructures semiconductrices ou le potentiel de désordre. Lorsque la distance typique sur laquelle varie ce potentiel reste grande devant la distance inter-atomique $u_{0}$, ce potentiel peut être traité dans l'approximation de la fonction enveloppe, qui consiste à chercher la fonction d'onde électronique sous la forme :

$$
\Psi_{n, \vec{k}}(\vec{r})=\frac{1}{\sqrt{V}} u_{n, \vec{k}}(\vec{r}) e^{i \vec{k} \cdot \vec{r}} F(\vec{r})
$$

où $F(\vec{r})$ est la fonction enveloppe, qui est la fonction d'onde solution du problème d'un électron de masse effective $m^{*}$ plongé dans un milieu de constante diélectrique $\varepsilon=\varepsilon_{0} \varepsilon_{r}$ ( $\varepsilon_{0}$ est la constante diélectrique du vide et $\varepsilon_{r}$ est la constante diélectrique relative du milieu) en présence du seul potentiel extérieur. C'est pourquoi, dans la suite, le potentiel cristallin n'est plus mentionné, mais est pris en compte par l'intermédiaire d'une masse effective $m^{*}$ et d'une constante diélectrique $\varepsilon=\varepsilon_{0} \varepsilon_{r}$ différentes de la masse de l'électron $m_{0}$ et de la constante diélectrique du vide $\varepsilon_{0}$.

\section{Désordre}

Le cas du cristal parfait n'est en général jamais rencontré dans les systèmes réels. Dans la majorité des cas le cristal comporte des défauts ou des impuretés qui viennent perturber l'arrangement périodique du potentiel cristallin. Ce désordre donne lieu à un potentiel extérieur statique (ou plus précisément dont les variations sont très lentes face au temps de réarrangement des électrons). On suppose ici que la température est suffisamment basse pour pouvoir négliger l'effet des phonons sur la diffusion des électrons.

Lorsque le désordre est faible, on peut le traiter dans une approximation semi-classique dans laquelle les trajectoires électroniques sont décrites par des paquets d'onde qui diffusent sur le désordre, décrit alors par une assemblée de centres diffuseurs. Bien entendu, cette approche n'est valable que dans la limite où la nature ondulatoire des électrons n'est pas impliquée dans les collisions sur les centres diffuseurs. Il faut donc que la longueur d'onde de De Brooglie $\lambda_{F}=1 / k_{F}$, où $k_{F}$ est le vecteur d'onde de Fermi, reste très inférieure à la distance moyenne entre diffusions successives, ou libre parcours moyen élastique $l_{e}$, soit $k_{f} l_{e} \gg 1$.

Dans le cas où $k_{F} l_{e} \sim 1$, il est nécessaire de prendre en compte la nature ondulatoire des électrons, conduisant à des effets de localisation des électrons par le désordre lorsque $k_{F} l_{e}<1$. Le produit $k_{F} l_{e}$ est une mesure du désordre effectif vu par les électrons. Pour un système électronique de densité $n$ en dimension $d, k_{F} \propto n^{1 / d}$, et donc la force du désordre effectif augmente quand la densité d'électrons diminue. 


\section{Interactions entre électrons}

Enfin, les électrons étant des particules chargées, ils interagissent entre eux par l'intermédiaire de l'interaction Coulombienne. Dans le cas où l'énergie d'interaction Coulombienne entre électrons $E_{\text {int }}$ est faible devant l'énergie cinétique des électrons, les interactions peuvent être négligées et chaque électron est considéré comme une particule indépendante des autres. Lorsque l'énergie d'interaction devient comparable ou même supérieure à l'énergie cinétique, les corrélations induites entre les électrons donnent lieu à des comportements collectifs des électrons. La résolution de ce problème d'électrons corrélés nécessite de prendre en compte les électrons dans leur ensemble, et passe donc par la résolution du problème à $N$ corps.

Dans un gaz d'électrons fortement dégénéré (pour lequel l'énergie de Fermi $E_{F}$ est grande devant l'énergie thermique $k_{B} T$, où $k_{B}$ est la constante de Bolzmann et $T$ est la température absolue du système), l'énergie cinétique des électrons est proche de l'énergie de Fermi. Il est alors possible de définir un critère de "force" des interactions comparée à l'énergie cinétique à l'aide du quotient $r_{s}$, défini comme le rapport entre l'énergie d'interaction et l'énergie de Fermi :

$$
r_{s}=\frac{E_{\mathrm{int}}}{E_{F}}
$$

Alors que l'énergie de Fermi d'un système d'électrons libres est proportionnelle à $n^{2 / d}$, où $n$ est la densité de porteurs et $d$ la dimensionnalité de l'espace, l'énergie d'interaction est proportionnelle à l'inverse de la distance entre particules, donc proportionnelle à $n^{1 / d}$. Le facteur $r_{s}$ est donc proportionnel à $n^{-1 / d}$. On s'attend donc à une augmentation des effets de corrélations entre électrons lorsque la densité d'électrons diminue.

\subsubsection{Propriétés de conduction}

\section{Métal ou isolant}

Le caractère métallique ou isolant caractérise la réponse de l'état fondamental d'un système électronique à un champ électrique appliqué. Cette réponse est, par définition, la conductivité $\sigma$ (ou la résistivité $\rho$ ) à température nulle. On définit alors un métal comme un système de conductivité non nulle (ou de résistivité finie), et un isolant comme un système de conductivité nulle (ou de résistivité infinie).

L'idée couramment admise par la physique du solide sur la nature d'un système électronique résulte de la théorie des bandes [6], développée dans le cas d'électrons sans interactions (voir les figures 1.1(a) et (b)). Si le nombre d'électrons est tel qu'il y a un nombre entier de bandes remplies, les bandes d'énergies supérieures étant complètement vides, la présence d'une bande d'énergie interdite rend le système isolant. Par contre si la dernière bande remplie est partiellement remplie, il existe des états libres juste au-dessus du dernier état occupé, et le système est un métal.

Cette condition sur l'énergie des états libres est nécessaire, mais pas suffisante. Pour obtenir un métal, il est en plus nécessaire que les fonctions d'onde électroniques soient délocalisées sur au moins toute la taille du système, puisqu'un électron dont la fonction 
(a)

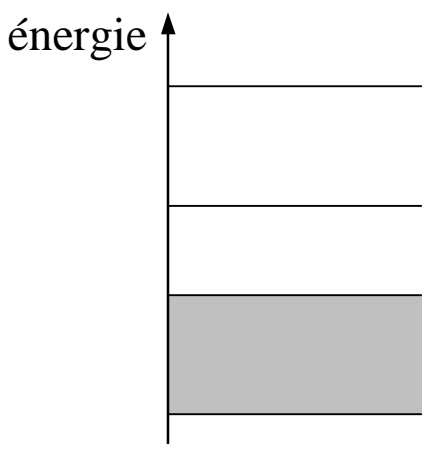

(c)

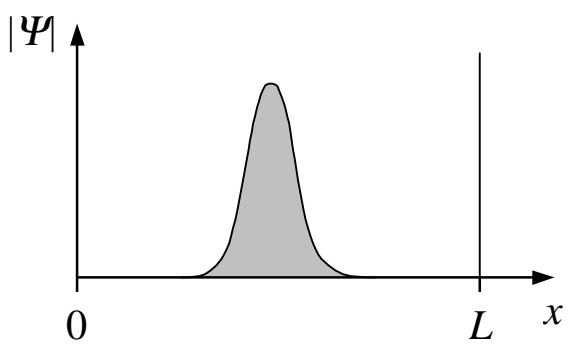

(b)



(d)

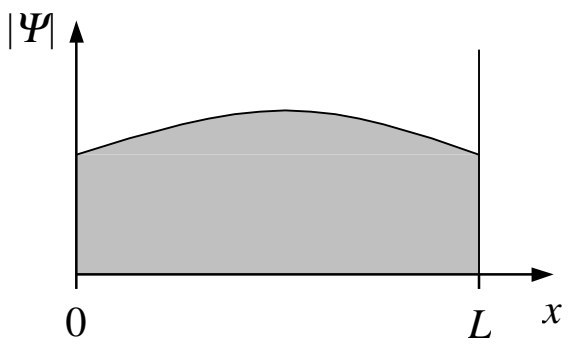

FIG. 1.1: Haut : Diagrammes d'énergie dans le cas (a) où l'énergie de Fermi tombe dans la bande d'énergie interdite entre les bandes de valence et de conduction (isolant de bande), ou (b) lorsque la bande de conduction est partiellement remplie (métal). Les parties grisées représentent le remplissage électronique. Bas : Amplitude des fonctions d'onde électroniques $|\Psi|$ dans le cas d'une bande de conduction partiellement remplie (cas (b)). Les fonctions d'ondes peuvent alors être (c) soit localisées (isolant), (d) soit délocalisées (métal). $x$ est l'abscisse, $L$ est la taille du système.

d'onde est localisée ne peut pas transporter du courant (voir les figures 1.1(b) et (c)). Nous verrons au §1.3.3 qu'il est même nécessaire d'avoir des fonctions d'ondes infiniment étendues.

\section{Conductivité de Drude}

Dans le cas où le désordre est faible, i.e. $k_{F} l_{e} \gg 1$, la conductivité peut être calculée par une approche semi-classique. On considère pour cela des paquets d'onde formés par la combinaison linéaire de fonctions d'onde électroniques. La résolution de l'équation de Bolzmann dans ce cas conduit à [6] :

$$
\sigma_{0}=\frac{1}{\rho_{0}}=\frac{n e^{2}<\tau>}{m^{*}},
$$

où $n$ est la densité de porteurs, $m^{*}$ la masse effective des porteurs, et $\langle\tau\rangle$ la valeur moyenne sur l'énergie du temps de collision entre l'électron et le centre diffuseur. Pour 
un système d'électrons fortement dégénéré (pour lequel $k_{B} T \ll E_{F}$ ), $<\tau>=\tau\left(E_{F}\right.$ ), que l'on notera $\tau$, temps de collision de transport. Dans ce régime, il est possible de définir la mobilité des porteurs $\mu=e \tau / m^{*}$.

\subsubsection{Théorie de Landau du liquide de Fermi}

Un grand nombre de théories décrivant les systèmes électroniques sont des théories de champ moyen prenant en compte les interactions entre électrons de manière perturbative. En toute rigueur, ces théories ne sont valables que pour des valeurs très faibles des interactions, autrement dit pour $r_{s} \ll 1$ (où le paramètre $r_{s}$ est défini au $§ 1.2 .1$ ). Ce cas correspond à des systèmes de très forte densité électronique, ce qui est rarement le cas pour les systèmes étudiés expérimentalement. Dans les métaux usuels, les valeurs typiques de $r_{s}$ sont plutôt de l'ordre de 1 à 10 . Pourtant il a été remarqué que les théories perturbatives rendent bien compte des propriétés expérimentales de ces systèmes, au moins qualitativement. Ainsi ces théories perturbatives semblent pouvoir s'appliquer au delà de leur domaine de validité. La justification d'une telle constatation a été donnée par la théorie du liquide de Fermi.

La connaissance de l'état fondamental d'un système d'électrons en interactions est complexe et l'idée de Landau pour décrire un tel système est de ne considérer que les excitations du système de faible énergie, $\delta E \ll E_{F}$, et à basse température, $k_{B} T \ll \delta E$. Dans les conditions expérimentales usuelles ce sont ces excitations qui déterminent les propriétés des systèmes électroniques. Landau fait alors l'hypothèse que, en raison du principe de Pauli qui limite la diffusion électron-électron, la durée de vie de ces excitations est très longue. On peut alors considérer ces excitations comme des quasiparticules faiblement couplées entre elles, pour lesquelles il est possible d'appliquer les théories de champ moyen. Les quasiparticules ainsi définies restent fermioniques et de charge $e$, mais les autres paramètres telle la masse doivent être redéfinis.

La validité de cette théorie réside dans l'hypothèse fondamentale suivante. Supposons que l'on parte d'un système sans interactions, et qu'on augmente les interactions de manière adiabatique jusqu'à atteindre le système réel. La théorie suppose qu'il existe une correspondance biunivoque entre les états du système réel et ceux du système sans interactions. En d'autres termes il faut que le spectre des excitations du système réel ait une forme identique à celui du système sans interactions, et en particulier il est nécessaire de pouvoir encore définir une surface de Fermi. Il a été montré que cette hypothèse est fausse dans un certains nombre de cas. Dans le cas d'interactions attractives entre électrons, il se produit une instabilité avec création de paires d'électrons et ouverture d'une bande d'énergie interdite au niveau de Fermi qui conduit à l'état supraconducteur. Cet état ne peut pas être obtenu de manière adiabatique à partir de l'état sans interactions. Dans le cas d'interactions répulsives trop fortes, on va voir dans le $\S 1.4$ que l'état fondamental du système ne peut pas non plus être obtenu à partir de l'état sans interaction. C'est le cas de l'isolant de Mott ou du cristal de Wigner. Enfin dans le cas de systèmes de basse dimensionnalité, les fluctuations quantiques rendent le système instable vis-à-vis d'une interaction aussi petite soit-elle. Ainsi il a été montré qu'en dimension un la théorie du liquide de Fermi ne peut plus s'appliquer, et il est nécessaire de faire appel à la théorie du liquide de Luttinger (voir une bonne introduction à cette théorie dans la thèse de 
V. Rodriguez [7]). Le cas de la dimension deux, qui nous intéresse plus particulièrement ici, est intéressant car la validité de l'hypothèse du liquide de Fermi n'y est pas encore complètement établie. Nous verrons cependant que cette hypothèse permet d'interpréter certaines mesures expérimentales.

Enfin la théorie de Landau est une théorie phénoménologique, et non perturbative, ce qui signifie qu'elle est à priori valable pour des valeurs des interactions aussi grande que l'on veut, tant que l'on reste dans l'hypothèse du liquide de Fermi. Cette théorie ne définit pas elle-même son domaine de validité, et seule la confrontation à l'expérience permet de savoir si la théorie peut s'appliquer ou non.

\subsection{Système électronique en présence de désordre}

Dans le calcul de la conductivité de Drude, l'hypothèse principale est qu'après une collision, l'électron a "oublié" les informations sur sa phase. Ainsi il n'y aura pas d'interférences possibles entre les fonctions d'ondes électroniques après plusieurs collisions. C'est cependant faux pour des collisions élastiques pour lesquelles les fonctions d'ondes électroniques pouvent interférer.

\subsubsection{Localisation faible}

\section{Corrections quantiques de la conductivité}

Il est possible d'écrire la conductivité à température nulle comme une somme de termes $\left|A_{n}\left(\vec{r}, \vec{r}^{\prime}\right)\right|^{2}$, où $A_{n}\left(\vec{r}, \vec{r}^{\prime}\right)$ représente la probabilité de transmission d'une position $\vec{r}$ à une position $\vec{r}^{\prime}$ après $n$ diffusions élastiques sur le potentiel de désordre. Parmi ces chemins possibles, un certain nombre forment des boucles. Considérons deux chemins, l'un parcourant la boucle dans un sens, l'autre dans l'autre sens (voir la figure 1.2). De par la symétrie d'inversion par renversement du sens du temps de la probabilité de transmission, ces deux chemins ont la même probabilité de transmission $|A|^{2}$. Dans une approche classique, la probabilité totale est la somme des probabilités : $P=2|A|^{2}$. Mais dans un calcul quantique, les deux chemins interfèrent, et il faut sommer directement les amplitudes : $P=|A+A|^{2}=4|A|^{2}$. On montre ainsi que la probabilité de rétrodiffusion $P_{c}=\sum\left|A_{n}(\vec{r}, \vec{r})\right|^{2}$ (chemins formant des boucles, encore appelés Coopérons) est augmentée par les interférences quantiques. Or ces chemins participent à une baisse de la conductivité. Ce mécanisme conduit donc à la localisation des porteurs en comparaison au cas de Drude.

Il est possible de calculer la contribution de ces chemins par un développement en perturbation en $1 / k_{F} l_{e}$, ce qui conduit à une correction de la conductivité $\delta \sigma=\sigma-\sigma_{0}$ par rapport à la conductivité de Drude $\sigma_{0}$ de l'équation (1.5) pour un système de taille $L$ 


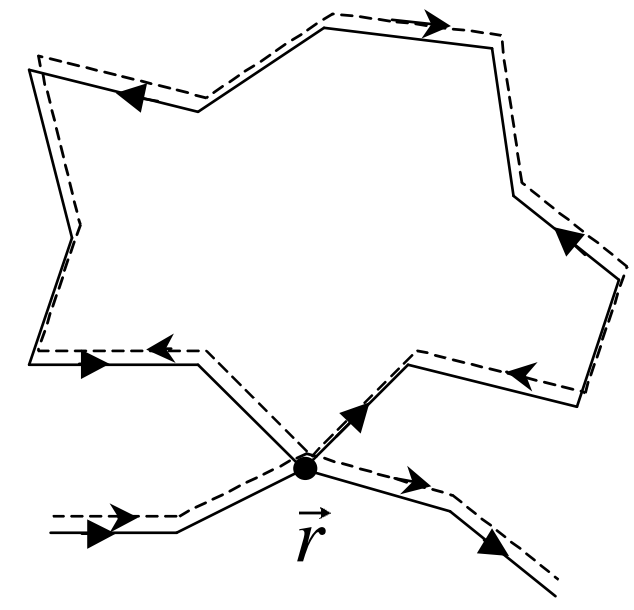

FIG. 1.2: Deux chemins rétrodiffusés (formant une boucle) partant et arrivant au point $\vec{r}$.

(voir par exemple [8]) :

$$
\begin{aligned}
& \delta \sigma_{L F}^{d=3}(L)=-\frac{e^{2}}{\hbar \pi^{3}}\left(\frac{1}{l_{e}}-\frac{1}{L}\right), \\
& \delta \sigma_{L F}^{d=2}(L)=-\frac{e^{2}}{\hbar \pi^{2}} \ln \left(\frac{L}{l_{e}}\right), \\
& \delta \sigma_{L F}^{d=1}(L)=-\frac{e^{2}}{\hbar \pi}\left(L-l_{e}\right),
\end{aligned}
$$

où $\sigma_{0}$ est la conductivité de Drude (équation (1.5)).

\section{Longueur de cohérence de phase}

Dans un système macroscopique, l'électron subit une succession de collisions dont certaines sont inélastiques. Ces collisions inélastiques ont pour effet de modifier la phase de la fonction d'onde électronique, rendant cette fonction d'onde incohérente. Étant donné que les effets de localisation faible font intervenir les interférences entre fonctions d'ondes électroniques, ils ne vont intervenir que sur des longueurs pour lesquelles les fonctions d'ondes sont cohérentes. Il est donc nécessaire ici d'introduire la longueur de cohérence de phase, qui est la longueur au-delà de laquelle la fonction d'onde électronique perd la mémoire de sa phase. Dans le cas de la localisation faible, la longueur de cohérence de phase $L_{\Phi}$ joue le rôle de la taille du système $L$ dans les équation (1.6) à (1.8).

\subsubsection{Localisation d'Anderson}

\section{Le modèle d'Anderson}

Lorsque le désordre devient important $\left(k_{F} l_{e}<1\right)$, le calcul précédent n'est plus valable. Anderson a proposé en 1958 [1] un modèle pour prendre en compte le désordre dans un système. Il considère des impuretés placées sur les sites d'un réseau périodique, mais l'énergie des niveaux électroniques diffère d'un site à l'autre. En dimension un, cela revient 
(a)

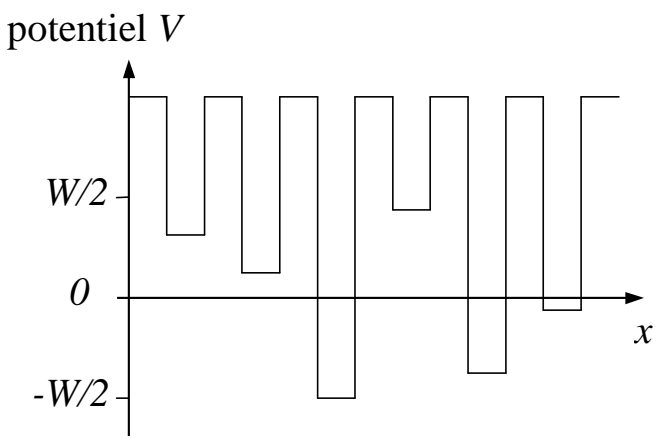

(b)

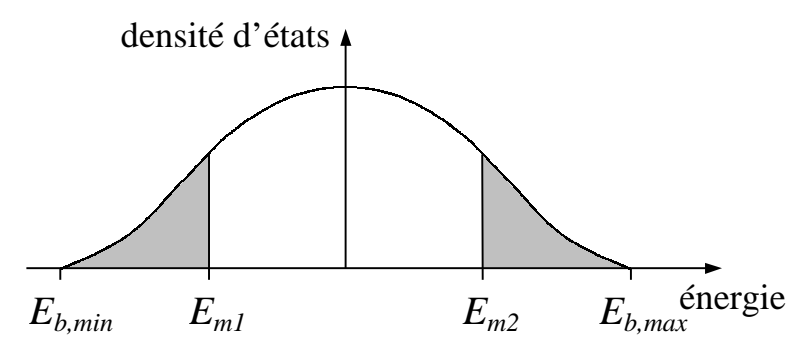

FIG. 1.3: (a) Puits de potentiel dans le modèle d'Anderson. (b) Densité d'états dans le modèle d'Anderson. Les régions grisées sont les régions d'états localisés, alors que la région centrale blanche correspond à des états délocalisés.

à considérer un potentiel du type de celui décrit sur la figure 1.3(a). L'énergie de chaque site prend une valeur comprise entre $-W / 2$ et $W / 2$, avec une distribution de probabilités uniforme dans cet intervalle d'énergie. $W$ est alors une mesure du désordre dans ce système. Notons $V$ l'intégrale de recouvrement entre les états sur sites plus proches voisins. La question d'Anderson est de savoir si les états électroniques du système sont délocalisés sur tout le système, ou si les états ont une extension spatiale finie. Un état délocalisé est décrit par la superposition d'un nombre infini d'états sur sites avec des poids équivalents, alors qu'un état localisé est décrit par la superposition d'états sur sites avec des poids décroissant exponentiellement avec la distance par rapport à un site. Si tous les états électroniques du système sont localisés, alors la conductivité du système à température nulle est nulle.

Anderson a montré que le paramètre critique est le rapport $W / V$. Pour une valeur suffisamment élevée de $W / V$, tous les états sont localisés. Lorsque ce rapport passe endessous d'une valeur critique $W_{c} / V$, des états délocalisés commencent à apparaître en milieu de bande. Pour une valeur faible de $W / V$, la région des états délocalisés devient large, recouvrant quasiment toute la bande (voir la figure 1.3(b)).

\section{Notion de bord de mobilité}

Mott a montré que, dans la bande, les états localisés et délocalisés sont séparés en énergie, comme le montre la figure 1.3 de droite. Les états en bord de bande ont une énergie cinétique plus faible que les états en milieu de bande, en raison de la densité d'états plus faible en bord de bande. Ainsi les états en bord de bande sont plus facilement localisés que ceux en milieu de bande. L'énergie séparant les états localisés et délocalisés est appelée bord de mobilité ( $E_{m 1}$ et $E_{m 2}$ sur la figure 1.3 de droite). Enfin Mott a montré que des états localisés et des états délocalisés ne peuvent pas coexister à la même énergie. En effet ces états se mélangeraient sous l'effet d'une interaction, aussi faible soit-elle, pour former un nouvel état. 


\section{L'approche de Thouless}

Thouless [9] a développé des arguments qui permettent de faire le lien entre le modèle d'Anderson et la conductance d'un système. De plus ces arguments vont nous conduire aux arguments d'échelle qui seront présentés dans la partie suivante.

Considérons un système de taille $L$ en dimension $d$. Les niveaux d'énergie d'un tel système sont espacés d'une largeur $\Delta$, l'espacement entre niveaux, qui est l'inverse de la densité d'états $\mathcal{D}(E)$. D'autre part, on considère un électron qui diffuse dans ce système de taille $L$. Le temps de diffusion, qui est le temps typique pendant lequel l'électron reste dans le système, est $\tau_{T}=L^{2} / D$, où $D$ est la constante de diffusion. Ainsi le système explorera les niveaux d'énergie contenus dans une bande de largeur $E_{T}=h / \tau_{T}=h D / L^{2}$. $E_{T}$ est appelée énergie de Thouless. Le rapport entre l'énergie de Thouless et l'espacement de niveaux, encore appelé nombre de Thouless et noté $g$, peut être relié à la conductance du système. En effet, on a :

$$
g=\frac{E_{T}}{\Delta}=\frac{h D \mathcal{D}(E)}{L^{2}} .
$$

Or la relation d'Einstein relie la constante de diffusion à la conductivité du système $\sigma$ :

$$
D=\frac{\sigma}{e^{2} \nu(E)},
$$

où $\nu(E)=\mathcal{D}(E) / L^{d}$ est la densité d'états par unité de volume. En notant que la conductance du système est donnée par $G=\sigma L^{d-2}$, on obtient :

$$
g=\frac{E_{T}}{\Delta}=\frac{h}{e^{2}} G .
$$

Le nombre de Thouless $g$ est encore appelé conductance généralisée sans dimension.

Revenons maintenant au modèle d'Anderson. En supposant que les niveaux d'énergie des impuretés sont régulièrement répartis entre $-W / 2$ et $W / 2$, l'espacement entre niveaux est $\Delta=W / N$, où $N$ est le nombre d'impuretés. D'autre part, pour un système contenant $N$ impuretés, l'énergie de Thouless est donnée par $V / N$, où $V$ est l'intégrale de recouvrement entre états. Le nombre de Thouless peut donc s'écrire :

$$
g=\frac{V}{W}=\frac{h}{e^{2}} G,
$$

et le critère d'Anderson donne que le système devient isolant lorsque le nombre de Thouless devient inférieur à une certaine valeur critique $g_{c}=V / W_{c}$ de l'ordre de 1 . Ce résultat montre donc que, dans le problème d'Anderson, c'est la conductance du système qui possède une valeur critique.

\subsubsection{Théories d'échelle}

\section{Influence de la dimensionnalité de l'espace}

Dans l'argument d'Anderson, la présence de la transition d'Anderson a lieu quelle que soit le dimensionnalité de l'espace. Des arguments simples permettent de montrer 
que cette vision est fausse en dimension un, pour laquelle un désordre, aussi faible soit-il, localise les états électroniques [10]. Pour le montrer, reprenons les arguments de Thouless. L'élargissement, en énergie, d'un paquet d'onde diffusant sur un longueur $L$ est égal à l'énergie de Thouless $E_{T} \propto L^{-2}$, alors que l'espacement entre niveaux d'un système de dimensionnalité $d$ varie comme $L^{-d}$. Ainsi le nombre de niveaux contenus dans un paquet d'onde est proportionnel à $L^{d-2}$. Pour $d=1$, lorsque $L$ augmente, le nombre de niveaux dans le paquet d'onde va diminuer jusqu'à ce qu'il ne reste plus qu'un état propre dans le paquet d'onde, conduisant à la localisation. Ainsi quel que soit le désordre, les états électroniques en dimensions un sont toujours localisés. Pour $d=2$, l'élargissement du paquet d'onde et l'espacement entre niveaux varient tous les deux comme $L^{-2}$, et aucune conclusion sur la localisation ne peut être donnée par cet argument. Il est alors nécessaire d'aller plus loin.

\section{Théorie d'échelle de la localisation}

Thouless a donc montré que l'on peut définir une conductance généralisée sans dimension $g=h / e^{2} \times G$. L'idée de la théorie d'échelle est de considérer la conductance $g(L)$ d'un système de taille $L$, et de se demander ce que devient cette conductance lorsqu'on multiplie par un entier $b$ la taille du système. Cette étape consiste donc à regarder la conductance d'un système contenant $b^{d}$ cubes de taille $L$. Thouless a montré que $g(b L)$ s'exprime uniquement en fonction de $g(L)$ et de $b$. A partir de cette démonstration, Abrahams, Anderson, Licciardello and Ramakrishnan (dits "bande des quatre") [4] ont montré que, lorsqu'on augmente la taille $L$ du système en combinant des blocs de taille donnée, la conductivité varie de manière à ce que

$$
\frac{d \ln (g(L))}{d \ln (L)}=\beta(g(L))
$$

i.e. que la fonction $\beta$ ainsi construite ne dépend que de $g$.

Il est possible de déterminer les limites asymptotiques de la fonction $\beta(g)$, à grandes et petites valeurs de $g$, par des arguments généraux de physique. Pour $g$ grand, la théorie macroscopique du transport est applicable, conduisant à :

$$
G(L)=\sigma L^{d-2} \quad \text { et } \quad \lim _{g \rightarrow \infty} \beta(g)=d-2
$$

Dans cette même limite, on peut aller plus loin en utilisant la théorie de la localisation faible (§1.3.1) qui donne un développement en $W / V=1 / g$ :

$$
\beta(g)=d-2-\frac{a}{g}+o\left(\frac{1}{g}\right) \text {. }
$$

Pour $g$ petit, on tombe dans le régime localisé, et on a :

$$
g=g_{a} e^{-L / a} \quad \text { et } \quad \lim _{g \rightarrow 0} \beta(g)=\ln \left[\frac{g}{g_{a}(d)}\right] \text {, }
$$




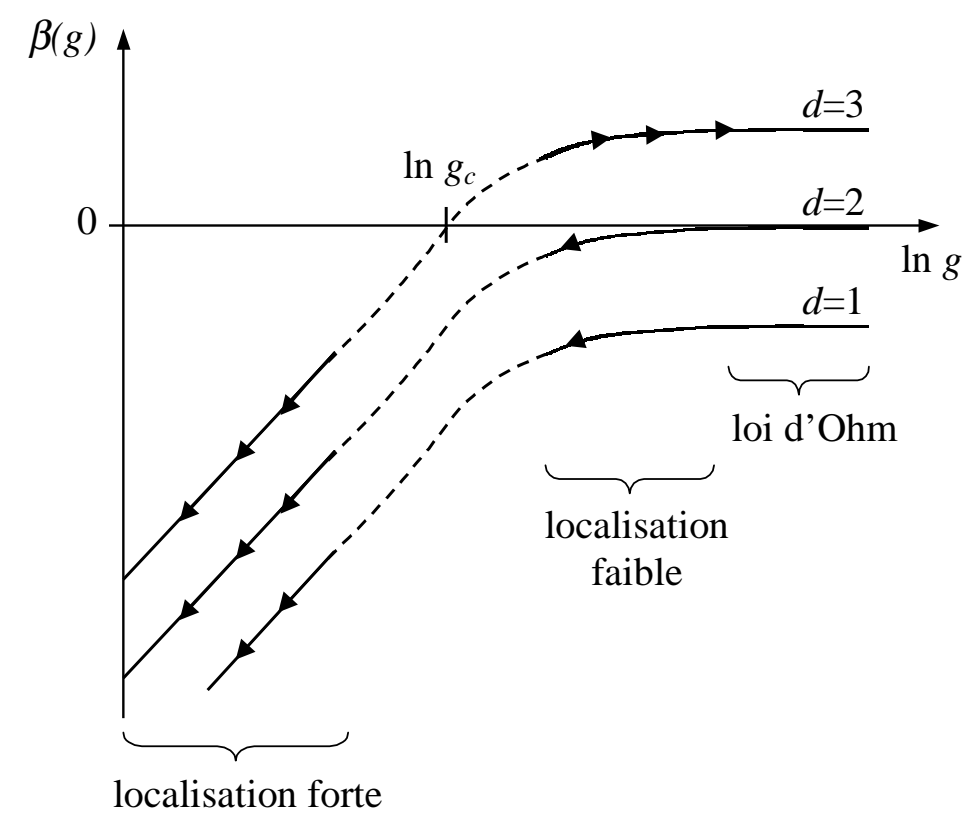

FIG. 1.4: Variation de la fonction $\beta(g)=d(\ln g) / d(\ln L)$ en fonction de $g$ pour différentes dimensionnalités $d$. Les flèches indiquent le sens de variation sur chaque courbe quand la taille $L$ du système augmente.

où $g_{a}$ est un rapport sans dimension de l'ordre de 1, et $a$ est la longueur de localisation. Il est là aussi possible d'aller plus loin en faisant un développement en perturbation en $V / W[1]$, donnant une correction positive :

$$
\beta(g)=\ln \left(\frac{g}{g_{a}}\right)\left[1+\alpha g+\mathcal{O}\left(g^{2}\right)\right]
$$

D'après ces considérations il est possible de tracer la fonction $\beta(g)$ en faisant l'hypothèse de continuité entre les deux limites (1.17) et (1.15), et en remarquant que $\beta(g)$ doit être monotone étant donné qu'une diminution de $V / W$ signifie plus de localisation. Cette fonction est tracée pour les dimensions d'espace égales à 1,2 et 3 dans la figure 1.4. On regarde le comportement de cette fonction lorsque la taille du système augmente (voir figure 1.4). Si $\beta>0, g$ augmente lorsque $L$ augmente, les électrons sont donc délocalisés et le système est métallique. Lorsque $\beta<0, g$ diminue lorsque $L$ augmente, le système est alors isolant. En dimension $d=3$, la courbe croise l'axe $\beta=0$ en un point $g_{c}$, ce qui signifie que le système peut subir une transition entre un état métallique (pour $g>g_{c}$ ) vers un état isolant (pour $g<g_{c}$ ). Par contre, pour $d=2$ et $d=1, \beta$ est une fonction toujours négative, ce qui signifie que le système ne rencontre pas de transition métal-isolant. D'après cette théorie d'échelle, un système d'électrons indépendants en dimension $d=2$ ou $d=1$ en présence d'un désordre arbitrairement petit est toujours isolant.

\section{4 Électrons en interactions}

Dans cette section on s'accupe de la limite opposée à celle de la section précédente. On considère en effet un système où l'énergie d'interaction Coulombienne domine, et le désordre est soit nul, soit faible. Nous avons vu au $§ 1.2 .3$ que, dans certains cas, il 
est possible de décrire le mouvement des électrons en interactions comme celui de quasiparticules indépendantes : c'est le modèle du liquide de Fermi. Dans la limite des très fortes interactions, nous verrons que l'état fondamental du système ne peut plus se déduire de l'état sans interactions de manière continue, et donc le modèle du liquide de Fermi n'est plus valable. Il est alors nécessaire de décrire le système en prenant explicitement en compte les corrélations entre électrons, conduisant à des états nouveaux tels l'isolant de Mott ou le cristal de Wigner. Pour ces deux modèles nous nous intéressons aux propriétés de conduction du système électronique, mais également la manière dont le système passe d'un état liquide de Fermi à un état fortement corrélé.

\subsubsection{Isolant de Mott}

Avant de prendre en compte les corrélations entre les électrons en interaction, il est intéressant de mentionner l'effet des interactions dans le cadre de la transition de Mott (voir par exemple [11]).

Considérons un réseau périodique de sites. Lorsque les sites sont suffisamment éloignés, et en l'absence d'interactions entre électrons, les électrons sont bien décrits par des fonctions d'ondes d'états liés sur sites. Toujours dans un cas sans interactions, si maintenant on rapproche les impuretés, il va se former des bandes d'énergie permises correspondant à l'élargissement des états liés (c'est le modèle des liaisons fortes), chaque état de chaque bande étant dégénéré deux fois en raison du spin de l'électron. Dans le cas où chaque site fournit un électron (c'est le cas pour les dopants dans les semiconducteurs), la première bande est à moitié remplie et le système est métallique.

Considérons maintenant les interactions entre électrons, et traitons le cas du premier état lié d'un site. Le premier électron autour de ce site a une énergie $E_{0}$ correspondant au premier état lié. Si on ajoute un deuxième électron autour de ce site, l'énergie de ce deuxième électron est augmentée d'une énergie d'interaction $U_{0}$ en raison de l'interaction Coulombienne. Si maintenant on rapproche les impuretés, les deux niveaux $E_{0}$ et $E_{0}+U_{0}$ s'élargissent, comme décrit dans la figure 1.5. Comme chaque état est maintenant dégénéré une seule fois, lorsque chaque impureté apporte un électron, la bande $E_{0}$ est complètement remplie alors que la bande $E_{0}+U_{0}$ est vide. Cette situation conduit à un état isolant. Si on rapproche encore les impuretés, les bandes d'énergies se croisent, conduisant à une seule bande. Et donc, pour un électron par atome et pour des atomes suffisamment rapprochés on aboutit à un état métallique.

Cette transition métal-isolant, appelée transition de Mott, montre les effets que peuvent avoir les interactions sur l'état d'un système électronique. Il est d'ailleurs important de noter que souvent, dans les expériences, à la fois le désordre et les interactions sont forts. Cette situation conduit à un effet combiné du modèle d'Anderson et de celui de Mott sur les états électroniques, et l'état atteint est souvent appelé "isolant de Mott-Anderson". 


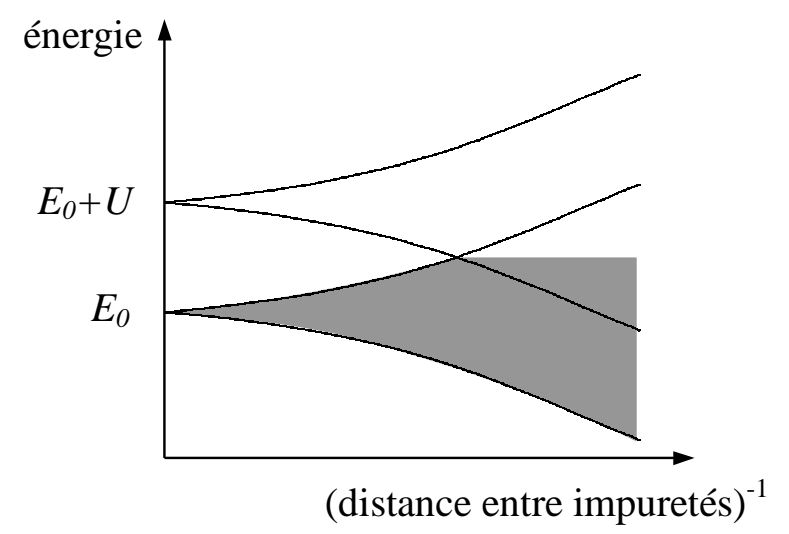

FIG. 1.5: Niveau d'énergie des états électroniques dans le modèle de Mott en fonction de l'inverse de la distance entre impuretés. La partie grisée représente le remplissage, égal à $1 / 2$ pour le modèle avec un électron par atome.

\subsubsection{Cristal de Wigner}

\section{Cristallisation de Wigner}

Dans un modèle d'électrons en interaction sans potentiel extérieur (i.e. sans sites localisés, comme dans le modèle de Mott, ni désordre dans un premier temps), trois énergies entrent en jeu : l'énergie d'interaction Coulombienne $E_{\text {int }}$, l'énergie cinétique $E_{c}$ et l'énergie thermique $k_{B} T$. Lorsqu'on se place à température nulle, l'énergie thermique n'entre pas en jeu, et il y a une compétition entre l'énergie cinétique qui tend à délocaliser les électrons et l'énergie Coulombienne qui tend à les éloigner les uns des autres, et donc à les répartir sur un réseau régulier. La transition qui en résulte a été décrite par Wigner en 1934 [2].

Pour un système d'électrons fortement dégénéré, l'énergie cinétique de confinement des électrons est égale à l'énergie de Fermi $E_{F}$. Lorsque l'énergie d'interaction Coulombienne entre électrons $\left(E_{\mathrm{int}}\right)$ est très grande devant l'énergie cinétique, i.e. lorsque le paramètre $r_{s}$ défini au paragraphe est très grand devant 1, l'interaction Coulombienne domine le comportement des électrons, et le système électronique cherche à minimiser cette énergie d'interaction. Le minimum d'énergie est atteint lorsque les électrons ont l'un par rapport à l'autre une distance maximale, donc lorsqu'ils sont positionnés aux noeuds d'un réseau périodique, d'où l'appellation cristal de Wigner pour un tel état électronique.

Cependant, de par leur nature quantique, si les électrons sont fortement localisés (si $x$ est la position moyenne, les mouvements $\Delta x$ par rapport à cette position moyenne sont faibles), leur énergie cinétique de confinement (proportionnelle à $p^{2}$, où $p$ est l'impulsion) devient très grande : d'après le principe d'Heisenberg, $p \cdot \Delta x \sim \hbar$, donc quand $\Delta x$ est faible, l'impulsion et l'énergie cinétique deviennent grandes, ce qui signifie que les électrons ne peuvent pas être localisés sur une surface aussi petite que l'on veut. La condition pour la formation d'un cristal de Wigner est alors que la taille de confinement minimisant la somme de l'énergie de confinement et l'énergie d'interaction est inférieure à la distance moyenne entre électrons plus proches voisins $a_{0}$. 


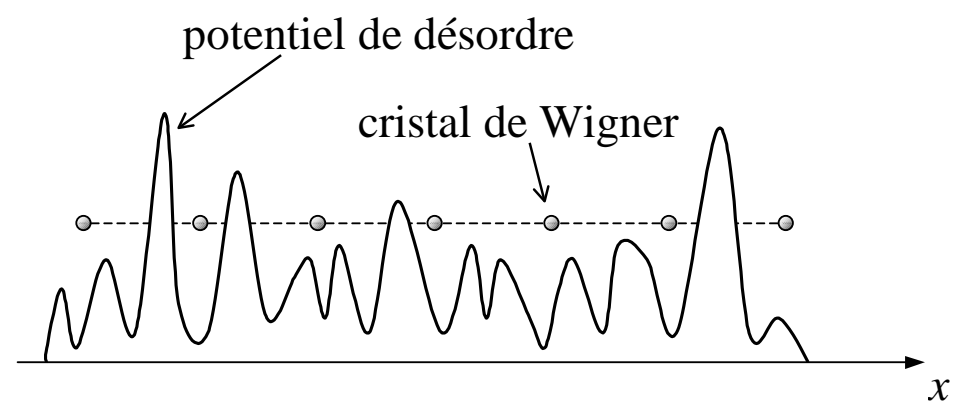

FIG. 1.6: Représentation schématique de l'accrochage d'un cristal d'électrons sur un potentiel de désordre.

\section{Effet du désordre}

On peut maintenant se poser la question de la stabilité d'une telle phase cristalline lorsqu'elle est en présence d'un potentiel de désordre. On définit généralement un cristal comme un système ayant un ordre à longue distance. En dimension trois les cristaux présents dans la nature prouvent qu'un cristal est stable même à température non nulle et en présence d'un faible désordre. Il n'en est pas de même pour un cristal en dimension deux. Il a été montré qu'en dimension deux, la phase ordonnée est détruite par le désordre, aussi petit soit-il (voir par exemple $[12,13]$ ). Cependant, lorsque le désordre est suffisamment faible, le système peut s'organiser en formant de cristallites de taille finie $L_{0}$. Par des études numériques sur des systèmes bidimensionnels d'électrons, Chui et Tanatar [14] ont même montré qu'un faible désordre aide à la localisation des électrons, et donc favorise la formation d'une phase corrélée de type cristal de Wigner, même si ce cristal est en fait un assemblage de cristallites.

On s'attend cependant à ce que la taille des cristallites diminue lorsque le désordre augmente. Ainsi on peut penser que la phase cristal de Wigner ne peut plus exister audelà d'une certaine valeur du désordre (lorsque la taille des cristallites s'approche de la maille du réseau d'électrons). Cette idée est encore compatible avec les études numériques de Chui et Tanatar [14] qui ont montré qu'alors le solide est bien ordonné près de la transition $\left(r_{s} \gtrsim 7,5\right)$, il devient plus désordonné à forte interactions $\left(r_{s} \gtrsim 20\right)$ pour aboutir à un état de verre de Wigner, i.e. un système fortement corrélé mais sans ordre. Le système électronique devient amorphe au-delà d'une transition de percolation due au fait que, lorsqu'on diminue la densité électronique sans modifier le désordre, le nombre d'impuretés par électron augmente, et il arrive un moment où le désordre l'emporte sur les interactions.

\section{Accrochage sur le désordre}

On a donc vu qu'un système d'électrons en interaction peut former une phase ordonnée lorsque les interaction sont suffisamment fortes, et que cette phase est stabilisée par un faible désordre. On s'intéresse maintenant aux propriétés de conduction d'un tel système électronique. En l'absence de désordre, le cristal se déplace sans dissipation, et un tel système a une résistivité nulle. Avec un faible désordre, et lorsque le champ électrique est suffisamment faible, le solide d'électrons est accroché sur le désordre, et le mouvement de translation est bloqué (voir la figure 1.6). 
Il est intéressant de remarquer que ce phénomène d'accrochage n'est possible que si on admet que le cristal n'est pas complètement rigide et qu'il peut se déformer. En effet, considérons un solide d'électrons complètement rigide plongé dans un potentiel de désordre aléatoire. Le calcul de la force de rappel exercée par les impuretés, réparties aléatoirement dans l'espace, sur le solide d'électron revient à un calcul de marche aléatoire. Cette force est proportionnelle à $\sqrt{N_{i}}$, où $N_{i}$ est le nombre d'impuretés, et, pour une densité constante d'impuretés, la force de rappel est donc proportionnelle à $\sqrt{V} \sim L_{0}^{d / 2}$, où $V \sim L_{0}^{d}$ est le volume du système de taille $L_{0}$ en dimension $d$. D'autre part, lorsqu'on applique un champ électrique sur le solide d'électrons, la force d'entraînement exercée par ce champ électrique est proportionnelle au nombre de charges, et donc au volume $V$ pour une densité de charges uniforme : $F_{e}=V n_{s} e E$. Le champ électrique seuil $E_{s}$ au-delà duquel le solide d'électrons peut se déplacer se calcule en égalisant la force de rappel et la force d'entraînement, conduisant à $E_{s} \sim V^{-1 / 2} \sim L_{0}^{-d / 2}$. Donc le champ seuil tend vers zéro quand la surface du système tend vers l'infini, ce qui signifie que ce système ne peut pas être accroché. Ainsi une bonne description du phénomène d'accrochage prend nécessairement en compte l'élasticité du solide d'électrons.

Dans ce cadre, l'accrochage du cristal résulte donc de la compétition entre l'énergie de déformation élastique et l'énergie de rappel due au désordre. De manière qualitative, pour un solide formé dans un domaine de taille $L_{0}$. On a vu que l'énergie gagnée par accrochage varie comme $L_{0}^{d / 2}$. D'autre part l'énergie élastique de ce domaine varie comme $L_{0}^{d-2}$. Ainsi, au-delà d'une certaine taille, l'énergie gagnée par l'accrochage domine l'énergie élastique et le domaine est accroché. On montre ainsi qu'un cristal de Wigner en présence d'un faible désordre est accroché, ce qui conduit à un comportement toujours isolant.

\section{Fusion du cristal de Wigner}

La question qui vient ensuite est le comportement du système lorsqu'on passe la transition entre la phase cristal de Wigner et la phase liquide de Fermi. La majorité des travaux existant concernent la fusion classique d'un cristal, il est donc intéressant de commencer par ces résultats. Nous nous limiterons ici au cas de la dimension deux.

Dans le cas de la fusion classique, l'énergie cinétique des porteurs est négligeable devant l'énergie d'interaction et l'énergie thermique. La fusion est gouvernée par la compétition entre l'énergie d'interaction $E_{\text {int }}$ et l'énergie thermique $k_{B} T$. Il a été montré que, dans un solide, un des effets de la température est de créer des défauts dans le cristal par activation thermique. Pour un cristal en dimension deux, Kosterlitz et Thouless [15], puis Nelson et Halperin [16] et Young [17], se sont intéressé à la création de dislocations dans un cristal (voir la figure 1.7(a)). Les contraintes créées par la dislocation décroissent comme l'inverse de la distance au cœur de la dislocation, donc l'énergie élastique diverge comme le logarithme de la taille du système. D'autre part, comme une dislocation peut se trouver sur n'importe quel site du réseau, l'entropie diverge également comme le logarithme de la taille du système. Il existe donc une température au-dessus de laquelle la création d'une dislocation est spontanée. De plus, alors que l'énergie de création d'une dislocation isolée est infinie pour un système de taille infinie, l'énergie de création d'une paire de dislocations de vecteurs de Burger opposés reste finie (voir la figure 1.7(b)). Kosterlitz et Thouless ont suggéré que la fonte du cristal se produit lorsqu'une petite partie de ces 
(a)

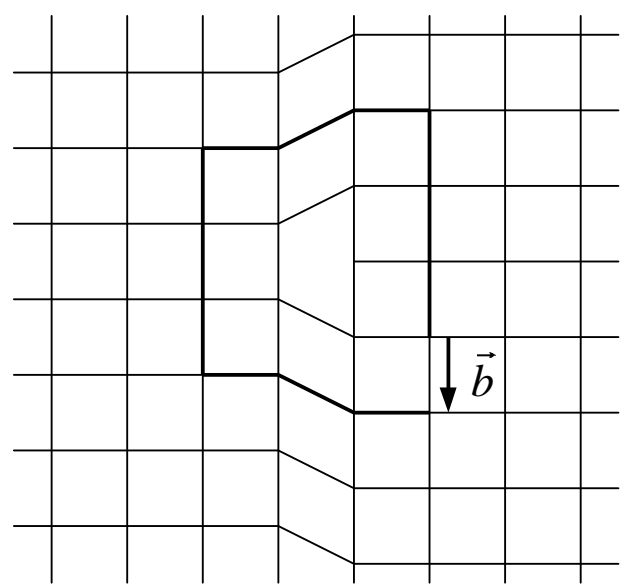

(b)

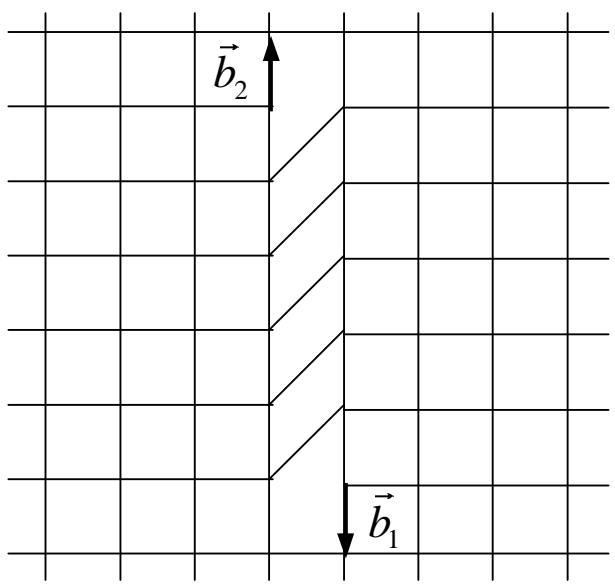

FIG. 1.7: (a) Schéma d'une dislocation dans un réseau carré en dimension deux. La dislocation peut être vue comme la suppression d'une demi-droite d'atomes. Elle est caractérisée par son vecteur de Burgers $\vec{b}$, qui est le vecteur nécessaire pour fermer un chemin composé d'un nombre de pas égaux à gauche, puis en haut, puis à droite et en bas. (b) Paire de dislocations de vecteurs de Burgers $\vec{b}_{1}$ et $\vec{b}_{2}$ opposés.

paires de dislocations se dissocient.

Dans le cas de la fusion quantique, pour laquelle l'énergie thermique est négligeable, il faut considérer la compétition entre l'énergie d'interaction $E_{\text {int }}$ et l'énergie cinétique $E_{c}=E_{F}$. Pour la fonte d'un tel cristal, Chui et Esfarjani ont proposé un mécanisme de création de paires de dislocations analogue au mécanisme de Kosterlitz-Thouless. Dans ce cas ce sont les fluctuations quantiques qui induisent la création de défauts (ici de dislocations) dans le cristal.

\section{5 Électrons en dimension deux}

Avec le développement de composants semiconducteurs tels que le transistor à effet de champ (FET), et les hétérostructures, il a été possible d'étudier expérimentalement des systèmes électroniques strictement bidimensionnels. Dans ces systèmes, le désordre et les interactions jouent un rôle particulier, et on peut se poser la question de la validité du modèle du liquide de Fermi dans ces systèmes. Les systèmes électroniques bidimensionnels ont été largement étudiés dans deux cas extrêmes d'interactions faibles et d'interactions très fortes, comme nous allons le voir dans cette section. Dans les deux cas les modèles ont pu rendre compte raisonnablement des expériences. Mais depuis quelques années sont étudiés des systèmes où désordre et interactions sont en compétition. Dans ces systèmes, les théories développées ne semblent plus pouvoir rendre compte de certains comportement, comme on le verra au chapitre 2 . 


\subsubsection{Particularités de la dimension deux}

\section{Désordre en dimension deux}

En dimension deux, la conductivité $\sigma$ et la conductance $G$ ont la même dimension. C'est également la dimension du quantum de conductance $e^{2} / h$. Ainsi, la conductivité de Drude peut s'écrire :

$$
\sigma_{0}=\frac{n_{s} e^{2} \tau}{m^{*}}=k_{F} l \frac{e^{2}}{h},
$$

avec $n_{s}$ la densité de porteurs par unité de surface, $\tau$ le temps de transport, $m^{*}$ la masse effective des porteurs, $k_{F}$ le vecteur d'onde de Fermi, $l$ le libre parcours moyen. En dimension deux, $\sigma_{0}$ est donc une mesure du désordre. Dans cette approximation, le critère de Ioffe-Regel sur la localisation se réduit à $\sigma_{0}=e^{2} / h$, et le système devient fortement localisé pour $\sigma_{0}<e^{2} / h$.

On a pu voir enfin que la théorie d'échelle pour des électrons indépendants prédit qu'en dimension deux, un système électronique en présence de désordre est toujours isolant. Ainsi, contrairement au cas de la dimension trois, on ne s'attend pas à observer de transition métal-isolant dans de tels systèmes, mais seulement une transition entre un régime de localisation faible lorsque la longueur de localisation $a$ est grande devant la longueur de cohérence de phase $L_{\Phi}$, et un régime de localisation forte lorsque $a<L_{\Phi}$.

\section{Interactions en dimension deux}

En dimension deux, l'énergie de Fermi d'un gaz d'électrons libres est donnée en fonction de la densité surfacique de porteurs $n_{s}$ par :

$$
E_{F}=\frac{\pi \hbar^{2}}{g_{v} m^{*}} n_{s}
$$

où $m^{*}$ est la masse effective des porteurs, et $g_{v}$ est la dégénérescence de vallée du matériau massif. La dégénérescence de spin est ici prise en compte. D'autre part, l'énergie Coulombienne d'interaction pour des électrons distants de $a_{0}=1 / \sqrt{\pi n_{s}}$ ( $a_{0}$ est le rayon de Wigner-Seitz), est :

$$
E_{\text {int }}=\frac{e^{2}}{4 \pi \varepsilon_{0} \varepsilon_{r}} \sqrt{\pi n_{s}},
$$

où $\varepsilon_{r}$ est la constante diélectrique relative du milieu. L'importance des corrélations entre électrons dues à l'interaction Coulombienne peut être évaluée par le rapport entre l'énergie d'interaction et l'énergie de Fermi du gaz d'électrons libres :

$$
r_{s}=\frac{g_{v} m^{*} e^{2}}{4 \pi \varepsilon_{0} \varepsilon_{r} \hbar^{2}} \frac{1}{\sqrt{\pi n_{s}}} \propto \frac{m^{*}}{\varepsilon_{r} \sqrt{n_{s}}} .
$$

Ce quotient peut également s'écrire $r_{s}=a_{0} / a_{B}$, où $a_{B}=\left(4 \pi \varepsilon_{0} \varepsilon_{r} \hbar^{2}\right) /\left(g_{v} m^{*} e^{2}\right)$ est le rayon de Bohr. Le rapport $r_{s}$ croît avec la masse effective des porteurs, et croît lorsque la densité de porteurs décroît, et les effets de corrélations entre électrons sont donc attendus plus favorablement à faible densité de porteurs. 


\subsubsection{Transport en dimension deux}

\section{Limite quasi-classique}

Le traitement quasi-classique du désordre conduit à la conductivité de Drude (voir l'équation (1.18)). Pour un liquide de Fermi, la résistivité de Drude peut se décomposer en deux termes (loi de Matthieusens) :

$$
\rho_{D}(T)=\rho_{i}+\rho_{p h}(T)
$$

où $\rho_{i}$ est la résistivité due à la diffusion des électrons sur les défauts statiques qui perturbent la périodicité du réseau. $\rho_{p h}(T)$ est la résistivité due aux phonons thermiques, qui dépend peu de la concentration en défaut (si celle-ci est faible). De façon empirique il a été montré que, dans les métaux, la résistivité due aux phonons peut se mettre sous la forme :

$$
\rho_{p h}(T) \propto \frac{T}{M \theta_{R}} f\left(\frac{T}{\theta_{R}}\right)
$$

où la fonction $f$ tend vers l'unité à haute température et est proportionnelle à $\left(T / \theta_{R}\right)^{4}$ à basse température. La température $\theta_{R}$ est proche de la température de Debye. Lorsque la température $T \ll \theta_{R}$, le terme $\rho_{p h}$ devient négligeable et la résistivité est dominée par la résistivité résiduelle $\rho_{i}$. Dans la suite, et tout au long de la thèse, on se place à suffisamment basse température pour pouvoir négliger la diffusion par les phonons (donc dans la limite $\left.T \ll \theta_{R}\right)$. Dans la conductivité de Drude (équation (1.18)), le temps de transport et le libre parcours moyens sont alors remplacés par les temps et longueur élastiques $\tau_{e}$ et $l_{e}$, dus uniquement sur les impuretés et défauts statiques.

Dans les systèmes bidimensionnels d'électrons dans les semiconducteurs, on peu citer les sources de diffusion suivantes (voir également Ando et al. [18]) : (a) diffusion sur les impuretés ionisées à l'interface [19]; (b) diffusion sur les rugosités d'interface [19]; (c) diffusion sur le désordre d'alliage [18]; (d) diffusion sur les impuretés dopantes [18]; (e) diffusion sur les impuretés ionisées en profondeur [20]. Nous verrons dans le chapitre 3 quels sont les sources de diffusion dominantes dans nos systèmes.

\section{Corrections quantiques}

A basse température, pour $T<T_{F}$, on a vu que les effets d'interférences quantiques peuvent modifier la conductivité (voir §1.3). La correction de conductivité due à la localisation faible est donnée par l'équation (1.7). Dans le cas où l'échantillon est macroscopique, la longueur $L$ doit être remplacée par la longueur de cohérence de phase $L_{\Phi}$. En régime diffusif, la correction de localisation faible est donc donnée par :

$$
\delta \sigma_{L F}=\alpha \frac{g_{V} e^{2}}{\pi h} \ln \left(\frac{\tau_{e}}{\tau_{\Phi}}\right)
$$

où $g_{V}$ est la dégénérescence de vallée, $\tau_{e}$ le temps de collision élastique ou temps de transport, et $\tau_{\Phi}=L_{\Phi}^{2} / D$ est le temps de cohérence de phase, relié à la longueur de cohérence de phase par le coefficient de diffusion $D=v_{F}^{2} \tau_{e} / 2$, avec $v_{F}$ la vitesse de Fermi (dans un système diffusif). $\alpha$ est une constante valant 1 pour une diffusion sur 
des impuretés (-1/2 pour de la diffusion spin-orbite et 0 pour de la diffusion de spin), mais peut être réduite par des effets comme la diffusion anisotrope (voir par exemple la référence [21]). A suffisamment basse température, il a été montré que la principale source de décohérence est liée aux interactions électron-électron [22]. Le temps de cohérence de phase s'exprime alors en fonction de la température, $\tau_{\Phi} \propto T^{p}$, où $p$ est un exposant proche de 1 [23], ce qui conduit à :

$$
\delta \sigma_{L F}=\alpha p \frac{g_{V} e^{2}}{\pi h} \ln \left(\frac{k_{B} T \tau_{e}}{\hbar}\right) .
$$

Les interactions Coulombiennes entre électrons, prises de façon perturbative, conduisent également à une correction logarithmique de la conductivité en fonction de la température $[24,25]$ :

$$
\delta \sigma_{i n t}=\frac{e^{2}}{\pi h}\left(1-\frac{3 F^{*}}{4}\right) \ln \left(\frac{k_{B} T \tau_{e}}{\hbar}\right),
$$

où $F^{*}$ est le paramètre du liquide de Fermi. Des interactions écrantée par le processus de Thomas-Fermi conduisent à $F^{*}<1$. Mais l'ajustement de courbes expérimentales a montré que ce paramètre peut être supérieur à 1 (voir par exemple Coleridge et al. [21] ou Senz et al. [26]).

L'application d'un faible champ magnétique perpendiculaire au plan du gaz d'électrons permet de séparer la localisation faible des effets d'interactions. En effet, un tel champ est suffisant pour déphaser les trajectoires électroniques et ainsi supprimer les effets d'interférences entre les chemins en boucles décrits dans la figure 1.2 et qui conduisent aux corrections de localisation faible. Par contre un faible champ magnétique n'a pas d'effet sur les corrections dues aux interactions. A faible champ magnétique, le déphasage dû à la localisation faible conduit à une magnétorésistance négative [27] :

$$
\Delta \sigma_{x x}^{L F}(B, T)=\alpha \frac{e^{2}}{\pi h}\left[\psi\left(\frac{1}{2}+\frac{\tau_{B}}{2 \tau_{\phi}}\right)-\psi\left(\frac{1}{2}+\frac{\tau_{B}}{2 \tau_{e}}\right)+\ln \left(\frac{\tau_{\Phi}}{\tau_{e}}\right)\right],
$$

où $\Psi(x)$ est la fonction digamma, $\tau_{\phi}(T)$ est le temps de cohérence de phase, $\tau_{B}=$ $\hbar /(2 e D B)$ le temps magnétique avec $D$ le coefficient de diffusion, $\tau_{e}$ le temps de collision élastique et $\alpha$ est un paramètre phénoménologique décrivant des mécanismes de diffusion additionnels (voir l'équation (1.25)).

Les corrections logarithmiques de la conductivité ont été observées pour la première fois en 1980 dans des films métalliques par Dolan et Osheroff [28] et dans des MOSFETs silicium par Bishop et al. et Uren et al. [29, 30] (voir figure 1.8). La séparation des deux corrections par l'effet d'un champ magnétique a été mesurée par Davies et al. et Uren et al. [31, 32]. Dans les MOSFETs Si, en l'absence de champ magnétique, le terme dominant provient de la localisation faible [32]. Ainsi, les résultats expérimentaux sur des gaz bidimensionnels d'électrons dans les semiconducteurs semblent en bon accord avec la théorie de la localisation (voir $§ 1.3$ ).

\section{Localisation forte}

Lorsqu'on diminue la densité de porteurs, le rapport $k_{F} l_{e}$ devient inférieur à 1 (et la conductivité de Drude devient inférieure à $\left.e^{2} / h\right)$. Expérimentalement, cette étude peut 


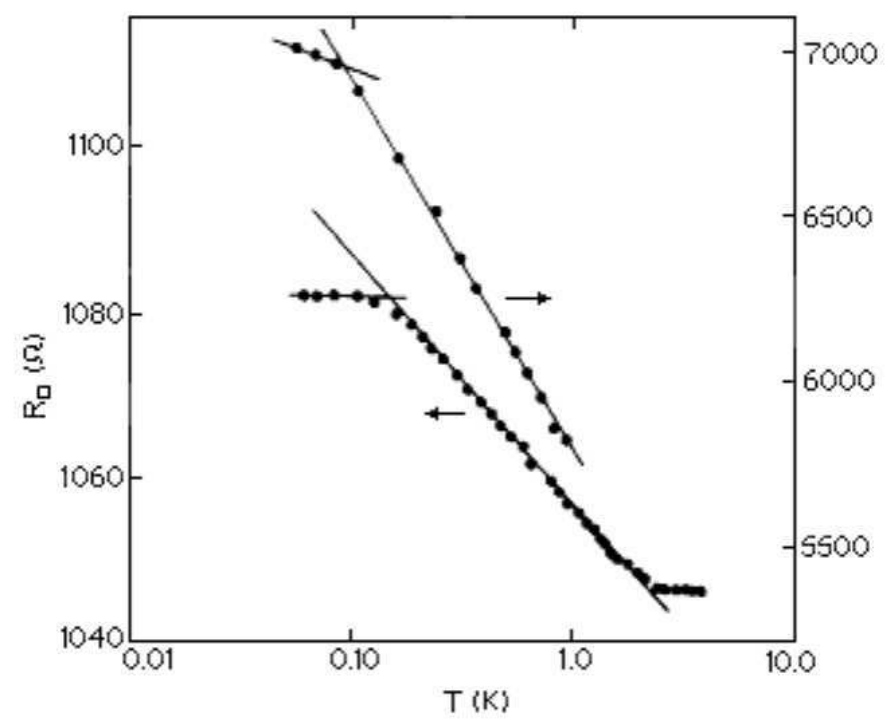

FIG. 1.8: Mesures de résistivité en fonction de la température dans des MOSFETs Si de mobilité $0,2 \mathrm{~m}^{2} / \mathrm{Vs}$, à deux densités électroniques proches de $10^{16} \mathrm{~m}^{-2}$, d'après Bishop et al. [29]. Dans cette échelle semi-log, les dépendances logarithmiques en température sont des droites, comme le montrent les ajustements dans la partie haute température.

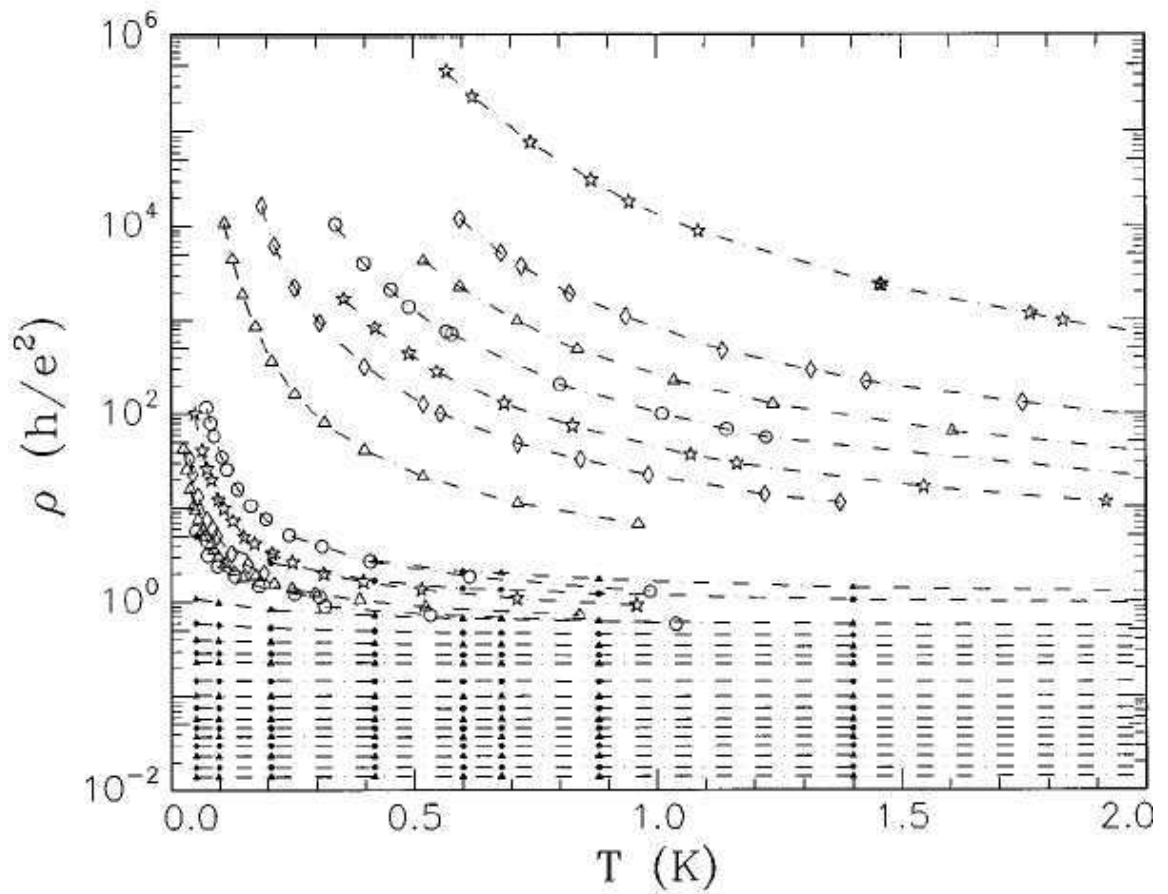

FIG. 1.9: Mesures de résistivité en fonction de la température dans des hétérostructures $n$-GaAs pour différentes valeurs de densités de porteurs, d'après Van Keuls et al. [33]. Les courbes à haute densité électroniques (courbes du bas) présentent une dépendance logarithmique de la résistivité en fonction de la température, caractéristique des corrections quantiques, alors que les courbes à faible densité électronique (courbes du haut) présentent une dépendance exponentielle, caractéristique de la localisation forte. 
être facilement menée dans les MOSFETs où la tension de grille permet de changer directement la densité de porteurs. Dans des MOSFETs Si et à très faible densité, Uren et al. ont noté une augmentation exponentielle de la résistivité quand la température décroît [30]. Cette dépendance est beaucoup plus forte que celle attendue pour la localisation faible, et a été attribuée à la localisation forte des porteurs. Nous verrons au chapitre 4 que, dans le cas d'un isolant d'Anderson (ou de Mott-Anderson), pour lesquels les électrons sont localisés sur des sites, les mécanismes de conduction envisagés conduisent effectivement à une divergence exponentielle de la résistivité à basse température.

La transition entre les régimes de localization faible et de localization forte a été étudiée dans des hétérostructures $n$-GaAs par Van Keuls et al. [33] par des mesures de longueurs de localisation dans les régimes de localisation faible et de localisation forte (voir la figure 1.9). La longueur de localisation diminue continûment quand on passe d'un régime de localisation forte vers un régime de localisation faible quand la densité d'électrons augmente, contrairement aux systèmes 3D présentant une divergence de la longueur de localisation à la transition métal-isolant. Ce comportement observé dans les hétérostructures $n$-GaAs est donc en accord avec la théorie d'échelle de la localisation, et montre que, dans ces systèmes bidimensionnels, il n'y a pas de transition métal-isolant.

\subsubsection{Effet d'un fort champ magnétique}

Le régime à fort champ magnétique ne sera pas étudié pendant cette thèse. Cependant il est intéressant de le mentionner ici pour deux raisons. La première est l'effet du désordre dans le régime d'effet Hall quantique qui conduit à des états localisés, et à l'observation de transitions métal-isolant en champ magnétique dans ces systèmes bidimensionnels. Nous verrons la seconde application d'un champ magnétique élevé dans le paragraphe suivant, traitant du cristal de Wigner en dimension deux, car c'est un moyen pour observer la cristallisation de Wigner dans les systèmes bidimensionnels dans les semiconducteurs.

\section{Effet Hall quantique}

En présence d'un champ magnétique, les tensions longitudinale et transverse $U_{x}$ et $U_{y}$ s'expriment en fonction des courants longitudinaux et transverses $I_{x}$ et $I_{y}$ par l'intermédiaire des tenseurs de résistivité et de conductivité :

$$
\left(\begin{array}{c}
U_{x} \\
U_{y}
\end{array}\right)=\left(\begin{array}{cc}
\rho_{x x} & \rho_{x y} \\
-\rho_{x y} & \rho_{x x}
\end{array}\right)\left(\begin{array}{c}
I_{x} \\
I_{y}
\end{array}\right) \quad \text { et } \quad\left(\begin{array}{c}
I_{x} \\
I_{y}
\end{array}\right)=\left(\begin{array}{cc}
\sigma_{x x} & \sigma_{x y} \\
-\sigma_{x y} & \sigma_{x x}
\end{array}\right)\left(\begin{array}{c}
U_{x} \\
U_{y}
\end{array}\right) \text {. }
$$

Dans l'approximation semi-classique (valable pour $\hbar \omega_{c} \ll E_{F}$, où $\omega_{c}=e B / m^{*}$ est la pulsation cyclotron et $E_{F}$ est l'énergie de Fermi), on montre que [6] :

$$
\sigma_{x x}(B)=\frac{\sigma_{0}}{1+\mu^{2} B^{2}} \quad \text { et } \quad \sigma_{x y}(B)=\mu B \sigma_{x x}(B)
$$

où $\sigma_{0}$ est la conductivité de Drude à champ magnétique nul, $\mu=\sigma_{0} / n_{s} e$ est la mobilité.

L'application d'un champ magnétique suffisamment fort $\left(\hbar \omega_{c} \gg E_{F}\right)$ conduit à la formation de niveaux de Landau (voir la figure 1.10 de gauche), et donne lieu à l'effet 


$$
B=0
$$

sans désordre

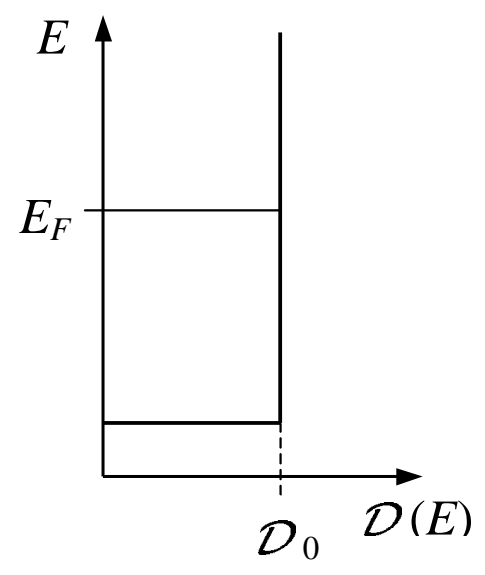

$$
B=0
$$

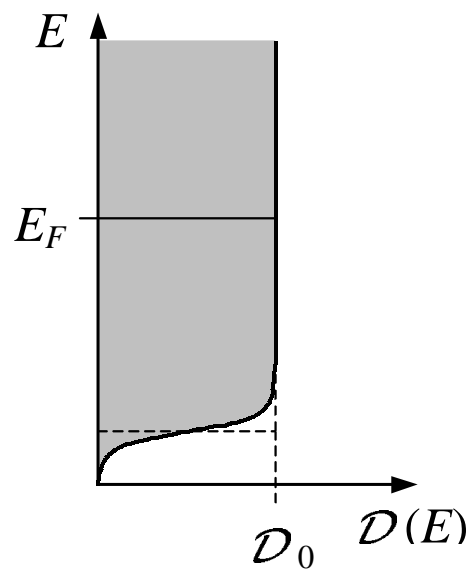

$B$ élevé



$B$ élevé

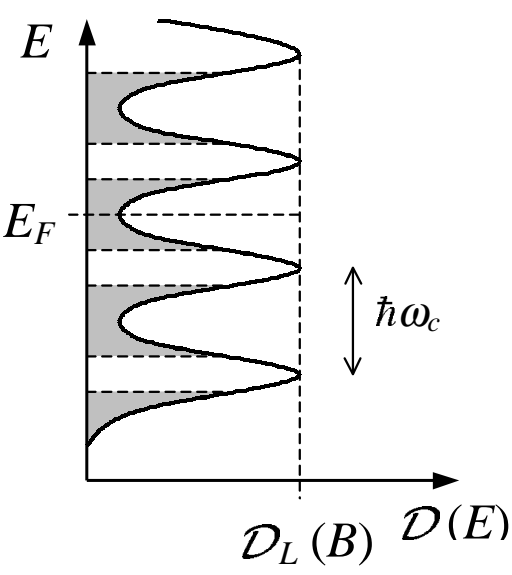

FIG. 1.10: Schéma de la densité d'états $\mathcal{D}(E)$ en fonction de l'énergie $E$, en dimension deux à champ magnétique nul et à champ magnétique élevé, avec formation des niveaux de Landau, pour un système sans désordre et un système désordonné. Les parties blanches (ainsi que les niveaux de Landau) correspondent à des états délocalisés, et les parties grisées correspondent à des états localisés par le désordre. Dans le cas des niveaux de Landau, l'énergie de Fermi correspond à un remplissage entier $\nu=2$ (2 niveaux de Landau remplis).

Hall quantique entier. Lorsque le remplissage des niveaux de Landau, $\nu$, est entier, le niveau de Fermi se trouve entre deux niveaux de Landau, la résistivité longitudinale $\rho_{x x}$ tend vers zéro et la résistivité transverse est quantifiée :

$$
\rho_{x y}=\frac{1}{\nu} \frac{h}{e^{2}}
$$




\section{Isolant de Hall}

En présence de désordre, les niveaux de Landau sont élargis pour donner des bandes. Lorsque le désordre est suffisamment fort, cet élargissement conduit à un continuum d'états sans réellement de bande d'énergie interdite entre états, comme le montre la figure 1.10 (en bas à droite). Cependant, les états en milieu de bande (correspondant à $\nu$ demientier) sont étendus [34], contrairement aux états entre niveaux (autour de $\nu$ entier) qui sont localisés. Ainsi on voit apparaître dans cette structure des bords de mobilité séparant états localisés et états délocalisés, caractéristique d'un système avec une bande interdite de mobilité.

Lorsque le remplissage est proche d'un remplissage demi-entier, le niveau de Fermi est dans une bande d'états délocalisés. Lorsque le remplissage est proche du remplissage entier, le niveau de Fermi est situé dans une bande d'états localisés, conduisant à l'isolant de Hall. Entre ces deux situations on s'attend à observer des transitions, qui ont été observées expérimentalement dans des MOSFETs Si et des hétérostructures GaAs (voir par exemple Shashkin et al. [35, 36]), et ont permis de tracer le diagramme de phase $n_{s}-B$ présenté dans la figure 1.11. Ce diagramme présente donc une successions de phases isolantes (parties grises) et métalliques (parties blanches). Il est alors possible de passer ces transitions de deux manières. La première en variant le champ magnétique : on augmente alors la dégénérescence de chaque niveau de Landau, et le niveau de Fermi passe d'un état localisé à un état étendu, puis inversement. Mais on peut également modifier la densité électronique, conduisant à l'observation de mêmes transitions. Dans ce dernier cas il est possible de faire passer le niveau de Fermi en dessous du premier niveau de Landau, conduisant à l'isolant basse densité de la figure 1.11. Les expériences semblent indiquer que les transitions entre état métallique et état isolant sont des transitions de percolation [36].

Dans cette approche, on s'attend à retrouver une phase isolante à champ magnétique nul. Ainsi la ligne de transition métal-isolant à basse densité doit tendre vers $n_{s}$ infini quand le champ magnétique tend vers zéro (ligne pointillé dans la figure 1.11). Nous verrons dans le chapitre 2 que cette vision n'est cependant pas complètement admise, et certaines expériences suggèrent que cette ligne de transition reste à densité finie à champ magnétique nul (ligne pleine dans la figure 1.11). Cette suggestion conduirait alors à l'existence d'un bord de mobilité dans un système bidimensionnel à champ magnétique nul, comme il a été proposé dans une étude théorique par Azbel [37], et qui contredit la théorie d'échelle de la localisation.

\subsubsection{Cristallisation de Wigner}

L'existence d'une phase collective de type cristal de Wigner a été observée expérimentalement dans la limite classique, pour laquelle l'énergie quantique (de point zéro) des électrons est négligée, et c'est pourquoi les résultats obtenus dans cette limites sont tout d'abord décrits. Ensuite nous verrons le cas de la limite quantique, qui nous intéresse plus particulièrement pour les gaz bidimensionnels d'électrons dans les semiconducteurs. En l'absence de champ magnétique, l'observation du cristal de Wigner est encore controversée, en raison principalement du désordre présent dans ces systèmes. Mais il existe une 




FIG. 1.11: Diagramme de phase hypothétique densité de porteurs $\left(n_{s}\right)$ - champ magnétique $(B)$ pour un système bidimensionnel d'électrons, déduit des mesures sur des MOSFETs Si et des hétérostructures (voir par exemple $[35,36]$ ). Pour simplifier, on n'a pas représenté tout le diagramme dans la région d'effet Hall quantique fractionnaire (une étude plus détaillée peut être trouvée dans la thèse de Perruchot [38]). La phase "métallique" (en blanc dans ce diagramme) est encore controversée. D'autre part la ligne de transition à basse densité et à faible champ magnétique n'est pas encore bien déterminée (notamment entre la ligne pleine qui reste finie et la ligne en pointillés qui diverge à faible champ magnétique). D'autre part l'existence du cristal de Wigner à basse densité a été observée à champ magnétique élevé, mais pas à faible champ magnétique.

alternative : en appliquant un champ magnétique suffisamment élevé il est possible de réduire fortement l'énergie cinétique des électrons et dans ce régime les mesures semblent indiquer l'existence d'une phase collective.

\section{Limite classique}

La transition de Wigner classique a été observée dans le cas d'électrons confinés à la surface de l'hélium. L'absence de défauts sur le substrat permet d'atteindre des mobilités très élevées $\left(\mu \sim 10^{3} \mathrm{~m}^{2} \cdot \mathrm{V}^{-1} \cdot \mathrm{s}^{-1}\right)$. En raison de la très faible densité de porteurs $\left(n_{s} \sim\right.$ $\left.10^{12} \mathrm{~m}^{-2}\right)$, l'énergie de Fermi est négligeable devant l'énergie thermique et le système électronique subit une transition vers un solide d'électrons quand la température devient inférieure à la température de fusion $T_{f}$. La cristallisation intervient lorsque le rapport entre l'énergie d'interaction et l'énergie thermique devient supérieure à un certain seuil :

$$
\Gamma=\frac{E_{\mathrm{int}}}{k_{B} T}=\frac{e^{2} \sqrt{\pi n_{s}}}{4 \pi \varepsilon_{0} \varepsilon_{r} k_{B} T}>\Gamma_{f},
$$


conduisant à une température de fusion $T_{f}=e^{2} \sqrt{\pi n_{s}} /\left(\Gamma_{f} 4 \pi \varepsilon_{0} \varepsilon_{r} k_{B}\right)$.

Expérimentalement, la phase cristal de Wigner à la surface de l'hélium a été observée pour la première fois par Grimes et Adams [39], et le seuil de fusion a été mesuré par Deville [40], $\Gamma_{f}=127 \pm 3$. Deville et al. [41] et Glattli et al. [42] ont ensuite montré, par des mesures de spectroscopie radiofréquences, que la cristallisation peut être décrite par la transition continue de Kosterlitz-Thouless (i.e. par la création de dislocations libres voir $\S 1.4 .2)$ : en effet, le module de cisaillement est bien décrit par le modèle de KosterlitzThouless, et la chaleur spécifique ne présente pas de changement brutal à la transition, caractéristique d'une transition continue (voir aussi Glattli et al. [43]). Typiquement, dans les systèmes étudiés, la température de fusion peut varier entre $700 \mathrm{mK}$ (électrons su l'hélium à $n_{s} \approx 10^{12} \mathrm{~m}^{-2}$ ) et $200 \mathrm{mK}$ (électrons dans les semiconducteurs à fort champ magnétique pour $n_{s} \approx 10^{14} \mathrm{~m}^{-2}$ ).

\section{Limite quantique}

Pour la transition de Wigner dans la limite quantique, on s'attend à une transition lorsque le rapport entre l'énergie d'interaction et l'énergie de Fermi dépasse un certain seuil :

$$
r_{s}=\frac{E_{\mathrm{int}}}{E_{F}}=\frac{g_{v} m^{*} e^{2}}{4 \pi \varepsilon_{0} \varepsilon_{r} \hbar^{2} \sqrt{\pi n_{s}}}>r_{w},
$$

conduisant à une densité critique en-dessous de laquelle on s'attend à observer la cristallisation de Wigner $n_{w}=\frac{1}{\pi}\left(g_{v} m^{*} e^{2}\right)^{2} /\left(4 \pi \varepsilon_{0} \varepsilon_{r} \hbar^{2} r_{w}\right)^{2}$.

Tanatar et Ceperley [44] ont calculé les énergies des états solide et fluide dans un gaz bidimensionnel d'électrons par des méthodes Monte-Carlo. Ils ont montré que le système électronique subit une transition de l'état fluide vers l'état solide pour $r_{s} \geq r_{w}=37 \pm 5$. Comme il a été noté au $\S 1.4 .2$, le désordre facilitant la localisation, $r_{w}$ peut varier en présence de désordre. Les simulations Monte-Carlo effectuées par Chui et Tanatar [14] donnent effectivement une réduction du paramètre critique en présence de désordre. Ils ont obtenu $r_{w} \approx 7,5$ en choisissant des paramètres correspondant aux expériences de Pudalov et al. [45] sur des MOSFETs Si.

La limite quantique de la cristallisation de Wigner ne peut pas être observée expérimentalement pour les électrons à la surface de l'hélium en raison de la faible densité de porteurs permise par la rigidité de la surface de l'hélium. Il a donc été envisagé d'observer une telle phase dans des gaz bidimensionnels d'électrons formés dans des MOSFETs silicium où dans des hétérostructures. Pour les gaz d'électrons dans GaAs, en raison de la faible masse effective des porteurs et de la constante diélectrique élevée $\left(m^{*}=0,067 m_{0}\right.$, où $m_{0}$ est la masse des électrons, et $\left.\varepsilon_{r}=13\right)$, il est nécessaire d'atteindre de très faible densités de porteur pour voir la cristallisation prédite par Wigner : $n_{w} \approx 3 \times 10^{12} \mathrm{~m}^{-2}$. Ces densités sont inaccessibles dans les systèmes fabriqués actuellement. Par contre, dans les MOSFETs silicium, la masse effective des porteurs est beaucoup plus élevée, la constante diélectrique est plus faible $\left(m^{*}=0,19 m_{0}, \varepsilon_{r}=7,7\right)$ et la dégénérescence de vallée $g_{v}=6$. Ainsi, on peut s'attendre à une transition de Wigner à des densités accessibles. Malgré certaines indications données par les mesures de transport et de bruit (voir par exemple Kravchenko et al. [46] et Pudalov et al. [45]), l'existence du cristal de Wigner dans ces systèmes n'est pas encore établie. Une discussion plus détaillée sera donnée dans le chapitre 


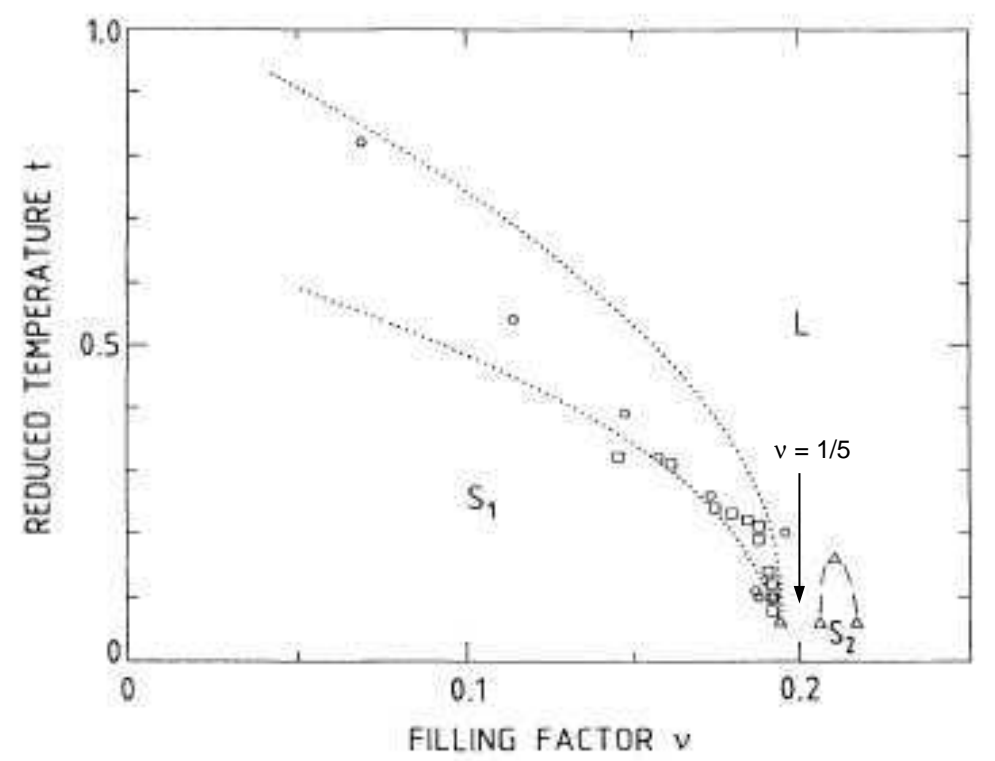

FIG. 1.12: Diagramme de phase du cristal de Wigner induit par le champ magnétique en fonction du remplissage des niveaux de Landau, $\nu$, et de la température réduite, $t$, montrant la phase liquide $\mathrm{L}$, la phase solide de Wigner $\mathrm{S}_{1}$, et la phase rentrante solide $\mathrm{S}_{2}$, d'après Williams et al. [48]. Les transitions sont déduites des mesures de tension seuil dans la caractéristique $I-V$ (voir $\S 4.3)$ pour plusieurs échantillons. La température réduite $t$ est le rapport entre la température et la température de fusion à $\nu=0$, et permet de tester l'universalité de ce diagramme de phase pour les différents échantillons.

2 .

\section{Limite quantique extrême}

L'existence du cristal de Wigner dans une hétérostructure ou un MOSFET à champ magnétique nul reste donc controversée. On dispose cependant d'un moyen pour augmenter la probabilité d'observer une phase très corrélée dans ces systèmes, c'est en appliquant un champ magnétique élevé. A suffisamment fort champ magnétique, lorsque le facteur de remplissage $\nu \ll 1$, les électrons sont confinés sur les orbites cyclotron, conduisant à un gel de l'énergie cinétique. Il a été montré par Lozovic et Yudson qu'en raison de l'interaction Coulombienne, ce mécanisme conduit à l'arrangement des centres des orbites cyclotron suivant un réseau périodique [47], et donc à la formation d'un cristal de Wigner induit par le champ magnétique, pour $\nu<\nu_{w}$. Cette transition en fonction de champ magnétique est indiquée dans le diagramme de phase $n_{s}-B$ de la figure 1.11. Lorsque le champ magnétique devient infini $(\nu \rightarrow 0)$, le système est supposé se comporter classiquement, avec une température de transition vers un cristal de Wigner égale à celle obtenue pour les électrons sur l'hélium, $T_{f}$.

Un certain nombre de mesures expérimentales suggèrent l'existence d'une phase cristal de Wigner induite par le champ magnétique dans des hétérostructures GaAs/AlGaAs et des MOSFETs silicium : des mesures de transport en fonction de la température par Willet et al. [49], des mesures de transport en fonction du champ électrique par Willet 
et al. [50] et Williams et al. [48], des mesures de bruit par Goldman et al. [51], Li et al. [52] et Kukushkin et al. [53], des mesures de spectroscopie radiofréquences [54], des mesures magnéto-optiques par Buhmann et al. [55] et Kukushkin et al. [53], des mesures de conductivité AC par Paalanen et al. [56]. Les mesures de spectroscopie Par des mesures simultanées de transport et de spectroscopie radiofréquences, Williams et al. [48] ont déterminé la formation de la phase ordonnée pour $\nu<\nu_{w}=0,192 \pm 0,004$, et ont tracé le diagramme de phase du cristal de Wigner à fort champ magnétique, avec un état de Hall quantique fractionnaire reentrant à $\nu=1 / 5$ (voir figure 1.12). Les différentes techniques ayant permis la mise en évidence de cette phase cristal de Wigner induite par le champ magnétiques sont décrites dans la thèse de François Perruchot [38, P. 66].

Ces observations suggèrent l'existence d'une phase collective accrochée à fort champ magnétique. Cependant, le mécanisme de fusion de la phase cristal de Wigner induit par le champ magnétique n'est toujours pas établi (voir Glattli et al. [43]). Ces considérations seront revues plus en détail dans le chapitre 4. De plus ils reste des interrogations sur une phase cristal de Wigner en présence de désordre : est-ce vraiment une phase corrélée ordonnée, où observe-t-on un état corrélé désordonné de type verre?

\subsection{Conclusion}

Nous avons donc vu les propriétés d'un système électronique en présence de désordre ou en fortes interactions. Dans le cas où l'un domine sur l'autre, les modèles théoriques semblent satisfaisant pour expliquer, au moins qualitativement, les résultats expérimentaux. Si l'on prend en compte le désordre, même avec de faibles interactions entre porteurs, ou un système fortement corrélé avec un faible désordre, ces deux limites conduisent à un état isolant, i.e. dont la conductivité s'annule à température nulle. On s'attend donc à retrouver cette caractéristique sur les systèmes expérimentaux. Les transitions entre les différentes phases isolantes peuvent être représentées dans un diagramme de phase désordre-interaction, comme le montre la figure 1.13.

Cependant ce diagramme de phase pose plusieurs questions qui n'ont pas été abordées dans cette partie :

- Quelle est la nature de la phase verre de Wigner (ou verre de Coulomb), pour laquelle on sait que le désordre et les interactions doivent tous les deux jouer un rôle important. Dans cette limite les calculs perturbatifs en interaction ou en désordre ne sont plus valables : un domaine nouveau est ouvert.

- On a vu qu'une transition entre le liquide de Fermi et le cristal de Wigner est attendue pour une valeur de $r_{s}$ proche de 37 . On peut se demander si la théorie du liquide de Fermi reste encore valable pour un paramètre $r_{s}$ compris entre 1 et 37, ou si une phase intermédiaire corrélée peut apparaître avant la cristallisation de Wigner, comme l'ont suggéré Benenti, Waintal et Pichard [3].

Ces questions sur le rôle de la compétition entre désordre et interactions en dimension deux est le sujet principal des études faites autour de la transition métal-isolant en dimension deux, dont une revue est donnée dans le chapitre 2 . 


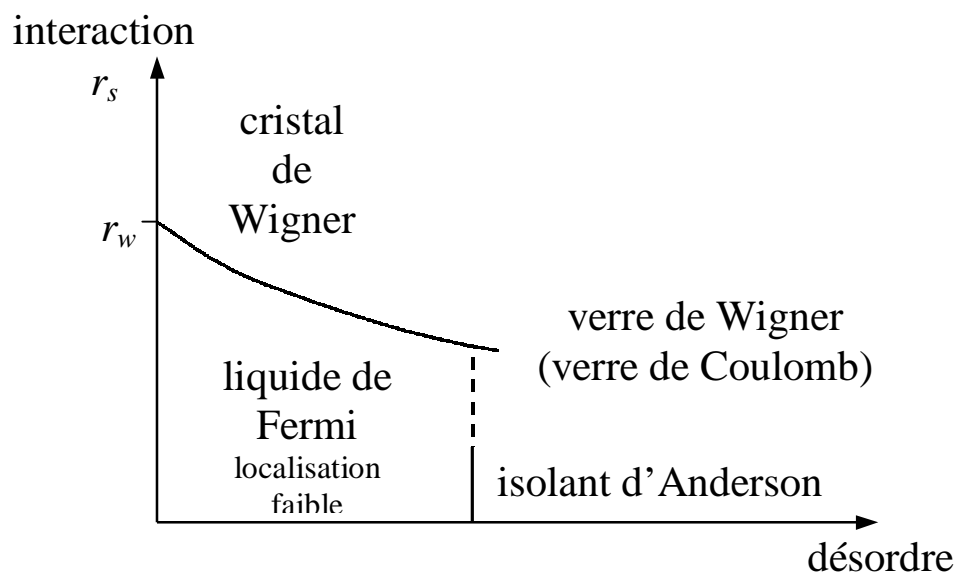

FIG. 1.13: Diagramme de phase désordre-interactions supposé pour un système d'électrons en dimension deux. 


\section{Chapitre 2}

\section{La transition métal-isolant en dimension deux}

\section{Sommaire}

2.1 Introduction . . . . . . . . . . . . . . . 48

2.2 Comportement métallique inattendu . . . . . . . 48

2.3 Observations expérimentales ............ 52

2.3.1 Transport à champ magnétique nul . . . . . . . . . . 52

2.3.2 Magnéto-transport . . . . . . . . . . . . 57

2.3.3 Mesures dans des systèmes originaux . . . . . . . . . . . 61

2.3.4 Autres observables . . . . . . . . . . . . . . . 63

2.4 Phénoménologie de la transition métal-isolant . . . . . . 64

2.4.1 Transition de phase quantique . . . . . . . . . 65

2.4.2 Origine quantique de la phase métallique . . . . . . . . . . 71

2.4.3 Rôle des interactions entre électrons . . . . . . . . . . 72

2.4.4 Rôle du désordre . . . . . . . . . . . . . . . . . 73

2.4.5 Rôle du spin . . . . . . . . . . . . . . . . . 75

2.4.6 Rôle d'un champ magnétique perpendiculaire . . . . . . 78

2.5 Interprétations théoriques de la transition métal-isolant • 78

2.5.1 Effet des interactions sur un système désordonné . . . . . 79

2.5.2 Cristallisation de Wigner . . . . . . . . . . . . . . . 80

2.5.3 Supraconductivité en dimension deux . . . . . . . . 84

2.5.4 Modèles de percolation . . . . . . . . . . . . 84

2.5.5 Interprétations classiques du comportement "métallique" . 87

2.6 Conclusion . . . . . . . . . . . . . . 92 


\subsection{Introduction}

Ce chapitre est une revue expérimentale de ce qui a été communément appelé la transition métal-isolant en dimension deux et à champ magnétique nul. Ce chapitre est important pour comprendre les interrogations, l'enthousiasme ou le rejet qu'a provoqué ce sujet dans le domaine de la matière condensée. De manière plus fondamentale, ce sujet pose le problème de l'état d'un système électronique à basse densité, dans lequel les interactions entre électrons ne peuvent plus être négligées.

Nous verrons quelles ont été les premières indications d'un comportement inexpliqué dans un certain nombre de systèmes. Après une description générale des résultats expérimentaux les plus marquants (voir §2.3), nous verrons plus particulièrement quelles indications permettent d'être déduites de ces résultats (voir §2.4). Enfin seront exposées plusieurs théories qui ont essayé d'interpréter le caractère nouveau des mesures expérimentales (voir $\S 2.5$ ).

\subsection{Comportement métallique inattendu}

\section{Premières observations}

Depuis le développement des théories sur la localisation (voir §1.3) et les mesures réalisées dans les années 80 sur des films métalliques et des MOSFETs Si (voir §1.5.2), il était couramment admis qu'un système d'électrons en dimension deux est toujours isolant. Dans ce contexte, les mesures de Kravchenko et al. [5] sur des gaz bidimensionnels d'électrons formés à l'interface entre l'isolant et le semiconducteur d'un MOSFET silicium en régime de forte inversion, et montrant un comportement métallique à basse température, ont soulevé de nombreuses questions. Les auteurs présentent des mesures de résistivité en fonction de la température pour plusieurs valeurs de la densité électronique $n_{s}$ (voir la figure 2.1-a). A haute densité électronique, la résistivité décroît quand la température décroît, et comme aucune remontée de la résistivité n'est observée aux températures les plus basses, on peut supposer que la résistivité reste finie à température nulle. Cette caractéristique est typique d'un état métallique, pour lequel les fonctions d'onde électroniques seraient délocalisées à température nulle (voir §1.2.2). Pour des valeurs faibles de la densité électronique, la résistivité diverge quand la température diminue, et semble tendre vers l'infini à température nulle, ce qui est caractéristique d'un comportement isolant. Enfin il existe une valeur définie de la densité d'électrons, notée $n_{c}$ (densité critique), à laquelle est observé ce changement de comportement, et pour laquelle la résistivité ne varie quasiment pas dans une certaine gamme de température.

Cette observation a posé de nombreuses questions. D'une part elle semble remettre en cause la théorie d'échelle de Abrahams et al. [4] (voir §1.3.3) qui exclut tout état métallique en dimension deux pour des électrons sans interaction. D'autre part, des caractéristiques analogues avaient déjà pu être observées pour d'autres transitions métal-isolant : la transition métal-isolant d'Anderson en dimension trois (voir par exemple la revue de Belitz et Kirkpatrick [57]), la transition supraconducteur-isolant dans des films minces (voir par exemple la revue de Goldman et Marković [58]) et la transition effet Hall quantique-isolant (voir par exemple Sondhi et al. [59]). Dans tous les cas, le passage de l'état isolant à l'état 


\begin{tabular}{|c|ccc|ccc|c|}
\hline système & $\begin{array}{c}m^{*} \\
\left(\text { en unité } m_{0}\right)\end{array}$ & $\varepsilon_{r}$ & $\begin{array}{c}\mu_{\max } \\
\left(\mathrm{m}^{2} / \mathrm{Vs}\right)\end{array}$ & $\begin{array}{c}n_{c} \\
\left(10^{15} \mathrm{~m}^{-2}\right)\end{array}$ & $r_{s, c}$ & $\begin{array}{c}\rho_{c} \\
\left(h / e^{2}\right)\end{array}$ & réf. \\
\hline MOSFET Si $n$ & 0,19 & 7,7 & 7,1 & 0,96 & 8,5 & 2 & {$[5]$} \\
& 0,19 & 7,7 & $\approx 1$ & 1,65 & 6,5 & $1,5-2$ & {$[60]$} \\
\hline$p$-SiGe & 0,4 & 13 & 0,11 & 2,2 & 7 & 0,5 & {$[63]$} \\
& $0,2-0,32$ & 13 & 1 & $\approx 1$ & 13 & 0,5 & {$[64]$} \\
\hline$p$-GaAs & 0,38 & 13,2 & 15 & 0,125 & 27 & 1,2 & {$[61]$} \\
& 0,3 & 13,2 & 25 & 0,51 & 11 & 0,5 & {$[62]$} \\
\hline$n$-AlAs & 0,46 & 10 & 7,7 & 0,7 & 18,5 & 2 & {$[65]$} \\
\hline$n$-GaAs ${ }^{\mathrm{a}}$ & 0,067 & 13,2 & 220 & 0,13 & 4,6 & 0,4 & {$[66]$} \\
\hline
\end{tabular}

${ }^{\text {a }}$ Contrairement au hétérostructures $n$-GaAs habituelles dont la croissance est faite sur un substrat GaAs orienté (100), la croissance de cette hétérostructure $n$-GaAs est faite sur un substrat orienté (311)A, technique utilisée pour faire croître les hétérostructures $p$-GaAs présentées ci-dessus.

TAB. 2.1: Paramètres des principaux systèmes bidimensionnels de porteurs (électron $n$, ou trous $p)$ présentant une transition métal-isolant. $\varepsilon_{r}$ est la constante diélectrique relative, $m^{*}$ la masse effective des porteurs, $\mu_{\max }$ la mobilité maximale, $n_{c}$ la densité critique déterminée par l'inversion de pente des courbes de résistivité en fonction de la température, $r_{s, c}$ le paramètre $r_{s}$ déterminé à la transition, et $\rho_{c}$ est la résistivité à la transition.

métallique est signé par un changement de comportement de la résistivité du système en fonction de la température. Les observations de Kravchenko et al. ont donc constitué un argument en faveur de l'existence d'une transition métal-isolant dans un système électronique en dimension deux et à champ magnétique nul.

\section{Observations dans différents systèmes}

Dans les quelques années qui ont suivi les expériences de Kravchenko et al., le comportement métallique de la résistivité en fonction de la température a tout d'abord été confirmé dans d'autres MOSFETs Si par Popović et al. [60], puis dans d'autres systèmes : des gaz bidimensionnels (G2D) de trous dans des hétérostructures GaAs/AlGaAs ( $p$ GaAs) par Hanein et al. [61] et Simmons et al. [62], des G2D de trous dans des hétérostructures $\mathrm{SiGe} / \mathrm{Si}(p-\mathrm{SiGe})$ par Lam et al. [63] et Coleridge et al. [64], des G2D d'électrons dans des hétérostructures AlAs/GaAs (n-AlAs) par Papadakis et al. [65], et des G2D d'électrons dans des hétérostructures GaAs/AlGaAs ( $n$-GaAs) par Hanein et al. [66]. Des exemples de ces observations sont reportés dans la figure 2.1, et une liste plus complète des systèmes étudiés avec leurs propriétés est donnée dans le tableau 2.1.

Il est intéressant de constater que ce comportement métallique est observé dans des systèmes variés, pour lesquels le gaz d'électrons est obtenu de différentes manières, par effet de champ dans le cas des MOSFETs, par dopage dans le cas des hétérostructures. De par son caractère universel, il a été suggéré que ce comportement métallique nouveau est une propriété générale des G2D d'électrons (et de trous), et non une propriété particulière des échantillons utilisés. 
(a)

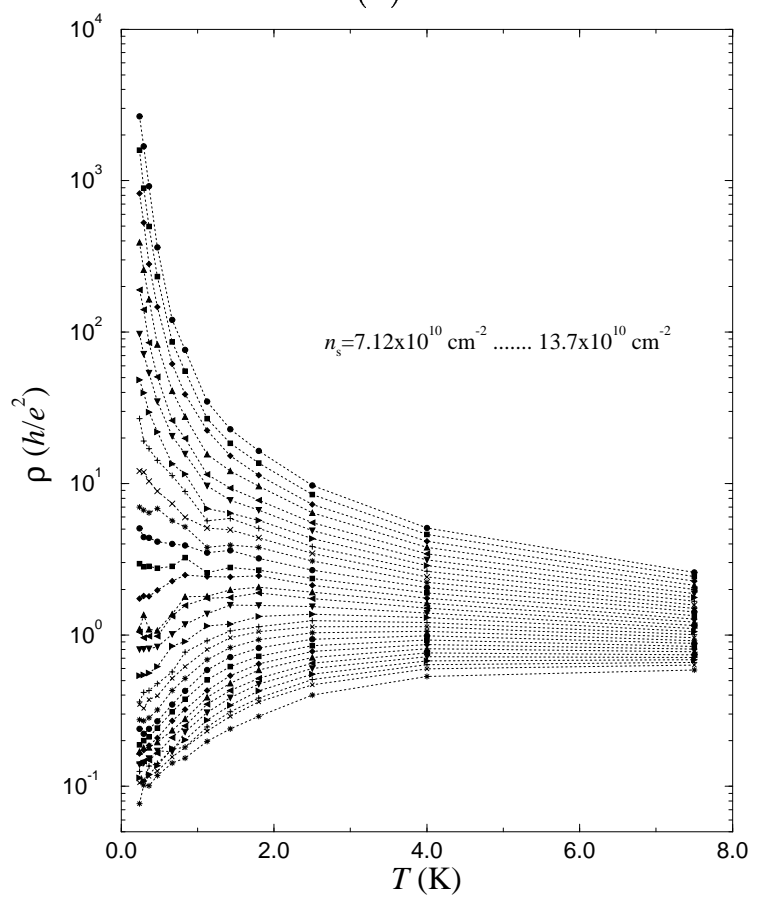

(c)



(b)

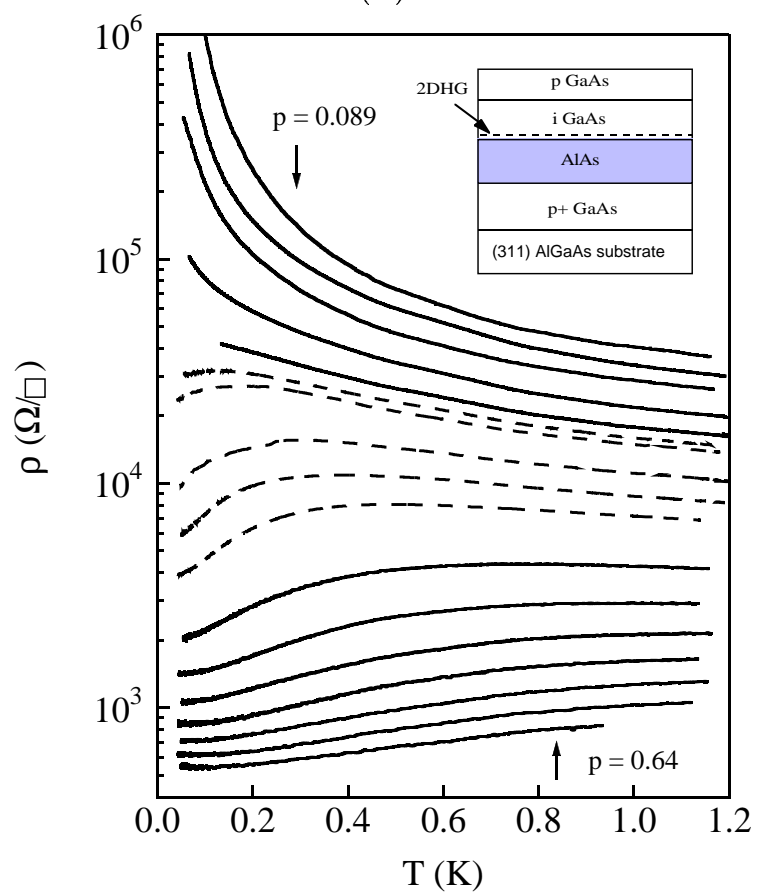

FIG. 2.1: Mesures de résistivité en fonction de la température pour des systèmes présentant un caractère métallique $(d \rho / d T>0)$. (a) dans un MOSFET Si [67], (b) dans une hétérojonction $p$-GaAs [61] et (c) dans un puits quantique $p$-SiGe [64]

\section{Rôle des interactions entre porteurs}

La première question posée par ces mesures est la raison pour laquelle un tel comportement est observé dans certains systèmes et pas dans d'autres. Tous les systèmes décrits dans le tableau 2.1 présentent deux caractères communs. Le premier est la faible densité de porteurs $n_{s}$. Le deuxième est la masse effective élevée des porteurs. On peut noter en particulier l'observation d'un fort comportement métallique dans des hétérostructures $p$-GaAs, alors que ce comportement est très faible, ou même absent, dans des hétérostruc- 
tures $n$-GaAs. La masse effective des trous est cinq fois plus élevée que la masse effective des électrons dans ce semiconducteur. Ainsi, si on calcule le paramètre $r_{s}$ donnant le rapport entre l'énergie d'interaction Coulombienne entre électrons et l'énergie cinétique (voir $\S 1.5)$ :

$$
r_{s}=\frac{E_{\text {int }}}{E_{F}} \propto \frac{m^{*}}{\sqrt{n_{s}}}
$$

ce paramètre est plus élevé dans les systèmes présentant un comportement métallique. On peut donc supposer que les interactions entre électrons jouent un rôle dans ce comportement nouveau.

Une autre remarque suggérant le rôle important des interactions est le comportement métallique, inattendu en dimension deux d'après la théorie d'échelle des systèmes désordonnés d'électrons indépendants (voir §1.3.3). Une manière de comprendre cette violation de la théorie d'échelle est d'introduire une nouvelle phase due aux interactions entre électrons.

\section{Faible densité et faible désordre}

L'autre paramètre important dans ce domaine est le désordre. Pour obtenir un système d'électrons de suffisamment basse densité électronique, et pour lequel les fonctions d'onde ne sont pas fortement localisées, il est nécessaire que le désordre soit faible. En effet, nous avons vu au chapitre 1.3 que, d'après le critère de Ioffe-Regel, les états électroniques sont fortement localisés pour $k_{F} l_{e}<1$ (on remarquera que $k_{F} l_{e}$ n'est en fait plus défini dans cette limite). Le temps de collision élastique $\tau_{e}$ est le temps qui caractérise le désordre, et il est relié au libre parcours moyen par $l_{e}=v_{F} \tau_{e}$, où $v_{F}$ est la vitesse de Fermi. En dimension deux, $v_{F}$ et $k_{F}$ sont proportionnels à la densité d'électrons $n_{s}$. Sur la figure 2.2 sont tracées la dépendance de $k_{F} l_{e} \propto n_{s}{ }^{2}$ en fonction de la densité électronique pour différentes valeurs du temps de collision. On montre ainsi que, dans des systèmes de faible désordre (pour lesquels le temps de collision est grand), la densité minimale avant d'observer la localisation forte est plus faible que dans les systèmes de fort désordre. Le fait de pouvoir atteindre une densité plus faible dans ces systèmes peu désordonnés montre qu'il est possible d'atteindre un nouveau régime pour lequel le facteur $r_{s}$ est plus élevé.

Les échantillons dans lesquels a pu être observé un comportement métallique de la résistivité présentent tous des mobilités élevées (pic de mobilité supérieur à $0,5 \mathrm{~m}^{2} \cdot \mathrm{V}^{-1} \cdot \mathrm{s}^{-1}$ ). Ces mobilités sont notamment plus élevées que celles obtenues dans les échantillons où seule la localisation avait été observée (voir §1.5.2).

\section{Validité du liquide de Fermi en dimension deux}

La question fondamentale est de savoir si le comportement nouveau est lié aux interactions entre électrons, ou s'il peut être expliqué dans le cadre de la théorie du liquide de Fermi. On a vu au paragraphe 1.2.3 que la théorie de Landau des liquides de Fermi est une théorie phénoménologique, et qu'elle ne permet pas de connaître son domaine de validité. Ainsi seule la confrontation avec l'expérience peut permettre de valider ou non cette théorie. On sait qu'en dimension trois l'hypothèse du liquide de Fermi est encore valable pour décrire un système électronique avec un paramètre $r_{s}$ supérieur à un. Est-ce encore le cas 


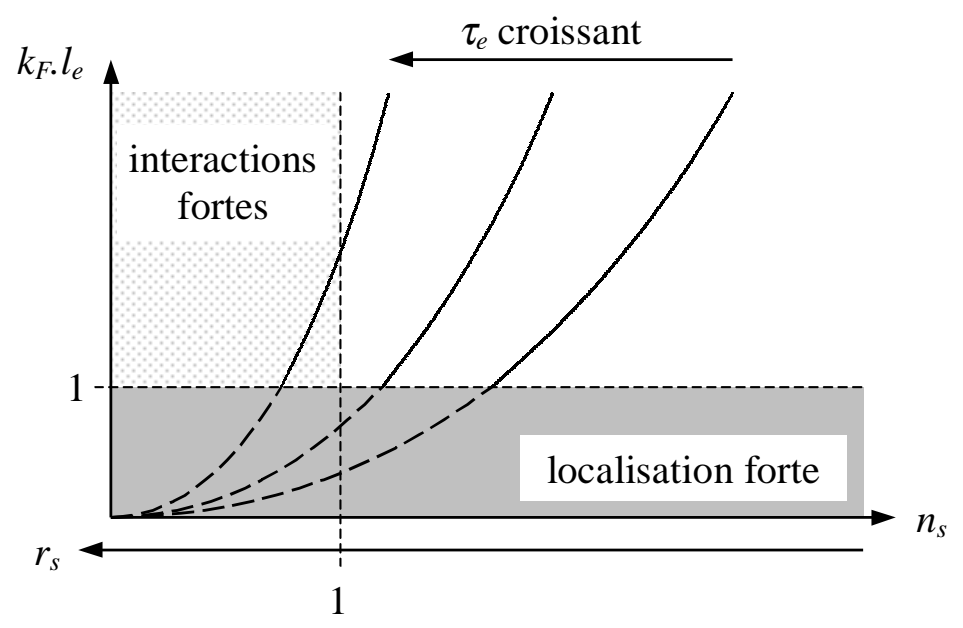

FIG. 2.2: Produit $k_{F} l_{e}$, caractérisant le désordre, en fonction de la densité électronique $n_{s}$, ou du paramètre $r_{s}$, pour différentes valeurs du libre parcours moyen $l_{e}$ dans le cas d'un système bidimensionnel d'électrons. On distingue différents régimes : le domaine de localisation forte est donné par le critère de Ioffe-Regel $k_{F} l_{e}<1$, le domaine d'interactions fortes est donné par $r_{s}>1$.

en dimension deux? Cette question montre que l'étude de la transition métal-isolant ne se réduit pas au seul problème de la nouvelle phase métallique, mais est relié au problème de la validité de la description d'un système bidimensionnel par la théorie du liquide de Fermi.

Pour interpréter les expériences, et tout au long des sections suivante, nous verrons que deux points de vue opposés peuvent être adoptés. Dans le premier le système est supposé décrit dans le cadre de la théorie du liquide de Fermi. Dans le second le système ne peut plus être décrit dans ce cadre, et il est nécessaire d'introduire une nouvelle phase.

\subsection{Observations expérimentales}

La grande majorité des mesures faites sur les gaz bidimensionnels de porteurs à faible densité sont des mesures de transport. A champ magnétique nul, il peut être étudié en fonction de trois paramètres, la densité de porteurs, la température, et le champ électrique appliqué pour la mesure. Il est également possible d'appliquer un champ magnétique, soit parallèle au plan formé par le gaz d'électron, conduisant à un effet sur le spin électronique, soit perpendiculaire à ce plan, ce qui induit des effets orbitaux. Les mesures de transport ne sont cependant pas suffisantes pour décrire toute la physique de ces systèmes. Des informations complémentaires ont pu être déduites de mesures thermodynamiques comme la compressibilité ou le pouvoir thermoélectrique, ou dynamiques comme les mesures de bruit.

\subsubsection{Transport à champ magnétique nul}

\section{Transport dans la phase métallique}

La caractéristique principale du comportement métallique (pour $n_{s}>n_{c}$ ) est la diminution de la résistivité $\rho$ lorsque la température $T$ diminue, i.e. $d \rho / d T>0$. Dans la majorité des systèmes étudiés, la résistivité sature à basse température. Le plus surprenant est alors qu'il n'y a pas d'indication de remontée de la résistivité à basse température, ce 
(a)

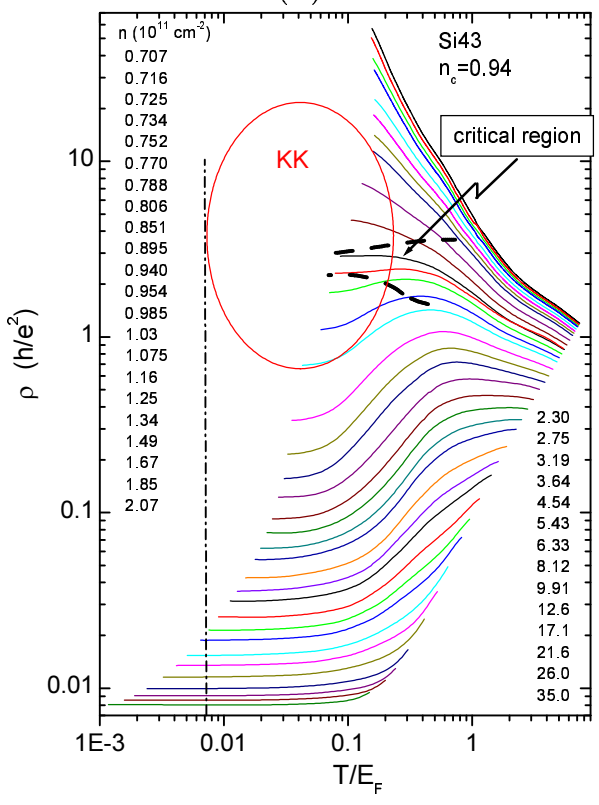

(b)

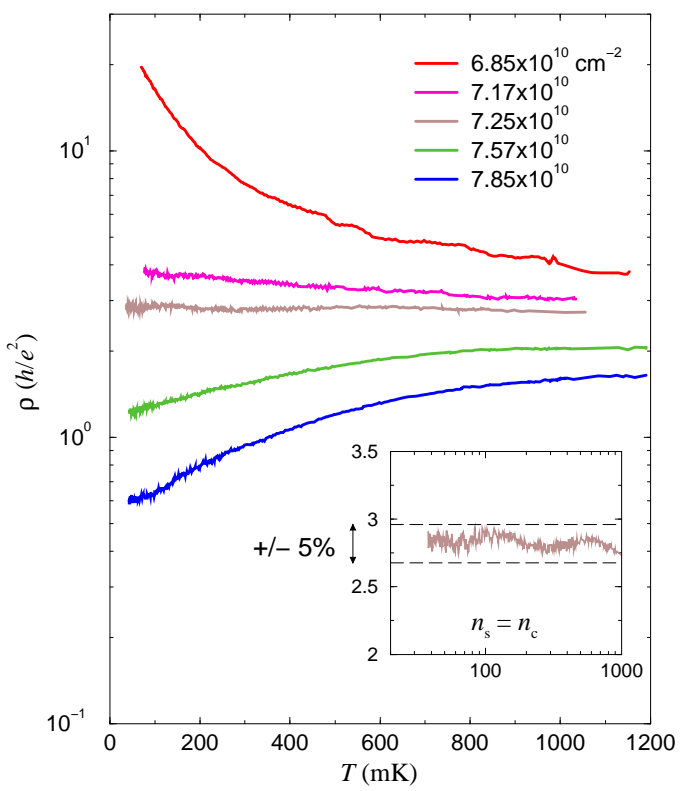

FIG. 2.3: Mesures de la résistivité en fonction de la température dans des MOSFETs Si pour différentes densités électroniques. (a) Mesure dans une large gamme de densités électroniques de $0,707 \times 10^{15} \mathrm{~m}^{-2}$ (courbe du haut) à $35,0 \times 10^{15} \mathrm{~m}^{-2}$ (courbe du bas), d'après [68]. (b) Mesure autour de la transition, pour une gamme de densités et de températures correspondant à la région entourée dans la figure de droite, d'après [69]. Il faut noter que ces deux résultats n'ont pas été obtenus sur le même échantillon, mais pour deux échantillons de mobilités et de densités critiques proches.

qui indique donc une absence de localisation. Dans des MOSFETs silicium, des mesures réalisées jusqu'à $35 \mathrm{mK}$ montrent même une diminution continue de la résistivité, sans saturation à basse température [69] (voir la figure 2.3(b)), alors que la température est très inférieure à la température de Fermi $\left(T<0,01 T_{F}\right)$ et que le rapport $k_{F} l_{e}$ est proche de 1. Dans ces conditions un effet de localisation important est attendu, mais est supplanté ici par le comportement métallique. Le comportement métallique est observé en dessous d'une température caractéristique $T^{*} \approx 2 \mathrm{~K} \approx 1 / 3 T_{F}$ dans les MOSFETs Si [69], où $T_{F}$ est la température de Fermi (voir la figure 2.3(a)). Cette température tend vers zéro quand on s'approche de la densité critique $n_{c}$. Dans les hétérostructures $p$-GaAs, Gao et al. ont noté que $T^{*}$ est également proche de $1 / 3 T_{F}$, mais ne semble pas varier avec la densité de porteurs [70]. Au-dessus de $T^{*}$, toutes les courbes présentent un comportement "isolant" $(d \rho / d T<0)$, qui peut être interprété par la transition entre le régime fortement dégénéré (pour $T \ll T_{F}$ ) et le régime non dégénéré (pour $T>T_{F}$ ).

En l'absence de théorie définitive pour décrire le comportement métallique, la dépendance en température a été étudiée par des lois empiriques. Pudalov a suggéré comme ajustement des courbes métalliques une loi activée de la forme [71] :

$$
\rho(T)=\rho_{0}\left(n_{s}\right)+\rho_{1}\left(n_{s}\right) \exp \left[-\left(\frac{T_{0}\left(n_{s}\right)}{T}\right)^{\gamma}\right]
$$


où $\rho_{0}, \rho_{1}$ et $T_{0}$ sont des paramètres indépendants de la température, mais dépendants de la densité, et $\gamma>0$ est un exposant, souvent choisi égal à 1. Cette loi rend compte de la chute de résistivité quand la température diminue, puis de la saturation à basse température à une valeur $\rho_{0}$ lorsque $T \ll T_{0}$. L'ajustement devient non valable à haute température, quand $T \gtrsim T_{0}$. Dans les MOSFETs Si [71], et les hétérostructures $p$-GaAs [61, 72], $n$-AlAs [65] et $n$-GaAs [66], $\gamma \approx 1$, ce qui est caractéristique d'un mécanisme de diffusion activé avec une énergie d'activation $E_{a}=k_{B} T_{0}$. Dans le $p$-SiGe, un exposant $\gamma \approx 0,5$ donne un meilleur résultat [64], ce qui rend compte de la dépendance plus faible de la résistivité en fonction de la température dans ce système.

Dans les hétérostructures $p$-GaAs et $n$-AlAs, il a été montré que les paramètres $\rho_{0}$ et $\rho_{1}$ décroissent quand la densité augmente [65,72], et le rapport $\rho_{1} / \rho_{0}$ décroît avec la densité de porteurs, traduisant le fait que la chute de résistivité est plus marquée à basse densité, i.e. proche de la transition. $\rho_{0}$ et $\rho_{1}$ divergent lorsque la densité se rapproche de la densité critique [61]. Hanein et al. [73] obtiennent en particulier $1 / \rho_{0}=\sigma_{0} \propto\left(n_{s}-n_{s}^{0}\right)$ avec $n_{s}^{0}$ proche de la densité critique $n_{c}$. Ce terme linéaire en $n_{s}$ peut s'interpréter comme une conductivité de Drude $\sigma_{D}=n_{e f f} e^{2} \tau / m^{*}$, où $n_{e f f}=n_{s}-n_{s}^{0}$ serait une densité effective de porteurs participant à la conduction. Enfin, très près de la transition, Coleridge et al. trouvent $\rho_{1}=\rho_{c}=0,5 h / e^{2}[64]$.

Dans les hétérostructures $p$-GaAs et $n$-AlAs, $T_{0}$ a une dépendance linéaire en $n_{s}$, et s'extrapole linéairement à 0 pour $n_{s}=0[61,74,65]$. La température de Fermi dépend également linéairement de $n_{s}$, et il est donc possible de calculer le rapport $T_{0} / T_{F}$, qui vaut environ 0,2 dans $p$-GaAs [74] et 0,1 dans $n$-AlAs [65]. Cela montre l'importance de ces deux grandeurs, $T_{0}$ et $T_{F}$, dans l'étude de la phase métallique.

Mills et al. ont étendu l'ajustement donné par l'équation 2.2 à haute densité en ajoutant la contribution des phonons [72] :

$$
\rho(T)=\rho_{0}+\rho_{e l}(T)+\rho_{p h}(T) .
$$

Cette formule est une extension de la loi de Matthieusens, avec $\rho_{0}$ la résistivité due aux impuretés et $\rho_{p h}$ la résistivité due aux phonons, mais en prenant en compte un terme supplémentaire de diffusion dû aux interactions entre électrons $\rho_{e l}$. Ce dernier terme est déduit expérimentalement de la dépendance $\rho(T)$ après extraction des termes dus aux phonons et aux impuretés :

$$
\rho_{e l}(T)=\rho_{1}\left(n_{s}\right) g(x) \exp \left[-\frac{T_{0}}{T}\right] \quad \text { avec } \quad g(x)=\frac{1}{x}\left(\frac{1}{\alpha^{2}}+\frac{1}{x^{2}}\right)^{-1 / 2},
$$

où $x=T / T_{0}$ et $\alpha$ est un paramètre. Cette relation est compatible avec la loi activée (2.2) $(\gamma=1)$ à basse température, et décroît en $1 / T$ à haute température. Dans cette description, à haute densité de porteurs $\left(>1,5 \times 10^{14} \mathrm{~m}^{-2}\right)$, la dépendance métallique de la résistivité est due aux phonons. Par contre, à basse densité de porteurs (près de la transition), le terme dû aux phonons est négligeable, et la dépendance métallique ne peut être expliquée que par le terme supplémentaire $\rho_{e l}$.

Des mesures réalisées dans une large gamme de densités électroniques dans des MOSFETs Si [68, 75, 76] (voir figure 2.3(a)) montrent un caractère identique aux expériences de Mills et al. [72], avec un comportement métallique observé à densité de porteurs élevée 
(jusqu'à $3,5 \times 10^{16} \mathrm{~m}^{-2}$ ) et donc pour un paramètre $r_{s}$ faible. Dans ce régime, les interactions ne permettent pas d'interpréter la dépendance en température de nature métallique, qui serait alors plutôt due à la diffusion par les phonons.

\section{Transport dans la phase isolante}

A basse densité électronique (pour $n_{s}<n_{c}$ ), la résistivité croît quand la température diminue $(d \rho / d T<0)$, caractéristique d'un comportement isolant. Dans ce régime, plusieurs théories peuvent rendre compte d'un tel comportement (ces théories seront vues en détails dans le chapitre 4), et conduisent de manière générale à une dépendance de la forme :

$$
\rho(T)=\rho_{0}\left(n_{s}, T\right) \exp \left[\left(\frac{T_{0}\left(n_{s}\right)}{T}\right)^{m}\right],
$$

où $\rho_{0}\left(n_{s}, T\right), T_{0}\left(n_{s}\right)$ et $m$ sont des paramètres. Nous verrons au chapitre 4 que les modèles de conduction par sauts pour des électrons indépendants localisés permettent de prévoir une telle dépendance en température, avec $m=1$ pour une conduction par sauts entre plus proches voisins, $m=1 / 3$ pour une conduction par sauts à portée variable (de Mott) et $m=1 / 2$ pour une conduction par sauts à portée variable avec bande interdite de Coulomb, dû aux effets des interactions entre électrons (Efros-Shklovskii). Le préfacteur $\rho_{0}$ dépend en général de la température $T$ suivant une loi de puissance, donc beaucoup moins rapidement que l'exponentielle.

Des mesures réalisées sur des MOSFETs silicium [67, 77, 78, 79], et dans des hétérostructures $p$-SiGe $[64,63]$ et $p$-GaAs [62] ont montré que, à faible densité $n_{s}$, la loi décrite dans l'équation (2.5) est bien suivie avec un exposant $m=1 / 2$ (pour $T \lesssim T_{0} / 4$ chez Kravchenko et al. [67]). L'exposant est donc celui attendu pour des sauts à portée variable en présence d'une bande interdite due aux interactions Coulombiennes entre électrons. Cependant ce comportement n'est pas propre aux systèmes présentant la transition métal-isolant puisqu'elle a été observée dans des hétérostructures $n$-GaAs ne présentant pas de comportement métallique [80]. Pour des courbes proches de la transition, Hamilton it et al. ont montré une dépendance plus faible en température, permettent d'ajuster les courbes avec $m=1 / 3$, et donc une loi de sauts à portée variable de Mott. A très basse densité de porteurs et à basse température, les mesures sur des MOSFETs Si conduisent à une loi simplement activée avec $m=1$ [45, 81, 82, 83].

On peut cependant noter que l'analyse des courbes $\rho(T)$ n'est pas toujours facile à faire en raison de la faible gamme de température explorée (en générale inférieure à une décade). Par exemple, Altshuler et al. [68] ont montré qu'il est possible d'ajuster les courbes obtenues par Kravchenko et al. [69] sur des MOSFETs silicium avec indifféremment un exposant $m=1 / 2$ ou $m=1 / 3$, sans pouvoir différencier le mécanisme le plus exact.

Le pré-facteur $\rho_{0}$ de la loi (2.5) obtenu sur les MOSFETs Si [67, 77, 79] est proche de $h / e^{2}$ et indépendant de la température et de la densité. Dans les hétérostructures $p$-SiGe $[63,64]$ et $p$-GaAs [62], $\rho_{0} \approx 0,5 h / e^{2}$ est indépendant de la densité. Ces observations sont incompatibles avec un mécanisme où les sauts sont engendrés par l'interaction électronphonon, pour lesquels on s'attend à une dépendance de $\rho_{0}$ en fonction de la température et de la densité, et peut être attribuée à un autre mécanisme de sauts lié à l'interaction 
électron-électron, confirmant là encore le rôle important des interactions dans les systèmes étudiés.

La température caractéristique $T_{0}$ décroît quand la densité augmente, et s'annule à une densité proche de la transition métal-isolant. En particulier, dans les MOSFETs Si, $T_{0}\left(n_{s}\right)$ est proportionnel à $\left|n_{s}-n_{c}\right|^{\beta}$, i.e. l'écart par rapport à la densité critique, avec $\beta \approx 1,6$ $[5,67,84]$. Dans les lois de sauts à portée variable, il est possible de relier la longueur de localisation à $T_{0}$ (voir le chapitre 4 ), et on montre alors que la longueur de localisation diverge quand $n_{s}$ se rapproche de $n_{c}$. Cette divergence n'est pas prévue par la théorie d'échelle de la localisation, qui prévoit une longueur de localisation variant continûment entre le régime de localisation forte et le régime de localisation faible (voir §1.5.2).

Les mesures présentant un comportement activé montrent que l'énergie d'activation décroît linéairement quand la densité diminue, et s'annule également à une valeur de la densité proche de $n_{c}[45,81,82,83]$. Cette observation avait déjà été faite par Shashkin et al. sur des MOSFETs Si [35] et des hétérostructures n-GaAs [36] à champ magnétique élevé dans le cadre des transition effet Hall quantique-isolant, mais également à champ magnétique nul. Dans le cadre des transitions effet Hall quantique-isolant, les transitions observées sont interprétées comme des transitions de percolation, et l'annulation de $T_{0}$ correspond alors à la transition.

\section{Comportement réentrant à haute densité}

Il a été observé sur des échantillons de faibles mobilité que, à haute densité de porteurs $\left(n_{s} \gtrsim 10 \times n_{c}\right)$, le comportement des courbes à basse température redevient isolant, avec une dépendance logarithmique caractéristique de la localisation faible. Ce comportement a été observé aussi bien dans des MOSFETs Si [76] que dans des hétérostructures $p$-GaAs [85], et est similaire au comportement observé dans des systèmes de densité électronique élevée [86] $\left(n_{s} \approx 10^{16} \mathrm{~m}^{-2}\right)$. Ce comportement isolant à haute densité peut être interprété comme une réentrance de la phase isolante observée à très basse densité, la phase métallique étant alors une phase intermédiaire.

\section{Transport non linéaire}

Les dépendances de la résistivité en températures sont réalisées dans la limite de faible champ électrique $(E \rightarrow 0)$, ou régime ohmique. Plusieurs mesures ont montré que la résistivité varie également fortement en fonction du champ électrique, conduisant à de fortes non-linéarités de la caractéristique $I-V$.

La figure 2.4(a) montre les dépendances en champ électrique de la résistivité dans le cas d'un MOSFET Si. Pour les densités de porteurs supérieures à la densité critique, $d \rho / d E>0$, ce qui est caractéristique d'un comportement métallique, et $d \rho / d E<0$ pour une densité de porteur inférieure à la densité critique, soit un comportement isolant. Il est à noter que, contrairement à la dépendance en température, les dépendances en champ électrique sont toujours monotones, comme il a été vu sur les MOSFETs Si [45, 5] et sur $p$-GaAs [87].

Dans des hétérostructures $p$-GaAs et à très basse densité, Yoon et al. [87] mesurent de très fortes non linéarités (voir la figure 2.4(b)). A la plus basse température, la caracté- 
(a)

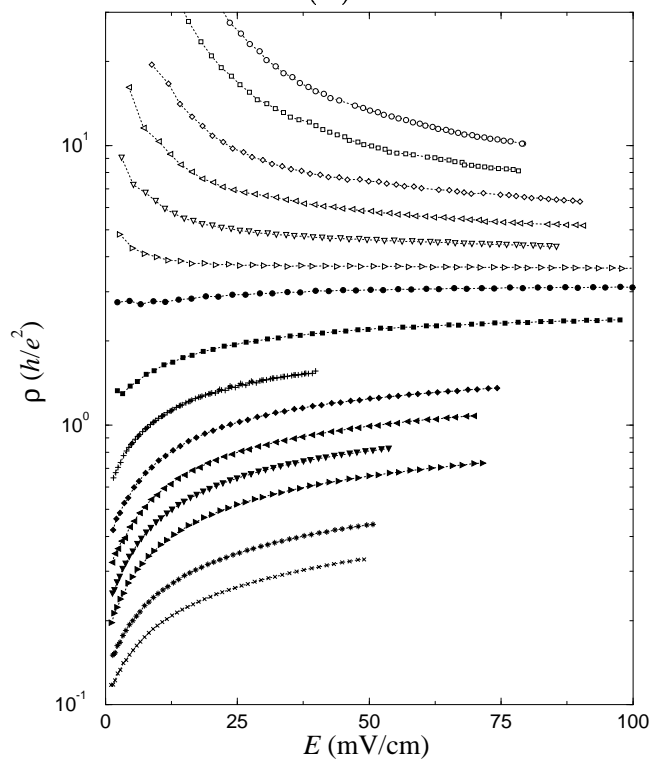

(b)

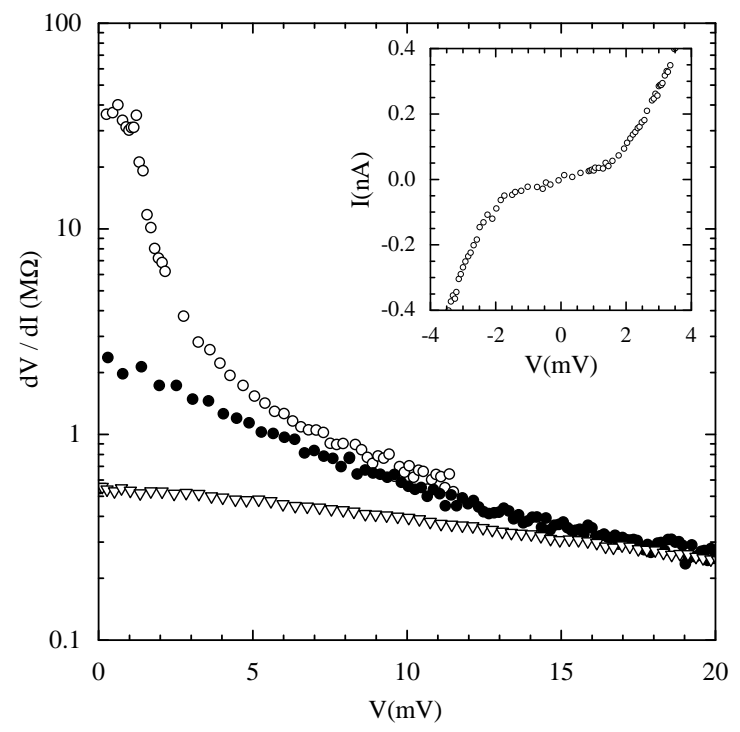

FIG. 2.4: Mesures de résistivité en fonction du champ électrique. (a) Dans le cas d'un MOSFET Si à différentes densités électroniques, d'après Kravchenko et al. [84]. (b) Dans le cas d'une hétérostructure $p$-GaAs dans la phase isolante à différentes températures, $T=50$ (ronds vides), 145 (ronds pleins), et $284 \mathrm{mK}$ (triangles), d'après Yoon et al. [87]. Encart : caractéristique $I-V$ pour la plus basse température, $T=50 \mathrm{mK}$.

ristique $I-V$ présente une tension seuil $V_{s}$ en-dessous de laquelle la courbe est linéaire, et au-dessus de laquelle ils observent une forte augmentation du courant (voir l'encart de la figure 2.4(b)). Cette caractéristique est analogue à celle obtenue sur les MOSFETs Si par Pudalov et al. [45] et Shashkin et al. [81], et a été interprétée comme le décrochage d'une phase collective. La tension seuil varie avec la densité, suivant une loi $\left(n_{s}-n_{c}\right)^{k}$, avec $k=1,5-2[45,88]$, et $n_{c}$ correspondant à la densité critique de la transition métalisolant. L'annulation de la tension seuil permet donc de déterminer la densité critique de la transition [81].

\subsubsection{Magnéto-transport}

Dans un G2D d'électrons, le champ magnétique a deux effets, un effet sur le mouvement orbital, et un effet sur le spin, ou effet Zeeman. Lorsque le champ magnétique est perpendiculaire au G2D, les deux effets sont combinés. Par contre, lorsque le champ magnétique est dirigé parallèlement au plan du G2D, le mouvement des électrons dans la direction orthogonale au plan étant bloqué, seul l'effet Zeeman continue à jouer. Ainsi un champ magnétique parallèle au plan ne joue que sur le spin des électrons. En particulier un champ magnétique parallèle suffisamment élevé est susceptible de polariser le spin des électrons sans modifier leurs trajectoires. Il faut cependant que la longueur magnétique $l_{m}=\sqrt{\hbar c / e B}$ reste faible devant l'étendue des fonctions d'ondes dans la direction orthogonale au plan du G2D. 
(a)

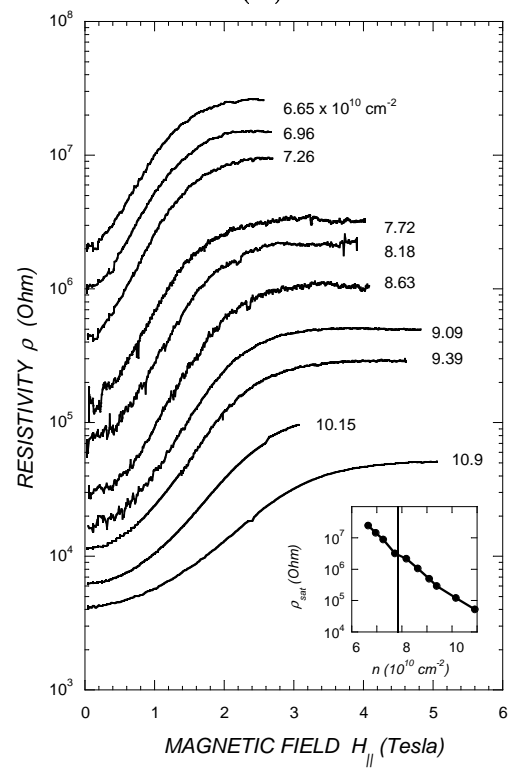

(b)

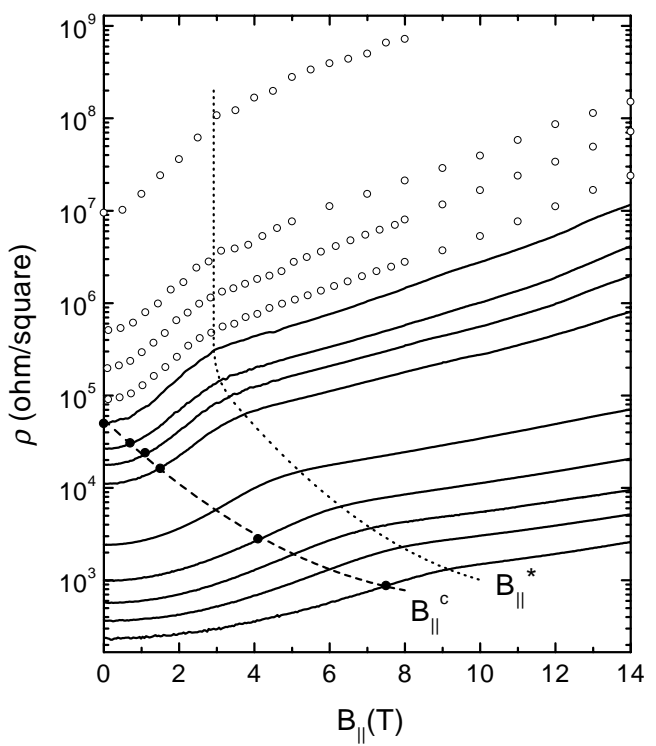

FIG. 2.5: Mesures de résistivité en fonction du champ magnétique appliqué parallèlement au gaz bidimensionnel d'électrons. (a) Dans un MOSFET Silicium, d'après Mertes et al. [79]. Encart : résistivité à fort champ magnétique $\rho_{\text {sat }}$, après la saturation des courbes $\rho\left(B_{\|}\right)$, en fonction de la densité de porteurs $n$. (b) Dans une hétérostructure $p$-GaAs, d'après Yoon et al. [89].

\section{Champ magnétique parallèle}

Après l'observation du comportement métallique de la résistivité à champ magnétique nul, le deuxième effet qui a posé de nombreuses question est une magnétorésistance positive géante observée par l'application d'un champ magnétique parallèle. Cet effet a été observé pour la première fois sur des MOSFETs Si par Dolgopolov et al. [90], puis a été reporté dans la majorité des systèmes présentant une transition métal-isolant, les MOSFETs Si [91, 92, 93, 79, 94, 95] et les hétérostructures p-GaAs [96, 89] (voir la figure 2.5). Cette magnétorésistance n'est cependant pas observée dans les hétérostructures $p$-SiGe, et ce point sera discuté en détail au paragraphe 2.4.5.

Dans les MOSFETs Si (voir la figure 2.5(a)), la résistivité augmente d'au moins un ordre de grandeur lorsque le champ magnétique augmente, cette augmentation atteignant quatre ordres de grandeur aux plus basses températures [97]. A faible champ magnétique, la résistivité augmente en $B_{\|}^{2}$, puis sature au-delà d'un certain champ magnétique $B_{\|}^{\text {sat }}$, dépendant de la densité de porteurs [91, 92, 98]. La résistivité reste alors constante jusqu'aux plus hautes valeurs de champ magnétique atteintes $(\approx 12$ Tesla $)$. Ce comportement est identique dans la phase métallique et dans la phase isolante, sans changement de comportement à la traversée de la transition métal-isolant [79]. $B_{\|}^{\text {sat }}$ augmente linéairement avec la densité de porteurs $n_{s}[92,79,94,99,100,101]$ (voir l'encart de la figure 2.5(a)).

La magnétorésistance est qualitativement semblable dans les hétérostructures $p$-GaAs [89] (voir figure 2.5(b)). Cependant la résistivité ne sature pas à fort champ magnétique, mais présente une rupture de pente au delà d'une certaine valeur $B_{\|}^{*}$ du champ magnétique. En dessous de $B_{\|}^{*}$, la résistivité croît comme $\rho=\rho_{1} \exp \left(B_{\|}^{2} / B_{1}^{2}\right)$, alors qu'au-delà de $B_{\|}^{*}$, La 
croissance de la résistivité est beaucoup plus lente, $\rho=\rho_{0} \exp \left(B_{\|} / B_{0}\right)$. Enfin, la magnétorésistance suit le même comportement dans la phase isolante $\left(n_{s}<n_{c}\right)$ et dans la phase métallique $\left(n_{s}>n_{c}\right)$ (voir en particulier la distinction traits pleins et symboles dans la figure 2.5(b)). Cette constatation suggère que l'origine de la magnéto-résistance est la même dans les deux phases. Les paramètres $B_{0}, B_{1}$ et $B_{\|}^{*}$ deviennent indépendants de $n_{s}$ quand $n_{s}$ diminue et s'approche de $n_{c}$.

L'effet le plus surprenant concerne l'application du champ magnétique parallèle lorsque le système présente un comportement métallique à champ magnétique nul (i.e. $n_{s}>$ $\left.n_{c}(B=0)\right)$. Dans les MOSFETs Si $[91,92]$ et les hétérostructures $p$-GaAs, le comportement métallique de la résistivité $(d \rho / d T>0)$ est inversé, donnant lieu à un comportement isolant, lorsqu'un champ magnétique parallèle supérieur à $B_{\|}^{c}$ est appliqué : cette observation a été interprétée par la destruction de la phase métallique pour un champ $B_{\|}>B_{\|}^{c}$. $B_{\|}^{c}$ est bien défini dans les hétérostructures $p$-GaAs [89], ce qui permet d'envisager une transition métal-isolant induite par le champ magnétique parallèle. Par contre, dans les MOSFETs Si [102], le champ magnétique critique varie avec la température est n'est pas bien défini. Il a même été suggéré que ce champ critique tende vers zéro quand la température tend vers zéro [91], ce qui signifie que la phase métallique est supprimée pour un champ magnétique très faible. Cependant, cette différence entre les deux systèmes peut être due au plus faible désordre dans les structures $p$-GaAs que dans les MOSFETs Si. Yoon et al. [89] et Gao et al. [70] ont montré que, dans des hétérostructures $p$-GaAs, $B_{\|}^{c} \propto\left(n_{s}-n_{c}\right)^{\alpha}$, avec $\alpha$ compris entre 0,7 [89] et 1 [70], et $n_{c}$ la densité critique déduite du changement de signe de $d \rho / d T$ à champ magnétique nul.

Pour $B_{\|}<B_{\|}^{c}$, il a été montré dans les MOSFETs Si que l'application d'un champ magnétique parallèle décale la densité critique de la transition $n_{c}$ (i.e. densité à laquelle il y une transition entre un comportement métallique et un comportement isolant de la résistivité en fonction de la température) vers les hautes densités [94, 102].

Nous verrons plus loin que $B_{\|}^{\text {sat }}$ (ou $B_{\|}^{*}$ dans le cas du $p$-GaAs) a été interprété comme le champ magnétique nécessaire pour polariser totalement le gaz d'électrons. Plusieurs auteurs se sont intéressés aux lois de transport à fort champ magnétique, i.e. $B_{\|}>B_{\|}^{\text {sat }}$ (ou $B_{\|}>B_{\|}^{*}$ ), donc dans le cas ou le système est supposé complètement polarisé. Dans les MOSFETs Si, Mertes et al. [103] et Shlimak et al. [104] ont montré que, à faible densité de porteur (phase isolante à $B_{\|}=0$ ), la loi de transport s'ajuste avec une loi $\rho\left(B_{\|}>B_{\|}^{\text {sat }}, T\right) \propto \exp \left[\left(T_{0} / T\right)^{0,7}\right]$, alors qu'une loi de transport de type Efros-Shklovskii est obtenue à champ magnétique nul, $\rho\left(B_{\|}=0, T\right) \propto \exp \left[\left(T_{0} / T\right)^{1 / 2}\right]$. Ce qui signifie que la dépendance en température est plus forte à champ magnétique élevé qu'à champ magnétique nul. Shlimak et al. ont cependant montré qu'il était également possible d'ajuster les courbes avec un exposant $1 / 2$ et un préfacteur dépendant de la température [104]. D'autre part Pudalov et al. [102] ont montré qu'un ajustement raisonnable pouvait aussi être obtenue avec un loi activée simple (exposant 1) dans tous les cas "isolants" (voir l'équation (2.5)). Il n'y aurait donc pas de comportement nouveau, mais un simple changement de désordre dû au champ magnétique.

Pour des densités élevées (comportement métallique à $B_{\|}=0$ ), et dans des hétérostructures $p$-GaAs, Gao et al. trouvent un comportement isolant, avec une correction logarithmique à la conductance, caractéristique de la localisation faible [70] : cette dé- 
pendance logarithmique, absente à $B_{\|}=0$, serait alors liée à la suppression de la phase métallique sous champ magnétique et au retour du comportement isolant attendu dans ces systèmes. D'autre part Gao et al. ont montré que, à faible champ magnétique (i.e. avant la suppression complète du comportement métallique), l'énergie d'activation $T_{0}$ de la loi $\rho(T)$ (voir l'équation (2.2)) dépend peu du champ magnétique [105]. Le seul effet du champ magnétique est de diminuer l'amplitude de l'effet, mais pas le comportement activé, indiquant que le champ magnétique parallèle ne joue pas sur la nature même de la phase métallique, mais semble diminuer sa proportion dans le système. Ces deux constatations sont compatibles avec des modèles de mélanges de phases.

Dans les MOSFETs Si, qui sont isotropes, l'effet d'anisotropie lié à l'orientation relative du champ magnétique avec le courant injecté ou l'orientation cristallographique est absent [91] ou très faible [101]. Par contre, dans les hétérostructures $p$-GaAs, la magnétorésistivité est anisotrope, comme l'ont montré Papadakis et al. en changeant les orientations relatives du champ magnétique, du courant et des directions cristallographiques [106]. Ce point sera détaillé au paragraphe 2.4.5.

\section{Champ magnétique perpendiculaire}

A faible champ magnétique et à basse température, les effets liés à la courbure de la trajectoire des électrons reste faible (régime d'effet Hall classique), et il est possible d'observer dans ce régime des effets liés aux corrections quantiques de localisation faible. Dans des systèmes sans ou à faibles interactions, ces corrections quantiques conduisent à une magnétorésistance négative (voir §1.5.3). Une magnétorésistance négative a été mesurée à haute densité $\left(n_{s}>n_{c}\right)$ dans des MOSFETs Si [92, 107], dans des hétérostructures $p$-GaAs/GaAlAs [108] et des hétérostructures $p$-SiGe [26, 21]. Ces mesures ont été interprétées par la présence de localisation faible dans ces systèmes. Nous verrons les conséquences de ces mesures au paragraphe 2.4.3.

A champ magnétique plus élevé, la magnétorésistance présente deux caractères différents suivant la densité électronique. A densité élevée $\left(n_{s} \geqslant n^{*}\right)$, une magnétorésistance positive parabolique supplante la correction de localisation faible, et est attribuée à la levée de dégénérescence Zeeman, alors qu'à faible densité $\left(n_{s} \leqslant n^{*}\right)$ est observée une forte magnétorésistance négative, attribuée à la correction due aux interactions électron-électron [92]. $n^{*}$ est plus élevée que la densité critique $n_{c}$ déduite des dépendances en température de la résistivité. Cependant les corrections attribuées aux interactions électron-électron observées ont une amplitude plus grande que celle attendue pour un métal. Cet écart peut être dû à la faible valeur de $k_{F} l_{e}$ (alors que les calculs de corrections sont fait pour $\left.k_{F} l_{e} \gg 1\right)$.

A fort champ magnétique, on entre dans le régime d'effet Hall quantique. Bien que ce régime ne concerne pas directement le domaine de la transition métal isolant décrite ici, nous verront au §2.4.6 qu'un certain nombre d'analogies peuvent être trouvées entre le comportement du système à champ magnétique nul, et à champ magnétique élevé où il a été observé une transition entre les états de Hall et des états isolants. Dans les MOSFETs Si faiblement désordonnés à faible densité de porteurs, les niveaux de Landau semblent coalescer en restant à une énergie finie quand le champ magnétique perpendiculaire tend vers zéro (phénomène de flottement des niveaux de Landau, qui est représenté par la ligne 
continue quand $B \rightarrow 0$ dans la figure 1.11) [109, 35, 110, 111]. Les mêmes résultats ont été obtenus sur $p$-GaAs [112, 113]. En particulier dans la référence [113], il a été montré que le point de croisement des courbes à différentes températures se déplace vers les faibles champs magnétiques quand la densité de porteurs augmente, menant de façon continue à une phase métallique à champ magnétique nul (voir la figure 2.6). Ces mesures semblent donc montrer que la transition métal-isolant à champ magnétique nul et les transitions effet Hall quantique-isolant ont une origine commune [114].

Il est intéressant de noter que ce comportement n'est pas observé dans des G2D sans interaction (par exemple dans $n$-GaAs où un comportement isolant est toujours observé à champ magnétique nul $[115,116])$ : dans ces système est observé un comportement réentrant isolant-EHQ-isolant, et la phase isolante est interprétée comme un isolant d'Anderson. Cette dernière transition est en accord avec la théorie de transition de "flottement des niveaux de Landau" [117, 118], et avec le diagramme de phase global proposé par Kivelson et al. [119].

\section{Effet d'un champ magnétique incliné}

Dans les MOSFETS Si, après application d'un champ magnétique parallèle suffisant pour supprimer le comportement métallique, le système présente une forte magnétorésistance négative en champ magnétique perpendiculaire [120, 121]. Cette magnétorésistance négative est similaire à celle obtenue dans des G2D d'électrons désordonnés avec de faibles interactions (voir par exemple les résultats sur le $n$-GaAs [122]), et remplace la magnétorésistance positive observée à partir dans phase métallique à faible. Ainsi le champ magnétique parallèle détruit la phase métallique en rétablissant le système dans une phase normale. Ce résultat est en accord avec les observations de Gao et al. dans des hétérostructures $p$-GaAs, qui retrouvent la correction logarithmique de conductivité liée à la localisation faible lorsqu'ils appliquent un fort champ magnétique parallèle [70].

Un champ magnétique incliné permet de mesurer le produit $g_{\mathrm{FL}} m_{\mathrm{FL}}$ obtenu dans la théorie du liquide de Fermi, la méthode étant décrite dans Okamoto et al. [94]. Il est alors possible de comparer ce produit avec le produit $g^{*} m^{*}$ obtenu pour des électrons libres (i.e. liés uniquement au potentiel cristallin). Okamoto et al. [94] ont montré que, dans des MOSFETs Si, le rapport $\alpha=g_{\mathrm{FL}} m_{\mathrm{FL}} / g^{*} m^{*}$ augmente avec le paramètre $r_{s}$, donc avec la force des interactions, et tend vers 1 quand $r_{s}$ tend vers 0 . Okamoto et al. [94] ont également mesuré la position de minimum de Shubnikov-de Haas $\left(B_{\perp \text { min }}\right)$ à différentes valeurs du champ magnétique parallèle $B_{\|} . B_{\perp \text { min }}$ augmente linéairement quand $B_{\|}$augmente, puis sature lorsque $B_{\|}$atteint la valeur critique correspondant à l'alignement des spins électroniques.

\subsubsection{Mesures dans des systèmes originaux}

Dans des hétérostructures $n$-AlGaAs, Ribeiro et al. ont créé un désordre "artificiel" en incorporant, près du gaz bidimensionnel d'électrons, une couche de plots quantiques InAs auto-organisés [123]. Cette technique leur permet de modifier le désordre, tout en gardant une valeur du paramètre $r_{s}$ faible $(<1)$. Alors que les échantillons à faible concentration de plot ne présentent qu'un comportement $\rho(T)$ isolant, les échantillons avec une forte 


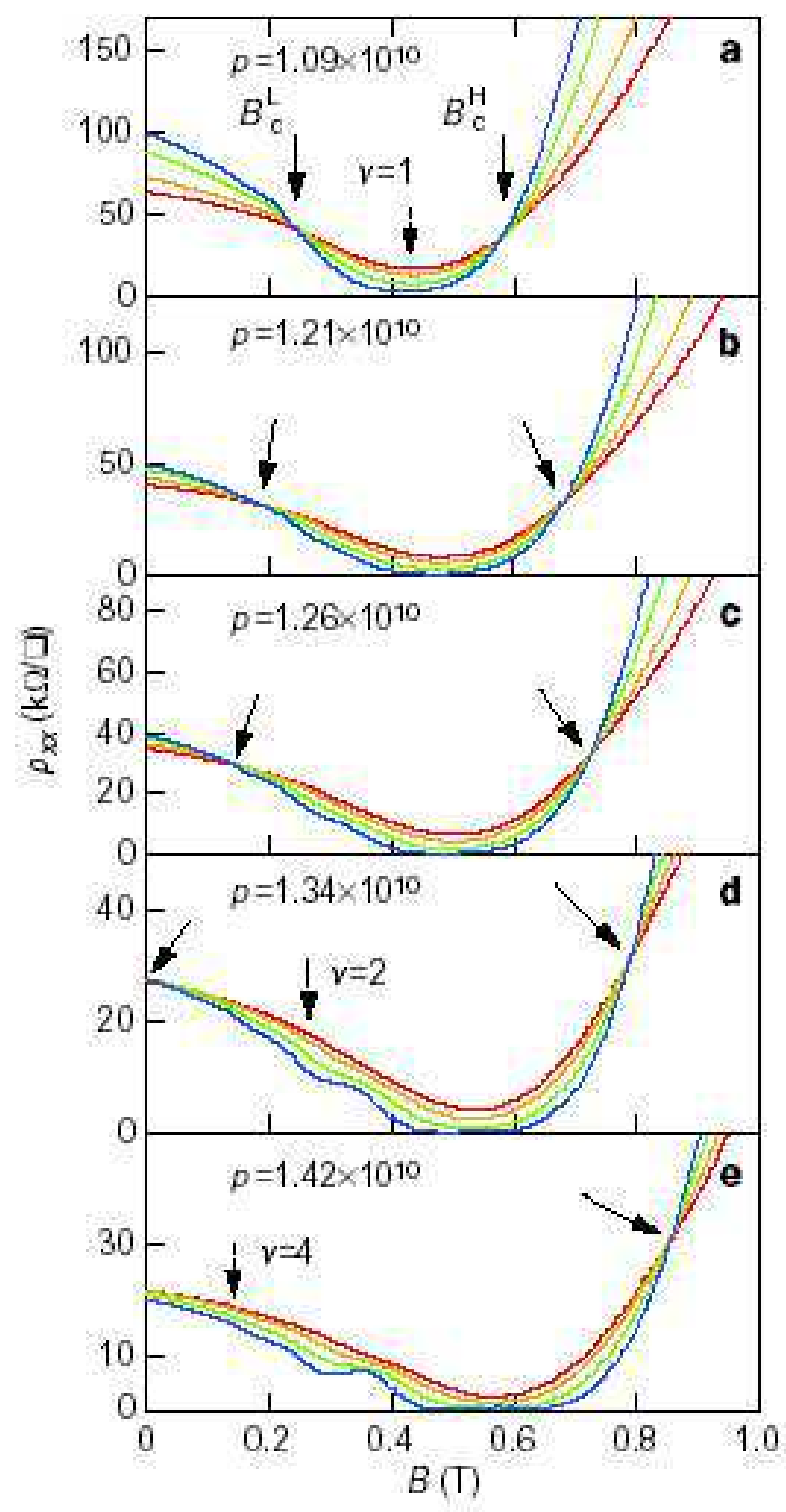

FIG. 2.6: Mesures de magnétorésistance à différentes températures et différentes densités électroniques $p$ sur une hétérostructure $p$-GaAs. Cette mesure montre le point de croisement des courbes, caractéristique d'une transition métal-isolant, et l'évolution de ce point de croisement en fonction de la densité. Ainsi la transition métalisolant observée à champ magnétique nul à une densité de $1,34 \times 10^{14} \mathrm{~m}^{-2}$ est reliée continûment à la transition métal-isolant en fonction du champ magnétique observée à plus basse densité. Extrait de Hanein et al. [113].

concentration de plots présentent les caractéristiques d'une transition métal-isolant (changement de signe de $d \rho / d T)$. Dans ces systèmes, l'existence d'une transition semble liée plus au désordre qu'aux interactions entre électrons (qui restent faibles), ou plutôt à la nature du désordre : dans les hétérostructures $n$-GaAs habituelles, la diffusion dominante est lié aux impuretés dopantes qui forment un désordre à longue portée, alors que les plots d'InAs créent un désordre à courte portée. 


\subsubsection{Autres observables}

\section{Compressibilité}

La compressibilité électronique d'un système est donnée par :

$$
\kappa=\frac{1}{n_{s}^{2}}\left(\frac{d n_{s}}{d \mu}\right)
$$

où $n_{s}$ est la densité électronique et $\mu$ est le potentiel chimique. Expérimentalement, il est possible de mesurer la compressibilité d'un gaz bidimensionnel d'électrons en mesurant la capacité entre le gaz d'électrons et une grille métallique placée à une distance donnée. La capacité par unité de surface est alors reliée à la compressibilité par [124] :

$$
c_{q}=e^{2}\left(\frac{d n_{s}}{d \mu}\right)=n_{s}^{2} e^{2} \kappa .
$$

À l'aide d'une deuxième grille, la compressibilité peut être mesurée en fonction de la densité électronique.

Dultz et al. [124] et Ilani et al. [125] ont réalisé des mesures de compressibilité dans des hétérostructures $p$-GaAs autour de la transition métal-isolant. Ils montrent que la compressibilité change de signe lorsque le système passe la transition, pour $n_{s}=n_{c}$, et devient négative à basse densité. Ce changement de signe est une signature thermodynamique d'une transition de phase, caractéristique d'une forte modification des propriétés d'écrantage du système électronique. A basse densité, la compressibilité devient négative et diverge, ce qui est caractéristique d'une phase incompressible. Dultz et al. [124] ont également montré que la phase isolante à champ magnétique nul a des caractéristiques identiques aux phase isolantes observées en champ magnétique pour des remplissages entiers, montrant une forte analogie entre l'isolant à basse densité à champ magnétique nul, et les phases isolantes dans le régime d'effet Hall quantique.

À l'aide d'un transistor à un électron (SET, pour "Single Electron Transistor"), Ilani et al. ont pu faire des mesures locales de compressibilité [125, 126]. Dans la phase métallique, la variation de la compressibilité en fonction de la densité est bien décrite dans l'approximation de Hartree-Fock. Au passage de la transition métal-isolant, en plus du changement de signe de la compressibilité quand la densité passe en-dessous de $n_{c}$, ils observent une forte augmentation des fluctuations du potentiel chimique quand la densité de porteurs varie. D'autre part, des mesures sur des SETs positionnés à différents endroits donnent des résultats différents, ce qui permet de déduire que la phase isolante est spatialement inhomogène [125]. Ils ont enfin montré que les événements conduisant aux fortes fluctuations du potentiel chimique sont liés aux réorganisations de charges, et concluent que les fortes interactions dans la phase isolante sont responsables de ces réorganisations [126].

\section{Pouvoir thermoélectrique}

Fletcher et al. ont mesuré le pouvoir thermoélectrique dans des MOSFETs Si [127]. Le pouvoir thermoélectrique diverge lorsque la densité de porteur s'approche de la densité critique, de la même manière que ce qui est prévu pour la transition d'Anderson 3D [128], ce qui semble indiquer l'existence d'un bord de mobilité en dimension deux. 


\section{Fluctuations de la résistance}

Les fluctuations de la résistivité dans un système électronique sont dues à la réponse du système aux fluctuations du désordre (les défauts et les impuretés). Il y a deux manières de changer le désordre. La première consiste à faire varier le potentiel chimique par l'intermédiaire d'une grille, et de mesurer les fluctuations de la résistivité du système en fonction de la tension de grille appliquée. Un autre moyen consiste à laisser le désordre fluctuer dans le temps en raison de l'activation thermique. On mesure alors les fluctuations de résistivité en fonction du temps qui, pour des échantillons macroscopiques, sont à l'origine du bruit basse fréquence en $1 / f$. Une étude plus détaillée de ce bruit est décrite dans la partie III.

Popović et al. ont mesuré les fluctuations de conductance lorsqu'ils changent la tension de grille sur des MOSFETs Si de petite taille (longueur de 1 à $8 \mu \mathrm{m}$ ) [129, 130]. Le spectre des fluctuations de conductance présente une partie basse fréquence qu'il n'est pas possible d'interpréter dans des modèles d'électrons indépendants. De plus, autour de la transition métal-isolant, ils observent l'apparition d'un terme supplémentaire à fréquence intermédiaire, correspondant à une nouvelle énergie caractéristique. Ce terme n'est présent ni dans la phase isolante, ni dans la phase métallique, et est également une preuve d'un changement de régime à la transition.

Bogdanovitch et Popović [82] et Jaroszynski et al. [83] ont mesuré les fluctuations de résistance à basse fréquence (bruit en $1 / f^{\alpha}$ ), respectivement dans des MOSFETs Si de faible longueur et de taille macroscopique. Ces deuxsystèmes présentent des caractéristiques très semblables. En-dessous d'une certaine densité critique $n_{g}$ (supérieure à la densité $n_{c}$ ), l'amplitude du bruit augmente très fortement quand la densité diminue. Cette augmentation du bruit est accompagnée par une augmentation de l'exposant du bruit entre $\alpha \approx 1$ à haute densité et $\alpha \approx 1,8$ à basse densité. C'est deux caractéristiques sont semblables aux mesures sur les verres de spin, et ont été interprétées comme un ralentissement de la dynamique lorsque la densité d'électron diminue. D'autre part le bruit augmente fortement quand la température diminue. Enfin la mesure du second spectre (qui est le spectre des fluctuations du spectre de bruit en résistance) n'est pas monotone en fréquence. Il présente une augmentation à basse fréquence, ce qui montre les fortes corrélations dans un tel système.

\subsection{Phénoménologie de la transition métal-isolant}

Dans la section précédente ont été présentés des résultats généraux obtenus dans des systèmes bidimensionnels de porteur de haute mobilité à faible densité de porteurs. Avant de présenter les théories qui ont essayer d'interpréter les comportements nouveaux autour de la transition métal-isolant, nous allons voir dans cette section comment les expériences peuvent répondre à certaines questions, notamment sur la nature de la transition observée, et en particulier si c'est une transition de phase, mais également sur le rôle que peuvent jouer les interactions, le désordre et le spin électronique. 


\subsubsection{Transition de phase quantique}

\section{Transitions de phase quantiques continues}

Dans le cadre de la TMI à 2D, nous verrons que certains auteurs ont évoqué la possibilité d'une transition de phase quantique entre un état métallique et un état isolant. Avant d'exposer les arguments en faveur d'une telle hypothèse, on rappelle ici les caractéristiques d'une telle transition. La majorité des points développés ici sont détaillés dans plusieurs revues, dans lesquelles on peut également trouver des exemples de transitions de phase quantiques continues. Voir en particulier Sondhi et al. [59] et Vojta [131, 132].

Définition d'une transition de phase quantique continue. Parmi les transitions de phase connues, on distingue deux types de transitions. Dans les transitions de phase du premier ordre, une variation infinitésimale d'un paramètre autour du point de transition s'accompagne d'une variation finie de l'énergie, appelée chaleur latente. Dans un transition de phase continue (ou du deuxième ordre), l'énergie est continue à la traversée du point de transition. Il a été montré que la taille et le temps caractéristiques des fluctuations divergent aux abord d'une transition de phase continue. Cette divergence associée aux observables physiques est appelé comportement critique, et a donné lieu à des développements théoriques très poussés, en particulier avec l'étude des comportements d'échelle.

Pour une transition de phase classique, le passage de l'état désordonné à l'état ordonné se produit en changeant la température du système : la transition est alors contrôlée par les fluctuations thermiques. Dans ce cas, la mécanique quantique n'est pas exclue car elle peut contrôler la formation de la phase ordonnée, comme c'est le cas dans l'état supraconducteur. Cependant, suffisamment près du point critique, les fluctuations quantiques sont importantes uniquement à l'échelle microscopique, alors que les fluctuations thermiques dominent à grande échelle, qui est l'échelle qui contrôle le comportement critique. À température nulle, les fluctuations thermiques sont absentes : il existe alors un certain type de transitions contrôlées par les fluctuations quantiques, ce sont les transitions de phase quantiques. Une transition de phase quantique se produit lorsque le changement d'un paramètre du Hamiltonien du système, tel le désordre ou la densité, change l'état fondamental du système. Dans une transition de phase quantique, les fluctuations quantiques dominent à grande échelle et contrôlent ainsi le comportement critique.

Rôle de la température dans une transition de phase quantique. Si $H$ est le Hamiltonien du système, l'opérateur densité $\exp \left(-H / k_{B} T\right)$ peut formellement être écrit comme un opérateur d'évolution $\exp (-i H \theta / \hbar)$ avec un temps imaginaire $\theta=-i \hbar / k_{B} T$. On voit ainsi que le calcul de la fonction de partition d'un système quantique :

$$
Z=\sum_{n}<n\left|\exp \left(-H / k_{B} T\right)\right| n>\equiv \sum_{n}<n|\exp (-i H \theta / \hbar)| n>
$$

se ramène formellement au calcul des amplitudes de transitions d'un état $|n\rangle$ au même état $\mid n>$ après un temps $\theta$. Le calcul de ces amplitudes de transition peut être fait grâce aux intégrales de chemin de Feynman, en sommant tous les chemins possibles sur 
des états intermédiaires séparés d'un temps imaginaire $\tau$. Le temps joue alors le rôle d'une dimension supplémentaire, une dimension infinie lorsque la température est nulle. Le système quantique en dimension $d$ peut ainsi se ramener à un système classique en dimension $d+1$, la dimension supplémentaire étant d'un caractère particulier puisqu'elle représente un temps imaginaire.

Comportement critique. Aux abords d'une transition de phase la longueur de corrélation $\xi$ diverge. Dans la dimension supplémentaire attachée au temps, il existe également une longueur de corrélation "temporelle" $\xi_{\tau}$. Ainsi, à température nulle, les deux longueurs de corrélation $\xi$ et $\xi_{\tau}$ divergent quand l'écart à la transition $\delta$ tend vers zéro suivant des lois :

$$
\xi \sim|\delta|^{-\nu} \quad \text { et } \quad \xi_{\tau} \sim \xi^{z}
$$

où $\nu$ est l'exposant critique de longueur de corrélation et $z$ l'exposant dynamique.

Une conséquence de la divergence des longueurs de corrélation aux abords de la transition est qu'il n'y a pas d'autres échelles de longueurs caractéristiques que $\xi$ et $\xi_{\tau}$. Ainsi les grandeurs physiques, telle la résistivité pour un système électronique, suivent une loi d'échelle dans laquelle $\delta$ n'intervient pas explicitement [59] :

$$
\rho(k, \omega, \delta)=\xi^{d_{\rho}} f\left(k \xi, \omega \xi_{\tau}\right),
$$

où $d_{\rho}$ est la dimension d'échelle, $k$ est le vecteur d'onde et $\omega$ la pulsation.

Lois d'échelle en dimension finie. L'équation (2.10) suppose que les dimensions du système sont infinies, aussi bien pour les dimensions dans l'espace réel que pour la dimension temporelle. Cette dernière condition impose une température nulle. Lorsque les dimensions du système sont limitées par une longueur $L$, et que la température est non nulle, les longueurs de corrélation sont limitées par ces longueurs :

$$
\xi \leqslant L \quad \text { et } \quad \xi_{\tau} \leqslant L_{\tau}=\frac{\hbar}{k_{B} T} .
$$

Alors, suffisamment près de la transition, les seules échelles de longueur caractéristiques deviennent $L$ et $L_{\tau}$.

Supposons maintenant que $L / \xi \gg L_{\tau} / \xi_{\tau}$, et on se place à une distance de la transition telle que $\xi$ est toujours la longueur caractéristique, mais la longueur temporelle caractéristique est maintenant $L_{\tau}$. Dans ce cas la dimension totale de l'espace n'est plus $d+1$, mais $d$. Cette réduction de la dimensionnalité de l'espace modifie les lois d'échelle, conduisant à une loi d'échelle en dimension finie qui, pour la résistivité, s'écrit [59] :

$$
\rho(k, \omega, \delta, T)=L_{\tau}^{d_{\rho} / z} f\left(k L_{\tau}^{1 / z}, \omega L_{\tau}, \frac{L_{\tau}}{\xi_{\tau}}\right) .
$$

Pour une mesure en continu, i.e. pour $k=0$ et $\omega=0$, en remarquant que $L_{\tau} / \xi_{\tau} \sim \delta^{\nu z} / T$, et en prenant en compte que, en dimension deux, $d_{\rho}=0$, cette loi d'échelle à température finie devient [59] :

$$
\rho(\delta, T)=f\left(\frac{\delta}{T^{1 / \nu z}}\right) .
$$


Dans la suite, cette loi d'échelle sera appelée loi d'échelle en température.

D'autre part, Sondhi et al. [59] ont suggéré que, suffisamment proche de la transition, les non-linéarités de la caractéristique $I-V$ ne peuvent pas provenir de propriétés à l'équilibre mais sont directement dues aux fluctuations critiques. Il est possible de montrer, par un argument dimensionnel, que le champ électrique agit comme une limite à la longueur de corrélation $\xi$. Supposons que l'on applique un champ électrique $E$ au système; il est alors possible d'associer une longueur caractéristique $l_{E}$ à ce champ électrique, et suffisamment proche de la transition $l_{E}$ est la seule longueur caractéristique. Pour évaluer cette longueur, il suffit de remarquer que, puisque $l_{E}$ est la seule longueur caractéristique, le seul temps caractéristique est $\sim l_{E}{ }^{z}$. Si on égalise l'énergie gagnée par un électron sur une distance $l_{E}$ avec l'énergie caractéristique liée au temps caractéristique, on obtient :

$$
e E l_{E} \sim \hbar l_{E}^{-z} \quad \Rightarrow \quad l_{E} \sim E^{-1 /(1+z)} .
$$

La loi d'échelle associée au champ électrique fini permet donc d'écrire que la résistivité (à $k=0$ et $\omega=0)$ n'est qu'une fonction du rapport $l_{E} / \xi$, soit :

$$
\rho(\delta, E)=g\left(\frac{\delta}{E^{1 / \nu(z+1)}}\right)
$$

Dans la suite, cette loi d'échelle sera appelée loi d'échelle en champ électrique.

\section{Paramètre critique de la TMI à 2D}

Dans le cadre de la transition métal-isolant en dimension deux, on suppose que le paramètre permettant de passer la transition est la densité de porteurs, et l'écart par rapport à la densité critique $n_{c}$ est donné par :

$$
\delta_{n}=\frac{n_{s}-n_{c}}{n_{c}} .
$$

Nous ne discuterons pas ici la validité de cette hypothèse, et nous verrons dans les paragraphes suivants que cette hypothèse est cohérente avec les lois d'échelles observées dans une majorité de G2D d'électrons et de trous. Il faut cependant mentionner que ce paramètre n'est pas couramment admis dans le cadre d'autres transitions métal-isolant. Dans la transition d'Anderson à $3 \mathrm{D}$, le paramètre est le désordre, comme dans la transition supraconducteur-isolant.

Un point important de l'étude de la TMI à $2 \mathrm{D}$ en temps que transition de phase consiste à déterminer le point critique de la transition, et donc la densité critique $n_{c}$. Ne connaissant pas à priori l'origine microscopique de la TMI, ce choix est en partie arbitraire. Dans une majorité de cas, c'est la dépendance en température de la résistivité qui permet de déterminer la densité critique de la transition [5]. À suffisamment basse température, le comportement $\rho(T)$ est monotone : la phase métallique est caractérisée par $d \rho / d T>0$, la phase isolante par $d \rho / d T<0$, et la densité critique par $d \rho / d T \approx 0$ sur une certaine plage de températures. L'objection la plus forte apportée à ce critère est le fait qu'une résistivité croissante quand la température diminue ne signifie pas que cette résistivité diverge à température nulle, et n'est donc pas nécessairement caractéristique 
d'un isolant. Une dépendance caractérisée par $d \rho / d T<0$ et qui sature à une valeur finie à température nulle peut aussi caractériser un métal. D'autre part une résistivité constante à la transition est contradictoire avec la notion de transition de phase de second ordre : le passage d'une résistivité finie à une résistivité infinie à température nulle impose une discontinuité de la résistivité, incompatible avec une transition de phase continue (voir [57] et les références incluses). Cette remarque a conduit à adopter un autre critère pour la transition métal-isolant à 3D : la phase isolante est caractérisée par une dépendance activée de la résistivité en fonction de la température, $\rho_{i}(T) \propto \exp \left[\left(T_{0} / T\right)^{m}\right]$, alors que le métal correspond à une loi en puissance de la température $\rho_{m} \propto T^{-\alpha}$ au-dessus d'une température $T_{\text {sat }}$ et qui sature à une valeur $\rho_{\text {sat }}$ en dessous de $T_{\text {sat }}$. Lorsque la densité tend vers la densité critique, $T_{\text {sat }}$ tend vers zéro et $\rho_{\text {sat }}$ tend vers l'infini, et aucune discontinuité n'est observée à la transition. L'exposant $\alpha$ attendu est $1 / 3$, mais peut être modifié par la prise en compte des interactions entre électrons. Expérimentalement deux valeurs ont été obtenues, $\alpha=1 / 3$ [133] et $\alpha=1 / 2$ [134, 135]. Certains auteurs ont employé un critère analogue à celui de la transition métal-isolant à $3 \mathrm{D}$ sur les résultats obtenus à $2 \mathrm{D}$. Altshuler et al. obtiennent ainsi un écart de $10 \%$ entre les deux critères, $d \rho / d T=0$ et loi activé dans l'isolant, pour des MOSFETs Si de mobilité élevée $\left(5,5 \mathrm{~m}^{2} . \mathrm{V}^{-1} . \mathrm{s}^{-1}\right)$ [68]. La différence se révèle encore plus importante dans le cas de MOSFETs Si très désordonnés [82].

Pour palier au problème du critère sur les courbes $\rho(T)$, et en particulier le fait que dans certains systèmes ces dépendances ne sont pas monotones, certains auteurs ont proposés d'utiliser la dépendance en champ électrique de la résistivité $[45,136,81]$. On a vu au paragraphe 2.3.1 que, dans la phase isolante, la caractéristique $I-V$ présente une très forte non-linéarité avec un seuil de conduction. L'existence de ce seuil de conduction a été interprété comme une caractéristique de la phase isolante, et nous verrons les origines possibles d'un tel comportement dans le paragraphe 2.5.2. Cela permet d'utiliser comme critère de transition l'annulation du seuil de conduction quand on passe dans la phase métallique. Pudalov et al. [137] et Shashkin et al. [81] ont montré que, dans les MOSFETs $\mathrm{Si}$, ce nouveau critère donne une densité critique voisine de celle obtenue par le critère sur la dépendance en température (à 1-5\% près d'après Altshuler et al. [68]).

Il est intéressant de comparer la densité critique obtenue par l'analyse des mesures de transport avec celle obtenue par les mesures de quantités thermodynamiques (voir §2.3.4). Les mesures de compressibilité dans $p$-GaAs ont montré que le changement intervenant à l'entrée de la phase isolante (changement de pente, fluctuations importantes et inhomogénéïtés de la compressibilité) se produisent à une densité proche de la densité critique obtenue par $d \rho / d T=0[125,126]$. Les mesures de pouvoir thermo-électrique dans des MOSFETs Si ont également mis en évidence une divergence de la pente de $S^{d}(T)$ à une densité proche de la densité critique de transport [127]. Cependant, dans les deux cas, la précision à laquelle sont obtenus ces résultats n'est pas précisée.

\section{Observation expérimentale de lois d'échelle}

Comme nous l'avons vu au début de ce paragraphe, une des caractéristiques importantes d'une transition de phase quantique est l'existence de lois d'échelle. Dans les premières observations faites par Kravchenko et al. sur des MOSFETs Si, il a été mis en 

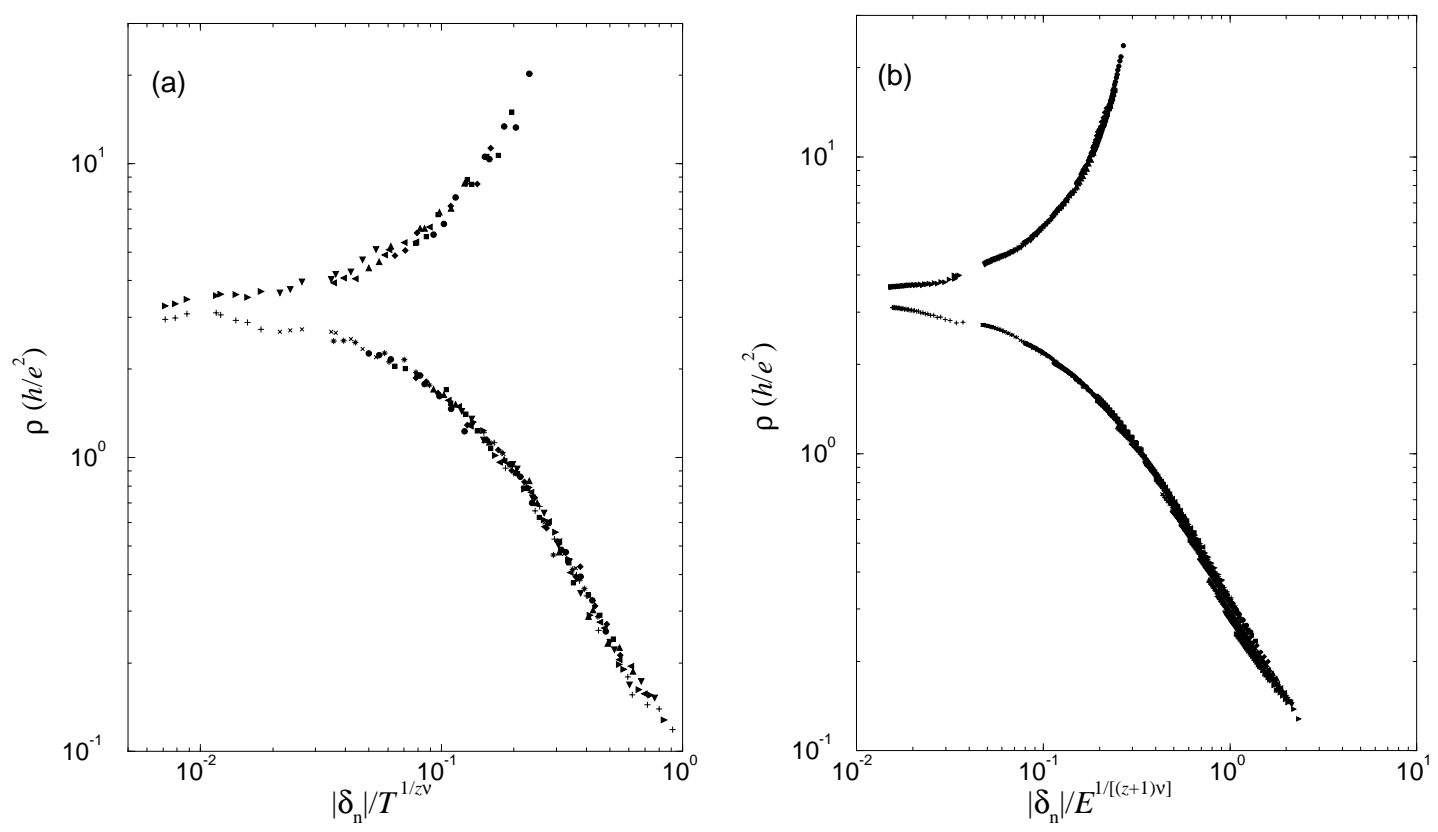

FIG. 2.7: Observation expérimentale de lois d'échelle (a) en fonction de la température et (b) en fonction du champ électrique. D'après Kravchenko et al. [84].

evidence une loi d'échelle en température [67], puis en champ électrique [84] (voir figure 2.7).

Expérimentalement, les lois d'échelle sont observées en traçant la résistivité en fonction du paramètre $T / T_{0}\left(n_{s}\right)$, où $T_{0}\left(n_{s}\right)$ est une valeur choisie empiriquement pour chaque valeur de la densité de façon à obtenir une superposition de toutes les courbes à différentes densités de porteurs. On a alors :

$$
\rho\left(T, n_{s}\right)=\rho_{c} f\left[\frac{T}{T_{0}\left(n_{s}\right)}\right]
$$

où $\rho_{c}$ est la résistivité à la densité critique. La dépendance de $T_{0}$ en fonction de $n_{s}$ est bien décrite, dans ces systèmes, par une dépendance en loi de puissance $T_{0}\left(n_{s}\right) \propto\left(n_{s}-n_{s}\right)^{a}$. Cette loi constitue donc une vérification de la loi d'échelle en température donnée dans l'équation (2.13) avec $a=z \nu$, comme le montre la figure 2.7(a). Dans certains systèmes, il a également été possible de tester la loi d'échelle en champ électrique (voir figure 2.7(b)). Ces lois d'échelle ont été confirmées dans les MOSFET silicium [138, 139, 140], dans des hétérostructures $p$-SiGe $[64,141]$ et dans des hétérostructures $p$-GaAs [62]. Les résultats de ces analyses sont résumés dans le tableau 2.2. On peut remarquer qu'aucun auteur ne présente de loi d'échelle en champ électrique dans $p$-GaAs. Enfin une étude plus précise sur les MOSFETs Si semble confirmer l'existence d'une loi d'échelle en champ électrique [142].

Le fait d'observer des lois d'échelle en température et en champ électrique a une double importance. D'une part cela permet de donner un argument en faveur de la transition de phase quantique. D'autre part cela permet de déterminer séparément les exposants critiques $\nu$ et $z$ (voir tableau 2.2). Un exposant dynamique $z$ proche de 1 a également été 


\begin{tabular}{|c|ccc|c|}
\hline système & $z$ & $\nu$ & $z \nu$ & référence \\
\hline MOSFET Si & $0,8 \pm 0,1$ & $1,5 \pm 0,1$ & $1,2 \pm 0,2$ & {$[84]$} \\
& $1,14 \pm 0,18$ & $1,87 \pm 0,24$ & $2,13 \pm 0,61$ & {$[138]$} \\
& $-^{\mathrm{a}}$ & $-{ }^{\mathrm{a}}$ & $1,5 \pm 0,1$ & {$[139]$} \\
& $-^{\mathrm{a}}$ & $-^{\mathrm{a}}$ & 0,8 à 1,6 & {$[140,137]$} \\
\hline$p$-GaAs & $-^{\mathrm{a}}$ & $-^{\mathrm{a}}$ & $4,8 \pm 0,4$ & {$[62]$} \\
\hline$p$-SiGe & $-^{\mathrm{a}}$ & $-^{\mathrm{a}}$ & $1,6 \pm 0,2 \mathrm{~b}$ & {$[63]$} \\
& $-^{\mathrm{a}}$ & $-^{\mathrm{a}}$ & 1,6 & {$[64]$} \\
& 1 & 2,85 & 2,85 & {$[141]$} \\
\hline
\end{tabular}

${ }^{\mathrm{a}}$ Lorsque seule la loi d'échelle en température a été testée, la détermination séparée de $\nu$ et $z$ n'est pas possible, et seul le produit $\nu z$ est présenté.

b Mesure établie dans la phase isolante.

TAB. 2.2: Valeurs des exposants critiques de longueur de corrélation $\nu$ et dynamique $z$ déterminés par l'observation de lois d'échelle en température et en champ électrique dans différents systèmes présentant les caractéristiques de la TMI.

obtenu dans la transition supra-conducteur-isolant [143] et dans la transition effet Hall quantique-isolant [144], et est attribué aux interactions électron-électron [59].

\section{Symétries entre phase métallique et phase isolante}

L'analyse des symétries des courbes de résistivité dans la phase isolante et la phase métallique a permi d'apporter un argument supplémentaire pour l'existence d'une transition de phase dans ces systèmes.

Kravchenko et al. ont remarqué qu'en traçant la résistivité en fonction de la variable $X=\delta_{n} / T^{1 / \nu z}$, la variation est continue et sans rupture de pente à la transition (i.e. pour $X=0$ ) [67]. Cette observation suggère que le mécanisme responsable des dépendances en température est le même dans les deux phases métallique et isolante. Altshuler et al. ont cependant remarqué que la symétrie de $\rho\left(T, n_{s}\right)$ autour de la courbe $\rho\left(T, n_{c}\right)=\rho_{c}{ }^{1}$ n'est pas vérifiée pour les résultats obtenus dans [69].

Les dépendances en champ électrique présentent également des symétries, se traduisant par la symétrie des courbes $\rho(E)$ par rapport à $\rho_{c} \approx h / e^{2}$ (par exemple pour Simmons et al. [114] dans des puits quantiques $p$-GaAs), ou par la symétrie des courbes I-V par rapport à la première bissectrice (voir Kravchenko et al. dans les MOSFETs Si [84]). Cependant la symétrie de la caractéristique $I-V$ n'est pas observée dans des hétérostructures $p$-GaAs par Yoon et al. [87].

\section{Lois d'échelle en champ magnétique}

Par des arguments généraux, Sachdev a montré que, pour un système dont le spin total est conservé, la conductivité autour d'une transition de phase d'ordre deux est une fonction universelle de $\left(g \mu_{B} B\right) /\left(k_{B} T\right)$ [145]. C'est ce qui est constaté dans les MOSFETs

\footnotetext{
${ }^{1}$ Cette symétrie peut être vérifiée en comparant $\rho\left(T, n_{s}^{\text {métal }}\right)$ et $\rho_{c}-\rho\left(T, n_{s}^{\text {isolant }}\right)$
} 
Si en champ magnétique parallèle [93, 146]. La loi d'échelle en champ magnétique parallèle suggère l'existence d'une transition de phase quantique [99].

\section{Universalité de la transition}

On peut terminer ce paragraphe en notant que, dans l'interprétation de la transition métal-isolant comme une transition de phase quantique, un point important est l'universalité de cette transition dans les différents systèmes étudier. Le caractère commun de la transition semble argumenter en faveur de cette universalité. D'autre part, la lecture des tableaux 2.1 et 2.2 montre que la densité critique, la résistivité critique, et les exposants critiques, sans être tout-à-fait universels, sont assez proches.

Cependant Pudalov et al. ont montré la variété des comportements observés dans les MOSFETs Si de différentes mobilités, ce qui laisse penser que le comportement métallique est lié plus aux échantillons utilisés qu'à une transition de phase quantique dans le système électronique [68, 137, 147].

Enfin l'existence d'une densité critique, et son universalité, peut constituer un argument en faveur de l'existence d'une transition de phase quantique. Coleridge et al. ont cependant montré dans des puits quantiques $p$-SiGe que cette densité critique dépend du désordre [64].

\subsubsection{Origine quantique de la phase métallique}

Dans la figure 2.3, où est représentée la résistivité en fonction du rapport entre la température et la température de Fermi, on remarque que le comportement métallique est observé pour $T \lesssim 0,3 \times T_{F}$. Ce résultat, observé dans les MOSFETs Si [69] et dans les hétérostructures $p$-GaAs [70], indique que le comportement métallique est une caractéristique d'un gaz d'électrons dégénéré, et a été attribué à la transition entre le régime classique pour $T \gg T_{F}$, lorsque la fonction de distribution est bien décrite par la distribution de Boltzmann, et le régime quantique pour $T \ll T_{F}$, lorsque le gaz d'électrons est fortement dégénéré. Dans des puits quantiques $p$-GaAs, Simmons et al. [62] ont montré que la chute relative de résistivité dans la phase isolante $[\sigma(T)-\sigma(T=0)] / \sigma(T=0)$ suit une loi d'échelle en fonction de $T / T_{F}$ pour des densités proches de la transition métal-isolant. Ces expériences montrent que le comportement métallique semble lié à l'état dégénéré du gaz d'électron. La résistivité calculée par la résolution de l'équation de Boltzman montre une transition entre un régime classique pour $T \gg T_{F}$, pour lequel on s'attend à une diminution de la résistivité quand la température augmente $\rho(T) \sim 1 / T$, et un régime quantique pour $T \ll T_{F}$, pour lequel la résistivité diminue avec une loi $\rho(T) \sim-T^{2}$. Das Sarma et Hwang ont montré que la partie haute température des courbes expérimentales peut être interprétée par une telle transition classique quantique pour $T \sim T_{F}[148,149]$. Par contre, il est nécessaire d'invoquer un autre mécanisme pour rendre compte de la partie basse température (comportement "métallique").

D'autre part, le comportement métallique a été observé jusqu'à des températures telles que $T / T_{F} \lesssim 10^{-2}$ dans les MOSFETs Si [69] et pour $T / T_{F} \lesssim 5 \times 10^{-3}$ dans les hétérostructures $p$-GaAs [150]. À des températures aussi basses, et pour les valeurs de conductivité observées $\left(\sim e^{2} / h\right)$, des effets importants de localisation quantique sont attendus (voir 
$\S 1.5 .2)$. Ainsi le comportement métallique semble lié à la suppression de ces effets de localisation.

Cependant, cette discussion sur la basse température atteinte dans les expériences nécessite d'être sûr de la température des porteurs. À basse température, les effets de chauffage de porteurs par un bruit extérieur ou par le courant injecté pour la mesure peuvent conduire à un gaz d'électrons dont la température est plus élevée que celle mesurée sur le réseau. Il a été envisagé que, sur certains système comme les MOSFETs silicium, la température des porteurs soit plus élevée que celle attendue [68], ce qui pourrait remettre en cause l'origine quantique du comportement métallique observé sur ces systèmes. Altshuler et al. ont discuté ce problème pour des MOSFETs Si de haute mobilité [68]. À basse température, due au faible couplage électron-phonon, il est fort probable que les électrons soient chauffés par une source extérieure. Ainsi une saturation de la résistivité en fonction de la température peut être interprétée comme un chauffage du gaz d'électron qui ne peut alors plus être refroidi à la température du substrat. Un calcul de dissipation de chaleur vers les contacts appliqué au cas des mesures de Kravchenko et al. [69] montre que, à une température de reseau de $35 \mathrm{mK}$ et pour une tension mesurée de $200 \mu \mathrm{V}$, la dissipation de chaleur ne permet de refroidir le système électronique qu'à une température de $360 \mathrm{mK}$, donc un facteur 10 au-dessus de la température annoncée.

\subsubsection{Rôle des interactions entre électrons}

En raison de la valeur élevée du paramètre $r_{s}$ dans les systèmes présentant les caractéristiques de la transition métal-isolant, et en raison de la contradiction observée par rapport à la théorie d'échelle de la localisation, les interactions semblent jouer un rôle important. Cependant, des mesures de magnétorésistance dans la phase métallique montrent que ces systèmes présentent les caractéristiques de la localisation faible, qui est une propriétés d'électrons indépendants. Ces mesures semblent donc indiquer que les interactions ne jouent pas nécessairement un grand rôle dans ce sujet, ou plutôt que la théorie du liquide de Fermi reste valable.

Une magnétorésistance négative caractéristique de localisation faible a été mesurée dans des MOSFET Si [98, 107, 151], les hétérostructures p-SiGe [26], et les hétérostructures $p$-GaAs $[152,108]$. À densité élevée (donc loin de la transition), la dépendance en température due à la localisation faible a pu être observée dans les MOSFETs Si [153, 76], le $p$-GaAs $[108,85]$ et le $p$-SiGe $[26,154]$. La raison pour laquelle ce comportement isolant n'est pas observé plus près de la transition serait alors dû à la température atteinte qui n'est pas suffisamment basse.

Pour réfuter cette dernière hypothèse, Mills et al. ont effectué des mesures à très basse température (jusqu'à $T=9 \mathrm{mK}$ ) sur des échantillons $p$-GaAs de haute mobilité $(\mu=20$ $\mathrm{m}^{2} / \mathrm{Vs}$ ). Ils ont observé une magnétorésistance présentant une forme compatible avec des effets de localisation faible, mais l'amplitude de l'effet est dix fois plus faible que celle attendue par la théorie [150]. D'autre part, dans la phase métallique, cette correction n'est pas visible sur la dépendance en température de la conductivité, et cela aux plus basses températures atteintes $\left(T<0,2 T_{F}\right)$ : cela indique que les effets de localisation faible doivent être de un à deux ordres de grandeur en-dessous de ce qui est prévu par la théorie. 
Dans les MOSFETs Si [155] et les puits quantiques $p$-GaAs [108] et $p$-SiGe [156], la contribution des interactions à la conductivité a pu être évaluée en mesurant la dépendance en température de la résistance de Hall. Dans les deux cas il a été montré que la contribution des interactions reste toujours très faible, malgré une valeur du facteur $r_{s}$ élevée (supérieure à 1). Il est cependant intéressant de noter que les théories permettant d'évaluer ces contributions ont pour base l'existence d'un liquide de Fermi, et ne prennent pas comme hypothèse la possibilité que les interactions soient fortes.

Dans les systèmes de mobilité élevée, Proskuryakov et al. ont suggéré que la théorie de localisation faible utilisée couramment pour un systèmes diffusif n'est plus valable, car alors la longueur magnétique $l_{B}$ devient inférieure au libre parcours moyen $l_{e}$ [152]. Ils ont alors analysé leurs mesures de magnétorésistance à faible champ magnétique à l'aide d'une théorie développée par Wittman et Schmid prenant en compte ce régime [157]. Ils déduisent alors le temps de cohérence de phase, et obtiennent pour $1 / \tau_{\Phi}$ une variation quadratique en $T$, ce qui est compatible avec un comportement dans le régime balistique $\left(k_{B} T \tau / \hbar \gg 1\right)$. Dans le régime diffusif $\left(k_{B} T \tau / \hbar \gg 1\right), 1 / \tau_{\Phi}$ est linéaire en $T$. Dans ces même mesures, Proskuryakov et al. montrent que la dépendance en température de la résistance de Hall est due à une dépendance en température de la densité de porteurs [152], et les corrections de conductance de Hall dues aux interaction sont très faible par rapport à ce que prévoit la théorie du liquide de Fermi.

Enfin, Ilani et al. ont montré que, dans la phase métallique, la dépendance en densité de porteurs de la compressibilité peut être ajustée en utilisant l'approximation de HartreeFock, ce qui est équivalent à considérer un liquide de Fermi [125]. Cependant les mesures dans la phase isolante montrent de fortes corrélations qui peuvent être attribuées aux effets de interactions entre électrons [126].

Ces résultats montrent que, malgré la valeur élevée du paramètre $r_{s}$ dans les systèmes étudiés, la plupart des résultats ne semblent pas contredire les prédictions faites dans le cadre de l'hypothèse du liquide de Fermi, qui semble donc encore valable dans la plupart de ces systèmes.

\subsubsection{Rôle du désordre}

On a vu que le comportement métallique peut être observé dans des systèmes présentant un désordre faible, et que les mesures dans des échantillons désordonnés conduit uniquement à des effets de localisation par le désordre. Il est possible d'étudier plus particulièrement l'effet du désordre sur les paramètres de la transition métal-isolant, soit en utilisant des échantillons différents, soit en modifiant le désordre en changeant la géométrie du potentiel de confinement par effet de champ. Plusieurs auteur ont suggéré que le comportement métallique n'est observé que pour des mobilités maximales élevées, en général supérieure à environ $1 \mathrm{~m}^{2} \cdot \mathrm{V}^{-1} \cdot \mathrm{s}^{-1}[60,71]$. L'augmentation du désordre (caractérisé par la mobilité maximale) induit une diminution de l'amplitude du comportement métallique (donné par $\Delta \rho=\rho\left(T_{\max }\right)-\rho\left(T_{\min }\right)$ ), une augmentation de la densité critique $n_{c}$ et une diminution de la résistivité à la densité critique $\rho_{c}[137,66,89]$, comme le montre la figure 2.8(a), issue de résultats de Pudalov [158], ainsi que la figure 2.8(b) regroupant des résultats sur différents systèmes.

On peut également remarquer que le désordre varie quand la densité de porteurs dimi- 
(a)

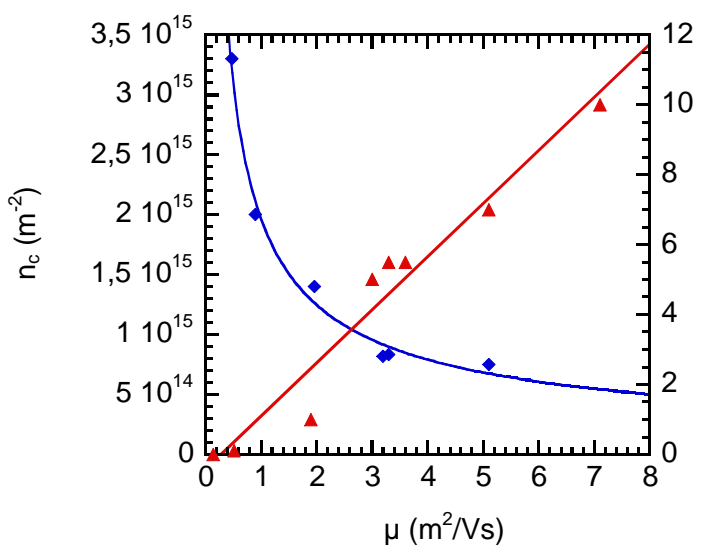

(b)

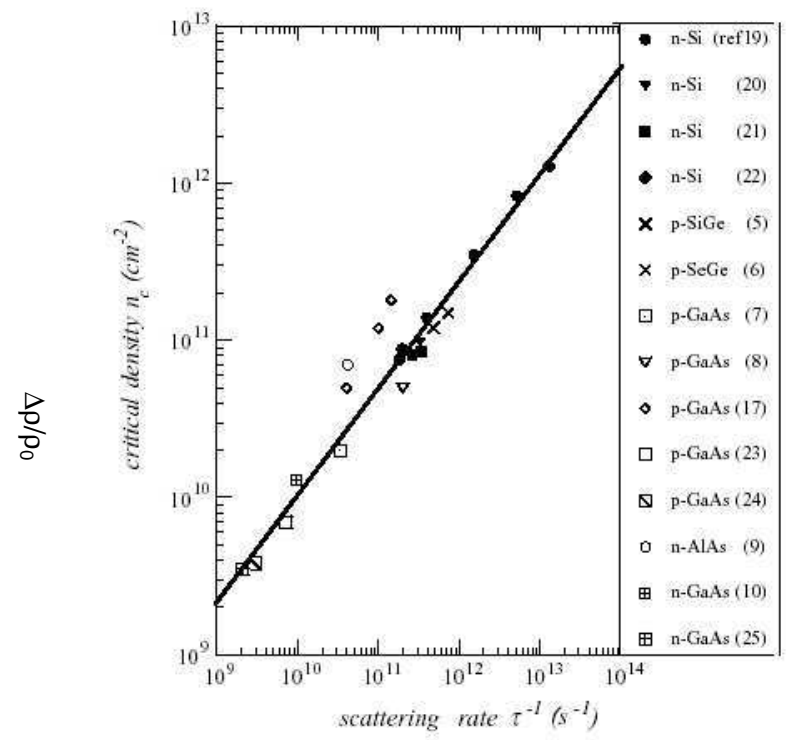

FIG. 2.8: (a) Densité critique $n_{c}$ et chute de résistivité $\Delta \rho / \rho_{0}$ en fonction de la mobilité maximale $\mu$ pour différents MOSFETs Si. Série de mesures prises dans les mêmes conditions par Pudalov [158]. (b) Densité critique de la TMI $\left(n_{c}\right)$ en fonction du désordre $(1 / \tau)$ pour différents systèmes cités dans la légende de droite. D'après Sarachik [159].

nue (par l'intermédiaire du produit $k_{F} l_{e}$ dans une approche diffusive) : ainsi la variation du désordre et des interactions entre électrons sont couplés quand on varie la densité. Il est cependant possible de faire varier le désordre indépendamment de la densité en modifiant la géométrie du potentiel de confinement des porteurs par l'intermédiaire de deux grilles situées de part et d'autre du G2D [160]. En effet, les impuretés sont en général situées à l'interface entre $\mathrm{Si}$ et $\mathrm{SiO}_{2}$ dans les MOSFETs $\mathrm{Si}$, ou à l'hétérojonction dans les hétérostructures. Le fait de modifier la forme du potentiel de confinement permet de rapprocher ou d'éloigner les porteurs par rapport à l'interface, et donc de modifier l'amplitude du désordre. Alors que, dans les hétérostructures de faible mobilité, Papadakis et al. ont montré que la modification de la forme du potentiel de confinement augmente le couplage spin orbite $[161,106,162]$, ce n'est pas le cas dans les hétérostructures de forte mobilité [160].

On peut également remarquer que les différents systèmes étudiés se comportent de manière analogue lorsque le désordre est élevé (voir la figure 2.9), ce qui souligne une certaine universalité des comportement à fort et à faible désordre. On s'attend donc à ce que le mécanisme conduisant au comportement métallique soit le même pour tous les systèmes.

Enfin, Pudalov et al. ont montré une forte analogie entre l'augmentation du désordre et l'application d'un champ magnétique parallèle [102]. Sur un système de faible désordre (mobilité de $4 \mathrm{~m}^{2} \cdot \mathrm{V}^{-1} \cdot \mathrm{s}^{-1}$ ), l'application d'un champ magnétique parallèle réduit l'amplitude du comportement métallique. Pour un champ magnétique de $12 \mathrm{~T}$, les courbes de résistivité en fonction de la température sont comparables à celles obtenues sur des échantillons à fort désordre (mobilité de $0,15 \mathrm{~m}^{2} \cdot \mathrm{V}^{-1} \cdot \mathrm{s}^{-1}$ ). Lorsque $B_{\|}$augmente, $n_{c}$ aug- 
(a)

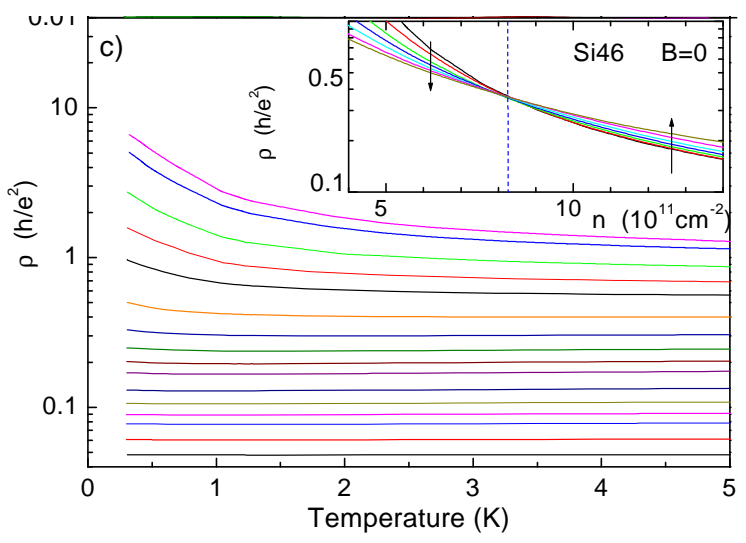

(c)

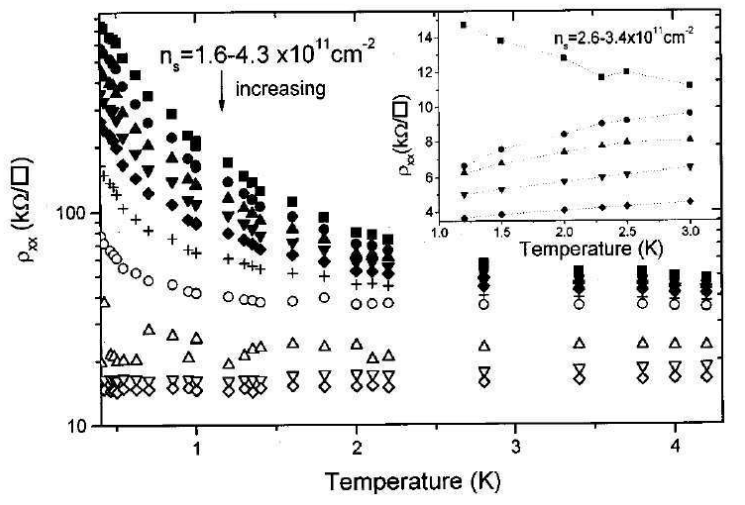

(b)

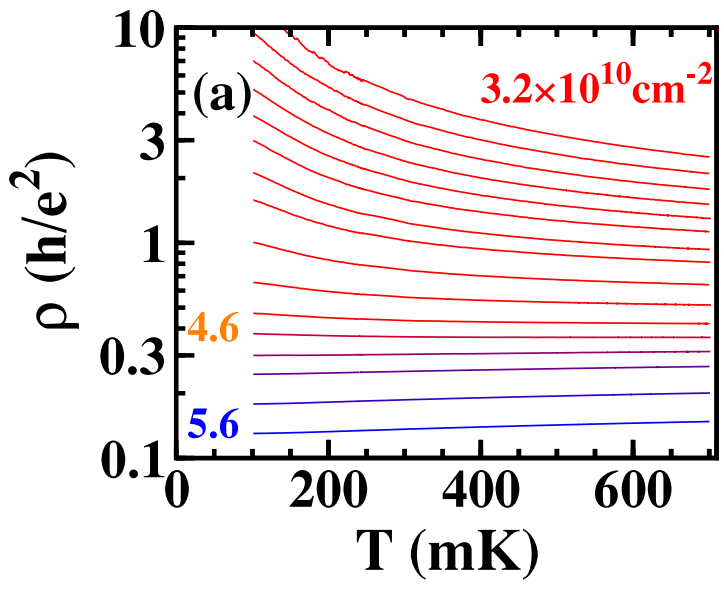

FIG. 2.9: Dépendance en température de la résistivité dans des systèmes de faible mobilité, pour les trois principaux systèmes présentant une transition métal-isolant. (a) MOSFET Si d'après Pudalov et al. [102], (b) hétérostructure $p$-GaAs d'après Simmons et al. [108], et (c) hétérostructure $p$-SiGe d'après Lam et al. [63].

mente et $\rho_{c}$ diminue, de la même manière que lorsqu'on augmente le désordre. De plus il y a un bon accord qualitatif entre les variations de $n_{c}$ et $\rho_{c}$ avec $B_{\|}$et avec le désordre (mobilité ou $k_{F} l_{e}$ ). Les auteurs en concluent que le champ magnétique parallèle induit une augmentation du désordre, qui serait dû à des impuretés magnétiques.

\subsubsection{Rôle du spin}

Pour un système bidimensionnel d'électrons, l'application d'un champ magnétique parallèle au gaz bidimensionnel ne se couple qu'au spin électronique, et n'a pas d'effet orbital.

Par des mesures en champ magnétique incliné sur des MOSFETs Si, Okamoto et al. ont montré que la saturation de la résistivité en fonction du champ magnétique parallèle dans les MOSFETs Si est due à l'alignement des spins du gaz d'électrons en interactions (liquide de Fermi) [94, 95]. Cette interprétation a été confirmée par des mesures d'oscillations de Shubnikov-de Haas dans un champ magnétique incliné avec un petit angle [99, 100]. Ainsi la valeur de la résistance semble influencée par l'aimantation des spins du gaz d'électrons. La dépendance linéaire du champ de saturation $B_{s a t}$ en fonction de la densité électronique 
$n_{s}$ semble confirmer cette interprétation $[92,94,99,100,101]$. On s'attend en effet à avoir :

$$
B_{s a t}=B_{p o l}=\frac{2 E_{F}}{g^{*} \mu_{B}}=\frac{h n_{s}}{e g^{*} m^{*}}
$$

où $\mu_{B}$ est le magnéton de Bohr, la dégénérescence de vallée $g_{v}=2$ étant incluse ici.

Tutuc et al. [163] ont effectué une mesure directe de la polarisation d'un gaz bidimensionnel de trous dans des puits quantiques $p$-GaAs en fonction du champ magnétique parallèle appliqué. Pour cela ils ont mesuré les oscillations de Shubnikov-de Haas dans un champ magnétique incliné pour lequel la composante parallèle au gaz de trous reste constante. La résistivité longitudinale tracée en fonction de $1 / B_{\perp}$ (où $B_{\perp}$ est la composante perpendiculaire du champ magnétique) présente des oscillations de Shubnikov-de Haas dont les fréquences caractéristiques (obtenues par transformée de Fourier) sont directement reliées au remplissage de chaque sous-bande de spin. Lorsque le champ magnétique augmente, ils montrent alors que le remplissage de la sous-bande inférieure (la plus remplie) augmente, puis sature à un champ magnétique parallèle $B_{\|}^{p}$ très proche du champ $B_{\|}^{*}$ caractérisant le changement de comportement de la magnétorésistance (de $\exp \left(B_{\|}^{2}\right)$ à $\left.\exp \left(B_{\|}\right)\right)$, confirmant que ce changement de comportement est bien lié à la polarisation complète du gaz d'électrons.

Cependant un champ magnétique parallèle peut également jouer sur le spin des états localisés et ainsi modifier la diffusion des électrons par ces états. Pudalov et al. [101] ont mesuré le champ magnétique de saturation $B_{s a t}$ sur des échantillons de différentes mobilités. À densité d'électron donnée, $B_{\text {sat }}$ varie d'un facteur 2-4 suivant les échantillons, ce qui semble indiquer que cette saturation ne correspond pas à l'alignement des spins des électrons libres, qui doit aboutir à une saturation ne dépendant que de la densité électronique [164], mais plutôt des spins des états localisés qui sont liés au désordre. Il faut cependant remarquer que la pente de $B_{\text {sat }}\left(n_{s}\right)$ est la même dans tous les échantillons de Pudalov et al. [101], ce qui est quand même en accord avec l'équation (2.18) si on admet l'existence d'un décalage sur la densité. Ce décalage a été interprété comme l'aimantation spontanée du gaz d'électrons [99]. Le fait que cet effet dépende fortement du désordre peut aussi signifier que cet effet provient du désordre et des états liés [101].

Dans les puits quantiques $p$-GaAs, le couplage spin-orbite associé à l'asymétrie du potentiel de confinement conduit à une levée de dégénérescence de spin à champ magnétique nul. Papadakis et al. [161, 106, 162] ont montré qu'il est possible de modifier le remplissage des sous-bandes de spin en modifiant la géométrie du potentiel de confinement par l'intermédiaire de deux grilles, l'une sur le dessus de l'échantillon et l'autre sur la face arrière, tout en conservant la densité totale de porteurs constante. ${ }^{2}$ Ils ont alors étudié l'influence du remplissage des sous-bandes de spin sur la transition métal-isolant, et plus particulièrement sur la phase métallique. Ils montrent que l'amplitude de la dépendance métallique (quantifiée par $(\rho(700 \mathrm{mK})-\rho(25 \mathrm{mK})) / \rho(25 \mathrm{mK}))$ est maximum lorsque la séparation des deux sous-bandes est maximale, et qu'elle est minimale lorsque la dégénérescence de spin n'est pas levée $[161,162]$. Cette variation suggère que la dépendance

\footnotetext{
${ }^{2}$ Les grilles modifient le champ électrique perpendiculairement au plan du gaz d'électrons, conduisant ainsi à un puits quantique carré ou triangulaire, avec une pente variable dirigée dans une direction ou l'autre.
} 
métallique est liée à l'existence de deux sous-bandes de spin séparées. Cette hypothèse est confirmée par les mesures en fort champ magnétique parallèle qui montrent que le comportement métallique est supprimé au-delà d'un champ critique.

Dans les puits quantiques p-GaAs, Papadakis et al. [106, 162, 165] ont étudié l'anisotropie de la magnétorésistance en champ magnétique parallèle quand ils appliquent un courant parallèle ou orthogonal au champ magnétique. Ils ont également étudié la magnétorésistance dans deux directions du plan, [011] et [233], qui présentent des mobilité différentes en raison d'une structure dans le désordre de surface conduisant à des mécanismes de diffusion différents. Le champ $B_{\|}^{*}$ de polarisation totale des électrons est plus faible que celui attendu par des calculs de bande. La différence est attribuée à une valeur élevé du produit $g^{*} m^{*}$ dans ces systèmes dont le facteur $r_{s}$ est élevé (entre 6,9 et 15,6). Ce résultat confirme le résultat d'Okamoto et al. [94]. Ces résultats suggèrent fortement que la magnétorésistance est due au dépeuplement d'une sous-bande de spin.

Papadakis et al. [165] ont étudié l'effet du potentiel de confinement sur le transport pour un courant appliqué dans les deux directions [01히 et [233]. Les mesures montrent que, alors que la résistivité résiduelle varie de manière très différente en fonction du potentiel de confinement dans les deux directions, l'amplitude du comportement métallique croît toujours lorsque l'écart entre sous-bandes de spin augmente. Cette expérience montre que le comportement métallique ne semble pas lié entièrement au mécanisme de diffusion, mais au remplissage des sous-bandes de spin. Cependant l'amplitude du comportement métallique est très différent dans les deux directions, ce qui suggère que le mécanisme de diffusion des porteurs a aussi un rôle à jouer.

On peut enfin remarquer que certains auteurs ont suggéré que la forte magnétorésistance peut aussi provenir d'un effet orbital, qui peut intervenir lorsque le champ magnétique est suffisamment fort pour que la longueur magnétique $l_{m}=\sqrt{\hbar c / e B}$ soit comparable à l'extension des fonctions d'ondes dans la direction orthogonale au G2D. Par des calculs de transport pour des électrons confinés dans un potentiel parabolique, Das Sarma et Hwang [166] montrent une forte magnétorésistance, et un changement de comportement lorsque la longueur magnétique devient inférieure à la taille du potentiel de confinement. Cette transition peut expliquer le changement de comportement observé dans les MOSFETs Si et les hétérostructures $p$-GaAs. Ils prédisent également une forte anisotropie entre la résistivité mesurée quand le courant est parallèle $\left(\rho_{\|}\right)$ou perpendiculaire au champ magnétique $\left(\rho_{\perp}\right), \rho_{\perp} \gg \rho_{\|}$. Cependant Pudalov et al. [101] n'ont pas observé, dans les MOSFETs Si, d'anisotropie pour un champ magnétique parallèle inférieur à 6 Tesla. Au-dessus de 6 Tesla, il existe une faible anisotropie de l'ordre de $10 \%$, qui peut être interprétée par un effet orbital. Dans les hétérostructures $p$-GaAs, Noh et al. [167] ont également observé une faible anisotropie, mais qui change de signe quand la densité diminue $\left(\rho_{\perp}>\rho_{\|}\right.$à haute densité et $\rho_{\perp}<\rho_{\|}$à faible densité). Leur conclusion est que l'anisotropie n'est pas lié à un effet orbital, mais est lié à une anisotropie du dépeuplement des sous-bandes de spin. De plus, Simonian et al. ont montré que la magnétorésistance ne dépend que du rapport $\left(g \mu_{B} B\right) /\left(k_{B} T\right)$, ce qui confirme que cet effet est lié au spin et non à un effet orbital $[93,146]$.

Enfin il est intéressant de citer le cas des puits quantiques $p$-SiGe, qui présentent un fort couplage spin orbite, dû à un facteur $g$ élevé et à l'asymétrie du potentiel de confinement. Ainsi, un champ magnétique faible est incapable de se coupler aux spins 
qui sont trop fortement couplés entre eux. Dans ce système, il a été montré qu'un champ magnétique parallèle n'a pas d'effet [141, 21]. Ce résultat vient confirmer le rôle du spin dans la magnétorésistance géante observée sur les MOSFETs Si et le p-GaAs.

\subsubsection{Rôle d'un champ magnétique perpendiculaire}

Les analogies entre la transition métal-isolant à champ magnétique nul et les transition effet Hall quantique-isolant pose la question de la nature commune des différentes phases. Nous avons déjà vu que Hanein et al. ont montré qu'il y a un passage continu entre la transition métal-isolant à champ magnétique nul et les transition à champ magnétique élevé dans le $p$-GaAs [113].

Il a été montré dans les MOSFETs Si que les mesures de résistivité en fonction de la température à différentes valeurs de densités électroniques présentent les mêmes caractéristiques à $B=0$ et à un champ magnétique correspondant à un remplissage $\nu=3 / 2$ [67]. La symétrie dans les courbes $I-V$ a été observée autour des transitions effet Hall quantique-isolant par Shahar et al. [168], et par Kravchenko et al. [84] et Simonian et al. [169] dans les MOSFETs Si autour de la transition métal-isolant à champ magnétique nul.

Ces analogies entre la TMI à 2D et champ magnétique nul et la transition EHQisolant à champ magnétique élevé est intéressante car elle peut être due à une origine commune des deux transitions. En particulier, il est maintenant connu que, entre les états de Hall, le système électronique en champ magnétique élevé présente des transitions de percolation. Plusieurs expériences dans des systèmes bidimensionnels de haute mobilité, tels les MOSFETs Si [35], les hétérostructures $n$-GaAs [36] et $p$-SiGe [170], ont montré que les exposants déterminés par l'annulation de l'énergie d'activation de la loi $\rho(T)$ ou du champ seuil dans les courbes $I-V$ conduisent à $\nu \approx 1$, compatible à $4 / 3$ attendu pour une transition de percolation classique [11].

\subsection{Interprétations théoriques de la transition métal- isolant}

La caractéristique nouvelle des systèmes dans lesquels est observée cette transition est la valeur élevée du paramètre $r_{s}$, et donc l'importance des interactions entre porteurs. Il est donc normal qu'un grand nombre d'approches aient voulu prendre en compte les fortes corrélations entre porteurs pour rendre compte de ces effets nouveaux. Mais d'autres approches ont essayé d'expliquer les résultats expérimentaux dans le cadre "classique" du liquide de Fermi. Deux questions se posent alors :

1. Quel est le rôle des interactions entre porteurs dans le nouveau comportement observé dans ces systèmes?

2. Le nouveau comportement peut-il s'expliquer dans le cadre de la théorie "classique" du liquide de Fermi où les interactions ne jouent qu'un rôle mineur?

Les observations expérimentales principales qui ont intrigué le plus la communauté scientifique et qui demandent des explications sont : 
1. Le comportement métallique de la dépendance en température de la résistivité jusqu'aux plus basses températures atteintes, alors q'un comportement isolant serait attendu à ces températures.

2. L'application d'un champ magnétique parallèle au plan du gaz bidimensionnel d'électron tend à localiser les électrons, supprimant le comportement métallique et ramenant les propriétés attendues pour un système bidimensionnel.

Étant donné l'aspect nouveau et inattendu du comportement métallique dans un système d'électrons en dimension deux, et de part la diversité des systèmes présentant un tel comportement, c'est cette phase qui a soulevé le plus d'intérêts quand à l'élaboration de modèles. Cependant la phase isolante pose également un certain nombre de questions : la très faible densité de porteurs laisse présager des effets de corrélations entre électrons encore plus forts que dans la phase métallique, et les nombreuses études menées pour la recherche d'une phase corrélée de type cristal de Wigner à champ magnétique nul montrent l'intérêt que peut susciter cette phase.

\subsubsection{Effet des interactions sur un système désordonné}

\section{Calculs perturbatifs}

La première suggestion qu'il puisse exister une phase métallique en dimension deux a été donnée par Finkel'shtein [171] par une étude des effets combinés des interactions et du désordre. Par une approche perturbative, il montre que l'analyse en loi d'échelle peut conduire à un comportement métallique à basse température, sans toutefois prévoir ni une transition métal-isolant en l'absence de champ magnétique, ni la nature de la phase métallique. Cependant, cette approche perturbative n'est valable que jusqu'à $r_{s} \approx 1$, valeur pour laquelle le comportement métallique commence à apparaître. Il est intéressant de noter que, dans ce calcul, l'application d'un champ magnétique extérieur conduit le système dans un état isolant en raison de l'effet Zeeman.

Cette théorie a été reprise dans le cadre de la TMI par Castellani et al. [172, 173] en montrant que, pour un faible désordre, elle peut être étendue au régime des très basses températures tout en restant valable. Cette théorie prévoit une résistance finie du système à température nulle et une dépendance en loi de puissance à basse température, celle-ci étant incompatible avec les dépendances couramment observées dans les systèmes expérimentaux (voir l'équation (2.2)). Cette théorie prédit une magnétorésistance en champ magnétique parallèle proportionnelle à $(B / T)^{2}$, compatible avec les expériences [86, 21] Cependant la théorie prédit une forte dépendance en température du coefficient de Hall qui n'est pas observée expérimentalement [155, 174].

Si et Varma [175] sont repartis de ce travail pour élaborer une théorie décrivant une transition métal-isolant, sans préciser la nature de la phase métallique. Cette théorie prédit que, à la transition, la conductivité et la compressibilité s'annulent. Il est intéressant de noter que la compressibilité a été mesurée sur du $p$-GaAs (voir §2.3.4) et qu'elle s'annule à la transition. 


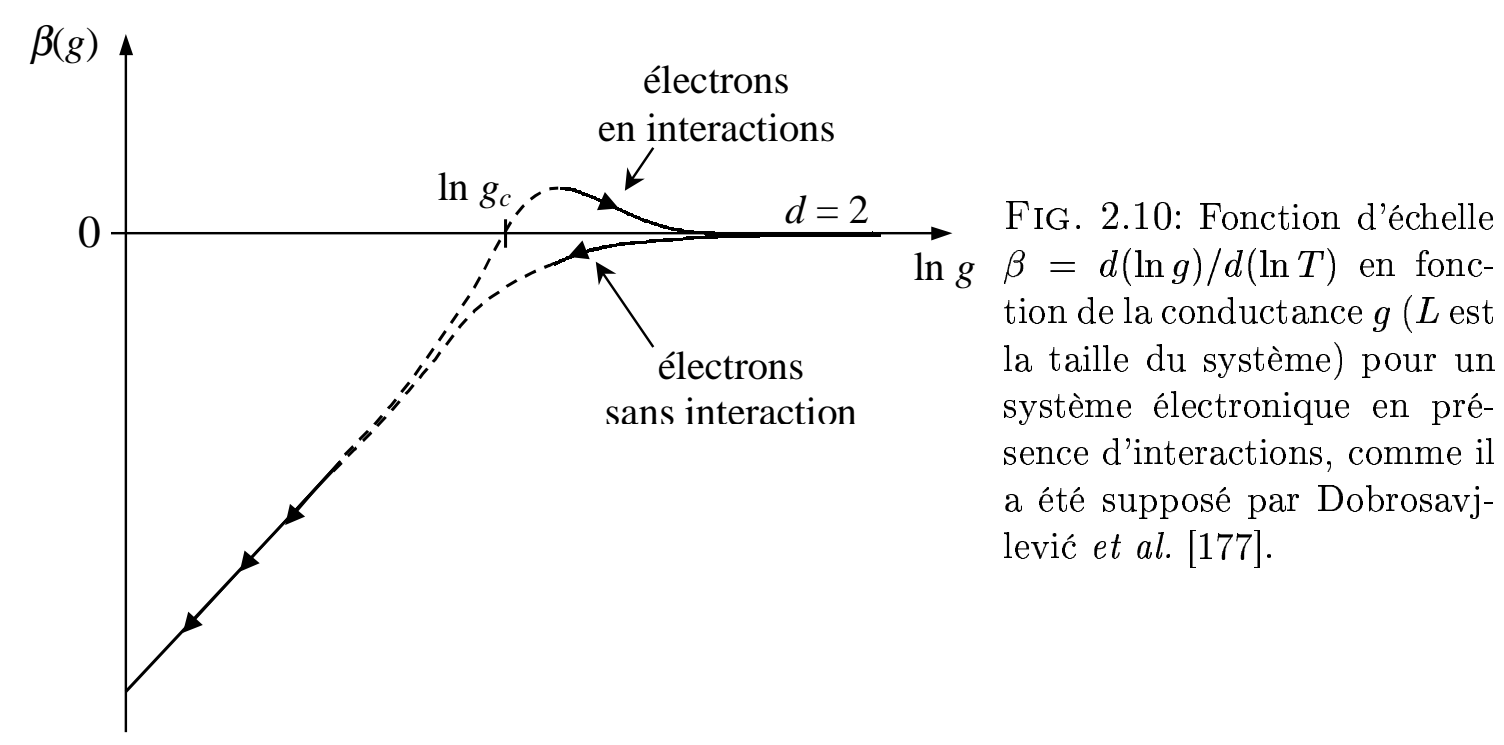

\section{Non liquide de Fermi}

La théorie d'échelle d'Abrahams et al. [4], montrant qu'un système bidimensionnel ne peut être qu'isolant, repose sur l'hypothèse essentielle que le système électronique peut être décrit par la théorie du liquide de Fermi. Cependant, à suffisamment forte interaction, on s'attend à ce que la théorie du liquide de Fermi ne soit plus valable [176]. Ainsi, Dobrosavjlević et al. [177] ont supposé que les interactions conduisent à une fonction $\beta=d(\ln g) / d(\ln L)$ positive pour une valeur de la conductance $g$ élevée ( $L$ est la taille du système), comme le montre la figure 2.10 , et conduit donc à une phase métallique pour laquelle les fonctions d'onde sont délocalisées à température nulle (voir aussi [178]). Dans ce nouveau schéma, il existe un point critique $g_{c}$ où une transition métal-isolant est attendue. On peut remarquer que la phase métallique supposée ne peut pas être un liquide de Fermi, puisque la limite sans interaction conduit à des fonctions d'ondes localisées.

L'étude du point critique $g_{c}$ a permis de montrer que la conductance suit une loi d'échelle analogue à celle observée dans les mesures expérimentales, et en particulier qu'il existe une symétrie $g^{*}\left(\delta_{n}, T\right)=1 / g^{*}\left(-\delta_{n}, T\right.$ ) (où $\delta_{n}=\left(n_{s}-n_{c}\right) / n_{c}$ est l'écart à la transition et $T$ est la température) également observée dans les mesures expérimentales sur les MOSFETs Si [67].

\subsubsection{Cristallisation de Wigner}

À suffisamment forte interaction, on s'attend à la formation d'une phase électronique fortement corrélée, tel le cristal de Wigner, ou un verre de Wigner en présence de désordre. Dans la phase basse densité, les expériences montrent en effet des comportements collectifs qui peuvent être interprétés par une telle phase. La question qui se pose alors est la manière dont s'effectue la transition entre la phase liquide de Fermi et la cristal (ou verre) de Wigner. Plusieurs auteurs ont envisagé que le comportement métallique puisse être lié à cette transition. 


\section{Cristal de Wigner à basse densité?}

Dans les expériences, plusieurs indications permettent d'envisager que la phase isolante est une phase collective cristalline ou vitreuse.

Dans ces systèmes propres, la caractéristique $I-V$ présente une tension seuil $V_{s}$, endessous de laquelle la caractéristique est linéaire avec une forte résistivité, et au-dessus de laquelle le courant augmente très fortement quand la tension augmente. Nous verrons plus en détail au paragraphe 4.3 que ces non-linéarités peuvent être interprétées comme dues à l'accrochage d'une phase électronique collective, telle le cristal de Wigner, et a été observée pour des ondes de densité de charge [179], le cristal de Wigner classique pour des électrons à la surface de l'hélium $[180,181]$ et pour le cristal de Wigner induit par un fort champ magnétique $[50,48,51]$. Une tension seuil dans la caractéristique $I-V$ à champ magnétique nul a été observée dans les MOSFETs Si $[45,81]$ et dans les hétérostructures $p$-GaAs [87]. Yoon et al. [87] ont comparé le seuil obtenu pour des hétérostructures $p$ GaAs avec les prédictions données par le modèle de Chui, prédisant la fonte d'un cristal de Wigner par la formation et la dissociation de paires de dislocations [182]. Alors que la tension seuil mesurée dans les échantillons de Yoon et al. est en accord avec ces calculs, la température de fusion qu'ils observe est un ordre de grandeur en dessous des prédictions de Chui [87].

L'analyse de la phase isolante en tant que cristal de Wigner a également été suggéré par Pudalov et al. qui ont mesuré le bruit large bande en fonction de la tension appliquée [45]. Ils observent une forte augmentation du bruit associée au seuil de décrochage, puis une diminution lente quand la tension augmente encore. Ces mesures renforcent l'idée que la phase isolante est un cristal de Wigner accroché sur le désordre.

L'existence d'une phase cristal de Wigner à faible densité est confortée par la valeur élevée du paramètre $r_{s}^{c}$ à la transition métal-isolant dans les systèmes les plus propres $\left(r_{s}^{c} \approx 35\right.$ dans les hétérostructures de Yoon et al. [87]), très proche de la valeur $r_{s}^{W} \geq 37$ prévue par les calculs Monte-Carlo de Tanatar et Ceperley [44] pour la cristallisation de Wigner. D'autre part, la dépendance en fonction du désordre valeur de $r_{s}$ à la transition métal-isolant, donnée par la figure 2.8(b), est en accord avec les prédictions de Chui et Tanatar qui ont montré que le cristal (ou verre) de Wigner est stabilisé par le désordre, donnant un paramètre $r_{s}^{W}$ décroissant quand le désordre augmente [14].

Enfin les mesures de compressibilité sur des hétérostructures $p$-GaAs ont montré que la phase isolante est incompressible [124, 125, 126], ce qui est caractéristique d'une phase solide.

\section{Fusion du cristal de Wigner}

Il existe donc de fortes indications pour que la phase isolante soit un cristal ou un verre de Wigner. On peut maintenant se demander comment se fait le passage de l'état de cristal à l'état de liquide.

Phase intermédiaire. Par les études Monte-Carlo, Tanatar et Ceperley [44] ont comparé le domaine de stabilité du liquide de Fermi et du cristal de Wigner lorsque l'interaction varie, et montre que le liquide de Fermi est l'état de plus basse énergie pour $r_{s}<r_{s}^{W}$, 
alors que le cristal de Wigner a une énergie plus faible pour $r_{s}>r_{s}^{W}$, retrouvant ainsi la transition du premier ordre décrite par Wigner [2]. Cependant, dans cette approche, Tanatar et Ceperley n'envisagent pas d'autre phase possible que ces deux limites : cette étude ne prouve donc pas qu'il ne puisse pas exister une phase de plus basse énergie différente de ces deux phases. Par des simulations exactes sur des systèmes de $N$ électrons dans un réseau carré $L \times L(N=4$ et $L=6$ dans la majorité de ces études $)$ en présence de désordre et avec des conditions de bords périodiques, Benenti et al. ont montré qu'il existe une phase intermédiaire entre le liquide de Fermi et le cristal de Wigner [3] (voir également la revue de Pichard et al. [183]).

Benenti et al. [3] ont tout d'abord montré que, pour $r_{s}>r_{s}^{F} \approx 5$, i.e. avant la transition de Wigner située à $r_{s}^{W} \approx 10$ en présence de désordre, les théories de champ moyens ne permettent plus de décrire le système, ce qui montre que les effets de corrélations deviennent importants. De plus, le calcul des courants permanent en présence d'un flux fait apparaître trois régimes [184] : pour $r_{s}<r_{s}^{F}$ les courants sont répartis aléatoirement, pour $r_{s}^{F}<r_{s}<r_{s}^{W}$ les courants sont orientés préférentiellement dans la direction du potentiel vecteur créé par le flux, et pour $r_{s}>r_{s}^{W}$ la valeur moyenne des courants s'annule (voir aussi [185]).

Des étude numériques sur les mêmes systèmes, mais en prenant en compte le spin, ont été réalisées par Selva et Pichard [186]. Ils ont montré que, pour $r_{s}<r_{s}^{F S} \approx 2,2$ (et donc proche de $r_{s}^{F} \approx 5$ déduit du courant permanent), l'instabilité de Stoner prévu par les calculs en champ moyen s'applique. Par contre, l'aimantation du système s'écroule au-delà de $r_{s}^{F S}$, et s'annule pour $r_{s}>r_{s}^{W}$, correspondant à la formation d'un cristal antiferromagnétique dû à la géométrie choisie pour ces simulations. Ces simulations permettent donc de mettre en évidence la phase intermédiaire par son comportement magnétique. Des études en fonction d'un champ polarisant (équivalent au champ magnétique parallèle dans les expériences) prévoient également une forte magnétorésistance positive et une diminution de $r_{s}^{W}$ quand le champ magnétique augmente, deux caractéristiques qui sont observées dans les expériences (voir §2.3.2). Ces observations, associées au fait que le comportement réantrant de la phase isolante pour $r_{s}<r_{s}^{F}$ a été observée dans certains systèmes [85, 76], suggèrent que ces simulations sur des petits systèmes reproduisent bien la physique des gaz d'électrons étudiés expérimentalement.

Enfin, Katomeris et al. ont réalisé des études sans désordre et sans spin afin de déterminer la nature de la phase intermédiaire observé [187]. Ils ont montré que cette phase ne peut pas se décrire correctement, ni dans une base d'ondes planes, ni dans une base d'états localisés. Mais elle se projette bien sur une base formée d'un nombre réduits d'états choisis parmi les états d'ondes planes et de particules localisées, et peut donc être décrite comme une superposition quantique d'un liquide et d'un solide. Ce résultat peut être interprété comme la fusion du cristal de Wigner par formation de défauts localisées, comme il a été envisagé par Andreev et Lifshitz [188].

Mélange de phases. Un autre scénario proposé pour la formation du cristal de Wigner repose sur l'idée que les deux phase liquide de Fermi et cristal de Wigner peuvent coexister. La nouvelle phase est alors un mélange classique de ces deux phases.

En supposant que la cristallisation de Wigner suit une transition de phase du premier 
ordre, Spivak [189] a montré que, en raison de l'interaction Coulombienne et de l'écrantage par la grille dans les MOSFETs, les deux phases liquide et solide ne se séparent pas complètement, mais forment un mélange de gouttelettes de la phase minoritaire dans la phase majoritaire. Dans la limite où la proportion de cristal de Wigner est faible, la conductance du système peut être calculée en considérant la diffusion du liquide de Fermi sur des gouttelettes de cristal de Wigner. Dans cette limite, il est possible de reproduire qualitativement la dépendance métallique de la résistivité en fonction de la température, ainsi que la dépendance en champ magnétique parallèle. Lorsque la proportion de parties cristal de Wigner devient suffisamment élevée, on s'attend à avoir une transition de percolation vers un état cristal ou verre de Wigner.

Shi et al. $[190,191]$ proposent également un scénario de cristallisation de Wigner par séparation de deux phase, une phase haute densité (liquide de Fermi) et une phase basse densité (cristal ou un verre de Wigner), favorisée par le désordre. Ce modèle conduit à une transition de percolation qui sera vue plus en détail au paragraphe 2.5.4.

Fusion en présence de désordre. Il est couramment supposé que, en l'absence de désordre, la transition de Wigner soir une transition du premier ordre. Cependant, Chakravarty et al. suggèrent que, en présence de désordre, la fusion du verre de Wigner soit continue, et donne donc lieu à une transition de phase du deuxième ordre [192]. Dans ce modèle, le liquide est dégénéré en spin. De plus, le verre de Wigner étant invariant par translation, cet état reste un liquide de spin (sans réelle formation d'une bande interdite de spin).

Dans ce modèle, Chakravarty et al. prédisent une compressibilité qui s'annule à la transition, comme il a été observé expérimentalement (voir §2.3.4). D’autre part ils expliquent la magnétorésistance positive en champ magnétique parallèle, avec un comportement qui varie continûment à la transition et en particulier une magnétorésistance qui reste positive dans la phase isolante.

\section{Verre d'électrons}

L'effet combiné des interactions entre électrons, qui tendent à former un système ordonné, et du désordre, qui tend à répartir les électrons de manière désordonnée, permettent d'envisager un état frustré de type verre d'électron (et donc sans création de bande d'énergie interdite) à la place du cristal de Wigner.

Les mesures de fluctuations de résistance effectuées sur des MOSFETs Si très désordonnés [82] et peu désordonnés [83], montrant un ralentissement de la dynamique du gaz d'électron en-dessous d'une valeur $n_{g}$ de la densité, semblent confirmer l'hypothèse d'une transition vers un état de verre. Dans ces expériences, le comportement vitreux apparaît à une densité plus élevée que la transition métal-isolant, ce qui semble favoriser un modèle de verre métallique.

Pour décrire la phase verre de Wigner, Pastor et Dobrosavljević ont utilisé un modèle d'électrons en interactions sans spin en présence de désordre, et utilisent une méthode de résolution basée sur une formulation en champ moyen dynamique [193]. L'hypothèse principale de ce modèle, justifiée par la longue portée des interactions, résulte dans la possibilité de traiter le verre d'électrons par un modèle de champ moyen. Lorsque l'énergie 
d'interaction est grande devant l'énergie de Fermi, il se forme une pseudo-bande interdite dans la densité d'états à une particule, conduisant à une phase vitreuse. Lorsque l'énergie de Fermi augmente au-delà d'un certain seuil, la phase vitreuse est supprimée et ils retrouvent les propriétés d'un liquide normal, caractérisant ainsi la transition isolant-métal observée dans les expériences. Dalidovitch et Dobrosavljević [194] ont montré que ce modèle peut rendre compte des observations expérimentales dans les MOSFETs Si [82, 83], et notamment le ralentissement de la dynamique à l'approche de la phase isolante (pour $n_{s}>n_{c}$ ) : avant l'observation d'une phase verre de Wigner, il existe une phase intermédiaire de verre métallique dont la conductivité est décrite par $\sigma(T) \propto T^{3 / 2}$. Cette phase intermédiaire est également décrite par Dobrosavljević et al. [195].

Sans entrer dans les détails, on peut cependant citer d'autres études théoriques évoquant la transition vers un verre d'électrons à basse densité, par exemple Thakur et Neilson [196, 197] ou Chakravarty et al. [192]. Sachdev [198] propose l'existence d'une phase verre de spin, pouvant interpréter les fortes dépendances en champ magnétique.

\subsubsection{Supraconductivité en dimension deux}

Pour expliquer la phase métallique au-delà d'une certaine densité critique $n_{c}$, certains auteurs ont évoqué la possibilité d'avoir une phase supraconducteur en dimension deux. Cette hypothèse a été suggérée par les nombreuses analogies entre les caractéristiques de la résistivité autour de la transition métal-isolant, et celles observée dans des films minces autour de la transition supraconducteur-isolant (cette transition est décrite par exemple dans la référence [58]). Les observations communes sont (a) les lois d'échelle sur la résistivité en fonction de la température et du champ électrique [199, 200], (b) la symétrie de la caractéristique $I-V$ par rapport à la droite $V=\rho_{c} I$ (où $\rho_{c}$ est la résistivité à la transition) [201] et (c) l'exposant dynamique déduit des lois d'échelle $z \approx 1$ [143]. Enfin, l'existence d'un champ magnétique critique qui supprime la phase métallique, montré par Yoon et al. [89] dans des hétérostructures $p$-GaAs, est compatible avec l'idée d'un état supraconducteur. En particulier, les mesures en champ magnétique parallèle ressemble à celles faites dans le cadre de la transition supraconducteur-isolant (voir [121] et les références incluses). On peut cependant noter que, contrairement au cas de la transition supraconducteur-isolant, les gaz bidimensionnels de porteurs ne présentent jamais de résistance nulle, et la présence de magnétorésistance négative dans certains échantillons autour de la transition métal-isolant en dimension deux contredit cette vision d'un état supraconducteur.

Un certains nombre d'études théoriques ont ainsi évoqué un état supraconducteur en dimension deux, notamment par Takada [202, 203], Hanke et Kelly [204, 205], Ren et Zhang [206]. D'autres auteurs ont montré que cet état pouvait être appliqué dans le cadre de la phase métallique, notamment Phillips et al. [207, 208], Kirkpatrick et Belitz [209, 210, 211], Thakur et Neilson [212].

\subsubsection{Modèles de percolation}

On a déjà vu que certains modèles de transition du liquide de Fermi vers le verre de Wigner proposent que la transition se fasse par l'intermédiaire d'une percolation [189, 
190, 191]. Ce mécanisme permettrait en particulier d'expliquer que l'on n'observe pas de transition de phase du premier ordre, mais plutôt une transition de phase du deuxième ordre, suggérée par l'observation de lois d'échelle. Mais d'autres types de percolation peuvent également être évoquées dans le cadre de gaz bidimensionnels d'électrons dilués. Ce théories ont été confortés par l'observation de fortes inhomogénéités spatiales dans les mesures de compressibilité locale aux abords de la transition métal-isolant [125, 126]. De même, les mesures de transport dans la phase métallique ont suggéré une conduction par l'intermédiaire de deux phases mélangées, une phase métallique et une phase isolante $[61,74,70]$.

Percolation classique. Le modèle de percolation proposé par Meir [213, 214, 215] ne prend pas en compte les interactions entre porteurs. Dans un système très dilué en présence de désordre, les homogénéités du potentiel conduisent à la formation de "lacs" d'électrons indépendants séparés entre eux par des points contact quantiques (PCQ). Le transport dans les PQC est cohérent et se fait par effet tunnel, alors que le temps de transit dans le un lac est supposé suffisamment pour conduire à un déphasage complet. On peut ainsi considérer que le système est un réseau de résistances classiques, chaque résistance étant déterminée par la résistance tunnel d'un PCQ, qui, à température nulle, vaut soit $h / e^{2}$, soit 0 , suivant la valeur du potentiel chimique. Un tel système présente une transition métal-isolant à une valeur critique de la densité électronique $n_{s}=n_{p}$, correspondant à la transition de percolation. Il est cependant important de noter que cette densité critique est plus faible que celle déduite de l'inversion de pente de $\rho(T)\left(n_{p}<n_{c}\right)$, comme il a été suggéré dans le paragraphe 2.4.1. L'effet de la température est de modifier la résistance de chaque lien, qui dépend exponentiellement de la température par l'intermédiaire de la distribution de Fermi.

Des considérations générales, ainsi que des simulations numériques basées sur ce modèles ont montré que plusieurs observations expérimentales sont reproduites par ce modèle. (1) Les comportements métallique et isolant de la résistivité en fonction de la température sont bien reproduits, et en particulier la loi (2.2) dans la phase métallique, et avec un bon ordre de grandeur de la résistivité $\left(\approx h / e^{2}\right.$ près de la transition). Cependant la dépendance métallique ne doit pas excéder un facteur 2 dans un système dégénéré, alors qu'elle peut atteindre un facteur 10 dans les mesures sur des MOSFETs Si. (2) Ce modèle prévoit une variation de la densité critique $n_{c}$ an fonction d'un champ magnétique perpendiculaire semblable à celle observée dans certains systèmes (voir §2.3.2) [215]. (3) Il prévoit enfin une suppression du caractère métallique en présence d'un champ magnétique parallèle [214].

Percolation de deux liquides quantiques. Le modèle de percolation proposé par He et Xie fait intervenir une phase liquide conductrice séparée par des régions d'une phase vapeur isolante [216]. Dans les MOSFETs Si, les charges négatives du gaz d'électrons sont compensées par des charges positives de la grille qui sont mobiles. Ainsi on peut considérer ce système comme deux gaz de porteurs en interaction, l'un étant le gaz $2 \mathrm{D}$ d'électrons, le deuxième peut être comme un gaz de trous. A haute densité électronique, le système est un liquide d'électrons-trous métallique, alors qu'à basse densité c'est un gaz d'excitons 
isolant. A densité intermédiaire il y a un mélange de ces deux phases, et la conductivité du système peut s'écrire comme la somme des conductivités : $\sigma=\sigma_{l}+\sigma_{g}$.

Ce modèle permet d'expliquer les dépendances en température métalliques de la résistivité, les non-linéarités de la caractéristique $I-V$ à faible champ électrique, ainsi que les dépendances de la résistivité en fonction d'un champ magnétique parallèle. Cependant ce modèle est spécifique au MOSFET Si et ne peut pas s'appliquer aux hétérostructures GaAs/GaAlAs pour lesquelles les donneurs chargés positivement sont fixés dans le cristal, et donc ne sont pas mobiles.

Méta-percolation. Shi et al. ont proposé un modèle de percolation quantique dans un réseau carré, avec une cohérence de phase finie [190]. Le transport à travers les liens se fait de manière cohérente, et un déphasage est introduit sur une certaine fraction des ces liens. La conductance d'un tel système a alors un contribution quantique et une contribution classique. Lorsqu'aucun déphasage n'est introduit, le système est toujours isolant, ce qui est en accord avec la théorie d'échelle de la localisation (voir §1.3.3). Lorsqu'un certain pourcentage de sites ont un déphasage, le système présente un caractère métallique audessus d'un taux de remplissage des sites $P_{c}$, alors qu'il est isolant en-dessous de $P_{c}\left(P_{c}\right.$ est différent de la probabilité de lien critique de la transition de percolation). Cette transition métal-isolant est accompagnée d'une divergence de la longueur de corrélation, ce qui est caractéristique d'une transition de phase du deuxième ordre.

Dans les systèmes réels, ce type de percolation peut être dû à l'existence d'un mélange de deux phases de densités différentes, la phase haute densité étant un liquide d'électrons, la phase basse densité un crystal ou un verre de Wigner. Enfin, comme la longueur de cohérence de phase doit tendre vers l'infini quand la température tend vers zéro, cette transition doit disparaître à température nulle, sauf si un mécanisme de déphasage persiste à température nulle.

Mélange de phases. Dans un gaz d'électrons en dimension deux, on s'attend à observer un liquide de Fermi à faible valeur de $r_{s}$, et un cristal de Wigner à forte valeur de $r_{s}$ (supérieure à 37 pour un système sans désordre [44]). D'autre part il a été montré que la phase liquide de Fermi devient instable pour $r_{s} \gtrsim 2$. Ainsi ces remarques conduisent naturellement à se poser la question de la nature de la phase intermédiaire pour $2 \leqslant$ $r_{s} \leqslant 37$ (voir $\S 2.5 .2$ ). Dans les modèles qui sont présentés dans ce paragraphe, il n'y a pas formation réellement d'une nouvelle phase, mais l'état intermédiaire est un mélange d'une phase liquide de Fermi de haute densité électronique, et une phase cristal de Wigner de basse densité électronique.

Shi et al. [217] ont suggéré qu'un gaz d'électrons pour $r_{s}>2$ étant instable, il est plus favorable qu'il réduise sa surface de façon à augmenter $r_{s}$, et forme ainsi des gouttelettes de liquide dense entourées de zones vides (ou de zones de plus faible densité, formant un cristal de Wigner désordonné, lorsque la température est non nulle). Cependant cette réduction de surface s'accompagne d'une augmentation de l'énergie de Fermi. Ils ont montré que, pour un système sans désordre, le mécanisme de formation des gouttelettes n'est pas favorable. Par contre, en présence de désordre, les électrons ont tendance à se rassembler dans les creux du potentiels, favorisant la formation de gouttelettes. Pour former 
ces gouttelettes, ils ont de plus montré qu'il est nécessaire que l'interaction Coulombienne soit écrantée, ce qui est le cas les systèmes expérimentaux, soit en raison d'une grille métallique dans les MOSFETs silicium, soit par le mécanisme de Thomas Fermi dans les hétérostructures. Un tel système physique est alors un très bon candidat pour le modèle de méta-percolation, développé par les mêmes auteurs [190], et qui a été présenté dans le paragraphe précédent. Shi et Xie [191] ont également montré que ce modèle de gouttelettes permet d'interpréter le changement de comportement de la compressibilité à la traversée de la transition métal-isolant observé par Dultz et Jiang [124], et Ilani et al. $[125,126]$.

On peut enfin noter que le modèle développé par Spivak [189] pour interpréter le comportement de la résistivité dans la phase métallique conduit à un scénario analogue de percolation d'un liquide de Fermi métallique dans un cristal de Wigner accroché isolant. Spivak considère également la formation de zones liquides mélangées à des cristallites, et stabilisées par l'interaction Coulombienne écrantée entre les électrons.

\subsubsection{Interprétations classiques du comportement "métallique"}

En considérant que certaines propriétés du gaz bidimensionnel d'électrons dans la phase "métallique" peuvent être interprétées dans le cadre de la théorie du liquide de Fermi (notamment la magnétorésistance négative à faible champ magnétique), certains auteurs ont essayé d'expliquer les observations expérimentales, principalement la dépendance en température de la résistivité et la magnétorésistance, par des modèles de particules indépendantes. Ces modèles ont permis de prouver qu'un comportement de type "métallique" (i.e. $d \rho / d T>0)$ n'est pas nécessairement lié à une délocalisation des fonctions d'onde électroniques à température nulle.

La plupart de ces modèles ne prennent pas en compte les corrections quantiques dues au désordre et aux interactions. La résistivité obtenue correspond alors à une résistivité "de Drude", à laquelle doivent s'ajouter ensuite les corrections quantiques, conduisant en particulier à un comportement métallique à suffisamment basse température. Le principal débat consiste alors à savoir si les températures atteintes dans les expériences sont suffisamment basses pour espérer observer le comportement isolant dû aux corrections quantiques.

\section{Corrections d'interactions du liquide de Fermi}

Zála, Narozhny et Aleiner [218] ont calculé les corrections de conductivité dues aux interactions dans l'hypothèse du liquide de Fermi et dans une large gamme en température, étendant ainsi le calcul d'Altshuler et al. [24, 25]. En particulier, dans le régime balistique pour lequel l'énergie thermique est supérieure à l'énergie caractéristique du désordre, $k_{B} T \gg \hbar / \tau$ (où $\tau$ est le temps de transport), cette correction aboutit à une dépendance métallique de la résistivité en fonction de la température $(d \sigma / d T>0)$. Dans ce régime, la conductivité varie linéairement avec $T$, alors qu'à basse température $\left(k_{B} T \ll \hbar / \tau\right)$, Zála et al. retrouvent la correction logarithmique isolante $(d \sigma / d T<0)$ prévue par les théories antérieures. Enfin, la théorie de Zála et al. prend en compte les effets d'écrantage dépendant de la température. Dans le même régime, Zála et al. ont également calculé la 
dépendance en champ magnétique parallèle de la résistivité afin de rendre compte de la forte magnétorésistance observée expérimentalement [219].

Plusieurs auteurs ont comparé les mesures expérimentales de conductivité en fonction de la température avec la théorie de Zála et al. . Ces expériences ont permis de déduire le paramètre du liquide de Fermi $F^{*}$, qui est compris entre -0, 14 et -0,5 dans les MOSFETs Si [220, 221, 222], entre $-0,3$ et $-0,7$ dans les hétérostructures $p$-GaAs [223, 224], et entre $-0,55$ et $-0,65$ dans les hétérostructures $p$-SiGe [21].

Dans des hétérostructures $p$-GaAs de très forte mobilité $\left(\mu_{\max } \approx 180 \mathrm{~m}^{2} .(\mathrm{Vs})^{-1}\right)$, Noh et al. ont montré que $\hbar / k_{B} \tau$ varie entre 16 et $80 \mathrm{mK}$ dans la gamme de densités où la transition métal-isolant est observée [224]. Dans ce système, la dépendance "métallique" linéaire observée à basse température peut donc être attribuée aux corrections calculées par Zála et al. Le paramètre du liquide de Fermi $F^{*}$ évalué à partir de la pente de la dépendance linéaire a une dépendance non monotone en fonction de la densité, et en particulier il diminue, en valeur absolue, quand la densité diminue, ce qui est l'inverse de la dépendance calculée dans le cas où le paramètre $r_{s}$ est faible. Cette dépendance, non prévue par la théorie, peut être attribuée à la forte valeur du paramètre $r_{s}(>22)$ dans les échantillons de Noh et al. Enfin, une confirmation de cette dépendance non prévue de $F^{*}$ est donnée par la mesure du facteur de Landé effectif,$g^{*}$, calculé à partir des courbes de magnétorésistance en champ magnétique parallèle.

Vitkalov et al. [221] ont comparé les dépendances en température et en champ magnétique parallèle de la résistivité dans des MOSFETs Si avec la théorie proposée par Zála et al. . Ils ont montré qu'il est possible d'obtenir un ajustement correct pour les dépendances en champ magnétique, mais les paramètres déduits de cet ajustement (le paramètre du liquide de Fermi $F^{*}$, le temps de transport $\tau$ et la levée de dégénérescence de vallées $\Delta_{V}$ ) ne permettent alors pas de rendre compte des dépendances en température. Le comportement métallique est plus prononcé que ne le prévoit la théorie, ce qui laisse penser qu'une source de diffusion manque dans le calcul de Zála et al..

\section{Écrantage dépendant de la température}

En prenant en compte l'écrantage du désordre par les interactions électron-électron dans le calcul de la conductivité, Gold et Dolgopolov ont montré que la conductivité peut présenter une dépendance "métallique" en température (i.e. $d \sigma / d T<0$ ) [19]. Dans l'expression de la conductivité de Drude, le libre parcours moyen élastique dépend de la température par l'intermédiaire de la constante diélectrique, conduisant à l'expression de la conductivité [19] :

$$
\sigma_{D}(T)=\frac{n_{s} e^{2} \tau_{e}(T)}{m^{*}}=\sigma_{D}(0)\left\{1-C\left(n_{s}\right) \frac{T}{T_{F}}-D\left(n_{s}\right)\left(\frac{T}{T_{F}}\right)^{3 / 2}+\mathcal{O}\left[\left(\frac{T}{T_{F}}\right)^{2}\right]\right\}
$$

où $C\left(n_{s}\right)$ et $D\left(n_{s}\right)$ sont des paramètres qui dépendent du mécanisme de diffusion, et augmentent quand la densité diminue. Cette équation traduit un comportement "métallique" linéaire au premier ordre en $T / T_{F}$ (donc à basse température). Dans ce modèle, Dolgopolov et Gold ont montré que l'application d'un champ magnétique parallèle induit une levée de dégénérescence de spin qui modifie la capacité d'écrantage du gaz d'électrons, 
conduisant à la magnétorésistance observée dans ces systèmes [225] : en effet, en raison du principe de Pauli, ce sont les électrons de spin up qui écrantent plus favorablement la diffusion des électrons de spin down, et inversement. Cet écrantage est donc diminué lorsque le système est polarisé.

Une dépendance "métallique" linéaire a été observée dans différents gaz bidimensionnels de porteurs de densité élevée : par Cham et al. [226] et par Vyrodov et al. [227] dans les MOSFETs Si, par Emeleus et al. dans des hétérostructures $p$-SiGe [228] et par Henini et al. dans des hétérostructures $p$-GaAs [229]. Dans tous les cas, la théorie de Gold et Dolgopolov permet d'expliquer raisonnablement la dépendance en température de la résistivité $(d \rho / d T>0)$.

Dans des puits quantiques $p$-SiGe, dont la mobilité (inférieure à $0,7 \mathrm{~m}^{2} / \mathrm{Vs}$ ) reste faible en comparaison des MOSFET Si et des structures $p$-GaAs, Senz et al. ont montré que le comportement métallique de la résistivité à basse température peut s'expliquer entièrement par ce modèle d'écrantage dépendant de la température [156]. Pour cela, il ont extrait des mesures en champ magnétique et de résistance Hall la valeur des corrections quantiques de conductivité dû à la localisation faible $\delta \sigma_{L F}$ et aux interactions entre électrons $\delta \sigma_{i n t}$. La conductance résultante, $\sigma_{D}(T)=\sigma(T)-\delta \sigma_{L F}-\sigma_{i n t}$, varie linéairement avec la température, comme attendue par la l'équation (2.19), et la constante $C$ déduite est compatible avec la théorie de Gold et Dolgopolov [19].

Ce modèle a été également évoqué par Hamilton et al. pour interpréter le comportement métallique dans des hétérostructure $p$-GaAs de haute mobilité $\left(\mu_{\max } \approx 110\right.$ $\mathrm{m}^{2}$. (Vs $\left.)^{-1}\right)[160]$ : en traçant la variation de conductivité, $(\sigma(T)-\sigma(T=0)) / \sigma(T=0)$, en fonction du rapport $T / T_{F}$, les courbes pour toutes les densités "métalliques" se regroupent en une courbe unique, linéaire pour $T / T_{F}<0,2$. Ainsi le mécanisme d'écrantage dépendant de la température semble pouvoir interpréter qualitativement les courbes expérimentales. Cependant le regroupement de toutes les courbes impose que les constantes $C\left(n_{s}\right)$ et $D\left(n_{s}\right)$ de l'équation (2.19) ne doivent pas varier avec la densité de porteurs, alors que le calcul analytique de Gold et Dolgopolov prévoient que $C$ et $D$ augmentent quand la densité diminue [19]. Enfin, cette interprétation en termes d'écrantage dépendant de la température ne permet pas d'expliquer la forte dépendance observée dans les MOSFETs silicium (chute d'un facteur 5 à 10 de la résistivité lorsque la température baisse de $4 \mathrm{~K}$ à quelques dizaines de $\mathrm{mK}$ ) [102]. De plus cette interprétation a été réfutée par Coleridge et al. [21] pour les hétérostructures $p$-SiGe qui disent qu'une telle théorie ne permet pas de justifiée la saturation de la résistivité à basse température dans tous les cas.

Des calculs plus complets, en prenant en compte les termes de Hartree et de Fock, ont été réalisés par Herbut dans le modèle de Hubbard, et donc pour des interactions sur sites [230]. Les dépendances en température et en champ magnétique parallèle sont qualitativement en accord avec les calculs de Gold et Dolgopolov.

L'idée de l'écrantage dépendant de la température a été reprise par Das Sarma et Hwang pour calculer la dépendance en température de la résistivité $\rho(T)$ dans la phase métallique en supposant qu'un nombre $n_{c}$ d'électrons sont gelés sur les impuretés à l'interface, mais sans donner de mécanisme physique conduisant à ce gel [148, 149]. Cette dernière hypothèse permet de trouver une forte dépendance en température de la résistivité, compatible avec les résultats sur les MOSFETs Si. Dans ce scénario, la transition métal-isolant intervient lorsque tous les porteurs sont gelés et qu'il ne reste plus de porteurs 
libres. Par une résolution de l'équation de Boltzman pour la diffusion sur des impuretés ionisées, en prenant en compte la dépendance en température de l'écrantage, Das Sarma et Hwang obtiennent une dépendance non monotone de la résistivité en fonction de la température, analogue à celle obtenue dans les expériences. Cependant, ce modèle prévoit une saturation de la résistivité en-dessous de la température de Dingle $T_{D}$, supérieure à ce qui est observé dans les MOSFETs Si. D'autre part, les mesures de densité par la pente Hall dans des MOSFETs Si [155, 174] sont incompatibles avec ce modèle supposant l'existence d'électrons gelés sur les impuretés.

\section{Pièges aux interfaces}

Altshuler, Maslov et Pudalov ont tenté d'expliquer le comportement métallique de la résistivité observé dans les MOSFETs Si par un mécanisme de piégeage de porteurs par des défauts situés à l'interface entre le silicium et l'oxyde $\mathrm{SiO}_{2}$ [231, 232]. Ils supposent que tous les pièges ont une énergie $\epsilon_{p}$, et cette énergie varie avec la tension appliquée sur la grille $V_{G}$. A température nulle, si $\epsilon_{p}\left(V_{G}\right)>\mu$ (où $\mu$ est le potentiel chimique), les pièges sont vides d'électrons et chargés positivement, ils sont donc de forts centres diffuseurs pour les électrons, conduisant à une forte résistivité. Par contre, si $\epsilon_{p}\left(V_{G}\right) \leqslant \mu$, les pièges ont tous piégé un électron et sont neutralisés, il ne diffusent donc pas les électrons, conduisant à une faible résistivité. Supposons maintenant que $\epsilon_{p}\left(V_{G}\right) \leqslant \mu$. A très haute température, pour $T \gg\left|\epsilon_{p}-\mu\right|$, la moitié des pièges sont vides, conduisant à une forte résistivité. Lorsque la température diminue, avec $T \geqslant\left|\epsilon_{p}-\mu\right|$ la densité de pièges neutralisés varie comme $\exp \left[\left(\epsilon_{p}-\mu\right) / T\right]$, ce qui conduit à une diminution exponentielle de la résistivité, comme il est observé expérimentalement. Altshuler et Maslov ont montré que cette théorie permet d'expliquer, outre le comportement métallique de la résistivité, les lois d'échelle en température. Ils interprètent les dépendances en champ électrique par le chauffage des porteurs, qui peut également conduire à des lois d'échelle. Enfin les dépendances en champ magnétique sont dues à un effet de spin sur les pièges. De plus cette théorie peut également s'appliquer aux hétérostructures munies d'une grille pour faire varier la densité de porteurs.

On peut cependant citer le commentaire de Kravchenko et al. [233], qui suggèrent que ce modèle ne peut pas rendre compte quantitativement des résultats expérimentaux en raison du faible nombre d'impuretés présentes dans le système. Cette suggestion a cependant été réfutée par Altshuler et Maslov [234]. On peut également noter que le modèle d'Altshuler et Maslov ne prend pas en compte les effets d'interférences quantiques qui sont attendues à basse température, et qui doivent redonné un comportement métallique de la résistivité. L'absence de comportement isolant à très basse température (jusqu'à 35 $\mathrm{mK}$ ) dans les MOSFETs Si étudiés par Kravchenko et al. [69] donnent un argument supplémentaire contre le modèle de pièges. Phillips donne également des arguments quand à la non validité de ce modèle dans le cas des hétérostructures $p$-GaAs [235].

Klapwijk et Das Sarma ont proposé un autre scénario, analogue au précédent, en prenant en compte la diffusion des électrons sur des ions chargés placés aux interfaces entre semi-conducteurs et présents en densité comparable à la densité électronique [236]. Ce scénario permet d'expliquer la magnéto-résistance importante observée dans la phase isolante. On peut également noter deux autres théories équivalentes proposées par Kozub 
et al. [237] et Pudalov et al. [238]

\section{Diffusion interbandes}

On a vu au paragraphe 2.4.5 que le spin électronique semble jouer un rôle sur la transition métal-isolant dans les systèmes bidimensionnels. Plus particulièrement, Papadakis et al. [161, 239], puis d'autres auteurs (voir §2.4.5), ont montré que le comportement métallique dans les hétérostructures $p$-GaAs est fortement influencé par la levée de dégénérescence de spin due au couplage spin-orbite. Plusieurs auteurs ont alors envisagé la diffusion interbandes comme source du comportement métallique de la résistivité [163, 71, 240, 102]. Alors que cet effet n'est pas prédominant dans les MOSFETs Si (voir Pudalov et al. [102]), il peut être important dans les hétérostructures $p$-GaAs en raison du fort coulage spin-orbite qui, associé au potentiel de confinement asymétrique, conduit à une levée de dégénérescence de bandes.

Yaish et al. [241, 242] ont effectué des calculs complets de résistivité en prenant en compte la diffusion interbandes, ainsi que les corrections de conductivité dues à la localisation faible, à l'antilocalisation faible (spin-orbite) et aux interactions. Ils montrent alors que la dépendance métallique observée dans ces systèmes peut être interprétée par l'antilocalisation et la diffusion interbandes. La transition métal-isolant est alors interprétée comme une transition entre un régime de forte levée de dégénérescence, et un régime de faible levée de dégénérescence quand la densité de porteurs diminue. En comparant des mesures de magnétorésistance et de résistivité en fonction de la température, ils obtiennent un bon accord pour deux types d'échantillons, des échantillons de mobilité élevée à forte densité de porteurs [241], et des échantillons de faible mobilité autour de la transition [242]. On peut cependant noter que, dans les deux cas, la chute de résistivité entre 2 $\mathrm{K}$ et $200 \mathrm{mK}$ ne dépasse pas $10 \%$, et leur modèle permet d'expliquer la transition dans les échantillons de faible mobilité (i.e. avec une densité critique élevée, $\approx 10^{11} \mathrm{~m}^{-2}$ ). Dans des échantillons $p$-GaAs de mobilité élevée $\left(\mu_{\max } \approx 110 \mathrm{~m}^{2}(\mathrm{Vs})^{-1}\right)$, pour lesquels la densité critique est environ dix fois plus faible $\left(n_{c} \approx 1 \times 10^{14} \mathrm{~m}^{-2}\right)$ et la chute de résistivité à basse température est de l'ordre de $50 \%$, Hamilton et al. ont montré que ce modèle ne permet pas de rendre compte du comportement métallique de la résistivité [160] : la levée de dégénérescence de spin conduirait à une magnétorésistance positive à faible champ magnétique, et à des oscillations de Shubnikov-de Haas présentant une double période, ce qui n'est pas observé expérimentalement.

\section{Autres modèles}

Pour compléter cette revue, on peut citer d'autres modèles spécifiques au système étudié, pour lesquels un mécanisme classique peut conduire à une dépendance "métallique" de la résistivité :

- Le couplage spin-orbite proposé par Pudalov [71].

- Dans le cas des MOSFETs Si, Punnoose et Finkel'stein ont évoqué la diffusion des électrons dans un système possédant plusieurs vallées [243].

- Chereminsin a montré que les corrections thermiques à la conductivité dues aux effets Peltier et Seebeck peuvent induire un comportement métallique [244, 245]. 


\subsection{Conclusion}

Le comportement expérimental des gaz bidimensionnels de porteurs de faible densité électronique et à champ magnétique nul n'est toujours pas bien compris. Dans ces systèmes désordonnés, les interactions entre électrons sont fortes et on s'attend à ce qu'elles jouent un rôle. Le fait que, pour certaines valeurs de la densité, la résistivité d'un tel système décroisse quand la température diminue, laisse penser que le système est métallique, i.e. que les fonctions d'ondes électroniques sont délocalisées à température. Cette caractéristique est très surprenante car elle contredit la théorie d'échelle de la localisation élaborée pour des électrons indépendants. A basse densité, ces systèmes présentent une transition métal-isolant.

Nous avons vu ici que plusieurs questions restent posées. Dans un premier temps l'origine réelle de la transition n'est pas bien déterminée. La première question concerne l'existence réelle d'une transition de phase dans ces systèmes. Bien que les interactions soient fortes dans ces systèmes, il ne semble pas établi que le système puisse être décrit dans le cadre du liquide de Fermi ou si un nouvel état électronique doit être introduit. Mais le désordre peut également jouer un rôle important dans cette transition. Enfin il a été observé un effet important du champ magnétique appliqué parallèlement au plan du gaz bidimensionnel, qui ne se couple alors qu'au spin de l'électron, et qui montre que le spin semble également jouer un rôle.

De manière plus générale, ce domaine assez nouveau de la transition métal-isolant en dimension deux pose des questions analogues à celles posée dans le cadre des électrons fortement corrélé. En particulier il a été montré que, lorsque l'interaction entre les électrons devient suffisamment forte, le système doit présenter une transition vers un état de type cristal de Wigner, ou verre de Wigner lorsque le désordre est important. Est-ce cet état qui est observé dans la phase isolante? Si oui, est-ce que la transition entre le cristal de Wigner se fait par une transition du premier ordre, comme il a été couramment supposé, ou est-ce qu'une nouvelle phase doit être considérée entre les deux? Enfin, est-ce que l'on ne peut pas envisager d'autres scénarios pour cette transition, comme le font les modèles de percolations?

Les travaux présentés dans cette thèse ont pour but de répondre à certaines de ces questions, et répondent en partie au manque d'observables explorées jusqu'à présent. Les mesures de transport, bien qu'indispensables pour décrire convenablement ces systèmes électroniques, ne permettent pas d'apporter toutes les informations nécessaires à la compréhension du sujet. En plus des mesures de fluctuations de résistance qui sont présentées dans cette thèse, on peut citer d'autres mesures envisageables dans ces systèmes, comme les mesures d'effet tunnel pour sonder la densité d'états, les mesures d'aimantation pour étudier le rôle réel du spin, les mesures thermodynamiques telles la capacité calorifique, etc.. Dans tous les cas, en raison du faible nombre d'électrons présents dans le système à faible densité $\left(\approx 10^{-13}\right.$ à $\left.10^{15} \mathrm{~m}^{-2}\right)$, ces expériences sont très délicates. Enfin d'autres systèmes semblent être prometteurs pour étudier les effets des interactions entre électrons, tels les systèmes mésoscopiques (boîtes quantiques ou anneaux), dans lesquels ont été observés des effets importants des interactions. 


\section{Chapitre 3}

\section{Systèmes étudiés et dispositif expérimental}

\section{Sommaire}

3.1 Gaz bidimensionnels de porteurs dans les semiconducteurs 94

3.1.1 Transistor à effet de champ . . . . . . . . . . . . . . 94

3.1 .2 Hétérostructure . . . . . . . . . . . . . . . . . . 94

3.1 .3 Electrons et trous . . . . . . . . . . . . . . . 95

3.2 Description des échantillons utilisés . . . . . . . 96

3.2 .1 Puits quantique $p$-SiGe/Si . . . . . . . . . . . . . 96

3.2 .2 Puits quantiques $p$-GaAs/AlGaAs . . . . . . . . . . . 99

3.2.3 Grandeurs caractéristiques . . . . . . . . . . . . . . 101

3.3 Techniques expérimentales . . . . . . . . . . . 102

3.3 .1 Basse température . . . . . . . . . . . . . . . 102

3.3.2 Mesures électriques bas niveau . . . . . . . . . . . 103 


\subsection{Gaz bidimensionnels de porteurs dans les semicon- ducteurs}

Pour une étude détaillée des gaz bidimensionnels d'électrons, en particulier dans les MOSFETs Silicium, il est possible de se référer à la revue de Ando, Fowler et Stern [18].

\subsubsection{Transistor à effet de champ}

Le principe du transistor à effet de champ (ou FET pour Field Effect Transistor) est décrit dans la figure 3.1 de gauche. L'application d'une tension sur la grille crée un champ électrique entre la grille et le semiconducteur, conduisant à une déformation de la structure de bande au voisinage de l'interface entre le semiconducteur ( $\mathrm{Si}$ ) et l'isolant $\left(\mathrm{SiO}_{2}\right)$. Lorsque le semiconducteur est dopé $p$ en volume (comme dans la figure 3.1 de gauche), l'application d'une tension positive sur la grille par rapport au semiconducteur conduit à une augmentation de la densité d'électrons au voisinage de l'interface $\mathrm{Si} / \mathrm{SiO}_{2}$. Pour une tension de grille suffisamment élevée, il se forme alors une couche d'inversion dans laquelle les électrons sont libres. En raison de la faible épaisseur du puits quantique ainsi créé, le vecteur d'onde des fonctions d'onde est quantifié dans la direction $z$ perpendiculaire à la surface. A suffisamment basse température, seul le premier niveau quantifié est rempli, et les électrons n'ont pas de composante de vitesse dans la direction $z$ : ils forment un gaz purement bidimensionnel. En variant la tension appliquée sur la grille, il est possible de modifier la profondeur du puits de potentiel, et ainsi de modifier la densité surfacique des électrons. Dans le cas où le semiconducteur est dopé $n$ en volume, et en appliquant une tension négative sur la grille, il est possible de former un gaz bidimensionnels de trous.

Le facteur limitant de la mobilité des porteurs dans cette structure vient de la présence d'une interface entre le semiconducteur ( $\mathrm{Si}$ ) et le matériau amorphe constituant la couche isolante $\left(\mathrm{SiO}_{2}\right)$. Les liaisons pendantes ainsi créées conduisent à des défauts à l'interface, dont une partie sont des pièges pour les électrons du gaz bidimensionnel.

\subsubsection{Hétérostructure}

Dans un transistor à effet de champ, le confinement des porteurs de charge est réalisé à l'interface entre un semiconducteur de faible largeur de bande interdite (en général de l'ordre de $1 \mathrm{eV}$ ) et un isolant, qui est un composé de grande largeur de bande interdite. Il est également possible de confiner des porteurs en utilisant des semiconducteurs de largeurs de bande interdite différentes. Le principe de l'hétérostructure est présenté dans la figure 3.1 de droite. Dans une telle structure, les porteurs de charge à l'interface ne sont pas créés par effet de champ, mais par dopage d'un des semiconducteurs (AlGaAs dans l'exemple de la figure 3.1 de droite). Deux types de structures sont couramment employées. Dans une hétérojonction, il n'y a qu'une seule interface, et le potentiel de confinement est triangulaire. Il est également possible de former deux interfaces très rapprochées (quelques dizaines de nanomètres) afin de créer un puits de potentiel carré ou puits quantique.

En ajoutant une grille à la surface d'une telle structure, il est possible de modifier la forme du puits de potentiel, et ainsi de modifier la densité surfacique de porteurs. Cette 

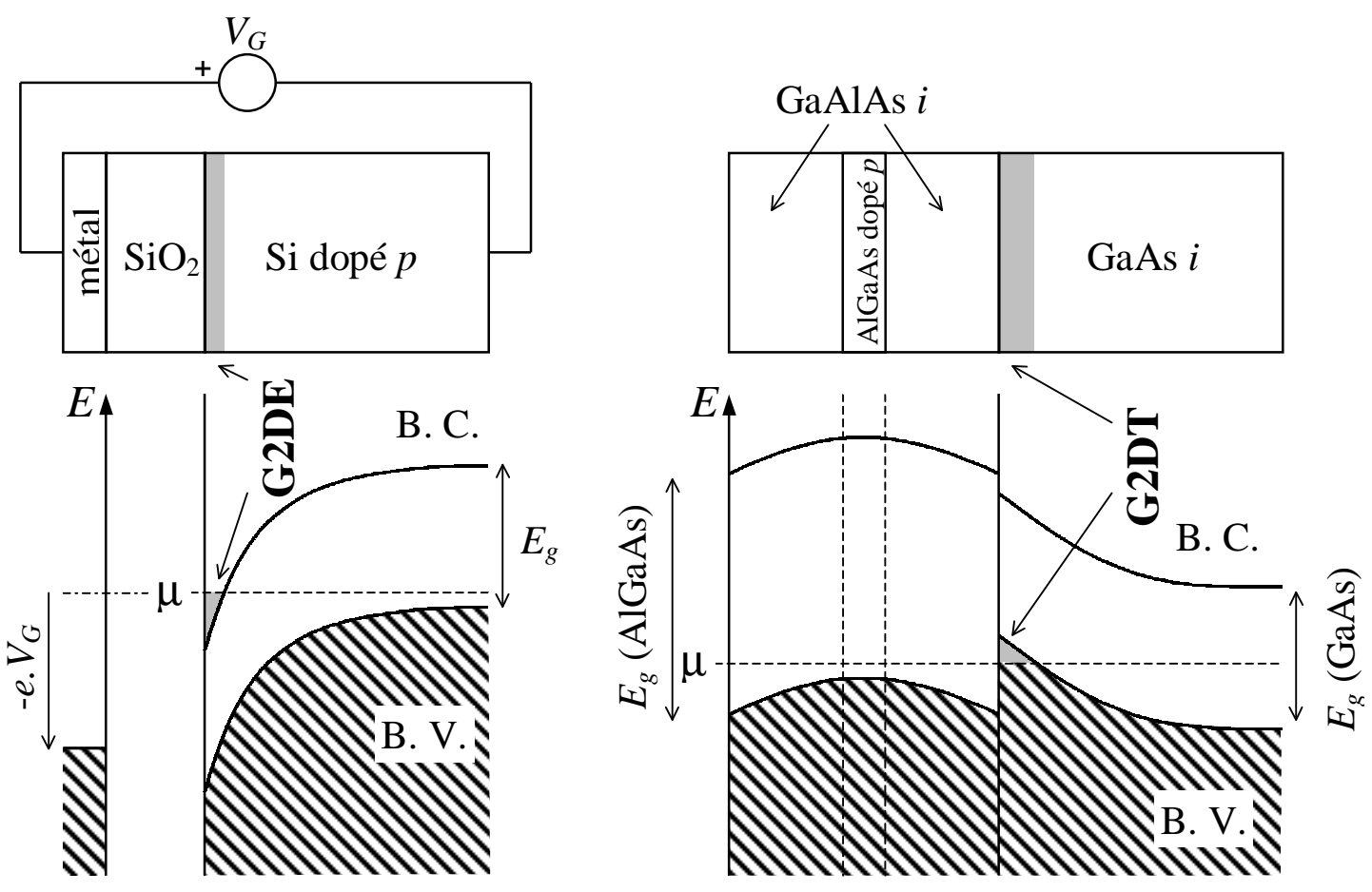

FIG. 3.1: Structure de bande schématique dans un MOSFET Si (à gauche) et une hétérojonction GaAs/AlGaAs (à droite) permettant ici d'obtenir respectivement un gaz bidimensionnel d'électrons (G2DE) et un gaz bidimensionnel de trous (G2DT).

densité peut également être modifiée par illumination, processus qui modifie le transfert de charge entre les impuretés dopantes et le gaz de porteurs.

Comparés aux MOSFETs, ces structures permettent de réaliser des gaz bidimensionnels beaucoup moins désordonnés, car les techniques de fabrication (épitaxie par jets moléculaires ou dépôt chimique en phase vapeur) permettent de former des interfaces très propres. D'autre part les semiconducteurs utilisés ont souvent un paramètre de maille très proche, réduisant ainsi les contraintes, et donc les défauts à l'interface. Enfin la technique de dopage dans un plan éloigné du gaz d'électron permet de réduire la diffusion des porteurs sur ces impuretés dopantes.

\subsubsection{Electrons et trous}

Suivant le signe de la tension de grille dans le cas des MOSFETs, ou suivant la nature du dopage dans les hétérostructures, il est possible de créer des porteurs libres, soit dans la bande de conduction, ce sont alors des électrons, chargés négativement, soit dans la bande de valence, ce sont des trous, chargés positivement (voir la figure 3.1). En raison de la forme très différente de la bande de conduction et de la bande de valence dans les semiconducteurs utilisés, les électrons et les trous ont des caractéristiques assez différentes, notamment en ce qui concerne leur masse effective. 


\subsection{Description des échantillons utilisés}

\subsubsection{Puits quantique $p$-SiGe/Si}

\section{Description du système}

Les premiers échantillons utilisés pendant la thèse sont des puits quantiques $\mathrm{SiGe} / \mathrm{Si}$, dont la structure est décrite dans la figure 3.2 du haut. Ils ont été réalisés par épitaxie par jet moléculaire au Paul Scherrer Institut (Villigen). Les conditions de fabrication ont été optimisées afin d'obtenir une mobilité maximale (température de croissance de $800{ }^{\circ} \mathrm{C}$, sans recuit après la croissance, pour plus de détails voir la référence [246]). Cette structure permet de réaliser un gaz bidimensionnel de trous dans le SiGe, à l'interface avec le Si. La structure a été gravée en forme de barre de Hall au Solid State Physics Laboratory de l'ETH Zürich, et les dimensions sont données dans la figure 3.2 du bas (les conditions de gravure et de métallisation sont décrites dans la référence [247]). Nous avons utilisé deux échantillons de géométries différentes, qui sont identifiés par les numéros B137 et B509.

La différence des paramètres de maille entre le silicium et le $\operatorname{SiGe}(0,5431 \mathrm{~nm}$ pour Si contre $0,5465 \mathrm{~nm}$ pour $\mathrm{Si}_{0,85} \mathrm{Ge}_{0,15}$, voir [247]) conduit à des contraintes importantes aux interfaces. Pour éviter la création de défauts à l'interface entre les deux matériaux, il est possible d'utiliser une couche de SiGe suffisamment fine pour que les contraintes ne puissent pas relaxer. Cependant, dans ce cas, la couche de SiGe est contrainte par le paramètre de maille du Si dans les directions parallèles au plan de l'interface, alors qu'elle garde le paramètre de maille du SiGe massif dans la direction orthogonale. Cette asymétrie conduit à un fort couplage spin-orbite qui, associé à l'asymétrie du potentiel de confinement, induit une levée de dégénérescence entre les bandes de trous lourds $(J=$ $\left.3 / 2, m_{J}= \pm 3 / 2\right)$ et de trous légers $\left(J=1 / 2, m_{J}= \pm 1 / 2\right)$. Cette contrainte impose ainsi que seule la bande de trous lourds est remplie à basse température (voir par exemple People [249]). D'autre part, en raison de la symétrie de cet état, le moment angulaire $\vec{J}$ est aligné perpendiculairement au plan du gaz de porteurs.

Dans les hétérostructures $p$-SiGe, le mécanisme de diffusion dominant est la diffusion par les charges d'interface (entre Si et SiGe). Mais les autres mécanismes cités au paragraphe 1.5.2 interviennent également. La contrainte dans le SiGe augmente la diffusion par les irrégularités d'interface, ce qui conduit à une mobilité plus faible que dans les hétérostructures GaAs/AlGaAs.

\section{Caractéristiques du SiGe}

Dans ce paragraphe sont données les caractéristiques du SiGe qui seront utilisées par la suite. On remarquera que, dans la plupart des cas, ces caractéristiques sont issues de données dans le SiGe massif, et ne prennent donc pas en compte la contrainte. Les principales caractéristiques sont données dans le tableau 3.1.

La constante diélectrique relative statique $\varepsilon_{r}$ est évaluée en faisant une interpolation linéaire entre les valeurs du silicium et du germanium [250] :

$$
\varepsilon_{r}=12+4 \cdot x
$$

où $x$ est la fraction de germanium dans l'alliage $\mathrm{Si}_{1-x} \mathrm{Ge}_{x}$. 

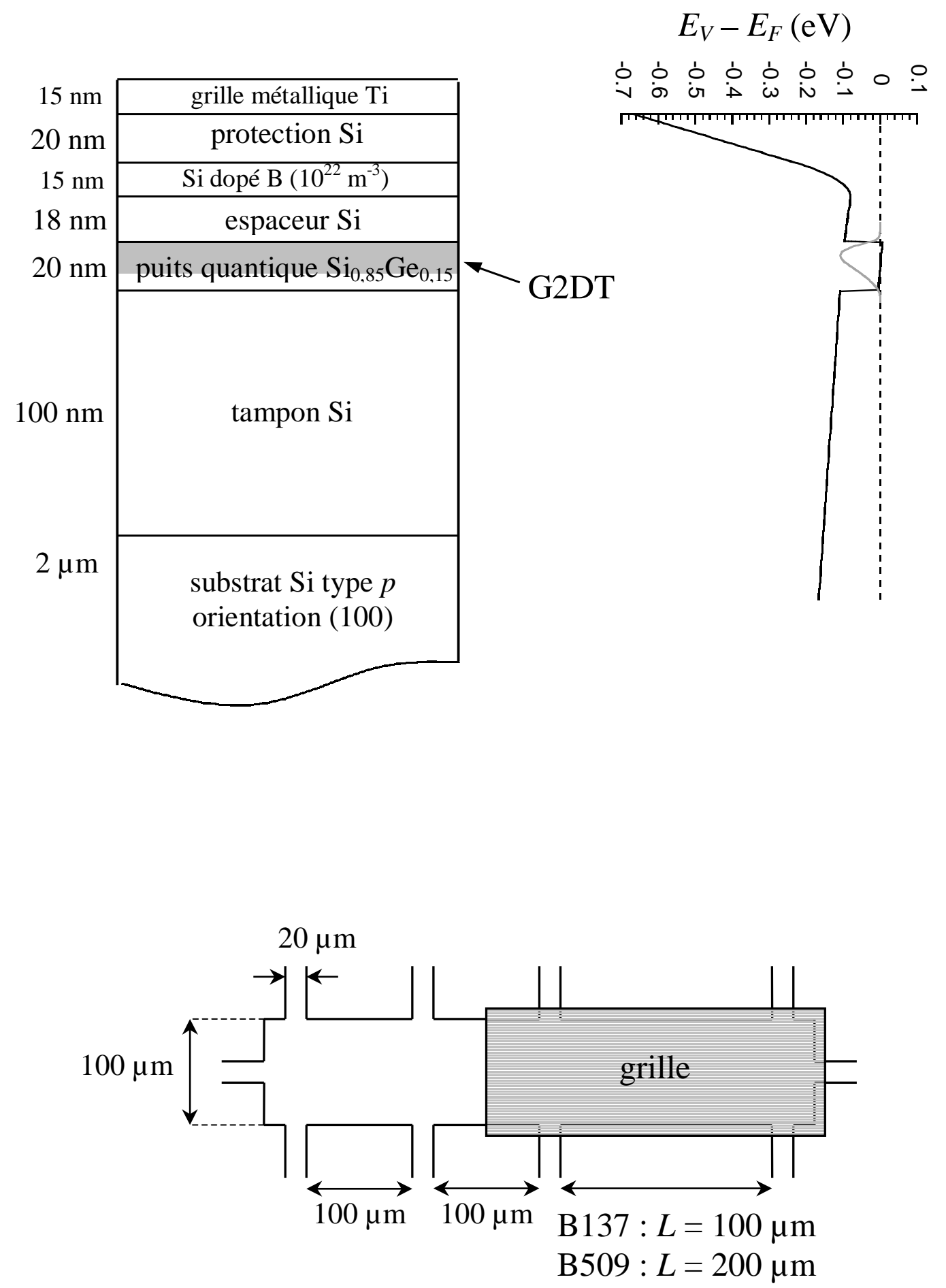

FIG. 3.2: Haut : Structure du puits quantique SiGe/Si B509 et B137 (à gauche), et diagramme de bande de valence et fonction d'onde du premier état lié du puits quantique calculés par la résolution de l'équation de Poisson-Schrödinger 1D (à droite) [248]. Bas : Géométrie des barres de Hall utilisées (B137 et B509). Les contacts ohmiques sont réalisés par un dépôt de $\mathrm{Ti} / \mathrm{Al}$ $(15 / 250 \mathrm{~nm})$, puis un recuit à $400^{\circ} \mathrm{C}$ (voir [247]). 


\begin{tabular}{|l|c|c|}
\hline $\begin{array}{l}\text { masse effective des trous lourds } m^{*} \\
\text { constante diélectrique relative } \varepsilon_{r}\end{array}$ & $\begin{array}{c}0,25 \times m_{0} \\
12,6\end{array}$ & $\begin{array}{c}\text { voir Senz } \text { et al. }[156] \\
\text { voir texte }\end{array}$ \\
\hline masse volumique $\rho$ & $2,778 \times 10^{3} \mathrm{~kg} \cdot \mathrm{m}^{-3}$ & voir texte \\
vitesse du son longitudinale $v_{L}$ & $8,100 \times 10^{3} \mathrm{~m} \cdot \mathrm{s}^{-1}$ & voir texte \\
vitesse du son transverse $v_{T}$ & $4,879 \times 10^{3} \mathrm{~m} \cdot \mathrm{s}^{-1}$ & voir texte \\
\hline
\end{tabular}

TAB. 3.1: Caractéristiques du $\mathrm{Si}_{0,85} \mathrm{Ge}_{0,15} \cdot m_{0}$ est la masse de l'électron.

Les vitesses du son longitudinale $v_{L}$ et transverse $v_{T}$ sont évaluées à partir des constantes élastiques $C_{11}, C_{12}$ et $C_{44}$, en effectuant une moyenne sphérique sur les directions cristallographiques [251]:

$$
v_{L}=\sqrt{\frac{3 C_{11}+2 C_{12}+4 C_{44}}{5 \rho}} \quad \text { et } \quad v_{T}=\sqrt{\frac{C_{11}-C_{12}+3 C_{44}}{5 \rho}}
$$

où $\rho$ est la masse volumique du cristal. Les constantes élastiques sont évaluées à partir des données dans le silicium et le germanium [252, p. 95] par interpolation linéaire :

$$
\begin{aligned}
& C_{11}=(1,650-0,378 \cdot x) \times 10^{11} \mathrm{~N} \cdot \mathrm{m}^{-2} \\
& C_{12}=(0,642-0,182 \cdot x) \times 10^{11} \mathrm{~N} \cdot \mathrm{m}^{-2} \\
& C_{44}=(0,794-0,119 \cdot x) \times 10^{11} \mathrm{~N} \cdot \mathrm{m}^{-2}
\end{aligned}
$$

où $x$ est la fraction en germanium dans $\mathrm{Si}_{1-x} \mathrm{Ge}_{x}$. Il en est de même de la masse volumique :

$$
\rho=(2,3283+2.9951 \cdot x) \times 10^{3} \quad \mathrm{~kg} \cdot \mathrm{m}^{-3}
$$

\section{Caractéristiques des échantillons}

Pour les deux échantillons, on a pu vérifier une loi de variation linéaire de la densité de trous $p_{s}$ en fonction de la tension de grille $V_{G}$ :

$$
p_{s}=\frac{C_{G}}{e} \cdot\left(V_{G}^{0}-V_{G}\right)
$$

avec $C_{G}=46 \mu \mathrm{F} . \mathrm{m}^{-2}$ pour l'échantillon B137 et $C_{G}=59 \mu \mathrm{F} . \mathrm{m}^{-2}$ pour l'échantillon B509. La valeur de $V_{G}^{0}$ dépend de l'état de charge des impuretés et varie d'un refroidissement à l'autre. A $V_{G}=0$, le gaz de trous est complètement déplété. En variant la tension de grille jusqu'à $-0,8 \mathrm{~V}$, il est possible d'augmenter la densité de trous jusqu'à environ $2 \times 10^{15}$ $\mathrm{m}^{-2}$ en limitant le courant de fuite grille-canal à quelques centaines de pA. La valeur élevée du courant de fuite est due au fait que la grille est réalisée par un contact Schottky, sans ajout d'un isolant entre le métal et le semiconducteur.

La mesure de résistivité Hall et des oscillations de Shubnikov-de Haas de la partie sans grille de l'échantillon B509 sont présentées dans la figure 3.3. Les densités déduites de la pente Hall et des oscillations de Shubnikov-de Haas coïncident à $4 \%$ près, et valent $p_{s}=4 \times 10^{15} \mathrm{~m}^{-2}$. La mobilité calculée à partir de la résistivité longitudinale à champ magnétique nul à $T=300 \mathrm{mK}$ est $\mu \approx 0,8 \mathrm{~m}^{2}$.(Vs) ${ }^{-1}$. Une même mesure effectuée sur l'échantillon B137 à une tension de grille $V_{G}=-0,8 \mathrm{~V}$ et à $T=130 \mathrm{mK}$ conduit à $p_{s}=2 \times 10^{15} \mathrm{~m}^{-2}$ et $\mu \approx 0,6 \mathrm{~m}^{2}$. $(\mathrm{Vs})^{-1}$, qui est la mobilité maximale dans cet échantillon. 


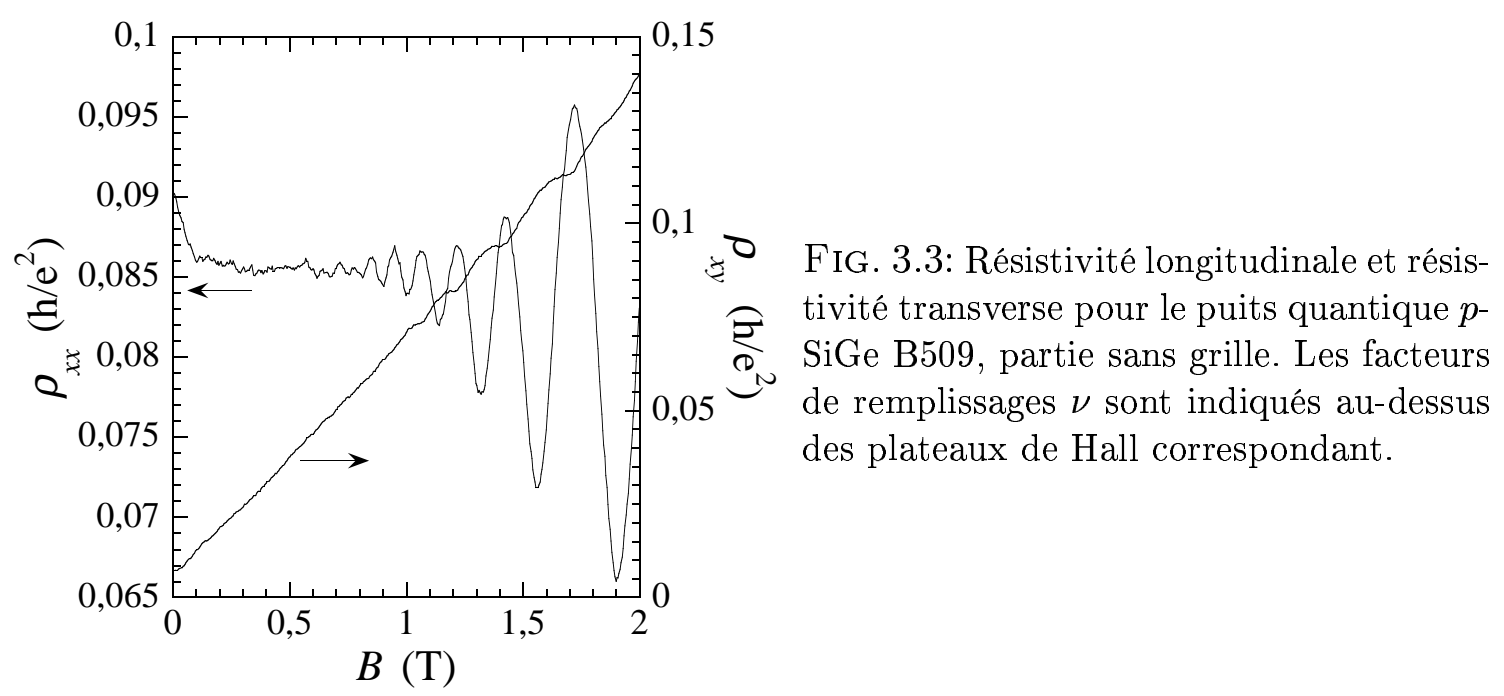

\subsubsection{Puits quantiques $p$-GaAs/AlGaAs}

\section{Description du système}

Le second système utilisé pendant la thèse est un puits quantique GaAs/AlGaAs, dont la structure est décrite dans la figure 3.4 du haut. Elle a été réalisée à l'Université de Nottingham. Dans cette structure, un gaz bidimensionnel de trous est réalisé dans le GaAs, à l'interface avec AlGaAs. La structure est gravée en forme de barre de Hall, dont les dimensions sont décrites dans la figure $3.4 \mathrm{du}$ bas.

Dans le matériau AlGaAs, les dopants Si sont soit donneurs, soit accepteurs, suivant l'orientation de la surface du substrat. Pour une orientation (100), les impuretés Si prennent la place de $\mathrm{Al}$ ou Ga et sont donc donneuses, donnant lieu à un gaz bidimensionnel d'électrons ( $n$-GaAs). Pour une orientation (311)A, comme c'est le cas pour les échantillons utilisés ici, les impuretés Si prennent la place de As et sont acceptrices donnant un gaz bidimensionnel de trous ( $p$-GaAs).

Dans le $n$-GaAs, ce sont les impuretés dopantes qui dominent la diffusion, donnant lieu à un désordre à longue portée. Dans les hétérostructures $p$-GaAs que nous avons utilisée, et à densité élevée, la diffusion est dominée par les défauts d'interface, donnant lieu à un désordre à courte portée. La surface du substrat GaAs, orientée dans la direction cristallographique (311)A, est reconstruite et présente une rugosité anisotrope, donnant lieu à une anisotropie de la mobilité du gaz bidimensionnel de trous, avec un axe de forte mobilité $(\overline{2} 33)$, et un axe de faible mobilité $(01 \overline{1})$. Les barres de Hall décrites dans la figure 3.4 de droite sont gravées dans différentes directions : Nu 944-1 dans la direction de forte

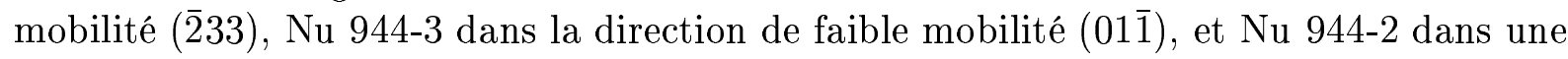
direction intermédiaire entre les deux précédentes.

Les caractéristiques principales du GaAs qui seront utilisées par la suite sont rappelées dans le tableau 3.2. 


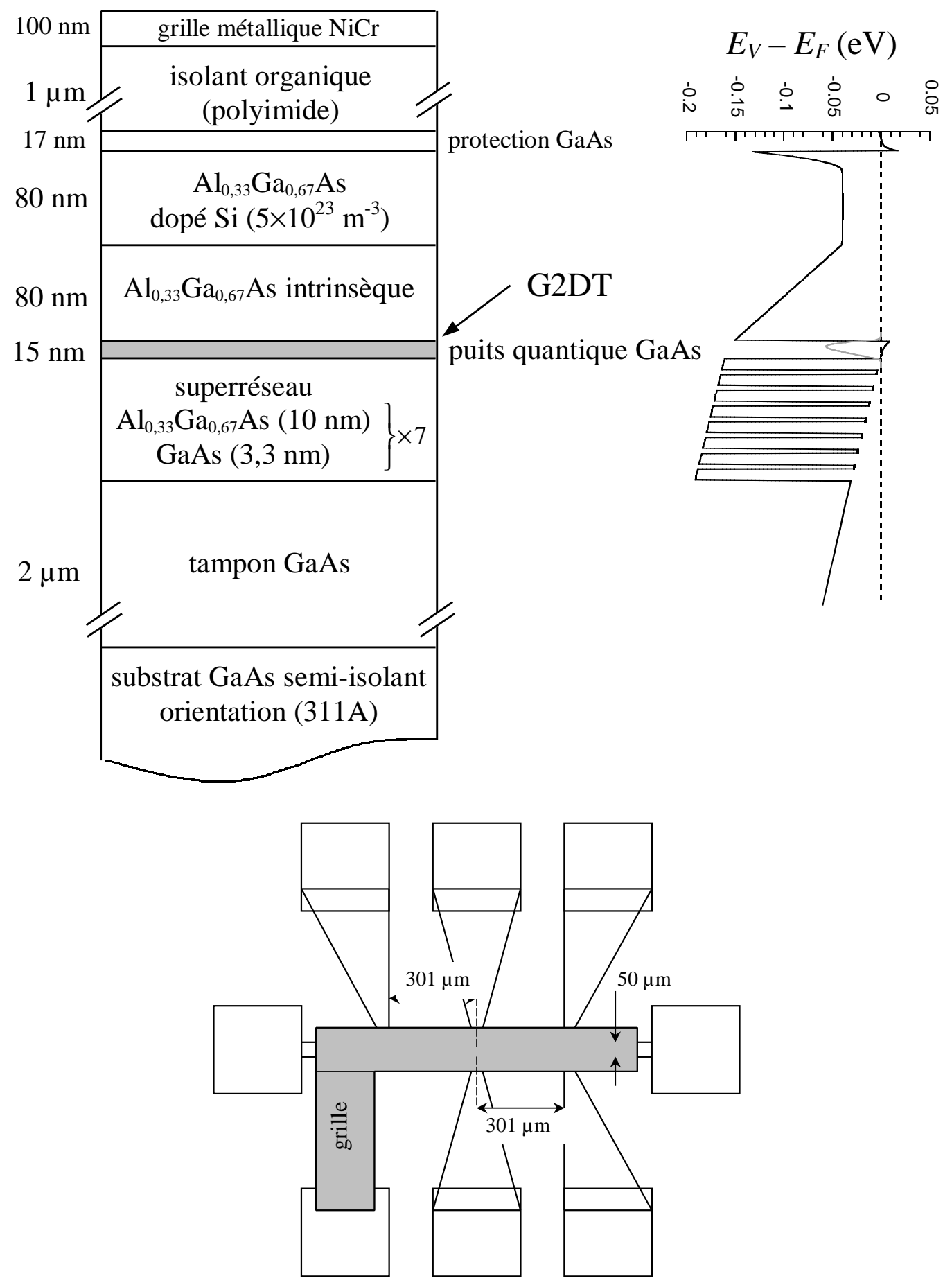

FIG. 3.4: Haut : Structure du puits quantique GaAs/AlGaAs Nu 944 (à gauche), et diagramme de bande de valence et fonction d'onde du premier état lié du puits quantique calculés par la résolution de l'équation de Poisson-Schrödinger 1D (à droite) [248]. Bas : Géométrie de la barre de Hall. Les contacts ohmiques sont réalisés en $\mathrm{Au} / \mathrm{Zn} / \mathrm{Au}(10 / 30 / 200 \mathrm{~nm})$ avec un recuit à 510 ${ }^{\circ} \mathrm{C}$. 


\begin{tabular}{|l|c|l|}
\hline masse effective des trous lourds $m^{*}$ & $0,37 \times m_{0}$ & réf. [253] \\
constante diélectrique relative $\varepsilon_{r}$ & 13,18 & réf. [254] \\
\hline
\end{tabular}

TAB. 3.2: Caractéristiques du GaAs, d'après les références citées. $m_{0}$ est la masse de l'électron.

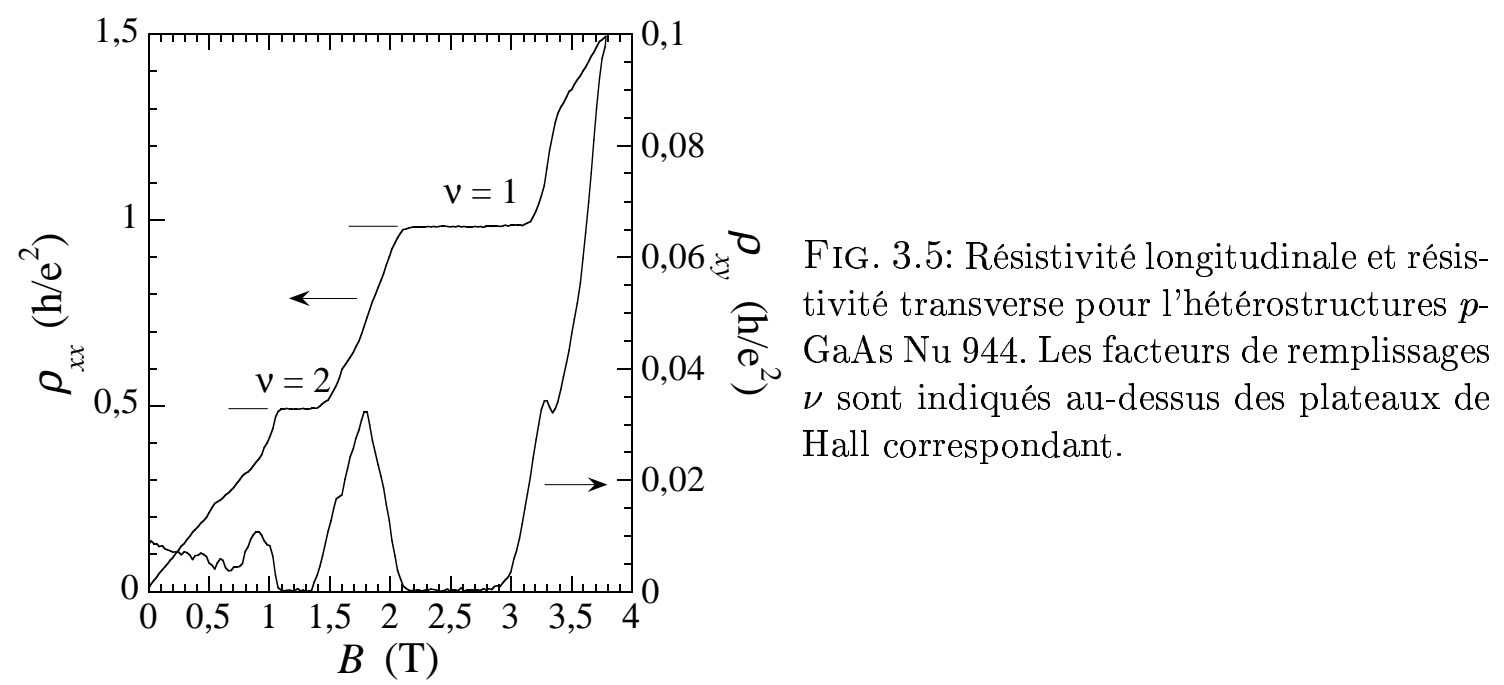

\section{Caractéristiques des échantillons}

L'étude faite sur l'échantillon Nu944-1 a montré une variation linéaire de la densité de trous $p_{s}$ en fonction de la tension de grille $V_{G}$ :

$$
p_{s}=\frac{C_{G}}{e} \cdot\left(V_{G}^{0}-V_{G}\right)
$$

avec $C_{G}=27,2 \pm 2 \mu \mathrm{F} . \mathrm{m}^{-2}$. La valeur de $V_{G}^{0}$ dépend du remplissage des impuretés, et dépend donc de la tension de grille appliquée lors du refroidissement. L'utilisation d'une grille a permis de faire varier la densité de porteurs entre $8,2 \times 10^{14} \mathrm{~m}^{-2}$ (pour $\left.V_{G}=-1,967 \mathrm{~V}\right)$ et $1,30 \times 10^{14} \mathrm{~m}^{-2}\left(\right.$ pour $\left.V_{G}=+2,45 \mathrm{~V}\right)$. On a vérifié que, dans cette gamme de variation de la tension de grille, le courant de fuite de grille reste au pA (résolution de la méthode de mesure).

Les mesures de résistivité Hall et des oscillations de Shubnikov-de Haas à tension de grille nulle sont présentées dans la figure 3.5. Les densité déduites de la pente Hall et des oscillations de Shubnikov-de Haas coïncident à $2 \%$ près, et valent $p_{s}=6,1 \times 10^{14}$ $\mathrm{m}^{-2}$. La mobilité calculée à partir de la résistivité longitudinale à champ magnétique nul à $T=200 \mathrm{mK}$ est $50 \mathrm{~m}^{2} \cdot(\mathrm{Vs})^{-1}$.

\subsubsection{Grandeurs caractéristiques}

Les principales grandeurs caractéristiques des échantillons étudiés pendant la thèse sont données dans le tableau 3.3. 


\begin{tabular}{|l|c|c|c|}
\hline & $p$-SiGe & $\begin{array}{c}p \text {-SiGe } \\
\text { sans grille }\end{array}$ & $p$-GaAs \\
\hline densité $p_{s}\left(10^{15} \mathrm{~m}^{-2}\right)$ & $1,6-0,5$ & 4,0 & $0,82-0,13$ \\
paramètre $r_{s}$ & $5,5-10$ & 3,5 & $10-27$ \\
rayon de Wigner-Seitz $a_{0}(\mathrm{~nm})$ & $14-25$ & 9 & $20-50$ \\
\hline mobilité maximale $\mu_{\max }\left(\mathrm{m}^{2} \cdot(\mathrm{Vs})^{-1}\right)$ & 0,6 & 0,8 & 50 \\
temps de collision élastique $\tau_{e}(\mathrm{~s})$ & $8,5 \times 10^{-13}$ & $1,12 \times 10^{-12}$ & $1,05 \times 10^{-10}$ \\
\hline température de Fermi $T_{F}(\mathrm{~K})$ & $18-6$ & 44 & $6-1$ \\
\hline
\end{tabular}

TAB. 3.3: Grandeurs caractéristiques des systèmes expérimentaux étudiés pendant la thèse. $p$-SiGe regroupe les données des échantillons B137 et B509, $p$-GaAs correspond à l'échantillon Nu944-1.

\subsection{Techniques expérimentales}

\subsubsection{Basse température}

\section{Le cryostat}

Les mesures présentées ont été réalisées dans un réfrigérateur à dilution ${ }^{3} \mathrm{He} /{ }^{4} \mathrm{He}$ avec échangeurs discrets réalisé au Service de Physique de l'État Condensé par le laboratoire de cryogénie. Il comporte 14 câbles coaxiaux. Sa température de base avec les câbles est d'environ $20 \mathrm{mK}$. Une cellule expérimentale reliée à la chambre à mélange par une impédance thermique en inox permet de monter la température de l'échantillon jusqu'à $2 \mathrm{~K}$ sans interrompre la dilution. La température de l'échantillon est mesurée par un thermomètre $\mathrm{RuO}_{2}$ étalonné entre $20 \mathrm{mK}$ et $4,2 \mathrm{~K}$. Une bobine supraconductrice permet d'ajouter un champ magnétique perpendiculaire à la surface de l'échantillon jusqu'à 8 T.

\section{Thermalisation de l'échantillon}

Une attention particulière a été portée à la thermalisation de échantillons. Il s'agit de minimiser l'impédance thermique entre la plaquette semiconductrice sur laquelle est gravée la barre de Hall, et la plaque de cuivre sur laquelle est collée la plaquette. Le thermomètre $\mathrm{RuO}_{2}$ utilisé pour la mesure de la température de l'échantillon est encapsulé dans la plaque de cuivre. L'enjeu d'une bonne thermalisation est que la température du thermomètre est bien celle de l'échantillon étudié, ce qui peut s'avérer essentiel pour les mesures de transport et les mesures de chauffage. Sur les échantillons $p$-SiGe, pour améliorer la thermalisation, des fils d'or ont été soudés par ultrasons, d'une part sur la plaquette de Si, d'autre part sur la plaque de cuivre.

La conductivité thermique de l'interface entre deux solides est souvent considérée comme étant de type Kapitza [255, p.246] :

$$
P=g\left(T_{1}^{4}-T_{2}^{4}\right)
$$

où $P$ est la puissance transférée, et $T_{1}$ et $T_{2}$ les températures des deux matériaux. Des estimations, et des mesures réalisées par L'Hôte et al. sur des interfaces semiconducteurmétal avec colle [256], permettent d'évaluer $g$ de l'ordre de $10^{-4}$ à $10^{-3} \mathrm{~W} . \mathrm{K}^{-4}$, ce qui 


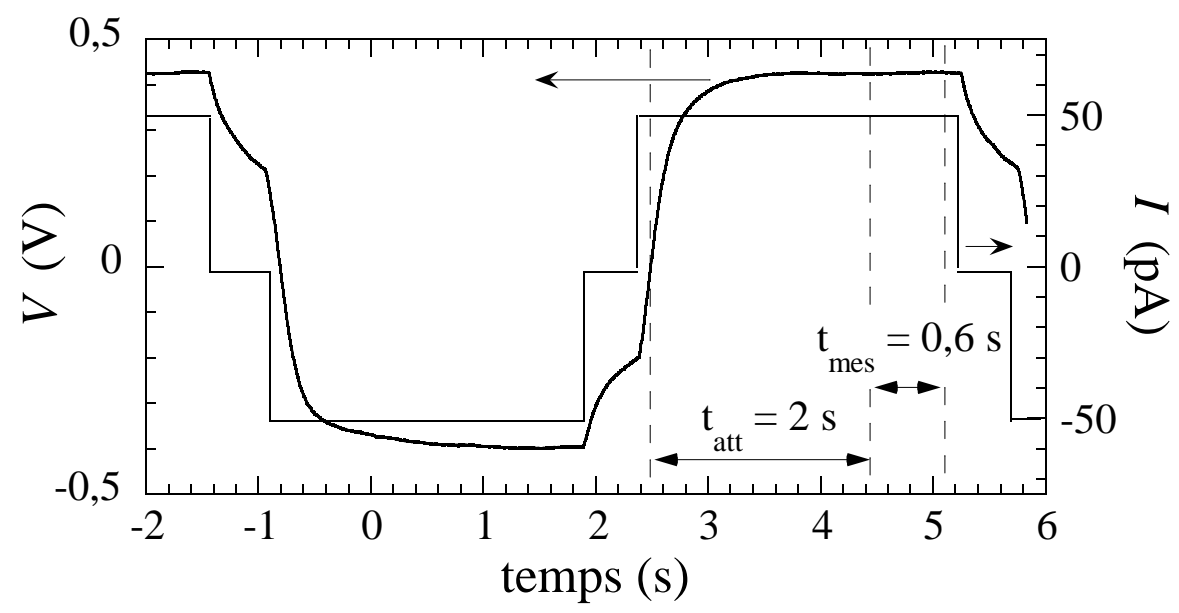

FIG. 3.6: Description temporelle d'une mesure typique de tension en sortie des amplificateurs. Exemple de mesure sur l'échantillon $p$-GaAs Nu944-1, présentant une résistance de 1,6 M $\Omega$. Le gain total des amplificateur est d'environ $5 \times 10^{3}$.

donne une élévation de température négligeable pour les puissances Joule que nous avons injectées dans nos échantillons.

\subsubsection{Mesures électriques bas niveau}

Pour les mesures de transport bas niveau, on a utilisé deux montages, une mesure quatre points pour mesurer des résistances inférieures à quelques $\mathrm{M} \Omega$ (voir la figure 3.7), et une mesure deux points pour des résistances supérieures à quelques $\mathrm{M} \Omega$ (voir la figure 3.8). Les filtres utilisés à l'entrée du réfrigérateur à dilution pour éliminer le bruit radiofréquence sont des filtres à traversée Murata de $10 \mathrm{nF}$.

On a utilisé une méthode de mesure en courant continu. Cependant, pour éliminer la tension de décalage provenant des amplificateurs, et qui peut varier au cours d'une expérience, le courant est inversé régulièrement. La tension est ensuite la moyenne (en valeur absolue) des tensions mesurées à courant positif et à courant négatif. En raison des constantes de temps du circuit dues au filtrage et à l'échantillon, nous avons apporté une attention particulière au temps d'attente après renversement du courant $\left(t_{a t t}\right)$, et au temps de mesure $\left(t_{m e s}\right)$. Un exemple de mesure est décrit dans la figure 3.6. La période de mesure varie de 5 à $20 \mathrm{~s}$ suivant la valeur de la résistance à mesurer (soit une fréquence de mesure de 0,2 à $0,05 \mathrm{~Hz}$ ).

Pour la mesure quatre points, on a utilisé des courants variant entre 1 pA et $10 \mathrm{nA}$ sur des résistances de $1 \mathrm{k} \Omega$ à quelques $\mathrm{M} \Omega$, avec une précision de mesure sur la tension inférieure $10 \mathrm{nV}$.

Pour la mesure deux points, on a utilisé des résistances $R_{m}$ calibrées de environ 100 $\mathrm{k} \Omega$ et $1 \mathrm{M} \Omega$. La résolution de la mesure de courant inférieure à $0,1 \mathrm{pA}$. 


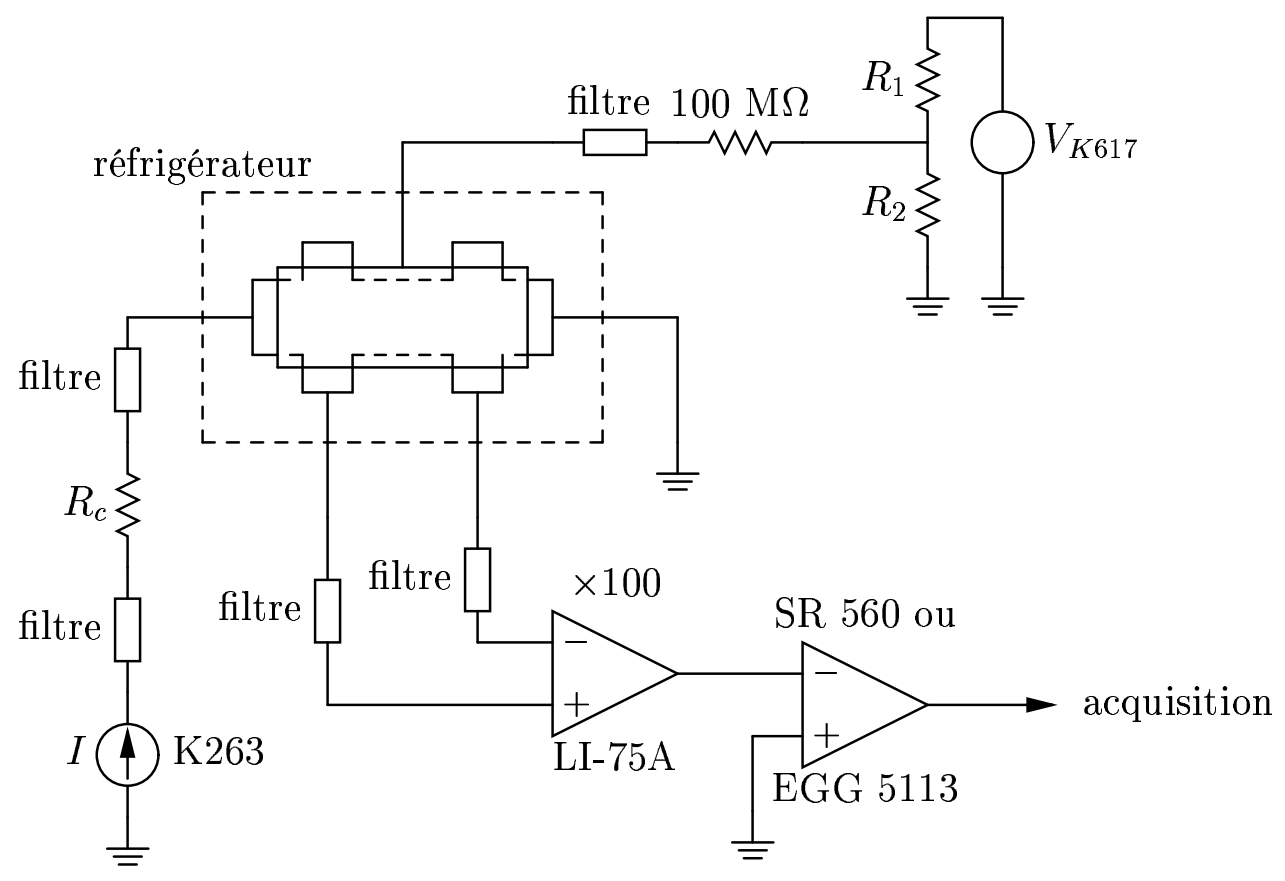

FIG. 3.7: Schéma de la mesure de résistance dans la configuration quatre points.

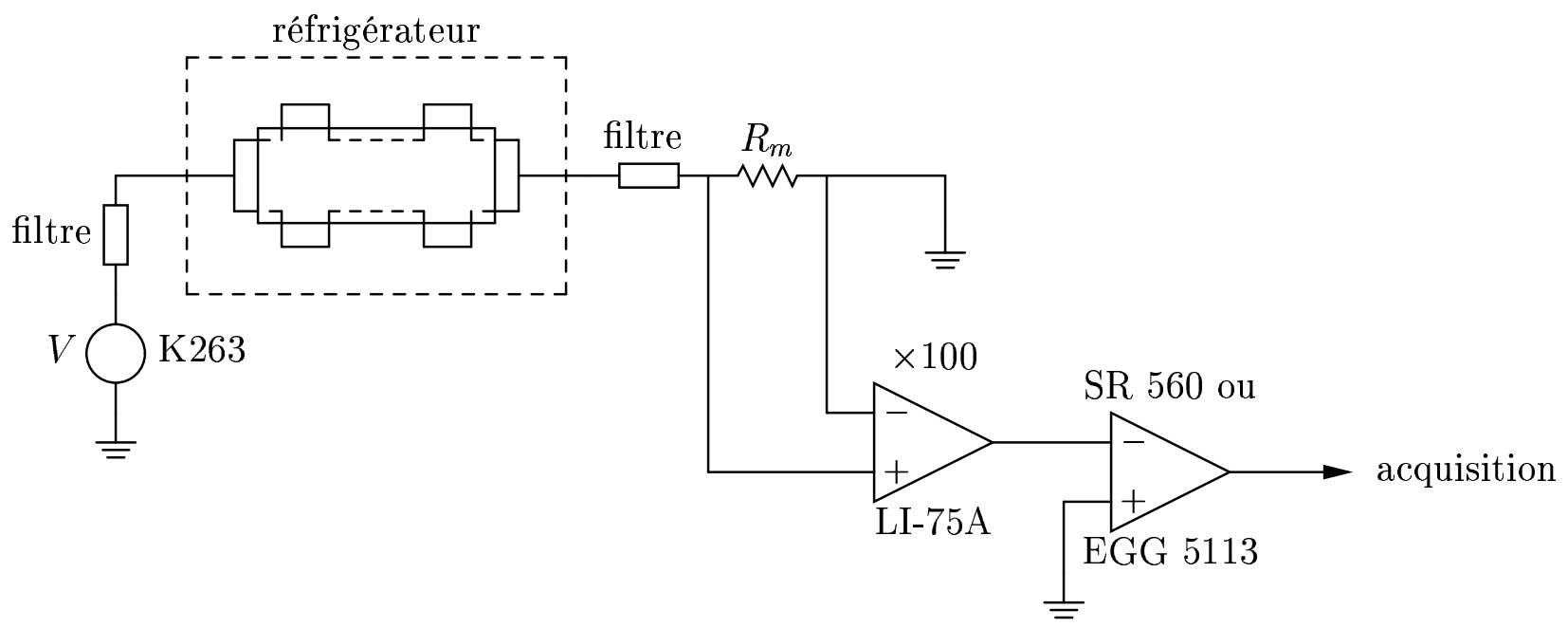

FIG. 3.8: Schéma de la mesure de résistance dans la configuration deux points. Le circuit pour la tension de grille est le même que dans la configuration quatre points. 


\section{Deuxième partie}

\section{Transport non-ohmique autour de la transition métal-isolant}





\section{Chapitre 4}

\section{Lois de transport autour de la transition métal-isolant}

\section{Sommaire}

4.1 Introduction . . . . . . . . . . . . . . . 108

4.2 Transport pour des électrons localisés . . . . . . . . 108

4.2 .1 Sauts assistés par les phonons . . . . . . . . . . 108

4.2.2 Effets collectifs dus aux interactions entre électrons . . . . 115

4.2 .3 Effet du champ électrique . . . . . . . . . . . . . . . . 118

4.3 Transport d'une phase collective ........... 121

4.3 .1 Modèles classiques . . . . . . . . . . . . . . . . . . 121

4.3.2 Accrochage par des impuretés chargées . . . . . . . . . 125

4.3.3 Conduction par création de paires de dislocations . . . . 126

4.3 .4 Expériences . . . . . . . . . . . . . . . . . . . . . . . . . . . . . . . . . . . . . .

4.4 Conclusion ..................... 129 


\subsection{Introduction}

Dans ce chapitre, nous nous intéressons plus particulièrement aux lois de transport attendues dans les régimes décrits dans le chapitre 1 . Ils concernent des particules indépendantes en présence de désordre, ou des particules fortement corrélées, et conduisent dans les deux cas à un comportement isolant. L'étude du transport dans le régime isolant s'est développé indépendamment du domaine de la transition métal-isolant en dimension deux. Ce sont pourtant des sujets très liés, car la compréhension de la phase basse densité ("isolante"), qui est la phase où les interactions entre électrons sont les plus fortes, peut apporter un argument décisif dans la compréhension de la transition métal-isolant en dimension deux, et en particulier en ce qui concerne le rôle des interactions. De plus, ces comportements isolants ont été largement étudiés autour de la transition effet Hall quantique-isolant à fort champ magnétique, qui présente des points communs avec la transition métal-isolant à champ magnétique nul (voir chapitre 2).

Pour des électrons indépendants, le désordre localise les électrons, conduisant à des mécanismes de transport par sauts entre états localisés (\$4.2). Pour des électrons fortement corrélés, l'état électronique attendu à basse densité est un cristal de Wigner accroché sur le désordre (§4.3). Ces deux limites conduisent à des lois de transport différentes en fonction de la température et du champ électrique, permettant ainsi, théoriquement, de distinguer ces deux comportements. Nous ne parlerons pas ici du régime de localisation faible qui a été décrit dans le paragraphe 1.5.2, ni des effets de la température et du champ électrique liés aux abords d'une transition de phase quantique qui ont été vus au paragraphe 2.4.1. De manière générale on ne débattra pas ici des dépendances en température liées à l'existence ou non n'une phase métallique en dimension deux. Les différents modèles traitant de ce sujet ont été présentés au chapitre 2 .

\subsection{Transport pour des électrons localisés}

Nous avons vu au chapitre 1 l'effet du désordre sur un système électronique en dimension deux, et en particulier le fait que les fonctions d'onde sont alors toujours localisées. Dans ce paragraphe, on considère le cas de la localisation forte, i.e. la longueur de localisation $a$ est faible devant la taille de l'échantillon $L$. Dans ce cas, et à suffisamment basse température, le transport électronique se fait par sauts entre états localisés. Nous détaillons ici les mécanismes de saut envisagés, en commençant par les sauts engendrés par l'interaction électron-phonon. Nous verrons ensuite le rôle des interactions électronélectron, qui peuvent modifier de façon importante le mécanisme de transport, puis nous verrons l'effet du champ électrique sur le transport. Il est possible de trouver une étude plus détaillée de ces mécanismes dans l'ouvrage de Shklovskii et Efros [11].

\subsubsection{Sauts assistés par les phonons}

\section{Sauts entre plus proche voisins}

Les états électroniques localisés peuvent être décrits par des fonctions d'onde hydrogénoïdes de rayon $a$, la longueur de localisation. Chaque état, indexé par un indice $i$, a 
une énergie $\epsilon_{i}$, déterminée par l'énergie du premier état lié, et une position $\vec{r}_{i}$ fixée. A suffisamment basse température, la conduction à travers un tel système se fait par sauts entre états localisés. La démarche pour calculer la résistance globale du système est la suivante. Connaissant le mécanisme de saut d'un état à l'autre, il est possible de calculer la résistance équivalente entre deux états localisés $i$ et $j$, notée $R_{i j}$. Le système peut alors être modélisé par un réseau de résistances entre tous ces sites. Ce modèle a été proposé pour la première fois pour traiter le problème du transport dans un isolant par Miller et Abrahams [257]. Une fois chaque résistance du réseau connue, la résistance globale peut être évaluée en combinant classiquement toutes les résistances. Dans le cas où les valeurs de ces résistances sont largement distribuées, la théorie de percolation permet d'effectuer ce calcul.

On s'intéresse donc dans un premier temps aux propriétés de transport entre deux états localisés, d'énergies respectives $\epsilon_{i}$ et $\epsilon_{j}$, séparés d'une distance $r_{i j}=\left|\vec{r}_{j}-\vec{r}_{i}\right|$. Le courant électrique $J_{i j}$ entre ces deux états peut être calculé à partir de la probabilité de saut d'un électron entre les deux états, $\Gamma_{i j}$. Le mécanisme induisant les sauts, proposé par Ambegaokar et al. [258], est le couplage électron-phonon : le phonon absorbé ou émis permet de fournir ou d'absorber la différence d'énergie entre les deux états électroniques. Si $f_{i}$ est la fonction de distribution au site $i$ et $f_{j}$ celle au site $j$, la probabilité de saut s'écrit [11] :

$$
\Gamma_{i j}=\gamma_{i j} f_{i}\left(1-f_{j}\right),
$$

où $\gamma_{i j}$, la probabilité de transition d'un état du site $i$ à un état du site $j$, est donnée par la règle d'or de Fermi :

$$
\gamma_{i j}=\frac{2 \pi}{\hbar} \frac{V_{0}}{(2 \pi)^{3}} \int\left|M_{\vec{q}}\right|^{2} \delta\left(\hbar \omega_{\vec{q}}-\left(\epsilon_{j}-\epsilon_{i}\right)\right) d \vec{q},
$$

où $V_{0}$ est le volume du système, $\hbar \omega_{\vec{q}}$ l'énergie d'un phonon de vecteur d'onde $\vec{q}$, et $M_{\vec{q}}$ est l'élément de matrice du couplage électron-phonon. Le calcul dans le cas du couplage entre les électrons et les phonons acoustiques conduit à [11] :

$$
\gamma_{i j}=\gamma_{i j}^{0} \exp \left(-2 r_{i j} / a\right) N\left(\epsilon_{j}-\epsilon_{i}\right)
$$

où $N\left(\epsilon_{j}-\epsilon_{i}\right)=\left[\exp \left(\frac{\epsilon_{j}-\epsilon_{i}}{k_{B} T}\right)-1\right]^{-1}$ est le facteur de Bose-Einstein, et $\gamma_{i j}^{0}$ dépend de la nature du couplage électron-phonon (le calcul dans le cas du couplage par le potentiel de déformation en dimension trois est présenté par Shklovskii et Efros [11]). Pour calculer la probabilité de saut à l'équilibre, les distributions électroniques utilisées sont les distributions de Fermi-Dirac, $f_{i}=\left[\exp \left(\frac{\epsilon_{i}-\mu}{k_{B} T}\right)+1\right]^{-1}$, où $\mu$ est le potentiel chimique. Enfin, lorsque la différence de potentiel entre les sites $i$ et $j, U_{i}-U_{j}$, est faible, le courant entre ces deux sites prend alors la forme de la loi d'Ohm :

$$
J_{i j}=R_{i j}^{-1}\left(U_{i}-U_{j}\right)
$$

où la résistance entre sites $R_{i j}$ est :

$$
R_{i j}=R_{i j}^{0} \exp \left(\xi_{i j}\right),
$$


avec

$$
\xi_{i j}=\frac{2 r_{i j}}{a}+\frac{\epsilon_{i j}}{k_{B} T} \quad \text { et } \quad R_{i j}^{0}=\frac{k_{B} T}{e^{2} \gamma_{i j}^{0}},
$$

avec $\epsilon_{i j}=\frac{1}{2}\left(\left|\epsilon_{i}-\epsilon_{j}\right|+\left|\epsilon_{i}-\mu\right|+\left|\epsilon_{j}-\mu\right|\right), \mu$ étant le potentiel chimique. Le premier terme de la résistance, $\exp \left(2 r_{i j} / a\right)$, correspond au recouvrement entre les fonctions d'onde électroniques des sites $i$ et $j$, alors que le deuxième terme, $\exp \left(\epsilon_{i j} / k_{B} T\right)$, est lié à l'activation thermique des sauts.

L'étape suivante consiste à calculer la résistance globale du réseau de résistances définies par l'équation (4.5). On remarque tout d'abord que, si les distances entre sites $r_{i j}$ et les énergies $\epsilon_{i j}$ ont une distribution uniforme sur tout le réseau, $\xi_{i j}$ a une distribution uniforme et les résistances composant le réseau de Miller-Abrahams sont exponentiellement distribuées. On peut ainsi les mettre sous la forme :

$$
R=R_{0} e^{\xi}
$$

où $R_{0}$ est une constante et $\xi$ est une variable aléatoire distribuée uniformément dans l'intervalle $-\xi_{0} \leq \xi \leq \xi_{0}$ avec $\xi_{0} \gg 1$. Ce réseau est très inhomogène, comportant des résistances très différentes. Ainsi toutes les résistances n'ont pas le même rôle dans la conduction. La résistance globale d'un tel réseau de résistances peut être traité avec la théorie de percolation. La première étape consiste à choisir une valeur donnée de $\xi$, et de remplacer toutes les résistances avec $\xi^{\prime}>\xi$ par des résistances infinies (circuits ouverts). On note $\sigma(\xi)$ la conductivité de ce nouveau système. On remarque que la conductivité du système global est la conductivité calculée pour $\xi=\xi_{0}$, soit $\sigma\left(\xi_{0}\right)$. Pour une valeur de $\xi$ suffisamment faible, le nombre de résistances dans le circuit est faible et elles sont très peu connectées entre elles, conduisant à une conductivité nulle. Si on augmente progressivement $\xi$, il arrive un moment où on forme un réseau infini de résistances connectées entre elles, conduisant à une conductivité non nulle : l'existence de ce seuil $\xi_{c}$ a été démontré par les théories de percolation, et est connu comme le seuil de percolation. Au delà du seuil, la conductivité augmente fortement, puis reste constante car les résistances qu'on attache ensuite au réseau infini initial (réseau percolant) ont des valeurs beaucoup plus élevées que les résistances incluses dans le réseau au seuil de percolation. On montre ainsi que la conductivité est déterminée par la conductivité du réseau au seuil de percolation [11]:

$$
\sigma\left(\xi_{0}\right)=\sigma\left(\xi_{c}\right)=\sigma_{0} e^{-\xi_{c}} \quad \text { avec } \quad \sigma_{0}=\frac{A}{R_{0} l_{0} \xi_{0}^{\nu}},
$$

où $l_{0}$ est le pas du réseau et $\nu$ est l'exposant de longueur de corrélation. Les calculs de $\xi_{c}$ et de $\nu$ dépendent des caractéristiques du réseau de percolation, sa dimensionnalité, sa forme. Des calculs exacts et des simulations numériques apportent ces réponses (voir par exemple dans [11]).

La résistivité totale prend donc une forme très analogue à la résistivité entre sites, et en particulier elle garde la dépendance exponentielle en température :

$$
\rho(T)=\rho_{0} \cdot \exp \left[\frac{T_{0}}{T}\right] \quad \text { avec } \quad T_{0}=\frac{C e^{2} n^{1 / d}}{k_{B} 4 \pi \varepsilon_{0} \varepsilon_{r}}
$$






FIG. 4.1: Schématisation de la répartition des états localisés en énergie $E$ et dans une direction de l'espace $x$, autour du potentiel chimique $\mu$. Les états vides sont représentés par des ronds vides, les signes - dans les autres états indiquent qu'il contiennent un électron. La bande grisée représente une bande d'énergie $\sim k_{B} T$ dans laquelle se trouvent les états accessibles pour des transitions. La flèche représente un saut.

où $C$ est une constante proche de $1, n=a^{-d}$ est la densité de sites localisés. Enfin, dans le cas du couplage par le potentiel de déformation en dimension trois, on s'attend à un préfacteur dépendant de la température suivant une loi linéaire, $\rho_{0}(T) \propto T$ [11], mais cette loi peut être modifiée suivant la dimensionnalité de l'espace et la nature du couplage électron-phonon.

\section{Sauts à portée variable : Mott}

Lorsque la température devient suffisamment faible, Mott a montré que la résistance entre deux proches voisins n'est plus la résistance la plus faible, mais qu'il faut prendre en compte des voisins plus éloignés [259]. Ce mécanisme est celui de sauts à portée variable.

On a vu dans le paragraphe précédent que la probabilité de sauts entre deux sites localisés $i$ et $j$, d'énergies respectives $\epsilon_{i}$ et $\epsilon_{j}$ et séparés par une distance $r_{i j}$, est donnée par :

$$
\Gamma_{i j} \propto \exp \left[-\left(\frac{2 r_{i j}}{a}+\frac{\epsilon_{i j}}{k_{B} T}\right)\right] .
$$

Le deuxième terme indique que les états énergétiquement accessibles par ce type de saut sont ceux situés à une énergie inférieure à quelques $k_{B} T$ au-dessus de l'énergie de l'état de départ (voir la figure 4.1). On peut noter que, pour une distribution aléatoire des états localisés en position et en énergie, plus les états sont éloignés et plus la probabilité de trouver un état proche en énergie est grande. Le deuxième terme est donc d'autant plus petit que la distance entre sites est grand. On note $\epsilon_{0}$ l'écart d'énergie moyen entre niveaux plus proche voisins. Lorsque la densité d'états par unité de volume $\mathcal{D}(E)$ est constante et vaut $\mathcal{D}\left(E_{F}\right)$, on a $\epsilon_{0}=1 /\left(\mathcal{D}\left(E_{F}\right) a^{d}\right)$, où $d$ est la dimensionnalité de l'espace. Il est donc possible d'évaluer l'écart moyen en énergie entre sites en fonction de la distance par :

$$
\epsilon_{M}=\epsilon_{0}\left(\frac{a}{r_{i j}}\right)^{d}
$$

En remplaçant $\epsilon_{i j}$ par $\epsilon_{M}$ dans l'expression de $\Gamma_{i j}$, puis en maximisant $\Gamma_{i j}$ par rapport à la distance entre sites, on obtient la distance typique d'un saut :

$$
r_{M}=a\left(\frac{T_{M}}{T}\right)^{\frac{1}{d+1}}
$$


avec $T_{M}=\epsilon_{0} / k_{B}$. Cette distance typique varie en $T^{-1 /(d+1)}$, donc croît quand la température diminue, ce qui justifie la dénomination de sauts à portée variable pour ce mécanisme de conduction. Enfin, puisque la résistance d'un tel système est proportionnelle à l'inverse de la probabilité de saut $\Gamma_{i j}$ (voir le paragraphe précédent), la résistance typique entre deux sites est :

$$
R_{M}=R_{M}^{0} \exp \left[\left(\frac{T_{M}}{T}\right)^{\frac{1}{d+1}}\right] .
$$

En remarquant que, comme pour le cas du réseau de résistance de Miller-Abrahams, les résistances sont exponentiellement distribuées, il est donc là aussi possible d'utiliser la théorie de percolation pour calculer la résistance globale du réseau. Un calcul détaillé conduit à la loi de Mott [11] :

$$
\rho(T)=\rho_{M} \cdot \exp \left[\left(\frac{T_{M}}{T}\right)^{\frac{1}{d+1}}\right] \quad \text { avec } \quad T_{M}=\frac{\beta}{k_{B} \mathcal{D}\left(E_{F}\right) a^{d}},
$$

où $\beta$ est un coefficient qui dépend du type de réseau de percolation et de la dimensionnalité de l'espace considéré. En dimension deux, $\beta=13,8$ [11]. Le préfacteur dépend de la température, mais avec une loi variant beaucoup plus lentement que la dépendance exponentielle [11] :

$$
\rho_{M}(T) \approx \rho_{M}^{\prime}\left(\frac{T_{M}}{T}\right)^{\frac{\nu-2}{4}}
$$

où $\nu$ est l'exposant de longueur de corrélation de la percolation. En dimension deux, cet exposant vaut $\nu=4 / 3[11]$.

\section{Bande d'énergie interdite de Coulomb : Efros-Shklovskii}

Le calcul de Mott suppose que la densité d'états $\mathcal{D}(E)$ est constante autour du niveau de Fermi. Cependant, Efros et Shklovskii ont montré que, en raison des interactions Coulombiennes entre porteurs, la densité d'états $\mathcal{D}(E)$ doit s'annuler au niveau de Fermi suivant une loi [260]:

$$
\mathcal{D}_{c}(E)=\frac{\alpha\left(4 \pi \varepsilon_{0} \varepsilon_{r}\right)^{d}}{e^{2 d}}\left|E-E_{F}\right|^{d-1},
$$

où $d$ est la dimensionnalité de l'espace, $\alpha$ est une constante numérique valant $d / \pi$ [11]. La bande d'énergie interdite ainsi créée est dite "douce" (soft gap) car la densité d'état ne s'annule qu'en un point unique, et non sur un intervalle d'énergie (voir la figure 4.2 de gauche pour le cas en dimension deux). L'ouverture de cette bande interdite au niveau de Fermi joue un rôle important pour le calcul de la résistivité. Dans un premier temps, l'ouverture d'une bande interdite dans la densité d'état ne sera pas "ressentie" si l'intervalle en énergie responsable des sauts $\epsilon_{M}$ (voir l'équation 4.11) est très grand devant la largeur typique de la bande interdite $\Delta_{c}$. Cette condition peut se ramener à une condition sur la température du système :

$$
T \gg T_{c}=\frac{a}{k_{B}}\left(\frac{e^{2(d+1)} \mathcal{D}_{0}^{2}}{\left(4 \pi \varepsilon_{0} \varepsilon_{r}\right)^{d+1}}\right)^{\frac{1}{d-1}}
$$



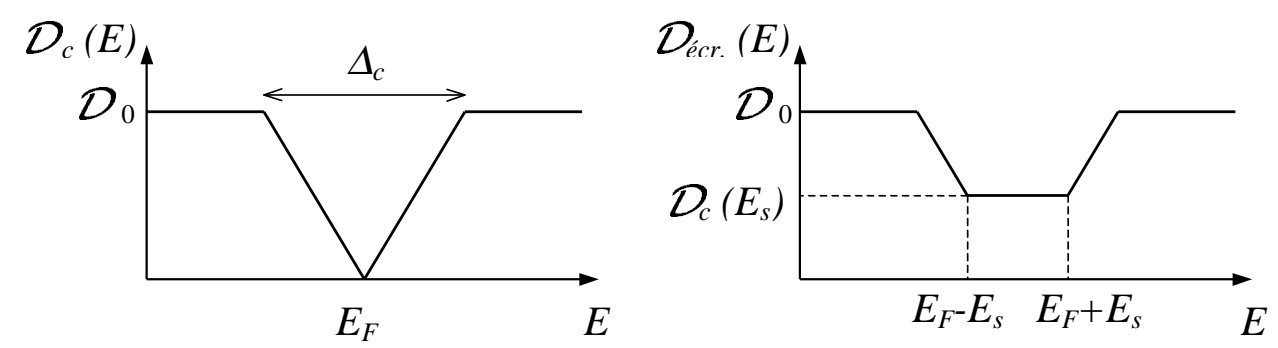

Fig. 4.2: Densité d'état autour du niveau de Fermi en dimension deux dans le cas d'interactions Coulombiennes non écrantées (à gauche) et écrantées par une grille (à droite).

où $\mathcal{D}_{0}$ la densité d'états au niveau de Fermi non perturbée (i.e. $\mathcal{D}\left(E_{F}\right)$ avant introduction des interactions). Lorsque la température est très supérieure à cette température $T_{c}$, on retrouve l'exposant $1 /(d+1)$ de Mott (équation (4.14)). Par contre, pour $T \leqslant T_{c}$, il est nécessaire de refaire le calcul de Mott avec la nouvelle densité d'état, ce qui conduit la loi d'Efros-Shklovskii pour la résistivité [11] :

$$
\rho(T)=\rho_{E S} \cdot \exp \left[\left(\frac{T_{E S}}{T}\right)^{\frac{1}{2}}\right] \quad \text { avec } \quad T_{E S}=\frac{C e^{2}}{4 \pi \varepsilon_{0} \varepsilon_{r} k_{B} a}
$$

où $a$ est la longueur de localisation et la constante $C$ dépend du type de réseau percolation utilisé et de la dimensionnalité de l'espace. En dimension deux, $C=6,2$ [261].

On peut noter enfin qu'une bande interdite "dure" (hard gap) au niveau de Fermi (i.e. l'annulation de la densité d'états sur une gamme d'énergie) conduit à une conduction de type activée, donc avec un exposant 1 dans l'exponentielle (ce mécanisme a été suggéré pour interpréter certaines expériences [262]).

\section{Écrantage des interactions entre porteurs par une grille métallique}

Pour des structures possédant une grille métallique, telles que les MOSFETs Si ou les hétérostructures dont la densité électronique peut être modifiée par effet de champ, Aleiner et Shklovskii ont montré que l'écrantage des interactions Coulombiennes entre les électrons, réduit la portée de ces interactions [263]. Étant donné que la bande interdite "douce" obtenue par Efros et Shklovskii [260] est due à la longue portée des interactions Coulombiennes, on s'attend à une modification de la forme de la densité d'états près du niveau de Fermi pour des interactions écrantées. Si l'interaction est écrantée au-delà d'une distance $r_{s}$, l'interaction entre des électrons séparés d'une énergie inférieure à $E_{s}=$ $e^{2} / 4 \pi \varepsilon_{0} \varepsilon_{r} r_{s}$ est négligeable. Ainsi la densité d'état est constante pour des états d'énergie $E$ telle que $\left|E-E_{F}\right|<E_{s}$, et croît ensuite linéairement comme prévu par Efros et Shklovskii (voir la figure 4.2 de droite). Pour l'écrantage par une gr9ille métallique située à une distance $d$ du gaz d'électrons, l'énergie Coulombienne écrantée prend la forme [263] :

$$
U(r)=\frac{e^{2}}{4 \pi \varepsilon_{0} \varepsilon_{r}}\left(\frac{1}{r}-\frac{1}{\sqrt{r^{2}+4 d^{2}}}\right) .
$$


Ainsi $d$ est la longueur d'écrantage et l'énergie d'écrantage s'écrit alors $E_{s}=e^{2} / 4 \pi \varepsilon_{0} \varepsilon_{r} d$. La densité d'états est constante pour des énergies inférieures à $E_{s}$ et vaut $\mathcal{D}_{c}\left(E_{s}\right)=$ $\alpha 4 \pi \varepsilon_{0} \varepsilon_{r} / e^{2} d$, comme on l'a représenté dans la figure 4.2 de droite. Ce modèle conduit à une augmentation de la conductance en présence d'écrantage en raison de la densité d'états plus élevée. De plus ce modèle explique qu'à basse température, lorsque la distance moyenne des sauts est maximale, la bande d'énergie interdite de Coulomb est supprimée et l'exposant dans la résistivité passe de $1 / 2$ (Efros-Shklovskii) à $1 / 3$ (Mott, en dimension deux). La température de transition en-dessous de laquelle le régime de Mott est retrouvé a été calculé par une méthode numérique [263] :

$$
\bar{T} \approx 0,013 \frac{e^{2} \xi}{k_{B} \epsilon d^{2}} .
$$

Le développement ci-dessus suppose que la longueur d'écrantage soit supérieure à la longueur de localisation $a$. La localisation $a$ dépendant de la densité de porteurs $n_{s}$, cette condition permet de déduire une valeur de densité au-delà de laquelle le régime d'EfrosShklovskii n'est plus observé, et on observe le régime de Mott quelle que soit la température.

\section{Expériences}

Dans ce paragraphe sont reportées certaines expériences ayant permis de vérifier les dépendances en température prévues par les mécanismes de sauts dans le régime d'électrons localisés.

Un grand nombre d'expériences sur des systèmes bidimensionnels d'électrons ont montré l'existence de lois de transport compatibles avec la loi de Mott (voir les références 7 à 11 et 17 à 20 dans $[264,265]$ ), mais aussi d'Efros-Shklovskii (voir les références 20 à 22 dans $[264,265]$, ainsi que les résultats sur des MOSFETs Si [67] et hétérostructures $n$-GaAs [266]). Enfin des lois de transport simplement activées ont également été observées, mais dans le régime quantique extrême, i.e. à fort champ magnétique (voir les références 23 à 25 dans [264, 265]). Dans certaines de ces expériences, la loi d'Efros-Shklovskii est retrouvé à basse température $[36,35]$. Les régimes de Mott et d'Efros-Shklovskii ont également été reportés à fort champ magnétique (voir références dans [264, 265]).

Malgré le bon accord de toutes ces expériences avec les lois de transport attendues par les sauts à portée variable, Ebert et al. [267], puis Van Keuls et. al. [264, 265] ont cependant fait remarquer que la recherche des exposants des lois de transport peut être biaisée par la dépendance en température du préfacteur. En effet, la majorité des mesures expérimentales considèrent un préfacteur indépendant de la température, alors que les lois de transport engendrées par le couplage électron-phonon induit une dépendance en température du préfacteur. Bien que cette dépendance soit algébrique, et donc plus faible que la dépendance exponentielle, elle peut conduire à un autre choix de l'exposant de la dépendance exponentielle. En particulier, si la gamme de résistance explorées n'est pas suffisamment grande (inférieure à trois ordres de grandeur), des lois de Mott ou EfrosShklovskii avec des préfacteurs bien choisis peuvent donner des ajustements aussi bons, et le seul test de la loi en température n'est pas une preuve en faveur d'un régime ou de l'autre. Dans des hétérojonctions $n$-GaAs, Van Keuls et al. montrent qu'en choisissant 
une dépendance du préfacteur en $T^{0,8}$, la loi d'Efros-Shklovskii est trouvée sur une très large gamme de résistivité (jusqu'à cinq ordres de grandeur) [264, 265]. Dans des hétérostructures $n$-GaAs et dans le régime d'effet Hall quantique, Ebert et al. ont obtenu un préfacteur $\rho_{E S} \sim T$ dans le régime d'Efros-Shklovskii [267].

La meilleure preuve de l'existence d'une bande d'énergie interdite "douce" a été donnée par des mesures directes de spectroscopie par effet tunnel. La bande d'énergie interdite de Coulomb décrite par l'équation (4.16) a ainsi été observée directement dans des échantillons Si dopés B (cas 3D) [268, 269], et dans des films métalliques Be (cas 2D) [270].

Kim et al. ont observé dans des films amorphes $\mathrm{In} / \mathrm{InO}_{x}$ (en dimension trois) une transition entre un exposant $1 / 2$ à haute température et 1 à basse température, interprété comme la transition entre une bande d'énergie interdite "douce" et une bande d'énergie interdite "dure" [262]. Ce comportement est attribué à des polarons à basse température, avec une énergie interdite de largeur environ $\Delta_{c} / 2$.

L'écrantage des interactions par une grille, avec une transition de la loi d'EfrosShklovskii à de la loi de Mott à basse température, a été observée expérimentalement dans divers systèmes, et a servi de preuve pour la présence d'interactions à longue portée dans ces systèmes [33, 271]. Van Keuls et al. ont étudié des hétérostructures $n$-GaAs avec différentes distances entre la grille et le gaz d'électron [264, 265], montrant la présence d'une bande interdite de Coulomb à basse densité électronique, et une conduction de Mott à haute densité électronique. Ces mesures suivent très bien la loi prévue par Aleiner et Shklovskii [263] pour l'écrantage des interactions entre électrons par la grille, et notamment l'évaluation de la température de transition entre les deux comportements. Yakimov et al. ont réalisé des mesures de transport dans des couches de silicium, dans lesquels les états localisés sont construits artificiellement grace à un réseau bidimensionnel de plot auto-organisés de germanium [271]. Alors que les échantillons sans grille métallique présentent une loi de conduction d'Efros-Shklovskii à toutes les températures, les échantillons avec grille présentent une transition entre le régime d'Efros-Shklovskii à haute température et le régime de Mott à basse température.

Cependant d'autres effets d'une grille métalliques peuvent être envisagés, par exemple pour interpréter les expériences de Entin-Wohlman et Ovadyahu dans des films $\operatorname{In}_{x} \mathrm{O}_{y}$ [272] Ils ont observé une réduction de la conductance en présence d'une grille, contrairement à ce que prévoit le modèle de Aleiner et Shklovskii. Dans leurs expériences, la présence d'une grille conduit à une conduction simplement activée, avec un exposant 1, et non 1/3. Un même comportement a également été observé par Adkins et Astrakharchik dans des films ultra minces de bismuth [273].

\subsubsection{Effets collectifs dus aux interactions entre électrons}

Dans le paragraphe précédent on a essentiellement considéré des électrons indépendants. L'interaction entre électrons est prise en compte dans la loi d'Efros-Shklovskii, mais le calcul est effectué en champ moyen, ce qui signifie que le mécanisme de sauts se fait encore par des particules indépendantes. Nous allons voir dans ce paragraphe deux effets des interactions qui peuvent conduire à des mécanismes nouveaux. Ces mécanismes ont été évoqués pour expliquer les écarts observés entre les résultats expérimentaux et les théories en électrons indépendants. 


\section{Sauts collectifs}

Dans les modèles proposés dans le paragraphe précédent, il est supposé que seuls les sauts à un électron interviennent. Dans le cas où les interactions entre électrons sont fortes, on peut se poser la question des sauts corrélés entre plusieurs électrons.

Les sauts à plusieurs électrons pour un seul phonon échangé ont été suggérés par Knotek et Pollak [274]. La prise en compte des sauts à deux électrons conduit à une diminution de l'énergie d'activation $k_{B} T_{0}$ dans les mécanismes par sauts simplement activés. PérezGarrido et al. [275] ont montré par des simulations numériques que, dans le transport par sauts de portée variable en présence d'une bande d'énergie interdite (régime d'EfrosShklovskii), les corrélations entre électrons ne peuvent pas être négligées. La prise en compte de ces sauts corrélés aboutit à une loi identique à la loi d'Efros-Shklovskii (4.18), mais avec une constante $C=0,61$, soit un facteur 10 en dessous de la valeur obtenue par la théorie de percolation [276]. Cette remarque est particulièrement importante dans le cas où on désire obtenir la longueur de localisation à partir de la température $T_{E S}$ et de l'équation (4.18). Cet effet des interactions a également été relevé dans Díaz-Sánchez et al. [277]. Cependant Tsiganov et Efros obtiennent un résultat contradictoire par des simulations numériques : ils ont montré que les sauts à deux électrons sont minoritaires [278].

Dans des expériences réalisées sur des hétérostructures n-GaAs, Van Keuls et al. ont comparé les longueurs de localisation avec la longueur d'écrantage due à la présence d'une grille métallique $[264,265]$. Ils ont constaté que la longueur de localisation est fortement surévaluée, ce qui peut être dû à une valeur trop élevée des constantes intervenant dans les lois de Mott ( $C$ dans l'équation (4.14)) et d'Efros-Shklovskii ( $\beta$ dans l'équation (4.18)). Il serait alors nécessaire de prendre des valeurs plus faibles d'un facteur environ 10 par rapport aux valeurs calculée dans le modèle de percolation. Ils ont interprété cette observation comme une preuve que les sauts se font de manière collective, mais que les corrélations entre électrons ne modifient pas la densité d'états calculée pour des électrons indépendants.

\section{Sauts assistés par les interactions entre électrons}

Le rôle des interactions entre électrons a également été évoqué pour expliquer un comportement anormal du préfacteur des lois de sauts. En particulier, Khondaker et al. [80] se sont interrogés sur le préfacteur obtenu, proche de la valeur universelle $h / e^{2}$, et indépendant de la densité et de la température. D'autre part un préfacteur également proche de $h / e^{2}$ a également été observé dans des MOSFETs Si par Mason et al. [77, 78], mais dans ce cas le préfacteur décroît quand la densité d'électrons augmente. Les expériences de Yakimov et al. sur des réseaux bidimensionnels d'îlots de Ge [271] ont montré que, dans le cas non écranté, le préfacteur $\rho_{E S}$ est indépendant de la longueur de localisation, et a une valeur universelle proche de $h / e^{2}$, ce qui est contradictoire avec le modèle de sauts induits par les phonons. Par contre, dans le régime écranté, le préfacteur $\rho_{M}$ dépend fortement de la longueur de localisation. Ainsi le caractère universel du préfacteur dans le régime non écranté semble être dû aux interactions entre électrons, ce qui permet d'envisager un mécanisme de sauts engendrés par les interactions entre électrons. Cependant un travail 
théorique récent de Dahm semble indiquer que le préfacteur ainsi obtenu est en accord avec le modèle d'Aleiner et Shklovskii, et ne nécessite pas de prendre en compte d'effets collectifs [279].

Le rôle des interactions entre électrons a également été suggéré par Marnieros et al. [280] dans des isolants 3D, et par Gershenson et al. [281] dans des hétérostructures $n$-GaAs, montrant que les lois de transport en régime de fort champ électrique ne dépendent que de la température des porteurs, et non de la température des phonons, alors que pour des sauts assistés par le couplage électron-phonon, la loi doit dépendre des deux températures.

Ces deux observations, associées au fait que, pour des systèmes électroniques de faible densité à basse température, le paramètre $r_{s}$ est élevé et le temps de collision électronélectron est beaucoup plus faible que le temps de collision électron-phonon, ont suggéré un mécanisme de sauts assistés par les interactions électron-électron. Dans ce cas, l'énergie nécessaire pour effectuer un saut ne provient pas des phonons, mais provient d'un autre électron couplé à l'électron effectuant le saut.

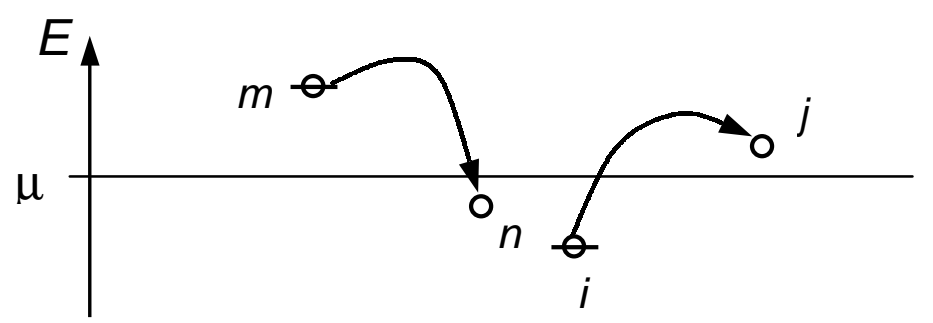

FIG. 4.3: Mécanisme de saut engendré par l'interaction Coulombienne directe.

Pour ce mécanisme, on suppose que l'énergie nécessaire pour effectuer un saut est fournie par la variation d'énergie d'un autre électron effectuant un saut simultanément (voir figure 4.3). La probabilité de saut s'écrit alors [282] :

$$
\begin{aligned}
& \Gamma_{i, m \rightarrow j, n} \propto \frac{1}{r_{i m}} \exp \left(-\frac{2\left(r_{i j}+r_{m n}\right)}{a}\right) f\left(\epsilon_{i}\right)\left(1-f\left(\epsilon_{j}\right)\right) f\left(\epsilon_{m}\right)\left(1-f\left(\epsilon_{n}\right)\right) \\
& \times \delta\left(\epsilon_{j}+\epsilon_{n}-\epsilon_{i}-\epsilon_{m}\right),
\end{aligned}
$$

et ne dépend donc que de la fonction de distribution des électrons $f(\epsilon)$, contrairement à l'équation (4.3) où apparaît également le facteur de Bose-Einstein pour les phonons. Ainsi seule la température des électron doit être prise en compte ici. Dans la limite basse température, i.e. $k_{B} T \ll\left|\epsilon_{i}-\mu\right|,\left|\epsilon_{j}-\mu\right|,\left|\epsilon_{m}-\mu\right|,\left|\epsilon_{n}-\mu\right|$, la probabilité s'écrit :

$$
\Gamma_{i, m \rightarrow j, n}=\gamma_{i, m \rightarrow j, n}^{0} \exp \left(-\frac{2 r_{i j m n}}{a}-\frac{\epsilon_{i j m n}}{k_{B} T}\right),
$$

avec

$$
\begin{aligned}
& r_{i j m n}=\left|r_{i}-r_{j}\right|+\left|r_{m}-r_{n}\right| \\
& \epsilon_{i j m n}=\frac{1}{2}\left(\left|\epsilon_{i}-\mu\right|+\left|\epsilon_{j}-\mu\right|+\left|\epsilon_{m}-\mu\right|+\left|\epsilon_{i}-\epsilon_{j}+\epsilon_{m}-\mu\right|\right) .
\end{aligned}
$$

La probabilité ainsi calculée prend une forme analogue à la probabilité de saut calculée pour le mécanisme de sauts par couplage électron-phonon. La résistivité peut donc être 
calculée de la même manière que pour la résistivité de Mott ou d'Efros-Shklovskii. La différence avec le mécanisme de sauts par couplage électron-phonon réside dans le préfacteur, et dans la température intervenant dans l'exponentielle. Cependant, un calcul plus poussé serait nécessaire pour voir si ce mécanisme est véritablement compatibles avec les expériences. La seule température des électrons qu'il faut considérer.

Enfin, Marnieros propose dans sa thèse d'autres mécanismes éventuels de sauts assistés par l'interaction entre électrons. Les calculs de ces processus conduisent également à une probabilité de sauts qui ne dépendant que de la température effective des porteurs de charge.

\subsubsection{Effet du champ électrique}

On s'intéresse maintenant à l'effet d'un champ électrique dans le régime de conduction par sauts. Les calculs décrits dans les paragraphes précédents supposent que le champ électrique est suffisamment faible pour pouvoir considérer la réponse linéaire du système. Mais ce calcul devient faux pour un champ électrique plus élevé. Deux effets principaux peuvent être considérés dans ce cas. Le premier consiste à prendre en compte les effets de champ électrique sur le saut lui-même, aboutissant au modèle des sauts dirigés. Mais à champ électrique plus élevé encore, le champ électrique va jouer sur la distribution des électrons qui ne pourra plus être considérée comme la distribution à l'équilibre, notamment par modification de la température effective des électrons.

\section{Sauts dirigés}

Dans ce paragraphe, on considère le cas des mécanismes de sauts à portée variable décrits par Mott ou Efros-Shklovskii. Dès qu'un champ électrique est appliqué, la conduction est plus favorable pour les électrons se propageant dans le sens opposé au champ électrique $\vec{E}$. Ainsi la probabilité de saut est modifiée :

$$
\Gamma_{i j} \propto \exp \left(-\frac{2 r_{i j}}{a}-\frac{\epsilon_{i j}-e \vec{E} \cdot \vec{r}_{i j}}{k_{B} T}\right) .
$$

Comme on l'a vu dans le cadre de la conductivité de Mott, il faut maximiser le terme dans l'exponentielle. Pour simplifier cette opération, on peut supposer que la conduction est dominée par les sauts les plus probables, et donc dans la direction opposée au champ électrique, et on néglige ainsi les sauts dans les autres directions. Dans le cas où $E<E_{c}=$ $\left(2 k_{B} T\right) /(e a)$, on aboutit à la loi :

$$
\rho(T, E)=\rho_{0} \exp \left[\left[\frac{T_{0}}{T}\left(1-\frac{e E a}{2 k_{B} T}\right)\right]^{m}\right],
$$

où $m$ est l'exposant caractéristique du mécanisme de sauts (Mott ou Efros-Shklovskii). Cette relation peut également se mettre sous la forme :

$$
\rho(T, E)=\rho_{0}(T, E=0) \exp \left(\frac{e E L(T, E)}{k_{B} T}\right),
$$


où $L(T, E)$ est la longueur de saut optimum en présence d'un champ électrique, qui est reliée à la longueur de saut optimum sans champ électrique $L(T, E=0)=a \frac{m}{2}\left(\frac{T_{0}}{T}\right)^{m}$ par :

$$
L(T, E)=L(T, E=0) \frac{1}{m} \frac{1-\left(1-\frac{e E a}{2 k_{B} T}\right)^{m}}{\frac{e E a}{2 k_{B} T}} \underset{E \ll E_{c}}{\approx} L(T, E=0) .
$$

Cependant certains auteurs ont proposés que l'approximation de sauts favorisés uniquement dans une direction est trop forte. Ainsi de manière plus générale il est possible d'écrire le courant engendré par les sauts sous la forme (voir par exemple dans Ladieu et al. [283]) :

$$
\begin{array}{r}
I(E, T)=I_{0} \exp \left[-\left(\frac{T_{0}}{T}\right)^{\gamma}\right] \\
\times \exp \left[1-A \frac{(e E a)^{\alpha}}{\left(k_{B} T\right)^{\alpha^{\prime}}}+B\left(\frac{e E a}{k_{B} T}\right)^{\beta}\right] .
\end{array}
$$

Dans la dépendance en champ électrique, le premier terme, contenant les paramètres $A$, $\alpha$ et $\alpha^{\prime}$, correspond aux sauts des électrons dans le sens opposé au champ électrique. Le second terme, contenant les paramètres $B$ et $\beta$, correspond aux sauts "en arrière", i.e. dirigés le long du champ électrique, c'est pourquoi leur contribution au courant est opposée par rapport aux sauts précédents. Les valeurs des paramètres obtenus dans les systèmes 3D sont reportés dans Ladieu et al. [283], qui ont en particulier montré l'importance de ces sauts "en arrière" dans le transport en champ électrique dans des semiconducteurs amorphes YSi.

\section{Limite fort champ électrique}

Lorsque $E\left(c_{s} a t\right) E_{c}=\left(2 k_{B} T\right) /(e a)$, le modèle précédent n'est plus valable. Un certain nombre de travaux théoriques (voir les références 7 à 12 dans [283]) ont montré que le courant ne dépend alors plus de la température, mais ne dépend que du champ électrique suivant une loi analogue aux lois de transport par sauts à portée variable :

$$
I=I_{0} \exp \left[-\left(\frac{E_{0}}{E}\right)^{m}\right]
$$

où $I_{0}$ et $E_{0}$ sont des paramètres, et $m$ est l'exposant obtenu dans la loi de transport à champ électrique tendant vers zéro.

Marianer et Shklovskii ont montré que'à température nulle et à fort champ électrique, la fonction de distribution des électrons est modifiée, et prend la forme d'une distribution de Boltzmann :

$$
f(\epsilon)=\exp \left(\frac{\epsilon-\epsilon_{F}}{k_{B} T_{e f f}}\right)
$$


où le paramètres $T_{\text {eff }}$ dépend du champ électrique $E$ :

$$
T_{e f f}=K \frac{e E a}{2 k_{B}}
$$

où $a$ est la longueur de localisation, et $K$ est une constante, évaluée numériquement en dimension trois $K=0,67 \pm 0,03$ [284]. Cette relation est modifiée à température finie, conduisant à :

$$
T_{e f f}^{2}=T^{2}+\left(K \frac{e E \xi}{k_{B}}\right)^{2} .
$$

Quel que soit le mécanisme de sauts, pour $T_{\text {eff }} \gg T$, on a $T_{\text {eff }} \propto E$.

Le calcul de la résistivité à fort champ électrique s'effectue en remplaçant la distribution de Fermi habituelle par la distribution de Boltzmann avec la température effective $T_{\text {eff }}$, conduisant à :

$$
\rho(T=0, E)=\rho_{0} \exp \left[\left(\frac{T_{0}}{T_{e f f}(E)}\right)^{m}\right]=\rho_{0} \exp \left[\left(\frac{E_{0}}{E}\right)^{m}\right] .
$$

\section{Chauffage des porteurs}

Il existe une autre manière de faire varier la distribution des électrons. Lorsque l'on injecte un courant dans le système, on fournit une puissance par effet Joule, et cette puissance doit être dissipée dans le réseau par le couplage électron-phonon. A basse température, le temps de collision électron-phonon est très faible, et on peut supposer qu'une partie de la puissance injectée sera distribuée dans le gaz d'électrons, conduisant à une distribution hors équilibre. Dans l'hypothèse de la température effective, on décrit les électrons par une distribution de Fermi à la température effective $T_{e}$ :

$$
f_{T_{e}}(\epsilon)=\left[\exp \left(\frac{\epsilon-\mu}{k_{B} T_{e}}\right)+1\right]^{-1} .
$$

Lorsque les sauts sont assistés par les phonons, la probabilité de sauts dépend en même temps de la température des phonons $T_{0}$ et de la température effective des électrons $T_{e}$ [282] :

$$
\Gamma_{i j}=\gamma_{i j}^{0} \exp \left(-\frac{2 r_{i j}}{a}\right) N_{T_{0}}\left(\epsilon_{j}-\epsilon_{i}\right) f_{T_{e}}\left(\epsilon_{i}\right)\left(1-f_{T_{e}}\left(\epsilon_{j}\right)\right),
$$

où $N_{T_{0}}\left(\epsilon_{j}-\epsilon_{i}\right)=\left[\exp \left(\frac{\epsilon_{j}-\epsilon_{i}}{k_{B} T_{0}}\right)-1\right]^{-1}$ est la distribution de Bose-Einstein pour les phonons. Ainsi on s'attend à une résistivité totale du système qui dépend des deux température $T_{0}$ et $T_{e}: \rho\left(T_{0}, E\right)=\rho\left(T_{0}, T_{e}\right)$. Le cas est différent lorsque les sauts sont assistés par les interactions électron-électron. On peut voir en effet au paragraphe 4.2.2 que la seule température qui intervient dans les probabilités de sauts est celle des électrons $T_{e}$ (par l'intermédiaire des distributions de Fermi). On en conclue que la résistivité d'un tel système ne va dépendre que de la température effective des électrons : $\rho\left(T_{0}, E\right)=\rho\left(T_{e}\right)$. Les effets de température peuvent alors se comprendre par une élévation de la température effective des porteurs. Le calcul de cette température effective nécessite de connaître le couplage électron-phonon (voir l'annexe A). 


\section{Expériences}

En dimension trois, une revue des expériences est donnée dans Ladieu et al. [283].

La dépendance en champ électrique due à une température effective (Marianer et Shklovskii) a été observée dans des MOSFETs Si de haute mobilité par Maslov et al. [78] avec un exposant $1 / 2$, et a servi de preuve supplémentaire en faveur de la formation d'une bande d'énergie interdite de Coulomb dans un tel système.

On peut noter que, dans des isolants de Mott-Anderson 3D, Ladieu, Sanquer et Bouchaud ont observé un effet de décrochage en fonction du champ électrique, résultant de la compétition entre le désordre et les interactions [285]. A faible champ électrique, les électrons sont accrochés collectivement sur désordre. Au-delà d'un champ électrique seuil, il existe des charges libres, donnant lieu à une augmentation brutale du courant. Dans ces mesures commencent à apparaître des effets collectifs analogues à ceux qui sont décrits dans le cadre du cristal de Wigner accroché présenté dans la section suivante.

\subsection{Transport d'une phase collective}

On a vu au chapitre 1 que les interactions entre électrons peuvent conduire à des états électroniques collectifs. On a vu également qu'en présence de désordre, un tel état est accroché sur le désordre, conduisant à un système isolant à température nulle. Nous allons voir ici qu'il existe deux moyen de créer un courant à travers un tel état, soit en applicant un champ électrique suffisamment fort, c'est le phénomène de décrochage, soit en augmentant la température, mais toujours dans une limite de basse température. Nous ne discuterons pas ici les effets de la température sur la fonte de l'état collectif (voir le chapitre 1). Nous verrons plus particulièrement le cas du cristal de Wigner. Et dans ce chapitre nous ne considérons pas non plus le rôle du spin électronique qui peut jouer sur la nature de cette phase basse densité.

Nous verrons dans un premier temps les modèles proposés pour décrire la conduction d'un cristal de Wigner classique, limite pour laquelle on néglige l'énergie cinétique des électrons. Comme on l'a vu au chapitre 1, cette limite ne correspond pas aux gaz d'électrons créés dans les semiconducteurs. Cependant, la majorité de théories ont été élaborées dans la limite classique, et cette limite permet de comprendre certains phénomènes mis en jeu. Dans un deuxième temps nous verrons le modèle de création de paires de dislocations qui, appliqué à la limite quantique, permet de rendre compte de phénomènes de transport. Enfin ces modèles seront confrontés aux expériences réalisées dans divers systèmes bidimensionnels d'électrons.

\subsubsection{Modèles classiques}

Dans les modèles classique de transport d'un cristal de Wigner, le cristal est considéré comme un système élastique. Nous avons vu dans le chapitre 1 que cette une condition nécessaire pour observer le phénomène d'accrochage en dimension deux. Cependant ces modèles ne prennent pas en compte les phénomène de déformation plastique. Le transport d'une telle phase collective accrochée résulte de la compétition entre l'énergie perdue pour déformer le cristal et l'énergie gagnée par l'accrochage sur le désordre, avec éventuellement 


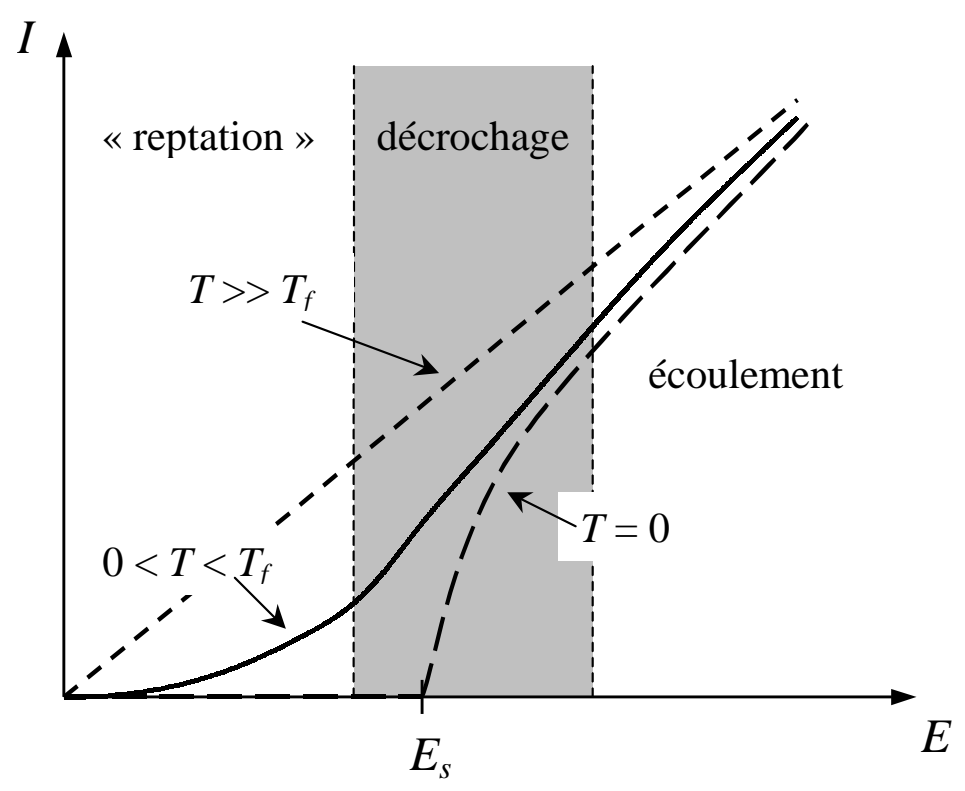

FIG. 4.4: Caractéristique champ électrique $(E)$ - courant $(I)$ typique pour une phase collective accrochée sur le désordre, à différentes températures, montrant les trois régimes de transport. D'après Chauve et al. [286, 287].

la contribution de l'énergie thermique à température non nulle. Pour l'étude des propriétés de transport dans le modèle classique, on pourra se référer à Chauve et al. [286, 287] et aux référence incluses.

Dans l'approche classique, on distingue plusieurs régimes de conduction (voir la figure 4.4). A faible champ électrique, le cristal est accroché sur le désordre. A température nulle, le cristal est immobile. Par contre, à température non nulle, le cristal peut se déplacer par activation thermique, conduisant au mécanisme de "reptation" (creep). Nous verrons qu'il existe un champ électrique seuil $E_{s}$ au-delà duquel le cristal peut se déplacer, même à température nulle, c'est le décrochage du cristal. Enfin, pour un champ électrique très élevé, le cristal se déplace sur un désordre moyenné, conduisant au mécanisme d'écoulement.

\section{Seuil de décrochage}

Principe du décrochage Dans le modèle le plus simple, le solide d'électrons se déforme pour profiter des creux du potentiel aléatoire, mais en payant une certaine énergie élastique de déformation. Ainsi au bout d'une certaine distance il perd sa cohérence, formant des domaines contenant un nombre fini $N_{D}$ d'électrons. Chaque domaine peut être décrit comme un solide rigide d'électrons de masse $N_{D} m^{*}$ et de charge $N_{D} e$ (voir la figure 4.5 de gauche). On peut remarquer que l'énergie d'un domaine plongé dans le potentiel de désordre est invariante par translation de $a_{0}$, la période du solide d'électrons, aux effets de bords près. Pour simplifier on peut prendre un potentiel sinusoïdal en dimension un (voir la figure 4.5 de droite) :

$$
V(x)=V_{0} \cos \left(2 \pi x / a_{0}\right) .
$$

Sous l'effet d'un champ électrique uniforme $E$ dans la direction $x$, le potentiel total devient :

$$
V^{\prime}(x)=V_{0} \cos \left(2 \pi x / a_{0}\right)-N_{D} e E x,
$$




$$
E=0
$$
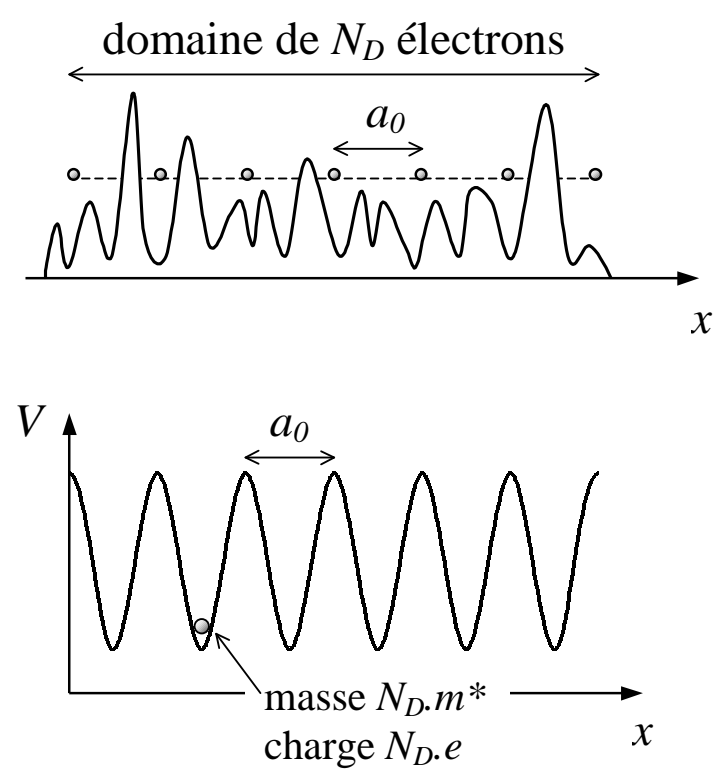

\section{$E>0$}

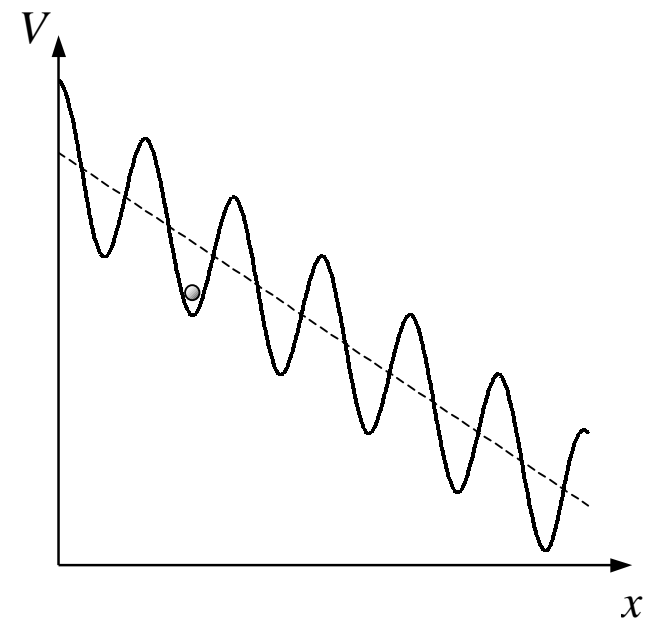

FIG. 4.5: Gauche : Schématisation en dimension un d'un cristal de Wigner comprenant $N_{D}$ électrons en présence d'un potentiel de désordre à champ électrique nul (en haut), et représentation de l'énergie de ce même cristal dans le potentiel périodique créé par le désordre (en bas). Droite : Représentation de l'énergie du cristal dans le potentiel de désordre pour un champ électrique non nul.

et le mouvement devient instable pour un champ électrique supérieur au champ seuil de décrochage :

$$
E>E_{s}=\frac{2 \pi V_{0}}{a_{0} N_{D} e} .
$$

Modèle de Larkin et Ovchinnikov Un modèle plus élaboré a été proposé par Larkin et Ovchinnikov $[12,13]$. Ils reprennent l'idée du volume corrélé, dans lequel le cristal n'est pas déformé. La taille typique de ce volume est donnée par une longueur $R_{c}$, et si $\xi$ est la portée des forces d'accrochage, la contrainte en bord de domaine est donnée par $s=\xi / R_{c}$. L'énergie libre élastique par unité de volume est donc $\frac{1}{2} C s^{2}$, où $C$ est le module élastique du cristal. Il est alors possible de déterminer la taille moyenne des domaines :

$$
R_{c}=\frac{C a_{0}}{n_{i}^{1 / 2} f},
$$

où $a_{0}$ le paramètre de maille du réseau d'électrons, $n_{i}$ la densité d'impuretés par unité de surface et $f$ la force d'accrochage. Enfin le champ seuil s'écrit :

$$
E_{s}=\frac{n_{i} f^{2}}{C \xi n_{s} e}
$$

où $\xi$ la portée des forces d'accrochage et $n_{s}$ la densité surfacique d'électrons. 


\section{Transport près du décrochage}

Le décrochage d'une phase collective accrochée peut être vu comme une transition de phase du deuxième ordre, pour laquelle le paramètre d'ordre est le courant (voir Chauve et al. $[286,287]$ par exemple). Ainsi, suffisamment près de la transition pour $E \gtrsim E_{s}$, le courant suit une loi d'échelle :

$$
I \sim\left(V-V_{s}\right)^{\beta}
$$

où $\beta$ est l'exposant de décrochage.

\section{Régime de reptation}

On se place maintenant dans le régime $E \ll E_{s}$ et à température finie, mais très faible devant la température de fusion du cristal. Un modèle naïf pour ce régime propose que la conduction suive une loi activée, avec une barrière d'activation $\Delta$ qui ne dépend que du désordre, et donc ne dépend pas du champ électrique appliqué :

$$
I \propto \exp (-\Delta / T) E .
$$

Ce mécanisme conduit donc à une résistivité qui varie de manière activée en fonction de la température :

$$
\rho(T) \propto \exp (\Delta / T) e x t
$$

Cependant cette idée est incorrecte car elle ne prend pas en compte le fait que la barrière doit diverger quand le champ électrique tend vers zéro.

Des arguments de lois d'échelle permettent de montrer que, dans le régime de reptation, la résistivité prend la forme (voir Chauve et al. [286, 287]) :

$$
\rho(T, E) \propto \exp \left[-\frac{T_{0}}{T}\left(\frac{E}{E_{s}}\right)^{-\mu}\right],
$$

où l'exposant $\mu$ peut être calculé en fonction de l'exposant de rugosité du potentiel de désordre.

\section{Cas du cristal de Wigner}

Les modèles développés dans cette partie permettre de bien rendre compte des résultats sur des systèmes désordonnés corrélés tels les ondes de densité de charge ou les vortex dans les supraconducteurs. Cependant les mesures de conductivité optique, réalisées par Li et al. [288, 289] dans la phase cristal de Wigner induite par le champ magnétique dans des hétérostructures $p$-GaAs, sont en désaccord avec les prédictions données par ces modèles (voir Giamarchi [290]). Chitra et al. [291, 292] ont calculé par des méthodes plus élaborées la conductivité optique, montrant un très bon accord avec ces expériences. Ces calculs montrent, qu'à fréquence nulle, la conductivité longitudinale tend vers zéro, et la conductivité transverse reste finie et égale à sa valeur classique. A plus haute fréquence, la conductivité longitudinale présente un pic à une fréquence correspondant à la fréquence d'accrochage $\omega_{p}$ (pour "pinning" en anglais). Le comportement de ce pic varie suivant la portée du potentiel de désordre. 
Giamarchi et Le Doussal [293] ont également montré que, dans le régime d'écoulement, le mouvement des électrons se fait à travers des canaux. Ces canaux sont eux-mêmes accrochés sur le désordre, ce qui conduit à une force de rappel transverse $F_{t r}$, même lorsque le seuil d'accrochage longitudinal est dépassé. Expérimentalement il est possible d'observer un tel mécanisme d'accrochage transverse en champ magnétique en mesurant la tension de Hall. En effet, en présence d'un champ magnétique, les électrons animé d'une vitesse $v$ sont soumis à la force de Lorentz dirigée perpendiculairement au mouvement $F_{L}=e v B$. A faible champ magnétique, quand $F_{L}<F_{t r}$, les canaux restent accrochés et aucune tension Hall ne doit être observée. Lorsque $F_{L}>F_{t r}$, la force de Lorentz est suffisante pour faire glisser les canaux sur le côté, et donc donner lieu à une tension de Hall.

\subsubsection{Accrochage par des impuretés chargées}

Dans les modèles précédents, le désordre est supposé à courte portée. Ce modèle peut s'appliquer de manière satisfaisante dans beaucoup de cas de systèmes collectifs accrochés (voir dans Chauve et al. [286, 287]), tels les vortex dans les supraconducteurs ou les ondes de densité de charge. Cependant, dans les matériaux semiconducteurs, le désordre provient principalement d'impuretés chargées, présentes soit en raison du dopage dans les hétérostructures, soit les impuretés résiduelles dues aux défauts aux interfaces ou dans le matériau massif. Ces impuretés créent alors un potentiel de désordre à longue portée qui peut modifier les propriétés d'accrochage du cristal de Wigner. Ruzin et al. ont montré en particulier que le modèle classique d'accrochage de domaines par un potentiel de désordre périodique n'est plus valable [294]. La raison principale est que, dans un régime de fort accrochage, les impuretés chargées induisent des défauts dans le cristal. Ils distinguent alors deux types d'impuretés chargées, donneuses ou acceptrices, et étudient l'accrochage en fonction de la distance entre ces impuretés et le cristal. A grande distance, une impureté crée un potentiel de désordre sur une grande échelle spatiale. Pour une impureté proche du cristal, si cette impureté est donneuse (et donc chargée positivement), un électron va se lié à cette impuretés et réduire fortement le potentiel créé par cette impureté. Au contraire, si cette impureté est acceptrice, elle va créé un fort potentiel d'accrochage.

Reichhardt et al. ont confirmé cette vision par des simulations numérique dans un modèle classique d'électrons en interaction en présence d'impuretés chargées placées en dehors du plan du gaz d'électrons [295]. Dans ce cas le désordre considéré est un désordre à longue portée. Avec un fort désordre, le cristal d'électrons subit des réarrangements lorsque la force exercée (i.e. le champ électrique) augmente, caractéristique de la création de défauts dans le cristal, et indiquant que le modèle de formation de domaines n'est pas valable ici. Lorsque la force est faible, seul un petit nombre d'électrons participe à la conduction, et le système est très désorganisé, avec un nombre important de défauts. Ces défauts sont des paires de disinclinations. Lorsque la force est très élevée, les électrons traversent l'échantillons par l'intermédiaire de canaux séparés, comme l'avait déjà montré Giamarchi et Le Doussal [293], et le cristal redevient organisé. Cette limite est alors compatible avec le régime d'écoulement dans les modèles classiques présentés dans le paragraphe précédent. A faible désordre, correspondant à la limite du cristal faiblement accroché, le cristal d'électrons se déforme élastiquement, sans créer de défauts. 


\subsubsection{Conduction par création de paires de dislocations}

Chui et Esfarjani ont montré que, même loin de la fonte, la conduction à travers un cristal accroché n'est pas nulle à température finie en raison des dislocations présentes dans le cristal [296]. Alors que la fonte du cristal intervient lorsqu'une paire de dislocations se dissocie, ils ont montré qu'une paire associée peut quand-même conduire le courant, car un déplacement de cette paire de dislocations conduit à un déplacement des électrons. De plus ils ont montré que les dislocations ainsi créées ne sont pas accrochées par le désordre pour un déplacement suffisant des dislocations. Ce mécanisme conduit à une conductivité qui croît exponentiellement avec la température suivant une loi activée :

$$
\sigma(T)=\sigma_{0} \exp \left(-E_{a} / k_{B} T\right),
$$

où $\sigma_{0}$ est une constante et l'énergie d'activation $E_{a}$ a été calculée dans le cadre du cristal de Wigner induit par le champ magnétique :

$$
E_{a}=2 E_{c}+\frac{\mu a_{0}^{2}}{2 \pi}\left(1-\frac{\nu}{0,2}\right)
$$

où $E_{c}$ est l'énergie de formation de la dislocation, $\mu$ est le module de cisaillement du cristal d'électrons, $a_{0}$ est le paramètre de maille du réseau d'électrons, et $\nu$ est le facteur de remplissage des niveau de Landau. Bien que cette théorie ne prenne en compte que la création de paires de dislocations, il se peut que d'autres défauts du cristal participent à la conduction.

Chui [182] a calculé l'énergie d'accrochage d'un cristal bidimensionnel d'électrons accroché sur un potentiel de désordre créé par des impuretés situées dans un plan à une distance $d$ du gaz d'électron, avec une concentration surfacique d'impuretés $n_{i}$. Dans la limite où la distance entre électrons plus proches voisins $a_{0}$ reste faible devant la distance $d$, l'énergie d'accrochage peut se calculer perturbativement en supposant que les écarts par rapport à la position d'équilibre restent faibles devant la distance entre électrons $a_{0}$ :

$$
m \omega_{p}^{2}=\frac{0,09 n_{i} a_{0}^{2} e^{2}}{4 \pi \varepsilon_{0} \varepsilon_{r} d^{3}}
$$

où $\omega_{p}$ est la fréquence d'accrochage.

Chui fait cependant remarquer que le champ électrique associé à cette énergie et au déplacement des électrons sur une distance $a_{0}, m \omega_{p}^{2} a_{0}$, est beaucoup plus élevé que les valeurs de champ électrique seuil observé expérimentalement [297]. De plus, des simulations numériques montrent que le mécanisme de décrochage ne fait pas intervenir une composante de Fourier particulière, mais tout une gamme de composantes de Fourier, ce qui est incompatible avec les idées de formation de domaines proposées dans le cadre classique (en particulier par Larkin et Ovchinnikov [182]). Chui a ainsi proposé un mécanisme de conduction en fonction du champ électrique par création de paires de dislocations, mécanisme qui est donc analogue à celui proposé pour la dépendance en température de la conductivité en-dessous du seuil de décrochage. Ce modèle conduit à un champ électrique de décrochage [297] :

$$
E_{d} \approx 0,35 n_{i}\left(d / a_{0}\right)^{-3} E_{\text {paire }} a_{0}
$$

où $E_{\text {paire }}$ est l'énergie de création d'une paire de dislocation. 


\subsubsection{Expériences}

Les lois de transport on été largement étudiées dans divers systèmes corrélés accrochés sur un désordre, en particulier dans le cas des ondes de densité de charge quasiunidimensionnelles (voir par exemple la revue de Grüner [179]) et dans le cas du cristal de Wigner à la surface de l'hélium (voir par exemple la revue de Dahm [180], et les résultats de Jiang et Dahm [181]). Dans ce paragraphe on se limitera uniquement au cas du cristal de Wigner formé dans les semiconducteurs. Comme on l'a vu au chapitre 1, il existe deux limites pour observer un cristal de Wigner dans les semiconducteurs. A fort champ magnétique, l'énergie cinétique des électrons est "gelée" dans l'orbite cyclotron et on s'attend à observer un cristal de Wigner classique. A champ magnétique nul, et à suffisamment basse densité électronique, l'énergie d'interaction devient largement supérieure à l'énergie cinétique, conduisant à la cristallisation de Wigner.

\section{Cristal de Wigner induit par le champ magnétique}

Le cristal de Wigner induit par le champ magnétique a été observé dans les hétérostructures $n$-GaAs pour un facteur de remplissage $\nu<1 / 5$ (avec également une phase réantrante à $\nu \gtrsim 1 / 5$, comme on l'a vu au chapitre 1 ). Il a également été évoqué pour interpréter les mesures réalisées dans les MOSFETs silicium à un remplissage intermédiaire entre 1 et 2 .

Des mesures de résistivité longitudinale à faible valeur de remplissage des niveaux de Landau $\nu$ ont été réalisées par Willett et al. [49] et Jiang et al. [298, 299] dans des hétérostructures $n$-GaAs. Ils observent, pour une valeur de $\nu$ inférieure à $1 / 5(\nu=1 / 5$ correspondant à un plateau de Hall quantique fractionnaire), une croissance exponentielle de la résistivité quand la température diminue. Cette dépendance est compatible avec une loi activée, et l'énergie d'activation diminue quand $\nu$ augmente, et s'annule au voisinage de $\nu=1 / 5$. Ce comportement activé est différent des lois de sauts à portée variable observées dans les phases isolantes à plus fort remplissage (par exemple $\nu=0,302$ situé entre les états de Hall fractionnaire à $\nu=1 / 3$ et $\nu=2 / 7$ [298, 299]), et est attribué à la conduction dans le régime de reptation d'un cristal de Wigner. D'autre part Willett et al. $[49,50]$ ont mesuré une énergie d'activation $E_{a}$ dépendant du champ magnétique par l'intermédiaire du facteur de remplissage des niveaux de Landau $\nu: E_{a}=E_{0}\left(1-\nu / \nu_{0}\right)$. Cette dépendance est compatible avec l'équation (4.47) donnant l'énergie d'activation dans le cas de la conduction par création d'une paire de dislocation. L'énergie d'activation s'annule pour $\nu=\nu_{0}$, où $\nu_{0}$ peut être interprétée comme le facteur de remplissage critique de formation du cristal. Cependant cette étude conduit à une valeur de l'énergie de formation d'une dislocation $E_{c}$ plus faible que ne le prédit la théorie de Chui et Esfarjani [296].

Dans la phase cristal de Wigner dans des hétérostructures $n$-GaAs, plusieurs auteurs ont observé de fortes non linéarités de la caractéristique $I-V$, compatibles avec l'idée du décrochage d'une phase collective. On peut citer en particulier Willett et al. [50], Williams et al. [48] et Goldman et al. [51]. La figure 4.6 présente un exemple de telles mesure. Ces non linéarités disparaissent quand le facteur de remplissage augmente et s'approche d'une valeur critique $\nu_{0}$, et quand la température augmente, caractéristiques de la fonte du cristal lorsque le champ magnétique diminue (i.e. l'énergie cinétique augmente) ou quand 


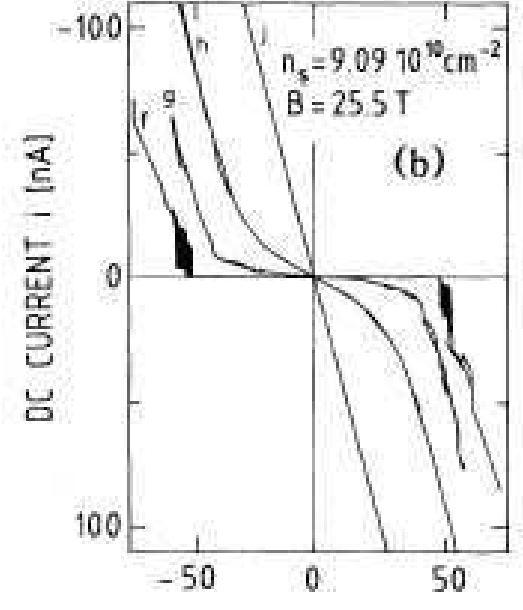

APPLIED POTENTIAL [mV]

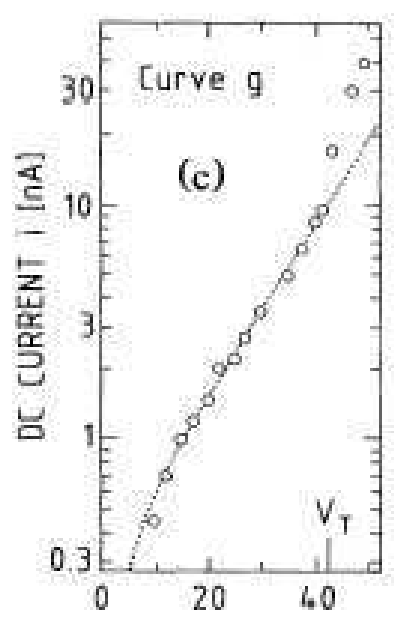

APPLIED POTENTIAL [mV]
FIG. 4.6: Non linéarités de la caractéristique $I-V$ dans la phase cristal de Wigner induite par le champ magnétique dans une hétérostructure $n$-GaAs, mesurées pour différentes températures, d'après Williams et al. [48].

l'énergie thermique augmente. Ces considérations permettent de tracer le diagramme de phase qui a été présenté au chapitre 1 (figure 1.12).

Williams et al. [48] ont pu interpréter les mesures de transport en champ électrique et de réponse radio-fréquence au voisinage du décrochage par la théorie classique des domaines interagissant avec un potentiel périodique.

Pudalov et Chui [136] obtiennent, dans des MOSFETs Si, à champ magnétique nul et champ magnétique élevé, des non-linéarités avec une tension seuil qui varie comme $\left(n_{s}-n_{c}\right)^{1,5-1,7}$, ce qui est compatible avec le décrochage par formation de paires de dislocations décrit par Chui. Cette tension seuil est beaucoup plus faible que l'estimation classique $m \omega^{2} a_{0}$ ( $\omega$ est la fréquence d'accrochage et $a_{0}=1 / \sqrt{\pi n_{s}}$ la maille de Wigner-Seitz du cristal de Wigner).

Enfin, l'existence d'un seuil de déplacement transverse a été observé par Perruchot et al. [300] dans des hétérostructures $p$-GaAs, comme l'on prédit Giamarchi et Le Doussal [293].

\section{Cristal de Wigner à champ magnétique nul}

A champ magnétique nul, pour espérer observer la cristallisation de Wigner, il est nécessaire que l'énergie d'interaction avec le désordre reste faible devant les énergies typiques mises en jeu dans la cristallisation (énergies cinétique et d'interaction), et c'est pourquoi cette phase n'est attendue que pour des systèmes de très haute mobilité. Lorsque le désordre devient important, il a pour conséquence de casser l'ordre du cristal, conduisant à une phase vitreuse, dont les propriétés sont encore peu connues. L'existence de cette phase cristal de Wigner à champ magnétique nul est donc encore controversée, mais les analogies entre les propriétés à fort champ magnétique et à champ magnétique nul (comme le seuil de la caractéristique $I-V$ ) suggèrent cependant l'existence d'une telle phase collective.

Comme on l'a vu dans le chapitre 2, les systèmes les plus propices pour observer une phase collective à basse densité sont les systèmes dans lesquels les porteurs ont la masse 
effective la plus élevée, comme les gaz bidimensionnels d'électrons dans les MOSFETs silicium ou les gaz bidimensionnels de trous dans les hétérostructures GaAs/AlGaAs ou $\mathrm{SiGe/Si}$. Kravchenko et al. [46], puis Pudalov et al. [45], ont montré que, dans les MOSFETs Si à très basse densité, les caractéristiques $I-V$ présentent de très fortes non linéarités avec l'existence d'une tension seuil. Cette tension seuil ne semble pas pouvoir être attribuée au chauffage des porteurs ou aux sauts dirigés pour des particules indépendantes localisées, et pourrait donc être attribuée au décrochage d'une phase collective telle que le cristal de Wigner.

Pudalov et al. [45] et Shashkin et al. [81] ont mesuré une dépendance activée de la résistivité en fonction de la température à faible champ électrique, compatible avec le transport dans une phase cristal de Wigner. On peut cependant remarquer que cette loi activée laisse place à une loi de transport par sauts à portée variable avec interactions Coulombiennes à suffisamment basse température, comme il a été mesuré par Mason et al. [77, 78]. Ce comportement est alors en fait plus proche des mesures de Shashkin et al. sur les transition effet Hall quantique-isolant dans des hétérostructures $n$-GaAs, qui attribuent ce comportement à la proximité de transitions de percolation [36].

Yoon et al. ont interprété les fortes non linéarités de la caractéristique $I-V$ dans des hétérostructures $p$-GaAs par le décrochage d'un cristal de Wigner [87]. Ils ont essayer de comparer la valeur de la tension seuil obtenue avec la théorie de création de paires de dislocations, et obtiennent un bon accord avec les résultats de Chui [297]. Cependant la théorie de Chui prédit alors une énergie d'accrochage très élevée comparée à la température de disparition des non linéarités observées dans ces hétérostructures.

\subsection{Conclusion}

Nous avons donc vu que le transport des électrons dans la phase basse densité peut revêtir des caractères particuliers pour des électrons indépendants localisés sur le désordre, ou pour une phase collective accrochée sur le désordre. Les mesures de transport en fonction de la température, et en fonction du champ électrique, constituent donc théoriquement un bon test quand à la nature de la phase étudiée. C'est cette étude que nous proposons de faire sur les systèmes expérimentaux dont nous disposons, et qui sera détaillée dans les chapitres 5, 6 et 7 . Il existe cependant une autre source de dépendances en champ électrique qui n'a pas été abordée complètement ici, même si elle a été évoqué dans le transport d'électrons indépendants localisés, c'est le phénomène de chauffage des porteurs. Nous verrons que dans certains cas ce mécanisme permet d'expliquer de très fortes non-linéarités. 


\section{Chapitre 5}

\section{Transport dans les puits quantiques $p$-SiGe et $p$-GaAs}

\section{Sommaire}

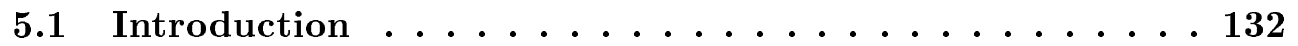

5.2 Présentation générale $\ldots \ldots \ldots \ldots \ldots \ldots \ldots \ldots$

5.3 Analyse du comportement métallique . . . . . . . . 134

5.3 .1 Puits quantique $p$-SiGe . . . . . . . . . . . . 134

$5.3 .2 \quad$ Puits quantique $p$-GaAs . . . . . . . . . . . . 137

5.4 Analyse du comportement isolant $\ldots \ldots \ldots \ldots \ldots$

$5.4 .1 \quad$ Principe de l'analyse . . . . . . . . . . . . . . . 138

5.4 .2 Puits quantique $p$-SiGe . . . . . . . . . . . . . . . 139

5.4 .3 Puits quantique $p$-GaAs . . . . . . . . . . . . . 143

5.5 Conclusion $\ldots \ldots \ldots \ldots \ldots \ldots \ldots \ldots \ldots$ 


\subsection{Introduction}

Nous nous intéressons dans ce chapitre aux lois de transport observées autour de la transition métal-isolant dans les deux systèmes qui ont été présentés au chapitre 3. Dans un premier temps, nous montrons que les dépendances en température de la résistivité présentent les caractéristiques d'un comportement métallique à haute densité de porteurs (i.e. $d \rho / d T>0)$, et d'un comportement isolant $(d \rho / d T>0)$ lorsqu'on diminue la densité de porteurs en dessous d'une valeur critique $p_{c}$. Nous étudions en détail ces dépendances en température, en montrant les points communs et les différences entre les deux systèmes. En particulier nous verrons comment certains modèles développés dans le cadre de la transition métal-isolant permettent de rendre compte de nos résultats.

\subsection{Présentation générale}

Les mesures de transport présentées ici sont faites à champ électrique suffisamment faible pour pouvoir négliger les effets non-linéaires, qui seront étudiés dans les chapitres

6 et 7. Dans la figure 5.1 sont reportées les dépendances en température de la résistivité pour les deux systèmes étudiés, pour des densités de porteurs $p_{s}$ autour de la transition métal-isolant. A faible densité de porteurs, $p_{s}<p_{c}$, la résistivité croît de façon monotone quand la température diminue $(d \rho / d T<0)$, et semble tendre vers l'infini à température nulle, ce qui est caractéristique d'un isolant. A haute densité de porteurs, $p_{s}>p_{c}$, la dépendance en température n'est plus monotone. Dans le cas des puits quantiques $p$ SiGe, on observe $d \rho / d T<0$ à basse température et $d \rho / d T>0$ à haute température. Ce dernier comportement est qualifié de "métallique". Dans les hétérostructures $p$-GaAs, le comportement est inversé, puisque $d \rho / d T<0$ à haute température, et le comportement métallique $d \rho / d T>0$ est obtenu à basse température. A plus haute densité, le comportement "métallique" est observé pour toutes les températures explorées. Les valeurs de densité critique $p_{c}$, ainsi que la résistivité $\rho_{c}$ obtenue à basse température à la transition, sont répertoriées dans le tableau 5.1.

Pour pouvoir comparer les comportements dans les deux systèmes, les variations de la résistivité ont été représentées en fonction du rapport entre la température et la température de Fermi, calculée à partir de l'énergie de Fermi d'un gaz d'électrons libres (voir l'équation (1.19)). Cette représentation permet de comparer des systèmes dont les densités critiques sont très différentes, comme c'est le cas pour nos deux systèmes. La figure 5.2 de droite montre une superposition des courbes de résistivité en fonction de $T / T_{F}$ pour les deux systèmes étudiés. Sur la figure 5.2 à gauche sont rappelées les courbes obtenues dans les MOSFETs Si de haute mobilité. La première observation est l'accord qualitatif entre les comportements observés dans les MOSFETs Si et nos hétérostructures $p$-GaAs, traduisant le caractère universel de la transition métal-isolant, même si dans les hétérostructures $p$-GaAs l'amplitude de la chute de résistivité est inférieure à celle observée dans les MOSFETs Si. Dans le puits quantique $p$-SiGe, le comportement semble différer des mesures dans les MOSFETs Si et les hétérostructures $p$-GaAs. Cependant la remontée de la résistivité observée à basse température intervient à une valeur de $T / T_{F}$ non atteinte dans les MOSFETs Si et les hétérostructures $p$-GaAs. En fait les puits quantiques $p$-SiGe 

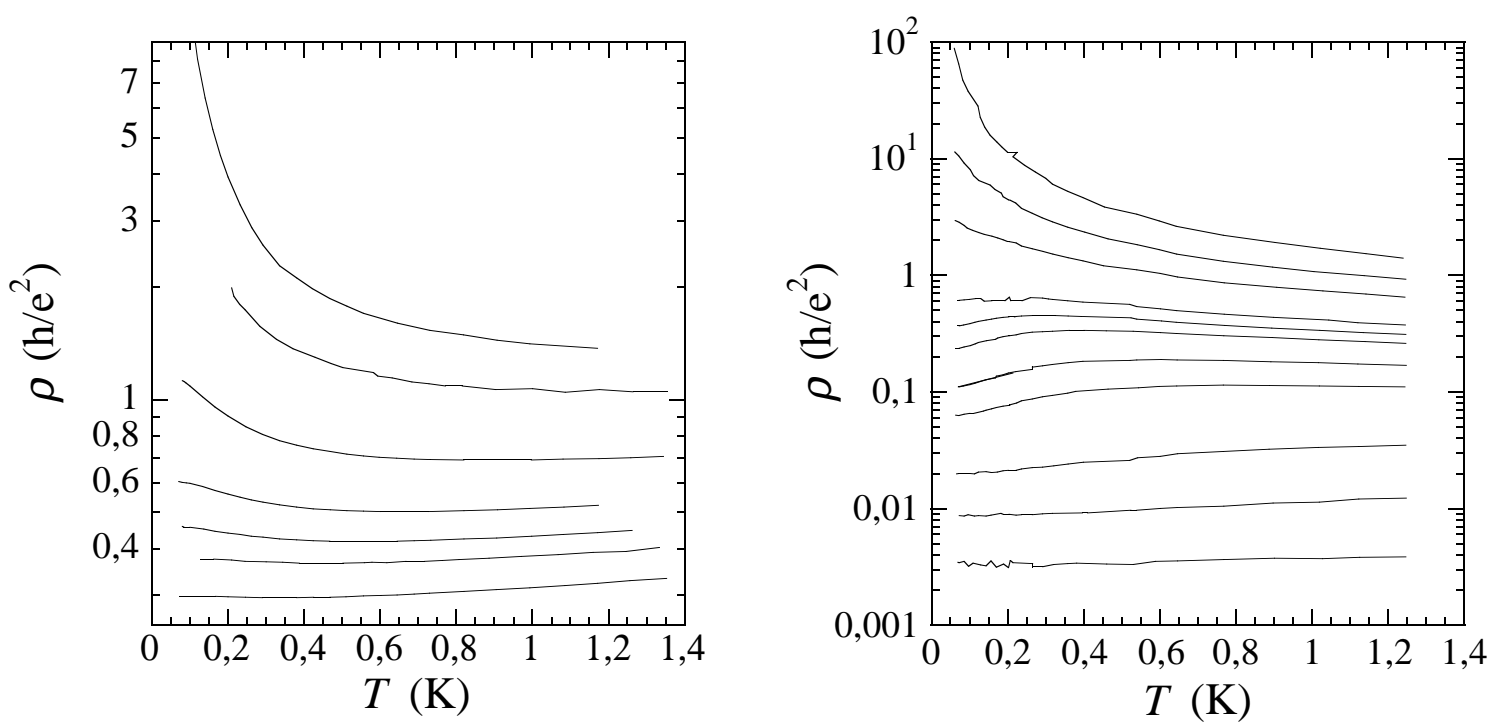

FIG. 5.1: Dépendances en température de la résistivité dans les deux systèmes étudiés. Gauche : Mesure dans un puits quantique SiGe de type $p$ (échantillon SiGe B509) pour les densités (de haut en bas) : $1,01-1,08-1,19-1,31-1,38-1,45-1,56 \times 10^{15} \mathrm{~m}^{-2}$. Droite : Mesure dans une hétérostructure GaAs de type $p$ (échantillon GaAs Nu944-1) pour les densités (de haut en bas) : $1,43-1,55-1,67-1,87-1,95-2,05-2,2-2,5-3,6-5,2-6,3 \times 10^{14} \mathrm{~m}^{-2}$.

\begin{tabular}{|cc|cc|}
\hline système & échantillon & $\begin{array}{c}\text { densité critique } p_{c} \\
\left(\mathrm{~m}^{-2}\right)\end{array}$ & $\begin{array}{c}\text { résistivité } \rho_{c} \\
\left(h / e^{2}\right)\end{array}$ \\
\hline$p$-SiGe & $\mathrm{B} 509$ & $\approx 1,3 \times 10^{15}$ & $0,4-0,5$ \\
\hline$p$-GaAs & $\mathrm{Nu} 944-1$ & $\approx 1,6 \times 10^{14}$ & $0,8-2$ \\
\hline
\end{tabular}

TAB. 5.1: Valeurs de la densité de porteurs critique $p_{c}$ à la transition métal-isolant, et de la résistivité $\rho_{c}$ mesurée à la densité $p_{c}$ à basse température.

sont assez proche des MOSFETs Si très désordonnés.

On remarque que, dans les échantillons $p$-GaAs pour $p_{s}>p_{c}$, la transition entre le comportement isolant à haute température et le comportement métallique à basse température se produit à $T / T_{F}$ compris entre 0,1 et 0,3 . Ce résultat a déjà été observé dans les hétérostructures $p$-GaAs par Mills et al. [72] et par Proskuryakov et al. [223], et a été interprété comme la transition entre le régime classique à haute température et le régime classique à base température. Dans les MOSFETs Si, cette transition est également vue pour $T / T_{F}$ compris entre 0,1 et 1 , bien que la densité de porteurs soit environ dix fois plus élevée que dans les hétérostructures $p$-GaAs. Cette comparaison renforce l'idée que le comportement métallique nécessite le caractère dégénéré du gaz d'électron. 

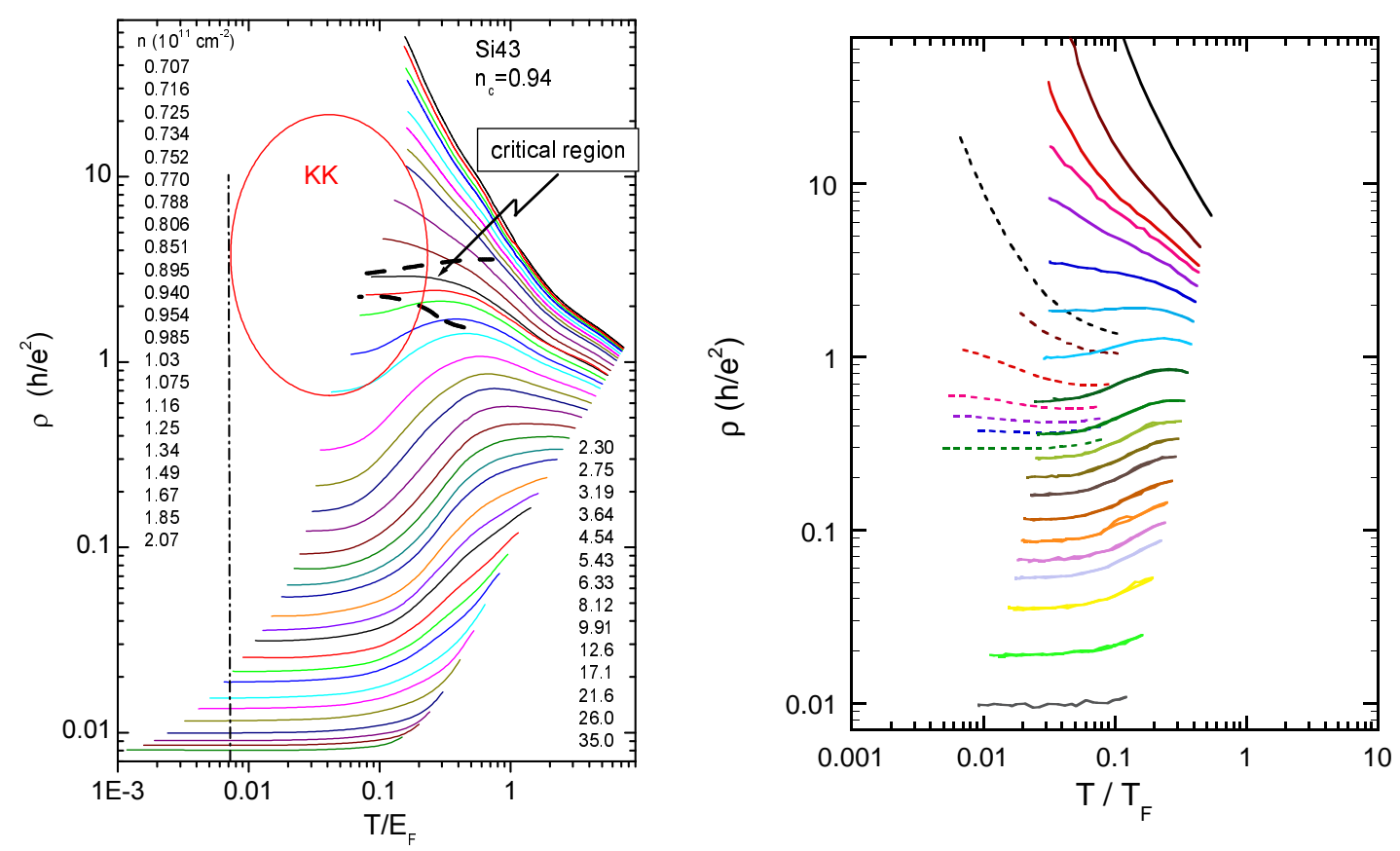

FIG. 5.2: Comparaison des dépendances de la résistivité en fonction du rapport entre la température et la température de Fermi, $T / T_{F}$. La figure de gauche donne les résultats obtenus sur des MOSFETs Si de haute mobilité (d'après [68]). Sur la figure de droite sont reportés nos résultats sur le puits quantique $p$-SiGe B509 (traits pointillés : les densités sont, respectivement de haut en bas, $1,01-1,19-1,31-1,38-1,45-1,56 \times 10^{15} \mathrm{~m}^{-2}$ ) et sur l'hétérostructure $p$-GaAs Nu944 (traits pleins : les densités sont, respectivement de haut en bas, $1,30-1,39-1,43-1,44-1,48-1,52-1,57-1,63-1,74-1,86-1,96-2,06-2,16-$ $\left.2,31-2,46-2,61-2,77-3,12-3,88-5,14 \times 10^{14} \mathrm{~m}^{-2}\right)$.

\subsection{Analyse du comportement métallique}

\subsubsection{Puits quantique $p$-SiGe}

\section{Résultats}

A haute densité électronique, là où est observé le comportement métallique des courbes $\rho(T)$, on se situe dans une limite de faible désordre $\left(k_{F} l_{e}>1\right.$, correspondant à $\left.\rho<h / e^{2}\right)$. Pour étudier la dépendance en température de la conductivité sur des puits quantiques $p$-SiGe dans le régime métallique, on peut reprendre l'analyse faite par Senz et al. sur ces mêmes systèmes [156]. Ils ont montré que la conductivité à basse température peut être expliquée par la somme de trois effets :

$$
\sigma(T)=\sigma_{D}(T)+\delta \sigma_{L F}(T)+\delta \sigma_{I}(T)
$$

où $\sigma_{D}(T)$ est la conductivité de Drude, $\delta \sigma_{L F}(T)$ la correction de conductivité due à la localisation faible et $\delta \sigma_{I}(T)$ la correction de conductivité due aux interactions électron-électron (voir §1.5.2). Cette analyse ne suppose pas à priori l'existence d'une phase métallique, et tous les termes présents ici supposent que le système électronique est décrit par des quasiparticules indépendantes (liquide de Fermi). Senz et al. ont également montré que, dans 

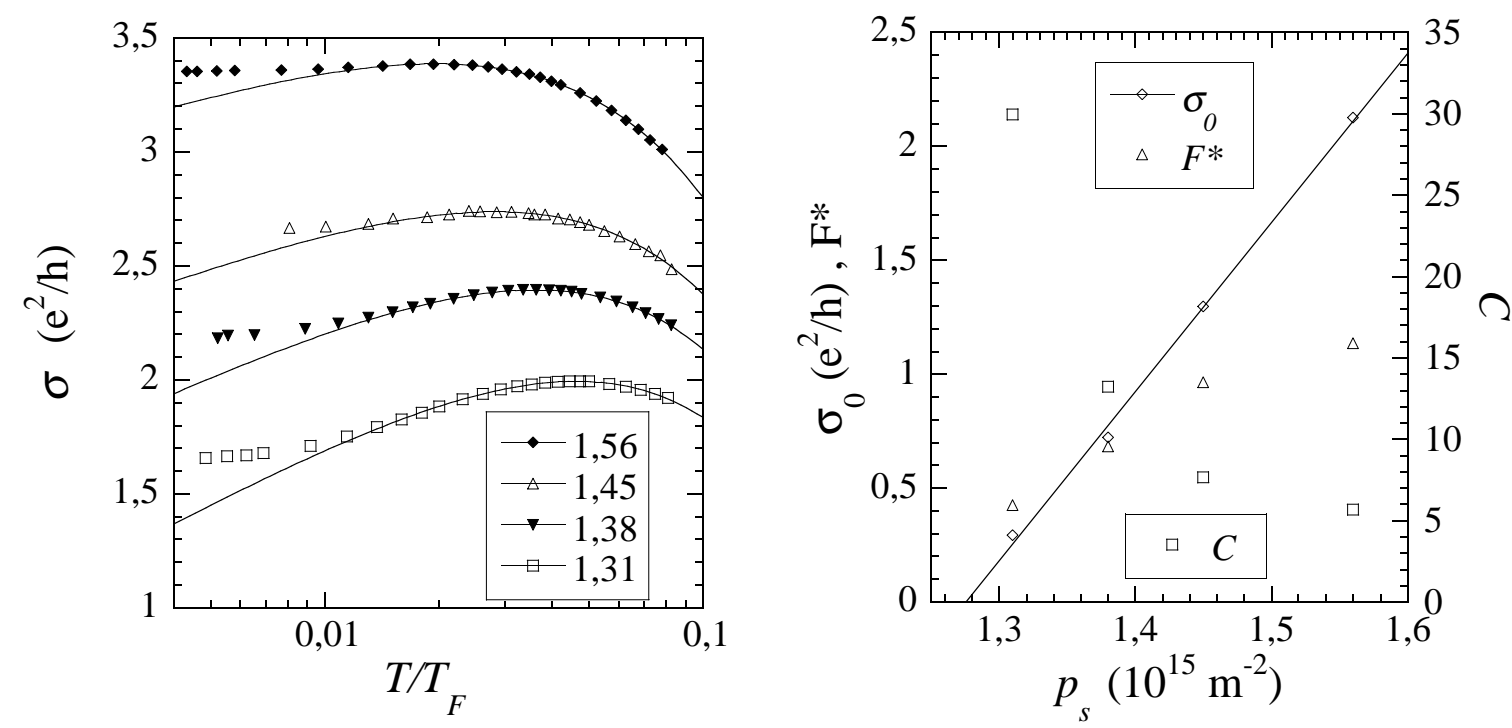

FIG. 5.3: Gauche : Conductivité en fonction de la température pour l'échantillon $p$-SiGe B509, et ajustement des courbes avec l'équation (5.4). Les paramètres de l'ajustement sont tracés en fonction de la densité de porteurs sur la figure de droite.

ces systèmes, la dépendance en température de la résistivité due au couplage électronphonon est négligeable en-dessous de environ $10 \mathrm{~K}$ [301]. Dans ce cas, la dépendance en température de la conductivité de Drude $\sigma_{D}(T)$ ne peut être expliquée que par un effet d'écrantage du désordre dépendant de la température, décrit par Gold et Dolgopolov [19], et conduisant à l'expression :

$$
\sigma_{D}(T)=\frac{p_{s} e^{2} \tau_{e}(T)}{m^{*}}=\sigma_{D}(0)\left\{1-C\left(p_{s}\right) \frac{T}{T_{F}}+\mathcal{O}\left[\left(\frac{T}{T_{F}}\right)^{3 / 2}\right]\right\}
$$

où $p_{s}$ est la densité de porteurs, $\tau_{e}$ le temps de collision de transport, qui dépend de la température par l'intermédiaire de l'écrantage, $C\left(p_{s}\right)$ un coefficient dépendant de la densité de porteurs et calculé par Gold et Dolgopolov [19]. Enfin, les corrections de conductivité dues à la localisation faible et aux interactions sont rappelées ici :

$$
\delta \sigma_{L F}=\alpha \frac{e^{2}}{\pi h} \ln \left(\frac{k_{B} T \tau_{e}}{\hbar}\right) \quad \text { et } \quad \delta \sigma_{I}=\frac{e^{2}}{\pi h}\left(1-\frac{3 F^{*}}{4}\right) \ln \left(\frac{k_{B} T \tau_{e}}{\hbar}\right)
$$

où $\tau_{e}$ est le temps de transport élastique et $F^{*}$ est un paramètre lié aux interactions. Expérimentalement, des mesures de magnétorésistance sur des puits quantiques $p$-SiGe $[26,21]$ ont obtenu $\alpha \approx 0,6-0,7$.

Pour vérifier que nos résultats obtenus sur les puits quantiques $p$-SiGe peuvent être interprétés par ce modèle, nous avons tracé sur la figure 5.3 de gauche la dépendance de la conductivité de l'échantillon B509 en fonction de $T / T_{F}$. Les points expérimentaux pour $T \geqslant 150 \mathrm{mK}$ ont été ajustés par la loi suivante :

$$
\sigma(T)=\mathcal{A}\left(p_{s}\right)-\mathcal{B}\left(p_{s}\right) \frac{T}{T_{F}}+\mathcal{C}\left(p_{s}\right) \ln \left(\frac{T}{T_{F}}\right)
$$


où $\mathcal{A}, \mathcal{B}$ et $\mathcal{C}$ sont les paramètres de l'ajustement. En choisissant $\alpha=0,6$, ces ajustements permettent alors de déduire les paramètres inconnus : $\tau_{e}$, ou plutôt $\sigma_{0}=p_{s} e^{2} \tau_{e}(T=0) / m^{*}$, $F^{*}$ et $C$. Les dépendances en densités de ces paramètres sont reportées sur la figure 5.3.

\section{Interprétation}

La conductivité de Drude à température nulle, $\sigma_{0}$, varie linéairement avec la densité, comme on peut l'attendre de la formule de Drude. Cependant cette conductivité tend vers zéro pour une valeur non nulle de la densité $p_{s}^{0} \approx 1,27 \times 10^{15} \mathrm{~m}^{-2}$, valeur proche de la densité critique de la transition métal-isolant $p_{c}$. Ce comportement a déjà été observé sur les hétérostructures $p$-GaAs à partir d'ajustements de courbes dans la phase métalliques [73], et a été interprétée par le fait que le nombre de porteurs mobiles est $p_{s}-p_{s}^{0}$, qui s'annule à la transition, les autres porteurs étant localisés et conduisant à la dépendance isolante de la résistivité à basse densité.

Le paramètre $F^{*}$ obtenu par Senz et al. [156] est 0,91 pour une densité de $2,6 \times 10^{15}$ $\mathrm{m}^{-2}$, et Emeleus et al. [228] obtiennent 0,85 pour une densité de $3,9 \times 10^{15} \mathrm{~m}^{-2}$. Les valeurs obtenus dans notre cas sont compatibles avec ces valeurs à haute densité, mais on observe une chute de $F^{*}$ à faible densité. Pour $C$, Senz et al. obtiennent 3,1 à $2,6 \times 10^{15}$ $\mathrm{m}^{-2}$ et 0,8 à $4,3 \times 10^{15} \mathrm{~m}^{-2}$, alors que Emeleus et al. obtiennent 1,5 à $3,9 \times 10^{15} \mathrm{~m}^{-2}$. Les valeurs obtenues dans notre cas sont compatibles pour les densité les plus élevées, et $C$ augmente quand la densité diminue, comme le prévoit le calcul de Gold et Dolgopolov [19]. Mais $C$ diverge à plus basse densité, près de la transition. On peut cependant remarquer que, à basse densité, les corrections de conductivité $\delta \sigma_{L F}$ et $\delta \sigma_{I}$ sont du même ordre que la conductivité de Drude. Ainsi, dans ce régime, les expressions de ces corrections ne sont plus valables, et l'écart de $F^{*}$ et de $C$ à basse densité peut se comprendre par la transition d'un régime de localisation faible vers un régime de localisation forte.

La saturation de la courbe $\sigma(T)$ à basse température $(T \leqslant 150 \mathrm{mK})$ n'est pas décrite dans le cadre de l'équation (5.4). Elle est vraisemblablement due à un chauffage du gaz de trous, dont la température effective devient supérieure la température du réseau mesurée par le thermomètre. L'origine la plus probable d'un tel chauffage est le courant de fuite grille canal, qui est négligeable à basse densité électronique car la tension de grille est faible (en valeur absolue), mais qui devient important aux valeurs de la tension de grille nécessaire pour atteindre la phase "métallique" (courant de fuite $I_{f} \approx 100 \mathrm{pA}$ pour une tension de grille donnant $p_{s}=1,4 \times 10^{15} \mathrm{~m}^{-2}$ ). Par effet Joule, ce courant de fuite induit un chauffage du gaz d'électrons.

En conclusion, les dépendances métalliques dans les puits quantiques $p$-SiGe peuvent s'expliquer par les théories déjà existantes et ne semblent donc pas présenter un caractère réellement nouveau. De plus on observe clairement dans ces courbes un comportement lié à la localisation à basse température, terme qui n'avait pas été vu auparavant en raison d'une valeur de $k_{F} l_{e}$ trop élevée [156, 21]. Néanmoins, ces échantillons sont plus désordonnés que les MOSFETs Si et les hétérostructures $p$-GaAs dans lesquels un comportement métallique plus marqué est observé, et l'interprétation proposée ici pour les puits quantiques $p$-SiGe n'est pas susceptible de marcher pour des systèmes plus "propres". On peut également objecter que la dépendance métallique observée dans les puits quantiques $p$ SiGe est suffisamment faible pour être expliquée par différents effets (voir en particulier les 
modèles "classiques" pour interpréter la dépendance métallique au §2.5.5) et que l'ajustement des courbes par un modèle à trois paramètres ne constitue pas une preuve que ce modèle est le seul à pouvoir interpréter les résultats. Enfin se pose la question du rôle des interactions, sachant la valeur élevée du paramètre $r_{s}$ et donc l'importance attendue des corrélations entre électrons. En particulier, le calcul de la correction due aux interactions, $\delta \sigma_{I}$, est issu d'un calcul en perturbation, ce qui suppose de faibles corrélations. Le bon accord des mesures avec les théories semble indiquer que la théorie du liquide de Fermi s'applique aux gaz bidimensionnels de trous dans le SiGe de type $p$ pour des valeurs de $r_{s}$ aussi élevées que 8.

\subsubsection{Puits quantique $p$-GaAs}

Nous avons ajusté les courbes métalliques obtenues dans les puits quantiques $p$-GaAs avec la loi empirique couramment employée dans l'étude de la transition métal-isolant en dimension deux [71] (voir §2.3.1) :

$$
\rho(T)=\rho_{0}+\rho_{1} \cdot \exp \left(-T_{0} / T\right)
$$

Cette loi rend compte de deux phénomènes de diffusion. Le premier terme $\rho_{0}$, indépendant de la température, peut être interprété comme la diffusion sur les impuretés et les défauts. Le second terme a une forme activée, avec une énergie d'activation $k_{B} T_{0}$, et peut être interprété par un mécanisme de diffusion pour lequel les centres diffuseurs sont activés thermiquement. Ce type de comportement a été interprété dans le cadre de la transition métal-isolant en dimension deux par un mécanisme de diffusion interbandes (voir §2.5.5 et Yaish et al. [241, 242]) : dans un système comportant plusieurs bandes remplies, les porteurs de la bande la plus remplie (d'énergie inférieure) sont diffusés par les porteurs de la bande la moins remplie (d'énergie plus élevée). Lorsque la température augmente, la bande d'énergie supérieure se remplie, ce qui contribue à une augmentation de la diffusion et donc de la résistivité. Dans ce cas l'énergie d'activation est la séparation entre les deux bandes. Cependant d'autres modèles permettent également de rendre compte d'un tel comportement activé, comme le modèle de percolation de Meir [214], le modèle de gouttelettes de Shi et al. [217, 302] (voir §2.5.4), ou le couplage spin-orbite évoqué par Pudalov et al. [71].

La figure 5.4 montre les ajustements des mesures sur l'échantillon Nu944-1 par la loi (5.5), ainsi que les dépendances des paramètres en fonction de la densité de trous. La résistivité $\rho_{0}$, souvent interprétée comme la résistivité résiduelle dues aux impuretés et aux défauts, varie en fonction de la densité de trous suivant une loi :

$$
\rho_{0}\left(p_{s}\right)=A_{0}\left(\frac{p_{s}-p_{0}}{p_{0}}\right)^{-\alpha_{0}}
$$

avec $A_{0}=0,068 h / e^{2}, p_{0}=1,31 \times 10^{14} \mathrm{~m}^{-2}$ et $\alpha_{0}=1,9$. L'exposant $\alpha_{0}$ est nettement différent de 1, valeur obtenue par Hanein et al. [73], ce qui nous permet d'exclure l'interprétation de ce terme comme une résistivité de Drude, avec la différence $p_{s}-p_{0}$ comme nombre de porteurs mobiles (voir $\S 2.3 .1$ ). 

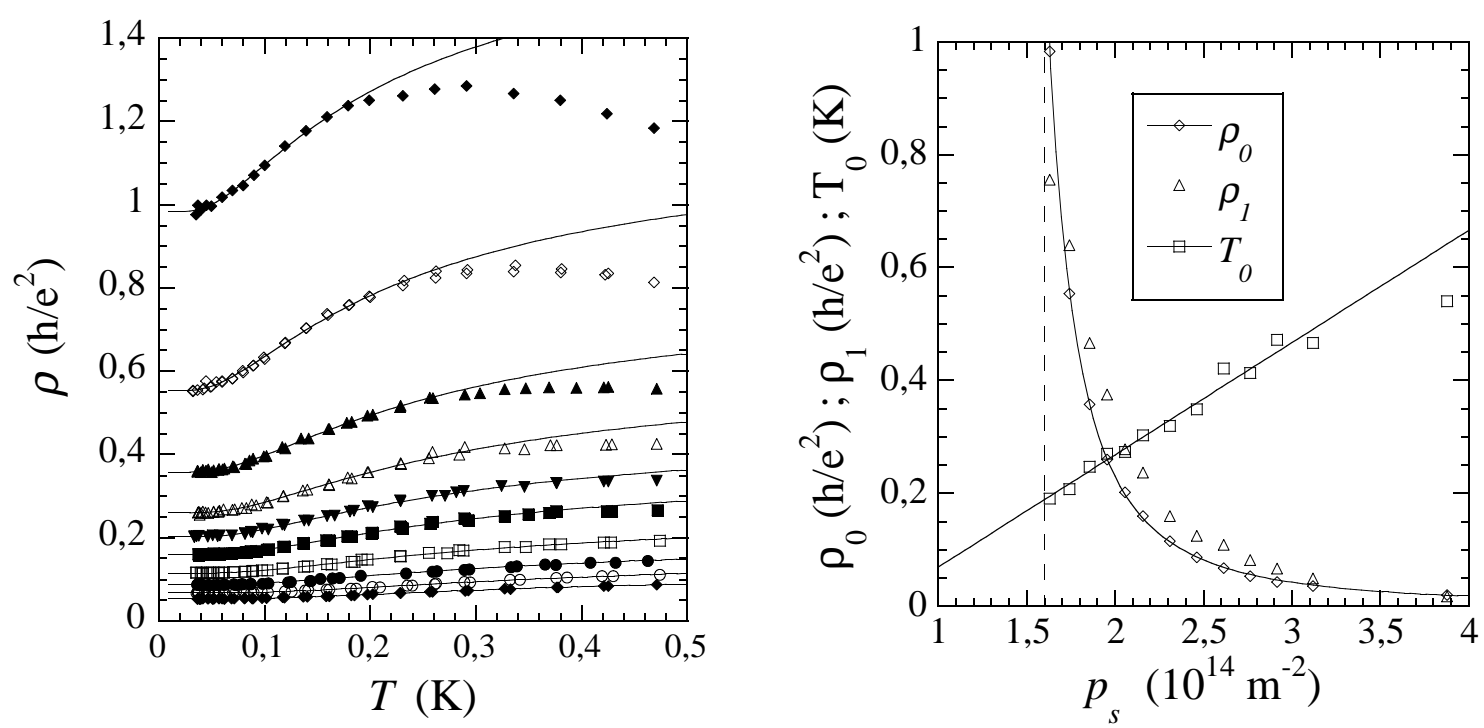

FIG. 5.4: Gauche : Résistivité en fonction de la température à haute densité de porteurs $\left(p_{s}>p_{c}\right)$ pour l'échantillon GaAs Nu944-1. Les densités sont, de haut en bas : 1,63-1,74-1,82-1,89$1,97-2,05-2,16-2,28-2,41-2,53 \times 10^{14} \mathrm{~m}^{-2}$. Les points expérimentaux sont ajustés à l'aide de l'équation (5.5). Droite : Paramètres de l'ajustement en fonction de la densité de porteurs. Les ajustements de $\rho_{0}$ et $T_{0}$ sont présentés dans le texte.

L'énergie d'activation $k_{B} T_{0}$ croît linéairement avec la densité suivant une loi $T_{0}\left(p_{s}\right) \propto$ $\left(p_{s}-p_{1}\right)$, comme il a déjà été observé dans différents systèmes $[71,61,72,65,66]$. Cependant, dans plusieurs expériences, cette énergie d'activation s'extrapole à 0 à une densité $p_{1}=0[61,74,65]$. Dans notre cas, l'extrapolation de cette énergie à faible densité montre qu'elle s'annule à une densité de porteurs $p_{1} \approx 6,55 \times 10^{13} m^{-2}$, correspondant à $r_{s}^{1}=37,7$.

\subsection{Analyse du comportement isolant}

\subsubsection{Principe de l'analyse}

Pour des électrons indépendants, et à faible densité, on se situe dans la limite $k_{F} l_{e}<1$ et un modèle semi-classique ne peut pas être appliqué. La théorie du transport entre états localisés par sauts à portée variable donne des prédictions précises sur les dépendances en température attendues (voir §4.2). Pour tester la validité de ces modèles dans notre cas, les courbes $\rho(T)$ ont été ajustée par la loi :

$$
\rho(T)=\rho_{0} \cdot \exp \left[\left(\frac{T_{0}}{T}\right)^{m}\right],
$$

où $\rho_{0}, T_{0}$ et $m$ sont les paramètres de l'ajustement. Nous verrons que dans certains cas il est nécessaire de pré-supposer la valeur de certains paramètres pour obtenir un ajustement correct. D'autre part, les théories de conduction par sauts prévoient un préfacteur $\rho_{0}$ dépendant de la température suivant une loi de puissance, $\rho_{0}(T) \propto T^{p}$. Bien que 


\begin{tabular}{|c|c|ccc|c|}
\hline échantillon & densité $\left(10^{15} \mathrm{~m}^{-2}\right)$ & $T_{0}(\mathrm{~K})$ & $T_{E S}(\mathrm{~K})$ & $T_{M}(\mathrm{~K})$ & $T^{*}(\mathrm{~K})$ \\
\hline B509 & 1,01 & 0,260 & 1,19 & 12,7 & 0,22 \\
& 1,08 & 0,166 & 0,409 & - & 0,25 \\
\hline \multirow{3}{*}{ B137 } & 0,50 & 0,672 & 6,06 & 98,0 & 0,29 \\
& 0,55 & 0,496 & 3,17 & 40,5 & 0,31 \\
& 0,60 & 0,351 & 1,64 & 15,1 & 0,30 \\
& 0,75 & 0,110 & 0,190 & 0,593 & 0,24 \\
\hline
\end{tabular}

TAB. 5.2: Température caractéristique issue des ajustements dans la phase isolante de $p$-SiGe avec un préfacteur indépendant de la température. $T^{*}$ est la température de transition entre le comportement $m=1$ et $m=1 / 2$.

cette dépendance soit plus faible que la loi exponentielle, elle peut parfois influencer les paramètres de l'ajustement.

Suivant le modèle, la température $T_{0}$ peut être reliée à différentes longueurs caractéristiques (voir $\S 4.2$ ). Dans le cas des sauts plus proche voisins $(m=1)$, on définit la distance entre sites plus proches voisins $a_{0}$ par :

$$
a_{0}=\frac{C e^{2}}{4 \pi \varepsilon_{0} \varepsilon_{r} k_{B} T_{0}},
$$

avec $C=1$. Dans le cas des sauts à portée variable de Mott $(m=1 / 3)$, la température caractéristique $T_{M} \equiv T_{0}$ conduit à la longueur de localisation $a_{M}$ :

$$
a_{M}=\left(\frac{2 \beta e^{2}}{\alpha 4 \pi \varepsilon_{0} \varepsilon_{r} k_{B} T_{M}}\right)^{1 / 2}
$$

avec $\beta=13,8$ et $\alpha=0,1$. Enfin, dans le cas du transport d'Efros Shklovskii $(m=1 / 2)$, la longueur de localisation $a_{E S}$ se calcule à partir de la température caractéristique $T_{E S} \equiv T_{0}$ par :

$$
a_{E S}=\frac{C e^{2}}{4 \pi \varepsilon_{0} \varepsilon_{r} k_{B} T_{E S}},
$$

avec $C=6,2$. Nous avons employé des notations différentes pour les températures caractéristiques et les longueurs de localisation dans les différents mécanismes afin de clarifier la présentation des résultats.

\subsubsection{Puits quantique $p$-SiGe}

\section{Résultats}

Dans ce paragraphe, on cherche à ajuster les courbes de résistivité en fonction de la température dans les échantillons $p$-SiGe pour des densités $p_{s}<p_{c}$ par la loi (5.7). Le paramètre important de cette loi est l'exposant $m$ qui permet de déterminer le mécanisme de transport des porteurs. Dans un premier temps, nous avons supposé un préfacteur $\rho_{0}$ indépendant de la température, et nous avons ajusté les courbes expérimentales $\rho(T)$ par la loi (5.7) avec $\rho_{0}, T_{0}$ et $m$ comme paramètres. Cette démarche conduit à un exposant 


\begin{tabular}{|c|c|ccc|c|}
\hline échantillon & densité $\left(10^{15} \mathrm{~m}^{-2}\right)$ & $a_{0}(\mu \mathrm{m})$ & $a_{E S}(\mu \mathrm{m})$ & $a_{M}(\mathrm{~mm})$ & $r_{m}^{*}(\mu \mathrm{m})$ \\
\hline B509 & 1,01 & 5 & 7 & 5,3 & 16 \\
& 1,08 & 8 & 20 & - & 25 \\
\hline \multirow{3}{*}{ B137 } & 0,50 & 1,9 & 1,3 & 1,9 & 6 \\
& 0,55 & 2,6 & 2,6 & 3 & 8 \\
& 0,60 & 3,7 & 4,9 & 5 & 12 \\
& 0,75 & 12 & 43 & 25 & 38 \\
\hline
\end{tabular}

TAB. 5.3: Longueurs caractéristiques associées aux différents mécanismes de transport, calculées avec $\varepsilon_{r}=12,6 . r_{m}^{*}$ est la distance moyenne des sauts d'Efros-Shklovskii calculée pour une température $T=T^{*}$.

\begin{tabular}{|c|c|ccc|}
\hline échantillon & densité $\left(10^{15} \mathrm{~m}^{-2}\right)$ & $\rho_{0}\left(h / e^{2}\right)$ & $\rho_{E S}\left(h / e^{2}\right)$ & $\rho_{M}\left(h / e^{2}\right)$ \\
\hline B509 & 1,01 & 1,07 & 0,344 & 0,0718 \\
& 1,08 & 0,877 & 0,475 & - \\
\hline & 0,50 & 2,07 & 0,224 & 0,0206 \\
B137 & 0,55 & 1,55 & 0,322 & 0,0493 \\
& 0,60 & 1,24 & 0,386 & 0,100 \\
& 0,75 & 0,885 & 0,579 & 0,367 \\
\hline
\end{tabular}

TAB. 5.4: Préfacteur obtenu dans l'ajustement des dépendances en température de la résistivité dans $p$-SiGe, et en considérant un préfacteur indépendant de la température. $T^{*}$ est la température de transition entre le comportement $m=1$ et $m=1 / 2$, et $r_{m}^{*}$ la distance moyenne des sauts d'Efros-Shklovskii calculée pour une température $T=T^{*}$.

$m$ qui varie en fonction de la densité : si on prend en compte toutes les courbes de type isolant, cet exposant est compris entre 0,49 et 1,1 dans l'échantillon B137, et entre 0, 16 et 1 dans l'échantillon B509. Il est possible d'ajuster les courbes avec un exposant $m \approx 0,7$ fixé. Nous allons voir dans la suite que cet exposant traduit une variation du régime de conduction en fonction de la température.

Pour s'en rendre compte, on a tracé dans la figure 5.5 la résistivité en fonction de $T^{-m}$ en échelle semi-log, avec différentes valeurs de $m$. Dans cette représentation, la loi de sauts avec un exposant $m$ et un préfacteur $\rho_{0}$ indépendant de la température donne une droite. Les ajustements ont été faits par la loi (5.7) en prenant un exposant $m$ correspondant à celui de l'axe des abscisses $T^{-m}$. Les paramètres des ajustements sont donnés dans les tableaux 5.2 et 5.4. Ces courbes montrent que l'exposant est proche de $m=1$ à haute température, et de $m=1 / 2$ ou $1 / 3$ à basse température, sans pouvoir distinguer le meilleur exposant.

Pour tester l'influence d'un préfacteur dépendant de la température, nous avons tracé $\rho(T) / T^{p}$ dans la figure 5.6, avec l'exposant $p=0,8$ utilisé par Van Keuls et al [264] ${ }^{3}$, en fonction de $T^{-1 / 2}$ et $T^{-1 / 3}$. On voit que le comportement activé ( $m=1$ avec un préfacteur indépendant de la température) peut être expliqué par du saut à portée variable et un préfacteur dépendant de la température. Dans ces ajustements, on remarque alors que

\footnotetext{
${ }^{3}$ En fait, l'exposant $p$ peut varier entre 0,6 et 1 , mais il a été montré que la différence ne modifie pas de façon très sensible cette analyse [264].
} 


\begin{tabular}{|c|c|cc|cc|cc|}
\hline échantillon & $\begin{array}{c}\text { densité } \\
\left(10^{15} \mathrm{~m}^{-2}\right)\end{array}$ & $\begin{array}{c}T_{E S} \\
(\mathrm{~K})\end{array}$ & $\begin{array}{c}T_{M} \\
(\mathrm{~K})\end{array}$ & $\begin{array}{c}a_{E S} \\
(\mu \mathrm{m})\end{array}$ & $\begin{array}{c}a_{M} \\
(\mathrm{~mm})\end{array}$ & $\begin{array}{c}A_{E S} \\
\left(h / e^{2} / K^{0,8}\right)\end{array}$ & $\begin{array}{c}A_{M} \\
\left(h / e^{2} / K^{0,8}\right)\end{array}$ \\
\hline B509 & 1,01 & 3,41 & 44,0 & 2,38 & 2,86 & 0,228 & 0,0341 \\
& 1,08 & 2,52 & 19,8 & 3,22 & 4,27 & 0,219 & 0,0706 \\
& 1,19 & 2,18 & 11,6 & 3,72 & 5,57 & 0,157 & 0,0716 \\
\hline \multirow{3}{*}{ B137 } & 0,50 & 10,3 & 176 & 0,789 & 1,43 & 0,151 & 0,0123 \\
& 0,55 & 6,89 & 92,7 & 1,18 & 1,97 & 0,180 & 0,0241 \\
& 0,60 & 4,60 & 55,5 & 1,76 & 2,55 & 0,209 & 0,0359 \\
& 0,75 & 2,23 & 15,5 & 3,63 & 4,82 & 0,230 & 0,0824 \\
\hline
\end{tabular}

TAB. 5.5: Paramètres des ajustements dans la phase isolante de $p$-SiGe avec un préfacteur dépendant de la température, $\rho_{0}=A_{0} T^{0.8}$.

l'exposant est proche de $m=1 / 2$ à haute température, et que l'exposant $m=1 / 3$ marche mieux à basse température. Les paramètres des ajustements réalisés dans ces conditions sont reportés dans les tableaux 5.5.

\section{Interprétation}

Le comportement de la résistivité dans la phase "isolante" dans les puits quantiques $p$-SiGe est donc qualitativement compatible avec les lois prévues par les théories d'électrons localisés sans interaction. En particulier, on observe une transition entre un régime de sauts plus proche voisins à haute température et de sauts à portée variable à basse température, comme le prévoit la théorie [11]. On peut cependant remarquer que, en utilisant un préfacteur dépendant de la température, les courbes peuvent être entièrement décrites par des lois de sauts à portée variable. Cette remarque ne modifie cependant pas la conclusion que le transport est bien décrit dans le cadre d'électrons indépendants.

Pour étudier de manière plus quantitative ces lois, nous avons déterminé les longueurs de localisation à partir des températures caractéristiques (voir les tableaux 5.3 et 5.5). Dans le cas des sauts de Mott, les longueurs de localisation obtenues (de l'ordre de quelques $\mathrm{mm}$ ) sont très élevées, et même une surévaluation de la constante $\beta$ ne permet pas d'expliquer ces valeurs élevées, ce qui nous permet d'exclure le mécanisme de Mott pour interpréter nos résultats. On peut également remarquer que la prise en compte du préfacteur dépendant de la température ne change pas cette conclusion.

Dans le cas Efros-Shklovskii, les longueurs ainsi calculées (de l'ordre de quelques $\mu \mathrm{m}$ ) sont assez élevées. D'autre part, en raison de la grille distante de $d=53 \mathrm{~nm}$ par rapport au gaz de trous, on s'attendrait alors à observer un écrantage des interactions et donc a trouver la loi de Mott [263]. Cependant de telles valeurs de longueurs de localisation ont déjà été observées dans les MOSFETs Si [78, 77], et ont été expliquées par une surévaluation de la constante $C$. Une constante 10 fois plus petite a été suggérée pour des sauts collectifs [276], et serait plus en accord avec nos valeurs de températures caractéristiques. On peut également s'interroger sur la valeur de la constante diélectrique $\varepsilon_{r}$ dans un isolant d'Anderson, qui peut être très différente de celle dans le semiconducteur massif. En particulier, Larkin et Khmel'nitskii ont montré théoriquement que, pour un système avec une grande longueur de localisation $a$, la constante diélectrique est proportionnelle à $a^{2}$, 



FIG. 5.5: Dépendances en température de la résistivité des échantillons $p$-SiGe B509 et B137 à faible densité de porteur (indiquée en encart en unité $10^{1} 5 \mathrm{~m}^{-2}$ ) en fonction de $T^{-m}$, où $T$ est la température et l'exposant $m$ varie d'une figure à l'autre. Les dépendances sont ajustées par des lois exponentielles (voir l'équation (5.7)).
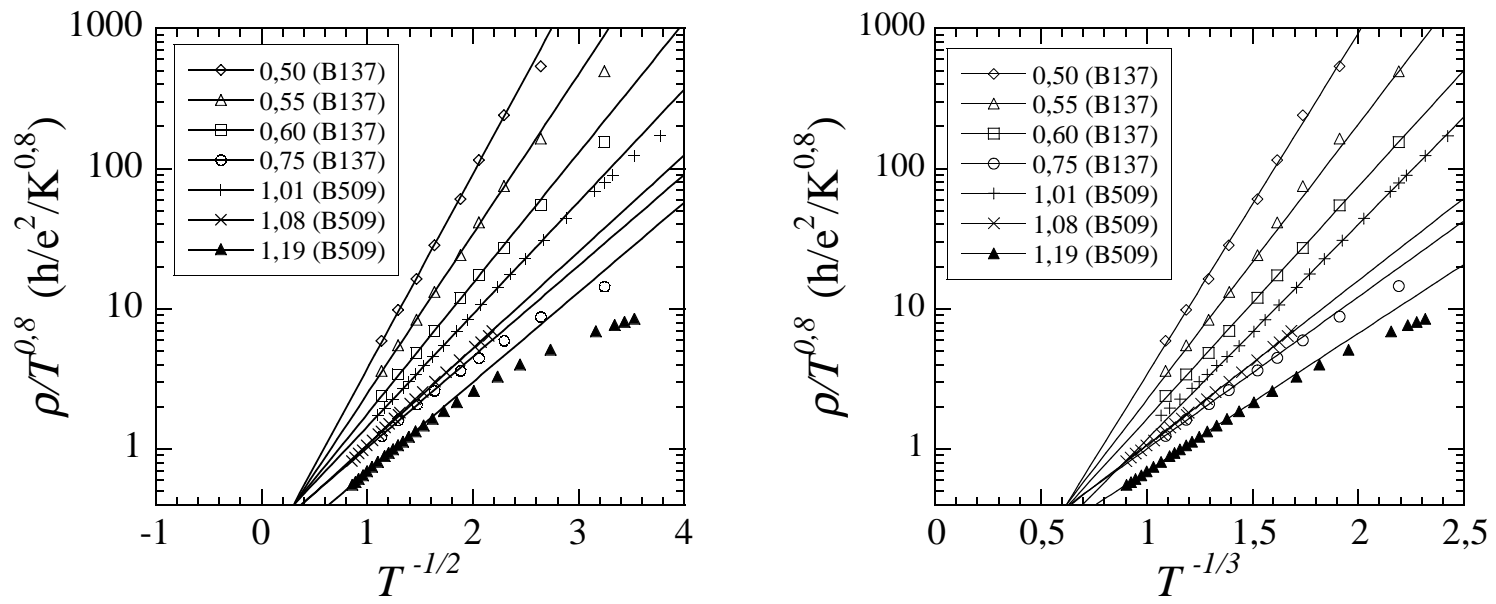

FIG. 5.6: Dépendances en température de la résistivité des échantillons p-SiGe B509 et B137 à faible densité de porteur (indiquée en encart en unité $10^{1} 5 \mathrm{~m}^{-2}$ ) en prenant en compte un préfacteur dépendant de la température dans la loi de transport. Les points sont ajustés par des lois exponentielles. 
ce qui conduit à une valeur élevée de cette constante [303].

Dans le cas des ajustements avec un préfacteur indépendant de la température, il est possible d'étudier la transition entre $m=1$ à haute température, et $m=1 / 2$ à basse température (nous avons volontairement exclu ici les sauts de Mott en raison de la valeur très élevée de la longueur de localisation obtenue). Cette transition peut être interprétée comme la transition entre un régime de sauts plus proches voisins à haute température et un régime de sauts à portée variable à basse température. La température à laquelle intervient la transition est donnée dans le tableau 5.2, et on s'attend à ce que cette température corresponde au point où la distance moyenne de sauts calculée dans le régime de sauts à portée variable est égale à la distance entre sites plus proche voisins [11]. Dans les théories de sauts à portée variable, la distance moyenne de saut s'évalue par :

$$
r_{m}(T)=a\left(\frac{T_{E S}}{T}\right)^{1 / 2} .
$$

Les distances moyennes de sauts à la transition, $r_{m}^{*}$, sont reportées dans le tableau 5.3. Le rapport $r_{m}^{*} / a_{0}=3,15 \pm 0,1$ pour toutes les densités, ce qui est en accord avec l'idée que c'est bien la longueur de sauts qui détermine la transition entre les régimes $m=1 / 2$ et $m=1$. Cette remarque permet de conforter l'analyse faite avec un préfacteur indépendant de la température.

Dans tous les cas, le préfacteur $\rho_{0}$ (dans le cas où il est indépendant de la température) dépend de la densité, comme le montre le tableau 5.4. Ce comportement est différent de ce qui a été observé dans les MOSFETs Si $[67,77,78]$, où un préfacteur universel indépendant de la température et de la densité a été obtenu, mais est en accord avec les théories de sauts à portée variable.

\subsubsection{Puits quantique $p$-GaAs}

\section{Résultats}

Pour ajuster les courbes expérimentales $\rho(T)$ dans l'échantillon $p$-GaAs, nous avons adopté la même démarche que pour les échantillons $p$-SiGe. On considère tout d'abord un préfacteur $\rho_{0}$ indépendant de la température dans la loi (5.7). Les figures 5.7-(a,b,c) présentent la résistivité en fonction de $T^{-m}$ en échelle semi-log. Dans cette représentation, la loi de sauts avec un exposant $m$ et un préfacteur indépendant de la température donne une droite. Les ajustements ont été faits par une loi (5.7) avec l'exposant $m$ correspondant à celui de l'axe des abscisses $T^{-m}$. Les paramètres des ajustements sont donnés dans les tableaux 5.6 et 5.7. Aux plus basses densités, on observe une transition entre un comportement simplement activé $(m=1)$ à basse température, et un comportement de sauts à portée variable $(m=1 / 2$ ou $1 / 3)$ à haute température, sans pouvoir distinguer le meilleur exposant en raison de la faible gamme de résistivités explorée. A densité plus élevée, la loi activée avec $m=1$ n'est plus observée, et seul un comportement avec sauts à portée variable est observé, toujours sans pouvoir différencier le type des sauts.

Pour tester l'influence d'un préfacteur dépendant de la température, nous avons tracé $\rho(T) / T^{p}$, avec $p=0,8$, en fonction de $T^{-1 / 2}$ et $T^{-1 / 3}$ (voir figures 5.7-(e,f)). Le comportement activé ( $m=1$ avec un préfacteur indépendant de la température) peut être en 


\begin{tabular}{|c|ccc|ccc|}
\hline densité $\left(10^{14} \mathrm{~m}^{-2}\right)$ & $T_{0}(\mathrm{~K})$ & $T_{E S}(\mathrm{~K})$ & $T_{M}(\mathrm{~K})$ & $a_{0}(\mu \mathrm{m})$ & $a_{E S}(\mu \mathrm{m})$ & $a_{M}(\mathrm{~mm})$ \\
\hline 1,30 & 0,25684 & 2,0912 & 21,027 & 4,9 & 3,8 & 4 \\
1,39 & 0,11532 & 0,65973 & 4,1920 & 11 & 12 & 9 \\
1,43 & 0,06999 & 0,32360 & 1,2822 & 18 & 24 & 17 \\
1,44 & - & 0,14182 & 0,67943 & - & 56 & 23 \\
1,48 & - & 0,036077 & 0,15843 & - & 220 & 47 \\
\hline
\end{tabular}

TAB. 5.6: Températures et longueurs caractéristiques déduites des ajustements des courbes de résistivité dans la phase "isolante" dans $p$-GaAs, pour un préfacteur indépendant de la température.

\begin{tabular}{|c|ccc|}
\hline densité $\left(10^{14} \mathrm{~m}^{-2}\right)$ & $\rho_{0}\left(h / e^{2}\right)$ & $\rho_{E S}\left(h / e^{2}\right)$ & $\rho_{M}\left(h / e^{2}\right)$ \\
\hline 1,30 & 6,99 & 0,945 & 0,00408 \\
1,39 & 5,99 & 1,41 & 0,00914 \\
1,43 & 4,81 & 1,56 & 0,0165 \\
1,44 & - & 2,24 & 0,0227 \\
1,48 & - & 3,04 & 0,0470 \\
\hline
\end{tabular}

TAB. 5.7: Préfacteurs déduits des ajustements de la résistivité dans la phase "isolante" dans $p$-GaAs, ce préfacteur étant indépendant de la température.

\begin{tabular}{|c|cc|cc|cc|}
\hline $\begin{array}{c}\text { densité } \\
\left(10^{14} \mathrm{~m}^{-2}\right)\end{array}$ & $\begin{array}{c}T_{E S} \\
(\mathrm{~K})\end{array}$ & $\begin{array}{c}T_{M} \\
(\mathrm{~K})\end{array}$ & $\begin{array}{c}a_{E S} \\
(\mu \mathrm{m})\end{array}$ & $\begin{array}{c}a_{M} \\
(\mathrm{~mm})\end{array}$ & $\begin{array}{c}A_{E S} \\
\left(h / e^{2} / K^{0,8}\right)\end{array}$ & $\begin{array}{c}A_{M} \\
\left(h / e^{2} / K^{0,8}\right)\end{array}$ \\
\hline 1,30 & 4,3694 & 78,854 & 1,8 & 2,1 & 0,800 & 0,0571 \\
1,39 & 1,9199 & 26,413 & 4,1 & 3,6 & 1,43 & 0,193 \\
1,43 & 0,99114 & 12,194 & 8 & 5,4 & 2,53 & 0,422 \\
1,44 & 0,53086 & 6,2651 & 15 & 7,5 & 5,04 & 0,884 \\
1,48 & - & - & - & - & - & - \\
\hline
\end{tabular}

TAB. 5.8: Paramètres déduits des ajustements de la résistivité dans la phase isolante dans $p$ GaAs avec un préfacteur dépendant de la température, $\rho_{0}=A_{0} T^{0.8}$.

partie expliqué par du saut à portée variable et un préfacteur dépendant de la température. Cependant le comportement activé reste présent à la plus basse densité.

Enfin, à haute densité de porteurs, la dépendance en température de la résistivité peut être également ajustée par une loi de puissance, comme le montre la figure 5.7-(d).

\section{Interprétations}

On peut remarquer que, dans nos résultats, on ne retrouve pas de manière univoque la loi d'Efros-Shklovskii, comme il a été observé dans les MOSFETs Si $[67,77,78]$ et les puits quantiques $p$-GaAs [62]. Il n'est en effet pas possible de distinguer la loi de Mott de celle d'Efros-Shklovskii. Pour comparer quantitativement nos résultats aux modèles de sauts à portée variable, nous avons extraits les longueurs de localisation à partir des ajustements (voir les tableaux 5.6 et 5.8). Dans le cas de la loi d'Efros-Schklovskii, la longueur de localisation varie de 4 à $200 \mu \mathrm{m}$ quand la densité augmente, alors que pour la loi de Mott, elle varie de 4 à $47 \mathrm{~mm}$. Ces longueurs sont donc très élevées comparées à la taille de 

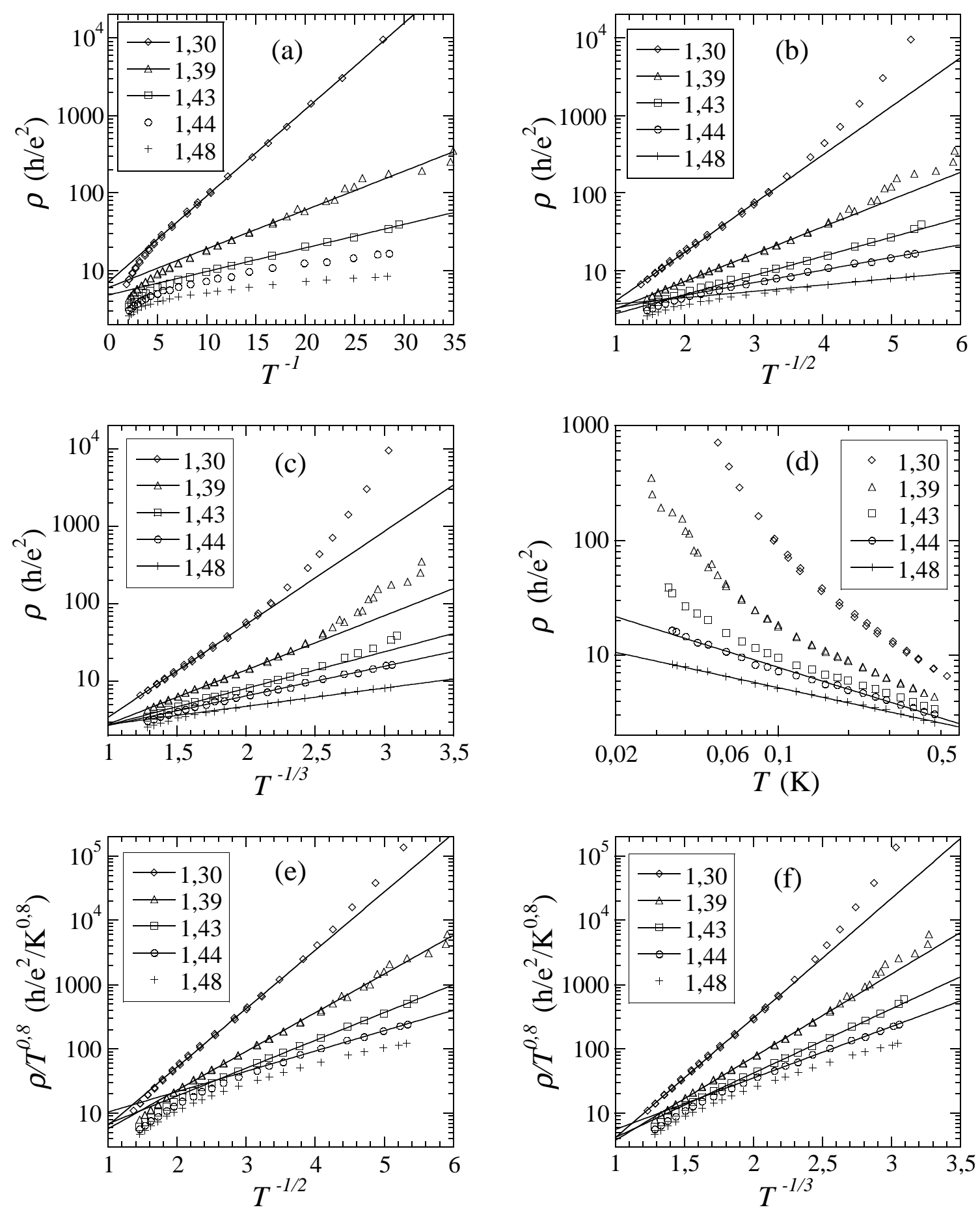

FIG. 5.7: Dépendances en température de la résistivité dans l'échantillon GaAs Nu944-1 dans la phase "isolante" (la densité de porteurs est donnée en encart en unité $10^{14} \mathrm{~m}^{-2}$ ) dans différentes représentations (voir texte). Les dépendances sont ajustées par des lois exponentielles (voir l'équation (5.7)), sauf pour (d) où les ajustements sont faits par une loi de puissance. 
l'échantillon, ce qui remet en cause, soit l'analyse en terme de sauts à portée variable, soit la valeur numérique des coefficient $C$ et $\beta$ dans l'évaluation des longueurs de localisation. Une raison à cette mauvaise évaluation peut être reliée à des effets collectifs [275], ce qui conduirait à une réduction d'un facteur environ 10 les longueurs de localisation dans le cas d'Efros-Shklovskii, conduisant à des valeurs plus raisonnables entre 0,2 et $20 \mu \mathrm{m}$. En fait, un des problèmes liés à cette analyse est la proximité de la transition métal-isolant. Même s'il n'existe pas de véritable transition de phase, on s'attend cependant à une transition entre la localisation faible (avec une divergence logarithmique de la résistivité) et la localisation forte (avec une divergence exponentielle de la résistivité). Entre ces deux régimes peuvent être rencontrés tout une gamme de comportements non déterminés, comme des lois de puissance, comme le montre la figure 5.7-(d). Le fait que les lois de sauts ne soient plus valables près de la transition métal-isolant a déjà été observé dans les MOSFETs Si par Bogdanovitch et Popović [82]. Cette remarque est une indication que les courbes aux densités comprises entre $p_{c}=1,57 \times 10^{14} \mathrm{~m}^{-2}$ et $1,44 \times 10^{14} \mathrm{~m}^{-2}$ ne sont pas caractéristiques d'un isolant, mais d'un métal.

La loi activée obtenue à basse température aux densités les plus faibles est le comportement le plus intéressant. En effet, ce comportement est très différent de celui observé dans les échantillons $p$-SiGe, dans lesquels l'exposant $m=1$ est obtenu à haute température. Dans le cadre de la conduction par sauts, on ne s'attend pas à trouver un mécanisme de sauts plus proches voisins à basse température, surtout si un mécanisme de sauts à portée variable est observé à température plus élevée, comme c'est le cas ici. Par contre, le comportement activé peut être interprété par l'ouverture d'une bande interdite "dure" au niveau de Fermi en raison de l'interaction Coulombienne, comme il a été suggéré par Kim et al. [262]. Cette bande interdite peut en particulier caractériser la formation d'une phase électronique ordonnée de type cristal de Wigner, pour laquelle une loi simplement activée est en effet attendue (voir $\S 4.3$ et Chauve et al. [286, 287]). L'énergie d'activation $k_{B} T_{0}$ déduite de l'ajustement avec $m=1$ à basse température décroît linéairement quand la densité augmente, et tend vers zéro à une densité $p_{a}^{0} \approx 1,47 \times 10^{14} \mathrm{~m}^{-2}$. Cette analyse, associée à l'observation de lois de puissance pour $p_{s}$ compris entre 1,57 et $1,44 \times 10^{14}$ $\mathrm{m}^{-2}$, permet de conclure que, dans le $p$-GaAs, la véritable transition métal-isolant semble plutôt être à la densité $p_{a}^{0}$.

La loi activée peut être également due au transport dans un système percolant : lorsque le système est constitué d'îlots métalliques séparés par des zones isolantes, le transport des porteurs se fait par sauts activés d'un îlot à l'autre. Cette constatation est intéressante dans le cadre de la transition métal-isolant en dimension deux pour laquelle des scénarios de percolation ont été évoqués (voir §2.5.4). Ce type d'analyse a déjà été faite dans le cadre des transitions dans le régime d'effet Hall quantique par Shashkin et al. [36, 35], pour lequel il est maintenant reconnu que le système présente des transitions de percolation (voir par exemple Girvin [304]).

On peut enfin remarquer que, pour une densité donnée, le régime activé n'est valable qu'à basse température. Nous avons tracé dans la figure 5.8 la dépendance de la température limite jusqu'à laquelle est observée le comportement activé en fonction de la densité de porteurs (où plus exactement la densité $p_{a}$ en-dessous de laquelle est observée le comportement activé à une température donnée), permettant de tracer un diagramme de phase concernant le comportement de la résistivité. La densité $p_{a}^{0}$ a été ajoutée car 


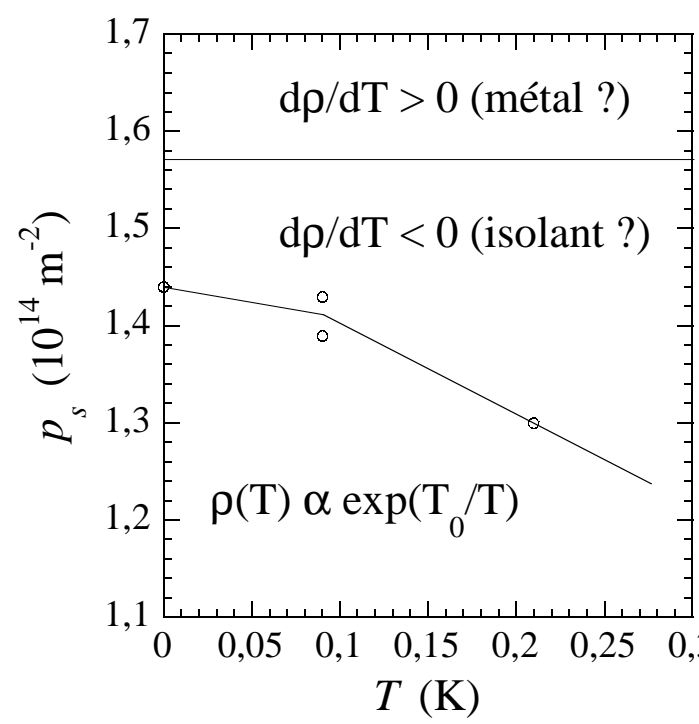

FIG. 5.8: Diagramme de phase des dépendances en température de la résistivité. La ligne horizontale à $p_{s}=1,57 \times 10^{14} \mathrm{~m}^{-2}$ correspond à $p_{c}$, alors que les points représentent $p_{a}$, la limite d'observation d'une loi activée sur la résistivité.

elle correspond à la densité à laquelle le comportement activé apparaît à température nulle. Comme il a déjà été évoqué pour le cas des MOSFETs Si par Bogdanovitch et Popović [82], il est possible de distinguer trois régimes : (a) un régime à haute densité où $d \rho / d T>0$, qui a été qualifié de régime métallique dans le cadre de la transition métalisolant en dimension deux; (b) un régime à densité intermédiaire où $d \rho / d T<0$, mais où la résistivité est une loi faiblement dépendante de la température (loi de puissance); (c) enfin un régime isolant pour lequel la résistivité varie exponentiellement en fonction de la température, et qui ne semble pas pouvoir être interprété dans le cadre du transport d'électrons indépendants localisés.

\subsection{Conclusion}

Nous avons donc étudié les lois de transport en fonction de la température dans le régime ohmique et dans les deux phases autour de la transition métal-isolant. Alors que les mesures dans les puits quantiques $p$-SiGe peuvent s'interpréter dans le cadre de théories pour des électrons indépendants, les mesures dans les puits quantiques $p$-GaAs présentent des caractères nouveaux. A haute densité, la chute brutale de résistivité quand la température diminue fait intervenir un mécanisme de transport avec une énergie d'activation qui n'est pas encore compris, et pour laquelle de nombreux modèles ont été évoqués. A basse densité, la loi de transport simplement activée ne peut pas être comprise dans le cadre du transport d'électrons indépendants localisés (sauts à portée variable), mais est compatible avec le transport d'une phase collective due aux interactions entre les porteurs.

Il ressort cependant de ces analyses que la seule dépendance en température de la résistivité ne permet de tirer une conclusion franche sur le mécanisme de transport dans ces systèmes, notamment dans la phase isolante. Pour cette raison il est nécessaire d'aller plus loin, en regardant les effets du champ électrique sur le transport. C'est l'objet des deux chapitres suivants, le chapitre 6 concernant les mesures faites sur les puits quantiques $p$-SiGe, et le chapitre 7 concernant les mesures dans les puits quantiques $p$-GaAs. 
Chapitre 5. Transport dans les puits quantiques $p$-SiGe et p-GaAs 


\section{Chapitre 6}

\section{Transport non-ohmique dans les puits quantiques $p$-SiGe}

\section{Sommaire}

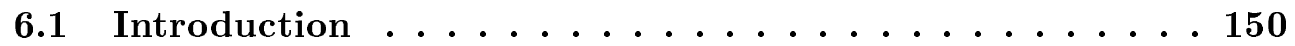

6.2 Etude du chauffage des porteurs . . . . . . . 150

6.2.1 Température effective des porteurs . . . . . . . . . . 151

6.2.2 Puissance dissipée par le couplage électron-phonon . . . . . 153

6.2.3 Paramètres du couplage électron-phonon . . . . . . . . . 155

6.2.4 Discussion des résultats . . . . . . . . . . . . . . . . 158

6.3 Dépendances en champ électrique expliquées par le chauf-

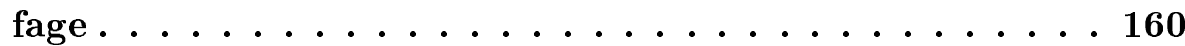

6.3.1 Observation de lois d'échelle sur la résistivité . . . . . . . 160

6.3.2 Fortes non linéarités de la caractéristique $I-V \ldots 163$

6.3.3 La diffusion de chaleur pour l'étude des non-linéarités . . . 166

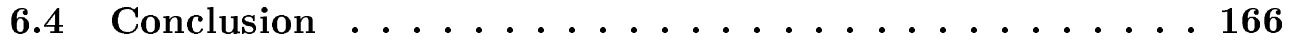




\subsection{Introduction}

Dans ce chapitre sont présentées les mesures de transport qui ont été réalisées sur des gaz bidimensionnels de trous dans des puits quantiques $\mathrm{SiGe} / \mathrm{Si}$, ces systèmes ayant été décrits dans le chapitre 3 . Nous avons vu au chapitre 5 que la variation de la résistivité en fonction de la température présente une transition entre un comportement métallique $(d \rho / d T>0)$ et un caractère isolant $(d \rho / d T<0)$ lorsque la densité de porteurs diminue. Ces dépendances en températures ont pu être interprétées par des théories d'électrons indépendants, et ne semblent donc pas présenter un caractère nouveau lié à l'existence d'une nouvelle phase métallique en dimension deux (voir le chapitre 2). Nous allons voir dans ce chapitre que les dépendances en champ électrique peuvent être interprétées par des effets de chauffage des porteurs en présence d'un champ électrique, et sont donc aussi compatibles avec des théories d'électrons indépendants.

Nous commençons par montrer que les dépendances en champ électrique sont dues à des effets de chauffage des porteurs, et cela pour toutes les densités électroniques que nous avons étudiées (voir §6.2). En particulier, nous n'observons pas de changement à la "transition métal-isolant" observée sur la dépendance en température de la résistivité. D'autre part, cette analyse nous a permis d'étudier le couplage électron-phonon dans un gaz bidimensionnel de trous dans $\mathrm{SiGe}$, dont les caractéristiques ne sont pas encore bien établies. Les mesures réalisées à très basse température permettent en particulier de mettre en évidence une contribution du couplage piézoélectrique, qui restait jusqu'alors controversée. On peut enfin remarquer que d'autres effets de champ électrique ont été évoqués dans le cadre de la transition métal-isolant en dimension deux. Nous verrons dans une deuxième section (voir $§ 6.3$ ) que le phénomène de chauffage permet d'expliquer les observations faites, et en particulier nous verrons comment le chauffage peut conduire des lois d'échelle en champ électrique, ou "imiter" une tension seuil dans la caractéristique $I-V$.

\subsection{Etude du chauffage des porteurs}

Lorsqu'on injecte une puissance électrique dans un système électronique, cette puissance est transférée aux porteurs de charge par effet Joule sous forme de chaleur. Dans un tel système, la dissipation de chaleur est faite principalement par l'intermédiaire de l'interaction entre les porteurs et les phonons du réseau (ou couplage électron-phonon, où le terme "électron" sera employé ici abusivement pour les trous comme pour les électrons). A basse température, le couplage électron-phonon est peu effectif, ce qui conduit à un échauffement du système électronique jusqu'à une température effective $T_{e}$ supérieure à la température du réseau $T_{0}$. Dans cette section nous mettons en évidence ce mécanisme dans les puits quantiques SiGe, en montrant qu'il est responsable des dépendances en champ électrique de la résistivité. 


\subsubsection{Température effective des porteurs}

La figure 6.1 présente les dépendances en température et en champ électrique de la résistivité dans l'échantillon SiGe B509. On constate que ces dépendances sont très semblables : la variation de la résistivité du système due à une augmentation du champ électrique correspond parfaitement à celle due à une augmentation de la température. D'autre part, lorsqu'on change la température du réseau $T_{0}$, la courbe $\rho(E)$ présente un plateau à la valeur $\rho\left(T_{0}\right)$, puis rejoint les courbes $\rho(E)$ à différentes températures. Ainsi, ces premières observations suggèrent que les dépendances en champ électrique sont liées au chauffage des porteurs : l'augmentation du champ électrique semble correspondre à l'augmentation de la température effective des porteurs $T_{e}$ plutôt qu'à un effet intrinsèque sur le mécanisme de transport.

Pour vérifier cette hypothèse de chauffage, nous avons mesuré la température effective des porteurs en comparant deux méthodes. Dans un premier temps on utilise la dépendance en température de la résistivité à champ magnétique nul. Dans le système étudié (trous dans $\mathrm{SiGe}$ ) et à densité élevée (phase métallique), nous avons vu que la dépendance en température de la conductivité à basse température peut être expliquée par la somme de trois effets (voir $\S 5.3$ ) :

$$
\sigma(T)=\sigma_{D}(T)+\delta \sigma_{L F}(T)+\delta \sigma_{I}(T)
$$

où la dépendance en température de la conductivité de Drude $\sigma_{D}(T)$ est due à la dépendance en température de l'écrantage, $\delta \sigma_{L F}$ est la correction de conductivité due à la localisation faible et $\delta \sigma_{I}$ la correction de conductivité due aux interactions entre porteurs. La dépendance due à la diffusion par les phonons est négligeable aux températures étudiées (cette diffusion devient non négligeable au-delà de environ $10 \mathrm{~K}$ [301]). Puisque ces trois effets ne dépendent directement que de la température effective des porteurs (et non directement de la température du réseau), il est possible d'utiliser la valeur de la résistivité à champ magnétique nul comme thermomètre, suivant la méthode décrite au $\S$ A.3.1. Dans la phase basse densité (phase isolante), nous avons vu que les courbes peuvent être ajustées par des lois de transport de type sauts à portée variable. Dans ce cas il est nécessaire de savoir qu'elle est l'origine des sauts, soit par l'intermédiaire du couplage électron-phonon comme le suggère le modèle de Mott, soit par l'intermédiaire du couplage électron-électron (voir la discussion dans le chapitre 4). Dans le cas de sauts assistés par les phonons, la résistivité est une fonction des deux températures $T_{0}$ et $T_{e}$. Dans le cas de sauts assistés par les interactions électron-électron, la résistivité est une fonction seulement de la température effective des porteurs $T_{e}$. Pour pouvoir utiliser la dépendance en température de la résistivité dans ce régime comme thermomètre, il est donc nécessaire de prendre en compte le deuxième cas de figure. Nous verrons que l'accord avec la théorie du couplage électron-phonon justifie à postériori cette hypothèse.

Pour montrer la validité du thermomètre utilisé (résistivité à champ magnétique nul), nous avons comparé la température effective déduite des mesures précédentes à champ magnétique nul avec celle obtenue par analyse des oscillations de Shubnikov-de Haas. Pour une densité de $1,19 \times 10^{15} \mathrm{~m}^{-2}$, nous avons mesuré la différence de résistivité $\Delta \rho_{x x}=$ $\rho_{x x}(B=1,03 \mathrm{~T})-\rho_{x x}(B=0,85 \mathrm{~T})$, où $B=1,03 \mathrm{~T}$ correspond à un minimum de $\rho_{x x}$ à un remplissage $\nu=5$, et $B=0,85 \mathrm{~T}$ est le maximum qui précède. La figure 6.2 



FIG. 6.1: Comparaison des courbes de résistivité en fonction de la température et du champ électrique dans l'échantillon SiGe B509. (a,b,c) Résistivité en fonction de la température du réseau mesurée à faible courant. (d,e,f) Résistivité en fonction du champ électrique pour différentes valeurs de températures de réseau $T_{0}$. (a) et (d) correspondent à de faibles valeurs de la densité de porteurs $p_{s}$, pour lesquelles le comportement est isolant, (b) et (e) correspondent à de fortes valeurs de la densité de porteurs $p_{s}$, pour lesquelles le comportement est métallique, enfin (c) et (f) correspondent à la partie sans grille.

montre que les deux thermomètres sont en très bon accord. Il est intéressant de remarquer que la dépendance en température des oscillations de Shubnikov-de Haas a une origine physique différente de celle de la résistivité à température nulle (voir §A.3), ce qui permet d'en déduire la validité de l'hypothèse de chauffage, selon laquelle toute la dépendance en champ électrique de la résistivité est due au chauffage des porteurs. Les mesures aux autres valeurs de densités ont été effectuées en utilisant $\rho(T)$ à champ magnétique nul 


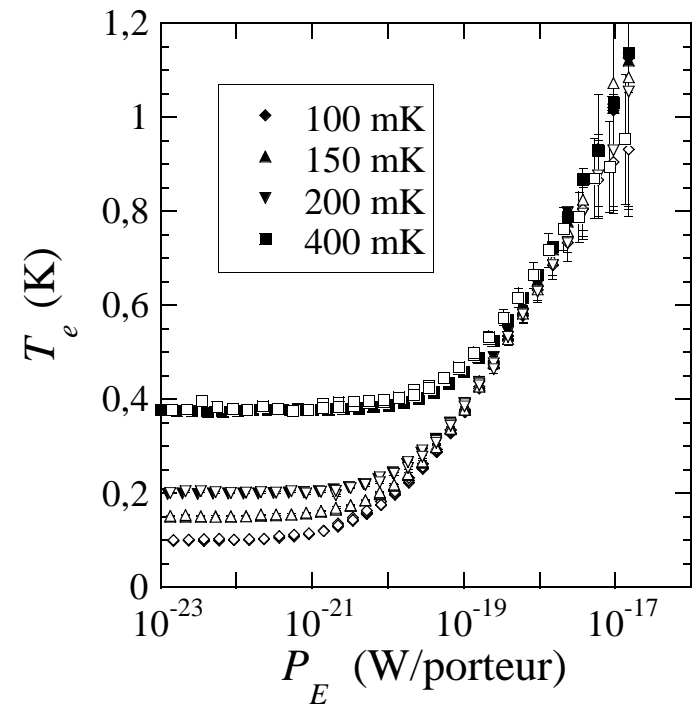

FIG. 6.2: Comparaison des deux thermomètres pour la mesure de la température effective $T_{e}: \rho$ en fonction de $T$ à champ magnétique nul (symboles pleins) et amplitude des oscillations de Shubnikov-de Haas (symboles vides). Mesure sur l'échantillon SiGe B509 pour une densité de porteurs de $1,19 \times$ $10^{15} \mathrm{~m}^{-2}$ et à différentes températures du réseau $T_{0}$ indiquées en encart. Les grandes barres d'erreurs à température effective élevée sont dues aux faibles dépendances des "thermomètres" dans cette gamme de température.

comme thermomètre.

\subsubsection{Puissance dissipée par le couplage électron-phonon}

La puissance électrique injectée par porteur est calculée par :

$$
P_{E}=\frac{R I^{2}}{S p_{s}},
$$

où $R=V / I$ est la résistance du système, $V$ et $I$ sont respectivement la tension et le courant, $S$ est la surface du système et $p_{s}$ la densité surfacique de porteurs. Des courbes typiques $P_{E}$ en fonction de $T_{e}$ pour différentes valeurs de la température du réseau $T_{0}$ sont présentée dans la figure 6.3 .

Nous avons vu au $§$ A.2 que, dans l'hypothèse du chauffage, la puissance injectée est égale à la puissance dissipée par le couplage électron-phonon. Dans le cas d'un gaz bidimensionnel de porteurs dégénéré, interagissant avec les phonons acoustiques tridimensionnels du réseau, la puissance dissipée peut se mettre sous la forme :

$$
P_{E}\left(T_{e}, T_{0}\right)=P_{d i s s}\left(T_{e}, T_{0}\right)=F\left(T_{e}\right)-F\left(T_{0}\right) .
$$

Ainsi, si on trace $P_{E}\left(T_{e}, T_{0}\right)+F\left(T_{0}\right)$ en fonction de $T_{e}$ pour plusieurs valeurs de $T_{0}$, cette fonction ne doit plus dépendre de $T_{0}$. De plus, ce tracé permet de déduire directement la fonction $F(T)$ qui, dans le calcul approché présenté au $\S$ A.2, est une fonction polynômiale :

$$
F(T)=A_{1} T^{\theta_{1}}+A_{2} T^{\theta_{2}},
$$

où $A_{i}$ et $\theta_{i}$ sont le coefficient et l'exposant du couplage électron-phonon, les deux termes indicés 1 et 2 correspondant aux deux types de couplages pouvant intervenir (déformation et piézoélectrique, voir le tableau A.1).

En pratique, ne connaissant pas à priori la fonction $F(T)$, il n'est pas possible de calculer $F\left(T_{0}\right)$ directement. Pour chaque valeur de $T_{0}$, on ajoute alors à la fonction $P_{E}\left(T_{e}, T_{0}\right)$ 

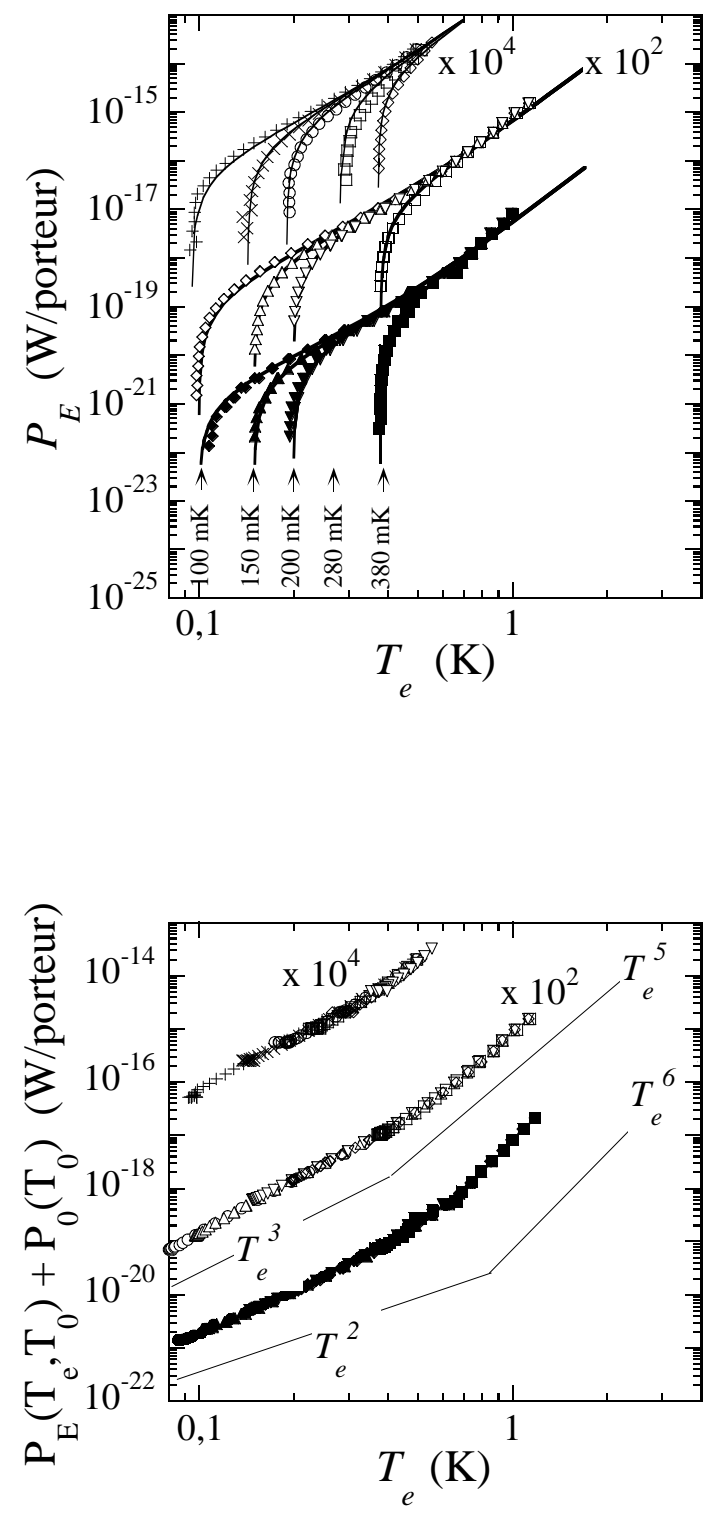

FIG. 6.3: Puissance injectée $P_{E}$ en fonction de la température effective des porteurs $T_{e}$ dans les deux échantillons SiGe (B509 et B137), pour différentes températures $T_{0}$ (indiquées en bas de la figure) et pour différentes valeurs de la densité de porteurs : de haut en bas, $0,55 \times 10^{15} \mathrm{~m}^{-2}$ (B137), $1,19 \times 10^{15} \mathrm{~m}^{-2}$ (B509) et $1,38 \times 10^{15} \mathrm{~m}^{-2}$ (B509). La puissance à $0,55 \times 10^{15} \mathrm{~m}^{-2}$ est multipliée par $10^{4}$, et celle à $1,19 \times 10^{15} \mathrm{~m}^{-2}$ par $10^{2}$. Les lignes sont des ajustements par les lois (6.3) et (6.4), avec des exposants 3 et 5 , et en prenant en compte la diffusion de chaleur par les contacts.

FiG. 6.4: La puissance dissipée ajoutée d'une valeur $P_{0}\left(T_{0}\right)$ choisie convenablement (voir texte) en fonction de la température effective des porteurs pour différentes températures $T_{0}$ et pour différentes valeurs de la densité de porteurs : de haut en bas, $0,55 \times 10^{15} \mathrm{~m}^{-2}$ (B137), $1,19 \times 10^{15} \mathrm{~m}^{-2}$ (B509) et $1,38 \times 10^{15}$ $\mathrm{m}^{-2}$ (B509). La puissance à $0,55 \times 10^{15} \mathrm{~m}^{-2}$ est multipliée par $10^{4}$, et celle à $1,19 \times 10^{15}$ $\mathrm{m}^{-2}$ par $10^{2}$. Les lignes représentent les dépendances en $T_{e}^{\theta}$, et sont données à titre indicatif. Les différents symboles représentent les différentes températures de réseau $T_{0}$, et sont identiques à ceux de la figure 6.3.

un terme $P_{0}\left(T_{0}\right)$ choisi de la manière suivante : pour la plus petite valeur de $T_{0}$, ce terme prend une valeur arbitraire $P_{0}\left(T_{0}^{\mathrm{min}}\right)$; ensuite on procède par valeurs croissantes de $T_{0}$, et on choisi $P_{0}\left(T_{0}\right)$ tel que toutes les courbes $\tilde{P}_{T_{0}}\left(T_{e}\right)=P_{E}\left(T_{e}, T_{0}\right)+P_{0}\left(T_{0}\right)$ se superposent. Pour affiner la méthode, on réitère le procédé en choisissant à nouveau $P_{0}\left(T_{0}^{\mathrm{min}}\right)$ de façon à améliorer la superposition. La figure 6.4 montre la superposition obtenue après quelques itérations. Le fait de pouvoir superposer toutes les courbes $\tilde{P}_{T_{0}}\left(T_{e}\right)$ pour toutes les valeurs de $T_{0}$ montre que $\tilde{P}_{T_{0}}\left(T_{e}\right) \equiv F\left(T_{e}\right)$ est indépendant de $T_{0}$, comme attendu dans la théorie du couplage électron-phonon. De plus, on peut alors vérifier que $P_{0}\left(T_{0}\right) \equiv F\left(T_{0}\right)$ suit bien la même fonction. Enfin, on peut ajuster la courbe $\tilde{P}_{T_{0}}\left(T_{e}\right)=F\left(T_{e}\right)$ pour obtenir la fonction $F(T)$. 


\subsubsection{Paramètres du couplage électron-phonon}

\section{Extraction des exposants}

A basse température $T_{e}$ et à faible densité de porteurs, $\tilde{P}_{T_{0}}\left(T_{e}\right) \propto T_{e}^{3}$. L'exposant 3 est caractéristique d'un couplage piézoélectrique non écranté (voir §A.2.2). Lorsque la densité augmente, cet exposant diminue, et tend vers 2 .

A haute température $T_{e}$, le nombre limité de points expérimentaux ne permet pas de déduire l'exposant avec exactitude. Cet exposant est proche de 5 à basse densité, mais est supérieur à 5 (voir 6$)$ à haute densité. D'autre part, si on ajuste la courbe $\tilde{P}_{T_{0}}$ vs. $T_{e}$ avec la loi (6.4) et les deux exposants libres, l'exposant fort augmente encore (entre 6 et 7 suivant la densité de porteurs). Cependant, l'ajustement est alors fortement contraint par la forme du coude séparant les deux régimes, et devient moins significatif. Dans tous les cas, le type de couplage intervenant à haute température est donc un couplage par déformation, mais l'exposant ne permet pas de conclure quand à la nature écrantée ou non de ce couplage. Pour déterminer l'amplitude du couplage, nous avons imposé dans la suite un exposant 5 à toutes les densités, donnant un bon résultat, comme le montrent les ajustements de la figure 6.3.

\section{Diffusion de chaleur par les contacts}

A haute densité et à basse température, l'exposant se rapproche de 2. Cet exposant faible peut être interprété par l'apparition d'une autre source de dissipation de chaleur, la diffusion de chaleur vers les contacts de mesure de tension (voir annexe B). Dans un modèle unidimensionnel simple, en supposant que les extrémités du système restent à la température du réseau et que la résistivité du système $\rho$ est homogène, on peut calculer la puissance dissipée par diffusion en fonction de la température effective des porteurs $T_{e}$ (voir annexe B) :

$$
P_{\text {cont }}=\frac{4 \mathcal{L}}{L^{2} p_{s} \rho}\left(T_{e}^{2}-T_{0}^{2}\right)
$$

où $\mathcal{L}=2,44 \times 10^{-8}$ W. $\Omega . \mathrm{K}^{-2}$ est la constante de Lorentz, $L$ la longueur du système, $p_{s}$ la densité de porteurs et $\rho$ la résistivité. Cette contribution permet donc d'expliquer l'exposant plus faible rencontré à haute densité.

Pour prendre en compte de manière plus exacte la contribution du refroidissement par les contacts, nous avons résolu numériquement l'équation de la chaleur en dimension deux (voir annexe B). Les résultats présentés dans la figure 6.5 montrent que l'effet de diffusion de chaleur est négligeable à basse densité, pour laquelle la résistivité est élevée et donc la conductivité thermique est faible, et il est important à densité élevée. La prise en compte des effets de chauffage par les simulations nous a permis d'évaluer plus exactement les préfacteurs des lois de chauffage.

\section{Déterminations des paramètres de couplage}

Avant de déterminer numériquement les paramètres de couplage, il est possible de faire une remarque sur la dépendance en densité de l'amplitude du chauffage. Sur la figure 6.6 de gauche est représentée la dépendance en densité de la puissance injectée à 

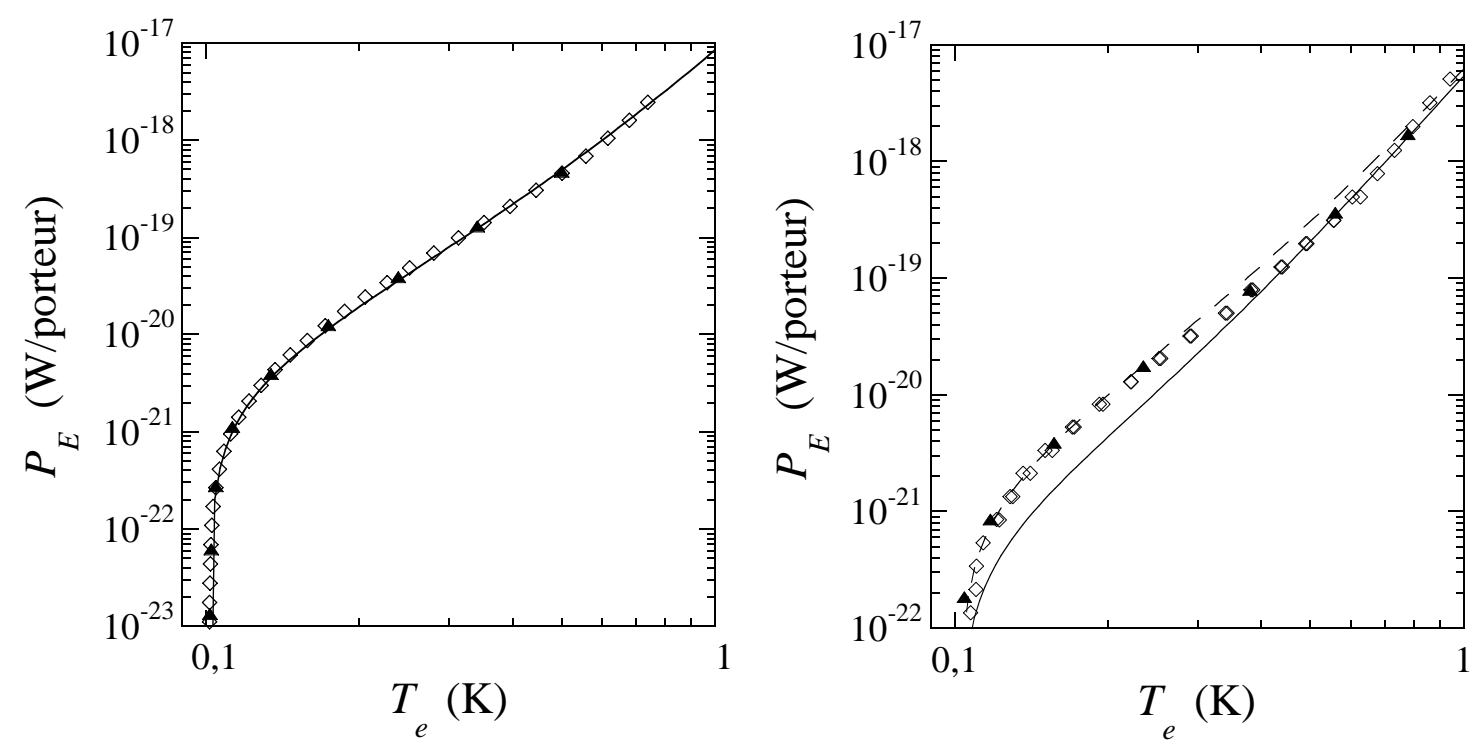

FIG. 6.5: Ajustement des courbes de puissance injectée en fonction de la température effective en prenant en compte la diffusion de chaleur par les contact à $T_{0}=0,1 \mathrm{~K}$, et pour $p_{s}=1,01 \times 10^{15}$ $\mathrm{m}^{-2}$ (gauche) et $p_{s}=1,38 \times 10^{15} \mathrm{~m}^{-2}$ (droite). Les losanges vides sont les points expérimentaux, le trait plein et les tirets sont deux ajustements avec deux termes en $T_{e}^{3}$ et $T_{e}^{5}$ (i.e. sans diffusion de chaleur vers les contacts), montrant la nécessité de prendre en compte la diffusion de chaleur par les contacts à densité élevée, et les triangles pleins sont issus d'une simulation 2D (où seuls les paramètres de couplage électron-phonon sont ajustables).

plusieurs températures effectives, en faisant attention à ne prendre que des températures pour lesquelles les effets de refroidissement par les contacts sont négligeables à toutes les densités. On remarque dans ces mesures que l'amplitude varie comme $p_{s}^{-3 / 2}$, comme on l'attend de la théorie du couplage électron-phonon (voir §A.2).

Nous avons enfin ajusté les courbes $P_{E}\left(T_{e}\right)$ par une loi prenant en compte les couplages piézoélectrique et par déformation non écranté :

$$
P_{E}=A\left(T_{e}^{3}-T_{0}^{3}\right)+B\left(T_{e}^{5}-T_{0}^{5}\right),
$$

où $A$ et $B$ sont les paramètres de l'ajustement. Pour prendre en compte le refroidissement par les contacts, nous utilisons la simulation 2D pour reproduire les courbes expérimentales. Les variations de $A$ et $B$ en fonction de la densité sont reportées dans la figure 6.6 de droite. Ces valeurs nous permettent alors de déterminer la constante piézoélectrique $e_{p z}$ et le potentiel de déformation $\Xi_{u}$.

Pour le couplage piézoélectrique avec écrantage faible, la fonction $F(T)$ donnée dans l'équation (A.12) peut se mettre sous la forme :

$$
F_{\text {n.ê. }}^{\mathrm{PZ}}(T)=K_{\text {n.ế. }}^{\mathrm{PZ}} \frac{\left(e_{p z}\right)^{2}}{\left(p_{s}\right)^{3 / 2}} T^{3},
$$

avec $e_{p z}$ la constante diélectrique, $p_{s}$ la densité de porteurs et $K_{\text {n.é. }}^{p z}$ un coefficient ne dépendant que des paramètres du matériau, et que l'on peut calculer à l'aide des valeurs 



FIG. 6.6: Gauche : Puissance injectée à différentes températures effectives (indiquées en encart) en fonction de la densité de porteurs. Droite : Dépendance en densité des paramètres de couplage électron-phonon déduit des ajustements sur l'échantillon B137 (losanges) et sur l'échantillon B509 (triangles). Les points supplémentaires (cercles) sont issus des mesures de Xie et al. [305] $\left(p_{s}=5 \times 10^{15} \mathrm{~m}^{-2}\right)$ et de Ansaripour et al. [306] $\left(p_{s}=3,5 \times 10^{15} \mathrm{~m}^{-2}\right)$ sur des hétérostructures $p$-Si $\mathrm{Ge}_{1-x}$ avec $x=0,2$. Dans les deux figures, les lignes représentent les dépendances en $p_{s}^{3 / 2}$.

données au $§ 3.2 .1$ pour le $\mathrm{Si}_{0,85} \mathrm{Ge}_{0,15}$ :

$$
K_{\text {n.é. }}^{\mathrm{PZ}}=\frac{3 \zeta(3)}{64 \sqrt{2} \pi^{5 / 2}} \frac{\left(m^{*}\right)^{2} e^{2}}{\hbar^{5}\left(4 \pi \varepsilon_{0}\right)^{2} \rho}\left[\frac{3}{v_{L}^{2}}+\frac{4}{v_{T}^{2}}\right]\left(k_{B}\right)^{3}=3,813 \times 10^{9} \mathrm{USI} \text {. }
$$

On obtient $\left|e_{\mathrm{pz}}\right|=(3,4 \pm 0,9) \times 10^{-3} \mathrm{C} \cdot \mathrm{m}^{-2}$. Cette valeur est plus faible que la valeur obtenue par Xie et al. dans une hétérostructure $p$-Si $\mathrm{Si}_{x} \mathrm{Ge}_{1-x}$ avec $x=0,2$ [305]. Cette différence est discutée dans le paragraphe suivant.

Pour le couplage par le potentiel de déformation avec écrantage faible, la fonction $F(T)$ donnée dans l'équation A.9 peut se mettre sous la forme :

$$
F_{\text {n.é. }}^{\mathrm{PD}}(T)=K_{\text {n.é. }}^{\mathrm{PD}} \frac{\left(\Xi_{u}\right)^{2}}{\left(p_{s}\right)^{3 / 2}} T^{5}
$$

avec $\Xi_{u}$ le potentiel de déformation, $n_{s}$ la densité de porteurs et $K_{\text {n.é. }}^{\mathrm{PD}}$ un coefficient ne dépendant que des paramètres du matériau :

$$
K_{\text {n.é. }}^{\mathrm{PD}}=\frac{3 \zeta(5)}{\sqrt{2} \pi^{5 / 2}} \frac{\left(m^{*}\right)^{2}}{\hbar^{7} \rho v_{L}^{4}}\left(k_{B}\right)^{5}=1,886 \times 10^{42} \mathrm{USI} .
$$

Si $\Xi_{u}$ est exprimé en $\mathrm{eV}$ (tous les autres paramètres étant en USI), on a $K_{\text {n.ê. }}^{\mathrm{PD}}=4,841 \times 10^{4}$. On obtient alors $\Xi_{u}=2,7 \pm 0,3 \mathrm{eV}$, qui est proche de $\Xi_{u} \approx 3 \mathrm{eV}$ obtenu par Xie et al. [305] et de Ansaripour et al. [306] sur des hétérostructures $p$-Si $\mathrm{G}_{x} \mathrm{Ge}_{1-x}$ avec $x=0,2$. 
On a vu enfin qu'il est possible d'ajuster les courbes $P_{E}\left(T_{e}\right)$ à haute température avec une loi de puissance en $T$ et un exposant supérieur à 5 . Si on choisi un exposant 7 , correspondant à un couplage par déformation fortement écranté, la valeur du potentiel de déformation, obtenue à partir des calculs du paragraphe A.2.2 et des données dans le $\mathrm{Si}_{0,15} \mathrm{Ge}_{0,85}$, est de $24 \mathrm{eV}$. Cette valeur est très élevée en comparaison de ce qu'on attend dans le SiGe, ce qui semble confirmer que le potentiel de déformation est faiblement écranté.

\subsubsection{Discussion des résultats}

\section{Couplage piézoélectrique}

A basse température, $F(T)$ suit donc une loi de puissance en $T^{3}$, caractéristique d'un couplage piézoélectrique non écranté. Un effet piézoélectrique ne peut être présent que pour des matériaux ne présentant pas de centre d'inversion. Un exemple typique est le GaAs qui présente une symétrie zinc-blende (groupe ponctuel $\overline{4} 3 \mathrm{~m}$, les atomes de gallium ayant une structure diamant et les atomes d'arsenic occupant les sites octaèdriques). Pour le $\mathrm{Si}_{1-x} \mathrm{Ge}_{x}$ massif, les atomes de silicium et de germanium sont répartis aléatoirement [307]. Le $\mathrm{Si}_{1-x} \mathrm{Ge}_{x}$ massif ne présente donc pas de propriétés piézoélectriques.

Cependant, d'autres études ont déjà mis en évidence des effets piézoélectriques dans des puits quantiques $p$-SiGe. Par des mesures de couplage électron-phonon analogues à celles que nous avons faites, Xie et al. avaient déjà mis en évidence un couplage piézoélectrique dans le $\mathrm{Si}_{0,8} \mathrm{Ge}_{0,2}$. Cependant ces mesures étaient faites à température élevée, dans un régime où le couplage piézoélectrique n'est pas dominant. De plus, ils ont obtenu une valeur très élevée de la constante piézoélectrique $\left(\left|e_{p z}\right|=1,6 \times 10^{-2}{\mathrm{C} . \mathrm{m}^{-2}}^{-2}\right.$, contestée par les mesures de Braithwaite et al. qui n'ont pas pu mesurer de terme piézoélectrique [251]. L'étude que nous avons faite constitue donc une confirmation qu'il existe un effet piézoélectrique dans les puits quantiques $p$-SiGe. Par rapport aux mesures précédentes [305, 308, 306], les mesures que nous avons faites ont été réalisées à plus basse température, température à laquelle l'effet du couplage piézoélectrique est dominant, et en tenant compte de la diffusion de chaleur vers les contacts. La constante de couplage que nous avons obtenue, $\left|e_{p z}\right|=(3,4 \pm 0,9) \times 10^{-3} \mathrm{C} . \mathrm{m}^{-2}$, est un facteur 5 en-dessous de la valeur obtenue par Xie et al. [305], mais est en meilleur accord avec les calculs présentés par Braithwaite et al. [251], qui donnent $e_{p z}=6,6 \times 10^{-3}$ C.m ${ }^{-2}$ pour le $\mathrm{Si}_{0,85} \mathrm{Ge}_{0,15}$ totalement ordonné.

L'existence d'un effet piézoélectrique dans le SiGe est encore très controversé, et on peut évoquer plusieurs raisons qui peuvent conduire à la brisure de la symétrie d'inversion dans les puits quantiques $p$-SiGe, et donc conduire à un effet piézoélectrique. Dans les couches de SiGe contraintes, et contrairement au SiGe massif, les paramètres de maille dans le plan du puits quantique et perpendiculairement à ce plan sont différents. Ces contraintes peuvent, selon les conditions de croissance, conduire à un ordre dans la couche de SiGe. Ainsi Ourmazd et Bean ont montré, sur des superréseaux Si/SiGe, qu'un recuit à température modérée $\left(450^{\circ} \mathrm{C}\right)$, puis un refroidissement lent, peut conduire à un ordre à courte distance [309]. Ils interprètent leur résultat par une transition ordre-désordre dont la température de transition est trop basse et la cinétique trop lente pour être observée 
dans le matériau massif, mais ces paramètres sont rendus plus favorables dans le cas de couches contraintes, la contrainte stabilisant la phase ordonnée. La structure proposée pour interpréter leurs résultats de diffraction d'électron est une structure diamant, avec un doublement de la période dans la direction $\langle 111\rangle$, conduisant à une alternance de plans SiSi-Ge-Ge-Si-Si-etc. En se basant sur des mesures de diffraction des rayons X, Tischler et al. ont réfutés cette structure et ont proposé une structure plus complexe [310]. La structure exacte d'une couche de SiGe contrainte n'est toujours pas tranchée, et le mécanisme conduisant à cet ordre non-plus (voir références dans [310]). Il est cependant essentiel de remarquer qu'un ordre peut exister dans ces couches, et donc cela peut conduire à un effet piézoélectrique. Outres les mesures de chauffage de porteurs [305, 308, 306], des mesures de spectroscopie acoustique [308, 311] sont venues confirmer la présence d'un effet semblable à un effet piézoélectrique dans des couches contraintes de $\operatorname{Si}_{1-x} \mathrm{Ge}_{x}$ (avec $x=0,2$ et 0,12$)$.

Dans notre cas, il n'est cependant pas clair que l'effet d'un ordre atomique soit dominant. En effet la température de croissance, choisie afin d'obtenir une mobilité élevée [246], est assez élevée $\left(800^{\circ} \mathrm{C}\right)$. Ces conditions de croissance ne sont donc pas favorable à l'obtention d'un ordre dans la couche contrainte de SiGe. Par contre, l'asymétrie du potentiel de confinement peut également briser la symétrie d'inversion dans les puits quantiques SiGe. Cet effet a été évoqué par Prus et al. pour interpréter un exposant 3 dans des mesures de couplage électron-phonon dans des MOSFETs Si [312], et peut se retrouver dans nos mesures.

\section{Écrantage faible}

L'écrantage conduirait à des exposants supérieurs à 3 et 5 pour, respectivement, le couplage piézoélectrique et le couplage par déformation. Les mesures montrent donc que, au moins à basse densité et à basse température pour le terme piézoélectrique, il n'y a pas d'écrantage. Cette observation est inattendue dans ces systèmes à très basse température car les phonons impliqués dans le couplage sont alors des phonons de faible vecteur d'onde. Cependant, l'écrantage faible est toujours observé dans les hétérostructures $p$-SiGe [305, 306] et les MOSFETs Si [312, 313, 314, 315, 316].

D'autre part, dans la gamme de densités $p_{c}<p_{s}<1,6 \times 10^{15} \mathrm{~m}^{-2}$, le produit $q l_{e}$ est compris entre 0,02 et 0,3 . Dans ce régime, on s'attend à observer un régime d'écrantage dynamique [317, 318] (voir §A.2.2), avec des exposants 4 et 6 , respectivement, pour le couplage piézoélectrique et le couplage par déformation. Ce mécanisme pourrait expliquer l'augmentation de l'exposant du couplage par déformation observé à haute densité de porteurs dans nos mesures. Cependant ce régime devrait également conduire à un changement sur le couplage piézoélectrique, ce qui n'est pas observé dans nos mesures.

\section{Dépendances en densité}

Il est important de remarquer que la dépendance en densité de porteurs des paramètres de couplage suit une loi proche de $\left(p_{s}\right)^{-3 / 2}$, attendue dans la théorie du couplage électronphonon. Aucune variation brutale des paramètres n'est observée à la densité critique de la transition métal-isolant. Ces mesures confirment la description du système SiGe dans 
le cadre du liquide de Fermi, déjà évoqué dans l'analyse des dépendances en température de la résistivité (voir chapitre 5 et Senz et al. [156]).

Dans la phase isolante (régime de conduction par sauts entre états localisés), on a montré que la température effective déduite de la mesure de résistivité à champ magnétique nul conduit à l'observation de lois de couplage électron-phonon en accord avec la théorie. Cette observation permet de justifier à postériori que la résistivité ne dépend en fait que de la température effective des porteurs, comme on l'attend pour un mécanisme de sauts assistés par l'interaction électron-électron. Cette idée a déjà été évoquée par Marnieros et al. dans des isolants d'Anderson 3D [280], et par Gershenson et al. dans des hétérostructures $n$-GaAs [281]. De plus cet effet des interactions est bien attendu dans notre système en raison de la valeur élevée du paramètre $r_{s}$. Cependant, le fait que la description en électrons indépendants localisés reste valable montre également que le désordre reste dominant dans ce système.

\subsection{Dépendances en champ électrique expliquées par le chauffage}

Cette analyse des dépendances en champ électrique en terme de chauffage des porteurs semble surprenante en regard des interprétations évoquées dans le cadre de la transition métal-isolant. Les lois d'échelle observées sur la résistivité dans les expériences sur la transition métal-isolant constituent un argument fort en faveur de l'existence d'une transition de phase quantique dans ces systèmes (voir §2.4.1). Dans les échantillons $p$-SiGe étudiés ici, nous verrons que notre analyse en terme de chauffage des porteurs est compatible avec l'observation de lois d'échelle en champ électrique. Dans la phase basse densité, les fortes non linéarités des courbes $I-V$ peuvent suggérer l'interprétation en terme d'effet du champ électrique sur le transport (voir le chapitre 4). Nous montrons ici aussi que les effets de chauffage peuvent également conduire à de très fortes non linéarités.

\subsubsection{Observation de lois d'échelle sur la résistivité}

\section{Lois d'échelle dans $p$-SiGe}

L'existence de lois d'échelle sur la résistivité dans les puits quantiques $p$-SiGe a été évoquée par Senz et al. [141], et une même analyse peut être faite sur nos mesures. Pour construire les courbes des figures 6.7 et 6.8 , nous avons procédé de la même manière que le décrivent Kravchenko et al. [5,67] (voir §2.4.1). Pour la loi d'échelle en température, il est nécessaire d'éliminer de la loi d'échelle les points à basse température en raison de la valeur finie du champ électrique, et les points à haute températures correspondant à de faibles longueurs (limitées par une longueur microscopique). Pour la loi d'échelle en champ électrique, les points à faible champ électrique sont exclus en raison de la température finie, et les points à fort champ électrique en raison d'une longueur microscopique. On peut enfin noter que ces coupures sont empiriques car la théorie d'échelle ne donne pas directement ces valeurs. Les figures 6.7 et 6.8 montrent donc que la résistivité suit des lois d'échelle qui peuvent être raisonnablement interprétées par l'approche d'une transition de 
phase quantique, avec des exposant critiques $\nu \approx 1,6$ et $z \approx 1,4$ en accord avec d'autres résultats (voir le tableau 2.2).

\section{Imitation des lois d'échelle par le chauffage}

On a vu au paragraphe 2.4.1 que, suffisamment près d'une transition de phase quantique continue, et pour une température et un champ électrique non nuls, la résistivité doit présenter un comportement d'échelle :

$$
\rho\left(\delta_{n}, T, E\right)=f\left(\frac{\delta_{n}}{T^{1 / \nu z}}, \frac{\delta_{n}}{E^{1 / \nu(z+1)}}\right),
$$

avec $\delta_{n}=\left(p_{s}-p_{c}\right) / p_{c}$ l'écart à la transition, $\nu$ et $z$ les exposants critiques de longueur de corrélation et dynamique. On a également vu que l'origine de ces lois d'échelle est liée au fait que les longueurs de corrélation $\xi_{\tau}$ et $\xi$ sont alors limitées respectivement par les longueurs associées à la température $L_{\tau} \sim 1 / T$ et au champ électrique $l_{E} \sim E^{-1 /(z+1)}$. D'autre part, la dimension temporelle est liée à la dimension spatiale par l'intermédiaire de l'exposant dynamique $z$. On peut donc définir la longueur "spatiale" liée à la température finie par $L_{\varphi} \sim L_{\tau}^{1 / z} \sim T^{-1 / z}$. Lorsque le champ électrique est suffisamment faible pour avoir $l_{E} \gg L_{\varphi}$, la seule longueur caractéristique est $L_{\varphi}$ et on s'attend à observer une loi d'échelle en température. Inversement lorsque $L_{\varphi} \gg l_{E}$, la seule longueur caractéristique est $l_{E}$ est on s'attend à observer une loi d'échelle en champ électrique.

Lorsque les électrons sont chauffés à une température supérieure à la température du réseau, la température qui intervient dans les lois d'échelle est la température du système électronique, donc la température $T_{e}$. Lorsqu'on applique un champ électrique élevé, la théorie du couplage électron-phonon donne une relation entre la température effective des porteurs et le champ électrique (voir §A.2) :

$$
T_{e} \propto E^{2 / \theta}
$$

dans la limite $T_{e} \gg T_{0}$ et dans le cas où on ne retient qu'une origine du couplage électronphonon, conduisant à un seul exposant $\theta$. Dans ces conditions, la loi d'échelle en température peut se réécrire :

$$
\rho\left(p_{s}, E\right)=f\left(\frac{\delta_{n}}{T_{e}^{1 / \nu z}}\right)=g\left(\frac{\delta_{n}}{E^{2 / \theta \nu z}}\right) .
$$

On remarque donc que, en présence de chauffage des porteurs, la loi d'échelle en température, exprimée en fonction de la nouvelle variable champ électrique, peut être vue comme une réelle loi d'échelle en champ électrique, avec l'exposant $1 / \nu(z+1)=2 / \theta \nu z$. On montre ainsi que, s'il existe une loi d'échelle en température, le chauffage conduit à une loi d'échelle en champ électrique.

\section{Etude des lois d'échelle malgré le chauffage}

On vient donc de voir que les effets de chauffage peuvent induire une loi d'échelle apparente en champ électrique. En fait, si une transition de phase quantique est réellement 

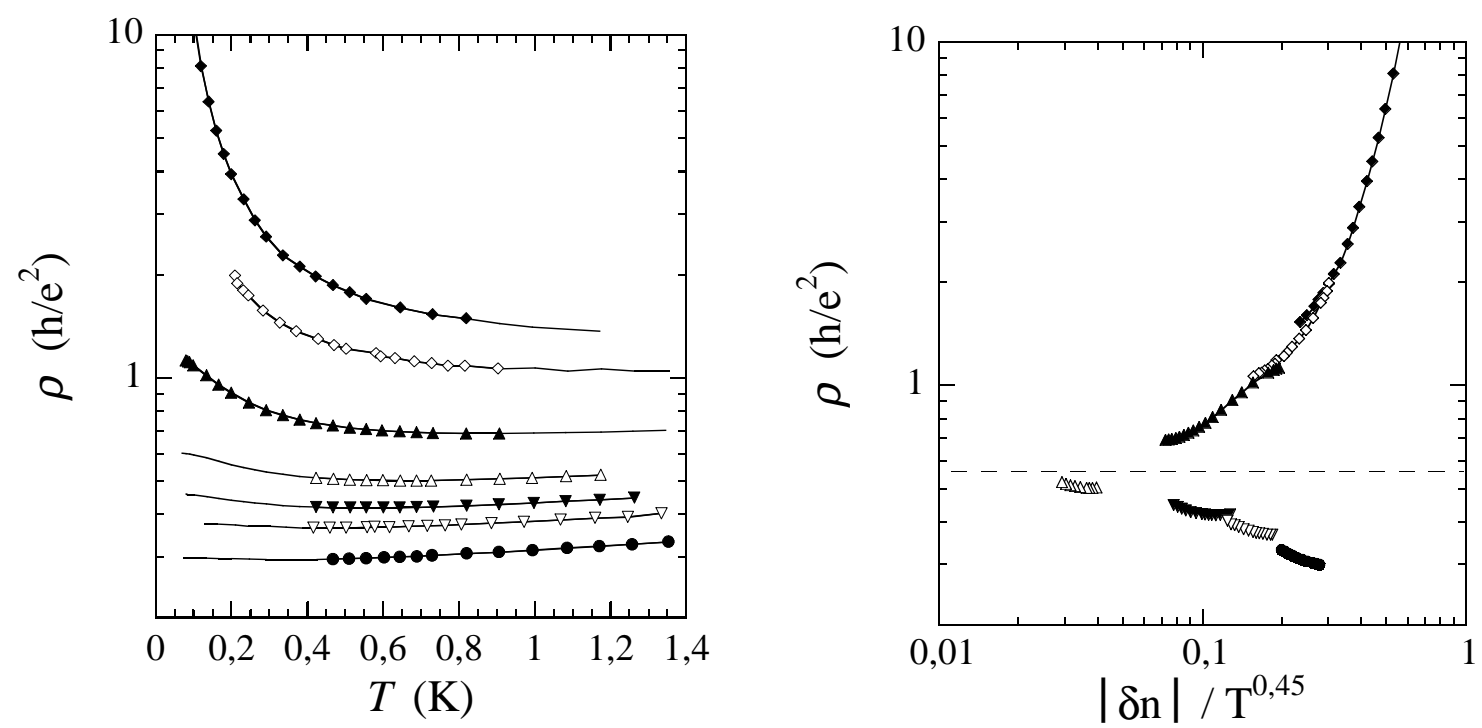

FIG. 6.7: Gauche : Résistivité en fonction de la température pour un puits quantique $p$-SiGe, et pour plusieurs densités (de haut en bas) : $1,01-1,08-1,19-1,38-1,45-1,56 \times 10^{15} \mathrm{~m}^{-2}$. Droite : Lois d'échelle obtenue à partir des courbes de la figure de gauche. Les lignes représentent l'ensemble des données expérimentales, les symboles représentent uniquement les points qui sont inclus dans la loi d'échelle de la figure de droite. La ligne horizontale représente la résistivité critique $\rho_{c}$.


FIG. 6.8: Gauche : Résistivité en fonction du champ électrique pour un puits quantique $p$-SiGe, et pour plusieurs densités. Droite : Lois d'échelle obtenue à partir des courbes de la figure de gauche. Les lignes représentent l'ensemble des données expérimentales, les symboles représentent uniquement les points qui sont inclus dans la loi d'échelle de la figure de droite. La ligne horizontale représente la résistivité critique $\rho_{c}$. 
présente dans un système, on a vu au paragraphe 2.4.1 qu'on s'attend à observer une loi d'échelle en température et en champ électrique. Ainsi les effets de chauffage peuvent conduire à un effet masquant la loi d'échelle réelle en champ électrique. Dans ce paragraphe nous allons voir s'il est possible de distinguer un effet de chauffage d'une loi d'échelle (figure $6.9)$.

La première méthode peut consister à comparer les exposants critiques obtenus. En effet, l'exposant intervenant dans la loi d'échelle en champ électrique $(1 / \nu(z+1))$ et celui intervenant dans le chauffage $(2 / \theta \nu z)$ peuvent être différents. Cependant cet argument ne permet pas de distinguer les deux effets si $\theta=2(z+1) / z$, soit environ 4 pour $z=1$. Dans le cas du $p$-SiGe, présentant deux exposants 3 et 5 , ce critère est encore plus difficile à appliquer, et ne permet donc pas de conclure.

On peut également utiliser une méthode plus rigoureuse, qui consiste à comparer les longueurs $L_{\varphi}\left(T_{e}\right) \equiv L_{\varphi}(E)$ et $l_{E}(E)$ intervenant dans les lois d'échelle. Les exposants respectifs du chauffage et des lois d'échelle permettent de connaître le comportement asymptotique des dépendances de ces deux longueurs en fonction du champ électrique $E$. La figure 6.9 représente la variation de $L_{\varphi}(E)$ déduite des mesures expérimentales dans le $p$-SiGe, en unité arbitraire car les théories d'échelle ne permettent pas de déduire la valeur de cette longueur dans l'absolu. Sur le même schéma, on peut indiquer la dépendance en $E$ de $l_{E}(E)$, en remarquant que $l_{E}>L_{\varphi}$ dans la gamme de champ électrique utilisée dans nos expériences car toute la dépendance en champ électrique provient du chauffage des porteurs. Si maintenant on extrapole ces courbes à forts champ électrique, et on remarque que, en raison des pentes différentes, $l_{E}(E)$ croise $L_{\varphi}(E)$ à suffisamment fort champ électrique, et donc que, si la loi d'échelle en champ électrique existe, elle doit devenir prépondérante à suffisamment fort champ électrique. Dans nos mesures, les effets électrostatiques liés à la présence de la grille ne nous ont pas permis d'augmenter le champ électrique jusqu'à cette limite. Pour espérer observer une éventuelle loi d'échelle, on peut également envisager des mesures sur des systèmes présentant un meilleur couplage électron-phonon, comme les MOSFETs $\mathrm{Si}$, ce qui permet de décaler la courbe $L_{\varphi}(E)$ vers la droite et donc de diminuer le champ électrique limite (voir la figure 6.9. Enfin nous verrons plus loin que l'utilisation de la diffusion de chaleur vers les contacts permet également de réduire les effets de chauffage, et ainsi d'explorer si le système présente effectivement une réelle loi d'échelle.

\subsubsection{Fortes non linéarités de la caractéristique $I-V$}

La figure 6.10 de droite montre que, à basse densité, la caractéristique $I-V$ est fortement non-linéaire, et pourrait être interprétée comme dû au décrochage d'une phase collective (voir §4.3). Nous avons donc montré que ces fortes non linéarités sont dues au chauffage des porteurs, associé à une forte dépendance en température de la résistivité. Pour aller plus loin, nous étudions le champ seuil qui peut être déduit de ces mesures, et nous allons montrer que les dépendances de ce champ seuil en fonction de la température ne sont pas compatibles avec le décrochage d'une phase collective, en donnant une méthode permettant de différencier par là les deux effets.

Pour déterminer la tension seuil, on a utilisé deux méthodes. La première consiste à ajuster les courbes $I-V$ à faible courant par une loi de puissance empirique, $I=A V^{\alpha}$, 




FIG. 6.9: Longueurs caractéristiques intervenant dans les lois d'échelle en dimension finie dans une transition de phase quantique. La longueur liée à la température, $L_{\varphi}$, est déduite de la température effective mesurée dans l'échantillon $p$ SiGe pour $p_{s}=1,19 \times 10^{15} \mathrm{~m}^{-2}(p$ $\mathrm{SiGe}$ ), ou des mesures de chauffage dans les MOSFETs Si [313], rapportées à la densité $10^{15} \mathrm{~m}^{-2}$. La dépendance en champ électrique de $l_{E}$ est donné par l'exposant $\nu(z+1)$.

puis à déterminer à partir de quelle valeur de la tension $V=V_{s, r}$ les points expérimentaux s'écartent de cette loi de puissance (voir la figure 6.10 de gauche). En pratique, la tension seuil $V_{s, r}$ est la tension pour laquelle la différence des courants devient supérieure à 10 \%. Dans les ajustements que nous avons effectués, l'exposant $\alpha$ est toujours proche de 1 (à moins de $5 \%$ près), ce qui signifie que la partie bas courant des courbes est linéaire, et que la tension seuil représente la fin du régime linéaire. Des méthodes analogues ont été couramment employées pour l'étude du décrochage d'ondes de densité de charge et du cristal de Wigner pour des électrons sur l'hélium (voir Grüner [179] et Dahm [180]). Dans une autre approche, la courbe $I-V$ à fort courant est ajustée par une loi linéaire, $I=B+C V$, et la tension seuil $V_{s, e}$ est l'abscisse à l'origine de cette droite (voir la figure 6.10 de droite). Cette approche est donc opposée à la précédente puisqu'elle utilise le régime d'écoulement de la phase collective. Cette méthode a été plusieurs fois employées pour l'analyse des non linéarités dans les hétérostructures (voir par exemple Shashkin et al. [81]).

A partir des tensions seuil et de la longueur du système, on déduit les champ électriques seuils $E_{s, r}$ et $E_{s, e}$, présentés en fonction de la température pour différentes densités électroniques dans la figure 6.11. $E_{s, r}$ décroît quand la température diminue, contrairement à ce qu'on attend dans les modèles classiques de décrochage (voir Grüner [179]), et $E_{s, e}$ croît quand la température diminue. On peut montrer que ces comportements sont facilement compréhensibles dans le cadre du chauffage des porteurs. On suppose pour cela que la dépendance "isolante" de la résistivité en fonction de la température peut s'écrit $\rho(T) \propto \exp \left(T_{0} / T\right)$, et on choisit une puissance dissipée par porteur par le couplage électron-phonon de la forme $P_{\text {diss }}=A\left(T_{e}^{\theta}-T^{\theta}\right) .{ }^{4}$ Nous ne considérons ici que la première

\footnotetext{
${ }^{4}$ Pour simplifier, on ne considère qu'un seul couplage, qui pourra être le couplage piézoélectrique dans la limite basse température effective, et le couplage par déformation dans la limite température effective élevée.
} 

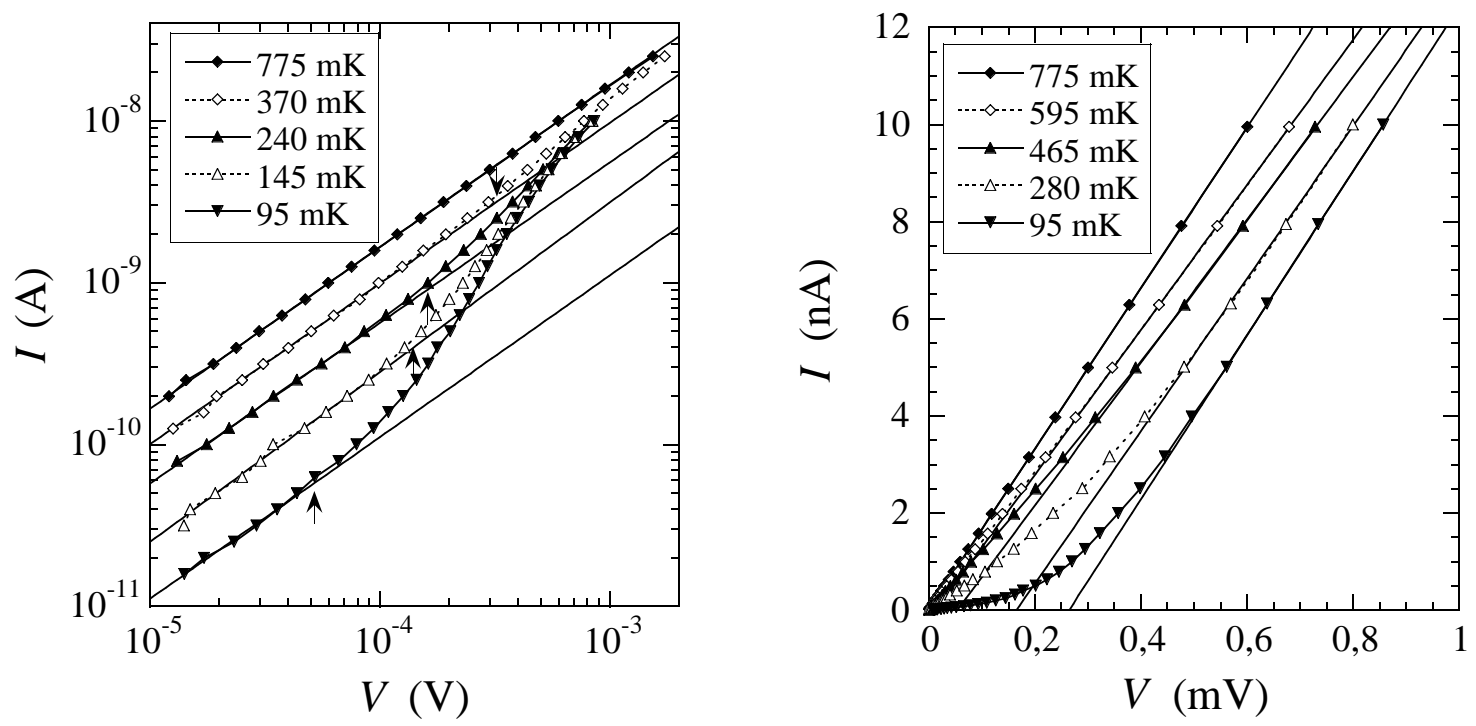

FIG. 6.10: Courant en fonction de la tension appliquée pour l'échantillon SiGe B137 à une densité $p_{s}=0,6 \times 10^{15} \mathrm{~m}^{-2}$ et à différentes température. Gauche : ajustement de la partie faible courant par une loi $I=A V^{\alpha}$, les flèches indiquent la tension seuil. Droite : ajustement de la partie fort courant par une loi $I=B+C V$.
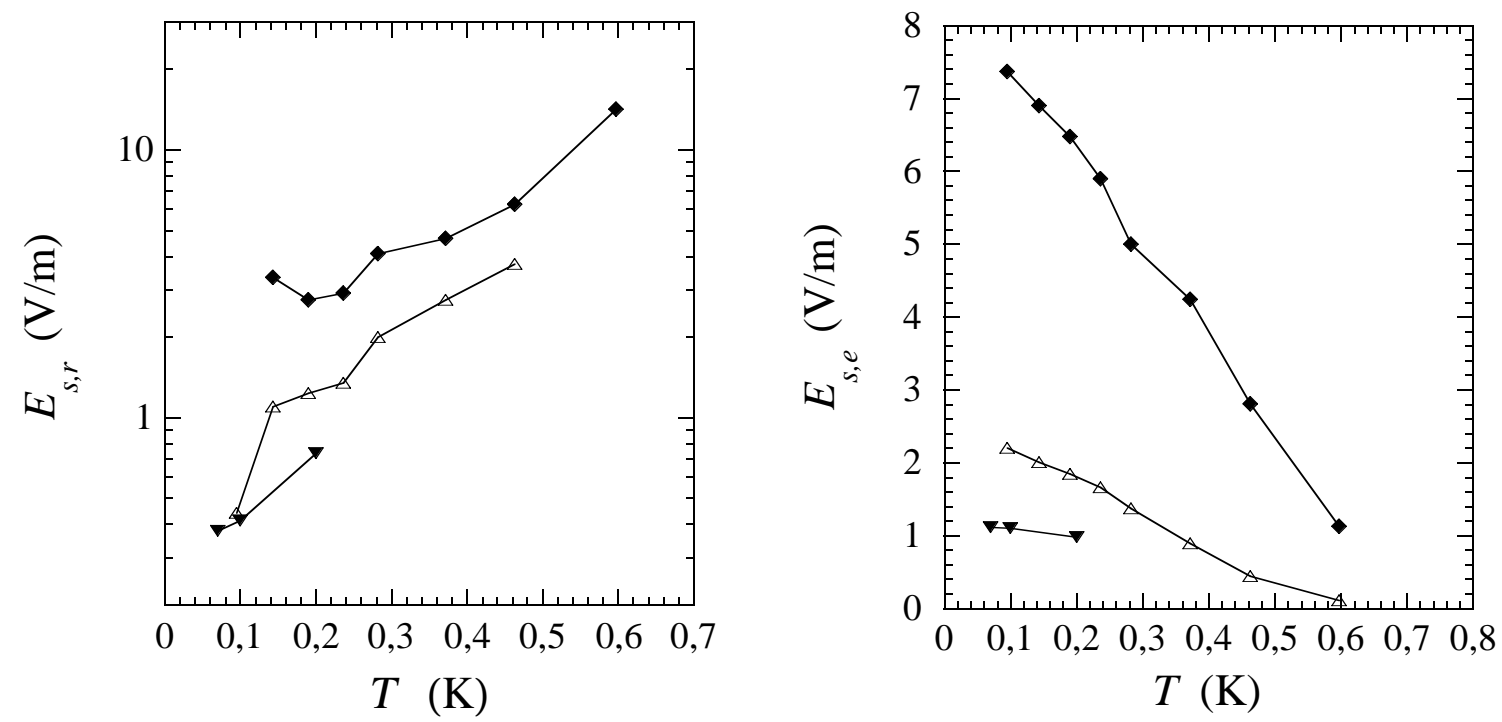

FIG. 6.11: Variation du champ électrique seuil, déterminées par les deux méthodes décrites dans le texte, en fonction de la température, pour l'échantillon B137 à $p_{s}=0,5 \times 10^{15} \mathrm{~m}^{-2}(\checkmark)$ et $0,6 \times 10^{15} \mathrm{~m}^{-2}(\Delta)$, et pour l'échantillon B509 à $p_{\times} 10^{15} \mathrm{~m}^{-2}(\boldsymbol{\nabla})$. 
manière de mesurer le champ seuil, $E_{s, r}$. Il est déterminé par :

$$
\frac{I\left(E_{s, r}\right)-I_{r e p t}}{I_{r e p t}}=\frac{\rho(T, E=0)-\rho\left(E_{s, r}\right)}{\rho\left(E_{s, r}\right)}=0,1 \quad \Rightarrow \quad \rho\left(E_{s 1, r}\right)=\frac{\rho(T, E=0)}{1,1},
$$

où $I_{\text {rept }}$ est le courant attendu pour le régime linéaire. On montre ainsi que le champ seuil s'écrit :

$$
E_{s, r}^{2} \propto T^{\theta+1} \rho(T)
$$

Ainsi $E_{s, r}$ est une fonction croissante de $T$ pour $T>T_{0} /(\theta+1)$, et est une fonction décroissante de $T$ pour $T<T_{0} /(\theta+1)$. Pour $p_{s}=0,5 \times 10^{15} \mathrm{~m}^{-2}$, on a déterminé $T_{0} \approx 0,7 \mathrm{~K}$, et avec $\theta=3$ à basse température, on obtient $T_{0} /(\theta+1) \approx 0,18 \mathrm{~K}$. Les dépendances en température de $E_{s, r}$ dans la figure 6.11 sont donc bien en accord avec des non-linéarités dues au chauffage des porteurs.

\subsubsection{La diffusion de chaleur pour l'étude des non-linéarités}

Nous avons donc vu jusqu'à présent que, dans les puits quantiques $p$-SiGe que nous avons étudiés, les dépendances en champ électriques sont entièrement dues au chauffage des porteurs. Dans ce cas, les éventuelles dépendances en champ électriques liées à d'autres effets sont masquées par les effets de chauffage. Dans ce paragraphe est proposée une méthode technologique permettant de réduire les dépendances en champ électrique liées au chauffage, et ainsi de tester l'existence ou non des autres dépendances en champ électrique.

La méthode envisagée repose sur l'utilisation du refroidissement par les contacts. L'utilisation d'un nombre important de contacts froids le long de la barre permet une utilisation de type "radiateur" (voir la figure 6.12 en bas). L'efficacité d'un tel dispositif a été tester numériquement par la résolution de l'équation de la chaleur (voir Annexe B). Sur la figure 6.12 sont comparés les profils de température dans une géométrie sans contacts de refroidissement et avec les contacts de refroidissement, montrant qualitativement le réduction de température dans une telle géométrie.

Un certains nombre de précautions sont à prendre pour que cet effet soit effectif. Dans un premier temps il est nécessaire que la présence des contacts ne perturbe pas les lignes de champ électrique dans la barre de Hall. Pour cela il est nécessaire d'utiliser des contacts de largeur la plus faible possible, et le facteur limitant est alors d'ordre technologique. D'autre part les contacts doivent présenter une bonne conductivité thermique, et donc une densité de porteurs élevée. Enfin il est important de relier les contacts à des réservoirs de température, comme des contacts métalliques.

\subsection{Conclusion}

Nous avons donc étudié les dépendances en champ électrique de la résistivité de gaz bidimensionnels de trous dans des puits quantiques $\mathrm{SiGe/Si}$. Nous avons montré que ces dépendances peuvent être entièrement expliquées par le chauffage des porteurs par le champ électrique. Cette étude nous a permis de déduire la nature du couplage électronphonon dans le $\mathrm{SiGe}$, avec un terme de déformation et un terme piézoélectrique. Notre 


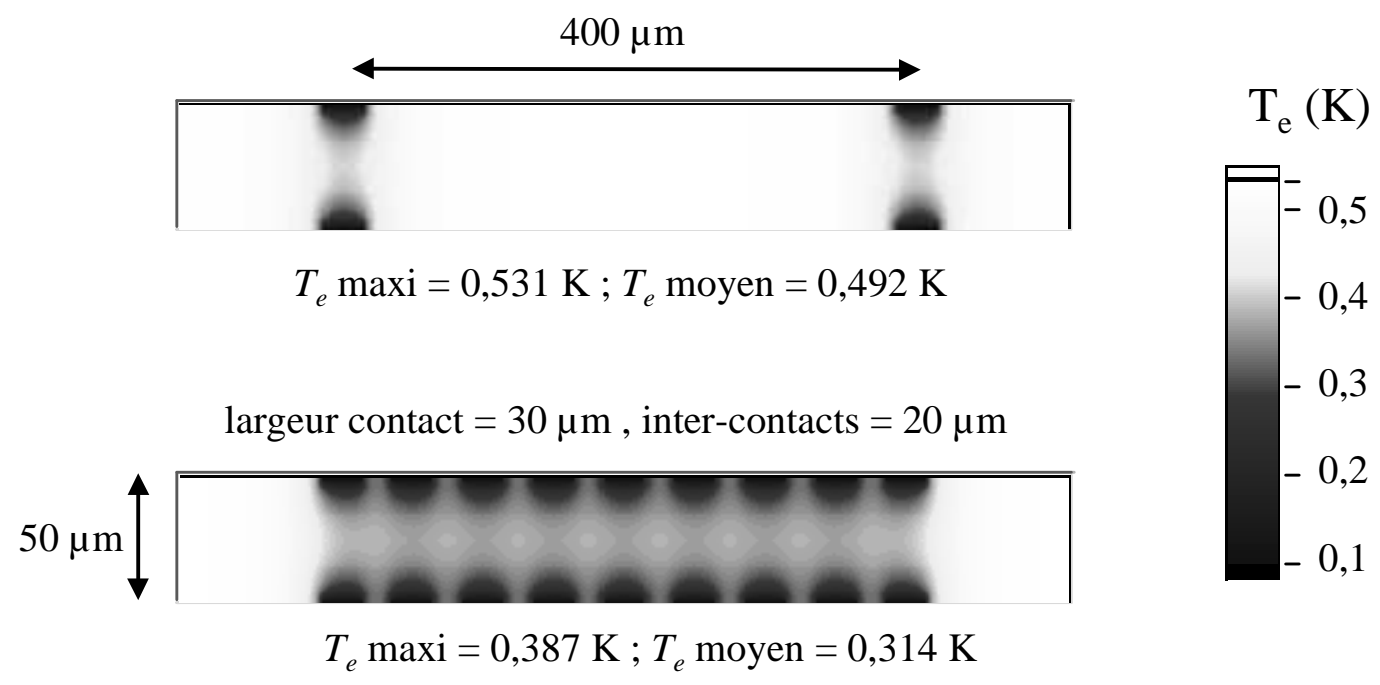

FiG. 6.12: Profil de température dans deux géométries différentes, en haut une barre de Hall conventionnelle, en bas la géométrie "radiateur" comportant un nombre important de contacts "froids". Résultat de simulation de diffusion de chaleur avec les paramètres correspondant au $p$-SiGe à une densité de $p_{s}=1,38 \times 10^{15} \mathrm{~m}^{-2}$ et une température de réseau $T_{0}=0,1 \mathrm{~K}$.

expérience à basse température montre ainsi l'existence du couplage piézoélectrique dans les gaz bidimensionnels de trous dans SiGe. On montre également que le potentiel de déformation est compatible avec les valeurs obtenues auparavant, et on calcul avec la constante piézoélectrique.

Cependant cette conclusion n'exclue pas d'autres effets de champ électrique dans ce système, mais qui sont masqués ici par les effets de chauffage. Il est ainsi possible d'envisager une géométrie particulière en "radiateur", où on utilise la diffusion de chaleur vers les contacts pour réduire les effets de chauffage. Cette méthode pourrait permettre de tester l'existence ou non d'effets de champ électriques dus à des lois d'échelle ou à une phase corrélée à basse densité. 
Chapitre 6. Transport non-ohmique dans les puits quantiques p-SiGe 


\section{Chapitre 7}

\section{Transport non-ohmique dans les puits quantiques $p$-GaAs}

\section{Sommaire}

7.1 Introduction . . . . . . . . . . . . . 170

7.2 Etude des effets de chauffage dans les puits quantiques $p$-GaAs ................... 170

7.3 Effets collectifs à faible densité de porteurs . . . . . . . 173

7.3.1 Comparaison avec des modèles d'électrons localisés . . . . . 173

7.3 .2 Tension seuil . . . . . . . . . . . . . . . . . 173

7.4 Conclusion $\ldots \ldots \ldots \ldots \ldots \ldots \ldots \ldots \ldots \ldots \ldots \ldots$ 


\subsection{Introduction}

Nous avons vu au chapitre 5 que les mesures de transport en fonction de la température dans les puits quantiques $p$-GaAs ne s'expliquent pas entièrement par les théories d'électrons sans interactions. C'est particulièrement le cas à très basse densité, où une interprétation possible des mesures est la formation d'une phase fortement corrélée de type cristal ou verre de Wigner. Pour confirmer une telle interprétation, nous avons étudié les dépendances en champ électrique de la résistivité. Nous avons tout d'abord étudié l'influence du chauffage des porteurs sur les non-linéarités de la caractéristique $I-V$. Ce modèle permet d'expliquer les effets de champ électrique à haute densité de porteurs (voir §7.2). Par contre, à basse densité de porteurs, on observe un écart par rapport au modèle de chauffage, qui peut s'interpréter comme un phénomène de décrochage d'une phase collective accrochée sur le désordre (voir $§ 7.3$ ).

\subsection{Etude des effets de chauffage dans les puits quantiques $p$-GaAs}

La figure 7.1 montre que la résistivité des échantillons $p$-GaAs présente une forte dépendance en champ électrique, comme il a déjà été remarqué par Yoon et al. [87] dans le même type d'échantillons, mais aussi dans les MOSFETs Si et les hétérostructures $p$-SiGe (voir le chapitre 6). L'étude sur les puits quantiques $p$-SiGe a montré que le chauffage des porteurs peut être important dans ce type de systèmes. Pour les mesures dans $p$-GaAs, nous avons commencé par étudier ces effets. Nous ne présentons pas ici une étude aussi complète que dans les puits quantique $p$-SiGe, cependant un certain nombre d'indications montrent qu'à haute densité de porteurs, les non-linéarités dans les puits quantiques $p$ GaAs peuvent être dues au chauffage des porteurs. La première indication est donnée par la comparaison qualitative des courbes de résistivité en fonction de la température et du champ électrique montrées dans la figure 7.1. Ces courbes présentent un comportement analogue quand la densité varie. La deuxième indication est donnée par la comparaison qualitative de la magnétorésistance à différentes températures et à différents champ électriques, comme le montre la figure 7.2. Le chauffage est le seul phénomène connu permettant d'expliquer la forte similarité des dépendances en température et en champ électrique de la totalité de la courbe de magnétorésistance, en particulier la résistivité à champ magnétique nul, la magnétorésistance négative à faible champ magnétique et les oscillations de Shubnikov-de Haas.

Pour compléter ces considérations qualitatives, nous avons évalué les effets de chauffage en étudiant la puissance dissipée dans l'hypothèse de chauffage, afin de la comparer aux études déjà réalisées dans ces systèmes. Pour $p_{s}<5 \times 10^{14} \mathrm{~m}^{-2}$, nous avons évalué la température effective des porteurs en comparant les dépendances en température et en champ électrique de la résistivité à champ magnétique nul (voir $\S$ A.3.1). Pour $p_{s}>5 \times 10^{14}$ $\mathrm{m}^{-2}$ (pour lesquelles $V_{G}<0$ ), la dépendance en température de la résistivité à champ magnétique nul est trop faible, et nous avons mesuré la résistivité à un champ magnétique de 0,2 T. La puissance injectée par porteur est ensuite donnée par $P_{E}=(V I) /\left(S p_{s}\right)$ ( $V$ est la tension, $I$ le courant, $S$ la surface et $p_{s}$ la densité de porteurs). Nous avons tracé sur la 

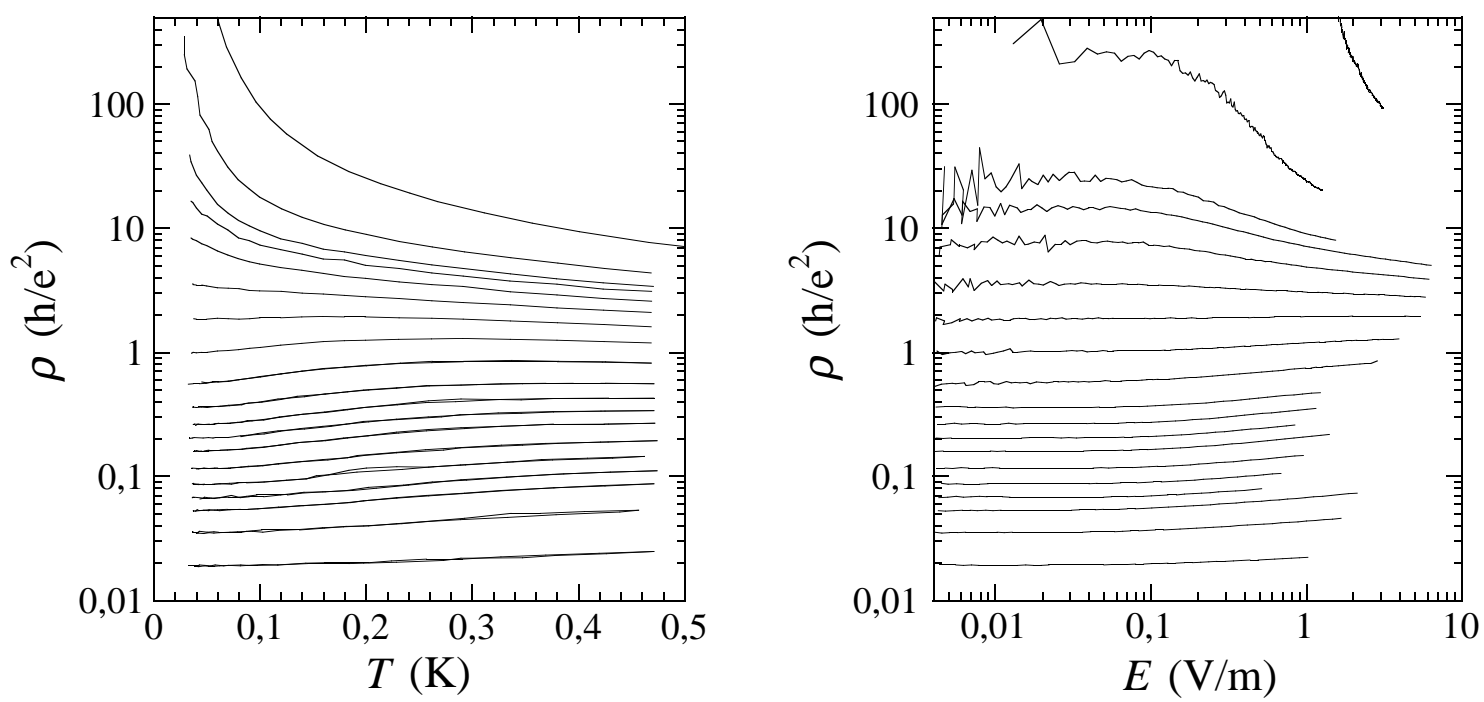

FIG. 7.1: Résistivité du puits quantique $p$-GaAs $\mathrm{Nu} 944$ en fonction de la température et du champ électrique, pour une densité de porteurs croissante (de haut en bas) : 1,30-1,39-1,43$1,44-1,48-1,52-1,57-1,63-1,74-1,86-1,96-2,06-2,16-2,31-2,46-2,61-$ $2,77-3,12-3,88 \times 10^{15} \mathrm{~m}^{-2}$. Gauche : Dépendances en température mesurées pour un champ électrique suffisamment faible (régime ohmique). Droite : Dépendance en champ électrique pour une température du réseau $T_{0}=35 \mathrm{mK}$.
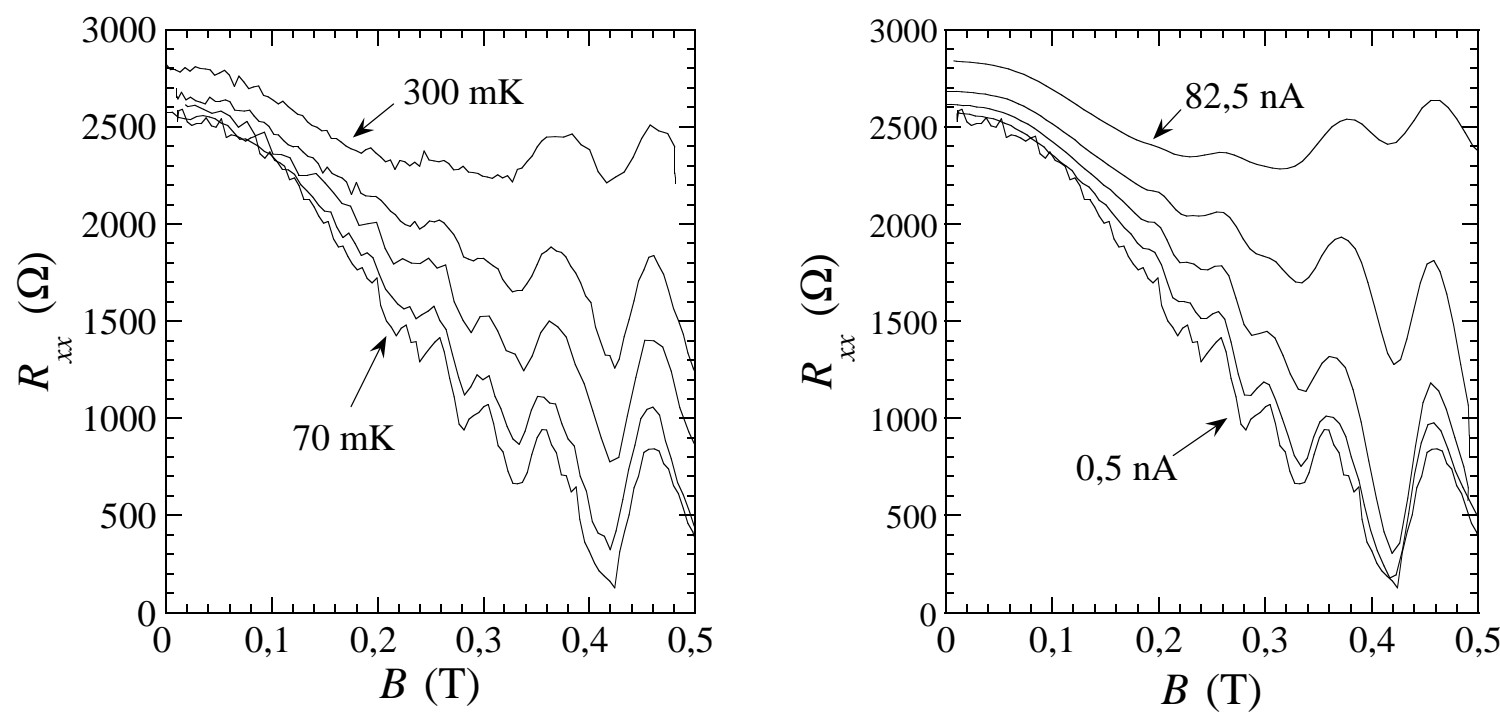

FIG. 7.2: Mesures de la résistance longitudinale $R_{x x}$ en fonction du champ magnétique sur une hétérostructure $p$-GaAs (échantillon Nu944-1) dans différentes conditions de température et de courant. Gauche : mesure à différentes températures (de haut en bas : $300-200-150-100-70$ $\mathrm{mK})$ et à faible courant $(I<0,8 \mathrm{nA})$. Droite : mesure à différents courants (de haut en bas : $82,5-27-13,05-6,65-0,5 \mathrm{nA})$ et à basse température $(T=70 \mathrm{mK})$. 



FIG. 7.3: Gauche : Puissance injectée pour une température effective de $100 \mathrm{mK}$, en fonction de la densité de porteurs, pour l'échantillon GaAs Nu944-1. La loi $p_{s}^{-3 / 2}$ est une indication de la dépendance attendue dans la théorie du couplage électron-phonon, et sont indiquées également les dépendances en densités attendues à partir des paramètres issus des résultats de Kent et al. [319] et à ceux de Gao et al. [320]. $p_{c}$ indique la densité à laquelle est observé le changement de comportement de la résistivité en fonction de la température ("transition métal-isolant"). Droite : Dépendance en température effective de la puissance injectée à $p_{s}=1,30 \times 10^{14} \mathrm{~m}^{-2}$ et $T_{0}=35 \mathrm{mK}$, montrant un loi de puissance proche de $T_{e}^{6}$ à haute température.

figure 7.3 de gauche la puissance effective donnant lieu à un chauffage des porteurs jusqu'à une température $T_{e}=100 \mathrm{mK}$, en fonction de la densité de porteurs. Quantitativement, si on compare nos points expérimentaux aux résultats obtenus pour le couplage électronphonon par Kent et al. à une densité $p_{s}=3,3 \times 10^{15} \mathrm{~m}^{-2}$ [319] et par Gao et al. à $p_{s}=1,2 \times 10^{15} \mathrm{~m}^{-2}[320]$, et extrapolés à nos valeurs de la densité en utilisant la loi $p_{s}^{-3 / 2}$ attendue pour le couplage électron-phonon, on remarque que nos mesures peuvent être interprétées par des effets de chauffage. On peut cependant remarquer que l'amplitude du couplage électron-phonon dans ce régime de température où la couplage piézoélectrique écranté domine n'est pas encore bien connue. La dépendance en densité de la puissance injectée est proche de la loi attendue $p_{s}^{-3 / 2}$ à densité élevée (voir la figure 7.3 de gauche).

A basse densité, on remarque un écart par rapport à la loi $p_{s}^{-3 / 2}$ : les points expérimentaux montrent une dépendance plus faible en fonction de la densité. Cette observation peut être interprétée de plusieurs manières. (a) La nature du couplage électron-phonon peut être modifiée par la transition vers un comportement fortement isolant à basse densité. (b) On peut également envisager d'autres effets conduisant à des non-linéarités plus fortes : s'il existe une autre source de non linéarités que l'effet de chauffage, l'hypothèse de chauffage utilisée pour déduire la température effective conduit, pour une puissance injectée donnée, à une température effective plus forte, ou pour une température effective donnée (comme il est considéré dans la figure 7.3) à une puissance injectée plus faible. (c) Enfin on peut également envisager que le nombre de porteurs participant effectivement 
au refroidissement diminue quand la densité diminue. Ce cas sera en particulier envisagé dans le chapitre 11 où les mesures de bruit seront interprétés par la présence d'une transition de percolation. Dans ce cas, pour une puissance injectée donnée, on s'attend à un chauffage plus important à basse densité de porteurs, ou pour une température effective donnée, on s'attend à une puissance injectée plus faible.

Une seconde indication qu'il existe un comportement qui diffère de la théorie attendue du couplage électron-phonon est donnée par la dépendance de la puissance injectée en fonction de la température effective $T_{e}$ à basse densité. Dans le $p$-GaAs et à suffisamment basse température, le couplage dominant est le couplage piézoélectrique écranté, qui doit donné une dépendance en $T_{e}^{5}$ de la puissance injectée. La figure 7.3 de droite montre que ce n'est pas le cas à basse densité, où une dépendance plus forte est observée, en $T_{e}^{6}$. Cette dépendance plus forte peu signifier que l'on sort des hypothèses du couplage électrons-phonon, ou qu'il existe une source de non-linéarités autre que le chauffage des porteurs.

\subsection{Effets collectifs à faible densité de porteurs}

En raison de la forte résistance à mesurer pour les faibles densités de porteurs, dans cette mesure on impose une tension et on mesure le courant à partir de la tension aux bornes d'une résistance de $100 \mathrm{k} \Omega$ ou $1 \mathrm{M} \Omega$ en série avec l'échantillon (voir le schéma $3.8)$.

\subsubsection{Comparaison avec des modèles d'électrons localisés}

Nous avons vu au paragraphe 4.2 que le transport dans les isolants d'Anderson est sensible au champ électrique. Dans le cas d'un fort champ électrique, la résistivité ne dépend plus de la température du réseau. On voit clairement sur la figure 7.4 de gauche que ce régime n'est pas atteint dans notre cas. A un champ magnétique intermédiaire, on s'attend à observer une loi du type (voir $§ 4.2)$ :

$$
\rho(T, E)=\rho_{0}(T, E=0) \exp \left(\frac{e E L}{k_{B} T}\right),
$$

où la longueur $L(T)$ dépend de la température. Dans la figure 7.4 de droite, nous avons tracé $\rho(T, E) / \rho_{0}(T, E=0)$ en fonction de $E / T$. On remarque alors que le logarithme de $\rho(T, E) / \rho(T, E=0)$ ne suit pas une loi linéaire en $E / T$, ce qui nous permet d'exclure ce modèle. On peut de plus remarquer que la dépendance en température de la résistivité $\rho(T, E=0)$ suit une loi simplement activée (à cette densité, voir §5.4.3), qui n'est donc pas compatible avec les lois de sauts à portée variable : on ne s'attend donc pas à ce que ce modèle soit valable dans ce cas.

\subsubsection{Tension seuil}

Les très fortes non-linéarités de la caractéristique $I-V$ peuvent également être interprétées par une tension seuil dans le mécanisme de transport. Dans un système d'électrons 

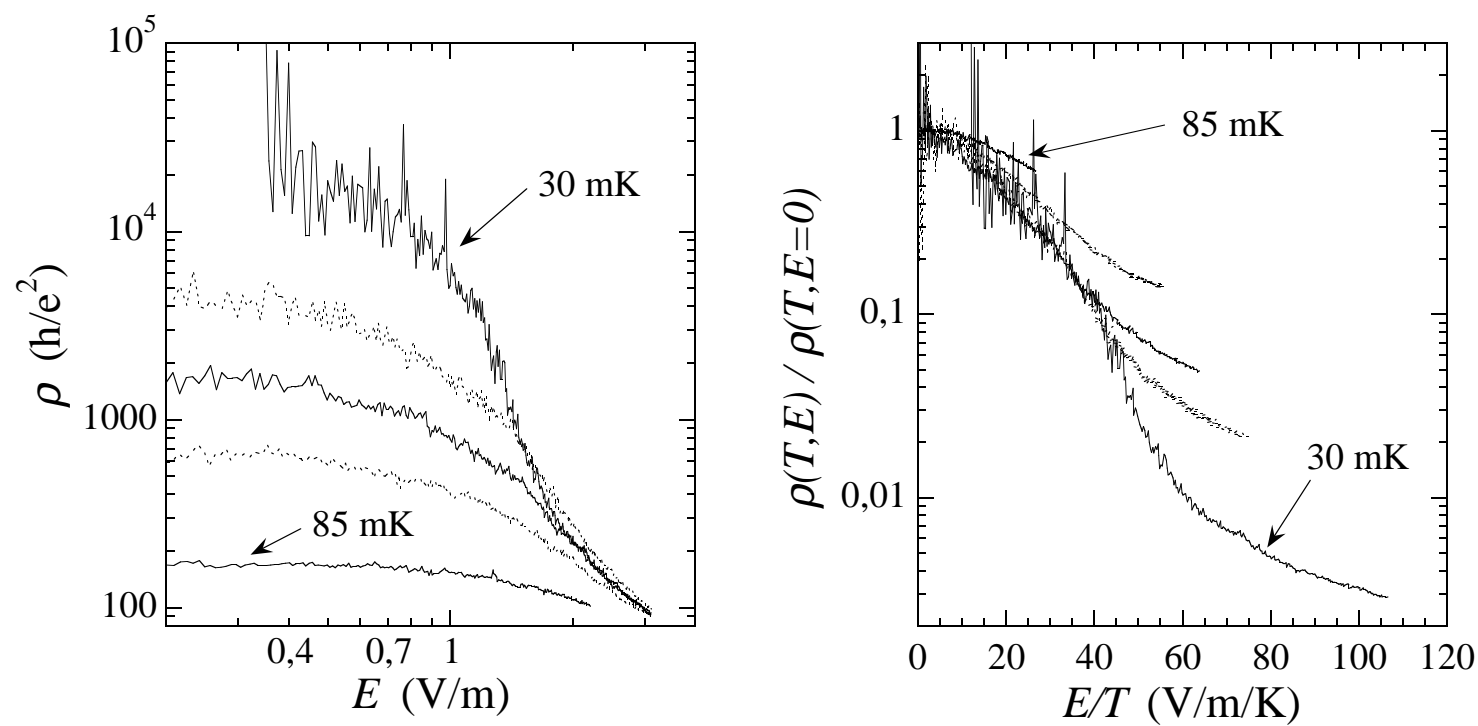

FIG. 7.4: Gauche : Dépendance en champ électrique de la résistivité à une densité $p_{s}=1,30 \times 10^{14}$ $\mathrm{m}^{-2}$, et pour différentes températures du réseau (de haut en bas, $T=30-40-50-55-85$ $\mathrm{mK})$. Droite : Dépendance du rapport entre la résistivité à champ électrique fini et la résistivité à champ électrique nul en fonction du rapport entre le champ électrique et la température, à une densité $p_{s}=1,30 \times 10^{14} \mathrm{~m}^{-2}$ et pour différentes températures du réseau (de haut en bas, $T=85-55-50-40-30 \mathrm{mK})$.

fortement corrélés, cette tension seuil est due à l'accrochage de la phase fortement corrélée (cristal ou verre de Wigner) sur le désordre (voir §4.3).

Pour déterminer la tension seuil, nous avons utilisé deux méthodes (ces méthodes on déjà été présentées pour les puits quantiques $p$-SiGe, au §6.3). La première méthode consiste à ajuster les courbes $I-V$ à faible courant par une loi de puissance empirique, $I=A V^{\alpha}$, puis à déterminer à partir de quelle valeur de $V=V_{s, r}$ les points expérimentaux s'écartent de cette loi de puissance (voir la figure 7.5 de gauche). Cette approche, qui prend en fait en compte le régime de "reptation" du cristal (voir la figure 4.4) pour les modèles classiques, donne une dépendance en courant de la forme (voir §4.3) :

$$
I \propto V \cdot \exp \left[-\frac{T_{0}}{T}\left(\frac{V}{V_{s}}\right)^{-\mu}\right]
$$

On s'attend donc à trouver un exposant $\alpha$ proche de 1 , mais qui peut être supérieur en raison de la contribution de la tension $V$ dans l'exponentielle. Avec un exposant 1, cette mesure revient à identifier la fin du régime linéaire, comme il a été employé par Goldman et al. [51]. On peut cependant remarquer que d'autres approches ont montré une dépendance différente dans ce régime (voir la thèse de Perruchot [38] pour une discussion de ce critère). Un exemple de détermination de cette tension seuil est donné dans la figure 7.5 de gauche. Le seconde méthode consiste à ajuster la courbe $I-V$ à fort courant par une loi linéaire, $I=B+C V$, et la tension seuil $V_{s, e}$ est l'abscisse à l'origine de cette droite (voir la figure 7.5 de droite). Cette approche est donc opposée à la précédente en ce sens qu'elle utilise le régime d'écoulement de la phase collective. 

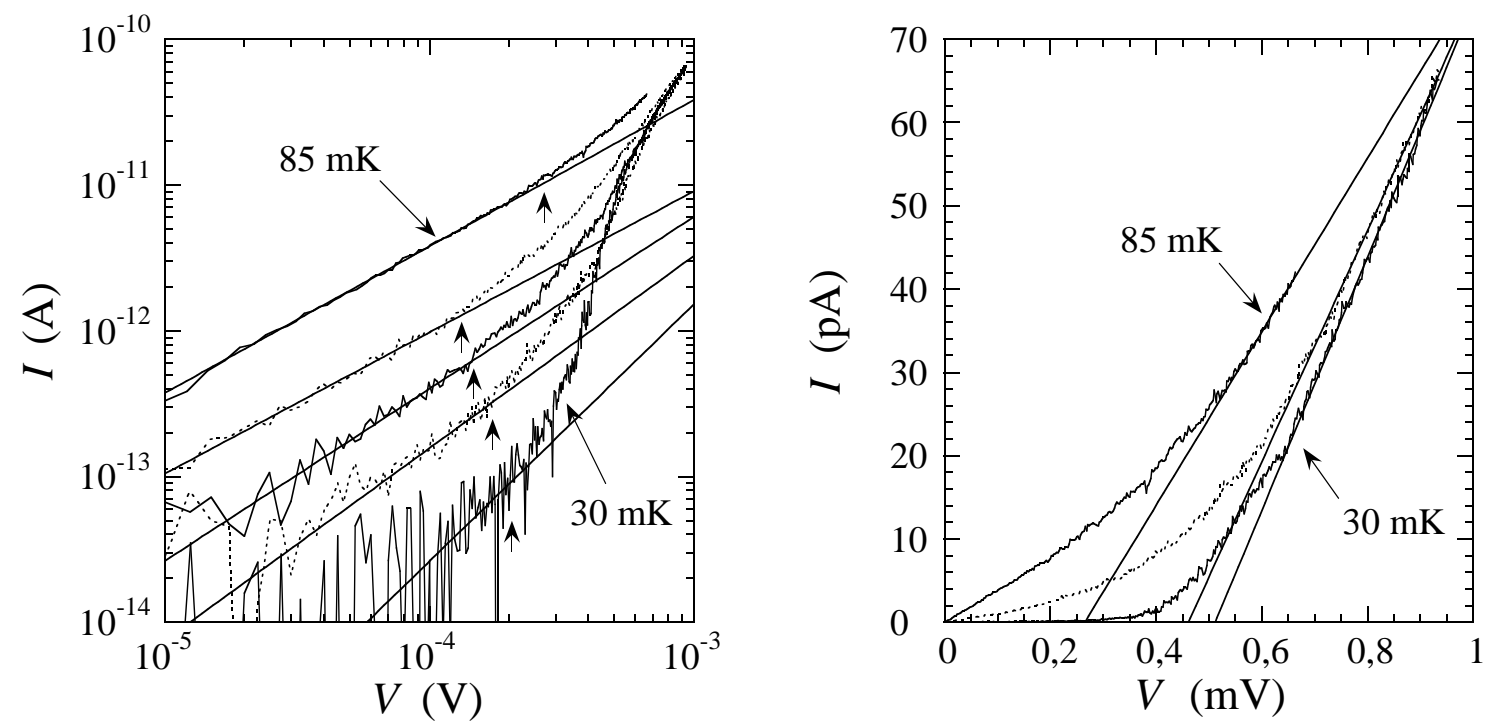

FIG. 7.5: Courant en fonction de la tension appliquée pour l'échantillon GaAs Nu 944-1 à une densité $p_{s}=1,30 \times 10^{14} \mathrm{~m}^{-2}$ et à différentes température. Gauche : Ajustement de la partie faible courant par une loi $I=A V^{\alpha}$ (de haut en bas, $T=85-55-50-40-30 \mathrm{mK}$ ), la tension seuil $V_{s, r}$ étant indiquée par un flèche verticale sur chaque courbe. Droite : Ajustement de la partie fort courant par une loi $I=B+C V$ (de haut en bas, $T=85-55-30 \mathrm{mK}$ ).

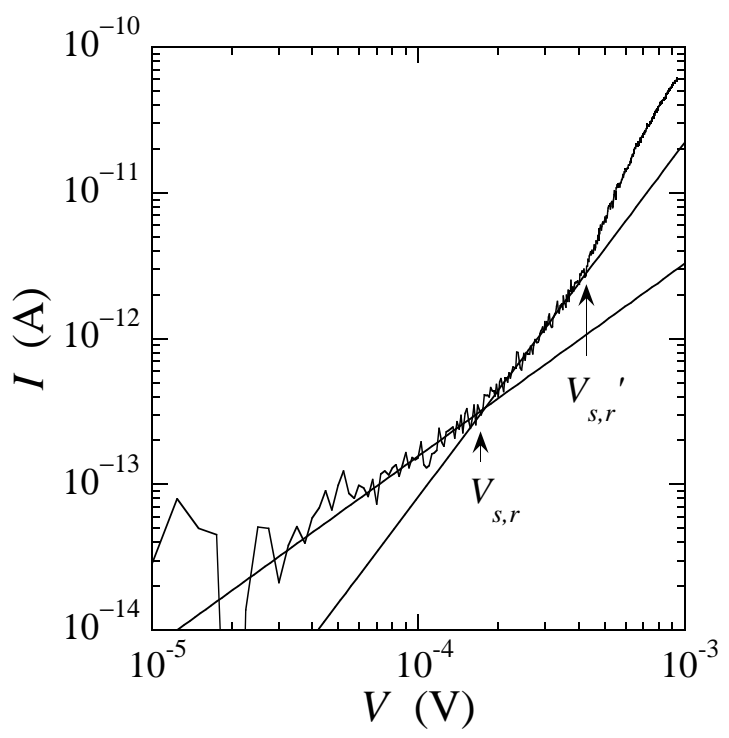

FIG. 7.6: Courant en fonction de la tension appliquée pour l'échantillon GaAs Nu 944-1 à une densité $p_{s}=1,30 \times 10^{14} \mathrm{~m}^{-2}$ et une température $T=40 \mathrm{mK}$. Ajustements des deux régimes par des lois $I=A V^{\alpha}$, avec $\alpha \approx 1,3$ à faible tension et $\alpha \approx 2,4$ à tension intermédiaire. Les flèches indiquent les deux tensions seuil décrites dans le texte. 
Avant de discuter les tensions seuil ainsi déterminées, on peut remarquer que, à la plus basse densité $\left(p_{s}=1,30 \times 10^{14} \mathrm{~m}^{-2}\right)$, la caractéristique $I-V$ semble en fait présenter plusieurs régimes en fonction de la tension appliquée (voir la figure 7.6). A faible tension, la caractéristique $I-V$ est proche d'une loi linéaire, ce qui nous a permis de déterminer la tension seuil $V_{s, r}$. A plus forte tension, le courant présente une dépendance proche de $V^{2}$, jusqu'à une deuxième tension seuil $V_{s, r}^{\prime}$. Cette deuxième tension seuil est retrouvée aux plus basses températures (jusqu'à $55 \mathrm{mK}$ ), et n'a pas été observée à plus haute densité.

Dans la figure 7.7 sont représentées les variations des tensions seuil, déterminées par les deux méthodes décrites, en fonction de la température pour différentes densités électroniques. La tension seuil déduite de la première méthode, $V_{s, r}$, augmente quand la température diminue à basse température, ce qui est compatible avec le décrochage d'une phase collective. En particulier on ne retrouve pas le comportement observé dans les puits quantiques $p$-SiGe pour lesquels les non-linéarités sont expliquées par le chauffage des porteurs (voir $\S 6.3$ ).

Sur la figure 7.8 de gauche sont représentées les variations des tensions seuil, déterminées à la plus basse température (environ $30-35 \mathrm{mK}$ ), en fonction de la densité de porteurs. Sur la figure 7.8 de droite sont indiquées les valeurs des énergies d'activation $T_{0}$ déduites de la dépendance en température de la résistivité (voir §5.4) en considérant une loi simplement activée $\rho(T) \propto \exp \left(T_{0} / T\right)$. Il est possible d'extrapoler la densité critique correspondant à l'annulation de l'énergie d'activation, $p_{s} \approx 1,44 \times 10^{14} \mathrm{~m}^{-2}$, ainsi que celle déduite de l'annulation de la tension seuil déduite par la première méthode, $p_{s} \approx 1,45 \times 10^{14} \mathrm{~m}^{-2}$. Ces deux valeurs sont très proches, ce qui permet de penser que le mécanisme conduisant à ces deux effets est le même. On peut en particulier remarquer que cette densité critique est plus faible que celle déduite de la dépendance en température de la résistivité, $p_{c}=1,57 \times 10^{14} \mathrm{~m}^{-2}$ (voir le chapitre 4). Or, pour des effets de chauffage, on s'attendrait plutôt à une annulation de la "tension seuil" apparente à la densité $p_{c}$. C'est donc un argument supplémentaire permettant de conclure que la dépendance en champ électrique observée à très basse densité dans nos puits quantiques $p$-GaAs ne sont pas dues au chauffage des porteurs.

L'existence d'une tension seuil sur la caractéristique $I-V$, ainsi que la dépendance en température de la résistivité suivant une loi simplement activée, montrent que ce système présente les caractéristiques d'une phase collective de type verre ou cristal de Wigner accroché sur le désordre. La densité à laquelle s'annulent la tension seuil et l'énergie d'activation $\left(1,45 \times 10^{14} \mathrm{~m}^{-2}\right)$ est alors la densité critique de fonte de la phase collective, qui correspond à $r_{s}=25$, en accord avec les simulations Monte-Carlo qui prévoient une densité critique entre 10 et 37 en fonction du désordre [44, 14]. Cependant ces caractéristiques sont également des signes du transport dans un système percolant en dessous du seuil de percolation [36]. Nous verrons au chapitre 11 que les mesures de bruit de résistance dans ce système sont compatibles avec cette deuxième hypothèse. Une étude plus poussée des non-linéarités semble cependant utile pour mieux comprendre ce régime, et en particulier pour comprendre l'origine du deuxième seuil $V_{s, r^{\prime}}$ à basse densité. 



FIG. 7.7: Variations des tensions seuil, mesurées par les deux méthodes décrites dans le texte, en fonction de la température, pour deux valeurs de la densité de porteurs, $p_{s}=1,30 \times 10^{14} \mathrm{~m}^{-2}$ $(\Delta)$ et $p_{s}=1,39 \times 10^{14} \mathrm{~m}^{-2}(\square)$. Dans la figure de gauche, les triangles pleins $(\boldsymbol{\Delta})$ représentent les mesures du second seuil $E_{s, r}^{\prime}$ pour $p_{s}=1,30 \times 10^{14} \mathrm{~m}^{-2}$.
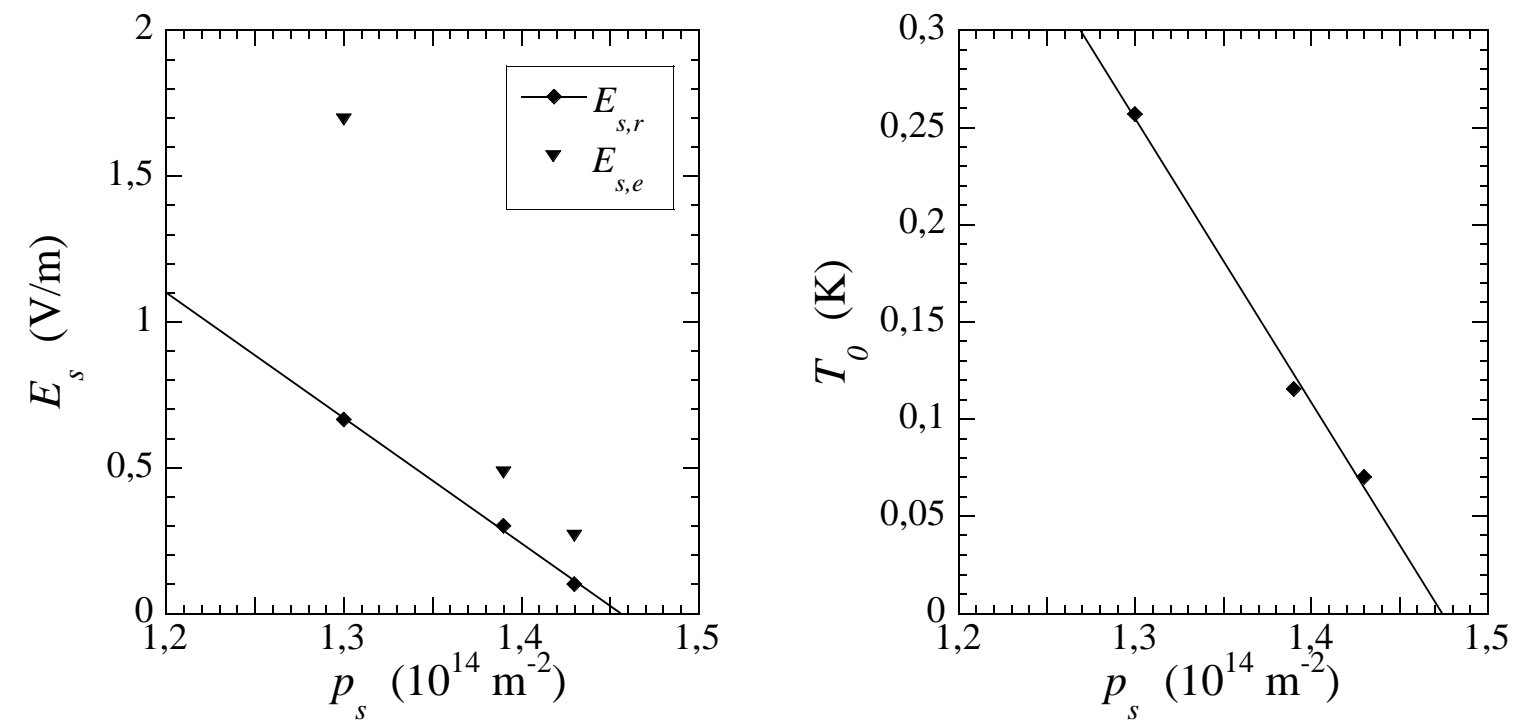

FIG. 7.8: Variations des tensions seuil mesurées par les deux méthodes décrites dans le texte (gauche) à la plus basse température (30-35 mK), et de l'énergie d'activation de la dépendance en température de la résistivité (droite), en fonction de la densité de porteurs. 


\subsection{Conclusion}

Nous avons étudié les non linéarités dans des puits quantiques $p$-GaAs dans une large gamme de densités de porteurs. A haute densité de porteurs, l'effet du champ électrique est qualitativement et quantitativement compatible avec des effets de chauffage des porteurs. A basse densité de porteurs, le chauffage des porteurs décrit par la théorie usuelle du couplage électron-phonon ne semble pas pouvoir expliquer les fortes non-linéarités observées. En particulier, l'existence d'une tension seuil sur la courbe $I-V$, et la dépendance activée de la résistivité en fonction de la température, sont compatibles avec l'existence d'une phase collective accrochée sur le désordre. Pour un système percolant, ces observations peuvent également décrire la conduction à température finie en-dessous du seuil de percolation. 


\section{Troisième partie}

\section{Fluctuations temporelles de résistance dans un gaz bidimensionnel de trous}





\section{Chapitre 8}

\section{Fluctuations temporelles de résistance dans un système d'électrons : bruit en $1 / f$}

Sommaire

8.1 Introduction . . . . . . . . . . . . . . 182

8.2 Généralités sur les fluctuations de résistance . . . . . . . 182

8.2 .1 Définitions . . . . . . . . . . . . . . . . . 182

8.2 .2 Résultat empirique de Hooge . . . . . . . . . . . . . . . 183

8.2 .3 Origine du spectre en $1 / f \ldots \ldots \ldots \ldots$

8.3 Description classique des fluctuations de résistance . . . . 184

8.3 .1 Modèle de piégeage . . . . . . . . . . . . . . . . . 185

8.3.2 Modèle de Dutta-Horn . . . . . . . . . . . . . . . . . 185

8.3.3 Modèles spécifiques . . . . . . . . . . . . . . . . 187

8.3.4 Résultats expérimentaux . . . . . . . . . . . . . 187

8.4 Fluctuations de résistance dans un isolant . . . . . 188

8.4.1 Modèles théoriques . . . . . . . . . . . . . . . . . 189

8.4 .2 Mesures expérimentales . . . . . . . . . . . . . . . 191

8.5 Fluctuations de résistance et interférences quantiques . 191

8.5.1 Fluctuations universelles de conductance . . . . . . . . . 192

8.5.2 Interférences quantiques dans un isolant . . . . . . . . . 195

8.6 Fluctuations de résistance et transition de phase . . . 195

8.6.1 Transition de percolation . . . . . . . . . . . . . 195

8.6.2 Transition d'Anderson en dimension trois . . . . . . . . . 199

8.6.3 Transition vitreuse . . . . . . . . . . . . . 200

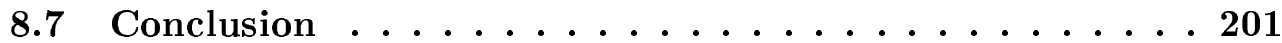




\subsection{Introduction}

Les fluctuations temporelles d'une grandeur physique, encore appelées bruit, sont couramment vues comme des sources de limitation dans les mesures. Mais le bruit d'une grandeur physique contient souvent une information supplémentaire par rapport à la valeur moyenne de la grandeur physique. Le premier intérêt des mesures de bruit est donc de comprendre la physique du système étudié. D'autre part, comprendre l'origine du bruit dans ces systèmes permet éventuellement de le réduire.

Dans plusieurs systèmes conducteurs, il a été observé que la tension mesurée aux bornes d'un conducteur traversé par un courant fixé fluctue, et que la puissance de bruit de ces fluctuations varie comme le carré du courant. Cette caractéristique signifie que c'est la résistance du système qui fluctue. D'autre part, dans la plupart des cas, le spectre des fluctuations croît fortement quand la fréquence diminue, ce qui a conduit à donner à ce bruit le nom de bruit en $1 / f$. L'existence d'un tel bruit dans un très grand nombre de conducteurs a conduit à s'y intéresser plus particulièrement, et à se poser la question de l'universalité d'un tel bruit.

Après avoir défini les grandeurs utilisées et les caractères généraux du bruit en $1 / f$ (§8.2), nous verrons les différentes origines possibles de ce bruit dans les systèmes électroniques. En particulier les mécanismes diffèrent notablement pour un système métallique (§8.3) ou pour un système isolant (§8.4). D'autre part les interférences quantiques peuvent également conduire à des fluctuations de résistance dans un système macroscopique (§8.5). Enfin, les fluctuations de résistance peuvent présenter un caractère particulier aux abords d'une transition de phase (§8.6).

Pour des informations plus complètes sur les fluctuations de résistance, mais aussi sur le bruit dans les systèmes électroniques, ou plus généralement en matière condensée, on pourra consulter les ouvrages de référence et revues de Kogan [321], Weissman [322], Kirton et Uren [323], et Imry [324].

\subsection{Généralités sur les fluctuations de résistance}

\subsubsection{Définitions}

Considérons un processus stochastique stationnaire $X(t)$ de valeur moyenne nulle, dont une réalisation $x(t)$ est mesurée sur un intervalle de temps $T$. Dans la limite $T \rightarrow \infty$, les composantes de Fourier de $X(t)$ sont des fonctions continues de la pulsation $\omega, A(\omega)$. On définit la puissance de bruit $S(\omega)$ par :

$$
S(\omega)=\left\langle|A(\omega)|^{2}\right\rangle,
$$

où $\langle\ldots\rangle$ représente la moyenne sur les réalisations.

Le théorème de Wiener-Khintchine montre que la puissance de bruit est la transformée de Fourier de la fonction d'autocorrélation :

$$
S(\omega)=\frac{1}{2 \pi} \int_{-\infty}^{+\infty}\left\langle X\left(t^{\prime}\right) X\left(t^{\prime}+t\right)\right\rangle e^{i \omega t} d t .
$$


Il est important de noter ici que l'on s'intéresse à un processus stochastique stationnaire, et que l'équation (8.2) n'est valable que dans cette hypothèse. Certains mécanismes physiques, tels la relaxation lente d'un système, peuvent remettre en cause cette hypothèse. Dans un tel cas il est nécessaire de calculer directement la fonction d'autocorrélation (c'est en particulier le cas des verres de spin).

Pour l'étude des fluctuations de résistance $\delta R(t)=R(t)-\bar{R}$, où $\bar{R}$ est la valeur moyenne de la résistance, on s'intéresse au spectre de bruit de résistance $S_{R}$ définit par :

$$
S_{R}(\omega)=\frac{1}{2 \pi} \int_{-\infty}^{+\infty}\left\langle\delta R\left(t^{\prime}\right) \delta R\left(t^{\prime}+t\right)\right\rangle e^{i \omega t} d t
$$

Pour mesurer ces fluctuations de résistance, il est possible de se placer à tension fixée et de mesurer le spectre des fluctuations de courant $S_{I}$, ou de se placer à courant fixé et de mesurer le spectre des fluctuations de tension $S_{V}$ :

$$
\left(\frac{S_{I}}{I^{2}}\right)_{V=c s t e}=\left(\frac{S_{V}}{V^{2}}\right)_{I=c s t e}=\frac{S_{R}}{R^{2}} .
$$

\subsubsection{Résultat empirique de Hooge}

Par une analyse empirique de plusieurs résultats expérimentaux sur des échantillons métalliques à température ambiante, Hooge a suggéré que le bruit de résistance en $1 / f$ observé dans différents échantillons homogènes puisse se mettre sous la forme [325] :

$$
\frac{S_{R}}{R^{2}}=\frac{A}{f}
$$

où $f$ est la fréquence et le coefficient $A$ est inversement proportionnel au nombre de porteurs de charges mobiles dans l'échantillon $N_{t o t}: A=B / N_{t o t}$. Les expériences ont établi que $B \approx 2 \times 10^{-3}$ dans des systèmes homogènes. Dans des échantillons non homogènes, le nombre de porteurs participant à la conduction peut être inférieur au nombre total de porteurs, conduisant à un coefficient plus élevé (voir les références dans [326]). Ainsi, dans de nombreux systèmes, il est nécessaire d'introduire une correction au coefficient $B$, ce qui a conduit à considérer $B$ comme un paramètre ajustable [322].

Les résultats importants de cette loi empirique sont, d'une part la dépendance en fréquence qui semble universelle, et d'autre la dépendance du bruit inversement proportionnellement aux nombre de porteurs, qui résulte d'une distribution homogène de sources de fluctuations indépendantes. Pour un échantillon dont la densité de porteurs de charges est uniforme, cette loi conduit à un coefficient $A$ inversement proportionnel au volume de l'échantillon, ce qui est un bon test pour vérifier l'origine du bruit mesuré.

\subsubsection{Origine du spectre en $1 / f$}

Un grand nombre de théories ont essayé de rendre compte du résultat de Hooge (équation (8.5)) dans les systèmes métalliques [322] et dans d'autres types de systèmes [321]. De manière générale, en superposant un grand nombre de spectres Lorentziens de la forme $S_{1} \propto f_{c} /\left(f_{c}^{2}+f^{2}\right)$ avec une distribution bien déterminée de fréquences caractéristiques 
$f_{c}$, il est possible de construire un spectre dont la dépendance en fréquence est $1 / f$. En effet, le spectre d'une assemblée de spectres Lorentziens non corrélés est :

$$
S(f) \propto \int_{-\infty}^{+\infty} \frac{f_{c}}{f_{c}^{2}+f^{2}} D\left(f_{c}\right) d f_{c} .
$$

Lorsque la distribution de fréquences caractéristiques a la forme $D\left(f_{c}\right) \propto 1 / f_{c}$, le spectre s'écrit alors :

$$
S(f) \propto \int_{-\infty}^{+\infty} \frac{1}{f_{c}^{2}+f^{2}} d f_{c}=\frac{\pi}{f},
$$

qui est bien en $1 / f$. Si la distribution de fréquences caractéristiques diffère légèrement de $1 / f_{c}$, le spectre de bruit est alors un spectre en $1 / f^{\alpha}$ avec $\alpha$ différent de un. L'exposant $\alpha$ est donc directement lié à la distribution des fréquences caractéristiques.

Avant de décrire en détails les modèles proposés pour expliquer les fluctuations de résistance, et en particulier le spectre Lorentzien et la distribution des fréquences caractéristiques, on peut comprendre sur un modèle simple l'origine possible du bruit en $1 / f$. Dans un système électronique, les fluctuations des impuretés et des défauts sont vues par les électrons comme des fluctuations du désordre. Les fluctuations de résistance traduisent donc la réponse du système électronique à ces fluctuations de désordre. Les variations spontanées du désordre dans le temps, par activation thermique ou par effet tunnel, conduit donc dans ce cas aux fluctuations temporelles de la résistance. Si on considère un système comportant une seule impureté, cette impureté pouvant fluctuer entre deux états, on observera des fluctuations de la résistance entre deux états $R_{1}$ et $R_{2}$ (système à deux niveaux). Si $\tau$ est le temps de vie moyen dans un état, la fonction d'autocorrélation des fluctuations de résistance prend la forme :

$$
\langle\delta R(0) \delta R(t)\rangle \propto(\overline{\delta R})^{2} e^{-t / \tau},
$$

dont la transformée de Fourier est un spectre Lorentzien, caractéristique du bruit télégraphique observé sur la trace temporelle. Si maintenant on considère une assemblée d'impuretés fluctuantes, avec une distribution bien choisie de temps caractéristiques, ces fluctuations du désordre peuvent conduire à un bruit de résistance en $1 / f$. Dans l'expression (8.8), $(\overline{\delta R})^{2}$ est la réponse du système électronique aux fluctuations du désordre. Dans ce cas, la mesure du bruit donne donc directement une information sur la physique du système électronique.

\subsection{Description classique des fluctuations de résistance}

Suivant les considérations présentées dans le paragraphe précédant sur l'origine du bruit en $1 / f$, les modèles proposés pour expliquer les fluctuations de résistance se sont attachés à donner une origine au spectre Lorentzien et à la distribution particulière des fréquences caractéristiques permettant d'obtenir un spectre en $1 / f$. Dans cette partie sont présentés les modèles qui permettent d'expliquer de manière classique les fluctuations de résistance dans des systèmes électroniques, et plus particulièrement les systèmes bidimensionnels qui nous intéressent. Seuls les modèles les plus couramment admis sont 
présentés dans cette partie, et les revues citées en introduction permettront d'avoir une liste plus complète. On s'intéresse ici à deux caractéristiques principales des fluctuations de résistance, la forme du spectre en $1 / f^{\alpha}$, qui peut différer d'un spectre purement en $1 / f$, et la dépendance en température du bruit qui renseigne sur le mécanisme physique mis en jeu.

\subsubsection{Modèle de piégeage}

L'un des premiers modèles pour expliquer les fluctuations de résistance dans des MOSFETs silicium a été développé par McWhorter [327]. Cette approche repose sur le piégeage des porteurs de charge par les pièges situés dans l'oxyde, induisant des fluctuations du nombre de charges mobiles et donc de la résistance. Le piégeage et le dépiégeage des porteurs est supposé se faire uniquement par effet tunnel entre les électrons du semiconducteur et les pièges dans l'oxyde. Si $l$ est la distance séparant un piège du gaz d'électrons, la fréquence caractéristique des sauts par effet tunnel peut s'écrire $f_{c}=f_{0} \exp \left(-l / l_{0}\right)$. La distribution des fréquences caractéristiques s'écrit alors en fonction de la distribution de distances :

$$
D\left(f_{c}\right) \propto\left|\frac{\partial l}{\partial f_{c}}\right| D(l) \propto \frac{l_{0} D(l)}{f_{c}} .
$$

Pour avoir une distribution en $1 / f_{c}$ des fréquences caractéristiques, il est donc nécessaire d'avoir une distribution spatiale uniforme de pièges dans l'oxyde $(D(l)=c t e)$. La distribution en énergie de ces pièges détermine alors la dépendance en température du spectre des fluctuations.

Ce modèle a une importance surtout historique car il est le premier à rendre compte des fluctuations de résistance dans des MOSFETs Si. Cependant il ne prend en compte que les fluctuations du nombre de porteurs, alors que les fluctuations du désordre peuvent aussi jouer.

\subsubsection{Modèle de Dutta-Horn}

Il est possible d'étendre le modèle de McWhorter a un cas plus général où la fréquence caractéristique $f_{c}$ dépend exponentiellement d'un paramètre quelconque, dont la distribution est uniforme. Un bon candidat est un mécanisme activé, pour lequel le paramètre est l'énergie d'activation $E_{a}$, avec $f_{c}=f_{0} \exp \left(-E_{a} / k_{B} T\right)$. Pour obtenir un bruit en $1 / f$, ce mécanisme nécessite une distribution uniforme des énergies d'activation.

Le modèle développé par Dutta, Dimon et Horn [328] peut-être vu comme une assemblée de systèmes à deux niveaux, chaque système étant caractérisé par la différence d'énergie entre les niveaux $\Delta E$ et par l'énergie moyenne d'activation $E_{a}$ (voir figure 8.1). L'assemblée de ces systèmes est caractérisée par la fonction de distribution $D\left(E_{a}, \Delta E\right)$. Il est possible de montrer que la densité spectrale des fluctuations résultant d'un système à deux niveaux est [322] :

$$
S_{1}(f, T)=\frac{1}{2 \pi} \operatorname{sech}^{2}\left(\frac{\Delta E}{2 k_{B} T}\right) \frac{f_{c}}{f_{c}^{2}+f^{2}},
$$




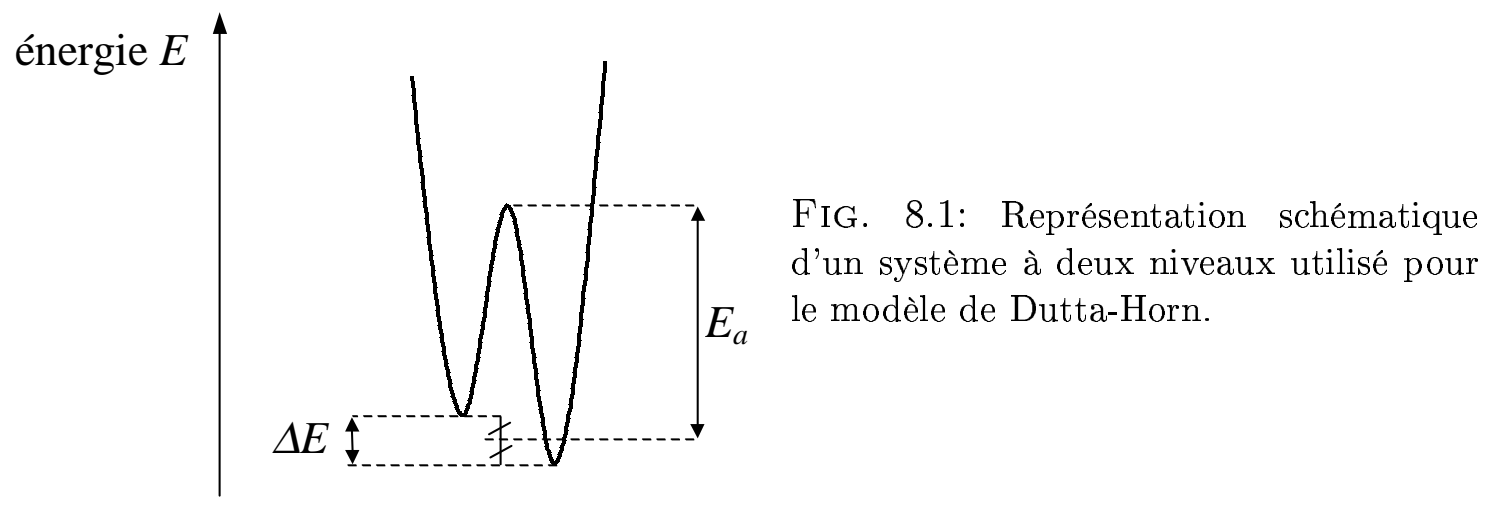

où $T$ est la température, et avec une fréquence caractéristique :

$$
f_{c}=f_{0}\left[\exp \left(-\frac{E_{a}+\Delta E / 2}{k_{B} T}\right)+\exp \left(-\frac{E_{a}-\Delta E / 2}{k_{B} T}\right)\right] .
$$

Pour obtenir une distribution en $1 / f_{c}$ des fréquences caractéristiques, il est nécessaire d'avoir une distribution $D_{1}\left(E_{a}\right)$ constante, avec :

$$
D_{1}\left(E_{a}\right)=\int_{-\infty}^{+\infty} D\left(E_{a}, \Delta E\right) \operatorname{sech}^{2}\left(\frac{\Delta E}{2 k_{B} T}\right) d(\Delta E) .
$$

Dutta, Dimon et Horn [328] se sont intéressés plus particulièrement au lien entre la forme spectrale du bruit et sa dépendance en température. Ils ont adopté pour cela les hypothèses suivantes : (1) les fluctuations ont une cinétique activée, (2) il existe une seule valeur de $f_{0} \gg f,(3)$ la variation de $D_{1}\left(E_{a}\right)$ est faible, (4) $D\left(E_{a}, \Delta E\right)=D_{1}\left(E_{a}\right) \delta(\Delta E)$ et (5) la résistance ne dépend pas de la température. Suivant ces hypothèses, ils ont établi la relation suivante, connue comme la relation de Dutta-Horn :

$$
-\frac{\partial \ln S(f, T)}{\partial \ln f}=1+\frac{1}{\ln \left(f_{0} / f\right)}\left[\frac{\partial \ln S(f, T)}{\partial \ln T}-1\right],
$$

où $S(f)=S_{R}(f) / R^{2}$. Cette relation relie donc la dépendance en fréquence du bruit en fonction de sa dépendance en température. En particulier, pour un bruit purement en $1 / f$, ce modèle conduit à $S(f, T)=S_{R} / R^{2} \propto T$. Mais, en général, le couplage entre les impuretés et la résistivité dépendent de la température, ce qui peut modifier de façon importante cette dépendance. Cependant, comme on considère des mécanismes dont l'énergie caractéristique est très petite devant $k_{B} T$, on s'attend à ce que la contribution de l'activation soit dominante, et donc on s'attend toujours à une augmentation de $S_{R} / R^{2}$ quand la température augmente.

Ce modèle repose sur le fait que les fluctuations de résistance sont dues à un processus activé. Plusieurs mécanismes permettent d'en rendre compte. On peut citer les fluctuations du nombre de porteurs, par exemple par piégeage par des défauts, les fluctuations de mobilités, les centres DX. Ces différents modèles sont détaillés dans le paragraphe suivant. 


\subsubsection{Modèles spécifiques}

\section{Fluctuations du nombre de porteurs}

Dans un système métallique, la formule de Drude montre que la conductivité est proportionnelle à la densité de porteurs de charge. Ainsi des fluctuations du nombre de porteurs dans un système conduisent à des fluctuations de la résistance. Différents mécanismes ont été envisagés pour interpréter ces éventuelles fluctuations du nombre de porteurs.

Dans les MOSFETs silicium, il existe des pièges dans la couche d'oxyde susceptibles de piéger des porteurs de charge. Ce mécanisme comme source de fluctuations du nombre de porteurs a déjà été vu dans le modèle de McWhorter (voir §8.3.1). Reimbold a calculé le bruit résultant du piégeage de porteur dans les MOSFETs Si [329] :

$$
\frac{S_{R}}{R^{2}}=\frac{B}{A f} \frac{N_{t}}{\left(C_{o x}+C_{D}-\beta Q_{n}\right)^{2}},
$$

où $A$ est la surface de l'échantillon, $N_{t}$ est la densité d'états de pièges $\left(\mathrm{eV}^{-1} \mathrm{~m}^{-3}\right), C_{o x}$ et $C_{D}$ les capacités par unité de surface de la couche d'oxyde et de la couche de déplétion, $B$ est un paramètre, $Q_{n}$ est la charge par unité de surface de la couche d'inversion et $\beta=e / k_{B} T$. Cette formule est proche de celle de Hooge (voir l'équation (8.5)), en particulier dans la dépendance en $1 / N_{t}$. Mais ici c'est le nombre de pièges, et non le nombre de porteurs de charges, qui est pris en compte. Cette considération peut expliquer les écarts souvent observés par rapport à la loi de Hooge.

\section{Fluctuations de mobilité}

Les fluctuations des impuretés ou des défauts du système induisent des fluctuations de la mobilité des porteurs de charge. Toujours dans le cas d'un métal, la formule de Drude conduit à une résistivité proportionnelle à la mobilité, et les fluctuations de mobilité induisent donc des fluctuations de la résistance. Ce mécanisme dépend en priorité du type de diffusion qui domine dans le système, et peut conduire à différents modèles. Cette source de fluctuations de résistance a été envisagée par Hooge [325, 330] pour expliquer la formule empirique de l'équation (8.5).

\subsubsection{Résultats expérimentaux}

Nous discutons ici les expériences faites dans des systèmes électroniques dans le régime métallique où le bruit peut être interprété par des modèles classiques. Nous allons voir comment les auteurs ont montré que le spectre en $1 / f$ provient bien d'une superpositions de processus de spectre Lorentzien, puis sont présentées les causes possibles des fluctuations de résistance dans ces divers systèmes.

\section{Caractérisation du spectre en $1 / f$}

Pour montrer que le bruit en $1 / f$ provient d'un grand nombre de fluctuateurs indépendants, il est nécessaire de connaître la dynamique d'un seul fluctuateur. Pour cette raison des études ont été faites sur des systèmes de petite taille. 
Des mesures ont été réalisées sur des MOSFETs Si par Ralls et al. [331] et Uren et al. [332]. En comparant le bruit télégraphique sur des échantillons de petite taille $\left(<1 \mu \mathrm{m}^{2}\right)$, et donc contenant un faible nombre de pièges, et le bruit en $1 / f$ sur des échantillons de grande taille $\left(>100 \mu \mathrm{m}^{2}\right)$, ces auteurs ont montré que le bruit en $1 / f$ observé dans les échantillons de grande taille est bien dû à la superposition de bruits télégraphiques de spectre Lorentziens.

Rogers et al. [333] confirme cette superposition de bruits Lorentzien comme source de bruit en $1 / f$ dans des jonctions tunnel métal-isolant-métal. Ils ont cependant montré qu'il est nécessaire d'introduire deux temps caractéristiques pour représenter les fluctuations des "fluctuateurs" individuels, et non un seul temps comme il est habituellement considéré dans un mécanisme activé.

\section{Origines des fluctuations de résistance}

Dans les expériences d'Uren et al., les dépendances des fluctuations de résistance en fonction du courant injecté et de la tension de grille permettent d'attribuer ce bruit aux fluctuations du nombre de porteurs [332]. Les auteurs ont en particulier vérifié que le bruit en $1 / f$ est compatible avec la théorie développée par Reimbold (équation (8.14)). C'est également l'origine envisagée dans les expériences de Ralls et al. [331], mais les fluctuations de mobilité ne peuvent pas être complètement exclues dans ces derniers résultats.

Chen et al. [334] ont mesuré le bruit en $1 / f$ dans un gaz bidimensionnel d'électron formé dans un plan de dopage $\delta$ dans GaAs. En comparant des conditions donnant des mobilités différentes, ils ont montré que les fluctuations de résistance produisant le bruit en $1 / f$ sont dues à des fluctuations de mobilité, comme le prévoit le modèle de Hooge et non à des phénomènes de piégeage dû aux défauts d'interfaces. De plus ces fluctuations de mobilité sont dues à la diffusion par les défauts du réseau, et non par les impuretés. Les fluctuations de mobilités ont également permis d'interpréter le bruit observé par Kurdak et al. dans des hétérostructures GaAs/GaAlAs de type $n$ à une température de $4,2 \mathrm{~K}$ [335]. Ce bruit est interprété par les fluctuations d'impuretés de dopage (donneurs Si dans AlGaAs). En effet, la mesure des fluctuations de résistance Hall sous champ magnétique permet d'évaluer directement les fluctuations du nombre de porteurs, qui est très faible dans les échantillons mesurés.

Enfin, un bon moyen de vérifier la nature activée des fluctuations de résistance est de regarder les dépendances en température du bruit mesuré. La formule de Dutta-Horn (8.13) constitue en effet un bon test, notamment en comparant les dépendances en fréquence et en température. Rogers et al. [333] ont mesuré sur des jonction tunnel un bruit qui augmente avec la température, donc compatible avec un mécanisme de Dutta-Horn.

\subsection{Fluctuations de résistance dans un isolant}

Dans un système fortement désordonné, les états électroniques sont localisés, et la formule de Drude pour la conductivité n'est plus valable : le système est isolant. On a vu au chapitre 1 qu'à basse température, le transport des électrons se fait alors par sauts entre états localisés. Plusieurs modèles ont été évoqués pour expliquer les fluctuations 
importantes de résistance observées dans ces systèmes isolants. Tous les modèles présentés ici sont des modèles classiques et s'inspirent de ceux développés dans la section précédente. Ils reposent sur le fait que la résistance du système isolant est donnée par la résistance du réseau critique de la théorie de percolation. Les fluctuations de résistance dans un tel système peuvent alors être expliquées soit par des fluctuations du nombre de porteurs contenus dans le réseau critique, soit par des fluctuations de la mobilité des porteurs, soit par des fluctuations de la densité d'états.

\subsubsection{Modèles théoriques}

\section{Fluctuations du nombre de porteurs}

Le premier à avoir envisagé des fluctuations de résistance dans le régime de sauts plus proche voisin est Shklovskii [336]. Dans ce modèle les fluctuations sont dues à des fluctuations du nombre d'électrons en raison d'un échange lent d'électrons entre le réseau conducteur infini et des zones isolées non conductrices. Ce modèle est en fait proche du modèle de McWhorter dans les MOSFETs (voir §8.3) qui considère l'échange d'électrons avec les pièges dans l'oxide. Comme le temps de relaxation des sauts dépend exponentiellement de la distance de saut, ce temps varie très largement entre les différentes impuretés, conduisant à un spectre en $1 / f^{\alpha}$ puis une saturation du bruit en-dessous d'une fréquence comprise entre 1 et $100 \mathrm{~Hz}$ en raison du nombre exponentiellement faible du nombre d'impuretés. Des simulations numériques [337] ont montré que l'exposant $\alpha$ est proche de 0,6, et diffère donc largement de 1.

\section{Fluctuations de mobilité}

Une autre origine possible pour ces fluctuations a été proposée par Kozub [338]. Dans ce modèle, des "fluctuateurs" (qui sont des porteurs qui sautent d'une impureté à une autre) produisent des fluctuations d'énergie des sites du système percolant. Les fluctuations sont donc ici des fluctuations de "mobilité", i.e. de probabilité de saut, et non des fluctuations du nombre de porteurs comme dans le modèle de Shklovskii. Ce mécanisme faisant intervenir tous les porteurs de charge du système, et en particulier ceux qui n'appartiennent pas à la conduction, contrairement au mécanisme proposé par Shklovskii, Kozub suggère que ce mécanisme est plus probable que celui de Shklovskii.

Suivant ce modèle, Kozub [338] aboutit à une évaluation du bruit :

$$
\frac{S_{R}}{R^{2}} \sim\left(\frac{r \xi_{c}}{L}\right)^{d} \frac{P(T) T}{\omega}\left[\frac{e^{2}}{4 \pi \varepsilon_{0} \varepsilon_{r} k_{B} T} r l(\omega)\right],
$$

où $r$ est la portée moyenne des sauts, $\xi_{c}$ est le paramètre critique de percolation sans dimension, représentant le nombre de sites inclus dans le réseau critique de percolation, et ainsi $r \xi_{c}$ est la longueur caractéristique d'un lien, $L$ la taille du système, $d$ la dimensionnalité de l'espace, $\omega$ est la fréquence, $P(T)$ est la densité d'état des "fluctuateurs" , et $l(\omega)$ est la distance entre sites formant un "fluctuateur". La quantité $\left(r \xi_{c} / L\right)^{d}$ est la fraction du volume du système qui contribue à la conduction. Ainsi l'inverse de cette grandeur représente la quantité de porteurs participant à la conduction. L'équation (8.15) 
est donc l'analogue de la formule de Hooge (8.5) pour le bruit en $1 / f$ dans un système percolant.

En supposant que $P(T)$ est indépendant de la température, la dépendance en température du bruit provient du seul terme $\left(r \xi_{c} / L\right)^{d}$ dans l'équation (8.15). Kozub [338] a évalué cette dépendance dans différents régimes de conduction en dimension trois. Mais il est possible de généraliser cette dépendance en dimension $d$ quelconque en utilisant les dépendances en température de $r$ et $\xi_{c}$ données dans le chapitre 1 :

sauts activés :

sauts à portée variable de Mott :

sauts à portée variable d'Efros-Shklovskii : $S_{R} / R^{2} \propto T^{-d}$

Dans les deux modèles de sauts à portée variable, le bruit augmente fortement à basse température. Cette dépendance peut se comprendre par la diminution du nombre de sites participant au réseau critique de percolation, et donc du nombre de résistances effectives dans le réseau de percolation, quand la température diminue. Cependant, à très basse température, il est nécessaire de prendre en compte la dépendance en température de $P(T)$ et on s'attend donc à une modification de ces dépendances.

\section{Verre d'électrons}

Dans le modèle proposé par Kogan [339], le système électronique est vu comme un système fortement corrélé et le bruit en $1 / f$ est expliqué par les transitions entre des états métastables du système d'électrons en interaction (verre).

\section{Fluctuations de densité d'états}

Yu a suggéré que les sauts de porteurs entre les sites, combinés à l'interaction Coulombienne entre ces porteurs, induisent des fluctuations de la densité d'états à une particule sur les sites [340]. Cette densité d'états intervenant dans le calcul des probabilités de saut, ces fluctuations induisent des fluctuations de la résistance.

Un calcul analytique basé sur ce modèle permet de montrer que le bruit de résistance a la forme $S_{R}(\omega) \sim 1 / \omega^{\alpha}$ [340]. L'exposant $\alpha$ est proche de 1, et dépend de la cinétique de la relaxation. Si la relaxation dépend logarithmiquement du temps, $\alpha=1$, alors que si elle dépend en loi de puissance du temps $t^{-\theta}, \alpha=1-\theta$ avec $\theta \ll 1$. Enfin ce calcul ne permet pas de prévoir les dépendances en température du bruit.

Une étude plus précise basée sur ce modèle a été réalisée par Shtengel et Yu [341] à partir de simulations numériques dans des conditions analogues à des expériences réalisées sur des isolants d'Anderson à 3D. Elle montre que l'exposant $\alpha$ augmente quand la température diminue, avec une valeur comprise entre 1,10 et 1,17 . De plus ce modèle conduit à une augmentation du bruit quand la température augmente, et donc à une variation opposée aux deux modèles précédents. 


\subsubsection{Mesures expérimentales}

Diverses expériences en dimensions trois et deux ont permis de tester les modèles décrits dans les paragraphes précédents. Il est cependant important de noter que ces modèles ne sont pas exclusifs, puisque des fluctuations de nombre de porteurs, de mobilité et de densité d'états peuvent intervenir simultanément.

Dans les MOSFETs Si, Voss [342] et Adkins et Koch (voir dans [83]) ont montré que le bruit en $1 / f$ est une propriété intrinsèque du gaz d'électrons, et n'est pas dû à des pièges à l'interface $\mathrm{Si} / \mathrm{SiO}_{2}$. Ces mesures ayant été faites dans le régime de conduction par sauts, les modèles cités dans cette section peuvent être appliquer pour interpréter les fluctuations de résistance. De plus Adkins et Koch ont montré que la puissance de bruit $S_{R}$ croît quand la température diminue, ce qui est opposé aux modèles classiques de piégeage-dépiégeage, mais est par contre compatible avec les modèles de Shklovskii et de Kozub. Enfin Voss a mesuré un spectre de bruit en $1 / f^{\alpha}$ avec $\alpha=0,8-1$, sans observer de saturation à basse fréquence, ce qui est incompatible avec le modèle de Shklovskii.

Pokrovskii et al. ont mesuré le bruit de résistance dans des MESFETs GaAs de différentes longueurs dans le régime de sauts à distance variable de Mott [343]. $S_{R} / R^{2}$ augmente fortement quand la densité diminue et quand la température diminue. Ils ont pu interpréter qualitativement et quantitativement ces dépendances dans le cadre du modèle de fluctuation d'énergie des sites [338]. Ils ont également observé sur le bruit une transition entre le régime de Mott et le régime de sauts entre plus proches voisins à plus haute température, se traduisant par une dépendance en température différente.

Shlimak et al. ont réalisé des mesures du bruit en $1 / f$ dans des échantillons 3D pGe :Ga et $n$-Ge :As dans le régime de sauts plus proches voisins (la résistivité varie comme $\left.\exp \left(T_{0} / T\right)\right)$ pour des températures entre 3 et $1 \mathrm{~K} . S_{V} / V^{2}$, pris à fréquence donnée, augmente quand la température augmente, mais moins vite que ne le prévoit le modèle de Shklovskii [336]. Dans ce régime, Kozub [338] prévoit un bruit indépendant de la température. Il est donc difficile ici de trouver un mécanisme dominant, mais le mécanisme de Shtengel et Yu semble exclu.

Enfin Massey et Lee [344] observent dans des isolants 3D de Si :B une décroissance du bruit quand la température diminue. D'autre part l'exposant $\alpha$ de la dépendance en fréquence augmente de 1,08 à 1,12 quand la température diminue de 10 à $1,7 \mathrm{~K}$. Ces deux observations sont compatibles avec les simulations réalisée par Shtengel et Yu qui prennent en compte les interactions électron-électron.

\subsection{Fluctuations de résistance et interférences quan- tiques}

Dans les deux sections précédentes, on a considéré des modèles décrivant de manière classique les fluctuations de résistance dans un métal puis dans un isolant. Cependant, à basse température, quand la longueur de cohérence de phase $L_{\Phi}$ devient suffisamment grande, on s'attend à observer des modifications de la conductance dues aux interférences quantiques. Nous allons voir ici que cet effet peut aussi être à l'origine de fluctuations de résistance qui, dans des systèmes de grande taille, conduisent à un bruit en $1 / f$. 


\subsubsection{Fluctuations universelles de conductance}

\section{Origine physique}

Nous avons vu au chapitre 1 que, dans un système métallique cohérent, la conductance est modifiée par les interférences quantiques. Ainsi toute grandeur qui peut modifier la phase des fonctions d'onde électroniques va modifier la conductance du système. En présence d'un potentiel scalaire $V$ et d'un potentiel vecteur $\vec{A}$, la variation de phase d'une trajectoire électronique d'impulsion $\vec{p}$ associée au parcours $d \vec{r}$ durant un temps $d t$ est donnée par :

$$
d \Phi=V \frac{d t}{\hbar}+\frac{e}{\hbar} \vec{A} \cdot d \vec{r}+\frac{1}{\hbar} \vec{p} \cdot d \vec{r} .
$$

Ainsi tout ce qui modifie soit le potentiel scalaire, soit le potentiel vecteur, soit l'impulsion est susceptible de modifier la conductance du système. Il a été montré que la modification du champ magnétique, directement relié au potentiel vecteur, ou du potentiel chimique par une grille, relié à l'impulsion, conduit à des fluctuations de la conductance de l'ordre de $e^{2} / h$, dites "fluctuations universelles de conductance" [345]. Il existe enfin un troisième moyen de modifier la conductance, le potentiel $V$, qui est relié au potentiel de désordre dans l'échantillon. Une modification de ce potentiel de désordre (en modifiant la configuration du désordre) induit donc des fluctuations de la résistance. A température non nulle, les impuretés et les défauts formant le désordre ne sont pas statiques, et ils peuvent fluctuer, conduisant à des fluctuations temporelles de la conductance. Feng, Lee et Stone [346] ont montré que ces fluctuations peuvent conduire à un spectre en $1 / f^{\alpha}$. De plus ils ont montré que, en dimension un et deux, la fluctuation d'une impureté conduit à des fluctuations de la conductance d'un système de taille $L_{\Phi}$ de l'ordre de $e^{2} / h$. Ces fluctuations sont alors suffisamment élevées pour être encore observées dans un échantillon macroscopique à température finie.

Considérons tout d'abord un système de taille $L$ complètement cohérent (i.e. $L_{\Phi}>L$ ), dans un espace de dimension $d$. Feng, Lee et Stone [346] ont montré que la variation de conductance $\delta G_{1}$ liée au déplacement d'une impureté sur une distance $\delta r$ dans ce système vaut, pour $d \geqslant 2$ :

$$
\left(\delta G_{1}\right)^{2} \approx\left(\frac{e^{2}}{h}\right)^{2} \frac{1}{n_{i} l_{e}^{d}}\left(\frac{L}{l_{e}}\right) \alpha\left(k_{F} \delta r\right),
$$

où $n_{i}$ est le nombre d'impuretés par unité de volume, $l_{e}$ le libre parcours moyen élastique, $k_{F}$ le vecteur d'onde de Fermi et $\alpha(x)=1-(\sin (x / 2) /(x / 2))^{2}$. On remarque alors que, en dimension deux, $\delta G_{1} \approx e^{2} / h$ est de l'ordre de la conductance du système $\left(G_{1} \approx e^{2} / h\right)$ et ne dépend pas de la taille $L$.

Dans le cas d'un échantillon macroscopique à température non nulle, le système n'est pas entièrement cohérent $\left(L_{\Phi}<L\right)$. Il est cependant possible de diviser l'échantillon macroscopique en un ensemble de domaines de taille $L_{\Phi}$. En supposant que chaque domaine contient $n_{T}$ impuretés fluctuantes indépendantes, la fluctuation de conductance d'un domaine sera donné par $n_{T}\left(\delta G_{1}\right)^{2}$. Si $\Omega$ est le volume de l'échantillon, le nombre de domaines dans l'échantillon est $\Omega / L_{\Phi}^{d}$, où $d$ est la dimensionnalité de l'espace. Les fluctuations des domaines sont donc moyennées sur la taille totale de l'échantillon, et les fluctuations 
globales de conductance prennent la forme :

$$
\frac{(\delta G)^{2}}{G^{2}}=\frac{(\delta G)_{c o h}^{2}}{G_{c o h}^{2}} \frac{L_{\Phi}^{d}}{\Omega} f\left(\frac{\hbar}{k_{B} T \tau_{\Phi}}\right)
$$

où $(\delta G)_{c o h}$ est la fluctuation de conductance dans un volume de taille inférieure à $L_{\Phi}, \tau_{\Phi}$ est le temps de cohérence de phase, avec $(\delta G)_{c o h}^{2}=A n_{T}\left(e^{2} / h\right)^{2}$ et $A \sim 1$ en dimension $\operatorname{deux}, G_{c o h} \approx e^{2} / h$, et la fonction $f$ est donnée par $f(x)=x$ si $x<1$ et $f(x)=1$ si $x \geqslant 1$. Lorsque $\hbar / k_{B} T \tau_{\Phi}>1$, on a donc finalement :

$$
\frac{(\delta G)^{2}}{G^{2}} \approx A n_{T} \frac{L_{\Phi}^{d}}{\Omega}
$$

Pour connaître le spectre des fluctuations de conductance, il est nécessaire de s'intéresser à la dynamique de fluctuation des impuretés. Supposons que ces impuretés fluctuent entre deux états avec une loi activée et un temps caractéristique $\tau$. Pour un seul défaut, le spectre de bruit est Lorentzien :

$$
\frac{S_{R}(\omega)}{R^{2}}=\frac{S_{G}(\omega)}{G^{2}}=\frac{(\delta G)^{2}}{G^{2}} \frac{2 \tau}{1+\omega^{2} \tau^{2}}
$$

$(\delta G)^{2} / G^{2}$ est donné par l'équation (8.19), et caractérise la réponse du système électronique aux fluctuations des impuretés. La moyenne sur un grand nombre de défauts avec une distribution large d'énergies d'activation conduit à un spectre de bruit en $1 / f$, comme il a été vu au paragraphe 8.2.3.

Enfin, on peut remarquer que la dépendance en température du bruit peut être déduite de la dépendance en température de $(\delta G)^{2} / G^{2}$ dans l'équation (8.19). En dimension deux, les seules termes dépendant de la température sont la densité d'impuretés fluctuantes $n_{T}$ et la longueur de cohérence de phase $L_{\Phi}$ :

$$
\frac{S_{R}}{R^{2}} \propto n_{T}(T)\left(L_{\Phi}(T)\right)^{2}
$$

À suffisamment basse température, la diffusion inélastique est dominée par l'interaction électron-électron, et $L_{\Phi} \propto T^{-1 / 2}$ (voir le chapitre 1). D'autre part, pour une densité d'états des impuretés constante, on s'attend à $n_{T}(T) \propto k_{B} T$ (c'est le nombre d'états dans une bande d'énergie de largeur $k_{B} T$ ). Ce mécanisme conduit donc à un bruit indépendant de la température. Cependant, à suffisamment basse température, le mécanisme activé est "gelé" et laisse place à un mécanisme d'effet tunnel indépendant de la température [346]. Dans ce cas, le bruit $S_{R} / R^{2} \propto T^{-1}$, i.e. il augmente quand la température diminue, comportement remarquable car il va à l'opposé de la dépendance classique donnée par le modèle de Dutta-Horn (voir §8.3.2). Ainsi, à suffisamment basse température, on s'attend donc à ce que les effets d'interférences l'emportent sur le mécanisme conduisant aux fluctuations des défauts. Enfin, à très basse température, lorsque $L_{\Phi}>L$, on s'attend à nouveau à une saturation du bruit. 

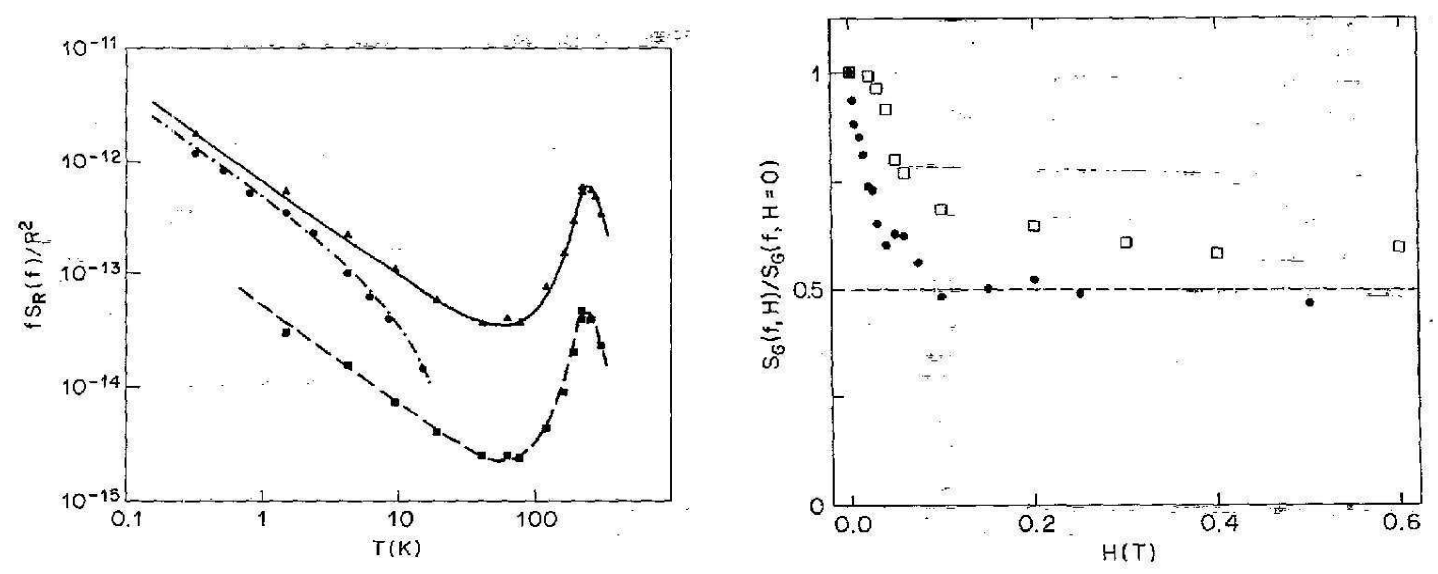

FIG. 8.2: (a) Amplitude du bruit de résistance en $1 / f$ mesuré à $1 \mathrm{~Hz}$ dans des films de Bi en fonction de la température $T$. Les trois courbes correspondent à des échantillons de taille et de géométrie différentes. (b) Bruit de conductance, défini par $S_{G}=S_{R} / R^{4}$ en fonction du champ magnétique $H$ mesuré à deux températures différentes. D'après Birge et al. [347]

\section{Observations expérimentales}

La prédiction la plus surprenante du modèle de Feng, Lee et Stone pour le bruit en $1 / f$ issu des fluctuations universelles de conductance est la dépendance en température qui est très différente de celle proposé dans les modèles classiques. C'est grâce à cette dépendance que Birge et al. ont interprété les mesures de bruit faites sur des films de bismuth [347, 348] (voir la figure voir la figure 8.2-a). A basse température, ce système est bidimensionnel au sens de la cohérence car l'épaisseur du film est alors plus faible que la longueur de cohérence de phase (qui est elle-même plus faible que les autres dimensions du film). Dans ce régime, l'amplitude du bruit $S_{R} / R^{2}$ augmente quand la température diminue, $S_{R} / R^{2} \propto T^{-1}$. Cette dépendance signifie qu'à basse température, les impuretés sont gelées et les effets quantiques dominent le bruit. A plus haute température, la transition vers un comportement 3D et les effets d'activation ne permettent plus d'interpréter facilement les résultats.

La confirmation des effets d'interférences quantiques sur le bruit en $1 / f$ est apportée par les mesures du bruit de conductance $S_{G}=S_{R} / R^{4}$ en fonction d'un champ magnétique. On a vu au chapitre 1 qu'un champ magnétique, supprimant la symétrie par renversement du sens du temps, supprime les effets d'interférences conduisant à la localisation faible. De la même manière il a été prédit que le champ magnétique réduit le bruit d'un facteur deux [347]. C'est effectivement ce qui est observé dans les films de bismuth, comme le montre la figure 8.2-a.

Mailly et Sanquer ont comparé les dépendances en champ magnétique des fluctuations universelles de conductance et du bruit en $1 / f$ dans des fils GaAs :Si mésoscopique à 45 $\mathrm{mK}$ [349]. Ils obtiennent un même facteur de réduction dans les deux cas, ce qui permet de conclure que, dans ces systèmes et à basse température, le bruit en $1 / f$ est dû aux fluctuations universelles de conductance. 


\subsubsection{Interférences quantiques dans un isolant}

Feng, Pichard ont montré que, dans un isolant d'Anderson, les interférences quantiques peuvent également conduire à des fluctuations mésoscopiques de la conductance du système en fonction du champ magnétique appliqué [350]. Suivant cette idée, et le raisonnement fait pour les fluctuations universelles de conductance, Feng, Pichard et Zeng prédisent l'existence d'un bruit macroscopique dû à ces fluctuations de conductance dans un isolant d'Anderson [351]. On s'attend alors également à observé un bruit qui augmente quand la température diminue.

Ces fluctuations de conductances ont été observées dans des isolants d'Anderson par Milliken et al. [352], et par Ladieu, Mailly et Sanquer [353] (voir également la thèse de Ladieu [354]).

\subsection{Fluctuations de résistance et transition de phase}

Jusqu'à présent nous avons vu les origines physiques possibles des fluctuations de résistances observées dans les conducteurs. Dans tous les cas, ces fluctuations résultent de la réponse du système à des fluctuations extérieures (en général des impuretés ou des défauts). Pour cette raison on peut se poser la question des évolutions de ces fluctuations lorsque le système subit une transition de phase et que ses propriétés subissent une modification fondamentale. Deux causes peuvent conduire à un comportement particulier des fluctuations de résistance. Dans une transition de phase du second ordre, les fluctuations du paramètre d'ordre et sa longueur de corrélation divergent à la transition, conduisant à des lois d'échelle. On peut donc s'attendre à une divergence du bruit à la transition, et à des lois d'échelle sur ce bruit. Plus généralement, le système subissant un changement d'état à la transition, on peut envisager que ce changement soit visible sur les mesures de bruit.

Cette étude est d'autant plus adaptée au cas des gaz bidimensionnels d'électrons très dilués car de nombreuses théories prévoient une transition de phase lorsqu'on baisse la densité électronique (voir chapitres 1 et 2).

\subsubsection{Transition de percolation}

Nous avons vu au chapitre 1 que la théorie de percolation permet de décrire des systèmes fortement désordonnés. En particulier, il est possible de calculer la conductivité d'un système très désordonné par cette méthode. Lorsqu'on augmente progressivement le paramètre de la percolation (la probabilité d'occupation $x$, comme nous le verrons après), cette théorie prédit que le système passe brusquement d'un état qui ne conduit pas le courant à un état qui conduit le courant à une valeur finie du paramètre $x$. Ainsi certaines transitions métal-isolant ont été interprétées comme des transition de percolation.

Dans les paragraphes qui suivent nous verrons les prédictions que donne la théorie de percolation sur la conductance et les fluctuations de résistance, et notamment les lois d'échelle vérifiées par ces deux grandeurs et les exposants critiques associés. Ensuite seront mentionnées des expériences ayant pu vérifier les prédictions théoriques. 


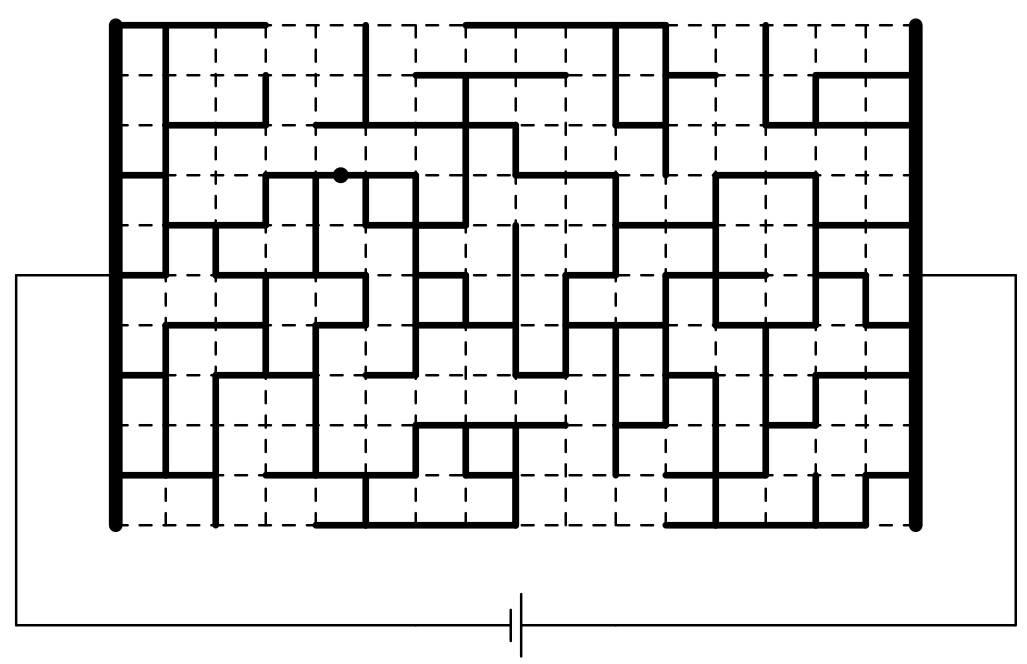

FIG. 8.3: Modèle de percolation sur un réseau carré en dimension deux. Les liens en gras sont les liens occupés par une résistance $r_{m}$, et donc qui conduisent le courant, alors que les liens en tirets sont les liens coupés. Dans cet exemple, $x>x_{p}$, tout en restant très proche du seuil de percolation $\left(x_{p}=1 / 2\right.$ dans ce réseau carré en dimension deux). Le lien marqué d'un point est le lien critique qui, s'il est supprimé, coupe le réseau percolant et annule la conductance globale du système.

\section{Conductance et fluctuations de résistance}

On a représenté sur la figure 8.3 un modèle simple de percolation sur réseau carré. On cherche à faire passer un courant entre les bords de gauche et de droite. Chaque lien peut être occupé par une résistance $r_{m}$ avec la probabilité $x$, ou être vide (i.e. le circuit est alors coupé) avec une probabilité $1-x$. On cherche alors à calculer la conductance de tout le système. Lorsque $x=0$, tous les liens sont coupés et la conductance du système est nulle. Si on ajoute des liens de manière aléatoire (ce qui revient à augmenter la probabilité d'occupation $x$ ), la théorie de percolation a montré que la conductance reste nulle jusqu'à une valeur critique de l'occupation, $x_{p}$, au-dessus de laquelle le système commence à conduire le courant et $G \neq 0$ (ce raisonnement est valable lorsque la taille du système tend vers l'infini). En particulier, pour un réseau carré en dimension deux, $x_{p}=1 / 2$. Au dessus du seuil de percolation, la conductance augmente progressivement. Le système passe donc d'un état isolant $\left(x<x_{p}\right)$ a un état métallique $\left(x>x_{p}\right)$, ce qui rend intéressante l'étude de la percolation dans le cadre d'une transition métal-isolant. La théorie de percolation permet ensuite de décrire le comportement de quantités physiques telle la résistance, ou les fluctuations de cette résistance, aux abords de la transition.

Pour évaluer la résistance $R=1 / G$ du système on égalise la puissance Joule dissipée dans tout le système et la somme des puissances dissipées par les liens de résistances $r_{m}$ :

$$
R I^{2}=\sum_{m} r_{m} I_{m}^{2} \quad \Rightarrow \quad R=\sum_{m} r_{m} i_{m}^{2}
$$

où $I$ est le courant total dans le système, $I_{m}$ le courant dans le lien $r_{m}$ et $i_{m}=I_{m} / I$. La seconde équation permet de calculer la résistance par simulation numérique. Dans une 
transition de percolation, la conductance s'annule à la transition (ou la résistance diverge) suivant une loi d'échelle [321] :

$$
G=\frac{1}{R} \propto\left(x-x_{p}\right)^{t}
$$

où l'exposant $t$ est l'exposant de conductivité.

Pour calculer les fluctuations de résistance $\delta R$ d'un système percolant, on suppose que ces fluctuations sont dues aux fluctuations de résistance des liens formant le réseau percolant $\delta r_{m}$. Les fluctuations de résistance peuvent s'exprimer en linéarisant l'équation (8.22) en $\delta r_{m}$ et en considérant que la puissance Joule est extrémale par rapport aux fluctuations de courant $\delta i_{m}$ (i.e. $\left.\delta i_{m}=0\right)$ :

$$
\delta R(t) \approx \sum_{m}\left(i_{m}\right)^{2} \delta r_{m}(t)
$$

Dans les systèmes désordonnés usuels, la longueur de corrélation microscopique (libre parcours moyen) est plus petit que l'espacement entre sites caractérisant les liens. On peut alors considérer que les fluctuations de résistance des liens ne sont pas corrélées. De plus, on suppose que les liens qui ne sont pas coupés ont tous une même résistance moyenne $r$. Si on note $s_{r}$ le spectre des fluctuation des résistances des liens, on montre que :

$$
\frac{S_{R}(f)}{R^{2}}=\frac{s_{r}(f)}{r^{2}} \frac{\sum_{m} i_{m}^{4}}{\left(\sum_{m} i_{m}^{2}\right)^{2}} .
$$

Le membre de droite de cette équation comporte deux termes de nature différente. Le premier terme, $s_{r}(f)$, est déterminé par les fluctuations de résistance des liens, et nécessite de connaître l'origine microscopique de ces fluctuations. Il ne dépend pas du réseau de percolation, et l'étude de ce terme sort donc de la théorie de percolation. Par contre c'est ce terme qui donne la dépendance en fréquence de $S_{R}(f)$. En général ces fluctuations ont un spectre en $1 / f$, conduisant à une dépendance de $S_{R}$ en $1 / f$. Le second terme dans le membre de droite de l'équation (8.25) est la grandeur qui dépend du réseau de percolation, et peut être évalué par simulation numérique. Lorsque $x$ s'approche de la valeur critique de percolation $x_{p}$ (avec $x>x_{p}$ ), le spectre des fluctuations de résistance diverge suivant la loi [355]:

$$
\frac{S_{R}}{R^{2}} \propto\left(x-x_{p}\right)^{-\kappa},
$$

où l'exposant $\kappa$ est l'exposant critique des fluctuations de résistance.

Un raisonnement qualitatif permet de comprendre cette divergence à la transition de percolation. Supposons que les éléments du réseau de percolation fluctuent de manière identique, et que ces fluctuations soient indépendantes. Loin de la transition $\left(x \gg x_{p}\right)$, un grand nombre de chemins participent à la conduction. Ainsi les fluctuations individuelles des éléments vont se moyenner, et donner lieu à un bruit faible. Quand on se rapproche de la transition, le nombre de chemins participant à la conduction diminue, et la moyenne se fait donc sur un nombre plus faible de chemins, conduisant à une augmentation du bruit quand $x \rightarrow x_{p}$. Le cas limite est atteint à $x \approx x_{p}$ quand la conduction est gouvernée par un nombre limité de liens critiques qui, s'ils sont supprimés, coupent le réseau percolant 


\begin{tabular}{|c|ccc|c|}
\hline \multicolumn{1}{|c}{$d=2$} \\
\hline modèle & $t$ & $\kappa$ & $w=\kappa / t$ & référence \\
\hline sur réseau & $1,32 \pm 0,05$ & 1,339 & $1,01 \pm 0,04$ & {$[321,355]$} \\
"gruyère Suisse" & - & - & $1,6-4,2$ & {$[356]$} \\
trous aléatoires & - & - & 3,2 & {$[357]$} \\
trous aléatoires inversé & - & - & 0,87 & {$[357]$} \\
réseau de résistances aléatoires & $1,2 \pm 0,1$ & 3,1 & 2,6 & {$[358]$} \\
\hline
\end{tabular}

\begin{tabular}{|c|ccc|c|}
\hline \multicolumn{7}{|c|}{$d=3$} \\
\hline modèle & $t$ & $\kappa$ & $w=\kappa / t$ & référence \\
\hline sur réseau & $1,95 \pm 0,1$ & 2,332 & $1,20 \pm 0,06$ & {$[321,355]$} \\
trous aléatoires & - & - & 2,1 & {$[357]$} \\
trous aléatoires inversé & - & - & 2,4 & {$[357]$} \\
\hline
\end{tabular}

TAB. 8.1: Exposants de la conductance, $t$, et du bruit, $\kappa$, pour une transition de percolation en dimension deux (tableau du haut) et en dimension trois (tableau du bas) obtenus par simulation numérique dans différents modèles.

et annulent la conductance (c'est le cas du lien marqué d'un point dans l'exemple décrit dans la figure 8.3). La fluctuation d'un lien critique, d'un état passant à un état bloqué, conduit à une résistance totale fluctuant entre une valeur finie et l'infini, et donc à une divergence des fluctuations.

Dans la pratique, il est souvent difficile de mesurer la probabilité d'occupation $x$, et il peut donc s'avérer difficile de vérifier les lois de puissance. Il est cependant possible de vérifier la relation entre le bruit et la résistance :

$$
\frac{S_{R}}{R^{2}} \propto R^{w} \quad \text { avec } \quad w=\frac{\kappa}{t} .
$$

Les exposants critiques de la conductance et des fluctuations de résistance dépendent fortement du modèle utilisé dans la simulation numérique. Le modèle le plus simple est le modèle sur réseau présenté dans la figure 8.3, mais il ne permet pas toujours de rendre bien compte des expériences, notamment lorsque le milieu est continu. Les résultats obtenus dans différents modèles en dimensions deux et trois sont résumés dans le tableau 8.1. On peut remarquer que les systèmes étudiés pendant la thèse sont en dimension deux.

\section{Résultats expérimentaux}

Observation du bruit dans des films métalliques par Garfunkel et Weissman [356]. L'épaisseur du métal est réduite par abrasion par du sable, conduisant à une modification du désordre dans ces films.

Koch et al. ont mesuré simultanément la résistance et les fluctuations de résistance dans des films fins d'or désordonnés afin d'obtenir le rapport des exposants $w=\kappa / t$ [359]. Dans ces échantillons, l'épaisseur du film est modifiée par abrasion par faisceau d'ions, permettant d'augmenter le désordre en gardant toujours le même échantillon. Ces expériences permettent de déterminer $w=2,0 \pm 0,1$. D'autre part la résistance $R$ et le 
bruit $S_{R} / R^{2}$ sont mesurés en fonction de la température. Dans la majorité des échantillons les moins résistifs, $R$ et $S_{R} / R^{2}$ augmentent quand la température augmente, ce qui est caractéristique d'un comportement métallique. Dans les échantillons les plus résistifs, $R$ et $S_{R} / R^{2}$ diminuent exponentiellement quand la température augmente, ce qui est caractéristique d'une conduction par sauts. La transition de percolation est estimée entre ces deux régimes.

Dans des échantillons $\mathrm{Pt} / \mathrm{Al}_{2} \mathrm{O}_{3}$, Mantese et al. trouvent également une loi d'échelle $S_{R} / R^{2}(T) \propto R(T)^{w}$, où la résistance est modifiée par l'intermédiaire de la température, avec $w \approx 4,22 \pm 0,06$ pour une fraction de $50 \%$ de Pt, et $w \approx 2,39 \pm 0,04$ pour une fraction de $46 \%$ de $\mathrm{Pt}$ [360].

Dans des composés manganite supraconducteurs, un pic de bruit est observé à la transition métal-isolant [361], ce qui permet aux auteurs de confirmer la nature percolative de cette transition en dimension trois. Les auteurs ont également mesuré l'exposant du rapport entre le bruit et la résistance, $w=1,2-3$, compatible avec des résultats de simulation en dimension trois.

\section{Systèmes à deux composantes}

Dans les modèles de percolation présentés dans les paragraphe précédents, on a supposé qu'une phase "liquide" percole dans un réseau de résistance infinie et non bruyant. Ainsi ces modèles conduisent à une résistance et à un bruit en résistance qui divergent à la transition. A partir de mesures faites sur des échantillons $\mathrm{Pt} / \mathrm{Al}_{2} \mathrm{O}_{3}$, Mantese et al. ont suggéré que, dans un système réel, il est possible d'observer de la conduction à travers les liens coupés par effet tunnel [360, 362]. Dans ce modèle, ils considèrent donc la percolation d'un système liquide à travers un système isolant. A densité élevée de liens, la conduction et le bruit sont contrôlés par la phase conductrice car la phase isolante est largement minoritaire. Dans ce régime, le comportement d'échelle dû à la percolation est bien observé. Par contre, lorsque la densité de lien se rapproche du seuil de percolation, la conductance du réseau percolant tend vers zéro, et la conduction commence à se faire à travers la partie isolante. Dans cette limites, Mantese et al. observent que la conductance et le bruit saturent à leurs valeurs dans l'isolant : dans ce régime, c'est donc la phase isolante qui contrôle le bruit.

\subsubsection{Transition d'Anderson en dimension trois}

Les fluctuations de conductances ont été mesurées à la traversée de la transition métalisolant d'Anderson par Cohen et al. [363, 364] dans des films d'oxyde d'indium et d'oxyde de zinc. La première remarque est que le bruit est entre 6 et 10 ordres de grandeur audessus du bruit mesuré dans des systèmes métalliques. A la traversée de la transition métal-isolant, pour $k_{F} l \sim 1$, le bruit augmente de environ trois ordres de grandeurs. Dans la phase métallique, le bruit et la résistance augmentent quand la température augmente. Ils ont interprété ces mesures par un modèle de bruit lié à l'approche de la transition d'Anderson. Ils ont en particulier réfuté l'interprétation en terme de transition de percolation classique : le bruit serait plutôt à un effet des interférences quantiques. 
Cependant, cette augmentation importante du bruit n'a pas été observée à la transition métal-isolant dans des échantillons cristallins de Si dopés. Les expériences de Ghosh et Raychaudhuri [365] ont en effet montré une légère augmentation du bruit lorsque $k_{F} l$ diminue (en restant supérieur à 1), et une diminution importante du bruit à l'approche de la transition métal-isolant.

Adkins et Koch [273] ont effectué des mesures dans des MOSFETs Si à travers la transition entre localisation faible et localisation forte. La variation $S_{R} / R^{2} \propto R$ est compatible avec la formule de Hooge. En effet, dans le cas où la conduction est assurée par les porteurs de charge injectés au-dessus du bord de mobilité, et en supposant que la mobilité des états délocalisés ne varie pas avec la densité de porteurs, la résistance est inversement proportionnelle aux nombres de porteurs libres. Ainsi le bruit est aussi inversement proportionnel au nombre de porteurs libres. Dans ces expériences, $S_{R} / R^{2}$ croît quand la température diminue de 4,2 à 1,5 K. Enfin le bruit varie continûment à la traversée de la transition du régime de localisation forte au régime de localisation faible. Mais la précision de la mesure n'a pas permis d'étudier en détaille le régime "métallique". Ils ont également vérifié que la longueur de corrélation du bruit est faible devant la taille des échantillons $(\approx 100 \mu \mathrm{m})$. Les caractéristiques expliquées plus haut indiquent que ces fluctuations ne peuvent pas être dues au transport diffusif des porteurs au-dessus du bord de mobilité, ni au piègeage des porteurs, ni aux fluctuations de température.

\subsubsection{Transition vitreuse}

Dans le cadre de la transition métal-isolant en dimension deux, certains modèles proposent la formation d'un verre à faible densité électronique en raison de la compétition entre les interactions Coulombiennes et le désordre (voir le chapitre 2). Des mesures de fluctuations de la résistance ont été faites dans des MOSFETs silicium présentant les caractéristiques de la TMI à 2D [82, 83]. Les constatations les plus surprenantes dans ces systèmes sont les nombreuses similitudes avec les mesures de fluctuations de résistance dans les verres de spin. Pour cette raison, et même si l'état du gaz d'électron dilué dans les MOSFETs silicium est encore controversé, on peut mentionner ici ces mesures.

Des mesures ont été réalisées dans des MOSFETs silicium de petite taille (longueur $\approx 1$ $\mu \mathrm{m}$ ) et de faible mobilité [82] ainsi que sur des MOSFETs Si macroscopiques faiblement désordonnés [83]. Les résultats sont qualitativement semblables dans les deux cas. Le bruit mesuré a un spectre en $1 / f^{\alpha}$, et présente une brusque variation lorsque la densité de porteur $n_{s}$ diminue en dessous d'une densité critique $n_{g}$ (qui diffère de la densité critique couramment employée dans le cadre de la TMI 2D, comme il a été précisé dans le chapitre 2). (1) Le bruit augmente fortement, avec une rupture de pente de la dépendance $S_{R}\left(n_{s}\right)$ à la transition, caractéristique d'un mouvement collectif des porteurs de charge 5. (2) Pour $n_{s}<n_{g}, S_{R}$ augmente fortement quand la température diminue (avec une dépendance exponentielle), alors que la dépendance en température du bruit est très faible pour $n_{s}>n_{g}$ (3) Quand la densité diminue, l'exposant $\alpha$ passe brutalement de

\footnotetext{
${ }^{5}$ Dans un modèle de verre, les états métastables du système correspondent à des minimums locaux de l'énergie libre, séparés par des barrières avec une large distribution de hauteurs, et donc de temps de relaxation. Les transitions inter-vallées, faisant intervenir la reconfiguration d'un nombre importants d'électrons, induisent alors un bruit en $1 / f$ corrélé d'amplitude élevée [83].
} 
$\approx 1$ pour $n_{s}>n_{g}$ à $\approx 1,8$ pour $n_{s}<n_{g}$, donc une valeur proche de 2 observée dans les verres de spin et interprété comme un ralentissement de la dynamique. Enfin le deuxième spectre $S_{2}\left(f_{2}, f\right)$, qui est le spectre de puissance des fluctuations de $S_{R}(f)$, varie en $1 / f_{2}^{1-\beta}$ avec $1-\beta=0$ pour $n_{s}>n_{g}$ et $1-\beta>0$ pour $n_{s}<n_{g}$. Cette variation signifie que les fluctuations de résistances ne sont pas gaussiennes, caractère qui indique que les fluctuations locales sont corrélées (une moyenne sur des fluctuations locales non corrélées conduiraient à des fluctuations globales gaussiennes). Toutes ces observations semblent donc indiquer que le gaz d'électrons subit une transition vers un état vitreux à une densité donnée $n_{g}$.

\subsection{Conclusion}

Nous avons donc vu les différents mécanismes qui peuvent conduire à des fluctuations de résistance dont la dépendance spectrale est proche de $1 / f$.

En particulier, le mécanisme mis en jeu peut varier de manière importante suivant le système considéré, s'il est métallique ou isolant, s'il est bien décrit classiquement ou si la mécanique quantique intervient, s'il présente une transition entre différents comportement. Tous ces modèles confirment donc notre idée initiale voulant que le bruit n'est pas seulement une quantité gênante pour la mesure, mais qu'il donne des informations importantes sur le système. Les fluctuations de résistance dans un conducteur sont particulièrement intéressantes car elles font intervenir la réponse du système électronique à des fluctuations extérieures, et constituent par là une observable supplémentaire par rapport aux mesures usuelles de transport. 


\section{Chapitre 9}

\section{Dispositif expérimental pour la mesure de bruit}

Sommaire



9.2 Principe de la mesure . . . . . . . . . . . . . 204

9.3 Bruits parasites ................... 206

9.3 .1 Bruits extérieurs . . . . . . . . . . . . 206

9.3.2 Bruit de la chaîne de mesure . . . . . . . . . . . . . 207

9.4 Suppression des bruits parasites ........... 211

9.4.1 Mesure en corrélation . . . . . . . . . . . . . . 211

9.4.2 Corrélation "amont-aval" . . . . . . . . . . . . . 214

9.4.3 Mesure synchrone . . . . . . . . . . . . . . . . . . . 214

9.4.4 Mesure synchrone en corrélation . . . . . . . . . . . 217

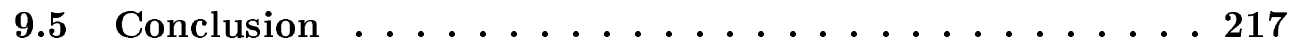




\subsection{Introduction}

Dans ce chapitre sont décrits les différentes méthodes permettant de mesurer les fluctuations de résistance à basse fréquence dans les gaz bidimensionnels de porteurs présentés dans le chapitre 3. En raison des nombreuses sources de bruits parasites, extraire le bruit provenant exclusivement de l'échantillon nécessite d'employer des montages élaborés. Après une description des montages de principe (§9.2) sont présentés les différents éléments pouvant perturber la mesure de bruit, i.e. produisant un bruit susceptible de masquer le bruit provenant de l'échantillon (§9.3). Ensuite sont décrites principalement deux méthodes permettant de réduire, ou même d'éliminer, certains de ces bruits parasites (§9.4), la méthode de corrélation et la mesure synchrone, et une méthode plus élaborée combinant ces deux techniques de mesure.

\subsection{Principe de la mesure}

Le but des expériences décrites ici est de mesurer les fluctuations de résistance $\delta R$, ou plus exactement la puissance de bruit $S_{R}$ liée à ces fluctuations. Expérimentalement il existe deux manières de mesurer ces fluctuations, soit en mesurant les fluctuations de tension à courant fixé, soit en mesurant les fluctuations de courant à tension fixée :

$$
\frac{S_{R}}{R^{2}}=\left(\frac{S_{V}}{V^{2}}\right)_{I=c s t e}=\left(\frac{S_{I}}{I^{2}}\right)_{V=c s t e} .
$$

Dans cette partie sont décrits ces deux montages, ainsi que la chaîne de mesure utilisée pour la mesure des spectres. Ces montages de principe sont idéaux, et nous verrons dans les parties suivantes les modifications à apporter pour prendre en compte les effets parasites.

\section{Mesure à courant fixé}

Le montage de mesure du bruit en tension à courant fixé est décrit dans la figure 9.1. Le courant est généré par une source de courant. La tension résultante aux bornes de l'échantillon est mesurée par une méthode quatre points pour minimiser l'effet des résistances de contact. Il est possible de remplacer la source de courant par une source de tension en série avec un résistance de charge de valeur très élevée devant la résistance de l'échantillon. Cette méthode permet de réaliser une source de courant moins bruyante que certaines sources de courant commerciales.

\section{Mesure à tension fixée}

Lorsque l'échantillon a une résistance très élevée, notamment comparée aux résistances d'entrée de l'amplificateur, la mesure ci-dessus n'est plus utilisable, en raison principalement du bruit en courant et de la résistance d'entrée finie des amplificateurs. On préfère alors réaliser la mesure de courant à tension fixée décrite dans la figure 9.2. La résistance de mesure $R_{m}$ doit être peu bruyante (comparé à l'échantillon) et de faible valeur devant la résistance $R$ de l'échantillon. Pour ces raisons on peut préférer à ce montage l'utilisation d'un amplificateur de courant. 


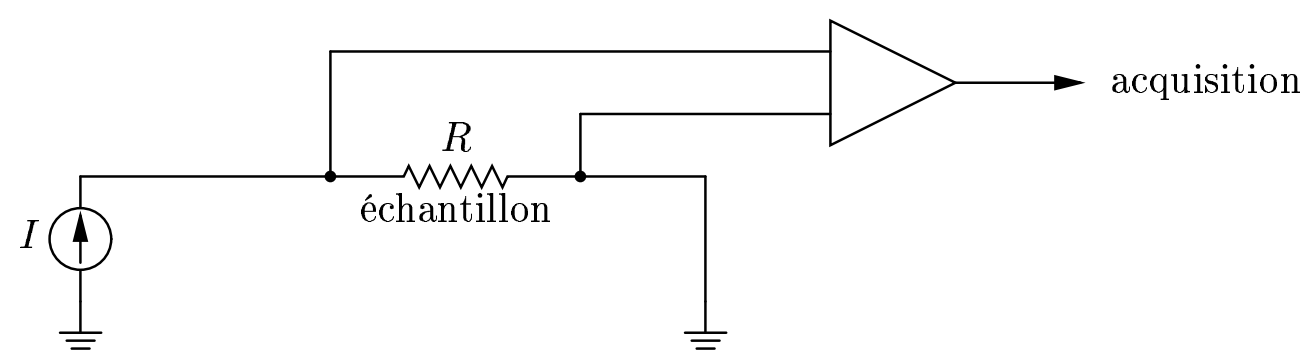

FIG. 9.1: Montage pour la mesure du bruit en tension à courant $I$ fixé.

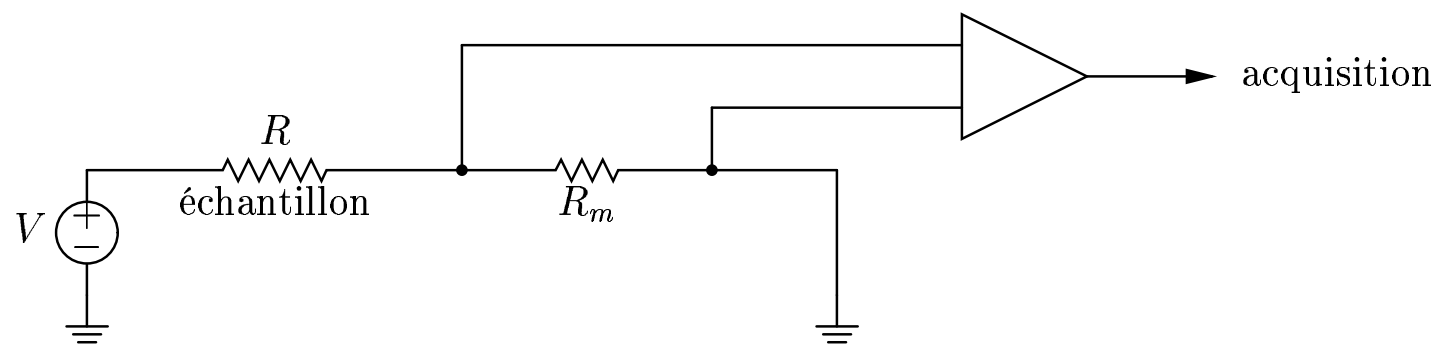

FIG. 9.2: Montage pour la mesure du bruit en courant à tension $V$ fixée.

\section{Amplification, filtrage et échantillonnage}

La partie amplification du montage utilisé pour les mesures comporte deux étages d'amplification. Le premier étage est réalisé par un amplificateur bas bruit LI 75 A (NF Electronic Instruments), dont le gain est fixé à 100. Le deuxième étage est réalisé par amplificateur un SR 560 (Standford Research Systems) ou EGG 5113 (EG\&G Instruments) (suivant la voie de mesure, comme il sera précisé dans la partie 9.4), qui ont un gain variable et la possibilité de filtrer le signal. Les filtres passe-bas de ces amplificateurs servent de filtre anti-repliement avant échantillonnage (de fréquence de coupure $f_{c}$, et de pente 6 ou $12 \mathrm{~dB} /$ octave). On se servira également dans la plupart des cas de filtres passe-haut pour éliminer la composante continue du signal (fréquence de coupure de $0,03 \mathrm{~Hz}$, pente $6 \mathrm{~dB} /$ octave). On a vérifié que les caractéristiques de ces filtres correspondent bien (à quelques pourcents près) aux données constructeur. D'autre part les gains sont étalonnés par mesure sur des résistances connues.

L'acquisition est réalisée grâce à une carte deux voies, interne à l'ordinateur (carte PCI 6111 E de National Instrument), qui permet de réaliser un échantillonnage sur 12 bits avec une fréquence d'échantillonnage comprise entre $1 \mathrm{kHz}$ et $5 \mathrm{MHz}$.

Les fréquences d'échantillonnage $f_{e}$ et de coupure anti-repliement $f_{c}$ sont choisies en fonction de la gamme de fréquences à explorer. Le filtre anti-repliement à $6 \mathrm{~dB} /$ octave conduit à une réduction du signal de $97 \%$ pour une fréquence égale à $30 \times f_{c}$. Pour une mesure de bruit en $1 / f$, ce signal est négligeable, et le théorème de Shannon nous autorise à choisir une fréquence d'échantillonnage $f_{e}=60 \times f_{c}$. En corrigeant l'effet du filtre anti-repliement, il est alors possible d'effectuer une mesure jusqu'à une fréquence de l'ordre de $f_{c}$. Enfin la fréquence minimale de mesure $f_{\text {min }}$ est déterminée par la fréquence d'échantillonnage $f_{e}$ et le nombre d'échantillons enregistrés $N_{e}$ par $f_{\text {min }}=f_{e} / N_{e}$. En 
résumé, la gamme de fréquences explorée est :

$$
\frac{f_{e}}{N_{e}} \leqslant f \lesssim f_{c}=\frac{f_{e}}{60}
$$

L'utilisation de filtres anti-repliement d'ordre plus élevé a permis d'augmenter $f_{c}$ pour la fin de nos expériences.

\subsection{Bruits parasites}

Dans cette parties sont décrites les différentes sources de bruits parasites peuvant perturber la mesure des fluctuations de tension dans la configuration de mesure simple décrite dans la figure 9.1. On peut classer ces bruits parasites suivant deux catégories, les bruits extérieurs au circuit de mesure qui pourraient, en théorie, être éliminés par un isolement total du montage expérimental vis-à-vis de l'extérieur, et les bruits intrinsèques au circuit de mesure.

\subsubsection{Bruits extérieurs}

\section{Bruits directs}

Certaines sources de bruit agissent directement sur la tension mesurée et peuvent être décrites par un terme général $\delta V_{\text {ext }}$. Ce terme peut dépendre de certains paramètres de la chaîne de mesure, et en particulier des gains des amplificateurs si ces bruits s'insèrent en amont des amplificateurs.

Le bruit d'origine électromagnétique est en général un bruit haute fréquence et ajoute directement à la mesure une tension fluctuante. Il a des conséquences qui peuvent être néfastes pour la mesure, en particulier en raison du chauffage du gaz d'électrons. Pour éliminer ce bruit, on utilise des filtres passe-bas à traversée sur les câbles électriques menant à l'échantillon, un blindage métallique étanche autour de la partie bas niveau du circuit de mesure, des cordons d'alimentation secteur filtrés et des mousses absorbant les radiofréquences.

Le bruit d'origine microphonique est un bruit basse fréquence, et peut donc gêner la mesure du bruit en $1 / f$. Il agit par l'intermédiaire de l'isolant des câbles coaxiaux par création de tensions induites, sur les composants électroniques des appareils, et directement sur l'échantillon. Les principales protections contre le bruit acoustique sont un isolement du banc expérimental vis-à-vis du reste du laboratoire par une dalle flottante et des amortisseurs hydrauliques, des plaques de mousse absorbante autour du cryostat, et l'optimisation du circuit de mesure pour éviter une trop grande mobilité des câbles. L'influence des bruits d'origine microphonique sur la mesure peut être étudiée par l'utilisation d'un accéléromètre.

Enfin le réseau électrique peut être la source d'un bruit à $50 \mathrm{~Hz}$ et aux harmoniques de $50 \mathrm{~Hz}$. Ce bruit est engendré par les appareils de mesure alimentés par le secteur, et est propagé soit par rayonnement, soit par les diverses connections entre les appareils, comme l'alimentation ou la communication par réseau GPIB (boucles de masse). Ce bruit 


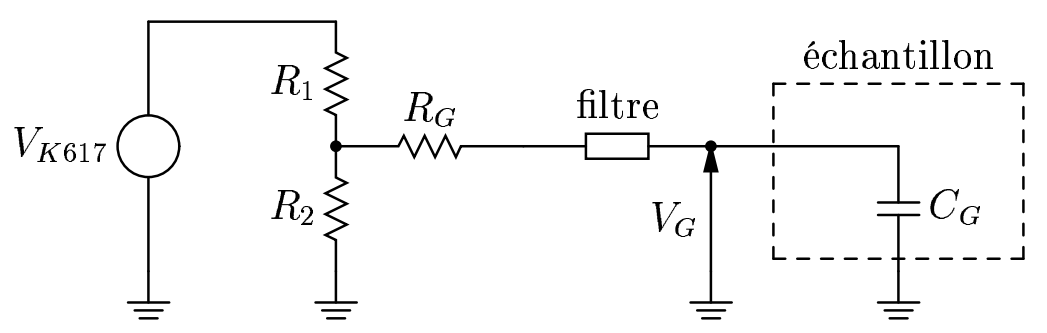

FIG. 9.3: Montage utilisé pour imposer la tension de grille. La tension est imposée par un générateur de tension Keithley K617, et la grille est symbolisée sur ce schéma par la capacité $C_{G}$.

peut être réduit par l'utilisation de batteries pour l'alimentation des appareils, ou de découpleurs entre les appareils. Dans l'expérience nous avons utilisé un transformateur pour découpler l'alimentation de certains appareils du secteur, un découpleur optique pour les communications entre l'ordinateur et les appareils par le réseau GPIB, et des cordons secteurs blindés.

\section{Bruits de modulation}

D'autres sources de bruit extérieures au système de mesure peuvent jouer sur les composants de la chaîne de mesure, et en particulier sur la résistance de l'échantillon, conduisant à des fluctuations de résistance $\delta R^{e x t}$. C'est le cas des fluctuations de température, notées $\delta T(t)$. Nous avons vu au chapitre 5 que la résistance des systèmes étudiés varie fortement avec la température, on s'attend donc à des fluctuations importante de la résistance globale qui pourra s'écrire $\delta R(t)=d R / d T \cdot \delta T(t)$. L'influence de ces fluctuations de température sur les mesures peut être testée en utilisant deux échantillons dont les résistances dépendent fortement de la température, séparés électriquement, mais tous les deux en contact thermique dans le cryostat. Une corrélation entre le bruit des deux échantillons permet de tester l'importance des fluctuations de température.

Dans le cas où la densité électronique peut être modifiée à l'aide d'une grille, les fluctuations de la tension de grille peuvent également conduire à des fluctuations de la résistance de l'échantillon. Afin de minimiser les fluctuations de tension de grille, on utilise le montage de la figure 9.3. L'utilisation d'une résistance $R_{G}$ de forte valeur (environ 100 $\mathrm{M} \Omega$ ) et d'un filtre passe-bas permet de réduire les fluctuations de la tension de grille $V_{G}$.

\subsubsection{Bruit de la chaîne de mesure}

Nous passons maintenant en revue les différentes sources de bruit inhérentes au circuit de mesure, qui peuvent être réduites par l'utilisation de composants adaptés, mais ne peuvent pas être éliminés directement.

\section{Bruit d'une résistance}

On peut modéliser une résistance $R(t)$ par trois dipôles placés en série : une résistance constante $\bar{R}$, une résistance fluctuante $\delta R(t)$ de valeur moyenne nulle dont le spectre de 
bruit à basse fréquence est en $1 / f$, et un générateur de tension fluctuante $\delta e(t)$ correspondant au bruit thermique (voir figure 9.4). Les spectres de bruit peuvent ainsi s'écrire $S_{R}(f)=A R^{2} / f$ (d'après la loi de Hooge, voir le chapitre 8 ) et $S_{e}(f)=4 k_{B} T R$ (voir $\S$ A.3.4), où $A$ est une constante que l'on pourra évaluer et $T$ est la température de la résistance.

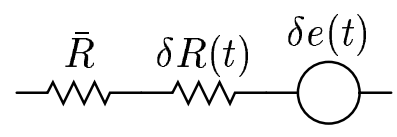

FIG. 9.4: Schéma équivalent en bruit d'une résistance

Les résistances utilisées dans le circuit de mesure sont des résistances bas bruit SFR 25 de marque Philips. Le bruit d'une de résistance de $1 \mathrm{M} \Omega$ à température ambiante a été mesuré par la méthode de corrélation (voir §9.4.1). A basse fréquence, il est bien décrit par $S_{R}(f)=A R^{2} / f$ avec $A \approx 5 \times 10^{-15}$ à $10^{-13}$. A plus haute fréquence, le spectre est constant et est le bruit thermique de cette résistance à température ambiante.

\section{Schéma équivalent de l'échantillon}

Pour les mesures de bruit, on utilise une barre de Hall comportant deux contacts de courant, et cinq contacts de tension, comme le montre la figure 9.5. On peut alors modéliser le système par deux résistances principales $R_{\text {éch }}$ et $R_{\text {aval }}$, et des résistances de contacts $R_{c i}$ et $R_{c v}$, suivant que ce sont des contacts de courant ou de tension. L'ensemble ces résistances peut être modélisé par le schéma de la figure 9.4. On remarquera en particulier que le seul terme qui nous intéresse est le terme de fluctuations de la résistance de l'échantillon $\delta R_{\text {éch }}(t)$, tous les autres termes étant alors considérés comme des bruits parasites à la mesure.

Pour les résistances de contact, l'évaluation du bruit thermique est aisé si on connaît la valeur de la résistance et la température. Par contre le bruit de résistance est à priori inconnu. Une évaluation très grossière peut cependant être donnée par la loi de Hooge (voir l'équation (8.5)) en connaissant le nombre de porteurs libres dans les contacts. Mais cette loi empirique a été vérifiée sur des systèmes métalliques homogènes, ce qui n'est pas nécessairement le cas des contacts de la barre de Hall.

\section{Bruit des amplificateurs de tension}

Les amplificateurs de tension peuvent être modélisés en bruit par le schéma de la figure 9.6. Les densités de puissance de $\delta e_{n}$, bruit en tension, et $\delta i_{n}$, bruit en courant, peuvent être calculées en fonction de la fréquence à partir de la figure de bruit $(F B)$ donnée dans les notices d'utilisation des amplificateurs :

$$
F B=10 \log \left[1+\frac{\left(\delta e_{n}\right)^{2}+\left(\delta i_{n} R_{s}\right)^{2}}{4 k_{B} T R_{s}}\right],
$$

où $R_{s}$ est la résistance aux bornes de laquelle est mesurée la tension. Pour la gamme de fréquences en dessous de $1 \mathrm{kHz}$, gamme de fréquences intéressante pour la mesure de 


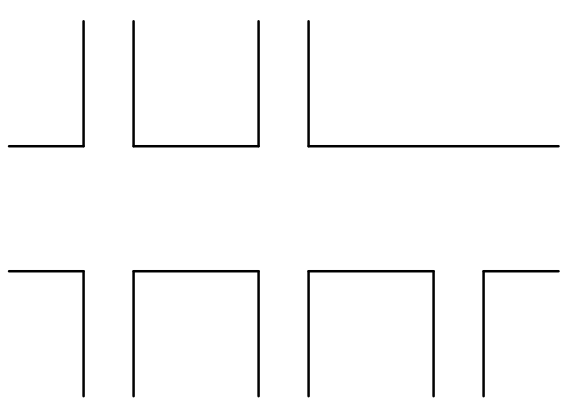

(a)

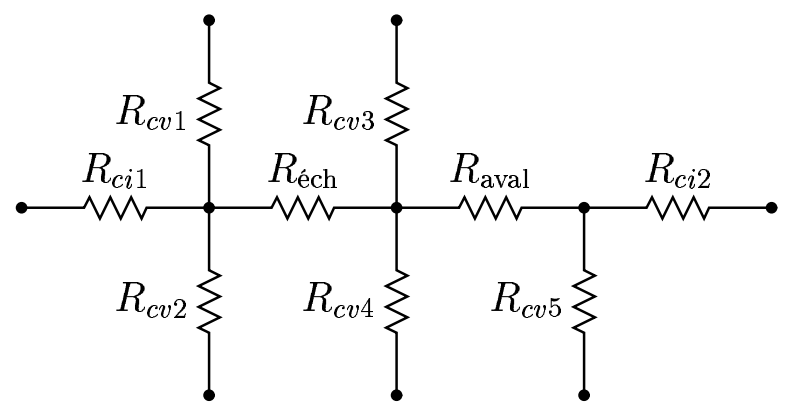

(b)

FIG. 9.5: Représentation schématique du système mesuré. (a) Géométrie de la barre de Hall. (b) Schéma électrique équivalent.



FIG. 9.6: Schéma équivalent en bruit de l'entrée d'un amplificateur de tension, où $R_{s}$ est la résistance sur laquelle est mesurée la tension, et $R_{c}$ la résistance connectée en sortie de l'amplificateur.

bruit basse fréquence, le bruit en courant est à peu prés constant à sa valeur minimale, alors que le bruit en tension augmente de façon importante. Les caractéristiques en bruit typiques des amplificateurs utilisés sont indiquées dans le tableau 9.1. On a vérifier par des mesures sur des résistances à température ambiante que le bruit des amplificateurs est compatible avec les données constructeur.

\section{Bruit de la source de courant}

Trois types de sources de courant ont été utilisées : (a) une source stabilisée réglable (modèle Keithley K 263), (b) une pile aux bornes d'un pont diviseur de tension en série avec une résistance de charge $R_{c}$ de forte valeur comparée à la résistance de l'échantillon

\begin{tabular}{|c|c|c|c|}
\hline marque & modèle & $\delta e_{n} @ 1 \mathrm{kHz}$ & $\delta i_{n} @ 1 \mathrm{kHz}$ \\
\hline NF Electronic Instruments & LI-75 A & $1,18 \mathrm{nV} / \sqrt{\mathrm{Hz}}$ & $12,7 \mathrm{fA} / \sqrt{\mathrm{Hz}}$ \\
Standford Research Systems & SR 560 & $<4 \mathrm{nV} / \sqrt{\mathrm{Hz}}$ & \\
EG\&G Instruments & EGG 5113 & $<4 \mathrm{nV} / \sqrt{\mathrm{Hz}}$ & \\
\hline
\end{tabular}

TAB. 9.1: Caractéristiques en bruit des pré-amplificateurs de tension utilisés, obtenues à partir des figures de bruit données par les constructeurs. 


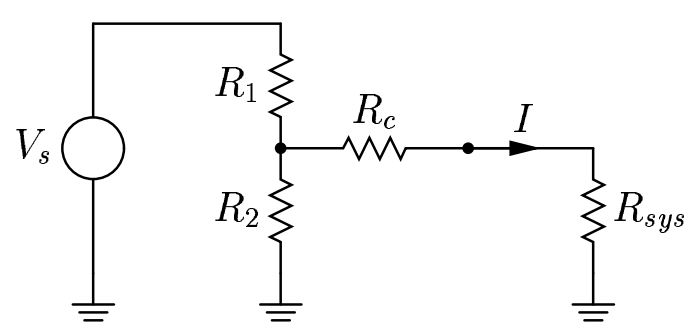

FIG. 9.7: Schéma d'une source de courant en utilisant une source de tension $V_{s}$, un pont de résistances diviseur de tension $\left(R_{1}, R_{2}\right)$ et une résistance de charge $R_{c}$. $R_{s y s}$ représente la résistance du système étudié.

(voir figure 9.7), (c) une source de tension alternative en série avec une résistance de charge $R_{c}$.

La source de courant stabilisée peut introduire des fluctuations intrinsèques du courant $\delta I(t)$, mais le courant moyen fourni $\bar{I}$ peut lui-même dépendre du temps car il peut dépendre de la résistance en sortie de la source (par exemple à cause d'un temps de réponse non instantané de la source aux fluctuations de cette résistance). Ces fluctuations peuvent être résumées par $I(t)=\bar{I}+\delta \bar{I}\left[\left(R_{c}+R\right)(t)\right]+\delta I(t)$.

La source de tension continue est sujette à des fluctuations de la tension imposée $\delta V_{s}(t)$, ce qui conduit à $V_{s}(t)=\bar{V}_{s}+\delta V_{s}(t)$. Le courant résultant de la configuration présentée dans la figure 9.7 peut s'écrire $I(t)=\bar{I}+\delta I(t)$. Pour $R_{c} \gg R_{1}, R_{2}, R_{s y s}$, le courant moyen est donné par :

$$
\bar{I} \approx \frac{\bar{R}_{2}}{\bar{R}_{1}+\bar{R}_{2}} \frac{\bar{V}_{s}}{\bar{R}_{c}} .
$$

Les fluctuations du courant sont alors reliées aux fluctuations de $V_{s}, R_{1}, R_{2}, R_{c}$ et $R_{\text {sys }}$.

Le principe de la source de courant alternative réalisée avec une source de tension alternative est le même qu'avec le source de tension continue, et les bruits parasites résultants s'évaluent de la même manière. Nous reviendrons sur l'intérêt de cette source dans la section 9.4 .

\section{Calcul global du bruit}

Le bruit provenant des câbles et des filtres est négligé dans ce calcul, mais on peut remarquer que leur contribution peut être incluse dans le bruit provenant des résistances de contact (c'est d'ailleurs la source essentielle de bruit thermique, l'échantillon étant à très basse température).

Dans la configuration de la mesure du bruit en tension avec une source de courant stabilisée, la tension mesurée en sortie de l'amplificateur peut alors s'écrire :

$$
\begin{aligned}
V_{m} & =G\left[\bar{R}_{\text {éch }} \bar{I}+\delta R_{\text {éch }} \bar{I}+\left(\bar{R}_{\text {éch }}+\delta R_{\text {éch }}\right)\left(\delta \bar{I}\left(R_{c}, R_{c i 1}, R_{\text {éch }}, R_{c i 2}\right)+\delta I+\delta i_{n}\right)\right] \\
& +G\left[\left(\bar{R}_{c v 1}+\delta R_{c v 1}+\bar{R}_{c v 3}+\delta R_{c v 3}\right) \delta i_{n}+\left(\delta e_{\text {éch }}+\delta e_{c v 1}+\delta e_{c v 3}+\delta e_{n}\right)\right]+\delta V_{e x t},
\end{aligned}
$$

où $G$ est le gain de l'amplificateur, et on a négligé le bruit des impédances d'entrée des amplificateurs. Les notations sont reprises des figures 9.4 et 9.5, et sont explicites, sachant que les contacts de tension sont les contacts $c v 1$ et $c v 3$. Le premier terme $G \cdot \bar{R}_{\text {éch }} \bar{I}$ est la valeur continue de la tension, qui sera en général éliminée par le filtre passe haut 
(voir $§ 9.2$ ). Le deuxième terme $G \cdot \delta R_{\text {éch }} \bar{I}$ est le terme qui nous intéresse puisqu'il ne fait intervenir que les fluctuations de résistance de l'échantillon. Les autres termes sont les bruits parasites que l'on essaie d'éliminer ou de réduire pour qu'ils ne soient pas gênants.

Lorsque le courant est fourni grâce à une source de tension $V_{s}$ en série avec une résistance $R_{c}$ de valeur élevée, et en omettant dans un premier temps le pont diviseur présenté dans la figure 9.7 (on considère que la tension délivrée en sortie du pont diviseur est $V_{s}=\bar{V}_{s}+\delta V_{s}$ ), la tension mesurée en sortie de l'amplificateur est donnée par :

$$
\begin{aligned}
V_{m} & =G \frac{\bar{R}_{\text {éch }}+\delta R_{\text {éch }}}{\left(\bar{R}_{c}^{\prime}+\delta R_{c}^{\prime}\right)+\left(\bar{R}_{\text {éch }}+\delta R_{\text {éch }}\right)}\left[\bar{V}_{s}+\delta V_{s}+\left(\delta e_{\text {éch }}+\delta e_{c}^{\prime}\right)\right] \\
& +G\left[\frac{\left(\bar{R}_{c}^{\prime}+\delta R_{c}^{\prime}\right)\left(\bar{R}_{\text {éch }}+\delta R_{\text {éch }}\right)}{\left(\bar{R}_{c}^{\prime}+\delta R_{c}^{\prime}\right)+\left(\bar{R}_{\text {éch }}+\delta R_{\text {éch }}\right)} \delta i_{n}+\left(\bar{R}_{c v 1}+\delta R_{c v 1}+\bar{R}_{c v 3}+\delta R_{c v 3}\right) \delta i_{n}\right] \\
& +G\left[\left(\delta e_{\text {éch }}+\delta e_{c v 1}+\delta e_{c v 3}+\delta e_{n}\right)\right]+\delta V_{e x t},
\end{aligned}
$$

où on a noté $\bar{R}_{c}^{\prime}=\bar{R}_{c}+\bar{R}_{c i 1}+\bar{R}_{c i 2}, \delta R_{c}^{\prime}=\delta R_{c}+\delta R_{c i 1}+\delta R_{c i 2}$ et $\delta e_{c}^{\prime}=\delta e_{c}+\delta e_{c i 1}+\delta e_{c i 2}$, $G$ est le gain de l'amplificateur, et les autres notations sont reprises des figures 9.4 et 9.5, et sont explicites. En négligeant $\bar{R}_{\text {éch }}+\delta R_{\text {éch }}$ devant $\bar{R}_{c}^{\prime}+\delta R_{c}^{\prime}$, et en ne gardant que les termes d'ordre un en $\delta R_{c}^{\prime} / \bar{R}_{c}^{\prime}$, cette expression se réduit à :

$$
\begin{aligned}
V_{m}= & G\left(\bar{R}_{\text {éch }}+\delta R_{\text {éch }}+\frac{\bar{R}_{\text {éch }}+\delta R_{\text {éch }}}{\bar{R}_{c}^{\prime}} \delta R_{c}^{\prime}\right)\left(\frac{\bar{V}_{s}}{\bar{R}_{c}^{\prime}}+\frac{\delta V_{s}}{\bar{R}_{c}^{\prime}}+\frac{\delta e_{\text {éch }}+\delta e_{c}^{\prime}}{\bar{R}_{c}^{\prime}}\right) \\
& +G\left(\bar{R}_{\text {éch }}+\delta R_{\text {éch }}+\bar{R}_{c v 1}+\delta R_{c v 1}+\bar{R}_{c v 3}+\delta R_{c v 3}\right) \delta i_{n} \\
& +G\left(\delta e_{\text {éch }}+\delta e_{c v 1}+\delta e_{c v 3}+\delta e_{n}\right)+\delta V_{e x t} .
\end{aligned}
$$

On distingue dans cette expression deux termes remarquables. Le premier est $G \cdot \bar{R}_{\text {éch }}\left(\bar{V}_{s} / \bar{R}_{c}^{\prime}\right)$ est la valeur continue de la tension, et est éliminé par le filtre passe haut (voir §9.2). Le second, $G \cdot \delta R_{\text {éch }}\left(\bar{V}_{s} / \bar{R}_{c}^{\prime}\right)$, contient l'information qui nous intéresse sur le bruit de résistance de l'échantillon. Tous les autres termes sont des bruits parasites que l'on essaie d'éliminer ou de réduire.

\subsection{Suppression des bruits parasites}

Dans cette section sont présentées des techniques de mesure permettant de supprimer ou de réduire les sources de bruits parasites qui ont été répertoriées dans la section précédente.

\subsubsection{Mesure en corrélation}

Parmi les sources de bruits parasites décrits dans la partie précédente, certaines proviennent directement de la partie mesure (que l'on distingue de la partie source), en particulier des résistances de contacts de tension et des amplificateurs. La méthode de corrélation présentée ici permet d'éliminer une grande partie de ces bruits. Le principe de cette méthode est de comparer les traces temporelles de deux voies de mesure prises comme l'indique le montage décrit dans la figure 9.8. Une fluctuation de tension provenant 


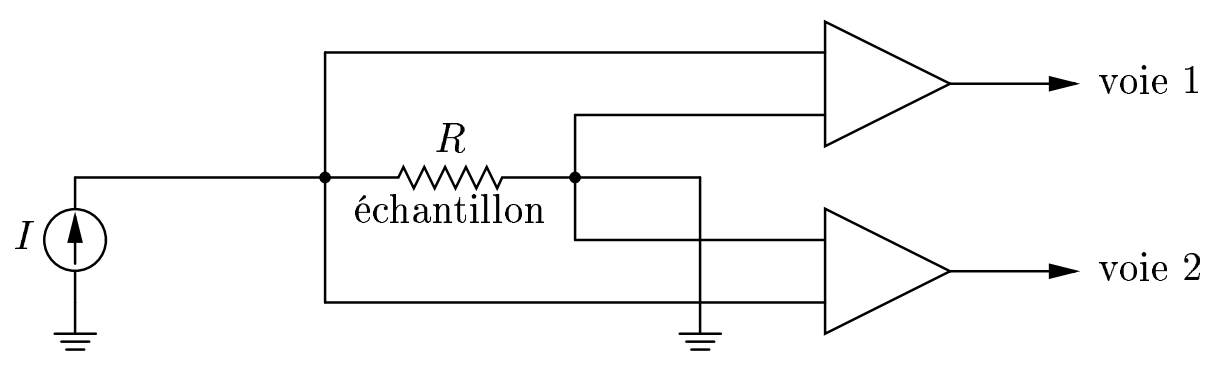

FIG. 9.8: Montage pour la mesure du bruit en tension avec corrélation.

de l'échantillon est enregistrée sur les deux voies de mesure en même temps, alors qu'une fluctuation de tension provenant d'une voie de mesure est absente sur l'autre voie. Cette méthode de corrélation supprime donc une grande partie des fluctuations dont l'origine est située dans une voie de mesure, tout en conservant inchangées les fluctuations provenant de l'échantillon. Nous allons voir ici le principe de cette méthode, puis nous verrons quels termes sont supprimés dans la corrélation.

Les tensions mesurées sur les voies 1 et $2, V_{m 1}(t)$ et $V_{m 2}(t)$, sont échantillonnées par la carte d'acquisition, puis une transformée de Fourier de ces signaux est réalisée, $V_{m 1}(\omega)$ et $V_{m 2}(\omega)$. Le spectre en corrélation est défini par :

$$
S_{\text {corr }}(\omega)=\left\langle V_{m 1}(\omega)^{*} \times V_{m 2}(\omega)\right\rangle
$$

où l'astérisque représente le complexe conjugué. On suppose que les signaux $V_{m 1}$ et $V_{m 2}$ sont composés d'une partie corrélée $V_{c}$ et de parties non corrélées $V_{1}$ et $V_{2}\left(V_{m 1}=V_{c}+V_{1}\right.$ et $\left.V_{m 2}=V_{c}+V_{2}\right)$. Lorsqu'on fait une moyenne sur un grand nombre $N$ de réalisations, on a donc :

$$
S_{\text {corr }}=\left\langle\left(V_{c}+V_{1}\right)^{*} \cdot\left(V_{c}+V_{2}\right)\right\rangle=\left\langle\left|V_{c}\right|^{2}\right\rangle+\left\langle V_{c}^{*} V_{2}\right\rangle+\left\langle V_{1}^{*} V_{c}\right\rangle+\left\langle V_{1}^{*} V_{2}\right\rangle .
$$

$V_{c}, V_{1}$ et $V_{2}$ étant non corrélés, les trois derniers termes s'annulent (dans la limite $N \rightarrow \infty$ ), et

$$
S_{\text {corr }}=\left\langle\left|V_{c}\right|^{2}\right\rangle,
$$

qui est la grandeur physique intéressante. Pour un nombre fini de réalisations $N$, il est possible d'évaluer l'erreur sur $S_{c o r r}$, donnée par l'écart type $\sigma_{c o r r}$ de la distribution $S_{c o r r}$ :

$$
\begin{aligned}
\sigma_{\text {corr }}^{2} & =\left\langle\left|V_{m 1}^{*} V_{m 2}\right|^{2}-S_{\text {corr }}^{2}\right\rangle \\
& =\left\langle\left|V_{c}\right|^{4}\right\rangle-\left\langle\left|V_{c}\right|^{2}\right\rangle^{2}+\left\langle\left|V_{c}\right|^{2}\right\rangle\left\langle\left|V_{1}\right|^{2}\right\rangle+\left\langle\left|V_{c}\right|^{2}\right\rangle\left\langle\left|V_{2}\right|^{2}\right\rangle+\left\langle\left|V_{1}\right|^{2}\right\rangle\left\langle\left|V_{2}\right|^{2}\right\rangle .
\end{aligned}
$$

On note $S_{1}=\left\langle\left|V_{1}\right|^{2}\right\rangle$ et $S_{2}=\left\langle\left|V_{2}\right|^{2}\right\rangle$ les spectres non corrélés. Si $\left|V_{c}\right|$ est une variable aléatoire gaussienne, on montre que $\left\langle\left|V_{c}\right|^{4}\right\rangle=3\left\langle\left|V_{3}\right|^{2}\right\rangle^{2}$, et on peut écrire :

$$
\begin{aligned}
\sigma_{c o r r}^{2} & =S_{c o r r}\left(S_{1}+S_{2}+2 S_{c o r r}\right)+S_{1} S_{2} \\
& =S_{m 1} S_{m 2}+S_{c o r r}^{2}
\end{aligned}
$$

en notant $S_{m 1}=\left\langle\left|V_{m 1}\right|^{2}\right\rangle=S_{\text {corr }}+S_{1}$ et $S_{m 2}=\left\langle\left|V_{m 2}\right|^{2}\right\rangle=S_{c o r r}+S_{2}$ les spectres des signaux mesurés sur les voies 1 et 2 respectivement. Pour $N$ réalisations, l'erreur sur $S_{\text {corr }}$ est donc :

$$
\operatorname{Err}\left(S_{c o r r}\right)=\left(\frac{\sigma_{c o r r}^{2}}{N}\right)^{1 / 2}=\frac{1}{\sqrt{N}}\left(S_{m 1} S_{m 2}+S_{c o r r}^{2}\right)^{1 / 2}
$$


Cette formule permet donc de calculer, à partir des spectres mesurés, l'erreur sur la partie corrélée lorsqu'on moyenne sur un nombre fini de réalisation. Pour avoir une bonne résolution, cette méthode nécessite de moyenner sur un nombre important d'acquisitions, ce qui allonge fortement le temps de mesure. D'autre part l'erreur est d'autant plus grande que le niveau des spectres non corrélés est grand. Ce qui signifie que cette méthode n'est efficace que si les parties non corrélées ne sont pas trop élevées comparé au bruit corrélé.

On peut maintenant reprendre les expressions des tensions $V_{m}$ comprenant toutes les sources de bruits (voir §9.3). Dans la configuration de mesure en corrélation (figure 9.8), les tensions en sortie des amplificateurs s'écrivent :

$$
V_{m 1}=V_{c}+V_{1} \quad \text { et } \quad V_{m 1}=V_{c}+V_{1}
$$

avec

$$
\begin{aligned}
V_{c}(I) & =G\left[\delta R_{\text {éch }} \bar{I}+\left(\bar{R}_{\text {éch }}+\delta R_{\text {éch }}\right)\left(\delta \bar{I}+\delta I+\delta i_{n 1}+\delta i_{n 2}\right)+\delta e_{\text {éch }}\right]+\delta V_{e x t}^{c o r r}, \\
V_{1} & =G\left[\left(\bar{R}_{c v 1}+\delta R_{c v 1}+\bar{R}_{c v 3}+\delta R_{c v 3}\right) \delta i_{n 1}+\left(\delta e_{c v 1}+\delta e_{c v 3}+\delta e_{n 1}\right)\right]+\delta V_{e x t}^{1}, \\
\text { et } V_{2} & =G\left[\left(\bar{R}_{c v 2}+\delta R_{c v 2}+\bar{R}_{c v 4}+\delta R_{c v 4}\right) \delta i_{n 2}+\left(\delta e_{c v 2}+\delta e_{c v 4}+\delta e_{n 2}\right)\right]+\delta V_{e x t}^{2}
\end{aligned}
$$

où les contacts de tension $c v 1$ et $c v 3$ correspondent à la voie 1 , les contacts $c v 2$ et $c v 4$ correspondent à la voie 2 , et les bruits des amplificateurs des voies 1 et 2 sont indicés respectivement par $n 1$ et $n 2$. La partie continue de chaque signal a été soustraite. Enfin les bruits d'origine extérieure ont été séparés suivant leur composante corrélée $\delta V_{e x t}^{\text {corr }}$, et leurs composantes non corrélées spécifiques aux deux voies de mesure, $\delta V_{e x t}^{1}$ et $\delta V_{e x t}^{2}$.

À courant appliqué nul, en supposant qu'il n'y alors plus de sources de fluctuations du courant, on a :

$$
V_{c}(I=0)=G\left[\left(\bar{R}_{\text {éch }}+\delta R_{\text {éch }}\right)\left(\delta i_{n 1}+\delta i_{n 2}\right)+\delta e_{\text {éch }}\right]+\delta V_{e x t}^{c o r r} .
$$

On remarque que cette méthode de mesure en corrélation permet de mesurer le bruit thermique de l'échantillon $\delta e_{\text {éch }}$, en se plaçant dans une gamme de fréquence où les bruits en courant des amplificateurs $\delta i_{n}$ et la partie corrélée du bruit extérieur $\delta V_{e x t}^{c o r r}$ sont faibles.

Enfin, pour la mesure du bruit de résistance $\delta R_{\text {éch }}$, on peut soustraire le bruit à courant donné et le bruit à courant nul, ce qui permet d'éliminer principalement le bruit thermique des résistances qui ne dépend pas du courant. Les seuls termes restant dans $V_{c}$ sont :

$$
\Delta V_{c}=G\left[\delta R_{\text {éch }} \bar{I}+\left(\bar{R}_{\text {éch }}+\delta R_{\text {éch }}\right)(\delta \bar{I}+\delta I)\right]
$$

Cette mesure est donc limitée principalement par le bruit de la source de courant. Il faut également noter que le terme $\delta R_{\text {éch }}$ contient aussi bien les fluctuations intrinsèques de résistance (qui sont celles qui nous intéressent) que les fluctuations dues à des sources extérieures (fluctuations de température ou de tension de grille). Pour valider la mesure il reste donc à vérifier que les bruits dues à la source de courant ou aux fluctuations externes de résistance sont faibles, ce qui est fait grâce à la mesure de corrélation "amont-aval" présentée dans la section suivante. 


\subsubsection{Corrélation "amont-aval"}

Cette mesure permet de vérifier que les fluctuations de la source de courant et les fluctuations de la résistance dues à des sources extérieures au système sont négligeables devant les fluctuations intrinsèques de résistance de l'échantillon $\delta R_{\text {éch }}^{\text {int }}$ et peuvent donc être négligés dans le spectre en corrélation. A défaut de pouvoir éliminer ces sources de bruit, il est possible de les réduire, d'évaluer leur grandeur par une méthode de corrélation dite "amont-aval", et éventuellement de les soustraire de la mesure en corrélation présentée au paragraphe précédent. Cette méthode utilise les deux parties de la barre de Hall intitulées $R_{\text {éch }}$ et $R_{\text {aval }}$ dans la figure 9.5 , et repose sur le fait que les fluctuations de courant, de température, ou toute autre fluctuation extérieure, sont vues de manières corrélées par les deux parties de la barre de Hall. Une mesure en corrélation entre la partie "échantillon" et la partie "aval" permet ainsi d'extraire ces contributions au bruit, sachant que la contribution intrinsèque à la résistance (i.e. les fluctuations de résistances recherchées) apparaissent sur les deux voies de mesure de manière non corrélée. Cette dernière remarque n'est cependant vraie que si la longueur de corrélation du bruit est faible devant la distance séparant les deux parties de l'échantillon.

Cette méthode reprend le principe de la mesure en corrélation présentée dans la partie précédente. Maintenant, une voie de mesure est connectée à la partie "échantillon" de la barre de Hall (i.e. entre les contacts $c v 1$ et $c v 3$ de la figure 9.5) alors que la seconde voie est connectée à la partie "aval" (i.e. entre les contacts $c v 4$ et $c v 5$ de la figure 9.5). La partie corrélée des tensions mesurées dans cette configuration appelée "amont-aval" est alors :

$$
\begin{aligned}
& V_{c, \text { éch }}^{a m-a v}(I)=G\left[\delta R_{\text {éch }}^{e x t} \bar{I}+\left(\bar{R}_{\text {éch }}+\delta R_{\text {éch }}^{e x t}\right)(\delta \bar{I}+\delta I)\right]+\delta V_{e x t}^{c o r r}, \\
& V_{c, \text { aval }}^{a m-a v}(I)=G\left[\delta R_{\text {aval }}^{e x t} \bar{I}+\left(\bar{R}_{\text {aval }}+\delta R_{\text {aval }}^{e x t}\right)(\delta \bar{I}+\delta I)\right]+\delta V_{e x t}^{c o r r},
\end{aligned}
$$

où $\delta R_{\text {éch }}^{\text {ext }}$ (resp. $\delta R_{\text {aval }}^{\text {ext }}$ ) est le terme de fluctuation de la résistance de l'échantillon (resp. de la partie avale) due à des sources extérieures (fluctuations de température, etc...). En comparant cette formule à l'équation (9.19), on remarque que les termes restant dans la mesure en corrélation sont également présents dans la mesure "amont-aval", à l'exception des fluctuations intrinsèques de résistance. La comparaison des deux spectres permet donc d'effectuer la vérification nécessaire à l'interprétation des résultats de la mesure en corrélation.

\subsubsection{Mesure synchrone}

Pour réduire les bruits parasites, il est possible d'envisager une autre configuration de mesure. Puisque l'on mesure des fluctuations de résistance, les fluctuations de tension sont directement corrélées au courant. Il est donc possible de faire une mesure synchrone en faisant varier le courant et en mesurant la partie du bruit qui varie de manière totalement corrélée au courant.

Le montage utilisé pour la mesure synchrone est présenté dans la figure 9.9. On utilise comme source de courant une source de tension $V_{\text {osc }}$ mise en série avec une résistance de charge $R_{c}$ de valeur élevée comparée à celle de l'échantillon. Ainsi on peut considérer que le courant dans l'échantillon vaut $I \approx V_{o s c} / R_{c}$. La tension $V_{o s c}$ est un signal sinusoïdal de 


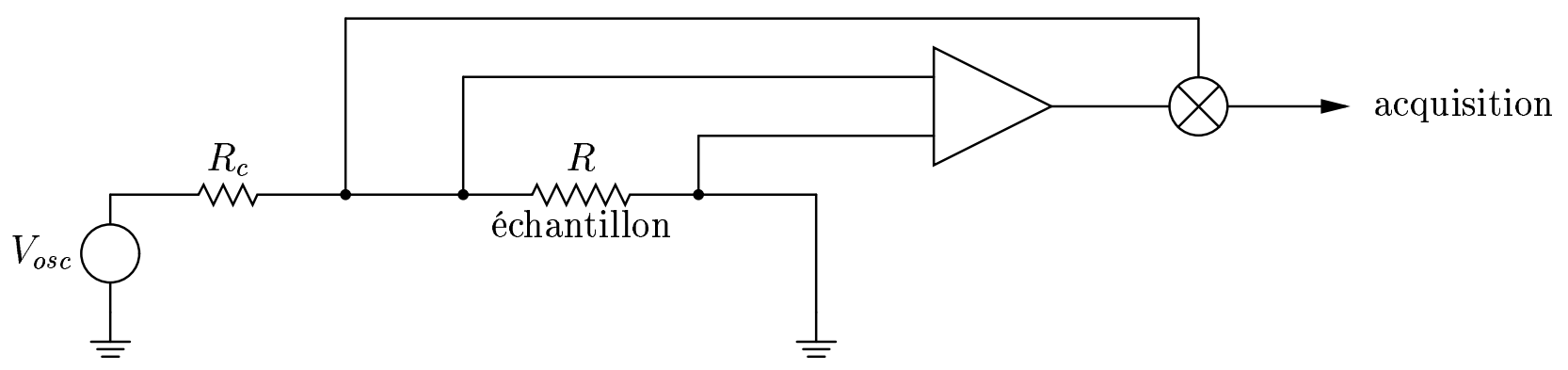

FIG. 9.9: Montage pour la mesure synchrone du bruit en tension.

fréquence $f_{o s c}=2 \pi \Omega, V_{o s c}=V_{0} \cos (\Omega t)$, ce qui conduit à un courant sinusoïdal à cette même fréquence. Le signal mesuré en sortie de l'amplificateur est ensuite multiplié par le signal $V_{o s c}$, puis est filtré par un filtre passe-bas de fréquence de coupure $\omega_{0}<\Omega$. On suppose dans cette partie que la pente du filtre est infinie.

Considérons maintenant la tension en sortie des amplificateurs qui se déduit de l'expression donnée par l'équation (9.7) en remplaçant $V_{s}$ par l'expression de $V_{o s c}$ :

$$
\begin{array}{r}
V_{m}(t)=G \bar{R}_{\text {éch }} \frac{V_{0}}{\bar{R}_{c}^{\prime}} \cos (\Omega t)+G\left(\delta R_{\text {éch }}(t)+\frac{\bar{R}_{\text {éch }}+\delta R_{\text {éch }}(t)}{\bar{R}_{c}^{\prime}} \delta R_{c}^{\prime}(t)\right) \frac{V_{0}}{\bar{R}_{c}^{\prime}} \cos (\Omega t) \\
+G\left(\bar{R}_{\text {éch }}+\delta R_{\text {éch }}(t)+\frac{\bar{R}_{\text {éch }}+\delta R_{\text {éch }}(t)}{\bar{R}_{c}^{\prime}} \delta R_{c}^{\prime}(t)\right)\left(\frac{\delta V_{\text {osc }}(t)}{\bar{R}_{c}^{\prime}}+\frac{\delta e_{\text {éch }}(t)+\delta e_{c}^{\prime}(t)}{\bar{R}_{c}^{\prime}}\right) \\
+G\left(\bar{R}_{\text {éch }}+\delta R_{\text {éch }}(t)+\bar{R}_{c v 1}+\delta R_{c v 1}(t)+\bar{R}_{c v 3}+\delta R_{c v 3}(t)\right) \delta i_{n}(t) \\
+G\left(\delta e_{\text {éch }}(t)+\delta e_{c v 1}(t)+\delta e_{c v 3}(t)+\delta e_{n}(t)\right)+\delta V_{e x t}(t),
\end{array}
$$

où on a également introduit un bruit provenant de la source de tension, noté $\delta V_{o s c}(t)$. Dans cette équation, on distingue trois types de termes. On détaille ici l'effet de la mesure en corrélation sur chacun de ces termes.

Le premier terme de l'équation (9.22) est de la forme $K \cos (\Omega t)$, où $K$ est un terme indépendant du temps. On a donc :

$$
K \cos (\Omega t) \times \cos (\Omega t)=\frac{K}{2}[1+\cos (2 \Omega t)] \underset{\omega_{0}<\Omega}{\stackrel{\text { filtrage }}{\longrightarrow}} \frac{K}{2} .
$$

Ce qui donne bien la valeur de la constante à un facteur $1 / 2$ près, comme on l'attend d'une mesure synchrone.

Avant de prendre en compte le deuxième terme de l'équation (9.22), considérons tous les autres termes qui sont de la forme $X(t)$, ne contenant pas de terme en $\cos (\Omega t)$. On introduit la transformée de Fourier de $X(t), X(\omega)$, définie par :

$$
X(\omega)=\frac{1}{2 \pi} \int_{-\infty}^{+\infty} X(t) e^{i \omega t} d t
$$


La mesure synchrone aboutit à :

$$
\begin{aligned}
X(t) \times \cos (\Omega t) & =\int_{-\infty}^{+\infty} X(\omega) e^{-i \omega t} \cos (\Omega t) d \omega \\
& =\int_{-\infty}^{+\infty} \frac{1}{2}[X(\Omega+\omega)+X(-\Omega+\omega)] e^{-i \omega t} d \omega \\
& \stackrel{\text { filtrage }}{\omega_{0}<\Omega} \int_{-\omega_{0}}^{+\omega_{0}} \frac{1}{2}[X(\Omega+\omega)+X(-\Omega+\omega)] e^{-i \omega t} d \omega .
\end{aligned}
$$

Cette mesure revient donc à décaler en fréquence le signal $X(t)$, et donc, au lieu de mesurer le spectre de $X(t)$ sur une bande de largeur $2 \omega_{0}$ autour de la fréquence nulle, on mesure le spectre de $X(t)$ sur une bande de largeur $2 \omega_{0}$ autour de la fréquence $\Omega$. En particulier si $X(t)$ est un bruit parasite, ce bruit est encore présent dans la mesure finale, mais est décalé en fréquence. Cette opération peut être très utile lorsque ce bruit parasite augment fortement à basse fréquence, tel le bruit en tension des amplificateurs et a une amplitude moindre à plus haute fréquence.

Considérons enfin le deuxième terme de l'équation (9.22), de la forme $Y(t) \cos (\Omega t)$. En introduisant la transformée de Fourier de $Y(t), Y(\omega)$, on montre :

$$
\begin{aligned}
Y(t) \cos (\Omega t) \times & \cos (\Omega t)=\int_{-\infty}^{+\infty} Y(\omega) e^{-i \omega t} \frac{1}{2}[1+\cos (2 \Omega t)] d \omega \\
& =\frac{1}{2} Y(t)+\int_{-\infty}^{+\infty} \frac{1}{4}[Y(2 \Omega+\omega)+Y(-2 \Omega+\omega)] e^{-i \omega t} d \omega \\
& \stackrel{\text { filtrage }}{\underset{\omega_{0}<\Omega}{\longrightarrow}} \frac{1}{2} Y_{|\omega| \leqslant \omega_{0}}(t)+\int_{-\omega_{0}}^{+\omega_{0}} \frac{1}{4}[Y(2 \Omega+\omega)+Y(-2 \Omega+\omega)] e^{-i \omega t} d \omega .
\end{aligned}
$$

Le premier terme obtenu est le signal $Y(t)$ filtré par le filtre passe-bas avec la fréquence de coupure $\omega_{0}$. Cependant on remarque qu'il existe également une deuxième composante correspondant au spectre de $Y(t)$ décalé de la fréquence $2 \Omega$. Dans le cas de la mesure du bruit en $1 / f$, et lorsque la valeur de $\Omega$ est suffisamment grande, le second terme est faible devant le premier.

$\mathrm{Si}$ on suppose que les spectres des signaux dépendants du temps sont négligeables autour des fréquences $\Omega$ et $2 \Omega$, la mesure synchrone conduit donc à :

$$
V_{\text {sync }}(t)=G \frac{V_{0}}{2 \bar{R}_{c}^{\prime}}\left[\bar{R}_{\text {éch }}+\left(\delta R_{\text {éch }}(t)+\frac{\bar{R}_{\text {éch }}+\delta R_{\text {éch }}(t)}{\bar{R}_{c}^{\prime}} \delta R_{c}^{\prime}(t)\right)_{|\omega| \leqslant \omega_{0}}\right] .
$$

Le premier terme est la mesure de la valeur moyenne de la résistance de l'échantillon. Dans le second terme on mesure les fluctuations de la résistance de l'échantillon $\delta R_{\text {éch }}$, qui peuvent provenir de sources intrinsèques (celles qui nous intéressent) ou externes, et les fluctuations de la résistance $R_{c}^{\prime}$, qui contient la résistance de charge et les résistances de contact de courant. Mais cette dernière contribution est multiplié par le rapport $\bar{R}_{\text {éch }} / \bar{R}_{c}^{\prime}$ qui est petit devant un, et qui affaiblit l'importance de cette contribution.

Dans la pratique, le choix de la fréquence de l'oscillateur $\Omega$ est très important car il faut que l'amplitude des bruits parasites soit faible sur une largeur de $2 \omega_{0}$ autour de cette fréquence. 




FIG. 9.10: Montage pour la mesure synchrone du bruit en tension avec corrélation.

\subsubsection{Mesure synchrone en corrélation}

On a vu que la mesure en corrélation permet de supprimer la plupart des bruits parasites. On a vu également que la mesure synchrone permet de réduire certaines sources de bruit en décalant la gamme de fréquences explorée vers des fréquences plus élevées, ce qui peut se révéler intéressant pour la mesure du bruit en $1 / f$ car les appareils du circuit ont souvent un bruit en $1 / f$ important. La combinaison des deux méthodes peut se révéler intéressante dans plusieurs cas.

La méthode en corrélation nécessite d'effectuer une moyenne sur plusieurs spectres pour être efficace. D'autre part, dès que les bruits non corrélés sont grands devant la partie corrélée, il est nécessaire de moyenner sur un très grand nombre de spectres pour avoir une résolution suffisante. Effectuer une mesure synchrone sur chacune des voies avant la corrélation, suivant le montage proposé en figure 9.10, permet de réduire de façon importante le bruit non corrélé, et ainsi d'améliorer l'efficacité de la corrélation. D'autre part, l'utilisation de deux fréquences différentes pour chacune des voies de mesure synchrone permet de supprimer la plupart des bruits parasites non-corrélés au courant, et à toutes les fréquences [368]. Enfin, cette méthode supprime les bruits non corrélés au courant, et ne nécessite donc pas d'effectuer une double mesure à courant fixé puis à courant nul, comme la mesure simple en corrélation l'impose.

\subsection{Conclusion}

Nous avons donc présenté dans ce chapitre les différentes méthodes de mesure des fluctuations de résistance à basse fréquence, ainsi que les avantages des méthodes pour supprimer les bruits parasites à la mesure que l'on désire effectuer. La mesure en corrélation permet de supprimer la plupart des bruits parasites, et nous avons vu qu'une mesure de corrélation "amont-aval" permet de vérifier que le bruit résultant provient bien des fluctuations intrinsèques de la résistance de l'échantillon. Cependant cette méthode a l'inconvénient d'être longue en raison de la moyenne à effectuer sur un grand nombre d'acquisitions, ce qui peut être rédhibitoire dans le cas où les bruits parasites ont une amplitude beaucoup plus élevée que les fluctuations qui nous intéressent. D'autre part cette méthode nécessite une géométrie bien particulière de l'échantillon, et en particulier il faut s'assurer que les deux côtés de mesure ne sont pas dissociés. La méthode de mesure synchrone ne permet pas de supprimer les bruits parasites, mais permet de décaler la 
fréquence de mesure par rapport à la fréquence nulle. Ceci peut se révéler utile lorsque le circuit présente un bruit basse fréquence important. Cette méthode présente l'avantage de ne nécessiter qu'une seule voie de mesure. Enfin il est possible d'améliorer encore d'avantage la méthode en corrélation en réduisant une partie du bruit en amont de la corrélation par une mesure synchrone. 


\section{Chapitre 10}

\section{Fluctuations temporelles de résistance dans les puits quantiques $p$-SiGe}

Sommaire

10.1 Introduction . . . . . . . . . . . . . . 220

10.2 Mesures sur la partie avec grille . . . . . . . . . . . 220

10.3 Mesures sur la partie sans grille . . . . . . . . 220

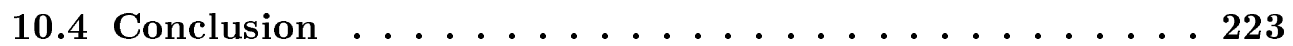




\subsection{Introduction}

Nous avons effectué des mesures de bruit basse fréquence sur les puits quantiques $p$-SiGe présentés au chapitre 3. Dans un premier temps (§10.2), nous avons mesuré le bruit dans la partie comportant une grille, afin de faire varier la densité de porteurs. Cependant nous montrons ici que la majeure partie du bruit mesuré est dû au courant de fuite grille-canal, ce qui ne permet pas de mesurer le bruit du gaz d'électrons. La seconde section $(\$ 10.3)$ est consacrée à la mesure de bruit dans la partie sans grille à haute densité de porteurs. La mesure synchrone corrélée permet d'effectuer une mesure $\mathrm{du}$ bruit de résistance du gaz bidimensionnel de trous avec une très bonne résolution. Nous attribuons le bruit mesuré aux fluctuations du désordre dans cette structure.

\subsection{Mesures sur la partie avec grille}

Les mesures sur la partie avec grille ont été faites par la méthode de corrélation pour une densité de $0,78 \times 10^{15} \mathrm{~m}^{-2}$. Un courant continu est injecté à l'aide d'une pile en série avec des résistances bas bruit (de valeur totale $10 \mathrm{M} \Omega$ ). Le filtre anti-repliement est un filtre passe-bas, $12 \mathrm{~dB} /$ octave, et la fréquence d'échantillonnage est de $6 \mathrm{kHz}$. La figure 10.1 de gauche montre la mesure du bruit corrélé à $4,2 \mathrm{~K}$ et pour cinq valeurs du courant injecté. La dépendance en fréquence $\mathrm{du}$ bruit basse fréquence est $1 / f^{\alpha}$, avec $\alpha$ toujours proche de 1 . On remarque d'abord que le bruit à courant nul présente une forte composante en $1 / f$, montrant ainsi qu'une grande part du bruit basse fréquence n'est pas lié aux fluctuations de résistance du système. D'autre part, nous avons mesuré le bruit dans la partie sans grille (voir §10.3) qui, pour un courant nul, est un facteur au moins 400 en-dessous du bruit mesuré dans la partie sous grille, et ne dépend pas de la fréquence. On peut donc attribuer ce bruit à courant injecté nul à la grille, et en particulier au courant de fuite grille-canal. On a vu en effet que ce courant vaut quelques centaines de pA dans la gamme de densités étudiées. On remarque alors que la différence $S_{V}(I)-S_{V}(I=0)$ varie comme $I^{2}$. Ainsi l'augmentation du bruit avec le courant est dû aux fluctuations de résistance de l'échantillon, et est donc le bruit recherché.

Nous avons également remarqué que le bruit à courant injecté nul varie dans le temps, et ces variations sont accentuées juste après la variation de la tension de grille (voir la figure 10.2 de gauche). Cela confirme que ce bruit a pour origine le courant de fuite grille-canal. Enfin $S_{V}(I=0, T)$ diminue lorsque la température augmente, comme le montre la figure 10.2 de droite. Cette observation prouve que le bruit ne provient pas de mécanismes activés thermiquement, qui auraient conduit à une augmentation du bruit quand la température augmente. On peut cependant remarquer que, à basse température, le changement du désordre peut également se faire par sauts tunnels. Enfin, entre $107 \mathrm{mK}$ et $4,2 \mathrm{~K}$, il y a quand-même une augmentation du bruit.

\subsection{Mesures sur la partie sans grille}

Étant donné les problèmes de mesure sur la partie avec grille de l'échantillon, en majeur partie en raison du courant de fuite grille-canal élevé, nous avons effectué des 


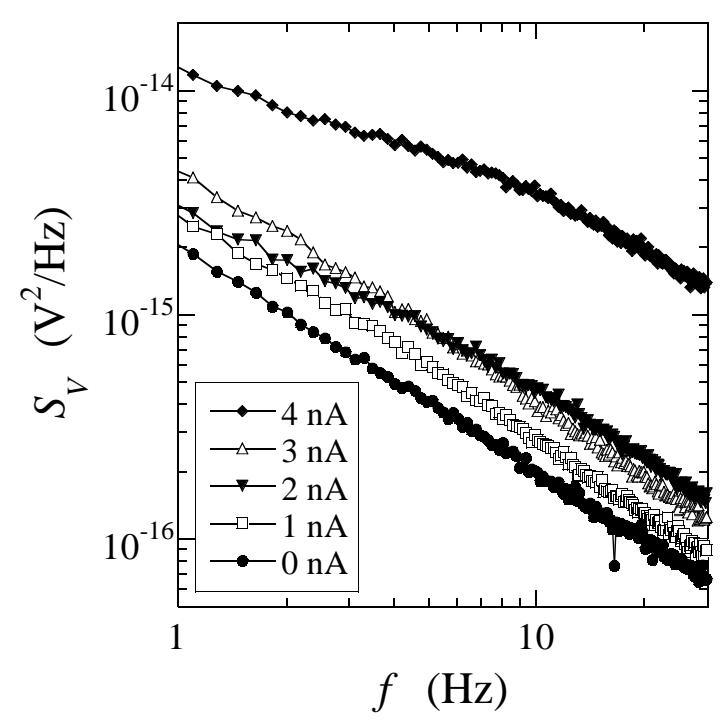

FIG. 10.1: Spectres de bruit en tension sur l'échantillon SiGe B509 avec grille, à une densité de porteurs $p_{s}=0,78 \times 10^{15} \mathrm{~m}^{-2}, T=$ $4,2 \mathrm{~K}$, et pour différentes valeurs du courant (indiquées en encart).
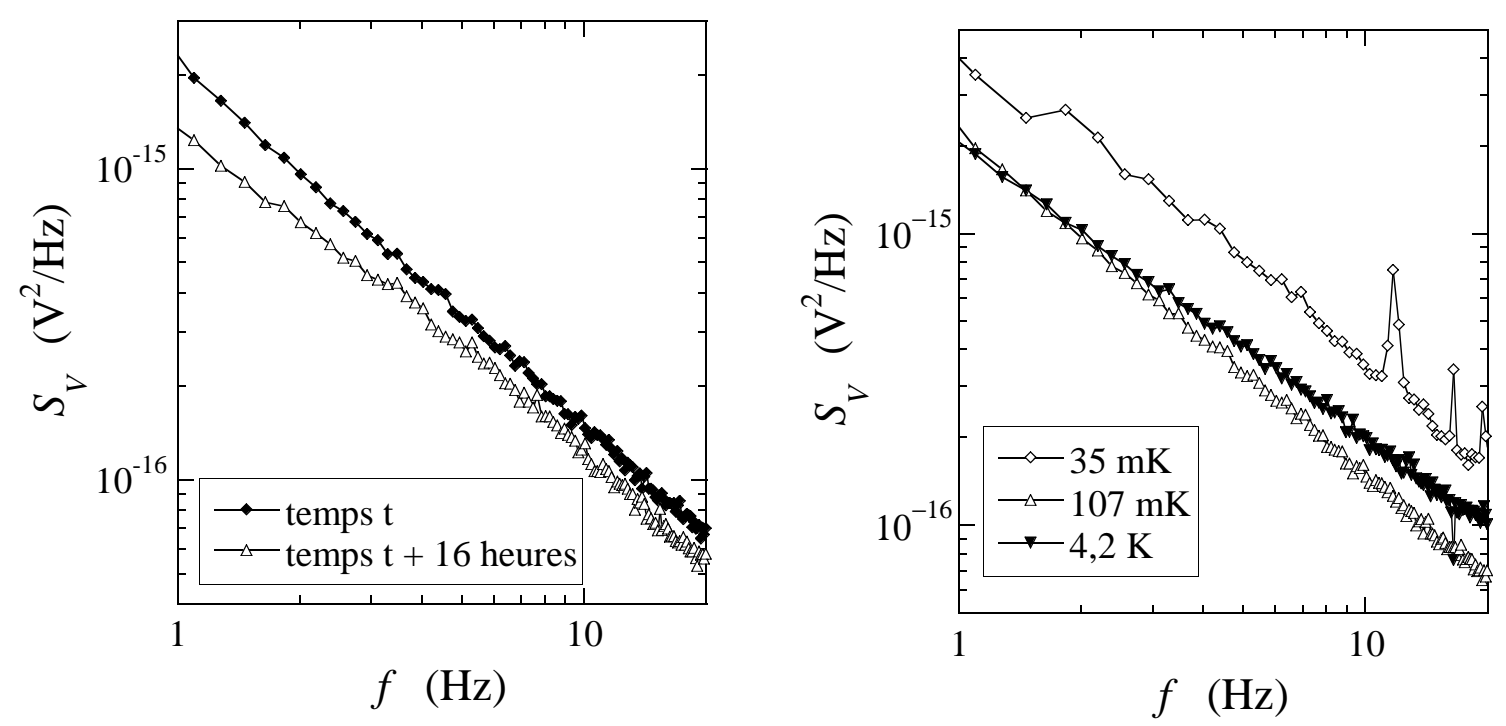

FIG. 10.2: Spectres de bruit en tension sur l'échantillon SiGe B509 avec grille, à une densité de porteurs $p_{s}=0,78 \times 10^{15} \mathrm{~m}^{-2}$ et à courant injecté nul. Gauche : dépendance en temps de la puissance de bruit, la température est fixée à $107 \mathrm{mK}$. Droite : Dépendance en température de la puissance de bruit. 



FIG. 10.3: Spectres de bruit en tension obtenus par la méthode de mesure synchrone en corrélation dans l'échantillon $p$-SiGe B509 à $T=830 \mathrm{mK}$. Gauche : Spectre de bruit sur les deux voies de mesure et en corrélé à un courant $I=250 \mathrm{nA}$. Droite : Spectre de bruit corrélé normalisé par la tension en à deux courant différents.

mesures sur la partie sans grille. En raison du faible niveau de bruit dans cette partie, au moins deux ordres de grandeur au-dessous du niveau de bruit dans la partie avec grille, nous avons utilisé la méthode de mesure synchrone avec corrélation (voir §9.4.4). Le courant est produit par une source de tension alternative reliée à une résistance de $20 \mathrm{M} \Omega$, et est modulé à une fréquence comprise entre 100 et $300 \mathrm{~Hz}$ pour la mesure synchrone. Dans cette mesure nous n'avons utilisé qu'une seule fréquence de modulation. Cette fréquence est très élevée comparée à la fréquence maximale d'étude du bruit, environ $5 \mathrm{~Hz}$. Une fréquence de modulation plus élevée conduit à une diminution du signal et une modification de la phase en raison des constantes de temps caractéristiques du circuit et du système étudié.

Un exemple de spectre de bruit mesuré par cette méthode est représenté sur la figure 10.3 de gauche. Cette méthode permet d'atteindre une très bonne résolution : il est possible de mesurer un bruit de l'ordre de $1 \mathrm{nV} / \sqrt{\mathrm{Hz}}$ à $1 \mathrm{~Hz}$. On peut remarquer que l'utilisation de deux fréquence de modulation, une pour chaque voie de mesure synchrone, pourrait permettre de réduire encore le niveau minimal du bruit. Le bruit des voies de mesure non corrélées provient du bruit en tension des amplificateurs, et du bruit Johnson des résistances de contact (de 40 à $120 \mathrm{k} \Omega$ ) : pour une résistance de $100 \mathrm{k} \Omega$, la puissance de bruit en tension est $4,6 \times 10^{-18} \mathrm{~V}^{2} / \mathrm{Hz}$. De plus le bruit en courant des amplificateurs donne à travers les contacts un bruit de quelques $10^{-18} \mathrm{~V}^{2} / \mathrm{Hz}$. La dépendance en fréquence $\mathrm{du}$ bruit est $1 / f^{\alpha}$ avec $\alpha$ proche de 1 . Dans la figure 10.3 de droite, on montre que la puissance de bruit en tension est proportionnel à $I^{2}$ (ou $S_{V} / V^{2}$ est indépendant de $I$ ).

Il est possible de comparer le bruit mesuré ici à la loi empirique de Hooge (voir §8.2) :

$$
\frac{S_{R}}{R^{2}}=\frac{S_{V}}{V^{2}}=\frac{A(T)}{N f}
$$


où $N=S p_{s}$ est le nombre de porteurs, et $A(T)$ est un préfacteur qui peut dépendre de la densité de défauts fluctuants, et de la température si les fluctuations de ces défauts sont induites thermiquement. Dans le cas de la partie sans grille, la densité de porteurs est $4,0 \times 10^{15} \mathrm{~m}^{-2}$, et à une température de $830 \mathrm{mK}$ on obtient $A(830 \mathrm{mK})=1,4 \times 10^{-4}$. Ce résultat peut être comparé à la mesure de fluctuations de résistance de Kurdak et al. [335] sur une hétérostructure $n$-GaAs à $4,2 \mathrm{~K}$. Ils montrent que les fluctuations sont dues à des fluctuations des défauts, et ils mesurent $A(4,2 \mathrm{~K})=3,2 \times 10^{-5}$. La valeur élevée de $A$ dans notre cas ne peut pas s'expliquer par un effet de la température, car, pour un mécanisme activé, on s'attend à un bruit croissant avec la température. Cette différence peut être attribuée à une plus grande densité de défauts ou d'impuretés dans nos échantillons $p$-SiGe comparé au $n$-GaAs.

\subsection{Conclusion}

Nous avons donc étudié les fluctuations de résistance dans un puits quantique $p$-SiGe à haute densité électronique. Par la méthode de mesure synchrone en corrélation, nous avons pu mesurer un bruit inférieur à $1 \mathrm{nV} / \sqrt{\mathrm{Hz}}$ à $1 \mathrm{~Hz}$. L'amplitude du bruit en $1 / f$ obtenu est compatible avec ce qui a déjà été trouvé dans des hétérostructure $n$-GaAs à plus haute température, et est attribué à un mécanisme classique de fluctuations de mobilité dues aux fluctuations du désordre. 


\section{Chapitre 11}

\section{Fluctuations temporelles de résistance dans les puits quantiques $p$-GaAs}

\section{Sommaire}

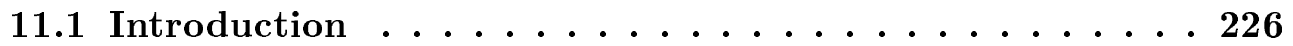

11.2 Mesures du bruit en $1 / f \ldots \ldots \ldots \ldots \ldots$

11.2 .1 Conditions de mesure . . . . . . . . . . . . . 226

11.2 .2 Mesures et vérifications . . . . . . . . . . . . . . 227

11.2 .3 Ajustement des spectres . . . . . . . . . . . . . 230

11.3 Dépendances du bruit en température et en densité . . . 231

11.3.1 Ajustements empiriques . . . . . . . . . . . . . . 231

11.3.2 Interprétations . . . . . . . . . . . . . . . . 233

11.4 Observation de lois d'échelle de percolation . . . . . 236

11.4.1 Loi d'échelle sur le bruit . . . . . . . . . . . . . . . . 236

11.4.2 Transition de percolation dans un gaz bidimensionnel dilué 238

11.4.3 Loi d'échelle sur la conductance . . . . . . . . . . . . . 242

11.4.4 Comparaison aux MOSFETs Si . . . . . . . . . . . 244

11.5 Conclusion . . . . . . . . . . . . . . 245 


\subsection{Introduction}

Nous avons vu au chapitre 8 que la mesure du bruit de résistance à basse fréquence, ou bruit en $1 / f$, peut donner des indications sur la nature du système électronique étudié. De plus ce bruit présente un comportement singulier aux abords d'une transition de phase. Si le changement de comportement de la résistivité (métallique ou isolant) observé dans les gaz bidimensionnels de porteurs dilués est réellement lié à une transition de phase, on s'attend donc à observer une signature de cette transition sur le bruit basse fréquence. Mais au-delà même de l'existence d'une transition de phase dans ces systèmes, la mesure de ce bruit peut également fournir des informations supplémentaires sur la nature du système à très faible densité, régime dans lequel on s'attend à un effet important des corrélations entre porteurs.

Nous avons effectué des mesures de bruit basse fréquence dans les gaz bidimensionnels de trous formés dans les hétérostructures GaAs/AlGaAs qui ont été présentés dans le chapitre 3 . Nous avons vu au chapitre 5 que ce système présente une transition entre un comportement métallique à haute densité de porteurs $\left(d \rho / d T>0\right.$ pour $\left.p_{s}>p_{c}\right)$ et un comportement isolant à faible densité de porteurs $\left(d \rho / d T<0\right.$ pour $\left.p_{s}<p_{c}\right)$.

Nous verrons tout d'abord les conditions expérimentales et les mesures obtenues dans ces conditions, en montrant que le bruit mesuré à basse fréquence, et dont la dépendance en fréquence est proche de $1 / f$, provient des fluctuations de résistance du gaz bidimensionnel (voir §11.2). Ensuite nous présenterons les dépendances de ce bruit en fonction de la densité de porteurs et de la température, autour de la densité $p_{c}$ à laquelle est observé le changement de comportement de la résistivité (voir §11.3). Enfin nous verrons la caractéristique la plus importante, qui est la loi d'échelle vérifiée par ce bruit lorsqu'il est présenté en fonction de la résistance du système (voir §11.4). Nous verrons que cette loi d'échelle peut être interprétée par l'approche d'une transition de percolation, conclusion qui est compatible avec des modèles proposés pour interpréter les mesures de transport dans les systèmes très dilués.

\subsection{Mesures du bruit en $1 / f$}

\subsubsection{Conditions de mesure}

Les mesures présentées dans ce chapitre ont été réalisées par la méthode de corrélation présentée dans le paragraphe 9.4.1. La géométrie de l'échantillon est rappelée dans la figure 11.1 de gauche. Un courant continu est délivré par un générateur de courant (Keithley K 263). Les mesures de tensions sont faites entre les contacts 3 et 4 , et entre les contacts 5 et 6. La mesure "amont-aval" est réalisée par corrélation des mesures de tension entre les contacts 3 et 4 , et entre les contacts 6 et 7 .

Pour cette mesure, on a utilisé les filtres passe-bande des amplificateur SR 560 et EGG 5113, avec des fréquences de coupures 0,03 et $10 \mathrm{~Hz}$, et des pentes $6 \mathrm{~dB} /$ octave. La coupure à basse fréquence permet de supprimer la composante continue du signal, ainsi que les dérives lentes de tension de décalage des pré-amplificateurs bas bruit (LI 75A), et ainsi d'augmenter la résolution de la mesure. La coupure haute est utilisée comme filtre 

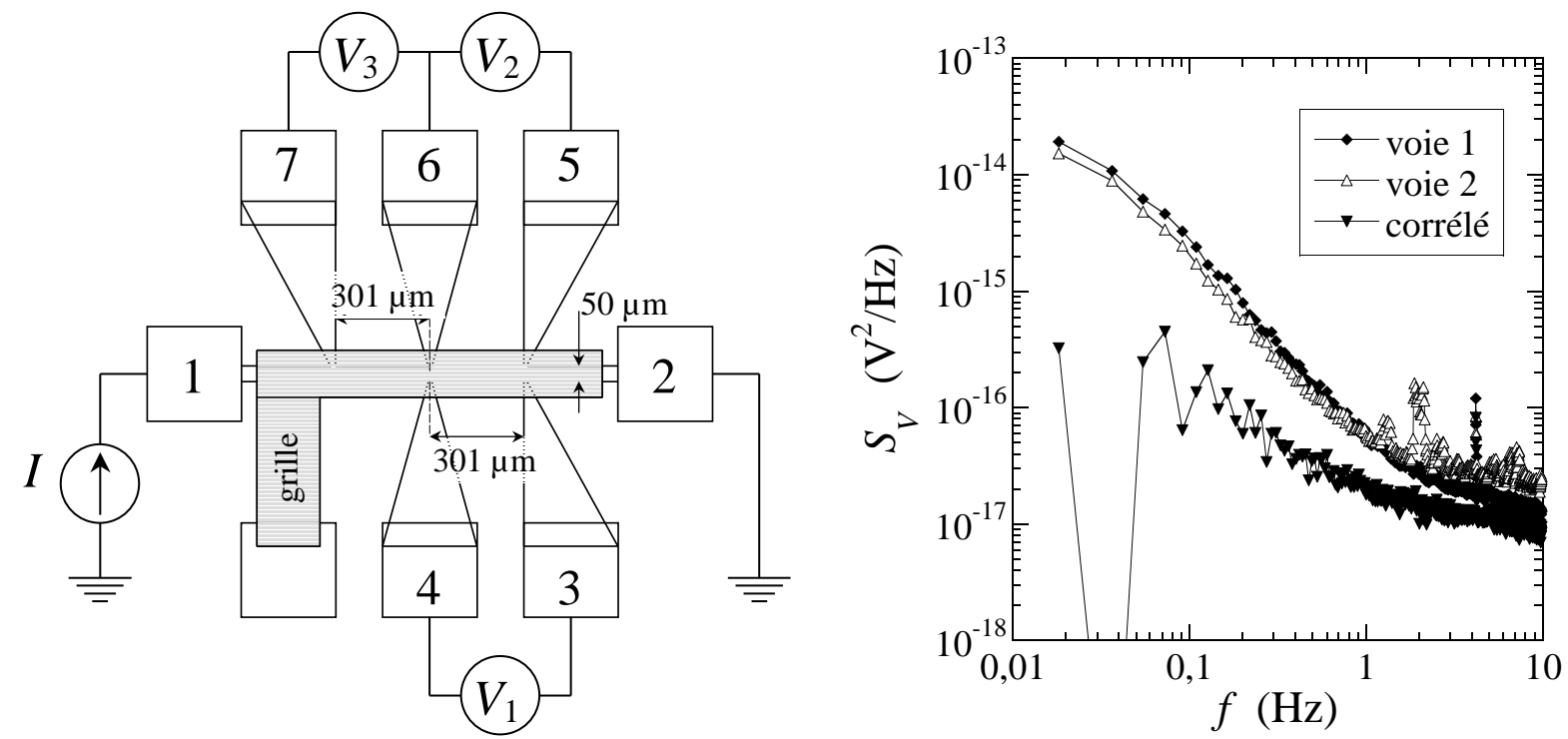

FIG. 11.1: Gauche : Géométrie de l'échantillon Nu 944 utilisé pour la mesure de bruit. Droite : Spectre de bruit en tension $S_{V}$ sur chaque voie de mesure (voie 1 et voie 2), et spectre de bruit résultant de la corrélation (densité de porteurs de $p_{s}=1,75 \times 10^{14} \mathrm{~m}^{-2}$, température $T=300$ $\mathrm{mK}$, courant injecté $I=1,5 \mathrm{nA})$.

anti-repliement. La fréquence d'échantillonnage est de $600 \mathrm{~Hz}$. Le gain total des deux étages d'amplification est d'environ $10^{4}$, gain étalonné dans la gamme de fréquences utile en utilisant un générateur de tension sinusoïdale, et vérifié par la mesure du bruit Johnson de résistances à température ambiante.

\subsubsection{Mesures et vérifications}

Un exemple de spectre mesuré par cette méthode est présenté dans la figure 11.1 de droite. Nous avons vérifié que le bruit basse fréquence mesuré sur les deux voies séparées provient principalement des amplificateurs : il est identique au bruit mesuré avec des résistances de faible valeur en entrée, et ne dépend pas des conditions de mesure sur l'échantillon (température, courant, etc...). La mesure en corrélation, associée à une moyenne sur un grand nombre de spectres (entre 50 et 500), permet d'éliminer le bruit provenant des amplificateurs. Le spectre de bruit résultant de la corrélation présente un partie plate à haute fréquence : ce bruit est indépendant du courant, et provient du bruit thermique de l'échantillon et des contacts, et du bruit en courant des pré-amplificateurs. La forte augmentation du bruit à basse fréquence dépend fortement du courant, et nous allons montrer qu'il provient des fluctuations de résistance de l'échantillon. La saturation $\mathrm{du}$ bruit à très basse fréquence est due à l'utilisation de filtres passe-haut (fréquence de coupure $0,03 \mathrm{~Hz}$ ), et est corrigée pour l'analyse des résultats. Les figures 11.2, 11.3, 11.4 et 11.5 de gauche montrent les spectres de bruits corrélés pris dans différentes conditions. Les régimes cités dans les légendes seront décrits au paragraphe 11.3.

Nous nous intéressons ici au bruit dépendant du courant, donc à la partie basse fréquence du bruit corrélé. La figure 11.6 montre que la dépendance en courant de la puis- 

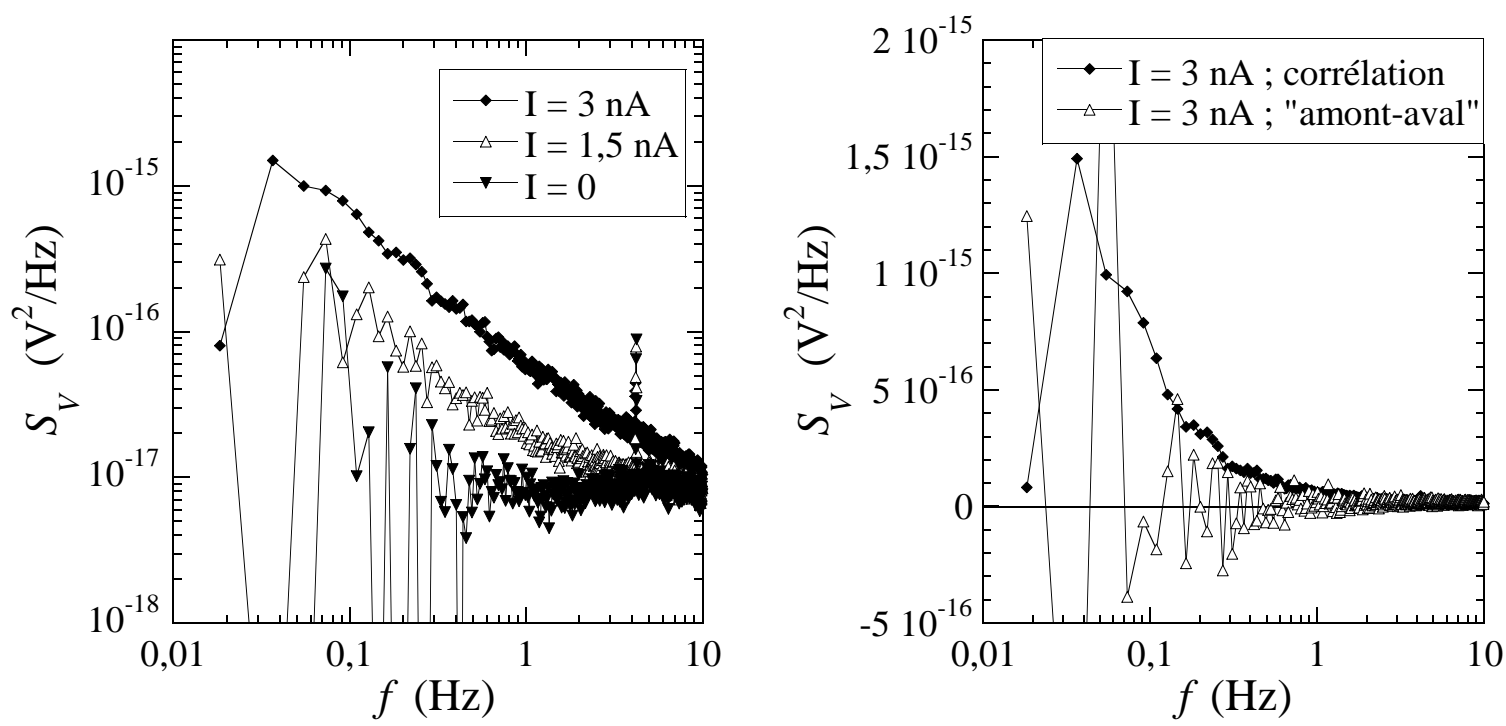

FIG. 11.2: Spectres de bruit en tension à $p_{s}=1,75 \times 10^{14} \mathrm{~m}^{-2}$ et $T=300 \mathrm{mK}$, dans le régime métallique pour lequel $d \rho / d T>0$ (comportement métallique) et $d\left(S_{R} / R^{2}\right) / d T>0$ (voir $\S 11.3)$. Gauche : mesure en corrélation. Droite : Comparaison entre la mesures en corrélation et la corrélation "amont-aval" à courant donné.
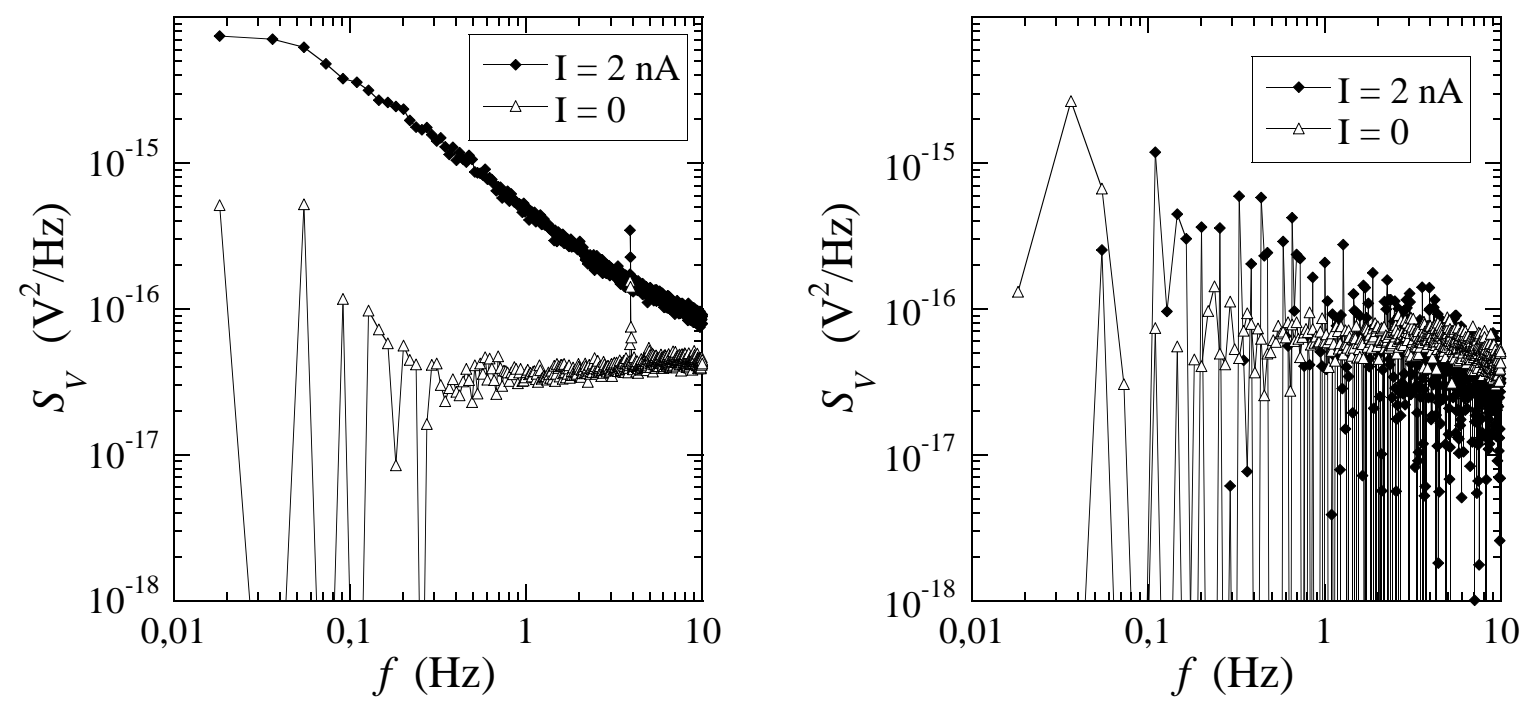

FIG. 11.3: Spectres de bruit en tension à $p_{s}=1,63 \times 10^{14} \mathrm{~m}^{-2}$ et $T=300 \mathrm{mK}$, dans le régime intermédiaire pour lequel $d \rho / d T>0$ (comportement métallique) et $d\left(S_{R} / R^{2}\right) / d T<0$ (voir $\S 11.3)$. Gauche : mesure en corrélation. Droite : Corrélation "amont-aval". 

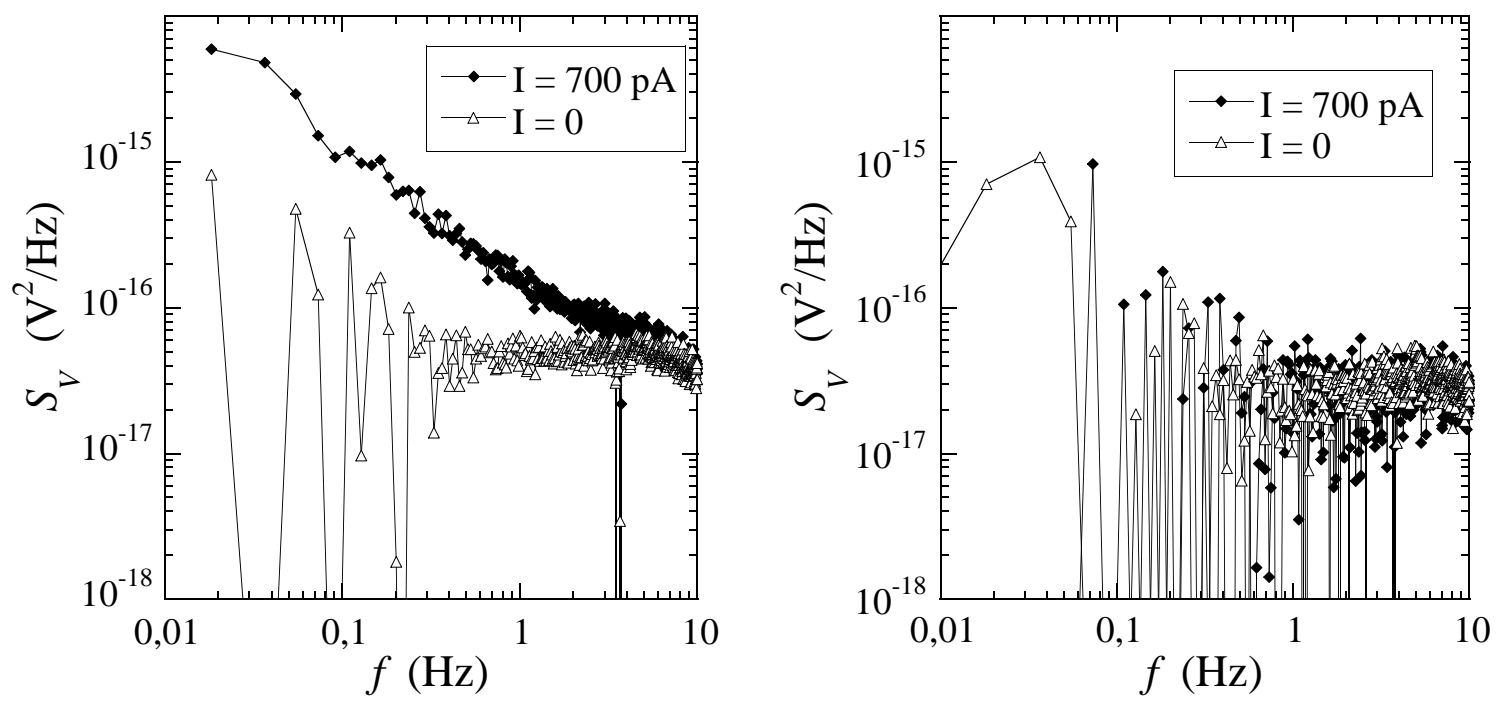

FIG. 11.4: Spectres de bruit en tension à $p_{s}=1,50 \times 10^{14} \mathrm{~m}^{-2}$ et $T=700 \mathrm{mK}$, dans le régime isolant pour lequel $d \rho / d T<0$ (comportement isolant) et $d\left(S_{R} / R^{2}\right) / d T<0$ (voir $\S 11.3$ ). Gauche : mesure en corrélation. Droite : Corrélation "amont-aval".
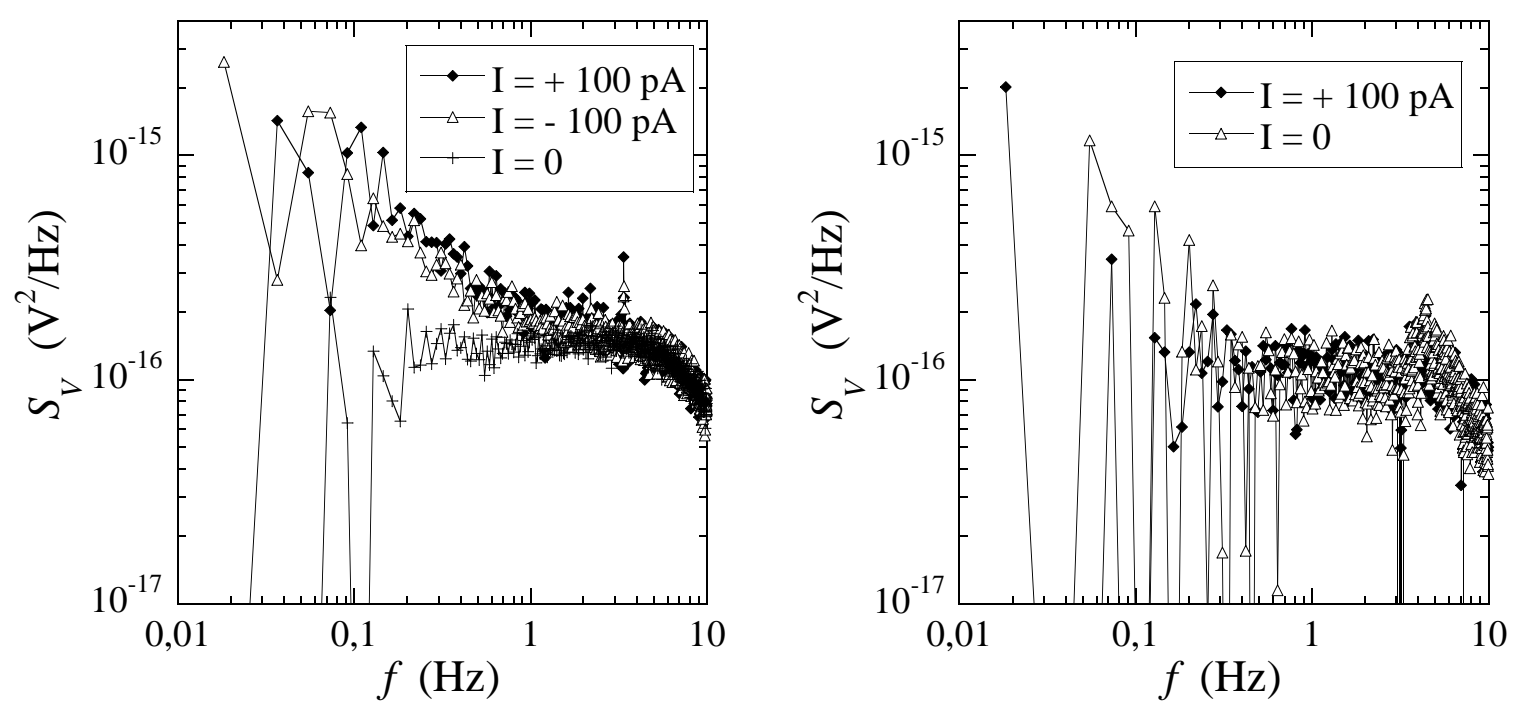

FIG. 11.5: Spectres de bruit en tension à $p_{s}=1,52 \times 10^{14} \mathrm{~m}^{-2}$ et $T=150 \mathrm{mK}$, dans le régime isolant pour lequel $d \rho / d T<0$ (comportement isolant) et $d\left(S_{R} / R^{2}\right) / d T<0$ (voir $\S 11.3$ ). Gauche : mesure en corrélation. Droite : Corrélation "amont-aval". 




FIG. 11.6: Dépendance en courant $I$ de la puissance de bruit en tension $\Delta S_{V}(I)=$ $S_{V}(I)-S_{V}(0)$. Exemple donné pour $p_{s}=$ $1,75 \times 10^{14} \mathrm{~m}^{-2}, T=300 \mathrm{mK}$, et $I=1,5$ et $3 \mathrm{nA}$.

sance de bruit en tension est proportionnelle au carré du courant, $\Delta S_{V}(I)=S_{V}(I)-$ $S_{V}(0) \propto I^{2}$, comme on l'attend d'un bruit provenant des fluctuations de résistance de l'échantillon. Cette vérification a été faite dans différentes conditions de mesure. On peut alors définir la puissance de bruit en résistance par $S_{R}=\Delta S_{V} / I^{2}$.

Pour vérifier que la source de courant n'induit pas un bruit parasite important, nous avons remplacé la source de courant par une source de tension continue (une pile) en série avec une résistance de $20 \mathrm{M} \Omega$, sans constater de modification du bruit mesuré. De même, le fait de mesurer le bruit corrélé d'un même côté ne modifie pas de façon conséquente le bruit corrélé : la seule différence observée est attribuée au bruit provenant des contacts (qui sont pris en compte dans la mesure corrélée d'un seul côté, mais pas dans la mesure habituelle effectuée des deux côtés), ce qui permet d'exclure d'éventuels effets géométriques dus à la configuration particulière de notre échantillon.

Enfin, pour vérifier que le bruit ne provient pas de fluctuations de courant, ou de fluctuations de résistance corrélées mais ne provenant pas du système (comme les fluctuations de température ou de tension de grille), nous avons comparé les mesures en corrélé avec les mesures en corrélation "amont-aval". Les figures 11.2, 11.3, 11.4 et 11.5 de droite montrent des spectres "amont-aval" pris dans les mêmes conditions que les spectres corrélés situés à gauche. La plus grande dispersion des points observée dans la mesure "amont-aval", par rapport à la mesure corrélée, est due à la statistique plus faible pour la moyenne des spectres, et à la faible valeur mesurée. On remarque cependant dans tous ces spectres que le bruit "amont-aval" est nettement inférieur au bruit corrélé, et qu'il ne dépend pas du courant. Cette dernière vérification permet donc d'attribuer le bruit basse fréquence observé dans ces mesures aux fluctuations de la résistance du gaz bidimensionnel de porteurs.

\subsubsection{Ajustement des spectres}

Avant d'effectuer un ajustement de la dépendance en fréquence du bruit, nous avons effectué les corrections de filtres à basse et haute fréquences. Les spectres de bruit $\Delta S_{V}(I)=$ 
$S_{V}(I)-S_{V}(0)$ obtenus pour chaque densité, chaque température et chaque courant ont été ajustés par la loi empirique :

$$
\Delta S_{V}=\frac{A}{f^{\alpha}}
$$

Pour obtenir la meilleure évaluation possible des paramètres d'ajustement $A$ et $\alpha$, ainsi que leurs erreurs, les points expérimentaux ont été ajustés avec une fenêtre de fréquences variables et nous avons calculé à chaque fois le $\chi^{2}$ de l'ajustement. Un exemple de variation des paramètres d'ajustement et $\mathrm{du} \chi^{2}$ en fonction de la fréquence maximale prise en compte est donné dans la figure 11.7 de gauche. On remarque alors que, pour les points à haute fréquence, l'ajustement n'est pas bon en raison principalement de la correction des filtres et de pics de bruit parasites à des fréquences fixes (notamment à environ 4 $\mathrm{Hz}$ ). Par contre, en dessous d'environ $2 \mathrm{~Hz}$, le $\chi^{2}$ sature à sa valeur minimale, et les paramètres d'ajustement sont alors très peu dépendant de la plage de fréquence retenue, caractéristique d'un bon ajustement. Enfin nous avons vérifié que la suppression des points à basse fréquence ne modifie pas l'ajustement.

Un exemple de meilleur ajustement est présenté dans la figure 11.7 de droite. Les ajustements effectués sur la totalité des mesures montrent que la dépendance en fréquence du bruit est $1 / f^{\alpha}$ avec $\alpha=1,1 \pm 0,2$, comme le montre la figure 11.8 de droite. L'exposant est donc toujours proche de 1 , caractéristique d'un bruit en $1 / f$. Quelle que soit la valeur de l'exposant, il est possible de comparer l'amplitude du bruit dans différentes condition en comparant le préfacteur $A$, égal à la valeur de la puissance de bruit à $1 \mathrm{~Hz}$. Pour pouvoir comparer les mesures faites à différentes valeurs du courant et à différentes valeurs de la résistance du système, on compare les valeurs de $A / V^{2}$, représentant les valeurs normalisées du bruit $S_{V} / V^{2}=S_{R} / R^{2}$ à $1 \mathrm{~Hz}$.

\subsection{Dépendances du bruit en température et en den- sité}

\subsubsection{Ajustements empiriques}

Le bruit basse fréquence a été mesuré pour différentes densités de porteurs entre 1,78 et $1,50 \times 10^{14} \mathrm{~m}^{-2}$, pour lesquelles la résistivité présente une transition entre un comportement métallique pour $p_{s}>p_{c} \approx 1,57 \times 10^{14} \mathrm{~m}^{-2}$, et un comportement isolant pour $p_{s}<p_{c}$ (voir la figure 11.8 de gauche). La température est comprise entre 35 et $700 \mathrm{mK}$. On s'intéresse ici aux variations de l'amplitude de bruit $S_{R} / R^{2}$ à $1 \mathrm{~Hz}$ (issu de l'ajustement du bruit en fonction de la fréquence, comme décrit dans le paragraphe précédent) en fonction de la densité de porteurs et de la température. Les résultats sont présentés dans les figures 11.9 et 11.10 de gauche.

Le bruit augmente très fortement lorsque la densité de porteur diminue. Cette dépendance est proche d'une loi exponentielle, comme le montre les ajustements faits à partir de la loi empirique (voir la figure 11.9 de gauche) :

$$
\frac{S_{R}}{R^{2}}(f=1 \mathrm{~Hz})=B_{0}(T) \exp \left[B_{1}(T) \times p_{s}\right]
$$





FIG. 11.7: Gauche : Paramètres d'ajustement de la dépendance en fréquence du spectre de bruit en tension, $S_{V}=A / f^{\alpha}$, et du $\chi^{2}$ par point de l'ajustement, en fonction de la gamme de fréquences prises en compte dans l'ajustement. La fréquence minimale est toujours $0,018 \mathrm{~Hz}$, et la fréquence maximale $f_{\text {max }}$ est variable. Exemple de traitement pour $p_{s}=1,63 \times 10^{14} \mathrm{~m}^{-2}$ et $T=500 \mathrm{mK}$, les valeur extraites de l'ajustement sont $A=(7,45 \pm 0,1) \times 10^{-16} \mathrm{~V}^{2}$ et $\alpha=1,09 \pm 0,03$. Droite : Meilleur ajustement de la dépendance en fréquence du spectre de bruit à basse fréquence, la fréquence maximale des points pris en compte dans l'ajustement est repérée par la ligne verticale (mêmes conditions expérimentales que la figure de gauche).
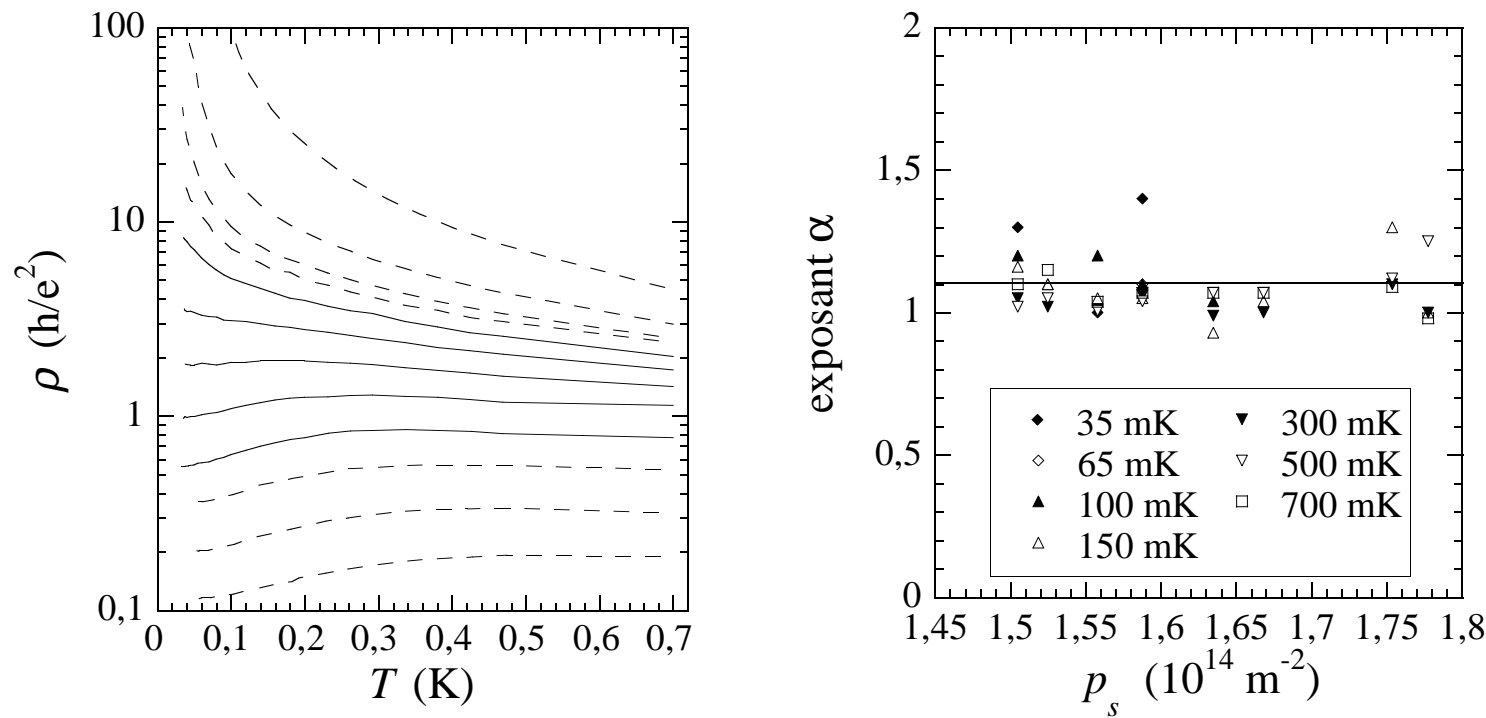

FIG. 11.8: Gauche : Résistivité en fonction de la température de l'échantillon $\mathrm{Nu} 944-1$. Les densités sont, de haut en bas : 1,$30 ; 1,39 ; 1,43 ; 1,44 ; 1,48 ; 1,52 ; 1,57 ; 1,63 ; 1,74 ; 1,86 ; 2,06$; $2,31 \times 10^{14} \mathrm{~m}^{-2}$. Les courbes en traits pleins repèrent la gamme de densités et de températures dans laquelle sont faites les mesures de bruit. Droite : Exposants $\alpha$ issus des ajustements des dépendances en fréquence du bruit basse fréquence par la loi $A / f^{\alpha}$. Les barres d'erreur (non représentées dans cette figure pour plus de clarté) sont équivalentes à la dispersion des points. 
Si on supprime les deux températures les plus basses ( 35 et $65 \mathrm{mK}$ ), on obtient un bon ajustement des paramètres par les deux lois $B_{0}=B_{0}^{0} \times T^{B_{0}^{1}}$ et $B_{1}=B_{1}^{0}+B_{1}^{1} \ln T$, comme le montre la figure 11.9 de droite.

Pour les dépendances en fonction de la température, on observe un comportement différent suivant la densité de porteurs (voir la figure 11.10 de gauche). Pour $p_{s}>p^{*}$ (avec $p^{*} \approx 1,7 \times 10^{14} \mathrm{~m}^{-2}$ ), le bruit croît avec la température, alors que pour $p_{s}<$ $p^{*}$, il décroît quand la température augmente. La valeur de $p^{*} \approx 1,7 \times 10^{14} \mathrm{~m}^{-2}$ est supérieure à la densité critique déterminée par le changement de pente de la résistivité à basse température, $p_{c} \approx 1,57 \times 10^{14} \mathrm{~m}^{-2}$. On peut donc distinguer trois régimes, un régime "métallique" à $p_{s}>p^{*}$ pour lequel $d R / d T$ et $d\left(S_{R} / R^{2}\right) / d T$ sont positifs, un régime "isolant" à $p_{s}<p_{c}$ pour lequel $d R / d T$ et $d\left(S_{R} / R^{2}\right) / d T$ sont négatifs, et enfin un régime intermédiaire à $p^{*}<p_{s}<p_{c}$ pour lequel $d R / d T>0$ à basse température et $d\left(S_{R} / R^{2}\right) / d T<0$. On peut cependant remarquer que, dans ce régime intermédiaire, la dépendance de la résistivité en fonction de la température n'est pas monotone $: d R / d T>0$ à basse température et $d R / d T<0$ à haute température (voir figure 11.8 de gauche). Ainsi la signature de ce régime intermédiaire semble déjà présente sur les courbes de résistivité.

Dans tous les régimes considérés, les dépendances en température de $S_{R} / R^{2}$ à $1 \mathrm{~Hz}$ peuvent être ajustées empiriquement par des lois de puissance (voir la figure 11.10 de gauche) :

$$
\frac{S_{R}}{R^{2}}(f=1 \mathrm{~Hz})=B_{2}\left(p_{s}\right) \times T^{B_{3}\left(p_{s}\right)}
$$

Les paramètres des ajustements sont présentés dans la figure 11.10 de droite. Le paramètre $B_{2}$ représente une extrapolation du bruit à une température de $1 \mathrm{~K}$, et l'exposant est la pente de la dépendance en température. Nous avons vérifié que les lois (11.2) et (11.3), avec les paramètres obtenus des ajustements, sont compatibles entre elles.

\subsubsection{Interprétations}

Sur les figures 11.9 de gauche et 11.10 de droite est indiquée la valeur de la densité critique $p_{c}$ déduite des dépendances en température de la résistivité. On remarque tout d'abord que la dépendance du bruit en fonction de la densité ne présente aucun comportement singulier autour de cette valeur critique, comme on l'attendrait d'une transition de phase. Nos résultats semblent donc infirmer l'existence d'une transition métal-isolant comme elle est habituellement définie (i.e. à $p_{s}=p_{c}$ ). Dans la suite sont décrits les modèles qui peuvent rendre compte des dépendances que nous avons observées sur le bruit. On remarquera cependant qu'aucun de ces modèles ne peut s'appliquer convenablement à nos résultats. Dans la section suivante, nous en verrons la raison : nos résultats montrent l'existence d'une transition de percolation à basse densité (densité inférieure aux densités explorées dans ces mesures de bruit), cette transition n'étant pas prévue par les modèles qui sont évoqués dans cette section.

L'augmentation du bruit avec la température $\left(d\left(S_{R} / R^{2}\right) / d T>0\right)$, observée à densité élevée, est à priori compatible avec les théories "classiques" donnant un bruit issu de processus activés qui font fluctuer les centres sur lesquels diffusent les électrons (voir $\S 8.3)$. Pour un exposant $\alpha$ proche de 1, Dutta, Dimon et Horn prévoient une dépendance $S_{R} / R^{2} \propto T$ [328]. Mais ils considèrent alors que le mécanisme de diffusion des électrons 
est indépendant de la température. La prise en compte des dépendances en température du mécanisme de diffusion (conduisant aux dépendances en température de la résistivité) pourrait expliquer que notre dépendance $S_{R} / R^{2} \propto T^{B_{3}}$ avec $B_{3} \approx 0,5$ et non 1 . On peut également remarquer que, à suffisamment basse température, on s'attend à ce que les effets d'interférences quantiques conduisent à une dépendance inverse du bruit en fonction de la température (voir §8.5). La prise en compte de ces effets peut également expliquer une diminution de l'exposant.

Le second comportement $\left(d\left(S_{R} / R^{2}\right) / d T<0\right)$ peut être interprété dans le cadre de différentes théories. Pour des électrons indépendants, un tel comportement peut être attribué aux interférences quantiques (voir §8.5) : en raison de l'augmentation de la longueur de cohérence de phase quand la température diminue, on s'attend à ce que le nombre de domaines cohérents dans le système diminue et donc que l'amplitude du bruit, moyennée sur le nombre de domaines, augmente quand la température diminue. Pour les fluctuations universelles de conductance, Feng, Lee et Stone prévoient un bruit variant en $S_{R} / R^{2} \propto T^{-1}$ [346] (dans le cas où le mécanisme créant les fluctuations n'est pas activé, mais se produit par effet tunnel), comportement qui a été observé expérimentalement dans des films de Bismuth par Birge et al. [347, 348]. Dans nos résultats, l'exposant de la dépendance en température varie continûment de $-0,5$ à $-1,5$ quand la densité diminue, et donc tous les résultats obtenus ne peuvent pas être interprétés entièrement par ce modèle. Les modèles d'électrons indépendants localisés prévoient également un bruit qui augmente quand la température diminue (voir §8.4). Cependant ces théories prédisent également une saturation du bruit à basse fréquence, ce qui n'est pas observé dans nos mesures. Pour un bruit provenant des fluctuations d'énergie des sites localisés, Kozub prédit une dépendance en $T^{-4 / 3}$ dans le régime de sauts de Mott, et $T^{-2}$ dans le régime d'Efros-Shklovskii [338]. Pokrovskii et al. ont réalisé des mesures de bruit de résistance dans des MESFETs GaAs de faible mobilité [343], et ont obtenu une dépendance du bruit en température en $T^{-1,9}$, compatible avec le modèle de Kozub. Les exposants obtenus dans nos mesures semblent trop faibles pour pouvoir être expliqués par ce modèle. Un troisième argument permet d'exclure cette origine du bruit : on a vu au chapitre 5 que les dépendances en température de la résistivité dans les puits quantiques $p$-GaAs que nous avons étudiés ne s'expliquent pas par les théories d'électrons indépendants localisés. Enfin, l'augmentation du bruit à basse température est également caractéristique d'une phase vitreuse (voir §8.6.3). Cette interprétation a en particulier été retenue pour les mesures de bruit de résistance faites sur des MOSFETs Si par le groupe de Popović $[82,83]$. Les dépendances en température et en densité de porteurs que nous observons sont qualitativement semblables à ces mesures sur les MOSFETs Si, et on peut donc envisager une même interprétation. Une comparaison plus détaillée de nos résultats avec ceux obtenus sur les MOSFETs Si est faite dans la section 11.4.4.

On peut faire une remarque sur les dépendances en densité du bruit : la dépendance exponentielle est beaucoup plus forte que la dépendance donnée par la loi empirique de Hooge $S_{R} / R^{2} \propto 1 / p_{s}$ (voir la dépendance donnée dans la figure 11.9 de gauche). On peut cependant noter que, dans notre système, une variation de la densité conduit également à une variation du désordre, et on s'attendrait donc dans un modèle classique à des écarts par rapport à la loi de Hooge. Dans les modèles d'électrons indépendants, il a également été suggéré que seul le nombre de porteurs participant à la conduction doivent intervenir 

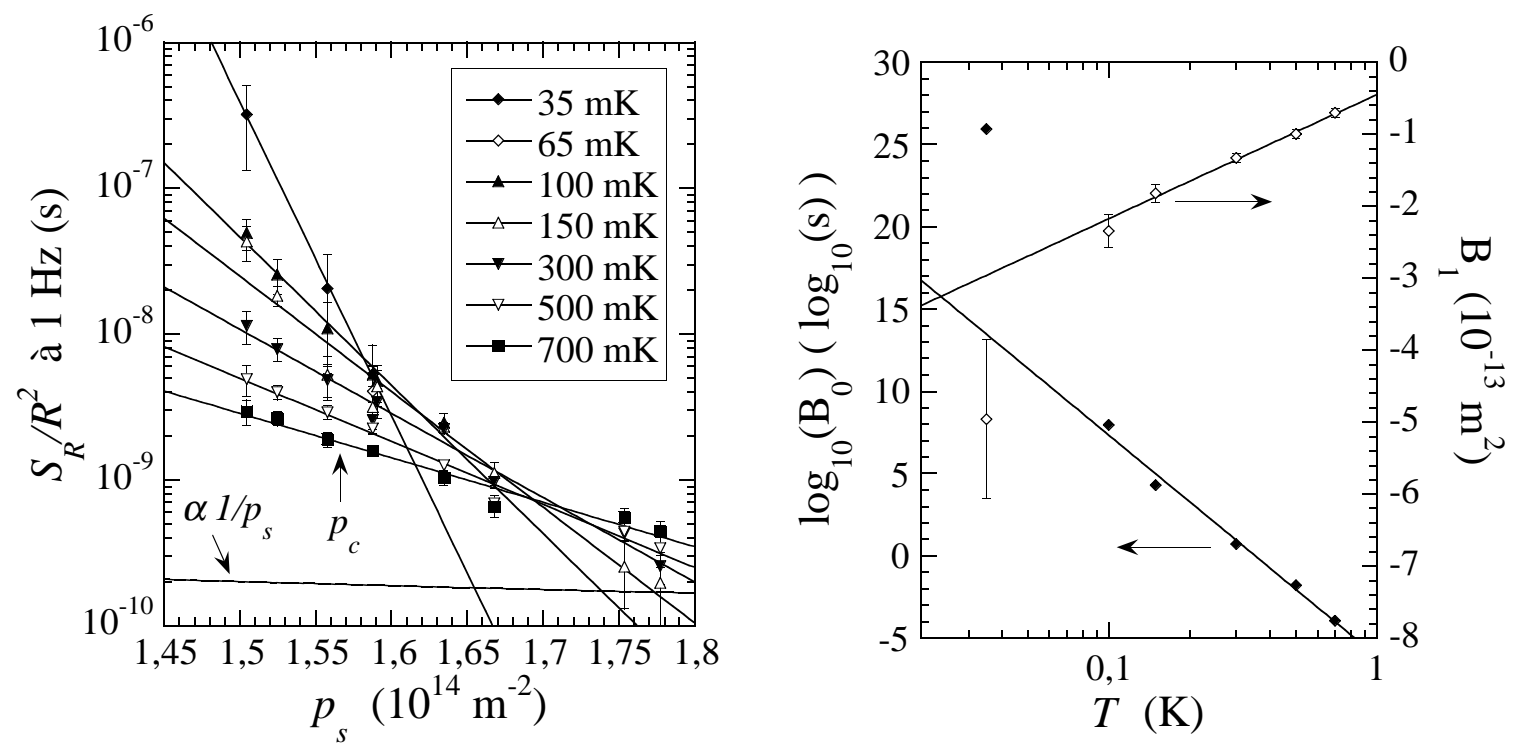

FIG. 11.9: Gauche : Dépendance en densité de porteurs de la puissance de bruit de résistance normalisée $S_{R} / R^{2}=S_{V} / V^{2}$ mesurée à $1 \mathrm{~Hz}$ sur l'échantillon $\mathrm{Nu} 944-1$, pour différentes valeurs de la température (données en encart). Les ajustements sont réalisés par des lois exponentielles (équation (11.2)), et la dépendance en $1 / p_{s}$ est donnée à titre indicatif. Droite : Paramètres des ajustements des dépendances en densité de $S_{R} / R^{2}$ à $1 \mathrm{~Hz}$ par l'équation (11.2).
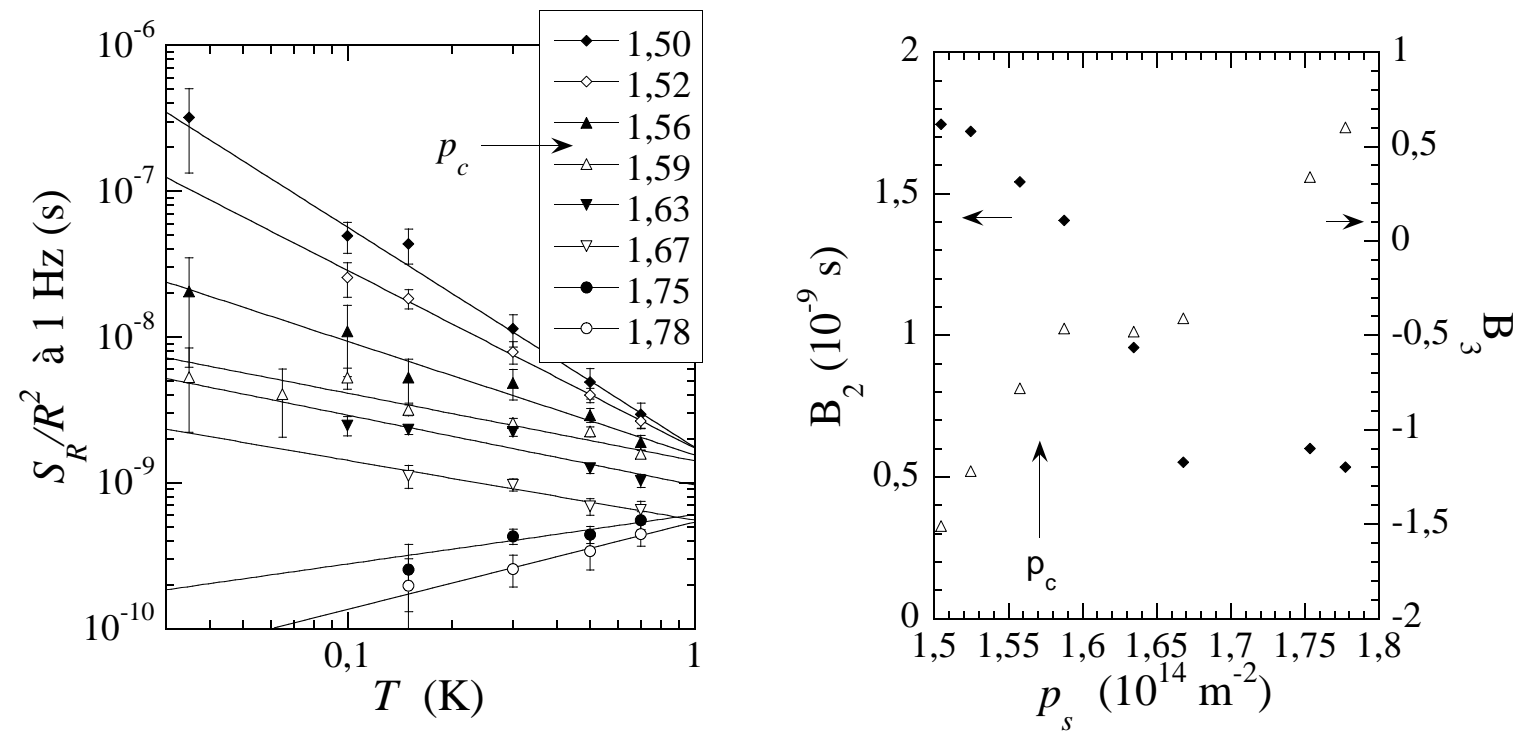

FIG. 11.10: Dépendance en température de la puissance de bruit de résistance normalisée $S_{R} / R^{2}=S_{V} / V^{2}$ mesurée à $1 \mathrm{~Hz}$ sur l'échantillon $\mathrm{Nu} 944-1$, pour différentes valeurs de densités (données en encart, en unité $10^{14} \mathrm{~m}^{-2}$ ). Les ajustements sont réalisés par des lois de puissance (équation (11.3)). Droite : Paramètres des ajustements des dépendances en température de $S_{R} / R^{2}$ à $1 \mathrm{~Hz}$ par l'équation (11.3). 




FIG. 11.11: Puissance de bruit normalisée $S_{R} / R^{2}$ à $1 \mathrm{~Hz}$ en fonction de la résistance $R$. Les symboles regroupent, de manière arbitraire, les points pris à température donnée et à densité variable. La ligne est un ajustement de la totalité des points par une loi de puissance $S_{R} / R^{2} \propto R^{2,4}$.

dans la conduction. En raison de la localisation des porteurs, on s'attend donc à ce que le nombre de porteurs participant à la conduction tende plus rapidement vers zéro que le nombre total de porteurs, ce qui pourrait expliquer une telle dépendance forte en fonction de la densité.

L'existence d'un régime intermédiaire, pour lequel certaines caractéristiques de la phase isolante sont présentes alors que la résistivité suit un comportement métallique, a déjà été mis en évidence dans divers systèmes. Dans les MOSFETs $\mathrm{Si}$, le groupe de Popović a montré que l'augmentation du bruit de résistance lorsque la densité diminue commence à une densité plus élevée que $p_{c}[82,83]$, comme dans nos mesures. De même, Ilani et al. ont montré que la compressibilité locales de G2DT dans GaAs présente des fluctuations importantes en fonction de la densité dans la phase isolante, mais que ces fluctuations sont déjà présentes pour un comportement métallique de la résistivité [125, 126]. Dans les deux cas, un tel comportement peut être lié à l'existence d'un état intermédiaire qui est le mélange de deux phases. Ce résultat est en particulier compatible avec la transition de percolation évoquée dans la section suivante.

\subsection{Observation de lois d'échelle de percolation}

\subsubsection{Loi d'échelle sur le bruit}

Dans la figure 11.11 est présentée la puissance de bruit normalisée $S_{R} / R^{2}$ à $1 \mathrm{~Hz}$ en fonction de la résistance, pour toutes les densités et toutes les températures étudiées. Toutes les mesures se regroupent sur une même courbe, alors que la résistance varie de 
plus d'un ordre de grandeur, et que la puissance de bruit varie de presque quatre ordres de grandeur. De plus, la courbe est une loi de puissance :

$$
\frac{S_{R}}{R^{2}} \sim R^{w}
$$

avec $w=2,40 \pm 0,06$.

Le fait que toutes les mesures se regroupent sur une même courbe est caractéristique d'un comportement d'échelle, pour lequel les grandeurs mesurées ne dépendent plus de différents paramètres de manière indépendante (par exemple la densité de porteurs et la température dans notre cas), mais ne dépendent plus que d'un seul paramètre. Un tel comportement est attendu près d'une transition de phase du deuxième ordre : suffisamment près de la transition, la seule longueur caractéristique est la longueur de corrélation $\xi$ qui contrôle le comportement de toutes les grandeurs physiques, et en particulier la résistance et le bruit de résistance. D'autre part, à l'approche d'une transition de phase du deuxième ordre, les grandeurs physiques divergent en même temps que la longueur de corrélation suivant une loi de puissance :

$$
R \sim \xi^{a} \quad \text { et } \quad \frac{S_{R}}{R^{2}} \sim \xi^{b}
$$

Ces deux relations montrent donc que, près d'une transition de phase du deuxième, il existe une relation entre le bruit de résistance et la résistance identique à celle de l'équation (11.4), avec $w=b / a$. Les résultats de la figure 11.11 constituent donc un argument fort en faveur de l'existence d'une transition de phase du deuxième ordre dans notre système. Cependant, la densité critique de la transition n'est pas $p_{c}$, densité à laquelle on observe un changement de comportement de la résistivité en fonction de la température, mais une densité inférieure à $1,50 \times 10^{14} \mathrm{~m}^{-2}$, densité la plus basse de nos mesures de bruit. La possibilité d'avoir une transition à une densité inférieure à $p_{c}$ a déjà évoquée dans le cadre de la transition métal-isolant en dimension deux (voir §2.4.1).

La divergence du bruit en fonction de la densité, qui est présentée dans la figure 11.9 de gauche, montre que la densité est un paramètre de contrôle de la transition. On peut également s'intéresser au rôle de la température sur les lois d'échelle. Deux comportements sont attendus. Pour une transition de phase quantique (à température nulle), la température conduit à changer la nature de la transition, et crée ainsi un régime où la transition de phase est encore ressentie, mais où le comportement critique est "adouci" (voir la figure 11.12). Dans une telle image, on s'attend à ce que le comportement critique (i.e. la divergence de la longueur de corrélation) soit atténué quand la température augmente et qu'on se rapproche de la densité critique. Ce comportement n'est pas observé dans notre cas, soit en raison d'une température trop faible, soit car on est encore loin de la densité critique, soit par un effet parasite ${ }^{6}$. Mais on peut également envisager une transition de phase à température finie, pour laquelle la ligne de "crossover" dessinée dans la figure 11.12 reste une ligne de transition avec un comportement critique conservé à toutes les températures. Dans ce cas, la densité et la température jouent un rôle analogue, car tous

\footnotetext{
${ }^{6}$ On peut en effet imaginer que, si le comportement critique sur la résistance et sur le bruit sont atténués de manière équivalente, le rapport entre $S_{R} / R^{2}$ et $R^{2,40}$ reste toujours constant.
} 


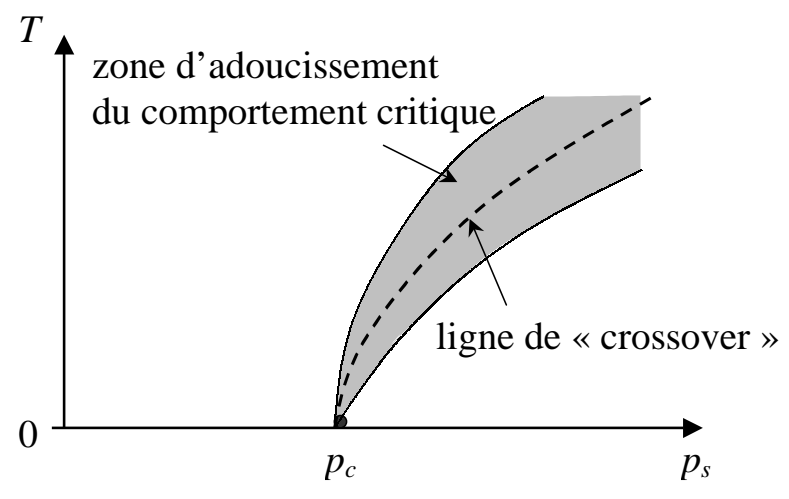

FIG. 11.12: Rôle de la température dans une transition de phase quantique : seule la transition à température nulle présente un réel comportement critique. A température finie, il est possible de définir une ligne de "crossover", autour de laquelle persistent des comportements critiques "adouci" par l'effet de la température.

les deux permettent de traverser la ligne de transition. Ce deuxième cas de figure semble mieux adapté pour expliquer le fait que, dans nos mesures, la loi d'échelle est observée pour toutes les températures.

Avant d'étudier plus en détail les modèles envisagés, on peut remarquer que cette loi d'échelle sur le bruit n'est pas observée dans des gaz bidimensionnels d'électrons désordonnés réalisés dans des MESFETs GaAs [343]. Pokrovskii et al. montrent en effet que, dans ces systèmes, le bruit de résistance peut être décrit par un modèle d'électrons indépendants localisés, et la dépendance en fonction de la résistance de la puissance de bruit est alors une loi différente de celle décrite dans l'équation 11.4. Ce résultat montre encore une fois que le système que nous étudions ne peut pas être décrit dans le cadre d'électrons indépendants localisés, comme c'est le cas pour des systèmes très désordonnés, pour lesquels le rôle des corrélations entre les électrons est moins important que dans notre système.

\subsubsection{Transition de percolation dans un gaz bidimensionnel dilué}

On a vu au paragraphe 8.6.1 que la loi d'échelle présentée dans l'équation (11.4) est prédite en particulier par les théories de percolation (qui est une transition de phase du deuxième ordre). Dans la suite nous analysons nos résultats en regard des développements faits autour des transitions de percolation. Nous allons voir que nos mesures sont en très bon accord avec une telle transition, mais qu'il n'est pas possible d'exclure d'autres types de transition de phase. Cependant il n'existe pas, à notre connaissance, d'étude assez poussée concernant le bruit de résistance autour d'autres types de transitions de phase, telle la transition d'Anderson ou la cristallisation de Wigner, c'est pourquoi ces autres transitions ne seront pas évoquées. On peut juste mentionner les mesures de Cohen et al. autour de la transition d'Anderson en dimension trois dans des films de $\mathrm{ZnO}[363,364]$ : les auteurs ont montré que le bruit en fonction de la résistance varie très fortement, proche d'une loi exponentielle, à l'approche de la transition. Le fait que nos résultats montrent une dépendance en loi de puissance nous permet de conclure que la transition observée n'est pas du même type que celle observée par Cohen et al..

Dans la théorie de percolation, l'exposant $w$ déduit de la loi d'échelle (11.4) est relié à l'exposant de la conductance $t$ et à l'exposant du bruit de résistance $\kappa$ par $w=\kappa / t$ (voir $\S 8.6 .1)$. Cet exposant peut donc nous renseigner sur les caractéristiques de la transition 
observée. Les calculs théoriques dans des modèles spécifiques, ainsi que des mesures expérimentales dans des systèmes présentant une transition de percolation, aboutissent à un exposant $w$ compris entre 0,87 et 4,2 (voir $\S 8.6 .1$, et plus particulièrement le tableau 8.1). On peut en particulier remarquer que l'exposant 2, 4 obtenu dans nos mesures est compris entre 1, exposant attendu pour un réseau carré de résistance, et 3,2 , attendu pour un réseau de sites séparés par des distances aléatoires ("random void"). Dans notre système, on s'attend effectivement à un cas intermédiaire entre ces deux limites, la distance entre sites n'étant pas constante, mais plutôt distribuée autour d'une valeur moyenne.

Dans le cadre de la transition métal-isolant en dimension deux, nous avons vu dans le chapitre 2 que le comportement métallique de la résistivité peut être interprété par des modèles de percolation (voir §2.5.4). La première remarque que l'on peut faire sur ces modèles est qu'ils décrivent une transition à densité plus faible que celle donnée par le changement de signe de $d \rho / d T$, et que le comportement métallique n'est qu'une conséquence du mélange de deux phases, et non un réel état métallique. Ces modèles ne proposent pas de prédiction sur le bruit de résistance, par contre ils présentent tous une transition de percolation et suivent donc les lois générales liées aux lois d'échelle. Certains modèles, tels celui de Meir [213, 214, 215] ou celui de He et Xie [216] sont des percolations à température nulle. Par contre le modèle de gouttelettes de Shi et al. [191, 217], ou le modèle de Spivak [189], sont des transitions à température finie. Dans notre cas, et en regard des lois d'échelle observées pour toutes les températures, ces derniers modèles semblent mieux s'accorder avec nos mesures.

La grande différence entre ces modèles est la manière de traiter les interactions entre électrons dans le système. Dans le modèle de Meir [213, 214, 215], le système est constitué de quasi-particules indépendantes, et le système percolant est dû au remplissage du potentiel de désordre par le "liquide" de quasi-particules, formant des poches d'électrons séparées par des barrières tunnel. Cependant, Shi et Xie [191] ont montré, par des simulations numériques, que les interactions écrantées entre électrons peuvent conduire à une configuration très différente de la répartition des électrons en comparaison du seul effet du désordre. La figure 11.13 montre la répartition de la densité électronique dans un système électronique dans une même configuration de désordre, pour un système sans ou avec interactions. On remarque alors qu'un système ne présentant pas de transition de percolation avec l'effet seul du désordre peut présenter une transition de percolation en raison de la formation de zones de faible densité (gouttelettes). Dans ce modèle, et à température finie, les zones de fortes densités sont constituées de quasi-particules indépendantes (liquide de Fermi), alors que les zones de faibles densité sont des zones d'électrons fortement corrélés formant des "cristallites de Wigner". Ce scénario est un des scénarios envisagés pour décrire la transition de Wigner dans un système désordonné. Dans une même idée, Spivak a montré que la formation d'un système percolant ne nécessite même pas la présence de désordre, et que les interactions seules peuvent conduire à des inhomogénéités de la densité électronique [366, 189]. 

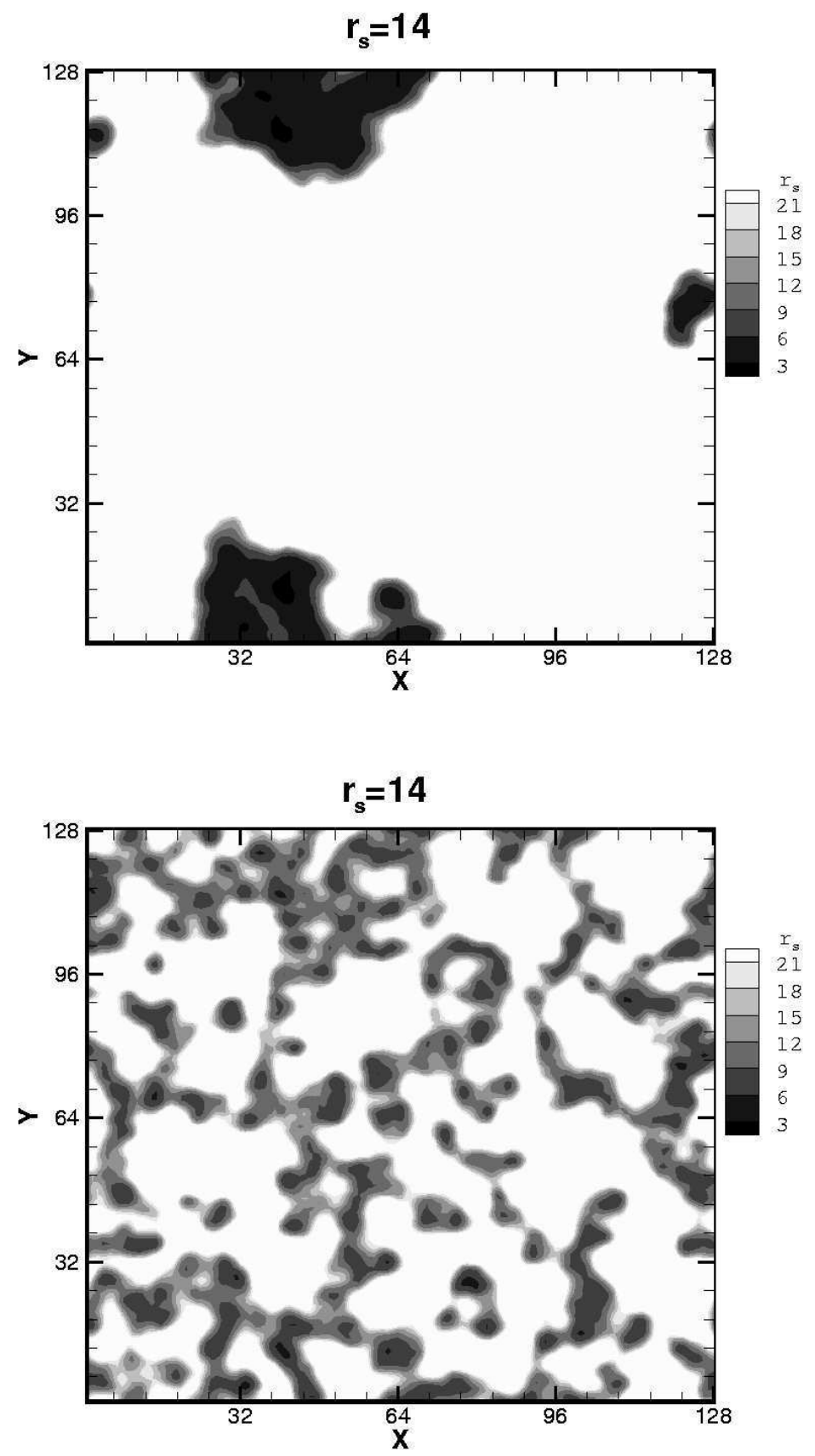

FIG. 11.13: Simulations de la densité électronique locale (exprimée en unités de $r_{s}$, d'après la définition donnée dans l'équation 1.32) dans un gaz bidimensionnel de porteurs à une densité électronique moyenne donnée (équivalente à un facteur $r_{s}$ moyen de 14) et pour une configuration donnée du désordre. La simulation est faite sans prendre en compte les interactions (en haut) et avec des interactions écrantées entre les porteurs (en bas). D'après Shi et Xie [191] 

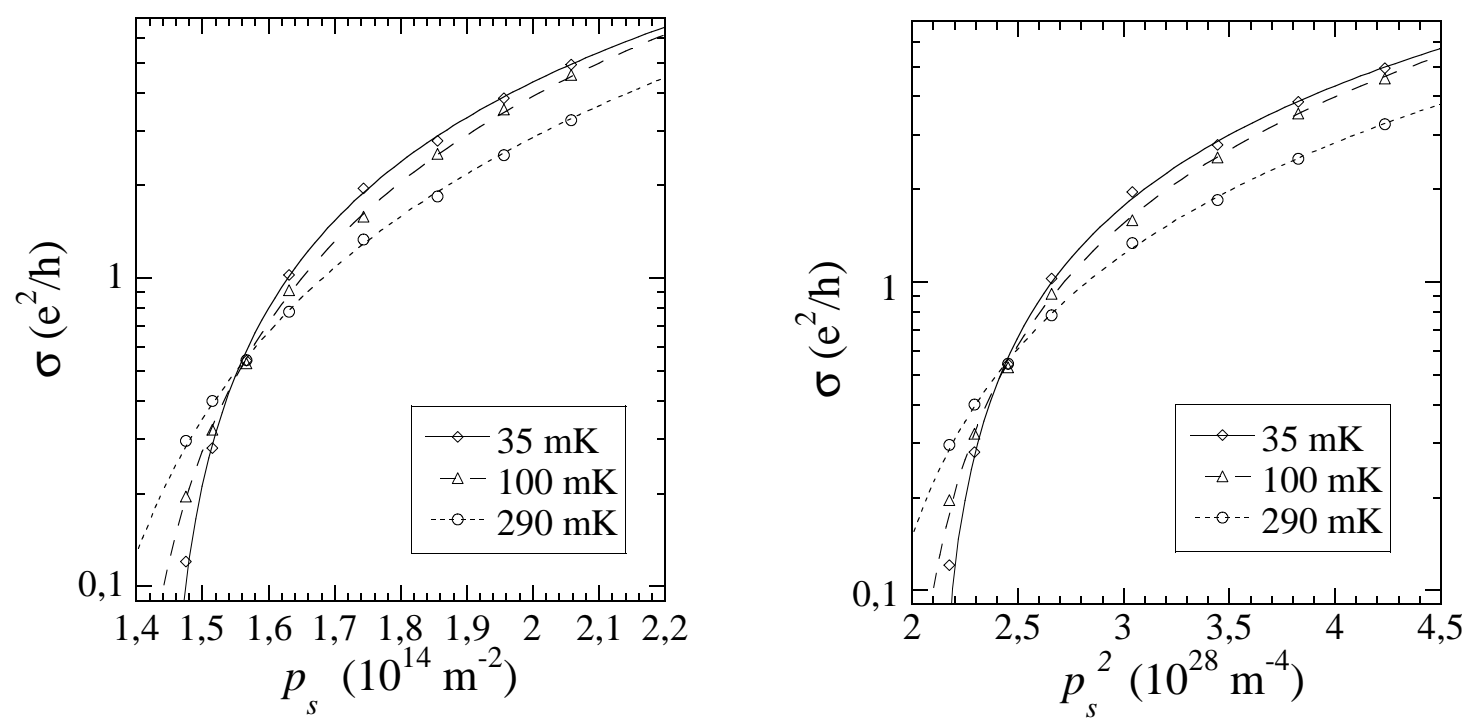

FIG. 11.14: Gauche (resp. droite) : Dépendances de la conductivité $\sigma$ en fonction de la densité $p_{s}$ (resp. de $\left.p_{s}^{2}\right)$ pour trois températures, et ajustements par $\sigma \propto\left(p_{s}-p_{p}\right)^{t}\left(\right.$ resp. $\left.\sigma \propto\left(p_{s}^{2}-p_{p}^{2}\right)^{t}\right)$, où $p_{p}$ et $t$ sont les paramètres de l'ajustement.
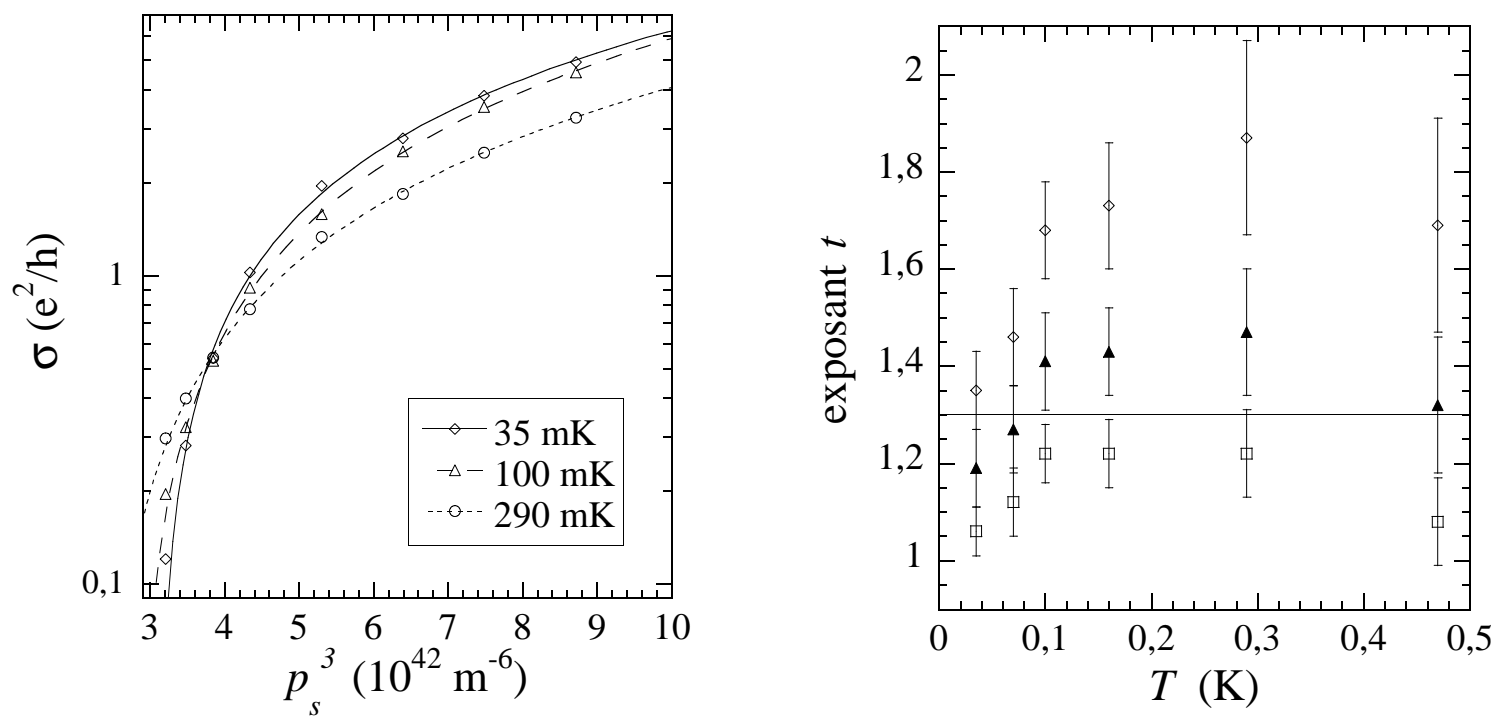

FIG. 11.15: Gauche : Dépendances de la conductivité $\sigma$ en fonction de $p_{s}^{3}$ pour trois températures, et ajustements par $\sigma \propto\left(p_{s}^{3}-p_{p}^{3}\right)^{t}$, où $p_{p}$ et $t$ sont les paramètres de l'ajustement. Droite : Dépendance en température de l'exposant $t$ des ajustements de la conductivité, dans les trois hypothèses : $x \propto p_{s}(\diamond), x \propto p_{s}^{2}(\mathbf{\Delta})$ et $x \propto p_{s}^{3}(\square)$. La ligne horizontale repère l'exposant 1,3 . 


\subsubsection{Loi d'échelle sur la conductance}

On a vu au paragraphe 8.6.1 que la théorie de percolation prévoit des lois d'échelle indépendantes sur la conductance et sur le bruit de résistance :

$$
\sigma \sim\left(x-x_{p}\right)^{t} \quad \text { et } \quad \frac{S_{R}}{R^{2}} \sim\left(x-x_{p}\right)^{-\kappa} .
$$

Cependant, pour vérifier ces lois d'échelle, il est nécessaire de connaître la probabilité de liens $x$, qui est le paramètre de la percolation. Dans notre système, $x$ dépend à priori de la densité de porteurs $p_{s}$ et de la température $T$, mais suivant une fonction qui est inconnue si on ne connaît pas le mécanisme microscopique conduisant à la transition de percolation. Dans le cadre de la transition métal-isolant, les modèles de percolation présentés dans le paragraphe 2.5.4 proposent différentes relations entre la densité de lien et la densité de porteurs. En nous basant sur la conductivité, et en supposant qu'elle doit suivre une loi d'échelle du type :

$$
\sigma \sim\left(x\left(p_{s}, T\right)-x_{p}\left(p_{s}, T\right)\right)^{t}
$$

où $t$ est l'exposant critique de la conductance, nous discutons dans cette partie la forme que peut prendre la fonction $x\left(p_{s}\right)$ à température fixée.

Dans un premier temps nous avons choisi la relation la plus simple, $x \propto p_{s}$, comme le propose le modèle de Meir [213, 214, 215], et nous avons testé l'existence possible de lois d'échelle de la conductivité à différentes températures. Les dépendances de la conductivité en fonction de la densité ont été ajustée avec une loi $\sigma=\sigma_{1}\left(p_{s}-p_{p}\right)^{t}, \sigma_{1}, p_{p}$ et $t$ étant les paramètres de l'ajustement. Ces ajustements sont présentés dans la figure 11.14 de gauche, et l'exposant $t$ déduit de l'ajustement est présenté en fonction de la température dans la figure 11.15 de droite. La loi d'échelle est donc bien vérifiée, mais on remarque que l'exposant $t$ varie en fonction de la température, et est supérieur à la valeur $t \approx 1,3$ attendue de manière générale pour une percolation en dimension deux (voir par exemple Shklovskii et Efros [11] ou Kogan [321]). Mis à part la valeur élevée de ce paramètre, sa dépendance en température est gênante car on a vu que l'exposant $w=\kappa / t$ (où $\kappa$ est l'exposant du bruit) est identique à toutes les températures.

Le choix $x \propto p_{s}$ est arbitraire. En particulier, si on considère que le gaz d'électrons s'organise en régions de forte densité "liquides" et régions de faible densité isolantes, comme il a été suggéré dans le modèle de gouttelettes par Shi et al. [217, 191], et plus récemment par Spivak [189], le paramètre de la percolation est la fraction de la surface occupée par les régions de forte densité, soit $x=p_{s} / p_{\text {eff }}$, où $p_{\text {eff }}$ est la densité effective des régions de forte densité (dans le cas où la densité effective des régions de faible densité est beaucoup plus faible). Shi et Xie [191] ont remarqué que, dans le cas où les électrons de la phase haute densité sont confinés par un potentiel de confinement harmonique (dû au désordre), $x \sim p_{s}$, alors qu'un potentiel de confinement plus "doux" conduit à $x \propto p_{s}^{\alpha}$ avec $\alpha>1$. Ils ont également montré qu'un exposant $\alpha$ plus grand que 1 est nécessaire pour interpréter les mesures de compressibilité de Dultz et al. [124] et Ilani et al. [125]. Pour étudier l'influence de cet exposant $\alpha$ sur la qualité de la loi d'échelle sur la conductivité, nous avons ajusté les courbes de conductivité en choisissant $\alpha=2$ (voir figure 11.14 de droite) et $\alpha=3$ (voir figure 11.15 de gauche). Dans tous les cas, et comme on l'a déjà remarqué pour $\alpha=1$, il est effectivement possible d'observer une bonne loi d'échelle. Par 


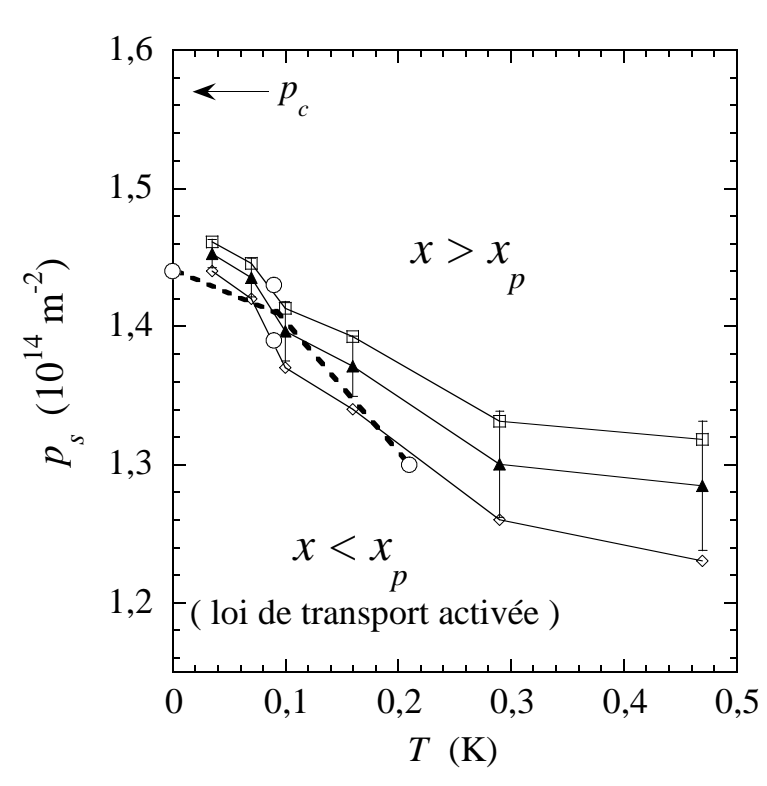

FiG. 11.16: Diagramme de phase représentant la densité critique $p_{p}$ déduite des lois d'échelle sur la conductivité en fonction de la densité de porteurs, pour les trois dépendances : $x \propto p_{s}(\diamond), x \propto p_{s}^{2}$ $(\boldsymbol{\Delta})$ et $x \propto p_{s}^{3}(\square)$. Pour plus de clarté, seules les erreurs pour $x \propto p_{s}^{2}$ sont indiquées (les autres erreurs sont semblables). Les lignes tracées sont des guides visuels, et permettent de définir une ligne de transition de phase séparant une phase pour laquelle $x>x_{p}$ et une phase pour laquelle $x<x_{p}$. Sur le même diagramme est tracé en cercles vides (o) (associés à la ligne en pointillés) la densité $p_{a}$ de transition entre un comportement activé de la résistivité en fonction de la température, pour $p_{s}<p_{a}$, et un comportement en loi de puissance, pour $p_{s}>p_{a}$ (voir §5.4.3).

contre la différence apparaît dans les exposants $t$ déduits de l'ajustement dans chaque cas, et qui sont reportés en fonction de la température dans la figure 11.15 de droite. Alors que dans le cas $\alpha=1$, l'exposant est supérieur à l'exposant 1,3 attendu, on remarque que pour $\alpha=2, t$ est toujours assez proche de 1,3 (aux erreurs près), et que pour $\alpha=3, t$ est toujours inférieur à 1,3 . Cette étude montre donc que le meilleur choix est l'exposant $\alpha=2$. Ce choix permet de réfuter le modèle de percolation classique de Meir, et semble plutôt favoriser les modèles de percolation décrits par Shi et al. et Spivak, pour lesquels le systèmes s'organise en régions de densités différentes. Cependant, dans tous les cas, $t$ varie en fonction de la température, ce qui laisse supposer qu'une fonction plus complexe $x\left(p_{s}\right)$ serait plus adaptée pour rendre compte du comportement critique.

Même si cette analyse ne permet pas de déterminer de manière univoque le type de percolation, il en important de mentionner que, quelque soit le choix de $\alpha$, il est possible de vérifier une loi d'échelle sur la conductivité, confirmant la validité du scénario de percolation. Dans la figure 11.16 sont reportées les valeurs de densités critiques de percolation $p_{p}$ déduites des ajustements de la conductivité. Même si on ne connaît pas exactement la fonction $x\left(p_{s}, T\right)$, nous avons donc démontré ici qu'il y une transition de percolation entre un domaine où $x>x_{p}$ à haute densité vers un domaine où $x<x_{p}$ à faible densité. Le rôle de la température est alors de déplacer la densité à laquelle se situe la transition. Comme il a déjà été évoqué lors de l'analyse de la loi d'échelle sur le bruit, la densité critique à laquelle se passe la transition est inférieure à la densité $p_{c}$ caractérisant la transition de comportement de la résistivité. On peut également comparer les densités critiques de percolation avec celles qui ont été déduites des analyses de la résistivité dans le régime isolant. On a vu en effet au chapitre 5 qu'il existe un régime à basse densité où la loi de transport est activée, et que cette loi devient une loi plus faiblement variable (loi de puissance ou loi de sauts à portée variable) au-delà d'une certaine densité $p_{a}$ qui dépend 
de la température. En comparant les valeurs ainsi obtenues avec les densités critiques de percolation (voir figure 11.16, on remarque que les deux frontières coïncident. Cette remarque est en accord avec le fait que, dans la zone pour laquelle $x<x_{p}$ on s'attend à observer un transport entre les zones conductrices isolées par sauts activés. Il reste cependant à savoir si cette image est vraie dans ce régime, où si le transport dans ce régime est dû aux zones isolantes (cristallites de Wigner accrochées), qui donneraient la même dépendance activée (voir §4.3).

\subsubsection{Comparaison aux MOSFETs Si}

Dans ce paragraphe, nous discutons nos résultats en regard des seuls autres résultats de mesure de bruit basse fréquence autour de la transition métal-isolant, réalisés dans les MOSFETs silicium. Bogdanovitch et Popović [82] ont étudié des MOSFETs très désordonnés et de petite taille (longueur de grille de l'ordre du $\mu \mathrm{m}$ ), dans lesquels le comportement "métallique" de la résistivité est faible, alors que Jaroszynski et al. [83] ont étudié des MOSFETs de forte mobilité, comparable aux expériences réalisées dans le cadre de la transition métal-isolant. Ils ont cependant montré que les caractéristiques du bruit mesuré dans les deux cas sont très semblables.

Certaines caractéristiques sont très semblables à celles que nous avons mesurées. Ils observent une forte augmentation du bruit lorsque la densité diminue, et cette augmentation débute à une densité électronique $n_{g}$ supérieure à la densité critique de la transition $n_{c}$. D'autre part, pour $n_{s}<n_{g}$ le bruit augmente fortement quand la température diminue. Ces deux caractéristiques sont également observées dans notre système, et on notera que, comme $n_{c}<n_{g}$, ils observent également un régime que nous avons qualifié d'intermédiaire, i.e. avec $d R / d T>0$ et $d\left(S_{R} / R^{2}\right) / d T<0$.

La grande différence avec nos mesures est l'augmentation de l'exposant $\alpha$ de la dépendance en fréquence du bruit lorsque la densité devient inférieure $n_{g}$ : il passe de environ 1 pour $n_{s}>n_{g}$ à environ 1,8 pour $n_{s}<n_{g}$. Nous n'observons pas un tel comportement. Cependant on peut noter que leurs mesures ont été réalisées à beaucoup plus basse fréquence (jusqu'à $10^{-5} \mathrm{~Hz}$ ), et il est possible qu'un tel comportement n'apparaisse plus à plus haute fréquence. Ils ont également mesuré le "second bruit" (i.e. le spectre des fluctuations du bruit $S_{R}$ ), et ont montré une forte dépendance en fréquence de ce bruit. Ce caractère est indicatif d'un bruit non gaussien, qui peut être interprété comme l'effet de corrélations. L'augmentation de l'exposant et la dépendance en fréquence du second bruit leur ont permis d'interpréter l'augmentation du bruit en-dessous de $n_{g}$ par une transition vers un état vitreux à basse densité électronique. On peut cependant noter qu'un bruit basse fréquence non gaussien a déjà été observé dans des échantillons amorphes de silicium dans la phase isolante par Parman et al. [367]. Dans ce système, le bruit non gaussien est interprété par le fait que les fluctuations de résistance sont déterminées par seulement un faible nombre de chemins conducteurs. On s'attend donc à un même effet dans un système percolant.

Une des raisons pour lesquelles l'augmentation de l'exposant $\alpha$ n'est pas observée dans nos mesures est la différence de gamme de fréquence observée. Il existe également des raisons physiques qui permettent d'interpréter une différence entre les mesures dans les MOSFETs Si et dans les hétérostructures $p$-GaAs. Les MOSFETs silicium utilisés sont 
plus désordonnés que les hétérostructures $p$-GaAs que nous avons utilisées, et la densité de porteurs dans les MOSFETs est environ dix fois plus élevée que celle dans le $p$-GaAs. D'autre part, la nature du désordre limitant le transport est différente dans les deux cas, puisque les impuretés dans les MOSFETs Si sont essentiellement des impuretés chargées (dans l'oxide), alors que ce sont principalement des défauts neutres (rugosité d'interface) dans le $p$-GaAs. Ainsi cette caractéristique peut conduire à une phase vitreuse dans les MOSFETs plutôt qu'à un mélange de deux phases avec une transition de percolation. Une autre différence provient de la distance entre le gaz bidimensionnel de porteurs et la grille métallique permettant de faire varier la densité de porteurs. Dans nos échantillons, cette distance est supérieure à $1 \mu \mathrm{m}$, en comparaison d'une distance moyenne entre porteurs de l'ordre de $50 \mathrm{~nm}$, ce qui permet de réduire les effets d'écrantage des interactions entre porteurs par la grille métallique. Dans les MOSFETs Si, la distance entre le daz bidimensionnel d'électrons et la grille est de 7 à 20 fois plus petite que dans notre système, et on s'attend donc à un effet plus important de l'écrantage.

Mais on peut également s'interroger sur la nature de la phase cristal de Wigner désordonné, qui constituerait la phase isolante du système percolant. Les mesures dans MOSFETs les plus désordonnés (Bogdanovitch et Popovic [82]) sont réalisées principalement dans la phase que l'on a qualifiée d'"isolante" dans nos échantillons, et le ralentissement de la dynamique est observé dans cette phase isolante. Les mesures sur les MOSFETs silicium semblent donc indiquer que la phase isolante est un verre plutôt qu'une phase d'électrons indépendants localisés, ce qui est compatible avec l'idée d'un mélange de phases aux abords de la cristallisation de Wigner.

On peut enfin mentionner les mesures de compressibilité locales de Ilani et al. sur des hétérostructures $p$-GaAs $[125,126]$. Ils ont montré que, dans la phase isolante, le système devient fortement inhomogène. Ces mesures sont en accord avec la description d'un système percolant dans les hétérostructures $p$-GaAs.

\subsection{Conclusion}

Ces mesures de bruit présentent une avancée importante dans la compréhension des mécanismes qui gouvernent la transition métal-isolant en dimension deux, et plus généralement de la physique des systèmes d'électrons en interaction forte. Elles ouvrent une voie de recherche nouvelle qui est l'étude de la transition autour de la densité critique $p_{p}$, inférieure à la densité $p_{c}$ habituellement utilisée dans le cadre de la transition métal-isolant.

Dans un premier temps, nous avons montré que la "transition métal-isolant" habituellement admise (avec changement de signe de $d \rho / d T$ ) n'est pas associé à un comportement singulier du bruit, et ne semble donc pas pouvoir être interprétée comme une réelle transition de phase. Par contre, le bruit suit une loi d'échelle en fonction de la résistance, et présente donc un comportement critique, mais la densité critique $p_{p}$ de cette transition est nettement inférieure à la densité critique déterminée par l'analyse des dépendances en température de la résistivité $p_{c}$. Dans la recherche de la nature de la transition $p_{s}=p_{p}$, nous avons montré que les lois d'échelle observées sont compatibles avec les théories de percolation. On ne peut cependant pas exclure un autre type de transition, mais seules les théories de percolation proposent des prédictions sur le bruit de résistance. 
L'analyse combinée des lois d'échelle suivies par le bruit de résistance et par la conductance est un outil essentiel pour déterminer la nature de la transition. En particulier, l'exposant $w=2,40 \pm 0,06$ obtenu peut être comparé à l'exposant prévu dans des modèles de percolation. Dans le cadre de la transition métal-isolant en dimension deux, certains modèles proposés prévoient l'existence d'une transition de percolation à basse densité, et il serait intéressant de déterminer cet exposant $w$ dans le cadre de ces modèles.

Dans une transition de percolation, il est nécessaire de connaître la probabilité de lien $x$ pour pouvoir vérifier les lois d'échelle séparées en bruit et en conductance, et en déduire les exposants critique $\kappa$ et $t$. Le fait que nous observions un loi d'échelle à toutes les densités et à toutes les températures indique que cette probabilité de lien doit varier avec la densité et avec la température, ce qui est caractéristique d'une transition à température finie. Enfin, par l'étude de la loi d'échelle sur la conductance, on a montré qu'une probabilité de lien $x \propto p_{s}^{2}$ décrit le mieux la transition observée.

Finalement, la comparaison de nos mesures avec celle de Bogdanovitch et Popović [82] et de Jaroszynski et al. [83] dans des MOSFET silicium, et les mesures de compressibilité locales de Ilani et al. $[125,126]$ dans des hétérostructures $p$-GaAs, semble conforter des théories qui donnent un scénario de passage d'une phase liquide de Fermi à une phase collective désordonnée (verre de Wigner) par une transition de percolation. 


\section{Annexe A}

\section{Couplage électron-phonon dans un gaz bidimensionnel de trous}

\section{Sommaire}

A.1 Introduction . . . . . . . . . . . . . . 248

A.2 Couplage électron-phonon dans un gaz bidimensionnel de porteurs ........................ 248

A.2.1 Puissance dissipée par le couplage électron-phonon . . . . . 248

A.2.2 Les différents types de couplages . . . . . . . . . . . . 250

A.2.3 Le couplage trou-phonon . . . . . . . . . . . . 252

A.2.4 Résultats expérimentaux . . . . . . . . . . . . 252

A.3 Détermination de la température effective des porteurs - 253

A.3.1 Résistivité à champ magnétique nul . . . . . . . . . . . . . . 254

A.3.2 Oscillations de Shubnikov-de Haas . . . . . . . . . . . 254

A.3.3 Localisation faible . . . . . . . . . . . . . . . 256

A.3.4 Bruit thermique . . . . . . . . . . . 256 


\section{A.1 Introduction}

Lorsqu'on fait passer un courant dans un système de résistance non nulle, une certaine partie de la puissance fournie est convertie en chaleur par effet Joule. Un gaz bidimensionnel d'électrons ne déroge pas à cette règle, et dès qu'un champ électrique $E$ est appliqué, une puissance $P_{E}=E^{2} / \rho$ est dissipée par unité de surface dans le système électronique. Dans un gaz bidimensionnel de porteurs formé à l'interface entre des semiconducteurs, cette puissance est dissipée dans le cristal par l'intermédiaire du couplage électron-phonon. À basse température, ce couplage est peu efficace, conduisant à une élévation de température du gaz de porteurs par rapport à la température du réseau.

La question du chauffage des porteurs est une question fondamentale dans l'étude des propriétés de systèmes électroniques à très basse température. En particulier, dans le cadre de la transition métal-isolant en dimension deux, la question de savoir si la température mesurée est la température réelle des porteurs est fondamentale pour comprendre les mécanismes mis en jeu [68]. D'autre part, la comparaison des courbes de résistivité en fonction de la température avec les prédictions théoriques ne peut être réalisée qu'en connaissant exactement la température des porteurs.

Pour mieux connaître les phénomènes de dissipations de chaleur, et ainsi mieux maîtriser la température des porteurs dans nos systèmes, il est tout d'abord important de connaître de manière fondamentale le mécanisme mis en jeu, le couplage électron-phonon (§A.2). Dans une deuxième section (§A.3) nous nous intéressons à la manière de mesurer la température effective des porteurs dans les systèmes étudiés.

\section{A.2 Couplage électron-phonon dans un gaz bidimen- sionnel de porteurs}

\section{A.2.1 Puissance dissipée par le couplage électron-phonon Hypothèses}

Pour décrire les électrons, on se place dans l'hypothèse de la température effective, dans laquelle le système électronique hors équilibre peut être décrit par une fonction de distribution de Fermi-Dirac à une température effective $T_{e}$ :

$$
f_{T_{e}}\left(\epsilon_{\mathbf{k}}\right)=\frac{1}{\exp \left(\frac{\epsilon_{\mathbf{k}}}{k_{B} T_{e}}\right)+1}
$$

avec $\epsilon_{\mathbf{k}}=E_{\mathbf{k}}-E_{F}$ l'écart à l'énergie de Fermi des électrons de vecteur d'onde $\mathbf{k}$. À basse température, il a été montré dans les MOSFETs Si [22] que le mécanisme de diffusion inélastique dominant est la diffusion électron-électron. On peut aussi vérifier que le temps de collision électron-électron est beaucoup plus court que le temps de relaxation de l'énergie [369]. Cette hypothèse est donc en général applicable dans les gaz bidimensionnels étudiés.

Les fonctions d'ondes électroniques sont des ondes planes dans les directions $x$ et $y$ définissant le plan du gaz d'électrons, et les états du puits de potentiel dans la direc- 
tion $z$ orthogonale au plan. De plus on ne considére qu'une seule bande d'énergie, dans l'approximation de la masse effective $m^{*}$. On verra que dans le cas de plusieurs bandes d'énergie (en particulier pour les trous de la bande de valence), il est possible de faire une approximation pour se ramener au cas à une seule bande. À basse température, pour $k_{B} T_{e} \ll E_{F}$, où $E_{F}$ est l'énergie de Fermi, on peut considérer le gaz d'électrons comme un système fortement dégénéré (régime de Bloch-Grüneisen).

À basse température, il y a très peu d'électrons d'énergie suffisamment élevée pour émettre des phonons optiques. Donc on peut considérer que seuls les phonons acoustiques interviennent dans le couplage électron-phonon. Cette hypothèse est satisfaite pour $k_{B} T_{e} \ll \hbar \omega_{\text {opt }}$, où $T_{e}$ est la température effective des électrons et $\omega_{\text {opt }}$ la pulsation des phonons optiques. D'autre part on ne prend en compte que les phonons du matériau massif, et non les déformations du réseau qui se propagent dans le plan des électrons [370]. Les phonons ont une fonction de distribution de Bose-Einstein à la température $T_{0}$ :

$$
N_{T_{0}}(\mathbf{q}, \lambda)=\frac{1}{\exp \left(\frac{\hbar \omega_{(\mathbf{q}, \lambda)}}{k_{B} T_{0}}\right)-1} .
$$

\section{Puissance dissipée par le couplage électron-phonon}

La puissance dissipée par le couplage électron-phonon est égale à la différence entre la probabilité d'émission et la probabilité d'absorption d'un phonon lors du couplage électron-phonon, multipliée par l'énergie transférée $\hbar \omega$ (où $\omega$ est la pulsation du phonon) lors de l'émission (resp. absorption) du phonon. Il faut ensuite sommer sur tous les vecteurs d'onde $\mathbf{q}$ et les polarisations $\lambda$ des phonons :

$$
P_{\mathrm{e}-\mathrm{ph}}=\sum_{\mathbf{q}, \lambda} \hbar \omega_{(\mathbf{q}, \lambda)}\left(w_{\text {émission }}-w_{\text {absorption }}\right) \text {. }
$$

Les probabilités d'émission (resp. d'absorption) d'un phonon, accompagnant la transition de l'électron d'un état de vecteur d'onde $\mathbf{k}$ à un état de vecteur d'onde $\mathbf{k}^{\prime}$ (resp. de $\mathbf{k}^{\prime}$ à k), sont données par :

$$
\begin{aligned}
w_{\text {émission }} & =f_{T_{e}}\left(\epsilon_{\mathbf{k}}\right)\left(1-f_{T_{e}}\left(\epsilon_{\mathbf{k}^{\prime}}\right)\right)\left(N_{T_{0}}(\mathbf{q}, \lambda)+1\right) \cdot W \\
\text { et } w_{\text {absorption }} & =f_{T_{e}}\left(\epsilon_{\mathbf{k}^{\prime}}\right)\left(1-f_{T_{e}}\left(\epsilon_{\mathbf{k}}\right)\right) N_{T_{0}}(\mathbf{q}, \lambda) \cdot W,
\end{aligned}
$$

où la probabilité de transition $W$ est donnée par la règle d'or de Fermi :

$$
W=\frac{2 \pi}{\hbar}|M(\mathbf{q}, \lambda)|^{2} \delta\left(\epsilon_{\mathbf{k}}-\epsilon_{\mathbf{k}^{\prime}}-\hbar \omega_{(\mathbf{q}, \lambda)}\right) .
$$

$M(\mathbf{q}, \lambda)$ est l'élément de matrice de l'interaction électron-phonon, et prend des formes différentes suivant la nature du couplage (voir le paragraphe suivant).

L'équation (A.3) est intégrable numériquement. Dans l'hypothèse du gaz d'électron fortement dégénéré, il est possible de mettre la puissance dissipée par le couplage électronphonon sous la forme [371] :

$$
P_{\mathrm{e}-\mathrm{ph}}=F\left(T_{e}\right)-F\left(T_{0}\right),
$$

où $F(T)$ est une fonction qui dépend de la nature du couplage électron-phonon. Dans les parties suivantes sont décrites les expressions de $F$ dans différents cas. 


\section{A.2.2 Les différents types de couplages}

\section{Couplage par déformation}

Les déformations du cristal (phonons) induisent un déplacement des charges du réseau cristallin. Lorsque ces déplacements d'atomes produisent des variations locales de volume du cristal, la densité locale de charge varie, et les électrons interagissent avec ce potentiel électrostatique. Ce mécanisme est à l'origine du couplage par déformation. Ce type de couplage faisant intervenir les variations locales de volume du cristal, seuls les modes longitudinaux de phonons vont être impliqués. En effet les modes transverses créent seulement un cisaillement sans variation de volume. Dans ce cas, l'élément de matrice de couplage prend la forme [372, p. 579] :

$$
\left|M^{P D}(q)\right|^{2}=\frac{\hbar \Xi_{u}^{2}}{2 \rho v_{L}} q
$$

où $\Xi_{u}$ et $\rho$ sont respectivement le potentiel de déformation et la masse volumique du matériau massif dans lequel le gaz d'électrons est localisé, et $v_{L}$ la vitesse des modes longitudinaux de phonons.

En l'absence d'écrantage du couplage électron-phonon, on peut calculer analytiquement la fonction $F(T)[371,305]$ :

$$
F_{\text {n.ê. }}^{\mathrm{PD}}(T)=\frac{3 \zeta(5)}{\sqrt{2} \pi^{5 / 2}} \frac{\left(m^{*}\right)^{2} \Xi_{u}^{2}}{\hbar^{7} n_{s}^{3 / 2} \rho v_{L}^{4}}\left(k_{B} T\right)^{5},
$$

où $\zeta(s)$ est la fonction de Riemann $(\zeta(5)=1,03692776), m^{*}$ la masse effective des porteurs, et $n_{s}$ la densité surfacique d'électrons.

\section{Couplage piézoélectrique}

Dans un cristal polaire, le déplacement en opposition de phase des charges de signes opposées du réseau induit la création de moments dipôlaires. L'interaction entre ces moments dipôlaires fluctuants et les électrons est à l'origine du couplage piézoélectrique. Pour que ce couplage ait lieu, il est nécessaire que le cristal ne comporte pas de centre d'inversion. En effet, dans le cas où le cristal présente un centre d'inversion, chaque moment dipôlaire va être compensé par son symétrique. Dans ce type de couplage vont intervenir aussi les bien les modes longitudinaux $(L)$ et transverses $(T)$ de phonons. L'élément de matrice de couplage s'écrit alors [373] :

$$
\begin{aligned}
\left|M_{L}^{P Z}(q)\right|^{2} & =\frac{\hbar\left(e \cdot e_{p z}\right)^{2}}{2 \rho v_{L}} \frac{9 q_{\perp}^{2} q_{\|}^{4}}{2 q^{7}} \\
\text { et }\left|M_{T}^{P Z}(q)\right|^{2} & =\frac{\hbar\left(e \cdot e_{p z}\right)^{2}}{2 \rho v_{T}} \frac{8 q_{\perp}^{4} q_{\|}^{2}+q_{\|}^{6}}{2 q^{7}}
\end{aligned}
$$

pour les deux types de polarisation, où $e_{p z}$ et $\rho$ sont la constante piézoélectrique et la masse volumique du matériau massif, $v_{L}$ et $v_{T}$ les vitesses des modes de phonons longitudinaux et transverses. $q_{\|}$et $q_{\perp}$ sont les normes des composantes parallèle et perpendiculaire du vecteur d'onde $\mathbf{q}$ des phonons par rapport au plan formé par le gaz d'électrons. 
En l'absence d'écrantage du couplage électron-phonon, on peut calculer analytiquement la fonction $F(T)[371,305]$ :

$$
F_{\text {n.ê. }}^{\mathrm{PZ}}(T)=\frac{3 \zeta(3)}{64 \sqrt{2} \pi^{5 / 2}} \frac{\left(m^{*}\right)^{2}\left(e \cdot e_{p z}\right)^{2}}{\hbar^{5}\left(4 \pi \varepsilon_{0}\right)^{2}\left(n_{s}\right)^{3 / 2} \rho}\left[\frac{3}{v_{L}^{2}}+\frac{4}{v_{T}^{2}}\right]\left(k_{B} T\right)^{3},
$$

avec $\zeta(3)=1,20205696, m^{*}$ la masse effective des porteurs et $n_{s}$ la densité de porteurs.

\section{Effet de l'écrantage statique}

En raison des interactions entre électrons, on s'attend à ce que le gaz d'électrons écrante le potentiel créé par les phonons. Les calculs présentés dans ce paragraphe prennent l'hypothèse de l'écrantage statique, pour lequel on suppose que le potentiel électrostatique lié aux phonons varie lentement comparé au temps de réaction des électrons. Cet effet peut être pris en compte en introduisant un facteur d'écrantage $S\left(q_{\|}\right)=1 / \varepsilon\left(q_{\|}\right)$dans l'élément de matrice de couplage électron-phonon. La fonction diélectrique d'écrantage statique $\varepsilon\left(q_{\|}\right)$s'écrit, pour $q_{\|}<2 k_{F}[374]$ :

$$
\varepsilon\left(q_{\|}\right)=1+H\left(q_{\|}\right) \frac{q_{s}}{q_{\|}},
$$

où $q_{s}=\left(m^{*} e^{2}\right) /\left(2 \pi \varepsilon_{0} \varepsilon_{r} \hbar^{2}\right)$ est le vecteur d'onde d'écrantage et $H\left(q_{\|}\right)$est une fonction qui rend compte de l'extension des fonctions d'onde électroniques dans la direction perpendiculaire au gaz d'électron (direction $\mathrm{z}$ ) :

$$
H\left(q_{\|}\right)=\iint \rho\left(z_{1}\right) \rho\left(z_{2}\right) e^{-q_{\|}\left|z_{1}-z_{2}\right|} d z_{1} d z_{2}
$$

avec $\rho(z)=|\Phi(z)|^{2}$, et $\Phi(z)$ est la fonction enveloppe de la fonction d'onde électronique dans la direction $z$.

Dans le cas où $q_{\|} \gg q_{s}, \varepsilon\left(q_{\|}\right) \sim 1$ et le calcul effectué sans tenir compte de l'écrantage est valable. On est alors dans la limite d'écrantage faible. Dans la limite de fort écrantage $\left(q_{\|} \ll q_{s}\right.$ et $q_{\|} \ll 1 / z_{0}$, avec $z_{0}$ l'extension spatiale typique des fonctions d'ondes suivant $z$ ), on peut négliger l'extension des fonctions d'ondes suivant $z$, et le facteur d'écrantage devient $S\left(q_{\|}\right) \approx q_{\|} / q_{s}$. Dans cette limite, il est possible de calculer analytiquement la fonction $F(T)$ [375, 371]. Le couplage par le potentiel de déformation devient :

$$
F_{\text {écr. }}^{\mathrm{PD}}(T)=\frac{360 \zeta(7)}{\sqrt{2 \pi}} \frac{\left(\varepsilon_{0} \varepsilon_{r}\right)^{2} \Xi_{u}^{2}}{e^{4} \hbar^{5} n_{s}^{3 / 2} \rho v_{L}^{6}}\left(k_{B} T\right)^{7},
$$

avec $\zeta(7)=1,008349277$, et pour le couplage piezoélectrique :

$$
F_{\text {écr. }}^{\mathrm{PZ}}(T)=\frac{\zeta(5)}{512 \sqrt{2} \pi^{5 / 2}} \frac{\varepsilon_{r}^{2}\left(e \cdot e_{p z}\right)^{2}}{e^{4} \hbar^{3} n_{s}^{3 / 2} \rho}\left[\frac{135}{v_{L}^{4}}+\frac{177}{v_{T}^{4}}\right]\left(k_{B} T\right)^{5} .
$$

Enfin il est intéressant de voir le rôle de la température et de la densité d'électrons sur l'écrantage. L'énergie des phonons excités à une température donnée est déterminée par $\hbar \omega_{q}=\hbar v q \sim k_{B} T$. Lorsque la température augmente, des phonons de plus grands vecteurs d'ondes sont excités. On s'attend donc à une réduction de l'écrantage à haute température, et à un écrantage maximum à basse température. 


\section{Modèle hydrodynamique}

Chow et al. ont montré qu'en présence de fort désordre, pour lequel $q l_{e}<1$ (où $q$ est le vecteur d'onde des phonons et $l_{e}$ le libre parcours moyen élastique), le modèle d'écrantage statique présenté au paragraphe précèdent ne peut plus s'appliquer [317, 318]. Ils ont ainsi introduit un modèle hydrodynamique, conduisant à :

$$
F_{\text {hydro. }}^{\text {PZ }}(T) \propto \frac{e^{2}}{h \sigma_{x x}}\left(k_{B} T\right)^{4} .
$$

Ils montrent alors que, tous paramètres étant égaux, ce modèle conduit à une augmentation de la puissance dissipée quand le désordre augmente.

\begin{tabular}{|c|c|c|c|}
\hline exposant $\theta$ & écrantage faible & écrantage fort & modèle hydrodynamique \\
\hline couplage piézoélectrique & 3 & 5 & 4 \\
potentiel de déformation & 5 & 7 & - \\
\hline
\end{tabular}

TAB. A.1: Tableau récapitulatif des exposants $\theta$ intervenant dans la puissance dissipée par le couplage électron-phonon pour un gaz bidimensionnel d'électron : $F(T) \propto T^{\theta}$.

\section{A.2.3 Le couplage trou-phonon}

Le calcul effectué jusqu'à présent est valable pour des électrons dans bande de conduction. Dans le cas où les porteurs de charge sont des trous, le calcul du couplage trou-phonon est plus compliqué en raison de la dégénérescence de bandes. Cela conduit à deux populations de trous, avec des masses effectives différentes, dits trous lourds et trous légers. Ces deux populations interagissent de manière différente avec les phonons, conduisant également à des paramètres de couplage différents (potentiel de déformation et constante piézoélectrique).

Alors que dans certains cas particuliers la dégénérescence peut être levée (par exemple par le couplage spin-orbite, ou en champ magnétique), dans la plupart des cas il est nécessaire de prendre en compte ces deux populations. La méthode la plus simple revient à prendre en compte une seule population de trous, et donc une unique bande de valence effective, avec une masse effective moyenne, ainsi que des paramètres de couplage moyens [376]. Il est donc possible de reprendre tout le calcul fait pour les électrons pour les appliquer aux trous. Dans ce calcul toutes les transitions électroniques doivent être des transitions intra-bandes, ce qui revient à néglige donc ici la diffusion entre trous lourds et trous légers. Dans la suite, le terme "couplage électron-phonon" sera employé indifféremment lorsque les porteurs de charge sont des trous ou des électrons, sachant que le calcul est alors complètement identique, aux paramètres de couplage près.

\section{A.2.4 Résultats expérimentaux}

Ne sont reportés ici que les cas qui nous intéressent, soit le couplage trou-phonon dans les puits quantiques $\mathrm{SiGe} / \mathrm{Si}$ et les puits quantiques GaAs/AlGaAs. De nombreuses expériences dans d'autres systèmes peuvent être trouvés dans la littérature. 


\section{Cas du SiGe de type $p$}

Le premier résultat dans le $\mathrm{Si}_{0,8} \mathrm{Ge}_{0,2}$ de type $p$ obtenu à basse température a été publié par Xie et al. [305]. La mesure a été faite à une densité de porteurs de $5 \times 10^{15} \mathrm{~m}^{-2}$ (mobilité $0,33 \mathrm{~m}^{2} / \mathrm{Vs}$ ) et pour des températures effectives de porteurs comprises entre 1,75 et 4,5 K. L'analyse des résultats permet aux auteurs d'exclure l'effet de l'écrantage sur le terme de déformation (absence de variation en $T^{7}$ ), et l'ajustement de la courbe nécessite l'introduction d'un terme en $T^{3}$ caractéristique d'un couplage piézoélectrique. Les paramètres de couplage obtenus sont $\Xi_{u}=3 \mathrm{eV}$ et $e_{p z}=-1,6 \times 10^{-2} \mathrm{C} / \mathrm{m}^{2}$.

L'absence d'écrantage sur le potentiel de déformation, ainsi que la valeur $\Xi_{u}=3 \mathrm{eV}$ ont été confirmés dans le $p$ - $\mathrm{Si}_{0,8} \mathrm{Ge}_{0,2}[308,251,306]$. Par contre ces mesures, réalisée à plus basse température (jusqu'à $0,3 \mathrm{~K}$ ) n'ont pas confirmé un couplage piézoélectrique aussi fort que dans [305]. L'existence d'un couplage piezoélectrique dans le SiGe est un question encore ouverte. Dans le cas d'une distribution aléatoire des atomes de silicium et de germanium dans $\mathrm{SiGe}$, on ne s'attend pas à observer d'effet piézoélectrique. Cependant des mesures de spectroscopie $[309,310]$ semblent indiquer la présence d'un ordre des couches de SiGe contraintes. D'autre part une mesure de spectroscopie acoustique a montré la présence d'un effet analogue à un effet piézoélectrique dans $\mathrm{Si}_{0,8} \mathrm{Ge}_{0,2}[308,311$.

En reprenant le calcul de Braithwaite et al., il est possible d'estimer la constante piézoélectrique attendue dans le cas d'un matériau totalement ordonné [251] :

$$
e_{p z}=-4,4 \times 10^{-2} \times x \quad\left(\mathrm{en} \mathrm{C} / \mathrm{m}^{2}\right),
$$

où $x$ est la proportion de germanium dans $\mathrm{Si}_{1-x} \mathrm{Ge}_{x}$ (valable pour $x \leq 0,5$ ). Dans le cas Xie et al. [305], ce calcul donne donc $e_{p z}=-8,8 \times 10^{-3} \mathrm{C} / \mathrm{m}^{2}$, valeur nettement plus faible que celle obtenue obtenue par les auteurs.

\section{Cas du GaAs de type $p$}

Le couplage électron-phonon a été étudié par Kent et al. dans des hétérostructures $p$-GaAs $[377,319,376]$. Ils montrent que le couplage électron-phonon, dans ce système, est dominé par le couplage piézoélectrique écranté, de la même manière que dans les hétérostructures $n$-GaAs.

Gao et al. ont étudié le couplage électron-phonon dans des hétérostructures de faible densité, présentant les caractéristiques de la transition métal-isolant en dimension deux [320]. Ils trouvent un couplage électron-phonon dominé par un terme en $T^{5}$ à haute densité (phase "métallique"), ce qui est compatible avec un couplage piézoélectrique écranté. Par contre, à faible densité, l'exposant du chauffage diminue fortement.

\section{A.3 Détermination de la température effective des por- teurs}

Pour l'étude du coulage électron-phonon, il est nécessaire de mesurer la température effective des porteurs $T_{e}$. Cette mesure consiste en général à utiliser une grandeur $G$ qui dépend de la température effective des porteurs $T_{e}$. Pour les mesures de $T_{e}$ à l'aide de 
ce "thermomètre à électrons", il est nécessaire de s'assurer que les paramètres variables (dans la majorité des cas le champ électrique $E$ ) ne modifient pas la grandeur mesurée autrement que par la modification de la température effective :

$$
G\left(T_{e}, T_{0}, E, \ldots\right) \equiv G\left[T_{e}\left(T_{0}, E, \ldots\right)\right]
$$

Cette condition est l'hypothèse de chauffage, et doit être vérifier pour qu'un thermomètre soit valable. Dans la suite de cette section sont décrites les différentes méthodes pour mesurer $T_{e}$.

\section{A.3.1 Résistivité à champ magnétique nul}

Pour utiliser les dépendances en température de la résistivité pour mesurer la température des électrons, il est nécessaire de vérifier qu'elle ne dépend que de la température effective $T_{e}$. A haute température, la résistivité d'un système électronique est limitée par la diffusion des électrons par les phonons. A basse température la diffusion par les phonons devient négligeable, et la résistivité est limitée par la diffusion sur le désordre. Le désordre ne dépendant pas de la température, on s'attend à ce que, à basse température, la résistivité ne dépende que de l'état du système électronique et donc que de la température effective des porteurs $T_{e}$. Cette condition est vérifiée dans le cas où la dépendance en température est due aux corrections quantiques de localisation faible et des interactions électron-électron car le mécanisme de déphasage dominant à basse température est la diffusion électron-électron, et ainsi les deux mécanismes de diffusion évoqués ne dépendent que de la température effective des porteurs $T_{e}$ [281]. Cependant, les effets du champ électrique $E$ sur la localisation faible [378, 379] peuvent compromettre la validité de cette méthode.

Dans le cas où ce thermomètre est valable, la température effective est déduite en comparant la valeur de la résistivité en fonction de la température à faible champ électrique (figure A.1 de gauche) et celle en fonction du champ électrique à basse température (figure A.1 de droite). Cette méthode a été employée dans un grand nombre de systèmes, par exemple dans le cas des MOSFETs Si [369] en utilisant la dépendance logarithmique de la conductivité en fonction de la température à champ magnétique nul (localisation faible et interactions), et dans le cas d'hétérostructures p-SiGe [306].

\section{A.3.2 Oscillations de Shubnikov-de Haas}

Un autre thermomètre possible est lié à la dépendance en température des oscillations de Shubnikov-de Haas à faible champ magnétique. Cette méthode est valable dans le cas d'un gaz d'électrons fortement dégénéré [380]. A faible champ magnétique, la densité d'états électronique présente des oscillations autour d'une valeur moyenne an fonction de l'énergie. La période de ces oscillations varie en fonction du champ magnétique, ainsi que la position du niveau de Fermi par rapport à ces oscillations. Ces oscillations de densité d'état au niveau de Fermi conduisent à des variations de la résistivité, et l'amplitude des oscillations diminue lorsque la température augmente en raison de l'élargissement de la fonction de distribution de Fermi. 

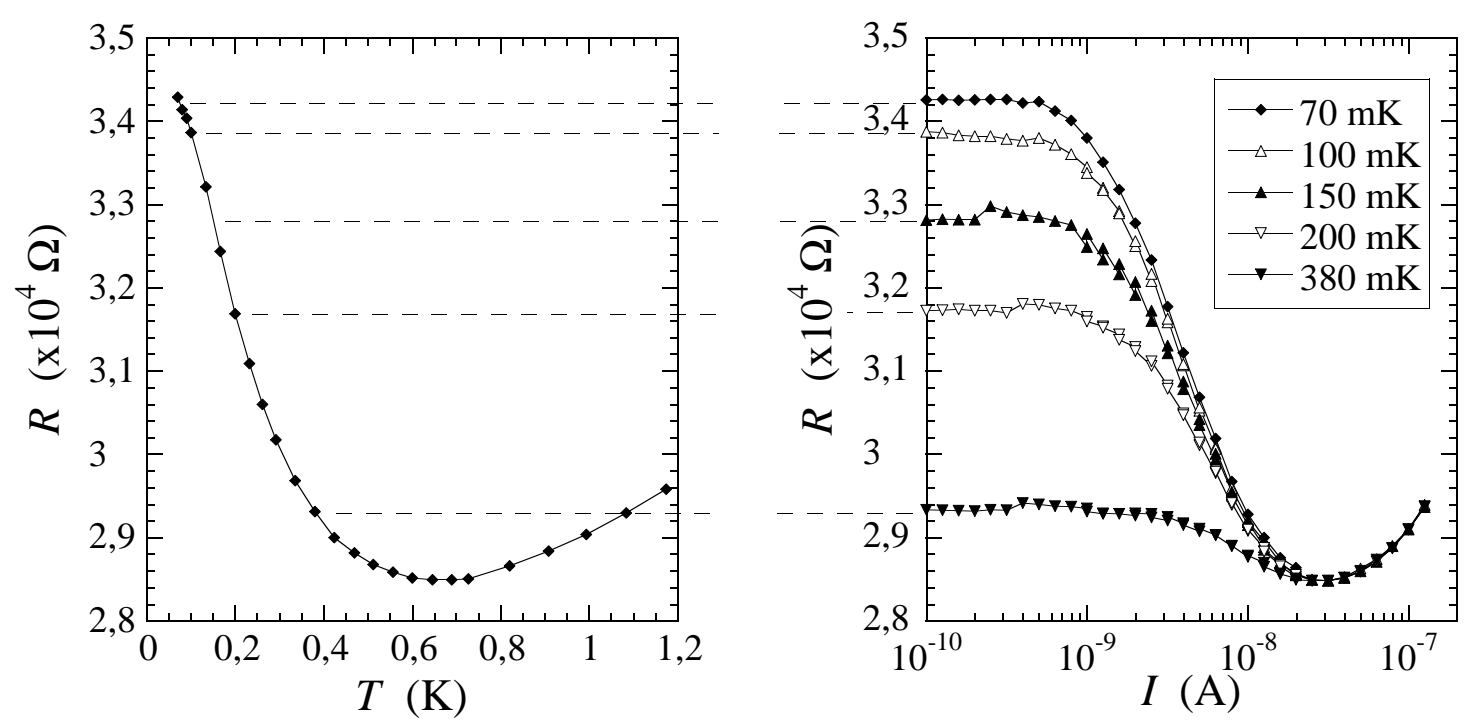

FIG. A.1: Mesure de résistance à champ magnétique nul sur un puits quantique $p$-SiGe (échantillon SiGe B509 pour une densité $p_{s}=1,31 \times 10^{15} \mathrm{~m}^{-2}$ ) en fonction de la température à faible courant $I=100 \mathrm{pA}$ (figure de gauche) et en fonction du courant à différentes températures (figure de droite). Les lignes horizontales donnent les correspondances entre les résistances mesurées à faible courant (figure de gauche) pour les températures indiquées sur la figure de droite.

La partie oscillante de la magnétorésistance $\rho_{x x}(B)$, notée $\Delta \rho_{x x}$, peut se mettre sous la forme [380] :

$$
\frac{\Delta \rho_{x x}}{\rho_{0}}=f\left(\omega_{c} \tau_{q}\right) \exp \left(-\frac{\pi}{\mu_{q} B}\right) \cos \left(\frac{2 \pi\left(E_{F}-E_{1}\right)}{\hbar \omega_{c}}-\pi\right) D(\chi)
$$

où $\rho_{0}$ est la résistivité à champ magnétique nul, $\omega_{c}=e B / m^{*}$ la pulsation cyclotron, $\tau_{q}$ le temps de vie quantique, $\mu_{q}=e \tau_{q} / m^{*}$ la mobilité quantique, $E_{F}$ l'énergie de Fermi et $E_{1}$ l'énergie de la première sous-bande. La dépendance en température de l'amplitude des oscillations est entièrement contenue dans la fonction enveloppe :

$$
D(\chi)=\frac{\chi}{\sinh \chi}, \quad \text { avec } \quad \chi=\frac{2 \pi^{2} k_{B} T}{\hbar \omega_{c}}
$$

Il est donc possible de déterminer la température effective des porteurs $T_{e}$ en comparant l'amplitude des oscillations $A\left(T_{e}\right)$ à champ magnétique donné avec celle à température $T_{0}$ connue $A\left(T_{0}\right)$. Mais cette méthode n'est valable que si le temps de vie quantique est indépendant de la température, ce qui peut être vérifié expérimentalement en vérifiant que la température de Dingle, $T_{D}=\hbar /\left(2 B \pi \tau_{q}\right)$, est indépendante de la température [380]. Cette méthode a été couramment employée pour des mesures de température effective à basse température, par exemple dans les MOSFET Si [381], dans les hétérostructures $n$-GaAs [380] et dans les hétérostructure $n$-SiGe [382]. 


\section{A.3.3 Localisation faible}

On a vu au paragraphe 1.3.1 que, pour un gaz fortement dégénéré, les interférences quantiques dues au désordre conduisent à des corrections à la conductivité de Drude dites corrections de localisation faible. A ces corrections s'ajoutent celles dues aux interactions électron-électron. Lorsqu'on ajoute un champ magnétique perpendiculaire au plan du gaz d'électrons, seules les corrections dues à la localisation faible sont affectées. Cette méthode permet de différencier les deux effets. La dépendance en température et en champ magnétique de ces deux corrections sont regroupées dans la formule d'Hikami [27] :

$$
\Delta \sigma_{x x}^{L F}(B, T)=\alpha \frac{e^{2}}{\pi h}\left[\psi\left(\frac{1}{2}+\frac{\tau_{B}}{2 \tau_{\phi}}\right)-\psi\left(\frac{1}{2}+\frac{\tau_{B}}{2 \tau_{e}}\right)+\ln \left(\frac{\tau_{\Phi}}{\tau_{e}}\right)\right],
$$

où $\Psi(x)$ est la fonction digamma, $\tau_{\phi}(T)$ est le temps de cohérence de phase, $\tau_{B}=$ $\hbar /(2 e D B)$ le temps magnétique avec $D$ le coefficient de diffusion, $\tau_{e}$ le temps de collision élastique et $\alpha$ est un paramètre phénoménologique décrivant des mécanismes de diffusion additionnels (voir l'équation (1.25)). L'ajustement des courbes de magnétorésistance permet de déduire le temps de cohérence de phase $\tau_{\phi}(T)$, qui, pour lorsque le mécanisme dominant de décohérence est l'interaction électron-électron, ne dépend que de la température effective des porteurs $T_{e}$. La température des porteurs a été mesurée par cette méthode dans des MOSFET Si [383] et des hétérostructures $n$-SiGe [382].

\section{A.3.4 Bruit thermique}

Dans un conducteur à l'équilibre, l'agitation thermique des charges est la source de fluctuations de courant : en l'absence d'une source extérieure de courant, le mouvement aléatoire des charges induit un courant non nul, car les charges allant dans un sens ne sont en général pas compensées par les charges allant dans l'autre. Pour un système d'impédance $Z(f)$, à l'équilibre avec un thermostat à la température $T$, le théorème de fluctuation-dissipation permet de relier les fluctuations du courant avec la partie réelle de l'impédance (i.e. sa partie dissipative).

A basse fréquence, lorsque $f \ll k_{B} T / h$, la puissance de bruit en courant $S_{I}(f)$ (ou en tension $\left.S_{V}(f)\right)$ est reliée à la partie réelle de l'impédance $Z(f)$ du système par le relation de Johnson-Nyquist [321] :

$$
S_{I}(f)=4 k_{B} T R e\left(Z(f)^{-1}\right) \quad \text { ou } \quad S_{V}(f)=4 k_{B} T R e(Z(f)),
$$

où $T$ est la température du système. On peut remarquer que même à une température de $1 \mathrm{~K}, k_{B} T / h=20 \mathrm{GHz}$, donc cette formulation du bruit thermique est valable dans une très large gamme de fréquences. Pour un système uniquement résistif, $\operatorname{Re}(Z(f))=R$ la résistance du système, ce bruit ne dépend pas de la fréquence. La mesure simultanée $\mathrm{du}$ bruit en tension et de la résistance d'un système permet donc de déduire de manière absolue la température du système.

Dans le cas d'électrons chauds, le système d'électrons n'est plus à l'équilibre avec le bain de phonons, ce qui conduit à la non validité du théorème de fluctuation-dissipation. En particulier, si on définit la température de bruit par :

$$
T_{N}=\frac{S_{V}(f)}{4 k_{B} \operatorname{Re}(Z(f))}
$$


le calcul conduit à des valeurs différentes du bruit suivant que les fluctuations de tension sont mesurées dans la direction parallèle au courant appliqué, $S_{V \|}$ donnant $T_{N \|}$, ou dans une direction perpendiculaire au courant, $S_{V \perp}$ donnant $T_{N \perp}$ [321] :

$$
\begin{aligned}
T_{N \|} & =T_{e}[1+X(f)], \\
T_{N \perp} & =T_{e},
\end{aligned}
$$

où $X(f)$ est un terme dépendant de la fréquence (voir [321]). La mesure de $T_{N \perp}$ donne donc directement la température effective des électrons. Cette mesure a été utilisée en particulier dans des fils 2D et quasi-1D [384]. 
Annexe A. Couplage électron-phonon dans un gaz bidimensionnel de trous 


\section{Annexe B}

\section{Diffusion de chaleur vers les contacts}

\section{Sommaire}

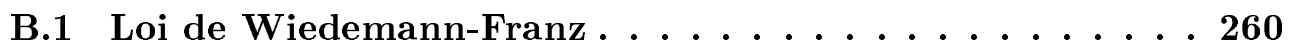

B.2 Modèle unidimensionnel . . . . . . . . . . . . 260

B.2.1 Équation de la chaleur en dimension un . . . . . . . 260

B.2.2 Résolution analytique . . . . . . . . . . . . . . . . . . 261

B.3 Modèle bidimensionnel . . . . . . . . . . . 261

B.3.1 Équation de la chaleur en dimension deux . . . . . . . . 261

B.3.2 Simulation numérique . . . . . . . . . . . . . . . 262 


\section{B.1 Loi de Wiedemann-Franz}

Dans un modèle d'électrons libres, la relation entre la conductivité thermique $\kappa$ et la conductivité (ou résistivité) électrique $\sigma$ (ou $\rho$ ) est donnée par la loi de Wiedemann-Franz [6] :

$$
\frac{\kappa}{\sigma T}=\frac{\kappa \rho}{T}=\frac{\pi^{2}}{3}\left(\frac{k_{B}}{e}\right)^{2}=\mathcal{L},
$$

où $T$ est la température locale des porteurs, et $\mathcal{L}=2,44 \times 10^{-8}$ W. $\Omega . \mathrm{K}^{-2}$ est le nombre de Lorentz.

Cette relation est bien vérifiée dans les métaux, et il a été montré qu'elle reste valable dans le cas d'un système d'électrons en interaction dans l'hypothèse du liquide de Fermi $[385,386]$. En effet, dans ce cas, la renormalisation due aux quasi-particules s'applique de la même manière sur le calcul de la conductivité électrique et de la conductivité thermique, conduisant à un rapport constant entre les deux grandeurs.

Par contre il peut exister des déviations dans des régimes où le liquide de Fermi n'est plus valable. Dans le cas du liquide de Luttinger il a ainsi été montré théoriquement [387] que la constante de Lorentz est modifiée à haute température, et que la loi de Wiedemann-Franz est violée à basse température car le désordre introduit alors des diffusions inélastiques, ce qui conduit à un nombre de Lorentz dépendant de la température. Dans le régime d'électrons localisés, une analyse par loi d'échelle montre que la constante de Lorentz peut varier avec la température, mais que cette dépendance reste beaucoup plus faible qu'une dépendance exponentielle [387].

\section{B.2 Modèle unidimensionnel}

\section{B.2.1 Équation de la chaleur en dimension un}

Dans une géométrie unidimensionnelle, l'équation de conservation de la chaleur s'écrit :

$$
\frac{d j_{Q}}{d x}=n_{s}\left(P_{E}(T)-P_{\mathrm{e}-\mathrm{ph}}(T)+P_{0}\right)=n_{s} P_{\text {totale }}(T)
$$

$j_{Q}$ étant le flux de chaleur, $n_{s}$ la densité de porteur (par unité de longueur pour un système purement 1D, ou par unité de surface pour un système 2D invariant par translation transverse), $P_{E}(T)=E^{2} /\left(\rho(T) n_{s}\right)$ la puissance injectée dans le système (par effet Joule) par unité de porteur, $P_{e-p h}(T)$ la puissance dissipée par l'interaction électron-phonon et $P_{0}$ la puissance parasite par unité de porteur, due à l'écrantage imparfait de l'expérience vis-à-vis des bruits parasites (radio-fréquence, etc...). La température du réseau $T_{0}$ est supposée uniforme sur toute la surface de l'échantillon.

$P_{0}$ est en général inconnue ou difficile à déterminer. Dans notre cas, on a démontré qu'elle restait faible aux températures étudiées $\left(T_{0}>30 \mathrm{mK}\right)$.

En utilisant (B.1), on obtient :

$$
j_{Q}=-\kappa \frac{d T}{d x}=-\frac{\mathcal{L}}{2 \rho} \frac{d\left(T^{2}\right)}{d x}
$$


et l'équation (B.2) s'écrit :

$$
\frac{d}{d x}\left[\frac{1}{\rho} \frac{d\left(T^{2}\right)}{d x}\right]=-\frac{2 n_{s} P_{\text {totale }}(T)}{\mathcal{L}} .
$$

\section{B.2.2 Résolution analytique}

Il est possible d'intégrer analytiquement cette équation en faisant deux approximations :

1. $P_{0}$ et $P_{e-p h}$ sont négligeables devant $P_{E}$,

2. La conductivité $\sigma$ ne dépend pas de $\mathrm{x}$ (i.e. ne dépend pas de la température $T(x)$ ), ce qui implique que $P_{E}$ ne dépend pas non plus de $T$.

L'intégration de cette équation avec les conditions aux limites aux bords $\mathrm{x}=0$ et $\mathrm{x}=\mathrm{L}$ de l'échantillon :

$$
T(x=0)=T_{0} \quad \text { et } \quad T(x=L)=T_{0},
$$

où $T_{0}$ est la température du réseau, donne le profile de température :

$$
T(x)=\left[\frac{n_{s} P_{E} \rho}{\mathcal{L}}\left(-x^{2}+L x\right)+T_{0}^{2}\right]^{\frac{1}{2}} .
$$

En définissant $T_{e}$ comme la température effective atteinte au milieu de la barre de Hall

$$
T_{e}^{2}=\max \left[T(x)^{2}\right]=T(L / 2)^{2}=\frac{n_{s} P_{E} \rho}{4 \mathcal{L}} L^{2}+T_{0}^{2},
$$

on retrouve le résultat donné par Zieve et al. [313] :

$$
P_{E}=\frac{4 \mathcal{L}}{L^{2} n_{s} \rho}\left(T_{e}^{2}-T_{0}^{2}\right)
$$

La diffusion de chaleur vers les contacts donne donc une terme en $T^{2}$, qui s'ajoute aux autres sources de dissipations (comme le couplage électron-phonon). La prise en compte du couplage électron-phonon (terme $P_{e-p h}$ qui dépend de $T$ et donc de $x$ ) et de la dépendance en $T$ (et donc en $x$ ) de la conductivité passe par une résolution numérique de l'équation de la chaleur.

\section{B.3 Modèle bidimensionnel}

\section{B.3.1 Équation de la chaleur en dimension deux}

En dimension 2, l'équation de la chaleur s'écrit :

$$
\vec{\nabla} \cdot \vec{j}_{Q}=n_{s}\left(P_{E}(T)-P_{\mathrm{e}-\mathrm{ph}}(T)+P_{0}\right)=n_{s} P_{\text {totale }}(T) .
$$

En utilisant l'équation (B.1) :

$$
\vec{j}_{Q}=-\kappa \vec{\nabla} T=-\frac{\mathcal{L}}{2 \rho} \vec{\nabla}\left(T^{2}\right)
$$


l'équation de la chaleur devient :

$$
-\frac{\mathcal{L}}{2} \nabla \cdot\left[\frac{1}{\rho} \nabla\left(T^{2}\right)\right]=n_{s}\left(P_{E}(T)-P_{\mathrm{e}-\mathrm{ph}}(T)+P_{0}\right)=n_{s} P_{\text {totale }}(T) .
$$

En utilisant les égalités $\partial \rho / \partial x=d \rho / d T \times \partial \rho / \partial x$ et $\partial \rho / \partial y=d \rho / d T \times \partial \rho / \partial y$, on peut écrire :

$$
\vec{\nabla} \cdot\left[\frac{1}{\rho} \vec{\nabla}\left(T^{2}\right)\right]=\frac{1}{\rho}\left\{\frac{\partial^{2}\left(T^{2}\right)}{\partial x^{2}}+\frac{\partial^{2}\left(T^{2}\right)}{\partial y^{2}}-\frac{2 T}{\rho} \frac{d \rho}{d T}\left[\left(\frac{\partial T}{\partial x}\right)^{2}+\left(\frac{\partial T}{\partial y}\right)^{2}\right]\right\} .
$$

Dans le cas où la résistivité dépend fortement de la température, il n'est pas possible de négliger $d \rho / d T$, et on doit donc conserver tous les termes de cette expression. Dans la suite on négligera cependant les termes en $d \rho / d T$, ce qui conduit à :

$$
\vec{\nabla} \cdot\left[\frac{1}{\rho} \vec{\nabla}\left(T^{2}\right)\right]=\frac{1}{\rho}\left[\frac{\partial^{2}\left(T^{2}\right)}{\partial x^{2}}+\frac{\partial^{2}\left(T^{2}\right)}{\partial y^{2}}\right] .
$$

\section{B.3.2 Simulation numérique}

Il est possible de résoudre numériquement l'équation (B.11) dans une géométrie donnée. Nous avons réalisé un programme permettant de simuler le profil de température résultant du refroidissement par les contacts dans les géométries de barre de Hall utilisées dans les expériences.

Pour les simulations, on utilise le calcul analytique approché de la puissance dissipée par le couplage électron-phonon dont les résultats sont donnés au paragraphe A.2. De manière générale on peut écrire :

$$
P_{\mathrm{e}-\mathrm{ph}}(T)=A_{1}\left(T^{\theta_{1}}-T_{0}^{\theta_{1}}\right)+A_{2}\left(T^{\theta_{2}}-T_{0}^{\theta_{2}}\right) .
$$

Pour le calcul de la puissance injectée, il est nécessaire de connaître le profil de champ électrique. Pour simplifier le calcul lors des simulations, on peut faire une des hypothèses suivantes :

1. On considère que la densité de courant est uniforme sur la barre de Hall, et est donnée par $j=I / W$, avec $I$ le courant injecté et $W$ la largeur de l'échantillon.

2. On suppose que le champ électrique est uniforme, et donné par $E=U / L$, avec $U$ la différence de potentiel aux bornes de la barre de Hall et $L$ la distance entre les contacts de mesure de tension.

La première (resp. seconde) hypothèse est valable lorsque le gradient de température dans la direction transverse (resp. longitudinale) n'est pas trop fort. Dans les deux cas cela nécessite une forte diffusion de la chaleur vers les contacts. La première hypothèse s'applique alors au cas d'une longue barre de Hall $(L>W)$, alors que la seconde est plus adaptée au cas d'une barre de Hall courte $(L<W)$.

Enfin on utilise deux conditions aux limites:

1. La température des porteurs aux contacts de mesure de tension est égale à la température du réseau $T_{0}$.

2. Le flux de chaleur s'annule sur les bords de la barre de Hall autres que les contacts (le gradient de température est alors nul sur ces bords). 


\section{Conclusion générale}

\section{Résultats}

Nous avons donc réalisé des mesures de transport, et des mesures de fluctuations de résistance à basse fréquence, sur des gaz bidimensionnels de trous à très faible densité de porteurs. Nous avons pour cela étudié deux types d'échantillons, des puits quantiques $\mathrm{SiGe} / \mathrm{Si}(p$-SiGe), et des puits quantiques GaAs/AlGaAs ( $p$-GaAs). Ces deux systèmes ont des mobilités suffisantes pour pouvoir observer les caractéristiques d'une transition métal-isolant, et la densité de porteurs est suffisamment faible pour pouvoir envisager des effets importants dus aux interactions entre électrons.

Les mesures de transport en fonction de la température ont permis de montrer que ces deux systèmes ont des propriétés différentes. Le transport dans les puits quantiques $p$-SiGe s'explique par des théories d'électrons indépendants, qui ne fait donc pas intervenir la nature corrélée du système électronique. Par contre, le transport électronique dans les puits quantiques $p$-GaAs ne semble pas pouvoir s'expliquer par les théories existantes d'électrons indépendants, ce qui laisse la place à une interprétation en termes d'électrons fortement corrélés. La grande différence entre ces deux systèmes, outre la nature différente des matériaux, est le désordre. Les puits quantiques $p$-SiGe ont une mobilité maximale environ cent fois plus faible que les puits quantiques $p$-GaAs, et la transition métal-isolant dans le $p$-SiGe se passe à une densité plus élevée que dans le $p$-GaAs. Le fait que des comportements collectifs ne soient pas observés dans le $p$-SiGe, alors qu'ils jouent un rôle dans le $p$-GaAs serait donc dû au désordre différent dans ces deux systèmes.

Dans les puits quantiques $p$-SiGe, le transport en champ électrique peut également être compris grâce à des théories d'électrons indépendants. Nous avons en effet montré que ces dépendances sont dues au chauffage des porteurs par effet Joule, en raison du faible couplage électron-phonon à basse température qui ne permet pas au gaz d'électron de se thermaliser suffisamment rapidement. D'autre part, l'étude du couplage électronphonon dans ce système montre qu'il n'y a pas d'écart avec la théorie développée pour des électrons indépendants. Cependant, le rôle des interactions dans ce système ne peut pas être complètement négligé : la validité des théories dans la phase isolante montre que le mécanisme de transport doit faire intervenir les interactions entre électrons. Enfin ces mesures sont également intéressantes du point de vue technologique puisqu'elle ont permis de confirmer l'existence d'un couplage piézoélectrique entre les électrons et les phonons, inattendu à priori dans le SiGe massif, mais qui peut être dû à l'absence de centre d'inversion dans le SiGe contraint. Enfin, l'étude des effets de chauffage dans ces systèmes est essentiel dans l'étude des non-linéarités dans le transport. Nous avons montré qu'en connaissant le couplage électrons-phonon dans un tel système, il est possible de 
distinguer les effets de chauffage des autres effets de champ électrique, plus intéressants pour étudier les propriétés de transport. Dans le $p$-SiGe, les mesures de bruit de résistance à haute densité électronique semblent également en accord avec des mécanismes classiques de fluctuations de résistance.

Dans les puits quantiques $p$-GaAs, les mesures de transport et les mesures de bruit révèlent un effet nouveau. Tout d'abord les mesures de transport ne semblent pas pouvoir s'interpréter par les théories d'électrons indépendants. Aux plus basses densités de porteurs, le transport en champ électrique montre une tension seuil, et la dépendance en température de la résistivité présente une loi simplement activée à très basse température, non prévue par les théories d'électrons indépendants localisés. Ces caractéristiques sont typiques du transport dans une phase collective accrochée sur le désordre, mais peuvent également être interprétées dans le cadre d'un système percolant au-delà du seuil de percolation. D'autre part, nous présentons le résultat tout-à-fait nouveau dans les gaz bidimensionnels d'électrons que le bruit de résistance basse fréquence présente une loi d'échelle en fonction de la résistance $S_{R} / R^{2} \sim R^{w}$, avec un exposant critique $w=2,40 \pm 0,06$, cette loi d'échelle étant caractéristique d'une transition de phase du second ordre, et en particulier d'un transition de percolation. Des lois d'échelle sont également observées sur la conductance. Cependant, une théorie de percolation simple ne permet pas directement de comprendre les lois d'échelle sur la conductance, et une théorie basée sur l'existence de deux phases (solide et liquide) est mieux adaptée. Ainsi il est possible d'évoquer un scénario de formation d'une phase fortement corrélée par un mécanisme de percolation, comme certains auteurs l'on suggéré dans le cadre de la transition métal-isolant en dimension deux.

\section{Perspectives}

Pour l'étude des non-linéarités de la caractéristique $I-V$, on a vu que les effets de chauffage des porteurs peuvent masquer d'autres effets du champ électrique, comme des lois d'échelle aux abords d'une transition de phase quantique ou le décrochage d'une phase collective. Pour diminuer les effets de chauffage, on a vu qu'il pourrait être intéressant d'utiliser une géométrie particulière de barre de Hall, avec un nombre important de contacts froids, de façon à bénéficier de la diffusion de chaleur vers les contacts pour mieux thermaliser le gaz d'électrons avec le réseau.

Il semble nécessaire de réaliser des mesures complémentaires de transport dans les échantillons $p$-GaAs dans la phase très basse densité, pour laquelle les théories de localisation pour des électrons indépendants ne peut pas s'appliquer. Une telle mesure précise permettrait de comparer quantitativement les mesures expérimentales à des modèles afin de déterminer la nature de la phase isolante.

Pour les mesures de bruit de résistance dans les puits quantiques $p$-GaAs, il serait intéressant d'étudier la nature du bruit, indépendamment de la transition de percolation, dans la phase haute densité et dans la phase très basse densité. En particulier, l'application d'un faible champ magnétique perpendiculaire permettrait de tester la nature quantique du bruit, notamment dans la phase métallique où les fluctuations universelles de conductance peuvent jouer un rôle. D'autre part une mesure de la statistique des fluctuations, par la mesure du second bruit ou par l'analyse des traces temporelles des fluctuations, 
peut permettre d'évaluer le rôle des corrélations dans ces deux phases.

Dans la phase fortement isolante, on s'attend à deux types de bruits, les fluctuations de résistance et le bruit de transport. Ce dernier peut caractériser le décrochage d'une phase collective. Dans cette phase on peut également envisager de mesurer les fluctuations de tension transverses au mouvement des électrons. La méthode expérimentale pour ces mesures a déjà été testée dans notre laboratoire.

Enfin, il peut être intéressant de mesurer le bruit de résistance en fonction d'un champ magnétique parallèle ou perpendiculaire. Il a été montré que le champ magnétique peut induire une transition entre un comportement métallique et un comportement isolant, au même titre que la variation de la densité électronique. On peut alors se demander si cette transition en champ magnétique est de même nature que la transition à champ magnétique nul. D'autre part, si ces transitions sont également des transitions de percolation, comme il a été suggéré dans le cas des transitions autour de l'effet Hall quantique [36], il serait intéressant de comparer les exposants critiques du bruit pour tester l'universalité de ces exposants.

Nous avons vu que le désordre semble modifier largement les effets des interactions dans ces systèmes bidimensionnels de porteurs. D'autre part, les systèmes étudiés expérimentalement dans le cadre de la transition métal-isolant présentent tous un caractère identique : dans les échantillons désordonnés, le faible comportement "métallique" peut être expliqué par des théories d'électrons indépendants, alors que dans le cas d'échantillons moins désordonnés, les théories d'électrons indépendants ne semblent plus pouvoir expliquer les mesures. On est alors mené naturellement à tester plus en détail l'influence du désordre sur les fluctuations de résistance. Par exemple, en utilisant des échantillons $p$-GaAs plus désordonnés que ceux que nous avons étudiés, on peut regarder à partir de quel moment la transition observée sur la mesure de bruit (et la loi d'échelle) est détruite, ou si elle persiste à fort désordre. De manière plus générale, la question qui reste entière est le rôle des interactions sur cette transition. Les progrès réalisés dans les techniques de fabrication peuvent nous permettre d'espérer obtenir dans un avenir proche des systèmes encore plus "propres" que ceux que nous avons étudiés (par exemple dans des systèmes tels que les MOSFETs organiques dans lesquels la masse effective des électrons est très élevées). Dans cette limite, et pour des densités encore plus faibles, on espère pouvoir observer la cristallisation de Wigner. Nous pourrons alors peut-être relier la transition de Wigner attendue à désordre très faible et la transition que nous observons actuellement dans nos systèmes. 


\section{Liste des publications}

R. Leturcq, D. L'Hote, R. Tourbot, V. Senz, U. Gennser, T. Ihn, K. Ensslin, G. Dehlinger and D. Grützmacher, "Heating of two-dimensional holes in SiGe and the $B=0$ metal-insulator transition", cond-mat/0107457 (non publié).

R. Leturcq, D. L'Hote, R. Tourbot, V. Senz, U. Gennser, T. Ihn, K. Ensslin, G. Dehlinger and D. Grützmacher, "Hot hole effects in a dilute two-dimensional gas in SiGe" (accepté à Europhysics Letters).

R. Leturcq, D. L'Hote, R. Tourbot, C. J. Mellor and M. Henini, "Scaling behavior of the resistance noise in a low density 2D hole gas in GaAs/AlGaAs quantum well", (accepté à Physical Review Letters).

R. Leturcq, V. Senz, D. L'Hote, U. Gennser, T. Heinzel, T. Ihn, G. Dehlinger and D. Grützmacher, "Experimental investigation of heating effects in $p$-SiGe 2DHG at milliKelvin temperatures", Proceedings of the $18^{\text {th }}$ General Conference of the Condensed Matter Division of the European Physical Society, Montreux, Suisse, 13 - 17 mars 2000.

R. Leturcq, V. Senz, D. L'Hote, U. Gennser, T. Heinzel, T. Ihn, G. Dehlinger and D. Grützmacher, "Etude expérimentale des effets de chauffage dans un gaz bidimensionnel de trous dans $\mathrm{SiGe} ", 7^{\text {èmes }}$ Journées de la Matière Condensée de la S.F.P., Poitiers, France, 29 août - 1er septembre 2000 (présentation orale).

R. Leturcq, D. L'Hote, R. Tourbot, "Etude expérimentale de la transition métal-isolant dans des gaz bidimensionnels de trous", $1^{\text {ère }}$ réunion du G.D.R. 2426 : Physique quantique mésoscopique, Orsay, France, juin 2001.

R. Leturcq, D. L'Hote, R. Tourbot, V. Senz, U. Gennser, T. Ihn, K. Ensslin, G. Dehlinger and D. Grützmacher, "Hole-phonon coupling in Si/SiGe two-dimensional hole systems", Proceedings of the $14^{\text {th }}$ International Conference on the Electronic Properties of Two-Dimensional Systems, Prague, République Tchèque, 30 juillet - 3 août 2001.

R. Leturcq, D. L'Hote, R. Tourbot, C. J. Mellor and M. Henini, "Resistance fluctuations of a two-dimensional hole system around the metal-insulator transition", Proceedings of the $19^{\text {th }}$ General Conference of the Condensed Matter Division of the European Physical Society, Brighton, Royaume Uni, 7 - 11 avril 2002.

R. Leturcq, D. L'Hote, R. Tourbot, C. J. Mellor and M. Henini, "Fluctuations de résistance dans un gaz bidimensionnel de trous autour de la transition métal-isolant en dimension deux", $8^{\text {èmes }}$ Journées de la Matière Condensée de la S.F.P., Marseille, France, 
$27-30$ août 2000 .

R. Leturcq, D. L'Hote, R. Tourbot, C. J. Mellor and M. Henini, "Resistance Fluctuations in a Low Density 2D Hole Gas in GaAs", International Worhshop on Electronic Crystals, Saint-Flour, France, 2 - 7 septembre 2002.

R. Leturcq, D. L'Hote, G. Deville, R. Tourbot, C. J. Mellor and M. Henini, "Fluctuations de résistance d'un gaz bidimensionnel électronique autour de la transition métalisolant", $2^{\text {èmes }}$ réunion du G.D.R. 2284 : Systèmes élestiques - du désordre à la plasticité, Carcassone, France, 23 - 26 septembre 2002. 


\section{Bibliographie}

[1] P. W. Anderson, 'Absence of Diffusion in Certain Random Lattices', Phys. Rev. 109, 1492 (1958).

[2] E. Wigner, 'On the Interaction of Electrons in Metals', Phys. Rev. 46, 1002 (1934).

[3] G. Benenti, X. Waintal et J.-L. Pichard, 'New Quantum Phase between the Fermi Glass and the Wigner Crystal in Two Dimensions', Phys. Rev. Lett. 83, 1826 (1999).

[4] E. Abrahams, P. W. Anderson, D. C. Licciardello et T. V. Ramakrishnan, 'Scaling Theory of Localization : Absence of Quantum Diffusion in Two Dimensions', Phys. Rev. Lett. 42, 673 (1979).

[5] S. V. Kravchenko, G. V. Kravchenko, J. E. Furneaux, V. M. Pudalov et M. D'Iorio, 'Possible Metal-Insulator Transition at $B=0$ in Two Dimensions', Phys. Rev. B 50, 8039 (1994).

[6] Ashroft et Mermin, Introduction to the Solid State Physics (Holt, Rinehart and Winston, New York, 1976).

[7] J. V. Rodriguez, 'Bruit dans les systèmes mésoscopiques', Doctorat, Université Paris VI (Novembre 2001).

[8] H. Bouchiat, 'Experimental signatures of phase coherent transport', dans : Mesoscopic Quantum Physics, Les Houches, Session LXI (Springer Verlag, 1994).

[9] D. J. Thouless, 'Electrons in Disordered Systems and the Theory of Localization', Phys. Rep. 13, 93 (1974).

[10] Y. C. Lee, C. S. Chu et E. Castaño, 'Effects of Finite Electric Field and Inelastic Scattering on Anderson Localization in Two Dimensions', Phys. Rev. B 27, 6136 (1983).

[11] B. I. Shklovskii et A. L. Efros, Electronic Properties of Doped Semiconductors (Springer-Verlag, 1984).

[12] A. I. Larkin, JETP Lett. 58,.

[13] A. I. Larkin et Y. V. Ovchinnikov, J. Low Temp. Phys. 34, 409 (1979).

[14] S. T. Chui et B. Tanatar, 'Impurity Effect on the Two-Dimensional-Electron FluidSolid Transition in Zero Field', Phys. Rev. Lett. 74, 458 (1995).

[15] J. M. Kosterlitz et D. J. Thouless, 'Ordering, metastability and phase transitions in two-dimensional systems', J. Phys. C : Solid State Phys. 6, 1181 (1973).

[16] D. R. Nelson et B. I. Halperin, 'Dislocation-mediated melting in two dimensions', Phys. Rev. B 19, 2457 (1979). 
[17] A. P. Young, Phys. Rev. B 19, 1855 (1979).

[18] T. Ando, A. B. Fowler et F. Stern, 'Electronic Properties of Two-Dimensional Systems', Rev. Mod. Phys. 54, 437 (1982).

[19] A. Gold et V. T. Dolgopolov, 'Temperature Dependence of the Conductivity for the Two-Dimensional Electron Gas : Analytical Results for Low Temperatures', Phys. Rev. B 33, 1076 (1986).

[20] A. Gold, 'Electronic transport properties of a two-dimensional electron gas in a silicon quantum-well structure at low temperature', Phys. Rev. B 35, 723 (1987).

[21] P. Coleridge, A. Sachrajda et P. Zawadzki, 'Weak localization, interaction effects, and the metallic phase in p-SiGe', Phys. Rev. B 65, 125328 (2002).

[22] M. J. Uren, R. A. Davies, M. Kaveh et M. Pepper, 'Magnetic Delocalisation of a TwoDimensional Electron Gas and the Quantum Law of Electron-Electron Scattering', J. Phys. C : Solid State Phys. 14, L395 (1981).

[23] B. L. Altshuler, A. G. Aronov et D. E. Khmelnitsky, 'Effects of electron-electron collisions with small energy transfers on quantum localisation', J. Phys. C : Solid State Phys. 15, 7367 (1982).

[24] B. L. Altshuler, A. G. Aronov et P. A. Lee, 'Interaction Effects in Disordered Fermi Systems in Two Dimensions', Phys. Rev. Lett. 44, 1288 (1980).

[25] B. L. Altshuler, A. G. Aronov et D. Khmel'nitzkii, 'Suppression of Localization Effects by the High Frequency Field and the Nyquist Noise', Solid State Commun. 39, 619 (1981).

[26] V. Senz, T. Heinzel, T. Ihn, K. Ensslin, G. Dehlinger, D. Grützmacher et U. Gennser, 'Coexistence of Weak Localization and a Metallic Phase in Si/SiGe Quantum Wells', Phys. Rev. B 61, R5082 (2000).

[27] S. Hikami, A. I. Larkin et Y. Nagaoka, 'Spin-orbit interaction and magnetoresistance in the two dimensional random system', Prog. Theor. Phys. 63, 707 (1980).

[28] G. J. Dolan et D. D. Osheroff, 'Nonmetallic Conduction in Thin Metal Films at Low Temperatures', Phys. Rev. Lett. 43, 721 (1979).

[29] D. J. Bishop, D. C. Tsui et R. C. Dynes, 'Nonmetallic Conduction in Electron Inversion Layers at Low Temperatures', Phys. Rev. Lett. 44, 1153 (1980).

[30] M. Uren, R. A. Davies et M. Pepper, J. Phys. C : Solid State Phys. 13, L985 (1980).

[31] R. A. Davies, M. Uren et M. Pepper, J. Phys. C : Solid State Phys. 14, L531 (1981).

[32] M. J. Uren, R. A. Davies, M. Kaveh et M. Pepper, 'Logarithmic Corrections to Two-Dimensional Transport in Silicon Inversion Layers', J. Phys. C : Solid State Phys. 14, 5735 (1981).

[33] F. W. V. Keuls, H. Mathur, H. W. Jiang et A. J. Dahm, 'Localization scaling relation in two dimensions : Comparison with experiment', Phys. Rev. B 56, 13263 (1997).

[34] V. T. Dolgopolov, G. V. Kravchenko, , A. A. Shashkin et S. V. Kravchenko, 'MetalInsulator Transition in Si Inversion Layers in the Extreme Quantum Limit', Phys. Rev. B 46, 13303 (1992). 
[35] A. A. Shashkin, V. T. Dolgopolov et G. V. Kravchenko, 'Insulating Phases in a Two-Dimensional Electron System of High-Mobility Si MOSFET's', Phys. Rev. B 49, 14486 (1994).

[36] A. A. Shashkin, V. T. Dolgopolov, G. V. Kravchenko, M. Wendel, R. Schuster, J. P. Kotthaus, R. J. Haug, K. V. Klitzing, K. Ploog, H. Nickel et W. Schlapp, 'Percolation Metal-Insulator Transitions in the Two-Dimensional Electron System of AlGaAs/GaAs Heterostructures', Phys. Rev. Lett. 73, 3141 (1994).

[37] M. Y. Azbel', 'Quantum particle in a random potential : Exact solution and its implications', Phys. Rev. B 45, 4208 (1992).

[38] F. Perruchot, 'Solide d'électron quantique en champ magnétique : réponse au champ électrique en présence de désordre', Doctorat, Ecole Polytechnique (Mars 1995).

[39] C. C. Grimes et G. Adams, 'Evidence for a Liquid-to-Crystal Phase Transition in a Classical, Two-Dimensional Sheet of Electrons', Phys. Rev. Lett. 42, 795 (1979).

[40] G. Deville, 'Dynamic Measurement of the Surface Tension of Liquid Helium with a Two-Dimensional Electron Probe', J. Low Temp. Phys. 72, 135 (1988).

[41] G. Deville, A. Valdes, E. Y. Andrei et F. I. B. Williams, 'Propagation of Shear in a Two-Dimensional Electron Solid', Phys. Rev. Lett. 53, 588 (1984).

[42] D. C. Glattli, E. Y. Andrei et F. I. B. Williams, 'Thermodynamic measurement on the melting of a two-dimensional electron solid', Phys. Rev. Lett. 60, 420 (1988).

[43] D. C. Glattli, G. Deville, V. Duburcq, F. I. B. Williams, E. Paris, B. Etienne et E. Y. Andrei, 'Experiments on Ordering in Classical and Quantum 2D Electron Systems', Surf. Sci. 229, 344 (1990).

[44] B. Tanatar et D. M. Ceperley, 'Ground State of the Two-Dimensional Electron Gas', Phys. Rev. B 39, 5005 (1989).

[45] V. M. Pudalov, M. D’Iorio, S. V. Kravchenko et J. W. Campbell, 'Zero-MagneticField Collective Insulator Phase in a Dilute 2D Electron System', Phys. Rev. Lett. 70, 1866 (1993).

[46] S. V. Kravchenko, V. M. Pudalov, J. Campbell et M. D'Iorio, 'Electron Solid at the Si Surface in Zero Magnetic Field?', JETP Lett. 54, 532 (1992).

[47] Y. E. Lozovik et V. I. Yudson, 'Crystallization of a two-dimensional electron gas in a magnetic field', JETP Lett. 22, 11 (1975).

[48] F. I. B. Williams, P. A. Wright, R. G. Clark, E. Y. Andrei, G. Deville, D. C. Glattli, O. Probst, B. Etienne, C. Dorin, C. T. Foxon et J. J. Harris, 'Conduction threshold and pinning frequency of magnetically induced Wigner solid', Phys. Rev. Lett. 66, 3285 (1991).

[49] R. L. Willett, H. L. Stormer, D. C. Tsui, L. N. Pfeiffer, K. W. West et K. W. Baldwin, 'Termination of the series of fractional quantum hall states at small filling factors', Phys. Rev. B 38, 7881 (1988).

[50] R. L. Willett, H. L. Stormer, D. C. Tsui, L. N. Pfeiffer, K. W. West, M. Shayegan, M. Santos et T. Sajoto, 'Current-voltage discontinuities in high-quality twodimensional electron systems at low Landau-level filling factors', Phys. Rev. B 40, 6432 (1989). 
[51] V. J. Goldman, M. Santos, M. Shayegan et J. E. Cunningham, 'Evidence for twodimentional quantum Wigner crystal', Phys. Rev. Lett. 65, 2189 (1990).

[52] Y. P. Li, T. Sajoto, L. W. Engel, D. C. Tsui et M. Shayegan, 'Low-frequency noise in the reentrant insulating phase around the $1 / 5$ fractional quantum Hall liquid', Phys. Rev. Lett. 67, 1630 (1991).

[53] I. V. Kukushkin, N. J. Pulsford, K. V. Klitzing, K. Ploog, R. J. Haug, S. Koch et V. B. Timofeev, 'Magneto-Optical Evidence for Depinning of the Wigner Crystal by an Electric Field', Phys. Rev. B 45, 4532 (1992).

[54] E. Y. Andrei, G. Deville, D. C. Glattli, F. I. B. Williams, E. Paris et B. Etienne, 'Observation of a Magnetically Induced Wigner Solid', Phys. Rev. Lett. 60, 2765 (1988).

[55] H. Buhmann, W. Joss, K. V. Klitzing, I. V. Kukushkin, G. Martinez, A. S. Plaut, K. Ploog et V. B. Timofeev, JETP Lett. 52, 306 (1990).

[56] M. A. Paalanen, R. L. Willett, P. B. Littlewood, R. R. Ruel, K. W. West, L. N. Pfeiffer et D. J. Bishop, 'rf conductivity of a two-dimensional electron system at small Landau-level filling factors', Phys. Rev. B 45, 11342 (1992).

[57] D. Belitz et T. R. Kirkpatrick, 'The Anderson-Mott transition', Rev. Mod. Phys. 66, 261 (1994).

[58] A. M. Goldman et N. Marković, 'Superconductor-insulator transitions in the twodimensional limit', Physics Today 51, 39 (1998).

[59] S. L. Sondhi, S. M. Girvin, J. P. Carini et D. Shahar, 'Continuous Quantum Phase Transitions', Rev. Mod. Phys. 69, 315 (1997).

[60] D. Popović, A. B. Fowler et S. Washburn, 'Metal-Insulator Transition in Two Dimensions : Effects of Disorder and Magnetic Field', Phys. Rev. Lett. 79, 1543 (1997).

[61] Y. Hanein, U. Meirav, D. Shahar, C. C. Li, D. C. Tsui et H. Shtrikman, 'The Metalliclike Conductivity of a Two-Dimensional Hole System', Phys. Rev. Lett. 80, 1288 (1998).

[62] M. Y. Simmons, A. R. Hamilton, M. Pepper, E. H. Linfield, P. D. Rose et D. A. Ritchie, 'Metal-Insulator Transition at $B=0$ in a Dilute Two Dimensional GaAsAlGaAs Hole Gas', Phys. Rev. Lett. 80, 1292 (1998).

[63] J. Lam, M. D'Iorio, D. Brown et H. Lafontaine, 'Scaling and the metal-insulator transition in Si/SiGe quantum wells', Phys. Rev. B 56, R12741 (1997).

[64] P. T. Coleridge, R. L. Williams, Y. Feng et P. Zawadzki, 'Metal-Insulator Transition at $B=0$ in $p$-Type SiGe', Phys. Rev. B 56, R12764 (1997).

[65] S. J. Papadakis et M. Shayegan, 'Apparent metallic behavior at $B=0$ of a twodimensional electron system in AlAs', Phys. Rev. B 57, R15068 (1998).

[66] Y. Hanein, D. Shahar, J. Yoon, C. C. Li, D. C. Tsui et H. Shtrikman, 'Observation of the metal-insulator transition in two-dimensional $n$-type GaAs', Phys. Rev. B 58, R13338 (1998).

[67] S. V. Kravchenko, W. E. Mason, G. E. Bowker, J. E. Furneaux, V. M. Pudalov et M. D'Iorio, 'Scaling of an Anomalous Metal-Insulator Transition in a TwoDimensional System in Silicon at $B=0$ ', Phys. Rev. B 51, 7038 (1995). 
[68] B. L. Altshuler, D. L. Maslov et V. M. Pudalov, 'Metal-Insulator Transition in 2D : Resistance in the Critical Region', Physica E 9, 209 (2001).

[69] S. V. Kravchenko et T. M. Klapwijk, 'Metallic Low-Temperature Resistivity in 2D over an Extended Temperature Range', Phys. Rev. Lett. 84, 2909 (2000).

[70] X. P. A. Gao, A. P. Mills, A. P. Ramirez, L. N. Pfeiffer et K. W. West, 'Weak-Localization-Like Temperature-Dependent Conductivity of a Dilute TwoDimensional Hole Gas in a Parallel Magnetic Field', Phys. Rev. Lett. 89, 016801 (2002).

[71] V. M. Pudalov, 'Unconventional Metallic State in a Two-Dimensional System with Broken Inversion Symmetry', JETP Lett. 66, 175 (1997).

[72] A. P. Mills, A. P. Ramirez, L. N. Pfeiffer et K. W. West, 'Nonmonotonic Temperature-Dependent Resistance in Low Density 2D Hole Gases', Phys. Rev. Lett. 83, 2805 (1999).

[73] Y. Hanein, D. Shahar, J. Yoon, C. C. Li, D. C. Tsui et H. Shtrikman, 'Properties of the apparent metal-insulator transition in two-dimensional systems', Phys. Rev. B 58, R7520 (1998).

[74] Y. Hanein, H. Shtrikman et U. Meirav, 'Very low density two-dimensional hole gas in an inverted GaAs/AlAs interface', Appl. Phys. Lett. 70, 1426 (1997).

[75] V. M. Pudalov, G. Brunthaler, A. Prinz et G. Bauer, 'Logarithmic Temperature Dependence of the Conductivity of the Two-Dimensional Metal', JETP Lett. 68, 534 (1998).

[76] V. M. Pudalov, G. Brunthaler, A. Prinz et G. Bauer, 'Maximum Metallic Conductivity in Si-MOS Structures', Phys. Rev. B 60, R2154 (1999).

[77] W. Mason, S. V. Kravchenko, G. E. Bowker et J. E. Furneaux, 'Experimental evidence for a Coulomb gap in two dimensions', Phys. Rev. B 52, 7857 (1995).

[78] W. Mason, S. V. Kravchenko et J. E. Furneaux, 'Experimental evidence of the Coulomb gap in a high-mobility 2D electron system in silicon', Surf. Sci. 361/362, 953 (1996).

[79] K. M. Mertes, D. Simonian, M. P. Sarachik, S. V. Kravchenko et T. M. Klapwijk, 'Response to Parallel Magnetic Field of a Dilute Two-Dimensional Electron System Across the Metal-Insulator Transition', Phys. Rev. B 60, R5093 (1999).

[80] S. I. Khondaker, I. S. Shlimak, J. T. Nicholls, M. Pepper et D. A. Ritchie, 'Twodimensional hopping conductivity in a $\delta$-doped $\mathrm{GaAs} / \mathrm{Al}_{x} \mathrm{Ga}_{1-x} \mathrm{As}$ heterostructure', Phys. Rev. B 59, 4580 (1999).

[81] A. A. Shashkin, S. V. Kravchenko et T. M. Klapwijk, 'Metal-Insulator Transition in a 2D Electron Gas : Equivalence of Two Approaches for Determining the Critical Point', Phys. Rev. Lett. 87, 266402 (2001).

[82] S. Bogdanovich et D. Popović, 'Onset of Glassy Dynamics in a Two-Dimensional Electron System in Silicon', Phys. Rev. Lett. 88, 236401 (2002).

[83] J. Jaroszynski, D. Popović et T. M. Klapwijk, 'Universal Behavior of the Resistance Noise Across the Metal-Insulator Transition in Silicon Inversion Layers', condmat/0205226. 
[84] S. V. Kravchenko, D. Simonian, M. P. Sarachik, W. Mason et J. E. Furneaux, 'Electric Field Scaling at a $B=0$ Metal-Insulator Transition in Two Dimensions', Phys. Rev. Lett. 77, 4938 (1996).

[85] A. R. Hamilton, M. Y. Simmons, M. Pepper, E. H. Linfield, P. D. Rose et D. A. Ritchie, 'Reentrant Insulator-Metal-Insulator Transition at $B=0$ in a TwoDimensional Hole Gas', Phys. Rev. Lett. 82, 1542 (1999).

[86] D. J. Bishop, R. C. Dynes et D. C. Tsui, 'Magnetoresistance in Si metal-oxidesemiconductor field-effect transitors : Evidence of weak localization and correlation', Phys. Rev. B 26, 773 (1982).

[87] J. Yoon, C. C. Li, D. Shahar, D. C. Tsui et M. Shayegan, 'Wigner Crystallization and Metal-Insulator Transition of Two-Dimensional Holes in GaAs at $B=0$ ', Phys. Rev. Lett. 82, 1744 (1999).

[88] V. M. Pudalov, M. D'Iorio et J. W. Campbell, 'Electric Field Induced Non-Ohmic Conduction in the 2D Insulating Phase', Surf. Sci. 361/362, 941 (1996).

[89] J. Yoon, C. C. Li, D. Shahar, D. C. Tsui et M. Shayegan, 'Parallel Magnetic Field Induced Transition in Transport in the Dilute Two-Dimensional Hole System in GaAs', Phys. Rev. Lett. 84, 4421 (2000).

[90] V. T. Dolgopolov, G. V. Kravchenko, A. A. Shashkin et S. V. Kravchenko, JETP Lett. 55, 733 (1992).

[91] D. Simonian, S. V. Kravchenko, M. P. Sarachik et V. M. Pudalov, 'Magnetic Field Suppression of the Conducting Phase in Two Dimensions', Phys. Rev. Lett. 79, 2304 (1997).

[92] V. M. Pudalov, G. Brunthaler, A. Prinz et G. Bauer, 'Instability of the TwoDimensional Metallic Phase to a Parallel Magnetic Field', JETP Lett. 65, 932 (1997).

[93] D. Simonian, S. V. Kravchenko, M. P. Sarachik et V. M. Pudalov, ' $H / T$ Scaling of the Magnetoconductance Near the Conductor-Insulator Transition in Two Dimensions', Phys. Rev. B 57, R9420 (1998).

[94] T. Okamoto, K. Hosoya, S. Kawaji et A. Yagi, 'Spin Degree of Freedom in a TwoDimensional Electron Liquid', Phys. Rev. Lett. 82, 3875 (1999).

[95] T. Okamoto, K. Hosoya, S. Kawaji, A. Yagi, A. Yutani et Y. Shiraki, 'MetalInsulator Transition and Spin Degree of Freedom in Silicon 2D Electron Systems', cond-mat/9906425.

[96] M. Y. Simmons et A. R. Hamilton, 'Real Metals, 2D or Not 2D ?', Nature 400, 715 (1999).

[97] E. Abrahams, S. V. Kravchenko et M. P. Sarachik, 'Metallic Behavior and Related Phenomena in Two Dimensions', Rev. Mod. Phys. 73, 251 (2001).

[98] V. M. Pudalov, G. Brunthaler, A. Prinz et G. Bauer, 'Breakdown of the Anomalous Two-Dimensional Metallic Phase in a Parallel Magnetic Field', Physica B : Cond. Mat. 249-251, 697 (1998). 
[99] S. A. Vitkalov, H. Zheng, K. M. Mertes, M. P. Sarachik et T. M. Klapwijk, 'Scaling of the Magnetoconductivity of Silicon MOSFETs : Evidence for a Quantum Phase Transition in Two Dimensions', Phys. Rev. Lett. 87, 086401 (2001).

[100] S. A. Vitkalov, M. P. Sarachik et T. M. Klapwijk, 'Spin Polarization of TwoDimensional Electrons Determined from Shubnikov-de Haas Oscillations as a Function of Angle', cond-mat/0101196.

[101] V. M. Pudalov, G. Brunthaler, A. Prinz et G. Bauer, 'Weak Anisotropy and Disorder Dependence of the In-Plane Magnetoresistance in High-Mobility (100) Si-Inversion Layers', Phys. Rev. Lett. 88, 076401 (2002).

[102] V. M. Pudalov, G. Brunthaler, A. Prinz et G. Bauer, 'Effect of the In-Plane Magnetic Field on Conduction of the Si-Inversion Layer : Magnetic Field Driven Disorder', cond-mat/0103087.

[103] K. M. Mertes, H. Zheng, S. A. Vitkalov, M. P. Sarachik et T. M. Klapwijk, 'Temperature Dependence of the Resistivity of a Dilute Two-Dimensional Electron System in High Parallel Magnetic Field', Phys. Rev. B 63, 041101(R) (2001).

[104] I. Shlimak, S. I. Khondaker, M. Pepper et D. A. Ritchie, 'Influence of parallel magnetic fields on a single-layer two-dimensional electron system with a hopping mechanism of conductivity', Phys. Rev. B 61, 7253 (2000).

[105] X. P. A. Gao, A. P. Mills, A. P. Ramirez, L. N. Pfeiffer et K. W. West, 'TwoDimensional Metal in a Parallel Magnetic Field', Phys. Rev. Lett. 88, 166803 (2002).

[106] S. J. Papadakis, E. P. De Poortere, M. Shayegan et R. Winkler, 'Anisotropic Magnetoresistance of Two-Dimensional Holes in GaAs', Phys. Rev. Lett. 84, 5592 (2000).

[107] G. Brunthaler, A. Prinz, G. Bauer, V. Pudalov, E. Dizhur, J. Jaroszynski, P. Glod et T. Dietl, 'Weak Localization in the 2D Metallic Regime of Si-MOS', Ann. Phys. (Leipzig) 8, 579 (1999).

[108] M. Y. Simmons, A. R. Hamilton, M. Pepper, E. H. Linfield, P. D. Rose et D. A. Ritchie, 'Weak Localization, Hole-Hole Interactions, and the "Metal"-Insulator Transition in Two Dimensions', Phys. Rev. Lett. 84, 2489 (2000).

[109] A. A. Shashkin, G. V. Kravchenko et V. T. Dolgopolov, 'Floating up of the extended states of Landau levels in a two-dimensional electron gas in silicon MOSFET's', JETP Lett. 58, 220 (1993).

[110] V. M. Pudalov, M. D'Iorio et J. W. Campbell, 'Termination of the quantized Hall effect by the electron solid', Surf. Sci. 305, 107 (1994).

[111] S. V. Kravchenko, W. Mason, J. E. Furneaux et V. M. Pudalov, 'Global Phase Diagram for the Quantum Hall Effect : An Experimental Picture', Phys. Rev. Lett. 75, 910 (1995).

[112] S. C. Dultz, H. W. Jiang et W. J. Schaff, 'Absence of floating delocalized states in a two-dimensional hole gas', Phys. Rev. B 58, R7532 (1998).

[113] Y. Hanein, N. Nenadovic, D. Shahar, H. Shtrikman, J. Yoon, C. C. Li et D. C. Tsui, 'Linking Insulator-to-Metal Transitions at Zero and Finite Magnetic Fields', Nature 400, 735 (1999). 
[114] M. Y. Simmons, A. R. Hamilton, T. G. Griffiths, A. K. Savchenko, M. Pepper et D. A. Ritchie, 'Metal-insulator transition at $B=0$ in an ultra-low density twodimensional hole gas', Physica B : Cond. Mat. 249-251, 705 (1998).

[115] H. W. Jiang, C. E. Johnson, K. L. Wang et S. T. Hannahs, 'Observation of magneticfield-induced delocalization : Transition from Anderson insulator to quantum Hall conductor', Phys. Rev. Lett. 71, 1439 (1993).

[116] I. Glozman, C. E. Johnson et H. W. Jiang, 'Fate of the Delocalized States in a Vanishing Magnetic Field', Phys. Rev. Lett. 74, 594 (1995).

[117] D. E. Khmelnitskii, Phys. Lett. A 182, 106 (1984).

[118] R. B. Laughlin, 'Levitation of Extended-State Bands in a Strong Magnetic Field', Phys. Rev. Lett. 52, 2304 (1984).

[119] S. Kivelson, D.-H. Lee et S.-C. Zhang, 'Global phase diagram in the quantum Hall effect', Phys. Rev. B 46, 2223 (1992).

[120] S. V. Kravchenko, D. Simonian, M. P. Sarachik, A. D. Kent et V. M. Pudalov, 'Effect of a Tilted Magnetic Field on the Anomalous $H=0$ Conducting Phase in High-Mobility Si MOSFET's', Phys. Rev. B 58, 3553 (1998).

[121] S. V. Kravchenko, D. Simonian, M. P. Sarachik, A. D. Kent et V. M. Pudalov, 'Magnetic Field Suppression of the Conducting State in Two Dimensions', Physica B : Cond. Mat. 256-258, 582 (1998).

[122] D. Shahar, D. C. Tsui et J. E. Cunningham, 'Observation of the $\nu=1$ quantum Hall effect in a strongly localized two-dimensional system', Phys. Rev. B 52, R14372 (1995).

[123] E. Ribeiro, R. D. Jäggi, T. Heinzel, K. Ensslin, G. Medeiros-Ribeiro et P. M. Petroff, 'Metal-Insulator Transition in a Disordered Two-Dimensional Electron Gas in GaAsAlGaAs at Zero Magnetic Field', Phys. Rev. Lett. 82, 996 (1999).

[124] S. C. Dultz et H. W. Jiang, 'Thermodynamic Signature of a Two-Dimensional MetalInsulator Transition', Phys. Rev. Lett. 84, 4689 (2000).

[125] S. Ilani, A. Yacoby, D. Mahalu et H. Shtrikman, 'Unexpected Behavior of the Local Compressibility Near the $B=0$ Metal-Insulator Transition', Phys. Rev. Lett. 84, 3133 (2000).

[126] S. Ilani, A. Yacoby, D. Mahalu et H. Shtrikman, 'Microscopic Structure of the Metal-Insulator Transition in Two Dimensions', Science 292, 1354 (2001).

[127] R. Fletcher, V. M. Pudalov, A. D. B. Radcliffe et C. Possanzini, 'Critical Behavior of Thermopower and Conductivity at the Metal-Insulator Transition in High-Mobility Si-MOSFET's', cond-mat/0002436.

[128] C. Castellani, C. Di Castro, M. Grilli et G. Strinati, 'Thermoelectric power in disordered electronic systems near the Anderson transition', Phys. Rev. B 37, 6663 (1988).

[129] D. Popović et S. Washburn, 'Mesoscopic Behavior Near a Two-Dimensional MetalInsulator Transition', Phys. Rev. B 56, R10048 (1997). 
[130] K. P. Li, D. Popovic et S. Washburn, 'Conductance Fluctuations Near the TwoDimensional Metal-Insulator Transition', Physica B : Cond. Mat. 249-251, 504 (1998).

[131] T. Vojta, 'Quantum phase transitions in electronic systems', cond-mat/9910514.

[132] T. Vojta, 'Quantum Phase Transitions', cond-mat/0010285.

[133] M. C. Maliepaard, M. Pepper, R. Newbury, J. E. F. Frost, D. C. Peacock, D. A. Ritchie, G. A. C. Jones et G. Hill, 'Evidence of a magnetic-field-induced insulatormetal-insulator transition', Phys. Rev. B 39, 1430 (1989).

[134] T. F. Rosenbaum, R. F. Milligan, M. A. Paalanen, G. A. Thomas et R. N. Bhatt, 'Metal-insulator transition in a doped semiconductor', Phys. Rev. B 27, 7509 (1983).

[135] S. Bogdanovich, D. Simonian, S. V. Kravchenko, M. P. Sarachik et R. N. Bhatt, 'Hopping conduction in uniaxially stressed Si :B near the insulator-metal transition', Phys. Rev. B 60, 2286 (1999).

[136] V. M. Pudalov et S. T. Chui, 'Character of the Phase Transition at the TwoDimensional Electron-Liquid-to-Solid Boundary', Phys. Rev. B 49, 14062 (1994).

[137] V. M. Pudalov, G. Brunthaler, A. Prinz et G. Bauer, 'Lack of Universal OneParameter Scaling in the Two-Dimensional Metallic Regime', JETP Lett. 68, 442 (1998).

[138] R. Heemskerk et T. M. Klapwijk, 'Nonlinear Resistivity at the Metal-Insulator Transition in a Two-Dimensional Electron Gas', Phys. Rev. B 58, R1754 (1998).

[139] D. Popović, A. B. Fowler et S. Washburn, 'Metal-Insulator Transition in a LowMobility Two-Dimensional Electron System', Physica B : Cond. Mat. 249-251, 701 (1998).

[140] V. M. Pudalov, G. Brunthaler, A. Prinz et G. Bauer, 'Metal-Insulator Transition in Two Dimensions', Physica E 3, 79 (1998).

[141] V. Senz, U. Dötsch, U. Gennser, T. Ihn, T. Heinzel, K. Ensslin, R. Hartmann et D. Grützmacher, 'Metal-Insulator Transition in a 2-Dimensional System with an Easy Spin Axis', Ann. Phys. (Leipzig) 8, 237 (1999).

[142] D. Lilliehöök et J. E. Furneaux, 'Scaling limits for the two-dimensional metalinsulator transition at $B=0$ in Si-MOSFET's', Phys. Rev. B 66, 165328 (2002).

[143] A. Yazdani et A. Kapitulnik, 'Superconducting-Insulating Transition in TwoDimensional $a$-MoGe Thin Films', Phys. Rev. Lett. 74, 3037 (1995).

[144] H. P. Wei, L. W. Engel et D. C. Tsui, 'Current scaling in the integer quantum Hall effect', Phys. Rev. B 50, 14609 (1994).

[145] S. Sachdev, Z. Phys. B 94, 469 (1994).

[146] D. Simonian, S. Kravchenko, K. Mertes, M. Sarachik et V. Pudalov, 'Magnetoconductance of the Anomalous 2D Conducting Phase in Parallel Field', Physica B : Cond. Mat. 256-258, 607 (1998).

[147] V. M. Pudalov, M. E. Gershenson et H. Kojima, 'Memory Effects in Electron Transport in Si Inversion Layers in the Dilute Regime : Individuality versus Universality', cond-mat/0201001. 
[148] S. Das Sarma et E. H. Hwang, 'Charged Impurity-Scattering-Limited LowTemperature Resistivity of Low-Density Silicon Inversion Layers', Phys. Rev. Lett. 83, 164 (1999).

[149] S. Das Sarma et E. H. Hwang, 'Calculated Temperature-Dependent Resistance in Low-Density Two-Dimensional Hole Gases in GaAs Heterostructures', Phys. Rev. B 61, R7838 (2000).

[150] A. P. Mills, A. P. Ramirez, X. P. A. Gao, L. N. Pfeiffer, K. W. West et S. H. Simon, 'Suppression of weak localization effects in low-density metallic 2D holes', cond-mat/0101020.

[151] G. Brunthaler, A. Prinz, G. Bauer et V. M. Pudalov, 'Exclusion of Quantum Coherence as the Origin of the 2D Metallic State in High-Mobility Silicon Inversion Layers', Phys. Rev. Lett. 87, 096802 (2001).

[152] Y. Y. Proskuryakov, A. K. Savchenko, S. S. Safonov, M. Pepper, M. Y. Simmons et D. A. Ritchie, 'Fermi-Liquid Behavior of the Low-Density 2D Hole Gas in a GaAs/AlGaAs Heterostructure at Large Values of $r_{s}$ ', Phys. Rev. Lett. 86, 4895 (2001).

[153] B. L. Altshuler, G. W. Martin, D. L. Maslov, V. M. Pudalov, A. Prinz, G. Brunthaler et G. Bauer, 'Weak-Localization Type Description of Conduction in the "anomalous" Metallic State', cond-mat/0008005.

[154] R. Leturcq, D. L'Hote, R. Tourbot, V. Senz, U. Gennser, T. Ihn, K. Ensslin, G. Dehlinger et D. Grützmacher, 'Heating of Two-Dimensional Holes in SiGe and the $B=0$ Metal-Insulator Transition', cond-mat/0107457.

[155] V. M. Pudalov, G. Brunthaler, A. Prinz et G. Bauer, 'Weak-Field Hall Resistance and Effective Carrier Density Measurements Across the Metal-Insulator Transition in Si-MOS Structures', JETP Lett. 70, 48 (1999).

[156] V. Senz, T. Ihn, T. Heinzel, K. Ensslin, G. Dehlinger, D. Grützmacher et U. Gennser, 'Analysis of the Metallic Phase of Two-Dimensional Holes in SiGe in Terms of Temperature Dependent Screening', Phys. Rev. Lett. 85, 4357 (2000).

[157] H.-P. Wittman et A. Schmid, 'Anomalous Magnetoconductance beyond the Diffusion Limit', J. Low Temp. Phys. 69, 131 (1987).

[158] V. M. Pudalov, 'On the Origin of the Metal-Insulator Transition in 2D', condmat/9707053.

[159] M. P. Sarachik, 'Disorder-dependence of the critical density in two-dimensional systems : An empirical relation', Europhys. Lett. 57, 546 (2002).

[160] A. R. Hamilton, M. Y. Simmons, M. Pepper, E. H. Linfield et D. A. Ritchie, 'Metallic Behavior in Dilute Two-Dimensional Hole Systems', Phys. Rev. Lett. 87, 126802 (2001).

[161] S. J. Papadakis, E. P. De Poortere, H. C. Manoharan, M. Shayegan et R. Winkler, 'The Effect of Spin Splitting on the Metallic Behavior of a Two-Dimensional System', Science 283, 2056 (1999).

[162] S. J. Papadakis, E. P. De Poortere, M. Shayegan et R. Winkler, 'Spin-Splitting in GaAs 2D Holes', cond-mat/0006497. 
[163] E. Tutuc, E. P. De Poortere, S. J. Papadakis et M. Shayegan, 'In-Plane Magnetic Field-Induced Spin Polarization and Transition to Insulating Behavior in TwoDimensional Hole Systems', Phys. Rev. Lett. 86, 2858 (2001).

[164] V. M. Pudalov, M. Gershenson, H. Kojima, N. Butch, E. M. Dizhur, G. Brunthaler, A. Prinz et G. Bauer, 'Low-Density Spin Susceptibility and Effective Mass of Mobile Electrons in Si Inversion Layers', Phys. Rev. Lett. 88, 196404 (2002).

[165] S. J. Papadakis, E. P. De Poortere et M. Shayegan, 'Confinement Symmetry, Mobility Anisotropy, and Metallic Behavior in (311)A GaAs 2D Holes', condmat/0008041.

[166] S. Das Sarma et E. H. Hwang, 'Parallel Magnetic Field Induced Giant Magnetoresistance in Low Density Quasi-Two-Dimensional Layers', Phys. Rev. Lett. 84, 5596 (2000).

[167] H. Noh, J. Yoon, D. C. Tsui et M. Shayegan, 'Non-Monotonic Magnetic Field and Density Dependence of in-Plane Magnetoresistance in Dilute Two-Dimensional Holes in GaAs/AlGaAs', cond-mat/0104240.

[168] D. Shahar, D. C. Tsui, M. Shayegan, E. Shimshoni et S. L. Sondhi, 'Evidence for Charge-Flux Duality near the Quantum Hall Liquid-to-Insulator Transition', Science 274, 589 (1996).

[169] D. Simonian, S. V. Kravchenko et M. P. Sarachik, 'Reflection Symmetry at a $B=0$ Metal-Insulator Transition in Two Dimensions', Phys. Rev. B 55, R13421 (1997).

[170] V. T. Dolgopolov, A. A. Shashkin, G. V. Kravchenko, C. J. Emeleus et T. E. Whall, 'Metal-insulator transition in a two-dimensional hole gas in Si/SiGe heterostructures. Quantum or classical percolation?', JETP Lett. 62, 168 (1995).

[171] A. M. Finkel'stein, Z. Phys. B 56, 189 (1984).

[172] C. Castellani, C. Di Castro, P. A. Lee et M. Ma, 'Interaction-driven metal-insulator transitions in disordered fermion systems', Phys. Rev. B 30, 527 (1984).

[173] C. Castellani, C. Di Castro et P. A. Lee, 'Metallic phase and metal-insulator transition in two-dimensional electronic systems', Phys. Rev. B 57, R9381 (1998).

[174] M. P. Sarachik, D. Simonian, K. M. Mertes, S. V. Kravchenko et T. M. Klapwijk, 'Hall Coefficient of the 2D Electron System in Silicon MOSFETs', Physica B : Cond. Mat. 280, 301 (2000).

[175] Q. Si et C. M. Varma, 'Metal-Insulator Transition of Disordered Interacting Electrons', Phys. Rev. Lett. 81, 4951 (1998).

[176] S. Chakravarty, L. Yin et E. Abrahams, 'Interactions and scaling in a disordered two-dimensional metal', Phys. Rev. B 58, R559 (1998).

[177] V. Dobrosavljević, E. Abrahams, E. Miranda et S. Chakravarty, 'Scaling Theory of Two-Dimensional Metal-Insulator Transitions', Phys. Rev. Lett. 79, 455 (1997).

[178] E. Abrahams, 'Scaling at the metal-insulator transition in two dimensions', Physica E 3, 69 (1998).

[179] G. Grüner, 'The Dynamics of Charge-Density Waves', Rev. Mod. Phys. 60, 1129 (1988). 
[180] A. J. Dahm, Electrons on helium films, e. y. andrei Édition (Kluwer Academic Publishers, 1997), pp. 281-316.

[181] H. W. Jiang et A. J. Dahm, 'Conduction Properties of a New Two-Dimensional Sliding Charge-Density Wave', Phys. Rev. Lett. 62, 1396 (1989).

[182] S. T. Chui, 'A Quantitative Study of Pinning of a 2D Electron Crystal in Heterojunctions', J. Phys. : Condens. Matter 5, L405 (1993).

[183] J.-L. Pichard, G. Benenti, G. Katomeris, F. Selva et X. Waintal, 'From the Fermi Liquid Towards the Wigner Solid in Two Dimensions', cond-mat/0107380.

[184] G. Benenti, X. Waintal et J.-L. Pichard, 'A new quantum phase in two dimensions', dans : Rencontres de Moriond (1999), cond-mat/9905028.

[185] F. Selva et D. Weinmann, 'Persistent currents for Coulomb interacting electrons on 2d disordered lattices', Eur. Phys. J. B 18, 137 (2000).

[186] F. Selva et J.-L. Pichard, 'Role of a parallel magnetic field upon a few correlated electrons trapped in 2d mesoscopic disordered clusters', Europhys. Lett. 55, 518 (2001).

[187] G. Katomeris, F. Selva et J.-L. Pichard, 'Andreev-Lifshitz conjecture revisited for a few electrons on a square lattice I', cond-mat/0206404.

[188] A. F. Andreev et V. M. Lifshitz, Sov. Phys. JETP 29, 1107 (1969).

[189] B. Spivak, 'Phase separation in the two-dimensional electron liquid in MOSFETs', cond-mat/0205127.

[190] J. Shi, S. He et X. C. Xie, 'Meta-Percolation and Metal-Insulator Transition in Two Dimensions', cond-mat/9904393.

[191] J. Shi et X. C. Xie, 'Droplet State and the Compressibility Anomaly in Dilute 2D Electron Systems', Phys. Rev. Lett. 88, 086401 (2002).

[192] S. Chakravarty, S. Kivelson, C. Nayak et K. Voelker, 'Wigner Glass, Spin-Liquids, and the Metal-Insulator Transition', Phil. Mag. B 79, 859 (1999).

[193] A. A. Pastor et V. Dobrosavljević, 'Melting of the Electron Glass', Phys. Rev. Lett. 83, 4642 (1999).

[194] D. Dalidovich et V. Dobrosavljević, 'Landau theory of the Fermi-liquid to electron glass transition', cond-mat/0205269.

[195] V. Dobrosavljević, D. Tanasković et A. A. Pastor, 'Glassy behavior of electrons near metal-insulator transitions', cond-mat/0206529.

[196] J. S. Thakur et D. Neilson, 'Frozen electron solid in the presence of small concentrations of defects', Phys. Rev. B 54, 7674 (1996).

[197] J. S. Thakur et D. Neilson, 'Phase diagram of the metal-insulator transition in two-dimensional electronic systems', Phys. Rev. B 59, R5280 (1999).

[198] S. Sachdev, 'Quantum Spin Glass Transition in the Two-Dimensional Electron Gas', cond-mat/0109309.

[199] Y. Liu, K. A. McGreer, B. Nease, D. B. Haviland, G. Martinez, J. W. Halley et A. M. Goldman, 'Scaling of the insulator-to-superconductor transition in ultrathin amorphous Bi films', Phys. Rev. Lett. 67, 2068 (1991). 
[200] Y. Liu, B. Nease, K. A. McGreer et A. M. Goldman, 'Scaling of the Electrical Conductivity of Ultrathin Amorphous Palladium Films', Europhys. Lett. 19, 409 (1992).

[201] M. P. A. Fisher et G. Grinstein, 'Presence of quantum diffusion in two dimensions : Universal resistance at the superconductor-insulator transition', Phys. Rev. Lett. 64, 587 (1990).

[202] Y. Takada, J. Phys. Soc. Jpn. 45, 786 (1978).

[203] Y. Takada, J. Phys. Soc. Jpn. 49, 1713 (1980).

[204] W. Hanke et M. J. Kelly, 'Surface Superconductivity and the Metal-OxideSemiconductor System', Phys. Rev. Lett. 45, 1203 (1980).

[205] M. J. Kelly et W. Hanke, 'Surface superconductivity and the MOS system', Phys. Rev. B 23, 112 (1981).

[206] Y. Ren et F. C. Zhang, 'Fermion analogy of anyon superconductivity in the twodimensional electron gas', Phys. Rev. B 49, 1532 (1994).

[207] P. Phillips et Y. Wan, 'Incipient p-wave Superconductivity in a Si-MOSFET', condmat/9704200.

[208] P. Phillips, Y. Wan, I. Martin, S. Knysh et D. Dalidovich, 'Superconductivity in a two-dimensional electron gas', Nature 395, 253 (1998).

[209] T. R. Kirkpatrick et D. Belitz, 'Disorder-induced triplet superconductivity', Phys. Rev. Lett. 66, 1533 (1991).

[210] D. Belitz et T. R. Kirkpatrick, 'Even-parity spin-triplet superconductivity in disordered electronic systems', Phys. Rev. B 46, 8393 (1992).

[211] D. Belitz et T. R. Kirkpatrick, 'Possible triplet superconductivity in MOSFETs', Phys. Rev. B 58, 8214 (1998).

[212] J. S. Thakur et D. Neilson, 'Superconductivity in a correlated disordered twodimensional electron gas', Phys. Rev. B 58, 13717 (1998).

[213] Y. Meir, 'Percolation-Type Description of the Metal-Insulator Transition in Two Dimensions', Phys. Rev. Lett. 83, 3506 (1999).

[214] Y. Meir, 'Two-species percolation and scaling theory of the metal-insulator transition in two dimensions', Phys. Rev. B 61, 16470 (2000).

[215] Y. Meir, 'From the Zero-Field Metal-Insulator Transition in Two Dimensions to the Quantum Hall Transition : A Percolation-Effective-Medium Theory', Phys. Rev. B 63, 073108 (2001).

[216] S. He et X. C. Xie, 'New Liquid Phase and Metal-Insulator Transition in Si MOSFETs', Phys. Rev. Lett. 80, 3324 (1998).

[217] J. Shi, S. He et X. C. Xie, 'Droplet state in an interacting two-dimensional electron system', Phys. Rev. B 60, R13950 (1999).

[218] G. Zala, B. N. Narozhny et I. L. Aleiner, 'Interaction corrections at intermediate temperatures : Longitudinal conductivity and kinetic equation', Phys. Rev. B 64, 214204 (2001). 
[219] G. Zala, B. N. Narozhny et I. L. Aleiner, 'Interaction corrections at intermediate temperatures : Magnetoresistance in a parallel field', Phys. Rev. B 65, 020201 (2001).

[220] A. A. Shashkin, S. V. Kravchenko, V. T. Dolgopolov et T. M. Klapwijk, 'Sharp increase of the effective mass near the critical density in a metallic $2 \mathrm{D}$ electron system', cond-mat/0111478.

[221] S. A. Vitkalov, K. James, B. N. Narozhny, M. P. Sarachik et T. M. Klapwijk, 'Inplane Magnetoconductivity of Si-MOSFET's : A Quantitative Comparison between Theory and Experiment', cond-mat/0204566.

[222] V. M. Pudalov, M. E. Gershenson, H. Kojima, G. Brunthaler, A. Prinz et G. Bauer, 'Interaction Effects in Conductivity of Si Inversion Layers at Intermediate Temperatures', cond-mat/0205449.

[223] Y. Y. Proskuryakov, A. K. Savchenko, S. S. Safonov, M. Pepper, M. Y. Simmons et D. A. Ritchie, 'Hole-Hole Interaction Effect in the Conductance of the TwoDimensional Hole Gas in the Ballistic Regime', Phys. Rev. Lett. 89, 076406 (2002).

[224] H. Noh, M. P. Lilly, D. C. Tsui, J. A. Simmons, L. N. Pfeiffer et K. W. West, 'Interaction Corrections to Two-Dimensional Hole Transport in Large $r_{s}$ Limit', cond-mat/0206519.

[225] V. T. Dolgopolov et A. Gold, 'Magnetoresistance of a Two-Dimensional Electron Gas in a Parallel Magnetic Field', JETP Lett. 71, 27 (2000).

[226] K. M. Cham et R. G. Wheeler, 'Temperature-Dependent Resistivities in Silicon Inversion Layers at Low Temperatures', Phys. Rev. Lett. 44, 1472 (1980).

[227] E. A. Vyrodov, V. T. Dolgopolov, C. I. Dorozhkin et N. B. Zhitenev, 'Temperature dependence of conductivity in figh mobility MIS structures on a base of (001) silicon', Sov. Phys. JETP 67, 998 (1988).

[228] C. J. Emeleus, T. E. Whall, D. W. Smith, N. L. Mattey, R. A. Kubiak, E. H. C. Parker et M. J. Kearney, 'Observation of Novel Transport Phenomena in a $\mathrm{Si}_{0.8} \mathrm{Ge}_{0.2}$ Two-Dimensional Hole Gas', Phys. Rev. B 47, 10016 (1993).

[229] M. Henini, P. J. Rodgers, P. A. Crump, B. L. Gallagher et G. Hill, 'Growth and Electrical Transport Properties of Very High Mobility Two-Dimensional Hole Gases Displaying Persistent Photoconductivity', Appl. Phys. Lett. 65, 2054 (1994).

[230] I. F. Herbut, 'The effect of parallel magnetic field on the Boltzmann conductivity and the Hall coefficient of a disordered two-dimensional Fermi liquid', Phys. Rev. B 63, 113102 (2001).

[231] B. L. Altshuler et D. L. Maslov, 'Theory of Metal-Insulator Transitions in Gated Semiconductors', Phys. Rev. Lett. 82, 145 (1999).

[232] B. L. Altshuler, D. L. Maslov et V. M. Pudalov, 'Metal-insulator transition in 2D : Anderson localization by temperature-dependent disorder?', Phys. Stat. Sol. (b) 218, 193 (2000).

[233] S. V. Kravchenko, A. A. Shashkin et V. T. Dolgopolov, 'Comment on "Fermi-Liquid Renormalization of the G-Factor and Effective Mass in Si Inversion Layers"', condmat/0106056. 
[234] B. L. Altshuler et D. L. Maslov, 'Altshuler and Maslov Reply', Phys. Rev. Lett. 83, 2092 (1999).

[235] P. Phillips, 'Deconstruction of the Trap Model for the New Conducting State in 2D', cond-mat/9910122.

[236] T. M. Klapwijk et S. Das Sarma, 'A few electrons per ion scenario for the $B=0$ metal-insulator transition in two dimensions', Solid State Commun. 110, 581 (1999).

[237] V. I. Kozub, N. V. Agrinskaya, S. I. Khondaker et I. Shlimak, 'A possible role of $\mathrm{D}^{-}$band in hopping conductivity and metal-insulator transition in 2D structures', cond-mat/9911450.

[238] V. M. Pudalov, G. Brunthaler, A. Prinz, G. Bauer et B. I. Fouks, 'Time-dependent Effects in the Metallic Phase in Si-MOS : Evidence for Non-Diffusive Transport', cond-mat/9907401.

[239] S. J. Papadakis, E. P. De Poortere, M. Shayegan et R. Winkler, 'Spin-splitting in GaAs two-dimensional holes', Physica E 9, 31 (2001).

[240] S. A. Vitkalov, H. Zheng, K. M. Mertes, M. P. Sarachik et T. M. Klapwijk, 'Hall Coefficient of a Dilute 2D Electron System in Parallel Magnetic Field', condmat/0008456.

[241] Y. Yaish, O. Prus, E. Buchstab, S. Shapira, G. B. Yoseph, U. Sivan et A. Stern, "Interband Scattering and the "Metallic Phase" of Two-Dimensional Holes in GaAs/AlGaAs', Phys. Rev. Lett. 84, 4954 (2000).

[242] Y. Yaish, O. Prus, E. Buchstab, G. B. Yoseph, U. Sivan, I. Ussishkin et A. Stern, 'Two Band Transport and the Question of a Metal-Insulator Transition in GaAs/GaAlAs Two Dimensional Holes', cond-mat/0109469.

[243] A. Punnoose et A. M. Finkel'stein, 'Dilute Electron Gas near the Metal-Insulator Transition : Role of Valleys in Silicon Inversion Layers', Phys. Rev. Lett. 88, 016802 (2002).

[244] M. V. Cheremisin, 'Peltier Effect Induced Longitudinal Resistivity of Ideal 2D Electron(Hole) Gas in Strong Magnetic Field', cond-mat/0008426.

[245] M. V. Cheremisin, 'Thermal correction to resistivity of $2 \mathrm{D}$ electron (hole) gas in low-temperature measurements at $B=0$ ', cond-mat/0203387.

[246] P. Schnappauf, 'Transportphänomenen in 2-dimensionalen Strukturen im Materialsystem Si-Ge-C', Maîtrise, Universität Regensburg (Juin 1997).

[247] V. Senz, 'Gate controlled two dimensional systems realized with group IV heterostructures', Maîtrise, Universität Regensburg (Novembre 1998).

[248] G. Snider, programme de résolution de l'équation de Poisson-Schrödinger 1D. Disponible sur Internet à l'adresse : http ://www.nd.edu/ gsnider.

[249] R. People, 'Indirect band gap of coherently strained $\mathrm{Ge}_{x} \mathrm{Si}_{1-x}$ bulk alloys on $<001>$ silicon substrates', Phys. Rev. B 32, 1405 (1985).

[250] Handbook of semiconductors, Vol. 2 (T. S. Moss and M. Balkanski). 
[251] G. Braithwaite, N. L. Mattey, E. H. C. Parker, T. E. Whall, G. Brunthaler et G. Bauer, 'Hot Hole Energy Relaxation in $\mathrm{Si} / \mathrm{Si}_{0.8} \mathrm{Ge}_{0.2}$ Two Dimensional Hole Gases', J. Appl. Phys. 81, 6853 (1997).

[252] Handbook of semiconductors, Vol. 1 (T. S. Moss and W. Paul).

[253] K. Hirakawa, Y. Zhao, M. B. Santos, M. Shayegan et D. C. Tsui, 'Anomalous cyclotron-resonance line splitting of two-dimensional holes in (311)A $\mathrm{Al}_{x} \mathrm{Ga}_{1-x} \mathrm{As} / \mathrm{GaAs}$ heterojunctions', Phys. Rev. B 47, 4076 (1993).

[254] S. Adachi, 'GaAs, AlAs and $\mathrm{Al}_{x} \mathrm{Ga}_{1-x} \mathrm{As}$ Material Parameters for Use in Research and Device Applications', J. Appl. Phys. 58, R1 (1985).

[255] O. V. Lounasmaa, Experimental Principles and Methods Below $1 \mathrm{~K}$ (Academic Press, 1974).

[256] D. L'Hôte, X. F. Navick, R. T. J. Mangin et F. Pesty, 'Charge and heat collection in a $70 \mathrm{~g}$ heat/ionization cryogenic detector for dark matter search', J. Appl. Phys. 87, 1507 (2000).

[257] A. Miller et E. Abrahams, 'Impurity conduction at low concentrations', Phys. Rev. 120, 745 (1960).

[258] V. Ambegaokar, B. I. Halperin et J. S. Langer, 'Hopping Conductivity in Disordered Systems', Phys. Rev. B 4, 2612 (1971).

[259] N. F. Mott, 'Conduction in glasses containing transition metal ions', J. Non-Cryst. Solids 1, 1 (1968).

[260] A. L. Efros et B. I. Shklovskii, 'Coulomb gap and low temperature conductivity of disordered systems', J. Phys. C : Solid State Phys. 8, L49 (1975).

[261] N. V. Lien, Sov. Phys. Semicond. 18, 207 (1984).

[262] J.-J. Kim et H. J. Lee, 'Observation of a nonmagnetic hard gap in amorphous $\mathrm{In} / \mathrm{InO}_{x}$ films in the hopping regime', Phys. Rev. Lett. 70, 2798 (1993).

[263] I. L. Aleiner et B. I. Shklovskii, 'Effect of screening of the Coulomb interaction on the conductivity in the quantum Hall regime', Phys. Rev. B 49, 13721 (1994).

[264] F. W. V. Keuls, X. L. Hu, H. W. Jiang et A. J. Dahm, 'Screening of the Coulomb Interaction in Two-Dimensional Variable-Range Hopping', Phys. Rev. B 56, 1161 (1997).

[265] F. W. V. Keuls, X. L. Hu, A. J. Dahm et H. W. Jiang, 'The Coulomb gap and the transition to Mott hopping', Surf. Sci. 361/362, 945 (1995).

[266] F. Tremblay, M. Pepper, R. Newbury, D. A. Ritchie, D. C. Peacock, J. E. F. Frost, G. A. C. Jones et G. Hill, 'Hopping in a low-mobility GaAs-AlGaAs heterojunction in the limit of low electronic concentrations', J. Phys. Cond. Matt. 2, 7367 (1990).

[267] G. Ebert, K. V. Klitzing, C. Probst, E. Schuberth, K. Ploog et G. Weimann, 'Hopping conduction in the Landau level tails in $\mathrm{GaAs}_{-} \mathrm{Al}_{x} \mathrm{Ga}_{1-x} \mathrm{As}$ heterostructures at low temperatures', Solid State Commun. 45, 625 (1983).

[268] J. G. Massey et M. Lee, 'Direct Observation of the Coulomb Correlation Gap in a Nonmetallic Semiconductor, Si :B', Phys. Rev. Lett. 75, 4266 (1995). 
[269] J. G. Massey et M. Lee, 'Electron Tunneling Study of Coulomb Correlations across the Metal-Isulator Transition in Si :B', Phys. Rev. Lett. 77, 3399 (1996).

[270] V. Y. Butko, J. F. DiTusa et P. W. Adams, 'Coulomb Gap : How a Metal Film Becomes an Insulator', Phys. Rev. Lett. 84, 1543 (2000).

[271] A. I. Yakimov, A. V. Dvurechenskii, V. V. Kirienko, Y. I. Yakovlev, A. I. Nikiforov et C. J. Adkins, 'Long-range Coulomb interaction in arrays of self-assembled quantum dots', Phys. Rev. B 61, 10868 (2000).

[272] O. Entin-Wohlman et Z. Ovadyahu, 'Modifications of hopping transport due to electrostatically enhanced Coulomb repulsion', Phys. Rev. Lett. 56, 643 (1986).

[273] C. J. Adkins et E. G. Astrakharchik, 'Screened hopping conduction in ultrathin metal films', J. Phys. : Condens. Matter 10, 6651 (1998).

[274] M. L. Knotek et M. Pollak, 'Correlation effects in hopping conduction : A treatment in terms of multielectron transitions', Phys. Rev. B 9, 664 (1974).

[275] A. Pérez-Garrido, M. Ortuño, E. Cuevas, J. Ruiz et M. Pollak, 'Conductivity of the two-dimensional Coulomb glass', Phys. Rev. B 55, R8630 (1997).

[276] V. L. Nguyen, Sov. Phys. Semicond. 18, 207 (1984).

[277] A. Díaz-Sánchez, M. Ortuño, M. Pollak, A. Pérez-Garrido et A. Möbius, 'Dielectric susceptibility of the Coulomb glass', Phys. Rev. B 59, 910 (1999).

[278] D. N. Tsigankov et A. L. Efros, 'Variable Range Hopping in Two-Dimensional Systems of Interacting Electrons', Phys. Rev. Lett. 88, 176602 (2002).

[279] A. J. Dahm, 'The prefactor for screened Mott hopping', cond-mat/0205193.

[280] S. Marnieros, L. Bergé, A. Juillard et L. Dumoulin, 'Dynamical Properties near the Metal-Insulator Transition : Evidence for Electron-Assisted Variable Range Hopping', Phys. Rev. Lett. 84, 2469 (2000).

[281] M. E. Gershenson, Y. B. Khavin, P. Schafmeister et A. D. Wieck, 'Hot-Electron Effects in Two-Dimensional Hopping with a Large Localization Length', Phys. Rev. Lett. 85, 1718 (2000).

[282] S. Marnieros, 'Couches minces d'isolants d'Anderson : application à la bolométrie à très basse température.', Doctorat, Université Paris XI (Mai 1998).

[283] F. Ladieu, D. L'Hôte et R. Tourbot, 'Non-Ohmic hopping transport in a-YSi : From isotropic to directed percolation', Phys. Rev. B 61, 8108 (2000).

[284] S. Marianer et B. I. Shklovskii, 'Effective temperature of hopping electrons in a strong electric field', Phys. Rev. B 46, 13100 (1992).

[285] F. Ladieu, M. Sanquer et J. P. Bouchaud, 'Depinning Transition in Mott-Anderson Insulators', Phys. Rev. B 53, 973 (1996).

[286] P. Chauve, T. Giamarchi et P. L. Doussal, 'Creep Via Dynamical Functional Renormalization Group', cond-mat/9804190.

[287] P. Chauve, T. Giamarchi et P. L. Doussal, 'Creep and Depinning in Disordered Media', Phys. Rev. B 62, 6241 (2000). 
[288] C.-C. Li, J. Yoon, L. W. Engel, D. Shahar, D. C. Tsui et M. Shayegan, 'Microwave Resonance and Carrier-Carrier Interaction in Two-Dimensional Hole Systems at High Magnetic Field', cond-mat/9810066.

[289] C.-C. Li, J. Yoon, L. W. Engel, D. Shahar, D. C. Tsui et M. Shayegan, 'Microwave resonance and weak pinning in two-dimensional hole systems at high magnetic fields', Phys. Rev. B 61, 10905 (2000).

[290] T. Giamarchi, 'Disordered Wigner crystals', dans : NATO ASI "Field Theory of Strongly Correlated Fermions and Bosons in Low - Dimensional Disordered Systems" (2001), cond-mat/0205099.

[291] R. Chitra, T. Giamarchi et P. L. Doussal, 'Dynamical Properties of the Pinned Wigner Crystal', Phys. Rev. Lett. 80, 3827 (1998).

[292] R. Chitra, T. Giamarchi et P. L. Doussal, 'Pinned Wigner crystals', Phys. Rev. B 65, 035312 (2002).

[293] T. Giamarchi et P. L. Doussal, 'Moving Glass Phase of Driven Lattices', Phys. Rev. Lett. 76, 3408 (1996).

[294] I. M. Ruzin, S. Marianer et B. I. Shklovskii, 'Pinning of a Two-Dimensional Wigner Crystal by Charged Impurities', Phys. Rev. B 46, 3999 (1992).

[295] C. Reichhardt, C. J. Olson, N. Grønbech-Jensen et F. Nori, 'Moving Wigner Glasses and Smectics : Dynamics of Disordered Wigner Crystals', Phys. Rev. Lett. 86, 4354 (2001).

[296] S. T. Chui et K. Esfarjani, 'Finite-Temperature Transport of a Pinned 2D Electron Lattice', Phys. Rev. Lett. 66, 652 (1991).

[297] S. T. Chui, 'Depinning, Defect Creation and Quantum Tunneling', Phys. Lett. A 180, 149 (1993).

[298] H. W. Jiang, R. L. Willett, H. L. Stormer, D. C. Tsui, L. N. Pfeiffer et K. W. West, 'Quantum Liquid versus Electron Solid Around $\nu=1 / 5$ Landau-Level Filling', Phys. Rev. Lett. 65, 633 (1990).

[299] H. W. Jiang, H. L. Stormer, D. C. Tsui, L. N. Pfeiffer et K. W. West, 'Magnetotransport studies of the insulating phase around $\nu=1 / 5$ Landau-level filling', Phys. Rev. B 44, 8107 (1991).

[300] F. Perruchot, F. I. B. Williams, C. J. Mellor, R. Gaal, B. Sas et M. Henini, 'Transverse threshold for sliding conduction in a magnetically induced Wigner solid', Physica B : Cond. Mat. 284-288, 1984 (2000).

[301] V. Senz, T. Ihn, T. Heinzel, K. Ensslin, G. Dehlinger, D. Grützmacher, U. Gennser, E. H. Hwang et S. Das Sarma, 'Analysis of the Resistance of Two-Dimensional Holes in SiGe over a Wide Temperature Range', Physica E 13, 723 (2002).

[302] J. Shi et X. C. Xie, 'Dephasing and the Metal-Insulator Transition', Phys. Rev. B 63, 045123 (2001).

[303] A. I. Larkin et D. E. Khmel'nitskii, 'Activation conductivity in disordered systems with large localization length’, Sov. Phys. JETP 56, 647 (1982). 
[304] S. M. Girvin, 'The Quantum Hall Effect : Novel Excitations and Broken Symmetries', dans : Topological Aspects of Low Dimensional Systems, Les Houches, Session LXIX (Springer Verlag, 1998).

[305] Y. H. Xie, R. People, J. C. Bean et K. W. Wecht, 'Power Loss by Two-Dimensional Holes in Coherently Strained $\mathrm{Ge}_{0.2} \mathrm{Si}_{0.8} / \mathrm{Si}$ Heterostructures : Evidence for Weak Screening', Appl. Phys. Lett. 49, 283 (1986).

[306] G. Ansaripour, G. Braithwaite, M. Myronov, O. A. Mironov, E. H. C. Parker et T. E. Whall, 'Energy Loss Rates of Two-Dimensional Hole Gases in Inverted $\mathrm{Si} / \mathrm{Si}_{0.8} \mathrm{Ge}_{0.2}$ Heterostructures', Appl. Phys. Lett. 76, 1140 (2000).

[307] C. D. Thurmond, J. Phys. Chem. 57, 827 (1953).

[308] O. A. Mironov, V. I. Khizhny, G. Braithwaite, E. H. C. Parker, P. J. Phillips, T. E. Whall et V. P. Gnezdilov, 'Observation of Piezoelectric-Like Behaviour in Coherently Strained B-Doped (100)SiGe/Si Heterostructures', J. Cryst. Growth 157, 382 (1995).

[309] A. Ourmazd et J. C. Bean, 'Observation of Disorder Transitions in StrainedSemiconductor Systems', Phys. Rev. Lett. 55, 765 (1985).

[310] J. Z. Tischler, J. D. Budai, D. E. Jesson, G. Eres, P. Zschack, J.-M. Baribeau et D. C. Houghton, 'Ordered Structures in $\mathrm{Si}_{x} \mathrm{Ge}_{1-x}$ Alloy Thin Films', Phys. Rev. B 51, 10947 (1995).

[311] V. I. Khizhny, O. A. Mironov, E. H. C. Parker, P. J. Phillips, T. E. Whall et M. J. Kearney, 'Direct Evidence for a Piezoelectriclike Effect in Coherently Strained SiGe/Si Heterostructures', Appl. Phys. Lett. 69, 960 (1996).

[312] O. Prus, M. Reznikov, U. Sivan et V. Pudalov, 'Cooling of Electrons in a Silicon Inversion Layer', Phys. Rev. Lett. 88, 016801 (2002).

[313] R. J. Zieve, D. E. Prober et R. G. Wheeler, 'Low-Temperature Electron-Phonon Interaction in Si MOSFETs', Phys. Rev. B 57, 2443 (1998).

[314] R. Fletcher, V. M. Pudalov, Y. Feng, M. Tsaousidou et P. N. Butcher, 'Thermoelectric and Hot-Electron Properties of a Silicon Inversion Layer', Phys. Rev. B 56, 12422 (1997).

[315] R. Fletcher, V. M. Pudalov, Y. Feng, M. Tsaousidou et P. N. Butcher, 'Erratum : Thermoelectric and Hot-Electron Properties of a Silicon Inversion Layer', Phys. Rev. B 60, 8392 (1999).

[316] R. Fletcher, Y. Feng, C. T. Foxon et J. J. Harris, 'Electron-Phonon Interaction in a Very Low Mobility GaAs/ $\mathrm{Ga}_{1-x} \mathrm{Al}_{x} \mathrm{As}$-Doped Gated Quantum Well', Phys. Rev. B 61, 2028 (2000).

[317] E. Chow, H. P. Wei, S. M. Girvin et M. Shayegan, 'Phonon Emission from a 2D Electron Gas : Evidence of Transition to the Hydrodynamic Regime', Phys. Rev. Lett. 77, 1143 (1996).

[318] E. Chow, H. P. Wei, S. M. Girvin, W. Jan et J. E. Cunningham, 'Effect of Disorder on Phonon Emissions from a Two-Dimensional Electron Gas in GaAs/ $\mathrm{Al}_{x} \mathrm{Ga}_{1-x} \mathrm{As}$ Heterostructures', Phys. Rev. B 56, R1676 (1997). 
[319] A. J. Kent, R. E. Strickland, K. R. Strickland et M. Henini, 'Phonoconductivity Measurement of the Phonon Absorption by a Two-Dimensional Hole Gas in a GaAs Heterojunction', Phys. Rev. B 54, 2019 (1996).

[320] X. P. A. Gao, A. P. Mills, A. P. Ramirez, S. H. Simon, L. N. Pfeiffer et K. W. West, 'Joule heating of dilute 2D holes in a GaAs quantum well', cond-mat/0203151.

[321] S. Kogan, Electronic Noise and Fluctuations in Solids (Cambridge University Press, 1996).

[322] M. B. Weissman, ' $1 / f$ Noise and Other Slow, Nonexponential Kinetics in Condensed Matter', Rev. Mod. Phys. 60, 537 (1988).

[323] M. Kirton et M. J. Uren, 'Noise in solid-state microstructures : A new perspective on individual defects, interface states and low-frequency $(1 / f)$ noise', Adv. Phys. 38, 367 (1989).

[324] Y. Imry, Introduction to Mesoscopic Physics (Oxford University Press, 1997).

[325] F. N. Hooge, ' $1 / f$ Noise is no surface effect', Phys. Lett. 29A, 139 (1969).

[326] I. Shlimak, Y. Kraftmakher, R. Ussyshkin et K. Zilberg, ' $1 / f$ hopping noise in crystalline germanium', Solid State Commun. 93, 829 (1995).

[327] A. L. McWhorter, Semiconductor Surface Physics (R. H. Kingston, 1957).

[328] P. Dutta, P. Dimon et P. M. Horn, 'Energy Scales for Noise Processes in Metals', Phys. Rev. Lett. 43, 646 (1979).

[329] G. Reimbold, IEEE Trans. Electron. Devices ED-31, 1190 (1984).

[330] F. N. Hooge, ' $1 / f$ noise sources', IEEE Trans. Electron. Devices 41, 1926 (1994).

[331] K. S. Ralls, W. J. Skocpol, L. D. Jackel, R. E. Howard, L. A. Fetter, R. W. Epworth et D. M. Tennant, 'Discrete Resistance Switching in Submicrometer Silicon Inversion Layers : Individual Interface Traps and Low-Frequency ( $1 / f$ ?) Noise', Phys. Rev. Lett. 52, 228 (1984).

[332] M. J. Uren, D. J. Day et M. J. Kirton, ' $1 / f$ and random telegraph noise in silicon metal-oxide-semiconductor field-effect transistors', Appl. Phys. Lett. 47, 1195 (1985).

[333] C. T. Rogers et R. A. Buhrman, 'Composition of $1 / f$ Noise in Metal-Insulator-Metal Tunnel Junctions', Phys. Rev. Lett. 53, 1272 (1984).

[334] X. Y. Chen, P. M. Koenraad, F. N. Hooge, J. H. Wolter et V. Aninkevicius, ' $1 / f$ noise in $\delta$-doped GaAs analyzed in terms of mobility fluctuations', Phys. Rev. B 55, 5290 (1997).

[335] Ç. Kurdak, C.-J. Chen, D. C. Tsui, S. Parihar, S. Lyon et G. W. Weimann, 'Resistance Fluctuations in GaAs $/ \mathrm{Al}_{x} \mathrm{Ga}_{1-x}$ As Quantum Point Contact and Hall Bar Structures', Phys. Rev. B 56, 9813 (1997).

[336] B. I. Shklovskii, 'Theory of $1 / f$ noise for hopping conduction', Solid State Commun. 33, 273 (1980).

[337] S. M. Kogan et B. I. Shklovskii, Sov. Phys. Semicond. 15, 605 (1981). 
[338] V. I. Kozub, 'Low-frequency noise due to site energy fluctuations in hopping conductivity', Solid State Commun. 97, 843 (1996).

[339] S. M. Kogan, 'Electron glass : Intervalley transitions and the hopping conduction noise', Phys. Rev. B 57, 9736 (1998).

[340] C. C. Yu, ' $1 / f$ Noise in a Coulomb Glass', cond-mat/0007085.

[341] K. Shtengel et C. C. Yu, ' $1 / f$ Noise in Coulomb Glasses', cond-mat/0111302.

[342] R. F. Voss, ' $1 / f$ noise and percolation in impurity bands in inversion layers', J. Phys. C : Solid State Phys. 11, L923 (1978).

[343] V. Y. Pokrovskii, A. K. Savchenko, W. R. Tribe et E. H. Linfield, 'Modulation origin of $1 / f$ noise in two-dimensional hopping', Phys. Rev. B 64, 201318 (2001).

[344] J. G. Massey et M. Lee, 'Low-Frequency Noise Probe of Interacting Charge Dynamics in Variable-Range Hopping Boron-Doped Silicon', Phys. Rev. Lett. 79, 3986 (1997).

[345] P. A. Lee et A. D. Stone, 'Universal Conductance Fluctuations in Metals', Phys. Rev. Lett. 55, 1622 (1985).

[346] S. Feng, P. A. Lee et A. D. Stone, 'Sensitivity of the Conductance of a Disordered Metal to the Motion of a Single Atom : Implications for $1 / f$ Noise', Phys. Rev. Lett. 56, 1960 (1986).

[347] N. O. Birge, B. Golding et W. H. Haemmerle, 'Electron Quantum Interference and 1/f Noise in Bismuth', Phys. Rev. Lett. 62, 195 (1989).

[348] N. O. Birge, B. Golding et W. H. Haemmerle, 'Conductance fluctuations and $1 / f$ noise in Bi', Phys. Rev. B 42, 2735 (1990).

[349] D. Mailly et M. Sanquer, 'Sensitivity of quantum conductance fluctuations and of $1 / f$ noise to time reversal symmetry', J. Phys. I France 2, 357 (1992).

[350] X. G. Feng, D. Popović, S. Washburn et V. Dobrosavljević, 'Novel Metallic Behavior in Two Dimensions', cond-mat/9903236.

[351] X. G. Feng, D. Popović et S. Washburn, 'Effect of Local Magnetic Moments on the Metallic Behavior in Two Dimensions', Phys. Rev. Lett. 83, 368 (1999).

[352] F. P. Milliken et Z. Ovadyahu, 'Observation of conductance fluctuations in large $\mathrm{In}_{2} \mathrm{O}_{3-x}$ films', Phys. Rev. Lett. 65, 911 (1990).

[353] F. Ladieu, D. Mailly et M. Sanquer, 'Conductance statistics in small insulating GaAs :Si wires at low temperature. II : experimental study', J. Phys. I France 3, 2321 (1993).

[354] F. Ladieu, 'Transport dans les isolants désordonnés en régime de cohérence quantique', Doctorat, Université Paris VI (1994).

[355] R. Rammal, C. Tannous, P. Breton et A.-M. S. Tremblay, 'Flicker $(1 / f)$ Noise in Percolation Networks : A New Hierarchy of Exponents', Phys. Rev. Lett. 54, 1718 (1985).

[356] G. A. Garfunkel et M. B. Weissman, 'Noise scaling in continuum percolating films', Phys. Rev. Lett. 55, 296 (1985). 
[357] A.-M. S. Tremblay, S. Feng et P. Breton, 'Exponents for $1 / f$ noise, near a continuum percolation threshold', Phys. Rev. B 33, 2077 (1986).

[358] C. Pennetta, G. Trefán et L. Reggiani, 'Scaling Law of Resistance Fluctuations in Stationary Random Resistor Networks', Phys. Rev. Lett. 85, 5238 (2000).

[359] R. H. Koch, R. B. Laibowitz, E. I. Alessandrini et J. M. Viggiano, 'Resistivity-noise measurements in thin gold films near the percolation threshold', Phys. Rev. B 32, 6932 (1985).

[360] J. V. Mantese et W. W. Webb, ' $1 / f$ Noise of Granular Metal-Insulator Composites', Phys. Rev. Lett. 55, 2212 (1985).

[361] V. Podzorov, M. Uehara, M. E. Gershenson et S.-W. Cheong, 'Giant Peak of the 1/f Noise at the Metal-Insulator Transitions in Low- $T_{c}$ CMR Manganites : Evidence of the Percolation Threshold at $T_{c}$ ', cond-mat/9912064.

[362] J. V. Mantese, W. A. Curtin et W. W. Webb, 'Two-component model for the resistivity and noise of tunneling metal-insulator composites', Phys. Rev. B 33, 7897 (1986).

[363] O. Cohen, Z. Ovadyahu et M. Rokni, ' $1 / f$ Noise and Incipient Localization', Phys. Rev. Lett. 69, 3555 (1992).

[364] O. Cohen et Z. Ovadyahu, 'Resistance noise near the Anderson transition', Phys. Rev. B 50, 10442 (1994).

[365] A. Ghosh et A. K. Raychaudhuri, 'Conductance fluctuations near Anderson transition', cond-mat/9906088.

[366] B. Spivak, 'Properties of the strongly correlated two-dimensional electron gas in Si MOSFET's', Phys. Rev. B 64, 085317 (2001).

[367] C. E. Parman, N. E. Israeloff et J. Kakalios, 'Conductance-noise power fluctuations in hydrogenated amorphous silicon', Phys. Rev. Lett. 69, 1097 (1992).

[368] H. Bouchiat, Communication privée.

[369] M. C. Payne, R. A. Davies, J. C. Inkson et M. Pepper, 'Energy Loss Rate in Silicon Inversion Layers', J. Phys. C : Solid State Phys. 16, L291 (1983).

[370] Y. Shinba, K. Nakamura, M. Fukuchi et M. Sakata, 'Hot Electrons in Si(100) Inversion Layer at Low Lattice Temperatures', J. Phys. Soc. Jpn. 51, 157 (1982).

[371] Y. Ma, R. Fletcher, E. Zaremba, M. D'Iorio, C. T. Foxon et J. J. Harris, 'EnergyLoss Rates of Two-Dimensional Electrons at a GaAs/ $\mathrm{Al}_{x} \mathrm{Ga}_{1-x}$ As Interface', Phys. Rev. B 43, 9033 (1991).

[372] J. Callaway, Quantum Theory of the Solid State, Vol. B (Academic Press, 1974).

[373] W. Xu et J. Mahanty, 'Acoustic-phonon emission angle in selectively doped AlGaAsGaAs-AlGaAs', J. Phys. : Condens. Matter 6, 6264 (1994).

[374] P. J. Price, 'Electron Transport in Polar Heterolayers', Surf. Sci. 113, 199 (1982).

[375] P. J. Price, 'Hot Electrons in a GaAs Heterolayer at Low Temperature', J. Appl. Phys. 53, 6863 (1982). 
[376] A. J. Kent, R. E. Strickland, K. R. Strickland, A. J. Cross, P. Hawker et M. Henini, 'Heat-pulse study of the phonon emission by hot two-dimensional holes in a gallium arsenide heterojunction', Semicond. Sci. Technol. 12, 849 (1997).

[377] A. J. Kent, A. J. Naylor, P. Hawker, M. Henini et B. Bracher, 'Observation of Giant Oscillations in the Phonon-Induced Conductivity of a GaAs Quantum Wire', Phys. Rev. B 55, 9775 (1997).

[378] M. Kaveh, M. J. Uren, R. A. Davies et M. Pepper, J. Phys. C : Solid State Phys. 14, L413 (1981).

[379] T. Tsuzuki, 'Electric Field Dependence of Non-Metallic Conduction in a TwoDimensional Random System', Solid State Commun. 38, 915 (1981).

[380] N. Balkan, H. Çelik, A. J. Vickers et M. Cankurtaran, 'Warm-Electron Power Loss in GaAs/ $\mathrm{Ga}_{1-x} \mathrm{Al}_{x} \mathrm{As}$ Multiple Quantum Wells : Well-Width Dependence', Phys. Rev. B 52, 17210 (1995).

[381] T. Neugebauer et G. Landwehr, 'Determination of the Phonon Modes Involved in the Carrier-Phonon Interaction in Silicon Inversion Layers at Low Temperatures by Nonohmic Transport Measurements', Phys. Rev. B 21, 702 (1980).

[382] G. Stöger, G. Brunthaler, G. Bauer, K. Ismail, B. S. Meyerson, J. Lutz et F. Kuchar, 'Shubnikov-de Haas Oscillations under Hot-Electron Conditions in $\mathrm{Si} / \mathrm{Si}_{1-\mathrm{x}} \mathrm{Ge}_{\mathrm{x}}$ Heterostructures', Phys. Rev. B 49, 10417 (1994).

[383] Y. Kawaguchi et S. Kawaji, 'A Study of Electron Mobility and Electron-Phonon Interaction in Si MOSFETs by Negative Magnetoresistance Experiments', Jpn. J. Appl. Phys. 21, L709 (1982).

[384] C.. Kurdak, D. C. Tsui, S. Parihar, S. A. Lyon et M. Shayegan, 'Electron Temperature in Low-Dimensional Wires Using Thermal Noise Measurements', Appl. Phys. Lett. 67, 386 (1995).

[385] C. Castellani, G. Kotliar et P. A. Lee, 'Fermi-liquid theory of interacting disordered systems and the scaling theory of the metal-insulator transition', Phys. Rev. Lett. 59, 323 (1987).

[386] M. Y. Reizer, 'Renormalization of the Thermoelectric Power Due to the ElectronElectron Interaction', Phys. Rev. B 44, 12701 (1991).

[387] M.-R. Li et E. Orignac, 'Heat conduction and Wiedemann-Franz Law in disordered Luttinger Liquids', cond-mat/0201291. 